\title{
Molecular Determinants of Spinal Hyperexcitability in Rat and Human Models of Pathological Pain
}

by

Annemarie Dedek

A thesis submitted to the Faculty of Graduate and Postdoctoral Affairs in partial fulfillment of the requirements for the degree of

Doctor of Philosophy

in

Neuroscience

Carleton University

Ottawa, Ontario

(C) 2021, Annemarie Dedek 


\section{Abstract}

Chronic pain is a health crisis with few safe and effective treatments. Understanding the mechanisms that drive chronic pain predicate the development of novel therapeutics for the disease. The superficial dorsal horn (SDH) is an integral part of the pain-processing circuit. Nociceptive output from the SDH is mediated by a homeostatic balance of inhibition and excitation, resulting in pain responses in healthy individuals that are proportional to noxious stimuli. In chronic pain, the balance between inhibition and excitation degrades, resulting in spinal hyperexcitability and an increase in pain outputs to the brain. To study the molecular processes that disrupt the balance of inhibition and excitation within SDH circuitry, we paired a male rat ex vivo pain model with in vivo models. We found that the loss of potassium-chloride cotransporter 2-dependent inhibition (disinhibition) and facilitated excitation (marked by potentiation of excitatory N-methyl-D-aspartate receptors, NMDARs) are linked. We determined that downregulation of the phosphatase $\mathrm{STEP}_{61}$ is the linker between disinhibition and NMDAR potentiation in male rats. To address the translational divide that exists between basic science rodent research and clinical trials, we developed an ex vivo pathological pain model using human organ donor tissue. Using this model, we found that the STEP 61 -mediated link between disinhibition and NMDAR potentiation is conserved in the SDH of male humans. Despite that chronic pain affects women more often than men, the molecular underpinnings of chronic pain have been studied almost exclusively in males. Surprisingly, we found that NMDARs are not potentiated in our female rodent pain models, and that protein markers of disinhibition and facilitated excitation remain unchanged. This demonstrates a sex difference in neuronal pain processing in rodents within the SDH. Further, we used human tissue models to discover that this sex difference is conserved between rats and humans. We conclude that loss of STEP ${ }_{61}$ links 
disinhibition and facilitated excitation in male rats and humans, but this mechanism of spinal hyperexcitability is sexually dimorphic. The lack of coupling of disinhibition to NMDAR potentiation suggests divergent neuronal signalling drives SDH hyperexcitability and chronic pain in females. This thesis highlights the importance of sex-inclusive research. 


\section{Acknowledgements}

Thank you to the patient group of educators at Bell High School. It is thanks to their belief in me that I channelled my teenage angst into a passion for academics. Thank you especially to Lisa $\mathrm{H}$, who has now become a dear friend.

I thank the members, past and present, of the Hildebrand and Tsai labs, as well as the many members of the Neuroscience Department that have made the journey fun and made me look forward to long days in the lab.

Thank you to my committee members, Dr. Hayley, Dr. Sun, and Dr. Bui. Your advice, time and feedback over these many years have been invaluable. Also thank you to my external examiner, Dr. Ross, for taking the time to join in this final part of the journey. Finally, thank you to my chair, Dr. McKinnel, for taking the time out of your busy schedule for my defense.

Thank you to my incredible supervisors, Dr. Hildebrand, and Dr. Tsai.

Dr. Hildebrand, thank you for offering me every possible opportunity to find success. Thank you for your support in each good and bad day. Thank you for your astounding patience and for leading by example in what it means to be a not just a good scientist, but a good person. Dr. Tsai, thank you for your inspiring, selfless dedication to helping others. Thank you for your trailblazing as a spinal cord researcher and physician. Thank you for your wisdom on good versus bad days - I remind myself of your outlook often.

My mother has supported me wholeheartedly my entire life. She taught me to be resourceful, work hard, improvise, and to never read the instruction manual. Thank you for never skipping a beat to offer love, help, and food.

My father was always my biggest cheerleader. He taught me to listen, to ask questions, and imparted on me an unwavering desire for independence. Thank you for being there every time I needed to talk, every time I needed comfort, and every time I missed the bus.

My younger brother has been a source of inspiration for me. He gave me confidence to not shy away from math and reminds me often to not become entrenched in my own views. Thank you for always making time to talk, for your problem-solving skills, and for checking the math on almost everything I have calculated since starting graduate school.

Děda Lád'a taught me to pay close attention to the natural world around me and inspired my pursuit of neuroscience. The memories I have walking with him in the forests surrounding Klabava are some of my most cherished memories. 
Babi Běla continues to teach me what it means to be strong, persistent, and independent. Strejda Lád'a taught me to persevere and to take a joke - thank you for the many inspiring visits to your work.

I thank all my family in the Czech Republic - I may no longer see them often, but they are in my thoughts daily.

And last, but not least, I thank my teammate and husband, Jeffrey. Thank you for teaching me to find balance in life, find strength in my body as well as my mind, and for reminding me to enjoy every moment of life. Thank you for your immense support in all my dreams and endeavors and thank you for bringing me ice cream to work. This would not have been possible without you. 


\section{I dedicate this work to my father, Frank Dedek}

My father had many dreams. In his youth, he dreamed of becoming a geneticist. His career goals pivoted, but after establishing a small business, he turned back to his love of learning. He started a math tutoring company in hopes of helping the many students struggling with mathematics, and he started writing a novel. Sadly, my father died before these dreams were fully realized. I lost my father one month before starting this project. I hope that the work in this thesis can act as a continuation for my father's inspiring pursuit of knowledge. 


\section{Table of Contents}

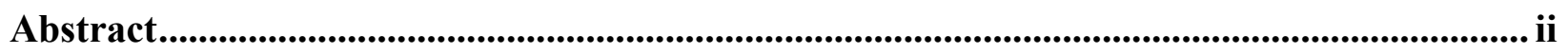

Acknowledgements ............................................................................................................................. iv

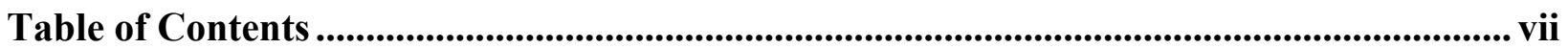

List of Abbreviations ..................................................................................................................... xii

List of Publications ............................................................................................................................. xiv

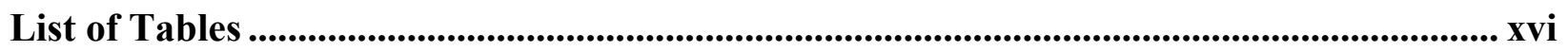

List of Figures...................................................................................................................................... xvii

Chapter 1: An introduction to spinal hyperexcitability in chronic pain .................................. 1

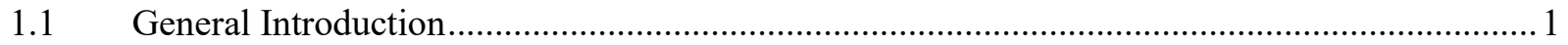

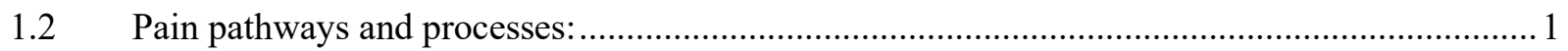

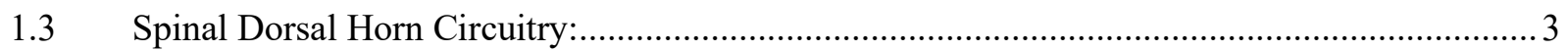

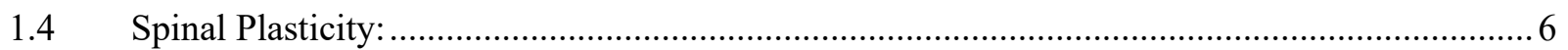

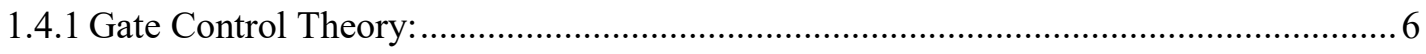

1.4.2 Central Sensitization:_......................................................................................

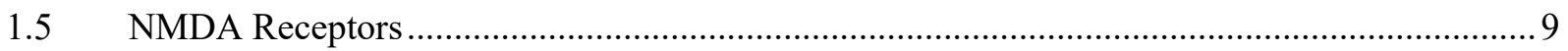

1.5.1 NMDAR background and stoichiometry ..............................................................

1.5.2 NMDAR expression in SDH circuits ....................................................................... 11

1.5.3 NMDAR modulation by Kinases ………………………………………………...... 14

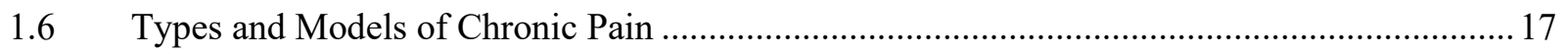

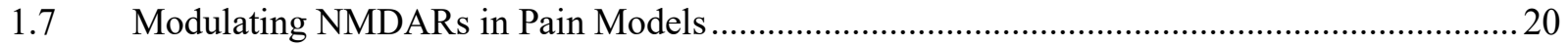

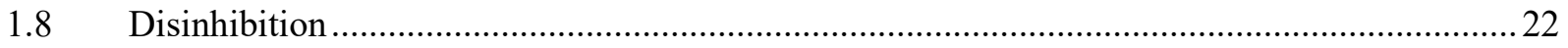

$1.9 \quad$ Linking Disinhibition and NMDAR Potentiation.................................................................

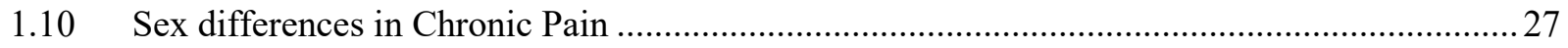




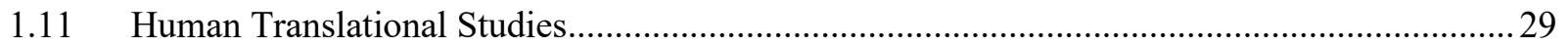

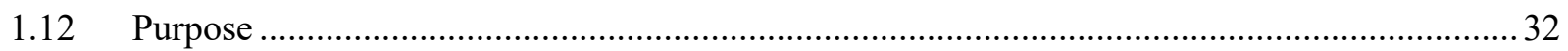

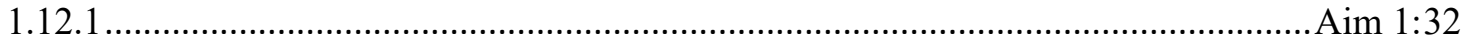

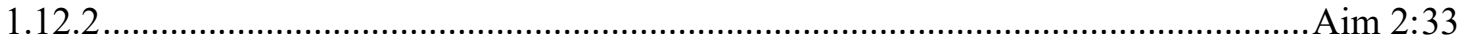

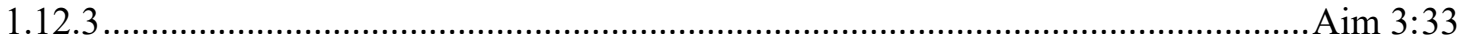

Chapter 2: Loss of STEP 61 couples disinhibition to $\boldsymbol{N}$-methyl-D-aspartate receptor potentiation in rodent and human spinal pain processing ............................................................... 35

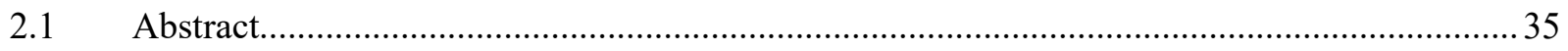

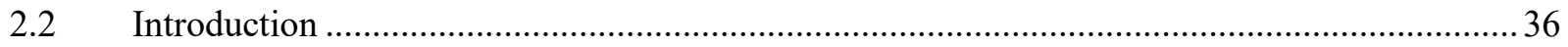

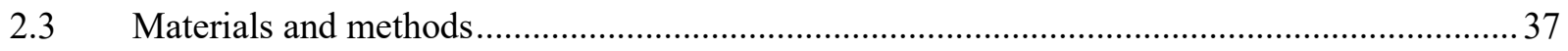

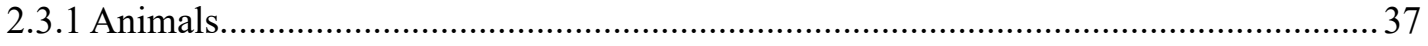

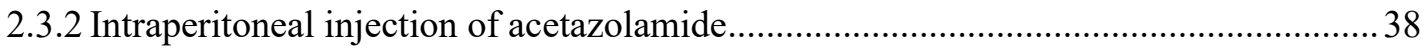

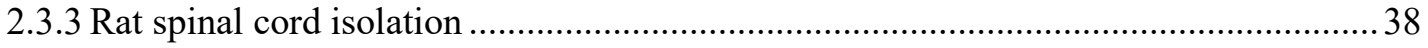

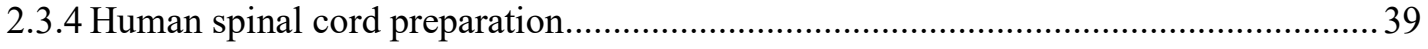

2.3.5 Ex vivo model of pathological pain processing ......................................................... 40

2.3.6 Electrophysiological recordings of lamina I neurons ............................................... 40

2.3.7 Isolation of synaptosome fractions and biochemical analysis of human and rodent spinal cord tissue.

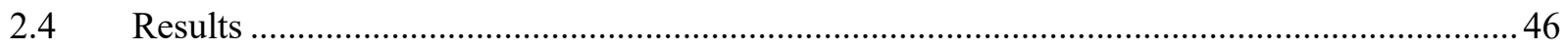

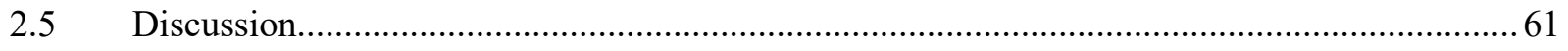




\section{Chapter 3: A neuronal mechanism of spinal hyperexcitability is sexually dimorphic in} rodent and human models of pathological pain............................................................ 88

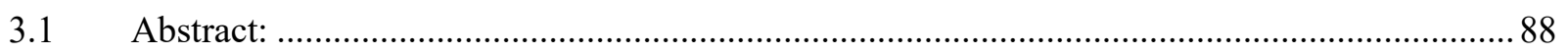

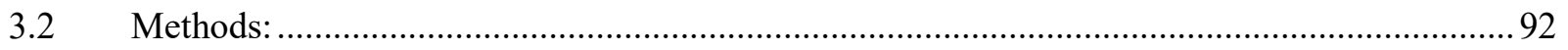

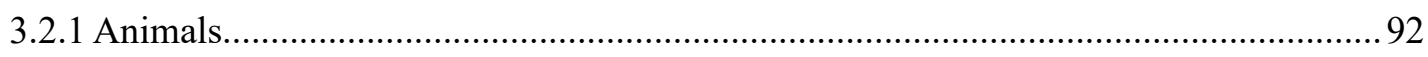

3.2.2 Freund's adjuvant model of inflammatory pain and von Frey behaviour testing......... 92

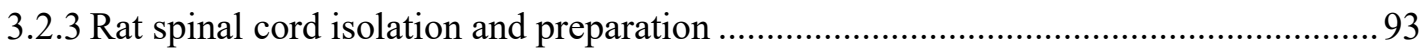

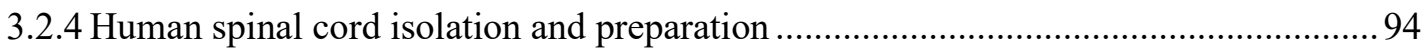

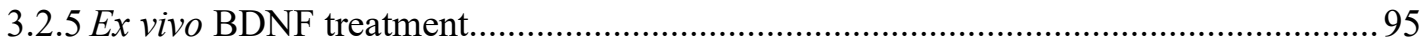

3.2.6 Lamina I electrophysiological recordings ................................................................ 95

3.2.7 Western blot analysis on synaptosome fractions of rat and human spinal tissue .........97

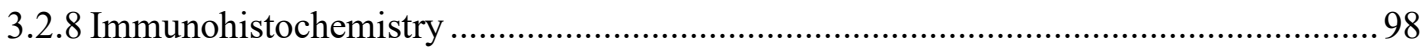

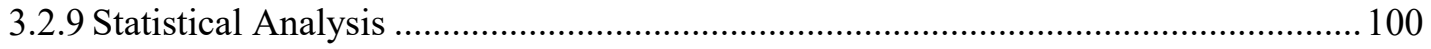

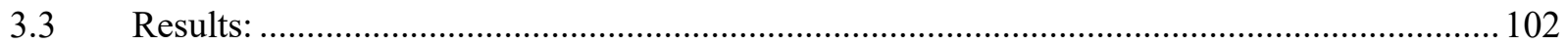

3.3.1 The CFA inflammatory pain model induces tactile allodynia in male and female adult rats, but only triggers the $\mathrm{STEP}_{61} / \mathrm{pFyn} / \mathrm{pGluN} 2 \mathrm{~B}$ spinal hyperexcitability pathway in males ........ 102

3.3.2 NMDARs in the female SDH are not potentiated or upregulated by ex vivo BDNF treatment or in the in vivo CFA model of pathological pain 105

3.3.3 Ex vivo BDNF treatment results in sexually dimorphic effects on markers of disinhibition and facilitated excitation at synapses in the human SDH 110

3.3.4 Ovariectomy initiates BDNF-sensitivity in female SDH neurons. 114

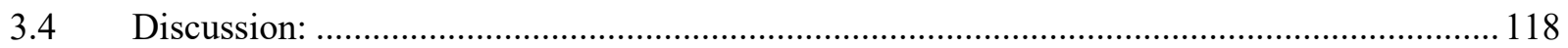

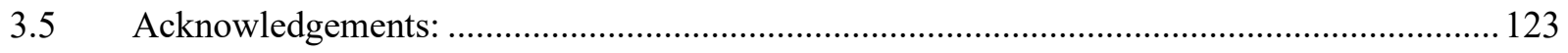

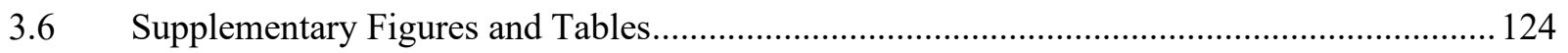




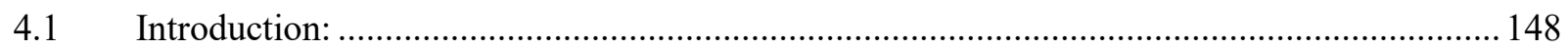

4.2 The role of STEP $_{61}$ as a molecular linker of disinhibition and NMDAR potentiation: ........... 149

4.3 Future directions for studying NMDAR function in spinal nociceptive networks:................ 152

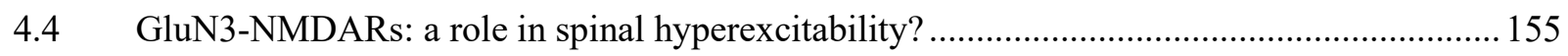

4.5 Lack of NMDAR potentiation in female CFA and BDNF treatment:............................... 156

4.6 First-ever patch-clamp electrophysiological recordings on human SDH tissue:................... 158

4.7 Ex vivo human spinal cord pathological pain models:.................................................... 161

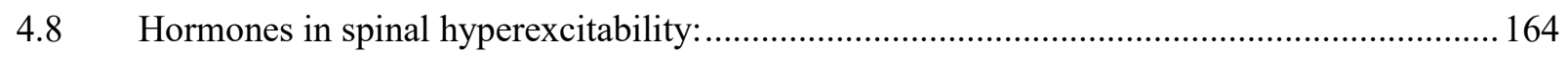

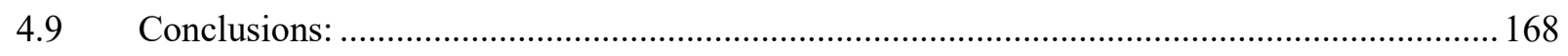

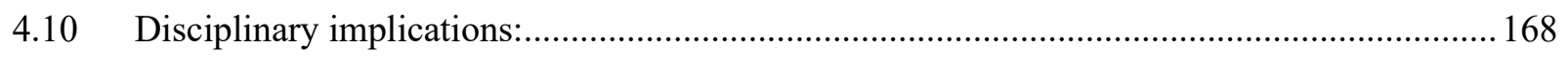

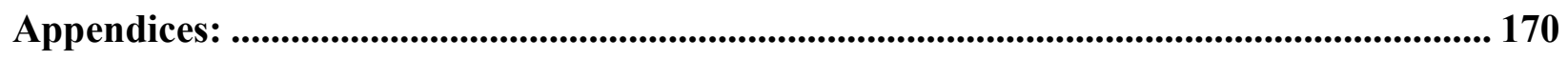

Appendix A - Potentiation of Synaptic GluN2B NMDAR Currents by Fyn Kinase Is Gated through

BDNF-Mediated Disinhibition in Spinal Pain Processing ........................................................... 170

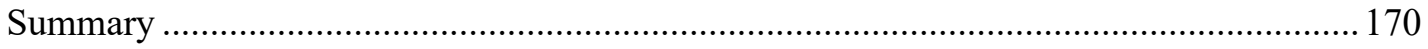

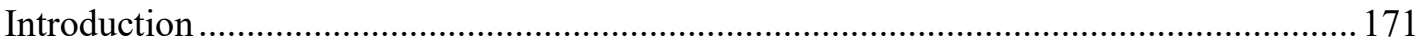

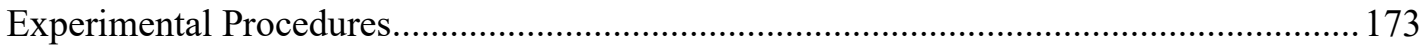

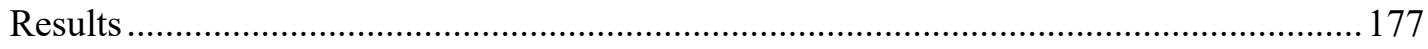

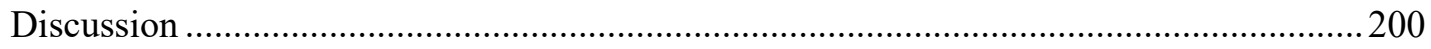

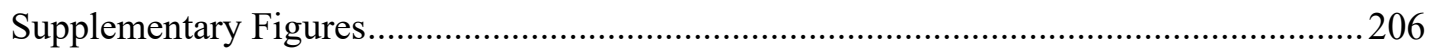

Appendix B - The T-type calcium channel antagonist, Z944, reduces spinal excitability and pain

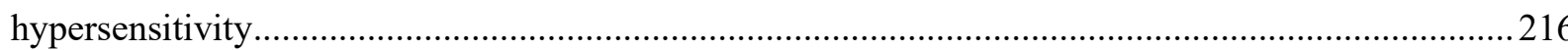

Abstract 


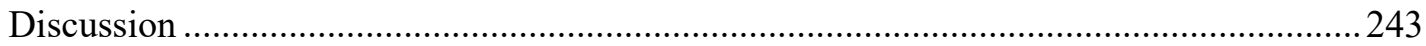

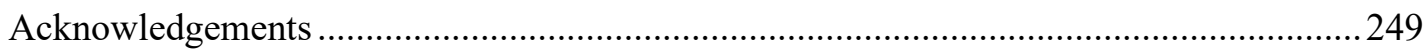

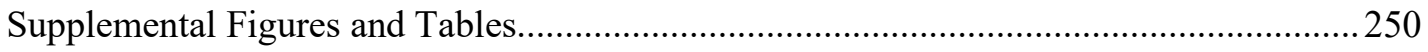

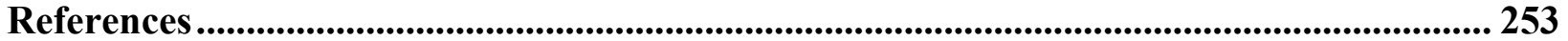




\section{List of Abbreviations}

AMPAR

ANOVA

ASCF

ATP

AUC

BDNF

CCI

Cdk5

CFA

CGRP

CIAT

CNS

CSF

DIC

DMSO

DRG

$\mathrm{EC}_{50}$

EGTA

EPSC

GABA

HEPA

HEPES

hERG

HVA

IC50

iGluR

IP

IR

$\mathrm{KCC} 2$

LTD

LTP

LVA

MEA

mEPSC

mGluR

NDD
- $\alpha$-amino-3-hydroxy-5-methyl-4-isohexazole propionate receptors

- analysis of variance

- artificial cerebrospinal fluid

- adenosine triphosphate

- area under curve

- brain derived neurotrophic factor

- chronic constriction injury

- cyclin dependant kinase-5

- complete Freund's adjuvant

- calcitonin gene-related peptide

- calcium imaging analysis toolbox

- central nervous system

- cerebrospinal fluid

- differential interference contrast

- dimethyl sulfoxide

- dorsal root ganglia

- half maximal effective concentration

- egtazic acid

- excitatory post-synaptic current

- gamma-aminobutyric acid

- high efficiency particulate air

- 4-(2-hydroxyethyl)-1-piperazineethanesulfonic acid

- human ether-a-go-go-related gene

- high voltage activate

- half maximal inhibitory concentration

- ionotropic glutamate receptor

-intraperitoneal

- infrared

-chloride potassium symporter 2

- long term depression

- long-term potentiation

- low voltage-activate

- multielectrode array

- miniature excitatory postsynaptic current

- metabotropic glutamate receptor

- neurological determination of death 


$\begin{array}{ll}\text { NGF } & \text { - nerve growth factor } \\ \text { NHP } & \text { - non-human primate } \\ \text { NMDAR } & \text { - N-methyl-D-aspartate receptor } \\ \text { nRT } & \text { - thalamic reticular nucleus } \\ \text { PACAP } & \text { - pituitary adenylate cyclase-activating peptide } \\ \text { pACSF } & \text { - protective artificial cerebrospinal fluid } \\ \text { PBS } & \text { - phosphate buffered saline } \\ \text { PKA } & \text { - protein kinase A } \\ \text { PKC } & \text { - protein kinase C } \\ \text { PMT } & \text { - photomultiplier tube } \\ \text { PNI } & \text { - peripheral nerve injury } \\ \text { PSNL } & \text { - partial sciatic nerve ligation } \\ \text { PTP } & \text { - protein tyrosine phosphatase } \\ \text { PWT } & \text { - paw withdrawal threshold } \\ \text { PWT } & \text { - paw withdrawal threshold } \\ \text { RDD } & \text { - rate-dependent depression } \\ \text { ScRNA-seq } & \text { - single-cell RNA sequencing } \\ \text { SD } & \text { - Sprague Dawley } \\ \text { SDH } & \text { - superficial dorsal horn } \\ \text { SEM } & \text { - standard error of the mean } \\ \text { SFK } & \text { - Src-family kinase } \\ \text { SNI } & \text { - spared nerve injury } \\ \text { SNL } & \text { - spinal nerve ligation } \\ \text { SNP } & \text { - single nucleotide polymorphism } \\ \text { STEP } 61 & \text { - striatal-enriched protein tyrosine phosphatase 61 } \\ \text { SUDO } & \text { - tetraethylammonium } \\ \text { TEA } & \text { - tetrodotoxin } \\ \text { TTX } & \text { VGCC }\end{array}$




\section{List of Publications}

Chapter 2: Loss of STEP61 couples disinhibition to N-methyl-D-aspartate receptor potentiation in rodent and human spinal pain processing. Annemarie Dedek*, Jian Xu*, Chaya M Kandegedara, Louis-Étienne Lorenzo, Antoine G

Godin, Yves De Koninck, Paul J Lombroso, Eve C Tsai, Michael E Hildebrand *These authors contributed equally to this work.

Published in Brain, Volume 142, Issue 6, June 2019, Pages 1535-1546.

doi.org/10.1093/brain/awz105

In this publication, Annemarie Dedek performed all in vivo experiments, performed almost all electrophysiological experiments, collected rat and human tissue for biochemical and immunohistochemical analysis, analysed data, and helped write and edit the manuscript.

\section{Chapter 3: A neuronal mechanism of spinal hyperexcitability is sexually dimorphic in} rodent and human models of pathological pain.

Annemarie Dedek, Jian Xu, Louis-Étienne Lorenzo, Antoine G. Godin, Chaya M. Kandegedara, Geneviève Glavina, Jeffrey A. Landrigan, Paul J. Lombroso, Yves De Koninck, Eve C. Tsai, Michael E. Hildebrand

In preparation for submission.

In this publication, Annemarie Dedek performed all in vivo and electrophysiology experiments, collected rat and human tissue for biochemical and immunohistochemical analysis, analysed data and helped write and edit the manuscript. 
Appendix A: Potentiation of Synaptic GluN2B NMDAR Currents by Fyn Kinase Is Gated through BDNF-Mediated Disinhibition in Spinal Pain Processing.

Michael E. Hildebrand, Jian Xu, Annemarie Dedek, Yi Li, Ameet S. Sengar, Simon Beggs, Paul

J. Lombroso, Michael W. Salter

Published in Cell Reports, Volume 17, Issue 10, December 2016, Pages 2753-2765.

doi.org/10.1016/j.celrep.2016.11.024

In this publication, Annemarie Dedek performed dissections and tissue preparation for electrophysiology and western blot analysis in a subset of experiments.

Appendix B: The T-type calcium channel antagonist, Z944, reduces spinal excitability and pain hypersensitivity.

Erika K. Harding*, Annemarie Dedek*, Robert P. Bonin, Michael W. Salter, Terrance P. Snutch,

Michael E. Hildebrand *These authors contributed equally to this work.

Published in The British Journal of Pharmacology, April 2021, Pages 1-16.

doi: $10.1111 /$ bph.15498

In this publication, Annemarie Dedek designed, performed, and analysed all the in vivo pharmacology experiments, and helped write and edit the manuscript. 


\section{List of Tables}

Chapter 2 Supplementary Table 1. Use of tissue samples collected from human organ donors.

Chapter 2 Supplementary Table 2. Antibodies used for western blots. $69-71$

Chapter 2 Supplementary Table 3. Statistical analyses performed in all Figures $73-76$

Chapter 3 Supplementary Table 1. Antibodies used for Western Blots $139-140$ Chapter 3 Supplementary Table 2. Statistics Summary Table $142-146$ Appendix A Supplementary Table 1. Initial NMDAR charge transfer values for various treatment conditions.... 211 Appendix A Supplementary Table 2. NMDAR charge transfer at two distinct points in patch clamp recordings at $+60 \mathrm{mV}$ 212 Appendix A Supplementary Table 3. NMDAR charge transfer values before and after intracellular or extracellular pharmacological treatment during recording at $+60 \mathrm{mV}$ Appendix B Supplementary Table 1. Table 6: Repeated measures one-way ANOVAs comparing effect of treatment over time in males. 248 Appendix B Supplementary Table 2. Post-hoc Bonferroni-corrected tests for all significant oneway repeated measure ANOVAs in males, comparing day 3 (post CFA injection) to each other time point. 248 Appendix B Supplementary Table 3. Repeated measures one-way ANOVAs comparing effect of treatment over time in females. $248-249$ Appendix B Supplementary Table 4. Post-hoc Bonferroni-corrected tests for all significant oneway repeated measure ANOVAs in females, comparing day 3 (post CFA injection) to each other time point. 249 


\section{List of Figures}

Chapter 2 Figure 1. GluN2B NMDARs are potentiated at lamina I synapses in a CFA model of chronic inflammatory pain, with an associated downregulation of synaptic $\mathrm{STEP}_{61} \ldots \ldots \ldots \ldots . . . .48$

Chapter 2 Figure 2. STEP 61 is necessary and sufficient to prime phosphorylation and potentiation of synaptic GluN2B-NMDARs by BDNF .............................................. 51

Chapter 2 Figure 3. Blocking KCC2-dependent disinhibition attenuates $\mathrm{STEP}_{61}$ downregulation by BDNF at superficial dorsal horn synapses and reverses CFA-mediated tactile allodynia..... 56

Chapter 2 Figure 4. The ex vivo BDNF treatment model of pathological pain processing drives a downregulation of $\mathrm{KCC} 2$ and $\mathrm{STEP}_{61}$ and an increase in pFyn and pGluN2B NMDARs at superficial dorsal horn synapses of viable adult human spinal cord $\ldots \ldots \ldots \ldots \ldots \ldots \ldots \ldots \ldots \ldots . \ldots . \ldots . \ldots . \ldots$

Chapter 2 Supplementary Figure 1. Ex vivo BDNF treatment model elicits no effect in SDH homogenate. 64

Chapter 2 Supplementary Figure 2. Ex vivo BDNF treatment model elicits no effect in non-SDH synaptosome fractions. 65

Chapter 2 Supplementary Figure 3. GluN2B dominates human lamina I mEPSCs

Chapter 2 Supplementary Figure 4. Western blots used for quantification in Figure 4C. 66 Chapter 2 Supplementary Figure 5. Ex vivo BDNF treatment model elicits no effect in human SDH homogenate. 67

Chapter 2 Supplementary Figure 6. Ex vivo BDNF treatment model elicits no effect in human nonSDH synaptosome fractions.

Chapter 2 Supplementary Figure 7. Supplementary information for human KCC2 immunohistochemistry..... 68

Chapter 2 Supplementary Figure 8. Western blots from 1C 77 


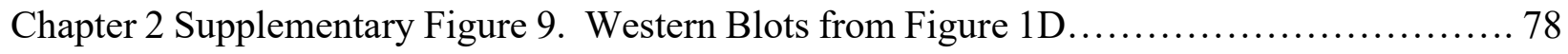

Chapter 2 Supplementary Figure 10. Western Blots from Figure 2E........................... 79

Chapter 2 Supplementary Figure 11. Western Blots from Figure 2E...................... 80

Chapter 2 Supplementary Figure 12. Western Blots from Figure 3A.......................... 81

Chapter 2 Supplementary Figure 13. Western Blots from Figure 3A....................... 82

Chapter 2 Supplementary Figure 14. Western Blots from Figure 4C........................ 83

Chapter 2 Supplementary Figure 15. Western Blots from Supplementary Figure 1........... 84

Chapter 2 Supplementary Figure 16. Western Blots from Supplementary Figure 2 .............. 85

Chapter 2 Supplementary Figure 17. Western Blots from Supplementary Figure 5 ............. 86

Chapter 2 Supplementary Figure 18. Western Blots from Supplementary Figure 6............. 87

Chapter 3 Figure 1. CFA elicits tactile allodynia in male and female adult rats, but only activates the $\mathrm{STEP}_{61} / \mathrm{pFyn} / \mathrm{pGluN2B}$ spinal hyperexcitability pathway in males...................... 104

Chapter 3 Figure 2. Female SDH NMDARs are not potentiated or upregulated by the ex vivo

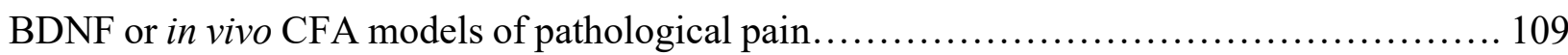

Chapter 3 Figure 3. Unlike in males, human female lamina I neurons do not show evidence of disinhibition or facilitated excitation following ex vivo BDNF treatment..................... 113

Chapter 3 Figure 4. Ovariectomy triggers BDNF-sensitivity in SDH neurons................. 117 Chapter 3 Supplementary Figure 1. Paw withdrawal threshold is decreased in male and female rats

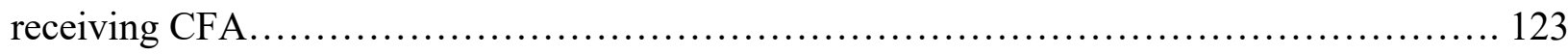

Chapter 3 Supplementary Figure 2. The CFA model of inflammatory pain elicits no change in our targets in crude synaptosome fractions of the portion of the spinal cord just ventral of the SDH in

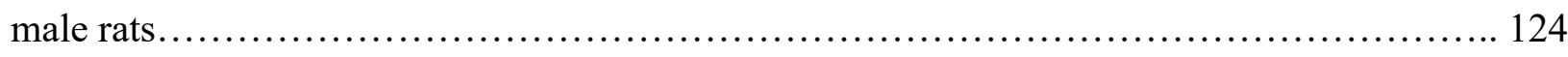


Chapter 3 Supplementary Figure 3. Male CFA SDH synaptosome gels from animals treated with either vehicle or CFA.

Chapter 3 Supplementary Figure 4. Male CFA VH synaptosome gels from animals treated with either vehicle or CFA. 126

Chapter 3 Supplementary Figure 5. The CFA model of inflammatory pain elicits no change in our targets in crude synaptosome fractions of the portion of the spinal cord just ventral of the SDH in female rats.

Chapter 3 Supplementary Figure 6. Female CFA SDH synaptosome gels from animals treated with either vehicle or CFA. 128

Chapter 3 Supplementary Figure 7. Female CFA VH synaptosome gels from animals treated with either vehicle or CFA.

Chapter 3 Supplementary Figure 8. BDNF incubation for 2-4 hours does not result in potentiation of female rat lamina I mEPSCs. .130 Chapter 3 Supplementary Figure 9. Incubating female rat tissue in $50 \mathrm{ng} / \mathrm{mL}$ BDNF elicits no change in our targets in crude synaptosome fractions of the portion of the spinal cord just ventral of the SDH. 130

Chapter 3 Supplementary Figure 10. Female BDNF SDH synaptosome gels from animals treated with either saline $(\mathrm{S})$ or BDNF (B) 131 Chapter 3 Supplementary Figure 11. Female BDNF VH synaptosome gels from animals treated with either saline (S) or BDNF (B). 132 Chapter 3 Supplementary Figure 12. Incubating female human tissue in $100 \mathrm{ng} / \mathrm{mL}$ BDNF elicits no change in KCC2, tSTEP, pFyn, Fyn, pGluN2B, or GluN2B in crude synaptosome fractions of the portion of the spinal cord just ventral to the SDH. aSTEP is significantly decreased.... 133 
Chapter 3 Supplementary Figure 13. Female BDNF human SDH synaptosome gels from human spinal samples treated with either saline (S) or BDNF (B)....

Chapter 3 Supplementary Figure 14. Female BDNF human VH synaptosome gels from human spinal samples treated with either saline (S) or BDNF (B) 135

Chapter 3 Supplementary Figure 15. Incubating female OVX rat tissue in $50 \mathrm{ng} / \mathrm{mL}$ BDNF elicits decreases in tSTEP and Fyn, and no change in KCC2, aSTEP, pFyn, pGluN2B, and GluN2B in crude synaptosome fractions of the portion of the spinal cord just ventral of the SDH..... 136 Chapter 3 Supplementary Figure 16. Female OVX rat SDH synaptosome gels from animals treated with either saline $(\mathrm{S})$ or BDNF (B)

Chapter 3 Supplementary Figure 17. Female OVX rat VH synaptosome gels from animals treated with either saline $(\mathrm{S})$ or BDNF (B) 138 Appendix A Figure 1. NMDAR mEPSCs in Lamina I Neurons Are Reversibly Potentiated by BDNF in the PNI Model of Neuropathic Pain. 177 Appendix A Figure 2. BDNF Is Sufficient to Facilitate NMDAR mEPSCs 181 Appendix A Figure 3. The Potentiation of NMDAR mEPSCs by BDNF/ PNI Requires Fyn but Not Src. 183

Appendix A Figure 4. Fyn but Not Src Directly Mediates the Potentiation of GluN2B-Containing NMDAR Responses at Lamina I Synapses. 188 Appendix A Figure 5. BDNF Activates Fyn but Not Src to Drive Trafficking and Phosphorylation of GluN2B NMDARs Selectively at Superficial Dorsal Horn Synapses..................... 190 Appendix A Figure 6. The Potentiation of NMDAR mEPSCs by BDNF/PNI Requires Disinhibition. 194 
Appendix A Figure 7. Downregulation of KCC2 Activity Gates Potentiation of NMDAR mEPSCs

by Exogenous BDNF.

196

Appendix A Supplementary Figure 1. Application of PP2 does not affect NMDAR mEPSCs... 205 Appendix A Supplementary Figure 2. BDNF does not alter phosphorylation of Fyn and GluN2B NMDARs in deep dorsal horn and ventral horn synaptosomes or total homogenates of superficial dorsal horn. 206

Appendix A Supplementary Figure 3. Tyrosine phosphorylation of TrkB by BDNF is not affected by the addition of $\mu \mathrm{M}$ acetazolamide (ACTZ).

Appendix A Supplementary Figure 4. BDNF, BDNF + acetazolamide, nor acetazolamide alone affects Fyn activation and GluN2B phosphorylation in the synaptic fraction from deep dorsal horn and ventral horn spinal tissue. 208 Appendix A Supplementary Figure 5. BDNF pretreatment did not increase calpain activity.... 209 Appendix A Supplementary Figure 6. Inward NMDAR mEPSCs remain constant during baseline records. 209

Appendix A Supplementary Figure 7. An increase in intracellular calcium is sufficient to gate potentiation of NMDAR mEPSCs by BDNF. 210 Appendix B Figure 1. Z944 selectively reduces inward current through low voltage-activated calcium channels in spinal cord lamina I neurons. 230 Appendix B Figure 2. Z944 selectively reduces inward current through low voltage-activated calcium channels in spinal cord lamina I neurons. Appendix B Figure 3. Figure 0 22: Z944 reduces excitability in a subset of laminae I/II spinal cord neurons 236 
Appendix B Figure 4. Figure 0 23: IP administration of Z944 reverses CFA-mediated tactile allodynia in both sexes, in a dose-dependent manner..................................... 240 Appendix B Supplementary Figure 1. Figure 0 24: Representative images of lamina I neuron

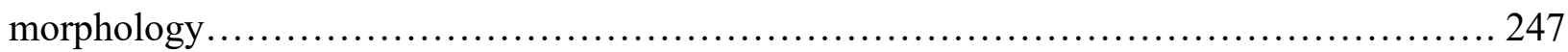




\section{Chapter 1: An introduction to spinal hyperexcitability in chronic pain}

\subsection{General Introduction}

Chronic pain is a debilitating disease that affects millions worldwide. Amidst the backdrop of the opioid epidemic, the need for new treatment strategies that are both safe and effective has never been greater. The development of new pain therapeutics has been plagued with difficulty, resulting in high failure rates of new therapeutics in clinical trials (Gewandter et al., 2020). A factor contributing to this high failure rate may be the predominant use of male rodent subjects in the foundational studies used to identify targets for therapeutic treatment. To address this problem, here, we will investigate mechanisms of spinal hyperexcitability using male and female rodent models, as well as preclinical human models of pathological pain. The goal of this body of work is to contribute to the foundation of knowledge of the mechanisms that lead to spinal hyperexcitability within the pain pathway, which may later lead to the development of novel therapeutics.

\subsection{Pain pathways and processes:}

Acute pain is a critical protective mechanism that alerts the body to tissue damage. The somatosensory nociceptive system is comprised of peripheral sensory neurons, circuits in the superficial dorsal horn (SDH) of the spinal cord and many brain regions. The peripheral sensory neurons, descending modulatory input to the spinal cord from the brain, and the SDH are responsible for nociception; once afferent signals are relayed to the brain, conscious perception of nociception results in the multifaceted experience of pain. Pain is defined as "an unpleasant 
sensory and emotional experience associated with, or resembling that associated with, actual or potential tissue damage" (Raja et al., 2020). Pain is modulated by experience, cognition, and even social constructs such as cultural expectation and gender (Gold and Gebhart, 2010). A large number of brain areas are involved in integrating and modulating pain (for review see Almeida et al., 2004). Additionally, the perception of pain can be blocked by inhibiting nociception at the peripheral or spinal level (Gracely et al., 1992). Thus, a practical treatment approach is modulating nociceptive input at the spinal cord level, before these nociceptive signals reach the brain.

Acute pain is protective; it is transient and has a high threshold for activation, meaning that normally only sensory input that poses a real or potential threat to tissue is encoded as painful (Grichnik and Ferrante, 1991). After sustained activation of peripheral nociceptors due to inflammation, tissue damage, or disease processes, acute pain signalling can provoke plastic changes in the SDH that lower the threshold for activation or even result in spontaneous pain. In the case of acute pain, a temporarily lowered pain threshold can be protective; for example, when tissue bruising becomes sensitive to the touch, it signals the body to protect the area so it can heal. These plastic changes are deemed pathological when they persist beyond a period of 3 months and are then deemed 'chronic' (Merskey and Bogduk, 2002). Chronic pain symptoms, which result from plastic changes in nociceptive circuitry, include hyperalgesia (increased pain response to noxious stimuli), allodynia (a painful response to a normally innocuous stimulus), and spontaneous or ongoing pain (Merskey and Bogduk, 2002). There are molecular and cellular changes associated with these pathological processes that form neural correlates of chronic pain as a disease in and of itself (Keller et al., 2007); however, these processes require further investigation. 


\subsection{Spinal Dorsal Horn Circuitry:}

The organization of circuitry in the dorsal horn lends itself to plastic changes that can increase the excitatory input from the peripheral sensory nervous system to the brain. Peripheral afferents have nociceptive receptors that detect tissue damage or inflammation by transducing chemical or mechanical stimuli into receptor potentials and then action potentials (Gold and Gebhart, 2010; Todd, 2010; Peirs and Seal, 2016). Peripheral afferents are made up of pseudounipolar neurons whose cell bodies are located in dorsal root ganglia (DRG). Nociceptors themselves may become sensitized by repeated stimulation, and thus increase their inputs onto the SDH (Gold and Gebhart, 2010).

Lamina I and II, the outer-most laminae of the SDH, are the main sites of entry for highthreshold nociceptive primary afferents (Todd, 2010; Peirs and Seal, 2016). A $\delta$ fibres are smalldiameter, myelinated primary afferent fibres that synapse onto lamina I and lamina $\mathrm{II}_{\text {outer }}$ neurons. $\mathrm{C}$ fibres also have a small diameter but are unmyelinated and synapse onto lamina I-II neurons. $\mathrm{A} \delta$ and $\mathrm{C}$ fibres can be categorized as peptidergic or nonpeptidergic. Peptidergic fibres contain neuropeptides, such as CGRP, substance P (SP) and galanin (Todd, 2010; Peirs and Seal, 2016). CGRP is of particular interest because primary afferents are the only cellular compartments containing CGRP within the SDH (Todd, 2015). Therefore, CGRP can be used as a marker of lamina I and II in rodents and humans (Shiers et al., 2021). Nonpeptidergic primary afferents in rodents can be labelled using $\mathrm{IB}_{4}$. $\mathrm{IB}_{4}$ is a plant lectin that binds to glycoconjugates on nonpeptidergic fibres in lamina I and II (Todd, 2015).

Within the SDH, primary afferents synapse onto second-order spinal neurons. Most neurons in laminae I and III, and virtually all neurons in lamina II, are interneurons, meaning that they make local synaptic connections within the dorsal horn. Interneurons within the SDH are vastly 
diverse. They can be divided into two general subtypes: excitatory (glutamatergic) and inhibitory (GABAergic ( $\gamma$-aminobutyric acid-ergic) and/or glycinergic) (Todd, 2010; Gatto et al., 2019). SDH neurons can also be categorized by morphology as either radial, vertical, fusiform, central and islet cells (Ran et al., 1998; Todd, 2010; Yasaka et al., 2010; Punnakkal et al., 2014; Dickie et al., 2019; Gatto et al., 2019), or by electrophysiological properties (delayed, tonic, initial bursting and single spiking cells, (Yasaka et al., 2010; Dickie et al., 2019; Gatto et al., 2019). Finally, neurons within the SDH can be categorized by neurochemical markers. For example, excitatory interneurons can be defined by the expression of markers such as neurokinin B, neurotensin, gastrin-releasing peptide, and substance P (Gutierrez-Mecinas et al., 2016; Dickie et al., 2017; Todd, 2017). Inhibitory interneurons can be classified by the presence of proteins such as galanin, neuropeptide Y, neuronal nitric oxide synthase, or parvalbumin (Iwagaki et al., 2013). Although these methods of categorization can be useful tools in describing broad cell types, there is overlap between the categorical features and functions of these interneuron categorization techniques (Todd, 2010; Gatto et al., 2019).

Recent advances in single-cell RNA sequencing techniques (scRNA-seq) have generated an extensive amount of data regarding the numerous subtypes of neurons in the SDH. Recently, Russ and colleagues have created a harmonized atlas of cell types from transcriptomic data from six separate studies of mouse spinal cord scRNA-seq data (Russ et al., 2020). Important findings from this large-scale approach include 20 distinct excitatory clusters and 14 inhibitory clusters within the dorsal horn (Russ et al., 2020). However, gaps still exist between the longstanding, well-established, functional classes of SDH neurons described above and the large number of clusters identified using scRNA-seq. Further investigation is needed to understand how these 
techniques converge to describe the subpopulations of neurons that contribute to specific functional subnetworks within the SDH (Gatto et al., 2019).

In addition to input from primary afferents, the SDH receives input from descending modulation from the brain. There is descending serotonergic input from the nucleus raphe magnus, as well as noradrenergic input from the locus coeruleus and pontine regions (Zoli et al., 1999; Todd, 2010). The SDH also receives descending input from a GABAergic pathway originating in the rostral ventromedial medulla (Kato et al., 2006; Todd, 2015).

The final neuronal subpopulation within the SDH is a small number of projection neurons. Nociceptive projection neurons are primarily located in lamina I, where they make up approximately $5 \%$ of the neuronal population of male rats (Spike et al., 2003). The axons of projection neurons are highly collateralized; some neurons project to three or more targets, including the lateral parabrachial nucleus, the thalamus, and the periaqueductal gray (Al-Khater and Todd, 2009). Understanding of organization and coding of nociceptive output through projection neurons is a developing topic. Recent developments show that there is heterogeneity in projection neurons, with some populations relaying monomodal sensory information, such as cold (Hachisuka et al., 2020), while most are polymodal (Häring et al., 2018; Choi et al., 2020). Projection neurons typically project contralaterally, crossing the midline to project rostrally, however, some project bilaterally as well (Spike et al., 2003). Connections between primary afferents, interneurons, and projections neurons in combination with descending input from the brain make up the circuitry in the SDH. The following section will highlight some of the phenomena that underlie plastic changes seen in this circuitry of the SDH. 


\subsection{Spinal Plasticity:}

\subsubsection{Gate Control Theory:}

Melzack and Wall proposed one of the first theories that postulate the importance of the complex circuits in the SDH, and how those circuits change to result in increased excitability (Melzack and Wall, 1965). They proposed that projection neurons in the SDH receive input from nociceptors as well as from low-threshold $A \beta$ fibres. The $A \beta$ fibres contribution to nociception is, however, gated by feed-forward activation of inhibitory interneurons. Repeated noxious stimulation was proposed to result in inhibition of these inhibitory interneurons, resulting in a transformation of low-threshold mechanosensation into a painful stimulus: allodynia (Melzack and Wall, 1965). Gate control theory spurred interest in interneuron function and how plasticity in spinal circuitry results in pain hypersensitivity.

Recent advances have expanded on Melzack and Wall's gate control theory to account for features of pain and itch processing and circuitry that were unknown when gate theory was first proposed (Braz et al., 2014). For example, interneurons within the SDH are diverse; recent advances have found that parvalbumin-expressing interneurons play a modality-specific role in gating mechanical, but not thermal input to the dorsal horn (Petitjean et al., 2015). Another development has been the understanding of how SDH neurons receiving monosynaptic excitatory $A \beta$ fibre input can be gated by disynaptic inhibition; it is the kinetic properties of excitatory synaptic glutamate receptors, in combination with filtering by potassium channels, that result in inhibition, or gating, within these neurons (Zhang et al., 2018). The discovery of the shared circuitry underlying pain/ itch signalling (Ross, 2011) presents a problem for gate theory. $\mathrm{Grp}^{+}$second-order neurons in the SDH integrate itch and pain transmission and code for pain in an intensity-dependent manner (Sun et al., 2017). This suggests that there is a "leaky gate" that 
controls pain transmission. This discovery accounts for crosstalk between pain and itch (Sun et al., 2017).

\subsubsection{Central Sensitization:}

Nociceptor sensitization is an important factor underlying pain hypersensitivity. This phenomenon, however, does not account for many of the complex symptoms of chronic pain, such as dynamic tactile allodynia and temporal summation of pain (Woolf, 2011). In the years that followed the description of Gate Theory, it became apparent that nociceptive synapses were shaped by use-dependant plasticity. Many years after Melzack and Wall's seminal theory, Clifford Woolf described a new mechanism called 'central sensitization' (Woolf, 1983). Central sensitization describes increased synaptic efficacy or excitability in nociceptive neurons in the SDH that lasted for a prolonged period (over twenty minutes) following brief, low-frequency bursts of action potentials into the SDH (Woolf, 1983, 2011). Central sensitization branches from two preceding concepts: 1 . Melzack and Wall's discovery of windup, a form of plasticity that results in increased amplitude of a response (depolarization) during repeated low-frequency stimulation, in SDH neurons (Melzack and Wall, 1965) and 2. Bliss and Lomo's discovery of long-term potentiation (LTP) in the hippocampus (Bliss and Lømo, 1973). Windup, LTP, and central sensitization are all the product of changes in synaptic plasticity - in each of the three processes, changes in the N-methyl D-aspartate receptor (NMDAR) are potentially responsible for increased depolarization of the post-synaptic neuron (Woolf and Salter, 2000; Latremoliere and Woolf, 2009; West et al., 2015). Central sensitization is distinguished from windup by its presence, measured by increased excitability after the stimulus is withdrawn. Windup, on the other hand, is characterized by progressively increased amplitude of depolarization during a course of repeated stimulation and is heterosynaptic; classic hippocampal LTP is homosynaptic 
and describes the magnitude of synaptic responses (Ji et al., 2003). In central sensitization, a conditioning input generates amplified subsequent responses to successive stimulation of discrete afferents, thus providing explanations for a variety of clinical symptoms of chronic pain (Ji et al., 2003).

Central sensitization describes a broad phenomenon that results in increased spinal and supraspinal responses in the nociceptive pathway to noxious or innocuous stimuli, as well as potential spontaneous activity in these circuits; however, many factors contribute to its initiation and maintenance. Glial supporting cells, immune factors such as cytokines, membrane excitability and gene transcription, along with activity-dependent plasticity, all contribute to increasing spinal hyperexcitability, which underlies chronic pain (Kuner, 2010, 2015; Woolf, 2011; Luo et al., 2014; Finnerup et al., 2021).

The output of increased excitability resulting from central sensitization can be produced at several levels within spinal circuits. At the molecular level, repeated activation leads to phosphorylation of receptors, kinases, and ion channels, thereby modulating their function $(\mathrm{Hu}$ and Gereau IV, 2003; Kawasaki et al., 2004; Ji et al., 2018). Another phosphorylation-dependent effect of central sensitization is the trafficking of receptors to the synapse (Liu and Salter, 2010; Tao, 2012; Finnerup et al., 2021). Molecular changes at the presynaptic membrane result in increases in quantal neurotransmitter release and increased probability of vesicle release (Moechars et al., 2006; Edwards, 2007; Toyoda et al., 2009; Chen et al., 2014b). At the postsynaptic level, alterations of receptor properties, as well as changes in the number and types of postsynaptic receptors, can result in an increased probability of evoking action potential through increases in EPSC magnitude (Lau and Zukin, 2007; Larsson and Broman, 2008; Choi et al., 2010; Ling et al., 2021). The resulting activation of metabotropic receptors and increases in 
intracellular calcium contribute to changes in gene transcription that further promote increases in excitability (Latremoliere and Woolf, 2009; Du et al., 2018; Zhang et al., 2019a). The molecular mechanisms that result in functional plastic changes in central sensitization can be observed at the structural level as well, with changes in the number of synaptic spines (Stratton and Khanna, 2020), changes in connectivity (Gong et al., 2019), as well as circuit-levels changes in

descending modulation from the brain that promote hyperexcitability (Mills et al., 2018). Despite the broad scope of mechanisms that lead to increases in spinal excitability, NMDARs are central mediators of many of these molecular mechanisms of hyperexcitability in spinal nociceptive networks.

\subsection{NMDA Receptors}

\subsubsection{NMDAR background and stoichiometry}

Glutamatergic signalling is the primary excitatory synaptic messenger system of the postnatal brain. This system contains both ionotropic glutamate receptors (iGluRs), including NMDARs, $\alpha$-amino-3-hydroxy-5-methyl-4-isohexazole propionate receptors (AMPARs), and kainate receptors as well as metabotropic glutamate receptors (mGluRs) (Henson et al., 2010; SanzClemente et al., 2013b; Yao et al., 2013). The iGluR family has a heterotetrameric structure. Each of the four subunits of an iGluR contains an extracellular N-terminal domain, a ligandbinding domain, a series of linked transmembrane domains that contribute to the receptor pore, and an intracellular C-terminal domain. The exact structure of the subunits depends on the type and subtype of iGluR (Cavara and Hollmann, 2008; Yao et al., 2013; Hansen et al., 2018). 
NMDARs have several subtypes that can make up the tetrameric structure of the receptor. Historically, it was thought that two main subtypes can compose an NMDAR: two obligatory GluN1 subunits (which have eight different functional splice variants) and two GluN2 subunits (which have four isoforms, GluN2A-GluN2D) (Schüler et al., 2008; Yao et al., 2013). In the last twenty years, a third subtype was discovered: GluN3 (with two isoforms: GluN3A and GluN3B) (Dunah et al., 1999; Cavara and Hollmann, 2008). NMDARs are composed of two GluN1 subunits and a combination of two other GluN2 and/or GluN3 subunits. The composition of the receptors varies across development and by location and dictates not only the physical structure of the receptor but also the physiological role that the receptor has. Due to a large number of possible subtype combinations, NMDARs vary greatly in their biophysical properties (Cavara and Hollmann, 2008; Paoletti et al., 2013).

Of the NMDAR subunits, the differences in biophysical properties between the GluN2A-D subunits have been studied most extensively. Each subunit has a distinct affinity for glutamate; the $\mathrm{EC}_{50}$ of GluN2A $(2.5 \mu \mathrm{M}$ in receptors containing two GluN1 and two GluN2A subunits (Erreger et al., 2005)) is five-fold greater than that of GluN2D (receptors containing two GluN1 and two GluN2D subunits), meaning that glutamate has a five-times higher binding affinity to GluN2D than to GluN2A. GluN2B and -2C have intermediate affinities for glutamate (Hansen et al., 2018). Additionally, the exponential deactivation time course ( $\left.\tau_{\text {decay }}\right)$ for the four subunits varies greatly: it is $40-50 \mathrm{~ms}$ for GluN2A-containing diheterotetrameric receptors, or receptors that contain two GluN1 subunits and two of the same type of non-obligatory subunit (GluN2A here), 200-400 ms for GluN2B-containing diheterotetrameric receptors, and approximately 2-4 s for GluN2D-containing diheterotetrameric receptors (Vicini et al., 1998; Paoletti et al., 2013). Diheterotetrameric GluN2A and GluN2B receptors have higher single-channel conductance and 
higher calcium permeability than GluN2C and GluN2D-containing receptors (Monyer et al., 1992; Retchless et al., 2012; Paoletti et al., 2013). Characterizing the properties of the many types of NMDARs is key to understanding how different NMDAR subunits contribute to the normal functioning of the central nervous system, as well as the role NMDAR subunit variation plays in the many neuropathologies that involve NMDARs (Pachernegg et al., 2012).

GluN1/GluN2-containing NMDARs are termed coincidence detectors. For the pore of the ion channel to open for GluN1/GluN2 receptors, four necessary events must occur: (i) the GluN1 subunits must bind glycine, (ii) the GluN2 subunits must bind glutamate at the same time as (iii) the depolarization of the membrane, and (iv) membrane depolarization forces a magnesium ion plug from the ion pore that then allows influx of cations. GluN3A/B subunits differ significantly in structure from GluN2 subunits and are more similar to GluN1 subunits (Cavara and Hollmann, 2008; Henson et al., 2010; Low and Wee, 2010). This results in GluN3A/B subunits binding glycine, not glutamate (Dunah et al., 1999; Cavara and Hollmann, 2008). Without a GluN2 subunit present, a GluN1/GluN3 heterodimeric receptor can open and allow ionic conductance by glycine binding and removal of the magnesium ion block alone. A heterotrimeric GluN1/GluN2/GluN3 receptor requires both glutamate and glycine binding (Cavara and Hollmann, 2008).

\subsubsection{NMDAR expression in SDH circuits}

Because NMDAR subunits have distinct physiological properties, it is important to consider their expression patterns. The GluN2A, 2B and 2D subunits are expressed in the rodent SDH (Bourinet et al., 2014; Russ et al., 2020). GluN2C has been found to be expressed at very low 
levels in the SDH, or not at all (Yung, 1998; Shibata et al., 1999). In the early postnatal brain, there is a high expression of GluN2B and GluN2D-NMDARs (Crair and Malenka, 1995; Hsia et al., 1998). The properties of GluN2B and GluN2D subunits promote plastic changes in development through synaptic integration due to their slow deactivation kinetics (Paoletti et al., 2013). In the weeks following birth, there is a developmental switch in the brain that promotes GluN2A-NMDAR expression, while expression and synaptic localization of GluN2B and GluN2D-NMDARs are decreased. This results in decreased synaptic strength and dampens the probability of functional circuit reorganization through NMDAR potentiation in the adult brain (Gray et al., 2011). This developmental switch does not occur in the SDH of male rats (females have not been studied) (Mahmoud et al., 2020). Instead, the relative contributions of GluN2Aand GluN2B-mediated NMDAR responses at lamina II synapses remain constant throughout the early developmental period of male rats (Mahmoud et al., 2020). In male adult rat lamina I neurons, GluN2B and GluN2D-containing NMDAR subunits make up the majority of NMDAR responses, with modest contributions from GluN2A-NMDARs (Hildebrand et al., 2014).

Although no comparison exists of synaptic NMDAR responses of SDH neurons by sex, a recent study used immunohistochemistry to examine the expression of NMDAR subunits across the SDH in juvenile rats of both sexes (Temi et al., 2021). Temi and colleagues found robust expression of GluN2A-NMDARs across both the SDH and the deep dorsal horn in both sexes. GluN2B subunits, however, were concentrated in the SDH in both sexes (Temi et al., 2021). In males, GluN2D was also enriched in the SDH, but tissue from females showed no differences between the SDH and the deep dorsal horn in GluN2D staining. This illustrates two key points: 1. that SDH circuitry is primed for central sensitization based on the functional properties of 
NMDAR subunits, and 2. That there are sex differences in the expression of GluN2D subunits within the juvenile rat SDH (Temi et al., 2021).

In addition to distinct distributions across the SDH, GluN2-NMDAR subunits have different localizations within neurons. Using immunohistochemistry, GluN2B and GluN2D are found to be expressed in both the neuropil and soma in the SDH in male and female juvenile rats (Temi et al., 2021). In contrast, the most robust expression of GluN2A-NMDARs is in the neuropil across both the SDH and the deep dorsal horn (Temi et al., 2021). In male adult rats, electrophysiological characterization of the relative contributions of GluN2-NMDAR subunits found that all spinally-expressed GluN2-NMDARs contribute to both synaptic and extrasynaptic signalling (Momiyama, 2000; Tong and MacDermott, 2014), with GluN2B and GluN2D making up the largest proportion of current (Hildebrand et al., 2014). Interestingly, investigation using unitary evoked EPSCs in male adult rat lamina I neurons found that individual synapses vary in their NMDAR subunit composition (Hildebrand et al., 2014).

Both GluN3A and GluN3B NMDAR subunits are expressed in the human spinal cord (Aguet et al., 2019). GluN3A is expressed in excitatory dorsal horn neurons of juvenile mice (Chamessian et al., 2018; Geus et al., 2020), while GluN3B-NMDARs appear to be expressed primarily in motoneurons within the adult rat spinal cord (Wee et al., 2008). Because there appears to be a developmental switch from GluN3A to GluN3B in the rat brain during development (Siaw et al., 2016), the expression patterns of GluN3-NMDAR subunits should be considered at multiple developmental timepoints in the SDH.

The subcellular distribution of GluN3-NMDAR expression remains unknown in the SDH. In the brain, GluN3A subunits differ in subcellular distribution from GluN2 subunits. 
GluN2 subunits are found primarily in the postsynaptic density, where most neurotransmission occurs. A study that used pre-embedded immunogold labelling found that GluN3A was present in the highest concentration in the perisynaptic membrane and decreased in concentration toward the postsynaptic density (Pérez-Otaño et al., 2006). This same study found that GluN3Acontaining NMDARs are selectively removed from the postsynaptic density by endocytosis in an activity-dependent manner, leaving the postsynaptic density rich in GluN1/GluN2 NMDARs (Pérez-Otaño et al., 2006). Another study used crude synaptosome fractions of forebrain and midbrain rat tissue found that both GluN3A and GluN3B are primarily associated with peripheral components of the postsynaptic density (Siaw et al., 2016). These findings demonstrate that GluN2-containing NMDARs make up the majority of synaptic NMDARs, while GluN3containing NMDARs are found extra-or-perisynaptically. The distributions of NMDAR subunits at normal physiological conditions both across the $\mathrm{SDH}$, as well as their distribution within neurons, sets the stage for modulation in response to stimuli.

\subsubsection{NMDAR modulation by Kinases}

Protein phosphorylation is an important mechanism for regulating NMDARs. Phosphorylation results in increased single-channel conductance (Levitan, 1994), as well as increased trafficking of NMDARs to synapses (Liu and Salter, 2010), and thus increases NMDAR currents. Protein kinases phosphorylate the cytoplasmic domains of NMDAR subunits; because these subunits have different $\mathrm{C}$-terminal domains, they are targeted by different kinases that have varied effects on the function of the receptors (Wang et al., 2014b). For example, GluN1 can be phosphorylated at three different sites. Protein kinase-C (PKC), a family of serine/threonine protein kinases that targets serine and threonine amino acid residues, 
phosphorylates S890 on GluN1, which results in the removal of the receptor from the synaptic membrane (Tingley et al., 1997). PKC can also phosphorylate S896. When S896 is phosphorylated along with S897 (which is phosphorylated by protein kinase A, PKA) surface expression of NMDARs increases (Scott et al., 2001). Interestingly, there are no known tyrosine modulation sites on GluN1 (Salter and Kalia, 2004).

GluN2A and GluN2B NMDAR subunits have notably long cytoplasmic domains. This results in several possible modulation sites by protein kinases (Wang et al., 2014b). On the GluN2A subunit, PKA phosphorylates S900 and S929; both these sites modulate receptor desensitization and resultant current decay times (Maki et al., 2013). PKC phosphorylates GluN2A S1291 and S1312, where phosphorylation potentiates GluN2A subunits (Jones et al., 2012). Phosphorylation of GluN2A S1232 by cyclin dependant kinase-5 (Cdk5) is linked to LTP (Li et al., 2001). Src, a member of the Src-family tyrosine kinase (SFK) family, phosphorylates tyrosine residues on GluN2A at Y1292, Y1325, and Y1387 (Salter and Kalia, 2004). Phosphorylation of these sites potentiates GluN2A NMDARs (Yang and Leonard, 2001; Salter and Kalia, 2004; Taniguchi et al., 2009).

GluN2B-NMDARs are also heavily regulated by protein kinases. S1303 is phosphorylated by both PKC and calcium/calmodulin-dependant protein kinase type II (CaMKII). S1323 is phosphorylated by PKC. S1480, which is phosphorylated by casein kinase 2 (CK2), results in the removal of the receptor from the synapse (Sanz-Clemente et al., 2013a). Y1252, Y1336, and Y1472 are phosphorylated by the SFK Fyn (Salter and Kalia, 2004), however, Y1472 is the main phosphorylation target of Fyn on recombinant GluN2B (Nakazawa et al., 2001) and promotes trafficking to the synapse (Goebel-Goody et al., 2009). 
Phosphorylation of GluN2B Y1336 promotes enrichment of extrasynaptic NMDARs (GoebelGoody et al., 2009).

Recent studies suggest that GluN2D and GluN3A receptor subunits are also regulated by phosphorylation, however, the effects of their phosphorylation remain unclear (Dunah et al., 1998; Chowdhury et al., 2013). GluN2D receptors undergo changes in phosphorylation throughout development, with a five to six-fold increase in tyrosine phosphorylation in the thalamus of unsexed rats seen from postnatal day 1 to postnatal day 49 (Dunah et al., 2002). The specific kinase that results in phosphorylation of GluN2D-NMDARs is unknown. Unlike GluN2B-NMDARs that show increased trafficking to synapses upon phosphorylation (GoebelGoody et al., 2009), GluN3A-NMDARs show increased endocytosis when phosphorylated at Y971 by Src (Chowdhury et al., 2013). This is particularly interesting, as both GluN2A and GluN3A are substrates of Src, but GluN3A decreases synaptic plasticity (Roberts et al., 2009), while GluN2A increases it (Salter and Kalia, 2004). Thus, the activity of Src may increase calcium permeability at synapses by concurrently increasing GluN2A receptor activity, while decreasing synaptic levels of GluN3A (Chowdhury et al., 2013).

There is an important functional significance of the differential regulation of NMDAR subunits by kinases. Because of differential expression of NMDAR subunits across the CNS (Yashiro and Philpot, 2008; Ewald and Cline, 2009), and because kinase activity can also be tissue-specific and is associated with specificity to distinct NMDAR subunits (Wang and Salter, 1994; Groveman et al., 2012), there are region-specific mechanisms of NMDAR modulation. For example, both GluN2A and GluN2B are implicated in synaptic plasticity in the CA1 region of the hippocampus (Lüscher and Malenka, 2012), however, differential mechanisms of activation by kinases result in GluN2A-mediated LTP by Src, while Fyn phosphorylation of GluN2B can 
result in LTD (Fox et al., 2006; Yang et al., 2012). Thus, determining the particular mechanisms that lead to region-specific synaptic plasticity can identify pharmacologic treatment targets that can precisely modulate a type of synaptic plasticity.

\subsection{Types and Models of Chronic Pain}

Chronic non-cancer pain can be classified into two categories: neuropathic pain or inflammatory pain. Neuropathic pain is the result of physical damage to the nervous system; for example, a lesion to a peripheral nerve during a surgical procedure (Vilim, 1999; Woolf and Mannion, 1999; Liu et al., 2008). Chronic inflammatory pain results from a prolonged inflammatory response to injury, infection, or as a result of an autoimmune disorder (Vilim, 1999; Liu et al., 2008; Dickie et al., 2017). A common animal model of neuropathic pain is peripheral nerve injury (PNI). In PNI, a nerve, most often the sciatic nerve (Figure 1), is mechanically damaged by lesion or constriction (Vilim, 1999). Widely used models of neuropathic pain include: spinal nerve ligation (SNL), where spinal nerves are tightly sutured (Ho Kim and Mo Chung, 1992), partial sciatic nerve ligation (PSNL), where a suture is placed partially through the sciatic nerve (Seltzer et al., 1990), chronic constriction injury (CCI), where multiple loose sutures are placed around the sciatic nerve (Bennett and Xie, 1988), and spared nerve injury (SNI), where both the common peroneal and tibial nerves are ligated (Decosterd and Woolf, 2000). In male rodents, the resulting injury from SNI or CCI results in the release of BDNF from microglia and the molecular cascade that results in pathological pain (Coull et al., 2005; Trang et al., 2009, 2012; Sorge et al., 2015). Recently, neuropathic pain has been successfully modelled using exogenous BDNF (Coull et al., 2005; Hildebrand et al., 2016). In these models, which we will refer to as the ex vivo model of pathological pain, naïve rodent 
spinal cords are removed and treated with $50 \mathrm{ng} / \mathrm{mL}$ recombinant BDNF for a minimum of 70 minutes (Coull et al., 2005; Hildebrand et al., 2016). Neurons from tissue treated with the ex vivo model show the same functional and biochemical hallmarks of neuropathic pain within lamina I: facilitated excitation, marked by potentiation of NMDAR mEPSCs and upregulation of total and phosphorylated GluN2B-NMDARs (Hildebrand et al., 2016), as well as disinhibition, marked by a depolarizing shift in GABA reversal potential (E $\left.E_{\mathrm{GABA}}\right)$ (Coull et al., 2005). Peripheral inflammatory pain is modelled in rodents using sub-plantar, intramuscular, or intraosseous injections of irritating substances that cause an inflammatory immune response to the site of injection (Zhang and Ren, 2011). In inflammatory pain models where peripheral inflammation is sustained and severe, such as in CFA-induced inflammatory pain, spinal mechanisms contribute to sustained pain hypersensitivity (Lin et al., 2002; Torsney, 2011). In such models, BDNF has been shown to be secreted from primary afferents, however, it is unclear whether disinhibition is involved and whether the inflammatory pain is linked to NMDAR potentiation (Mannion et al., 1999; Lever et al., 2001; Zhao et al., 2006; Sikandar et al., 2018). 


\section{Inflammatory Pain Models:}

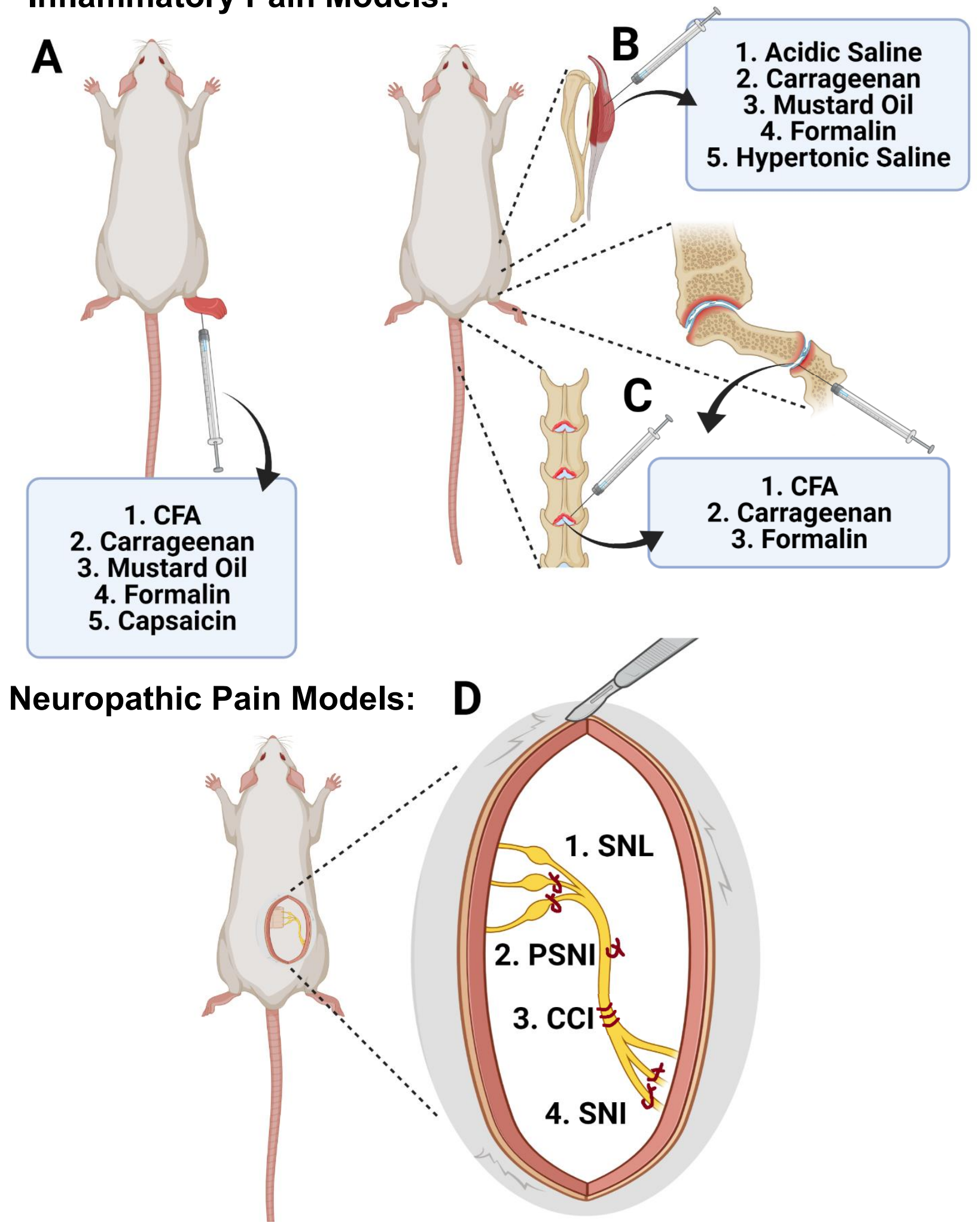

Figure 1. Common in vivo animal models of inflammatory and neuropathic pain. A) Cutaneous and subcutaneous inflammatory pain models exemplified by injection of an inflammatory agent (1. Complete Freund's 
adjuvant, CFA (Iadarola et al., 1988) 2. Carrageenan (Vinegar et al., 1969) 3. Mustard Oil (Inoue et al., 1997$) 4$. Formalin (Dubuisson and Dennis, 1977) 5. Capsaicin (LaMotte et al., 1991)) into the footpad of a rat. B) Muscle inflammation can be modelled using intramuscular injections of 1. Acidic saline (Sluka et al., 2001), 2. Carrageenan (Diehl et al., 1988), 3. Mustard oil (Ro et al., 2003), 4. Formalin (Ro et al., 2003), or 5. Hypertonic saline (Ro et al., 2003 ) into, for example, the gastrocnemius muscle. C) Joint inflammation can be modelled using intraosseous or intraarticular injections of 1. CFA (Wú Lin et al., 1998), 2. Carrageenan (Radhakrishnan et al., 2003), or 3. Formalin (Martins et al., 2006). D) Neuropathic pain can be modelled using: 1. spinal nerve ligation (SNL), tight ligation of spinal nerves (Ho Kim and Mo Chung, 1992), 2. partial sciatic nerve injury (PSNI) performed by suturing partway through and ligating the sciatic nerve (Seltzer et al., 1990), 3. Chronic constriction injury (CCI) is performed by placing several loose sutures around the sciatic nerve (Bennett and Xie, 1988), 4. Spared nerve injury (SNI), where the common peroneal and tibial nerves are ligated (Decosterd and Woolf, 2000). Figure made using BioRender.com

\subsection{Modulating NMDARs in Pain Models}

Studies examining the effects of blocking NMDARs have demonstrated that NMDARs are critically involved in spinal hyperexcitability in neuropathic pain. An early study found that intrathecal injection of the NMDAR antagonist MK-801 reverses mechanical and heat hyperalgesia in male rats with neuropathic pain (Yamamoto and Yaksh, 1992a). Since recent studies have found that phosphorylation and upregulation of GluN2B-NMDARs in the SDH drive neuropathic pain in male mice and rats (Hildebrand et al., 2016; Xie et al., 2016; Zhou et al., 2019), it is of particular interest to specifically target the GluN2B subunit-containing NMDARs. Intrathecal administration of the GluN2B-NMDAR antagonist Ro 25-6981 reversed thermal hyperalgesia and mechanical allodynia in male SNL rats (Qu et al., 2009). In addition, intrathecal injection of the GluN2B-specific antagonist ifenprodil before SNL inhibited mechanical allodynia, but not thermal hyperalgesia, in male SNL rats (Qu et al., 2009). 
Modulating NMDARs has also been found to be successful in alleviating inflammatory pain in animal models. The NMDAR antagonist MK-801 administered intrathecally reduces phase I of the formalin model of inflammatory pain in male rats (Yamamoto and Yaksh, 1992b). Other studies investigated the knockdown of the GluN1 subunit of the NMDAR in the spinal cord in mice of both sexes. Selective deletion of GluN1 subunits by localized injection of adenoassociated virus reduced the GluN1 mRNA levels by $60-80 \%$ in the SDH and prevented the induction of inflammatory pain, but did not affect pain thresholds in uninjured animals (South et al., 2003; Garraway et al., 2007). Because NMDARs serve critical roles across the CNS, it is important to target pathological NMDAR signalling, while leaving basal NMDAR signalling intact. Lui and colleagues found that targeting Src-NMDAR anchoring within the NMDAR complex could reverse both inflammatory (formalin and CFA) and neuropathic (PNI) pain in male rats (Liu et al., 2008).

In the clinic, NMDAR antagonists have shown some efficacy as analgesic agents (Collins et al., 2010; Zhong et al., 2010; Aiyer et al., 2018). Clinically available NMDAR antagonists include ketamine, methadone, amantadine, memantine, dextromethorphan, valproic acid, and carbamazepine, and all have shown efficacy for treating certain clinical pain populations (Collins et al., 2010; Zhou et al., 2011; Aiyer et al., 2018). Of the clinically used NMDAR antagonists mentioned here, none are effective for treating all types of pain. In many cases, a given treatment alleviates pain in specific modalities of a type of pain. For example, dextromethorphan treatment reduces neuropathic pain in patients with diabetic neuropathy, but not in postherpetic neuralgia (Nelson et al., 1997; Sang et al., 2002; Aiyer et al., 2018). Many have high risks of adverse side effects: ketamine must be administered carefully at low doses to prevent dissociative or hallucinogenic effects (Niesters et al., 2014). Methadone, which is both an opioid receptor 
antagonist and NMDAR antagonist, is associated with cardiac side effects such as QT

prolongation and has large variability in absorption, metabolism and potency (Palat and Chary, 2018). Finally, NMDAR antagonists administered systemically interfere with cellular mechanisms of plasticity and learning, thereby producing unwanted side effects (Ilkjaer et al., 1996). These findings indicate that NMDARs could serve as a useful pharmacological target for the treatment of chronic pain, however, it is necessary to selectively target pathological painrelated NMDAR processes to avoid unwanted side effects in the brain, such as inability to form new memories.

\subsection{Disinhibition}

Loss of $\gamma$-aminobutyric acid type $\mathrm{A}\left(\mathrm{GABA}_{\mathrm{A}}\right)$ receptor-mediated inhibitory synaptic transmission can result in increased output from the dorsal horn through a process called disinhibition. Coull and colleagues were the first to describe the mechanism underlying disinhibition (Coull et al., 2003). Using a male rat PNI model of neuropathic pain, they found that lamina I neurons from PNI animals had a significant reduction in anion reversal potential $\left(\mathrm{E}_{\mathrm{GABA}}\right)\left(\right.$ Coull et al., 2003). The change in $\mathrm{E}_{\mathrm{GABA}}$ can result in a net excitatory effect of $\mathrm{GABA}_{\mathrm{A}^{-}}$ receptor/glycine receptor (GlyR)-mediated postsynaptic currents, as was measured using calcium imaging and electrophysiology. Exogenous application of GABA increased intracellular calcium, indicating that GABA application resulted in action potential firing in lamina I neurons from PNI animals. (Coull et al., 2003). The depolarizing shift in $\mathrm{E}_{\mathrm{GABA}}$ is a result of potassium chloride cotransporter 2 (KCC2) downregulation; pharmacological blockade of KCC2 using the selective KCC2 blocker [(dihydroindenyl)oxy]alkanoic acid (DIOA) resulted in an increase in intracellular calcium following GABA application, indicating that KCC2 blockade was sufficient to drive loss 
of inhibition within lamina I neurons. Furthermore, selective knockdown of KCC2 resulted in a decrease in both mechanical and thermal nociceptive threshold (Coull et al., 2003). Thus, Coull and colleagues concluded that $\mathrm{KCC} 2$ downregulation in male PNI animals drives changes in the transmembrane anion gradient, resulting in loss of inhibition, or disinhibition (Coull et al., 2003).

In their 2005 paper, Coull and colleagues expanded on the findings of their 2003 work to investigate the driving factors of disinhibition (Coull et al., 2005). They found that intrathecal administration of ATP-activated microglia resulted in not only decreases in PWT but also resulted in a significant increase in $\mathrm{E}_{\text {anion, }}$, resembling the changes in $\mathrm{E}_{\text {anion }}$ observed in PNI animals (Coull et al., 2005). They next tested if BDNF, which is known to be secreted by microglia, was sufficient to induce a PNI-like phenotype. They found that intrathecal BDNF administration reduced PWT and that when BDNF was bath-applied to spinal slices for 90 minutes or more, there was a resulting shift in $\mathrm{E}_{\text {anion }}$ in lamina I neurons (Coull et al., 2005). Using the function-blocking antibody against TrkB (anti-TrkB), the cognate receptor for BDNF, as well as a BDNF-sequestering fusion protein, TrkB-Fc, Coull and colleagues found that BDNF is necessary to sustain tactile allodynia and the depolarizing shift in $\mathrm{E}_{\text {anion }}$ that results from PNI in male rats (Coull et al., 2005). In summary, Coull and colleagues' work established that when BDNF is secreted from microglia, TrkB receptors trigger the downregulation of the potassium chloride co-transporter $\mathrm{KCC} 2 . \mathrm{KCC} 2$ is responsible for maintaining a low concentration of intracellular chloride, which supports normal GABAergic transmission. A high concentration of intracellular chloride also promotes the efflux of negatively charged bicarbonate through $\mathrm{GABA}_{\mathrm{A}}$ channels, which can lead to overall membrane-depolarizing events. Disinhibition results in a decrease in normal inhibitory GABAergic signalling and promotes excitatory efflux of bicarbonate (Coull et al., 2003, 2005). 
Important recent developments in the understanding of disinhibition reveal that there is a gradient of ionic plasticity within the SDH (Ferrini et al., 2020). At baseline levels, KCC2 is expressed at lower levels in the SDH than in deeper laminae, with the lowest levels seen in lamina I (Ferrini et al., 2020). In addition, because peptidergic primary afferents synapse onto lamina I neurons, lamina I have a higher degree of TrkB activation than deeper areas of the SDH. This results in lower levels of KCC2 compared to lamina II, and thus a reduced ability to maintain chloride homeostasis. This results in lamina I neurons being more susceptible to activity-dependant metaplasticity that can result in disinhibition (Ferrini et al., 2020).

Given the role of disinhibition in mediating spinal hyperexcitability, disinhibition is a possible therapeutic target for the treatment of neuropathic pain. One component for pharmacological intervention is blocking carbonic anhydrase using acetazolamide (Asiedu et al., 2010; Lee and Prescott, 2015). KCC2 downregulation reduces chloride extrusion capacity in the $\mathrm{SDH}$, which results in a depolarizing efflux of bicarbonate when $\mathrm{GABA}_{\mathrm{A}}$ receptors are activated (Coull et al., 2003). Blocking carbonic anhydrase decreases bicarbonate efflux and thus restores inhibitory tone following chloride dysregulation (Asiedu et al., 2010; Lee and Prescott, 2015). Acetazolamide has been effective in reversing SNL-induced neuropathic pain in male rats and SNI-induced neuropathic pain in male mice (Asiedu et al., 2010, 2014). The discovery of KCC2mediated disinhibition was made using exclusively male animals. Lee-Kubli and Calcutt performed experiments demonstrating that disinhibition occurs in female rats as well (Lee-Kubli and Calcutt, 2013). They examined rate-dependent depression (RDD), which is a measure of the decline in the amplitude of the spinal Hoffman reflex following repeated stimulation (Ishikawa et al., 1966; Meinck, 1976; Lee-Kubli and Calcutt, 2013) and can be used as a measure of disinhibition (Lee-Kubli and Calcutt, 2013). Using female neuropathic rats and female rats that 
received an intrathecal injection of BDNF, they found deficits in RDD, indicating disinhibition occurred in both neuropathic female rats and female rats that had received intrathecal BDNF (Lee-Kubli and Calcutt, 2013). Mapplebeck and colleagues recently used in vivo recordings of

brush-evoked spiking in lamina I neurons and found that both male and female SNL rats display KCC2-mediated disinhibition and that acetazolamide reverses the effect of SNL on increases in brush-evoked spiking (Mapplebeck et al., 2019).

In addition to targeting disinhibition through inhibition of carbonic anhydrase using acetazolamide, Gagnon and colleagues have developed chloride extrusion enhancers, CLP257 and CLP290, that increased KCC2 plasma expression and reversed tactile allodynia in male SNI and CCI neuropathic rats (Gagnon et al., 2013; Lorenzo et al., 2020). Intrathecal administration of CLP290 successfully reversed SNI-induced neuropathic tactile allodynia in both male and female rats, suggesting that rescuing $\mathrm{KCC} 2$ function can alleviate neuropathic pain in both sexes (Mapplebeck et al., 2019).

\subsection{Linking Disinhibition and NMDAR Potentiation}

Although disinhibition and facilitated excitation were first described as separate processes (Coull et al., 2003; Liu et al., 2008), evidence is mounting that they may, indeed, be linked. For example, modelling disinhibition using intrathecal administration of bicuculline to block $\mathrm{GABA}_{\mathrm{A}}$ receptors in the spinal cord results in pain hypersensitivity, but this hypersensitivity can be reversed using NMDAR antagonist D-APV (Cao et al., 2011). In addition, bicuculline-induced disinhibition resulted in a PKA-dependent increase in synaptic GluN2B-NMDARs in male rodents (Cao et al., 2011). In a male SNL model, restoring KCC2 using intrathecal $\mathrm{KCC} 2$ gene transfer not only reversed allodynia but also reversed NMDAR 
potentiation in SDH neurons ( $\mathrm{Li}$ et al., 2016). Conversely, increases in NMDAR activity resulting from neuropathic pain have been found to lead to degradation of $\mathrm{KCC} 2$ through calpain activity (Zhou et al., 2012). These findings suggest an intertwined relationship between KCC2 downregulation and NMDAR potentiation, but the linker between these processes in the SDH in pathological pain remains unclear.

Some key molecular players known to mediate pathological pain processes can hint at possible linkers between disinhibition and NMDAR potentiation. For example, the Src-family kinase Fyn, and not Src kinase, potentiates excitatory GluN2B-containing NMDARs within lamina I in a model of neuropathic pain (Hildebrand et al., 2016). Additionally, increasing Fyn activity in the SDH of male mice results in persistent tactile allodynia (Liu et al., 2014). Fyn's direct action on GluN2B and not GluN2A is responsible for NMDAR potentiation within lamina I (Abe et al., 2005; Yang et al., 2011; Hildebrand et al., 2016; Li et al., 2017a). Fyn is activated by BDNF-TrkB signalling; indeed, behavioural sensitization induced by PNI can be reversed using BDNF-TrkB antagonists (Coull et al., 2005; Wang et al., 2009). Thus, a linker between disinhibition and NMDAR potentiation would likely be tied to BDNF-TrkB-Fyn NMDAR signalling, as well as BDNF-TrkB-KCC2 signalling.

Fyn kinase and NMDAR activity can both be downregulated by the enzyme 'striatalenriched protein tyrosine phosphatase-61' (STEP 61 ) (Pelkey et al., 2002; Paul et al., 2003, 2007; Valjent et al., 2005; Xu et al., 2015a). $\mathrm{STEP}_{61}$ is a membrane-associated phosphotyrosine phosphatase (PTP) that has been found to oppose levels of NMDAR tyrosine phosphorylation, promotes internalization of GluN2B-NMDARs, and has been found to deactivate Fyn by dephosphorylating at $\mathrm{Y}^{420}$ (Pelkey et al., 2002; Goebel-Goody et al., 2012; Won and Roche, 2021). STEP $_{61}$ activity thus decreases NMDAR currents Interestingly, BDNF downregulation 
increases $\mathrm{STEP}_{61}$ levels in the cortical cultures, while the same study found that increasing BDNF-TrkB signalling triggered decreases in $\mathrm{STEP}_{61}$ activity (Xu et al., 2016). In addition to ties to mechanisms of facilitated excitation, $\mathrm{STEP}_{61}$ is also linked to disinhibition. In a study using intrathecal bicuculline to model disinhibition in the $\mathrm{SDH}$, loss of GABAergic inhibition resulted in a loss of $\mathrm{STEP}_{61}$ function (Li et al., 2015). This opposing interaction of BDNF and STEP $_{61}$ leaves these two players poised to play modulatory roles in neurologic and neuropsychiatric diseases.

Research on $\mathrm{STEP}_{61}$ in the brain implicates $\mathrm{STEP}_{61}$ signalling in several NMDAR-linked pathologies. For example, STEP ${ }_{61}$ has been implicated in Alzheimer's disease (Xu et al., 2014; Castonguay et al., 2018; Lee et al., 2021), Huntington's disease (Gladding et al., 2014), and schizophrenia (Xu et al., 2016). In the spinal cord, $\mathrm{STEP}_{61}$ is highly expressed in the SDH in naïve male and female rodents (Li et al., 2015; Azkona et al., 2016). STEP 61 is downregulated in the SDH by CFA-induced inflammatory pain (Li et al., 2015; Xu et al., 2015b; Azkona et al., 2016). STEP 61 restoration has reversed tactile allodynia in CFA-induced inflammatory pain ( $\mathrm{Li}$ et al., 2015), and CFA injection resulted in increased levels of phosphorylated, deactivated STEP $_{61}$ (Azkona et al., 2016). This evidence suggests $\mathrm{STEP}_{61}$ signalling may link the processes of disinhibition and NMDAR potentiation.

\subsection{Sex differences in Chronic Pain}

It comes as no surprise that both males and females experience both acute and chronic pain. In part due to this shared experience of pain and in part due to bias across a large proportion of scientific research, the majority of studies on pain have been performed only using male rodents (Mogil, 2012; Shansky and Murphy, 2021). And thus, it was assumed that 
nociceptive pathways are conserved between males and females. However, clinical evidence suggests divergent pain signalling pathways. Women show increased pain sensitivity compared to men in experimentally induced electrical, thermal, and chemically-induced pain (Bartley and Fillingim, 2013). Differences in mechanical pain threshold have been reported in rodents as well, with females showing lower mechanical pain thresholds than males (Li et al., 2009). In the clinic, women are more likely to report low back pain, neck pain and orofacial pain, and twice as many women report migraines or headaches (Pleis et al., 2010; Chen et al., 2017). These observations lay the foundation for investigating possible differences in nociceptive signalling.

Until recently, the existence of physiological sex differences in pain was a contested issue (Mogil, 2012). Now there is a growing body of literature that shows sex differences in brain regions associated with descending pain modulation (Wang et al., 2014a), as well as differences in resting-state functional connectivity within the dynamic pain connectome that links the many brain areas that regulate pain, attention and cognition (Fauchon et al., 2021; Kim et al., 2021; Osborne et al., 2021). In the periphery, sex differences in immune modulation of high mobility group box 1 protein (HMGB1) have been observed in a mouse arthritis model, with macrophage TLR4 involvement in males, but not females (Rudjito et al., 2021). In mouse DRG, differences in nociceptor-enriched translatomes show sex differences in prostaglandin signalling (TavaresFerreira et al., 2020), while transcriptomic differences in mouse DRG and trigeminal ganglia suggest male mice have differential expression of genes in sensory neurons that are protective against effects of neuropathic pain (Mecklenburg et al., 2020).

In spinal cord nociception, sex differences in immune mediators of pain have been at the forefront. In males, microglia are activated and secrete BDNF, which in turn results in neuronal hyperexcitability. Unlike males, SDH hyperexcitability in females appears to be caused by a 
downstream result of T-lymphocyte activation (Sorge et al., 2015; Chen et al., 2017; Mapplebeck et al., 2017). These studies show different immune triggers for the initiation of SDH hyperexcitability; however, it is unclear whether there is a sexually dimorphic response within SDH neurons themselves. Sexually dimorphic signal transduction pathways dependant on protein kinase $\mathrm{A}, \mathrm{C} \varepsilon$, and $\mathrm{C} \delta$ have been found to be regulated by sex hormones in several pain models (Dina et al., 2001, 2007; Joseph and Levine, 2003; Hucho et al., 2006). Thus, it is conceivable that these signal transduction pathways may regulate sexually dimorphic neuronal mechanisms of nociception within the SDH.

\subsection{Human Translational Studies}

In the pharmaceutical development of therapeutics for pain, there is a massive jump from studies in animal models of pain to testing potential therapeutic candidates in clinical trials. This jump has contributed to the failure of a large proportion of analgesic clinical trials (Staahl and Drewes, 2004; Gereau et al., 2014). The development of human preclinical models for pain research is necessary for the development of new analgesic treatments. In recent years, genetic tools have begun to be used to characterize human CNS tissue and compare it to rodent models. For example, mRNA analysis has been used to link the presence of neuropeptide FF, which is believed to play a role in pain modulation and opiate function, to humans, cows, rats, and mice (Vilim, 1999). RNA-sequencing has also been used to conduct similar comparisons where mouse, rat, and human tissues were compared to look for expression of itch-associated peptides (Goswami et al., 2014). More recently, Parisien and colleagues have examined single nucleotide polymorphisms (SNPs) associated with expression levels of a gene or exon (expression quantitative trail loci, eQTLs) in human DRGs. They found pain-related genetic associations 
implicating the human leukocyte antigen locus, and were able to use manipulations in a mouse model of inflammatory pain to confirm these findings (Parisien et al., 2017). Such experimental designs highlight the powerful potential to identify genetic targets in human genetic studies and make manipulations in rodent models. The field of human pain genetics research is quickly evolving. To summarize the extensive list of genetic contributors to human pain, Meloto and colleagues created the Human Pain Genetics Database (Meloto et al., 2018). This database has allowed for associations of genetic variants with analgesia and nociception-related pathways, as well as enabled comparisons between multiple pain pathways (Meloto et al., 2018).

Functional analysis of human pathophysiology has been undertaken in several fields other than pain research. For example, hippocampal tissue from epileptic patients who underwent partial unilateral resection of the hippocampus has been used for electrophysiological characterization of neurons and comparisons to rat models (Kann et al., 2005). Although an extremely useful approach for the study of epilepsy, similar approaches cannot be used in the study of human spinal cord physiology, as no clinical procedures involve excision of spinal cord tissue from patients. Some researchers call for an increase in the use of non-human primate (NHP) models of pain, but many research facilities are not equipped for NHPs, and these models still represent inter-species comparisons (Hama et al., 2013).

One pain research area that has successfully compared functional properties of human and rodent nociceptive tissue is DRG research. Using DRG extracted from human organ donors, researchers have used acute cultured human DRG neurons to study electrophysiological responses to chemical and electrical stimuli (Davidson et al., 2014; Waxman and Zamponi, 2014; Zhang et al., 2015, 2017, 2019b; Enright et al., 2016). For example, Davidson and colleagues used whole-cell patch-clamp recordings to examine membrane properties of nociceptive neurons 
and found that small-diameter DRG neurons display an inflection on the descending slope of the action potential. This inflection is a defining feature of rodent nociceptive neurons (Rose et al., 1986) and indicates that this feature is conserved between species and that most small-diameter DRG neurons in humans can be classified as nociceptors. (Davidson et al., 2014). Studies characterizing nociceptor responses to compounds that produce pain or itch across species may also pave the way for this preparation to be used as preclinical target validation in human tissue (Davidson et al., 2014; Zhang et al., 2015, 2017, 2019b; Enright et al., 2016).

These pivotal studies have laid the foundation for use of human organ donor tissue in pain research; however, human DRG preparations leave questions of human CNS nociceptive physiology unanswered. An important recent study used immunohistochemical approaches to examine protein staining distributions in the human spinal cord (Shiers et al., 2021). They found that central projection patterns of nociceptor populations differ in spinal laminar distribution from rodents (Shiers et al., 2021). Future studies will need to examine the synaptic connectivity of primary afferents and SDH neurons in humans. These findings are critical for understanding the organization of the human nociceptive network but do not allow for functional characterization of the human SDH, or the functional changes associated with chronic pain. One possibility for translational, pre-clinical models for the study of human pathological pain is a spinal cord ex vivo model, such as our previously validated rodent model where spinal slices are incubated in BDNF (Hildebrand et al., 2016). The use of such an ex vivo model would provide a pivotal tool for translational research in the field of chronic pain that would help bridge the gap between basic science and clinical research. 


\subsection{Purpose}

We aim to identify molecular players that drive spinal hyperexcitability in the superficial dorsal horn following inflammation in both rodent and human models of pathological pain. We will use a combination of electrophysiological, biochemical, and behavioural analyses in human and rat tissue to examine mechanisms of spinal cord pain amplification. In our electrophysiological approach, we will record spontaneous synaptic NMDAR responses as a readout of excitatory synaptic activity. My research aims to: (1) identify the molecular mechanism underlying dorsal horn hyperexcitability in male rats, (2) determine if molecular mechanisms are conserved in the male human spinal cord, and (3) investigate sex differences in the pathological pain pathway in both rats and humans and examine underlying causes of any identified sex differences.

\subsubsection{Aim 1:}

To identify molecular mechanisms underlying SDH excitability in male rats, in Chapter 2, we will use the CFA model of inflammatory pain to determine if mechanisms are conserved between rodent models of neuropathic and inflammatory pain. We will perform biochemical analysis to compare changes in specific protein levels between synaptosomes isolated from rats subjected to the CFA model of inflammatory pain and the ex vivo model of pathological pain. If inflammatory pain shows the same hallmark features as neuropathic and ex vivo models, we hypothesize that CFA will drive increased levels of GluN2B-NMDARs, active Fyn and decreased levels of KCC2 and $\mathrm{STEP}_{61}$. Further examining this pathway, we will perform electrophysiological recordings on lamina I neurons from rats post-CFA injection to determine whether synaptic NMDAR responses are potentiated in an inflammatory model of pain. We will 
then use the ex vivo BDNF treatment model of pathological pain in combination with electrophysiological recordings to test $\mathrm{STEP}_{61}$ 's involvement in spinal hyperexcitability. We will test whether blocking disinhibition in spinal slices treated with the CFA model of inflammatory pain reverses any effects produced by CFA alone. Finally, we will use von Frey behaviour testing to determine whether blocking disinhibition affects pain hypersensitivity in vivo.

\subsubsection{Aim 2:}

We will investigate whether mechanisms of spinal hyperexcitability are conserved between rat and human males. This aim will be addressed in Chapter 2. We will perform electrophysiological recordings on human lamina I neurons to characterize which NMDAR subtypes mediate synaptic NMDAR responses. We will then develop a human ex vivo model of pathological pain in human spinal tissue. We will use this model to determine whether the ex vivo BDNF treatment model results in immunohistochemical and biochemical markers of disinhibition and facilitated excitation in human spinal cord tissue.

\subsubsection{Aim 3:}

To investigate sex differences in spinal hyperexcitability, in Chapter 3, we will first perform western blots to compare changes in synaptosome protein levels between tissue from male and female rats subjected to the CFA model of inflammatory pain and the ex vivo BDNF model of pathological pain. If inflammatory pain shows the same hallmark features as in males, I hypothesize that CFA will increase levels of GluN2B-NMDARs, active Fyn and decreased levels of $\mathrm{KCC}_{2}$ and $\mathrm{STEP}_{61}$ at superficial dorsal horn synapses in comparison to control-treated female rats. We will next determine whether CFA-mediated inflammatory pain results in lamina I 
NMDAR mEPSC potentiation in female rats. We will compare these CFA-animal recordings to recordings on female rat tissue that has been treated with the ex vivo BDNF model to determine if BDNF pre-treatment results in NMDAR potentiation of lamina I neurons. Next, we will compare the effects of ex vivo BDNF treatment in males and females using immunohistochemical and biochemical approaches on human spinal card tissue. Finally, we will use female rats that were ovariectomized before reaching sexual maturity, then left to age-match our other animals, to determine whether identified sex differences are hormonally mediated. 


\section{Chapter 2: Loss of STEP $_{61}$ couples disinhibition to $\boldsymbol{N}$-methyl-D-aspartate receptor potentiation in rodent and human spinal pain processing}

\subsection{Abstract}

Dysregulated excitability within the spinal dorsal horn is a critical mediator of chronic pain. In the rodent nerve injury model of neuropathic pain, BDNF-mediated loss of inhibition (disinhibition) gates the potentiation of excitatory GluN2B N-methyl-D-aspartate receptor (NMDAR) responses at lamina I dorsal horn synapses. However, the centrality of this mechanism across pain states and species, as well as the molecular linker involved, remains unknown. Here, we show that $\mathrm{KCC} 2$-dependent disinhibition is coupled to increased GluN2Bmediated synaptic NMDAR responses in a rodent model of inflammatory pain, with an associated downregulation of the tyrosine phosphatase $\mathrm{STEP}_{61}$. The decreased activity of $\mathrm{STEP}_{61}$ is both necessary and sufficient to prime subsequent phosphorylation and potentiation of GluN2B NMDAR by BDNF at lamina I synapses. Blocking disinhibition reversed the downregulation of $\mathrm{STEP}_{61}$ as well as inflammation-mediated behavioural hypersensitivity. For the first time, we characterize GluN2B-mediated NMDAR responses at human lamina I synapses and show that a human ex vivo BDNF model of pathological pain processing downregulates $\mathrm{KCC}_{2}$ and $\mathrm{STEP}_{61}$ and upregulates phosphorylated GluN2B at dorsal horn synapses. Our results demonstrate that $\mathrm{STEP}_{61}$ is the molecular brake that is lost following $\mathrm{KCC}$-dependent disinhibition and that the decrease in $\mathrm{STEP}_{61}$ activity drives the potentiation of excitatory GluN2B NMDAR responses in rodent and human models of pathological pain. The ex vivo human BDNF model may thus form a translational bridge between rodents and humans for the identification and validation of novel molecular pain targets. 


\subsection{Introduction}

Chronic pain is a widespread, debilitating disease with few safe and effective treatments. To develop novel therapeutic approaches, we must identify the molecular determinants of pathological pain (Gereau et al., 2014; Yekkirala et al., 2017). However, the vast majority of basic science pain research ends at target identification and validation in rodent models of chronic pain. Typically, findings from rodent models form the scientific rationale for a large jump to direct testing of individual compounds in specific human pain syndromes. When candidate molecules fail in clinical testing, their targets are often abandoned even when the underlying reasons for failure remain unexplored (Morgan et al., 2012). Thus, proof-of-concept human tissue studies are urgently needed to test assumptions on cross-species similarity and to bridge the rodent-to-human translational divide (Gereau et al., 2014).

Pathological pain arises when neurons in the nociceptive pathway become sensitized by repeated exposure to noxious stimuli. Within the spinal dorsal horn, sensitization disrupts the balance between excitation and inhibition, leading to a sustained increase in nociceptive transmission from lamina I output neurons to the brain (Latremoliere and Woolf, 2009; Todd, 2010; Bourinet et al., 2014; Kuner, 2015; Alles and Smith, 2018). We have recently found that two mechanisms of dorsal horn hyperexcitability_BDNF-mediated disinhibition (Coull et al., 2005) and GluN2B N-methyl-D-aspartate receptor (NMDAR) potentiation by the Src family kinase, Fyn (Abe et al., 2005) — are directly linked following nerve injury (Hildebrand et al., 2016). However, the molecular linker that drives the coupling between disinhibition and facilitated excitation remains unidentified. Here, we investigate this critical problem in both rodent and human spinal models of pathological pain. 


\subsection{Materials and methods}

\subsubsection{Animals}

All rodent experiments were performed on male adult Sprague-Dawley rats supplied by Charles River Laboratories, weighing 350-450 g. Animals were housed and cared for in accordance with the recommendations of the Canadian Council for Animal Care and from regulations and policies of Carleton University and the University of Ottawa Heart Institute. Animals were housed in pairs, had free access to food and water and were randomly assigned to their respective experimental groups.

Freund's adjuvant model of inflammatory pain and behaviour testing Complete Freund's adjuvant (CFA, Sigma) was used to model inflammatory pain. Rats were given a $0.4 \mathrm{ml}$ plantar injection of either phosphate-buffered saline (PBS: control) or CFA (a $50 \%$ by volume mixture of CFA and PBS) under isoflurane anesthesia.

Behaviour testing for pain hypersensitivity was performed using von Frey filaments to measure mechanical paw withdrawal threshold. Withdrawal threshold was measured using the simplified up-down method (SUDO), as described by Bonin et al. (2014). The SUDO method standardizes the number of stimuli per test to five, thus ensuring that each animal receives a constant number of trials while minimizing the number of stimuli to prevent sensitization during testing. Measurements were taken at baseline (pre-injection), and then every $24 \mathrm{~h}$ until the endpoint of the study. Animals used for biochemical analysis were sacrificed $120 \mathrm{~h}$ post-CFA injection. Animals used for electrophysiological recording were sacrificed $72-120 \mathrm{~h}$ post-CFA injection. For both biochemical analysis and electrophysiological recording, only the ipsilateral side of the lumbar spinal cord was used. 


\subsubsection{Intraperitoneal injection of acetazolamide}

Seventy-two hours following sub-plantar injection of CFA and following behaviour testing (see above), male Sprague-Dawley rats were given an intraperitoneal injection of 300 $\mathrm{mg} / \mathrm{kg}$ acetazolamide (Sigma) dissolved in Ringer's solution ( $\mathrm{pH}$ 8.28). Behaviour testing was then performed every $10 \mathrm{~min}$, with the experimenter blinded to treatment conditions. A small pilot of 10 animals was used to identify the time point of maximal behaviour reversal, which was 40 min post-intraperitoneal injection. All further cohorts were sacrificed after the behaviour testing at $40 \mathrm{~min}$ post-intraperitoneal injection.

\subsubsection{Rat spinal cord isolation}

Rats were anesthetized using an intraperitoneal injection of $3 \mathrm{~g} / \mathrm{kg}$ urethane (Sigma). Once under deep anesthesia, spinal cords were rapidly dissected via ventral laminectomy and immediately placed in ice-cold oxygenated protective sucrose artificial CSF solution (referred to as 'saline': $50 \mathrm{mM}$ sucrose, $92 \mathrm{mM} \mathrm{NaCl}, 15 \mathrm{mM}$ D-glucose, $26 \mathrm{mM} \mathrm{NaHCO} 3,5 \mathrm{mM} \mathrm{KCl,} 1.25$ $\mathrm{mM} \mathrm{NaH}_{2} \mathrm{PO}_{4}, 0.5 \mathrm{mM} \mathrm{CaCl}_{2}, 7 \mathrm{mM} \mathrm{MgSO}_{4}, 1 \mathrm{mM}$ kynurenic acid, bubbled with $5 \% \mathrm{CO}_{2} / 95 \%$ $\mathrm{O}_{2}$ ). The L3-L6 lumbar region was isolated and dorsal and ventral roots were removed under a dissection microscope.

The spinal cords were then sliced parasagittally to $300 \mu \mathrm{m}$ for electrophysiological recording using a Leica VT1200S vibratome at an amplitude of $2.75 \mathrm{~mm}$ and a speed of 0.1 $\mathrm{mm} / \mathrm{s}$ through the dorsal horn. Kynurenic acid was washed out by incubating slices in $34^{\circ} \mathrm{C}$, kynurenic acid-free saline for $40 \mathrm{~min}$. Previous control experiments have shown no difference in NMDAR synaptic responses in lamina I neurons from slices that were sectioned in saline with or without kynurenic acid as long as slices were recovered in kynurenic acid-free saline (data not 
shown). Following this recovery period, the incubation chamber was removed from the heated water bath and allowed to cool to room temperature. For biochemical analysis, a $\sim 400 \mu \mathrm{m}$ horizontal section containing the dorsal horn was removed. The following $1200 \mu \mathrm{m}$ of spinal tissue containing the deep dorsal horn and ventral horn was used for control comparisons. Following slicing, tissue for biochemical analysis was either flash-frozen using histo-freeze (Fisher Super Friendly Freeze'It) or was treated according to the ex vivo model of pathological pain processing (see below) and then flash frozen.

\subsubsection{Human spinal cord preparation}

Spinal tissue was collected from adult (18-69-years-old) male human organ donors identified by the Trillium Gift of Life Network. Donors were pre-screened to exclude patients with communicable diseases (hepatitis, HIV/AIDS or syphilis) or chronic conditions such as morbid obesity that could negatively affect health of the donor's organs. Tissue from donors that had spinal cord damage or that were taking chronic pain medications were also excluded from the study. The most common cause of death was compromised blood flow to the brain (hemorrhage or ischemia). For the collection and experimentation with human spinal cord tissue, approval was obtained from the Ottawa Health Science Network Research Ethics Board. Hypothermia was induced using a cooling bed and the body was perfused with high magnesium protective solution (Celsior or Belzer UW) before organ collection. Spinal cords were isolated via ventral laminectomy within $114 \pm 25 \min (n=14)$ of cross-clamping the heart after organs were removed for donation. Once the spinal cord was removed, the thoracic and lumbar regions were isolated and placed in ice-cold saline in preparation for biochemical, immunohistochemical 
and/or electrophysiological experiments. A summary of which experimental procedures were performed on each human spinal cord sample can be found in Supplementary Table 1.

In a subset of donors, human spinal tissue for electrophysiological recording was sectioned in the transverse plane into $400-500 \mu \mathrm{m}$ sections using a Leica vibratome at an amplitude of $2.75 \mathrm{~mm}$ and a speed of $0.1 \mathrm{~mm} / \mathrm{s}$ through the dorsal horn. As done for rodent slices, spinal sectioning was performed in oxygenated, ice-cold saline including $1 \mathrm{mM}$ kynurenic acid, followed by recovery in saline without kynurenic acid at $34^{\circ} \mathrm{C}$ for $40 \mathrm{~min}$. Human tissue for biochemical analysis was left unsectioned and was treated according to the ex vivo model of pathological pain processing (see below). Following treatment according to the ex vivo model, tissue was either flash-frozen, and the dorsal horn was removed using a scalpel blade or tissue was fixed with $4 \%$ paraformaldehyde.

\subsubsection{Ex vivo model of pathological pain processing}

Following removal of the spinal cord from the subject (rat or human) according to the above-stated procedures, tissue was placed in oxygenated, room temperature saline containing 50-100 $\mathrm{ng} / \mathrm{ml}$ recombinant BDNF (Alomone Labs) or saline alone for $70-80 \mathrm{~min}$. This same approach was used for treatment of spinal tissue with BDNF and TAT-STEP, BDNF and acetazolamide, acetazolamide alone, or TC-2153.

\subsubsection{Electrophysiological recordings of lamina I neurons}

After slice preparation, cells were viewed using brightfield optics. Lamina I neurons were located dorsal to the substantia gelatinosa, within the $50 \mu \mathrm{m}$ portion of tissue directly ventral of 
the white matter. As described previously (Hildebrand et al., 2016), the extracellular recording solution used was an artificial CSF solution containing (in $\mathrm{mM}$ ): $125 \mathrm{NaCl}, 20 \mathrm{D}$-glucose, 26 $\mathrm{NaHCO}_{3}, 3 \mathrm{KCl}, 1.25 \mathrm{NaH}_{2} \mathrm{PO}_{4}, 2 \mathrm{CaCl}_{2}$, and $1 \mathrm{MgCl}_{2}$ in addition to $500 \mathrm{nM}$ TTX, $10 \mu \mathrm{M} \mathrm{Cd} 2^{+}$, $10 \mu \mathrm{M}$ strychnine and $10 \mu \mathrm{M}$ bicuculline to block voltage-gated $\mathrm{Na}^{+}$channel, voltage-gated $\mathrm{Ca}^{2+}$

channel, glycinergic and GABAergic currents, respectively. We used borosilicate glass patchclamp pipettes with resistances of 6-12 M . The internal patch pipette solution contained (in mM): 105 Cs-gluconate, $17.5 \mathrm{CsCl}, 10$ BAPTA or 10 EGTA, 10 HEPES, 2 MgATP, 0.5 $\mathrm{Na}_{2} \mathrm{GTP}$ and had a $\mathrm{pH}$ of 7.25 and an osmolarity of $295 \mathrm{mOsm}$.

Criteria for recorded neurons were as follows: an access resistance below $30 \mathrm{M} \Omega$, and leakage currents less than $-100 \mathrm{pA}$ at a holding potential $\left(\mathrm{V}_{\mathrm{h}}\right)$ of $-70 \mathrm{mV}$. We also selected for the larger neurons in the area, to favour projection neurons. After establishing a whole-cell patch at -60 $\mathrm{mV}$, the holding potential was slowly increased to $+60 \mathrm{mV}$ to record NMDAR miniature excitatory postsynaptic currents (mEPSCs). Selection criteria for mEPSCs included: no events that completely decay within $100 \mathrm{~ms}$, no outlier events with an amplitude $>100 \mathrm{pA}$ for rat cells and 200 pA for human cells, and events must decay to at least $50 \%$ of their overall amplitude by 500 ms. For analysis, mEPSC traces were detected and averaged together for each given treatment in Clampfit 10.7 (Molecular Devices). Traces were then transferred to Origin Pro (Northampton) for graphing.

\subsubsection{Isolation of synaptosome fractions and biochemical analysis of human and rodent spinal cord tissue}


The human spinal cord was treated according to the ex vivo model of pathological pain processing following tissue collection. The tissue was flash-frozen with liquid nitrogen following treatment and stored at $-80^{\circ} \mathrm{C}$. Approximately $4 \mathrm{~mm}$ of the superficial dorsal horn was separated from the rest of the cord using a scalpel blade on dry ice. For rats, the lumbar region of the rat spinal cord was sectioned using a vibratome to obtain a $\sim 400 \mu \mathrm{m}$ superficial dorsal horn section and another section consisting of the remainder of the spinal cord.

The isolation of synaptosomal fractions was performed as described previously (Xu et al., 2009). Tissue was first homogenized using Wheaton dounce tissue grinders in $300 \mu 1$ of ice-cold TEVP-320 mM sucrose buffer containing (in mM): 320 sucrose, 10 Tris-HCl (pH 7.4), 1 EDTA, 1 EGTA, $5 \mathrm{NaF}$, and $1 \mathrm{Na}_{3} \mathrm{VO}_{4}$ with complete protease inhibitor and phosphatase inhibitor cocktails (Roche) to obtain total homogenates. A fraction of the homogenates was used for analysis. The remaining total homogenate lysates were centrifuged at $4^{\circ} \mathrm{C}$ for $10 \mathrm{~min}$ at $1000 \mathrm{~g}$ and a further $15 \mathrm{~min}$ at $12000 \mathrm{~g}$ to obtain the crude synaptosome pellet. The pellet was resuspended in TEVP $320 \mathrm{mM}$ sucrose buffer by brief sonication.

The protein content of the homogenates and the synaptosomal fractions was determined by the Pierce BCA protein assay kit (Thermo Scientific). Thirty micrograms of total protein from each sample were loaded on $8 \%$ SDS-PAGE and transferred to PVDF membranes (Bio-Rad). Membranes were blocked in 5\% bovine serum albumin (BSA) in Tris-buffered saline (TBS) $+0.1 \%$ TWEEN-20 (TBS-T) and incubated overnight in 5\% BSA + TBS-T plus primary antibodies [anti-STEP (1:1000), anti-KCC2 (1:1000), anti-Fyn (1:1000) and anti- $\beta$-actin (1:10 000) from Santa Cruz; anti-non-phospho-STEP (1:1000) and anti-pY416-Src (or pY420-Fyn) (1:1000) from Cell Signaling; anti-pY ${ }^{1472}$ GluN2B (1:1000) and anti-p ${ }^{1325}$ GluN2A (1:1000) 
from PhosphoSolutions; anti-GluN2B (1:2000) and anti-GluN2A (1:1000) from Millipore; for further details on antibodies used in western blots, see Supplementary Table 2]. Membranes were washed three times with TBS-T and incubated in horseradish peroxidase (HRP)-conjugated secondary antibodies (anti-mouse and anti-rabbit (1:5000) from Pierce for $2 \mathrm{~h}$ at room temperature. Membranes were developed using Chemiluminescent Substrate kit (Pierce) and visualized using G:BOX with the GeneSnap software (Syngene). All densitometric bands were quantified using ImageJ (NIH).

\subsubsection{Immunohistochemistry and antibodies}

Transverse fixed human spinal sections were cut at $25 \mu \mathrm{m}$ on a sledge freezing microtome Leica SM2000R (Leica Microsystems). Sections were permeabilized in PBS (pH 7.4) with $0.2 \%$ Triton (PBS+T) for $10 \mathrm{~min}$, washed twice in PBS and incubated for $12 \mathrm{~h}$ at $4{ }^{\circ} \mathrm{C}$ in primary anti-KCC2 antibody and anti-CGRP antibody (see below) diluted in PBS+T containing $10 \%$ normal goat serum. After washing in PBS, the tissue was incubated for $2 \mathrm{~h}$ at room temperature in a solution containing a mixture of the goat-Cy3 anti-rabbit purified secondary antibody (1:500, Jackson ImmunoResearch Laboratories, Cat. \#111-165-144) goat anti-mouse Alexa Fluor ${ }^{\circledR} 647$ cross-adsorbed secondary antibody (1:500, Thermo Fisher Scientific Cat. \#A21235) and DAPI (1:500, Thermo Fisher Scientific; Cat. \#D1306) diluted in PBS+T (pH 7.4) containing $10 \%$ normal goat serum. Sections were mounted on SuperFrost ${ }^{\mathrm{TM}}$ gelatin-subbed slides (Fisherbrand) and cover-slipped using fluorescence mounting medium (Dako, Cat. \#S3023).

\subsubsection{Markers of the peptidergic small diameter afferent terminals}


Calcitonin gene-related peptide (CGRP) immunoreactivity was used as a specific marker of nociceptive peptidergic afferent terminals (not present in any other types of axons in the dorsal horn) (Rosenfeld et al., 1983; Gibson et al., 1984; Hunt and Rossi, 1985; Ju et al., 1987) using a monoclonal anti-CGRP antibody (1:5000; Sigma \#C7113) raised in mouse. This antiserum detects human $\alpha$-CGRP and $\beta$-CGRP but does not cross-react with any other peptide (data supplied by Sigma).

\subsubsection{Anti-KCC2 antibody}

A polyclonal antibody raised in rabbit (1:1000, Millipore/Upstate, Cat. \#07-432) was used in this study. This antibody was raised against a His-tag fusion protein corresponding to residues $932-1043$ of the rat KCC2 intracellular C-terminal (Williams et al., 1999; Mercado et al., 2006). This antibody is highly specific for rat $\mathrm{KCC} 2$ (KCC2a and $\mathrm{KCC} 2 \mathrm{~b}$ isoforms) and does not share any homologous sequences with other KCCs or co-transporters.

\subsubsection{Confocal laser scanning microscopy}

All confocal images were acquired using a Zeiss LSM 880 Confocal Laser Scanning Microscope. Acquisitions were 12-bit images of size $2048 \times 2048$ pixels with a pixel dwell time of $10 \mu$ s. An oil-immersion $\times 63$ plan-apochromatic objective was used for high magnification confocal laser scanning microscopy images, which were processed for quantification. Laser power was adequately chosen to avoid saturation and limit photobleaching. All the acquisitions were performed with the same laser settings [laser, power, photomultiplier tube (PMT) settings, image size, pixel size and scanning time]. During the acquisitions, the experimenter was blind to the slice conditions (i.e. saline versus BDNF treatment). 


\subsubsection{Ex vivo subcellular KCC2 distribution in human dorsal horn neurons}

We developed homemade MATLAB routines to quantify and monitor the changes in the KCC2 intensity distributions in subcellular compartments. This new method is based on a modified version of an already published algorithm used to detect receptor membrane internalization (Ferrini et al., 2017). A user has to delineate the membrane of neuronal cell bodies present in the acquired confocal image. For this analysis, we use CGRP as a marker of the superficial dorsal horn. Only neurons present in the regions of the dorsal horn expressing CGRP were considered. The imaging and analysis were both done blind to the experimental conditions. For each pixel in the region of interest, the distance to the closest membrane segment was calculated. Using this distance map, the mean pixel intensity and standard deviation of KCC2 fluorescence signal were quantified as a function of the distance to the neuron membrane. A negative position value represents the region outside of the labelled neuron. A total of 118 neurons for saline and 108 neurons for BDNF from nine adult males were analyzed. Two types of cells were observed during the analysis. The first type showed a strong membrane staining, while the other type exhibited a larger intracellular KCC2 labelling. Because of this, we divided all analyzed cells into two groups using the position of the maximum $\mathrm{KCC} 2$ intensity in each subcellular profile. Cells for which the KCC2 intensity maximum was at a distance $<0.5 \mu \mathrm{m}$ and $>0.5 \mu \mathrm{m}$ were pooled in two distinct groups. For saline, 108/116 neurons are in the first group $(<0.5 \mu \mathrm{m})$; in contrast, 36 of 108 neurons analyzed for the BDNF conditions are in the second group $(>0.5 \mu \mathrm{m})$. Averaged profiles were obtained for each subject and condition (saline and $\mathrm{BDNF}$ ) and from those averages, the global KCC2 intensity profiles were obtained for each condition. The KCC2 membrane intensity (at position $0 \mu \mathrm{m}$ ) and the $\mathrm{KCC} 2$ intracellular 
intensity $(0.5 \mu \mathrm{m}<$ position $<2.5 \mu \mathrm{m})$ were extracted from each subject's averaged KCC2 intensity profiles. We carried out 2-way parametric paired t-tests on the KCC2 intensity at the membrane and in the intracellular compartment and a $\chi 2$ test to test the statistical difference between the distributions of cells in the two conditions.

\subsubsection{Statistical analysis}

All data are presented as means \pm standard error of the mean (SEM). Comparison of means were performed using Student's paired and unpaired t-tests (Microsoft Excel Office 365), and one-way ANOVAs (IBM SPSS Statistics 25.0). Tukey's significant difference test followed ANOVAs, as appropriate. A Pearson's $\chi 2$ test was used in Supplementary Fig. 7D. In all tests, P $<0.05$ were considered statistically significant. Supplementary Table 3 lists all tests performed, the comparison made, and the associated P-values.

\subsection{Results}

To test whether NMDAR responses at lamina I synapses are potentiated across pathological pain models, we measured mEPSCs in adult male rats that received a hind-paw injection of CFA. The CFA injection model of persistent inflammation induces prolonged pain hypersensitivity that is mediated by dorsal horn sensitization (Ren et al., 1992). We selected rats that had a sustained reduction in withdrawal threshold of the ipsilateral paw 3-5 days after CFA injection (Fig. 1A). As observed for the nerve injury model (Hildebrand et al., 2016), we found that the NMDAR component of mEPSCs in lamina I neurons was significantly increased in CFA injected rats (NMDAR charge transfer $=6.54 \pm 0.32 \mathrm{pC}, \mathrm{n}=8$ neurons from six animals) 
compared to naïve rats (NMDAR charge transfer $=3.01 \pm 0.18 \mathrm{pC}, \mathrm{n}=10$ neurons from eight animals, $\left.\mathrm{P}=2.92 \times 10^{-8}\right)($ Fig. $1 \mathrm{~B}$ and Supplementary Table 3$)$. Moreover, the component of NMDAR mEPSCs that was potentiated in CFA-injected rats compared to naïve rats had a decay constant of $210 \mathrm{~ms}$, which is consistent with GluN2B-mediated NMDAR responses (Hildebrand et al., 2014). GluN2B-containing NMDARs are potentiated by Fyn-dependent phosphorylation at Tyr1472, while Fyn itself is activated by phosphorylation at Tyr420 (Trepanier et al., 2012). To test for CFA-induced changes in total and phosphorylated levels of GluN2B and Fyn, we performed western blot analysis on the crude synaptosomal fraction of the superficial dorsal horn. As found in the nerve injury model (Hildebrand et al., 2016), CFA injection led to a significant increase in activated $(\mathrm{pY} 420, \mathrm{P}=0.032)$ but not total $(\mathrm{P}=0.42)$ Fyn and an associated increase in total $(\mathrm{P}=0.047)$ and phosphorylated $(\mathrm{pY} 1472, \mathrm{P}=0.016)$ GluN2B in superficial dorsal horn synaptosomes ( $\mathrm{n}=4$; Fig. $1 \mathrm{C}$ and Supplementary Table 3). 
A
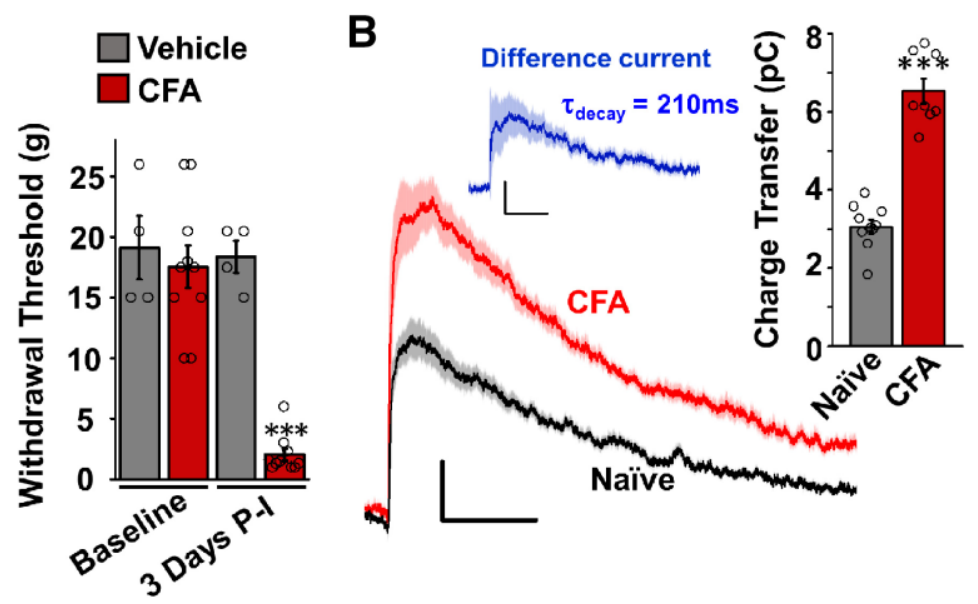

\section{C}

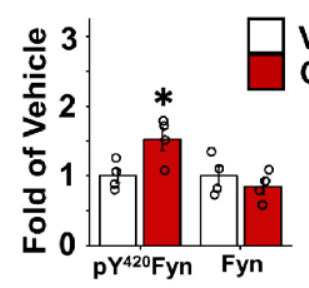

Vehicle (V)

CFA (C)
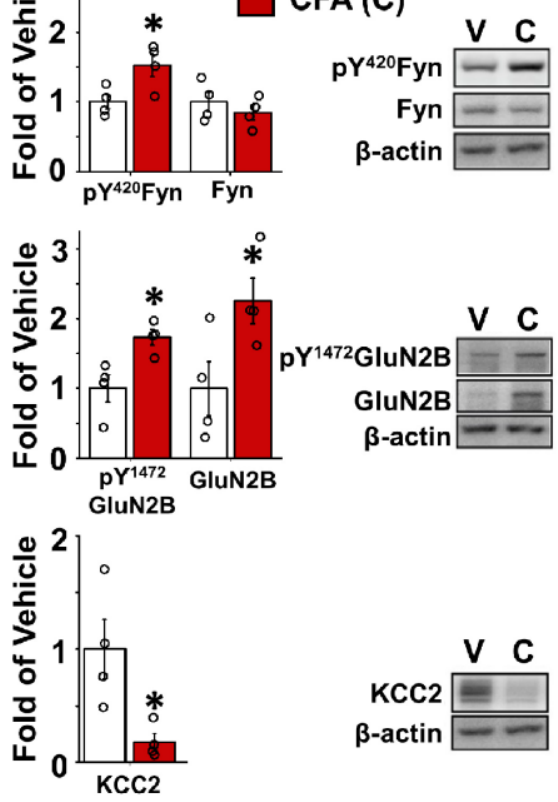

V C
Kcc2
$\beta$-actin

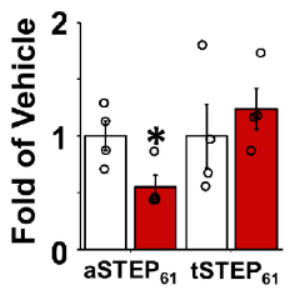

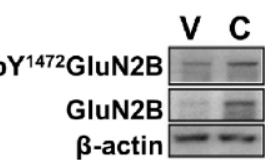

D

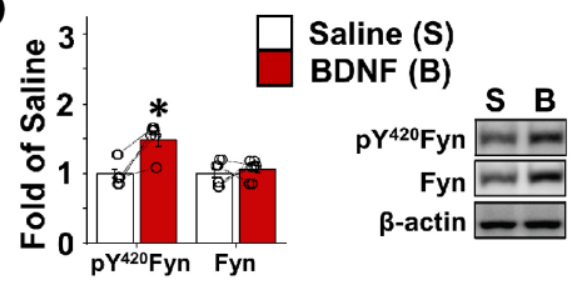

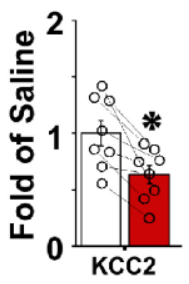
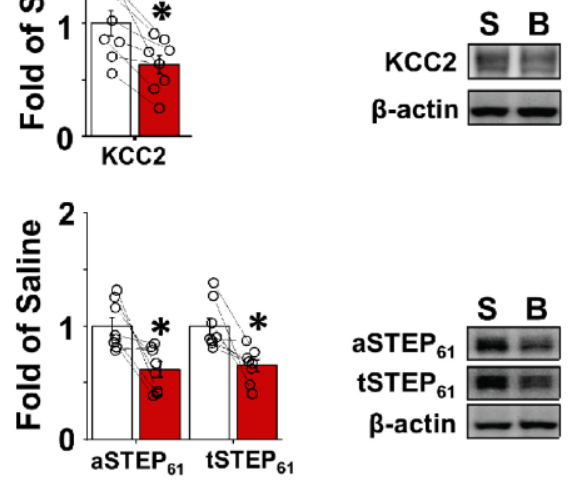

Chapter 2 Figure 1. GluN2B NMDARs are potentiated at lamina I synapses in a CFA model of chronic

inflammatory pain, with an associated downregulation of synaptic STEP 61 . GluN2B NMDARs are potentiated at lamina I synapses in a CFA model of chronic inflammatory pain, with an associated downregulation of synaptic STEP $_{61}$. A) Left: Illustration of the in vivo CFA injection model of inflammatory pain. The window over the rat's 
back depicts the area of spine removed for either electrophysiological or biochemical analysis. Right: Ipsilateral paw withdrawal threshold $(\mathrm{g}$ ) at baseline before and 3 days after hindpaw injection with vehicle (grey, $\mathrm{n}=4$, spinal cords used for C) or CFA (red, $\mathrm{n}=10$ animals, spinal cords used for B and C) comparing vehicle to CFA 3 days postinjection. B) Left: average NMDAR mEPSCs at $+60 \mathrm{mV}$ from lamina I neurons in ipsilateral spinal cord of untreated (black, $\mathrm{n}=10$ cells from eight animals) and CFA-injected (red, $\mathrm{n}=8$ cells from six animals) adult male rats. Top: Difference current for NMDAR mEPSCs from CFA rats subtracted from NMDAR mEPSCs from control rats to reveal the 'potentiated' NMDAR mEPSC component. Right: Plot of NMDAR charge transfer (from 40 ms to $500 \mathrm{~ms}$ ) for untreated (grey) versus CFA-injected (red) rats. All current traces in Figs 1, 2 and 4 represented as means (dark lines) \pm SEM (lighter bars) of mEPSCs from all averaged cells. Scale bars $=100 \mathrm{~ms}(\mathrm{x}$-axes); $5 \mathrm{pA}(\mathrm{y}-$ axes). C) Plots (left) and representative western blots (right) from synaptosome fractions of ipsilateral superficial dorsal horn from CFA-injected rats (red, $n=4)$ and vehicle-injected rats (white, $n=4)$. For quantification, all targets were normalized to beta-actin as a loading control. D) Plots (left) and representative western blots (right) from synaptosome fractions of superficial dorsal horn treated with either control saline (white, $\mathrm{n}=8$ ) or $50 \mathrm{ng} / \mathrm{ml}$ recombinant $\mathrm{BDNF}$ for $70 \min ($ red, $\mathrm{n}=8$ ). $* \mathrm{P}<0.05, * * \mathrm{P}<0.01 * * * \mathrm{P}<0.001$; exact $\mathrm{P}$-values can be found in Supplementary Table 3.

The BDNF-dependent disinhibition of lamina I neurons is mediated by a downregulation of the $\mathrm{KCC} 2$ chloride transporter and subsequent loss of the chloride gradient required for GABA $_{A}$-dependent synaptic inhibition (Coull et al., 2005). We found that KCC2 was significantly decreased in superficial dorsal horn synaptosomes following CFA injection ( $\mathrm{n}=4$, $\mathrm{P}=0.023$; Fig. 1C). We also found that $\mathrm{KCC} 2(\mathrm{P}=0.0064)$ was downregulated and $\mathrm{pY}^{420}$ Fyn $(\mathrm{P}=0.0011)$ as well as total $(\mathrm{P}=0.026)$ and $\mathrm{pY}^{1472} \mathrm{GluN} 2 \mathrm{~B}(\mathrm{P}=0.016)$ were upregulated in the ex vivo BDNF model of pathological pain processing $(\mathrm{n}=8$; Fig. 1D and Supplementary Table 3), where spinal sections from naïve rats are treated with recombinant $(50 \mathrm{ng} / \mathrm{ml})$ BDNF (Coull et al., 2005; Hildebrand et al., 2016) (Fig. 2A). The KCC2-Fyn-GluN2B signalling pathway was 
restricted to superficial dorsal horn synaptosomes, as no BDNF-mediated changes were observed in either the homogenate fraction of superficial dorsal horn (Supplementary Fig. 1) nor in the synaptosome fraction of the remainder of the spinal cord (Supplementary Fig. 2). Taken together, our results show that GluN2B-containing NMDARs are phosphorylated and potentiated by a KCC2- and Fyn-dependent pathway at lamina I synapses in CFA-injected rodents. We therefore propose that KCC2-dependent disinhibition is coupled to NMDAR potentiation in both neuropathic (Hildebrand et al., 2016) and inflammatory models of chronic pain. However, what is the molecular linker that couples these two distinct pathological mechanisms? 
A Ex vivo BDNF Model B
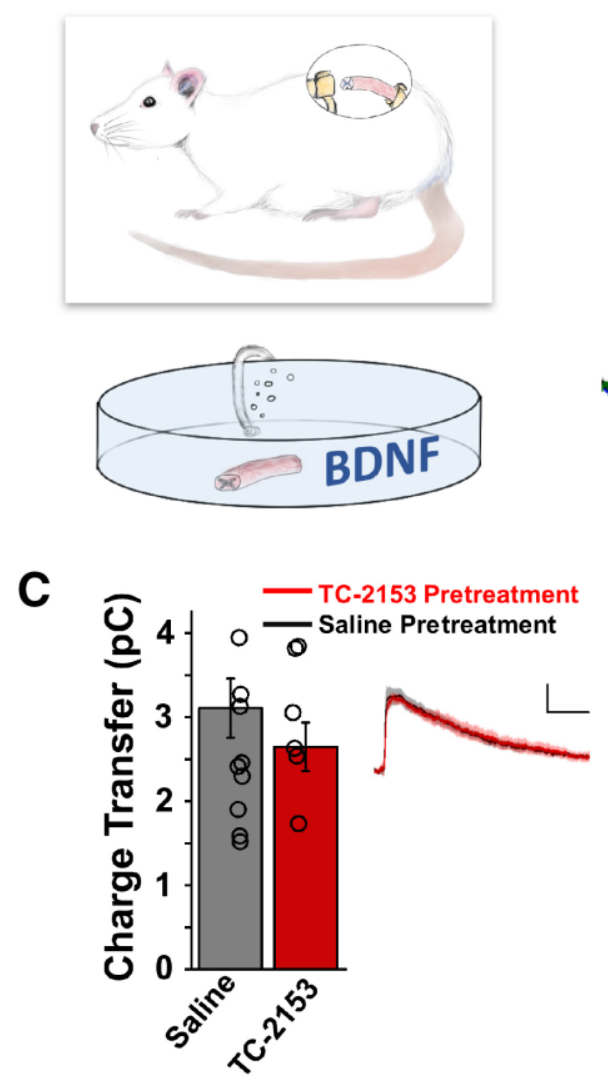
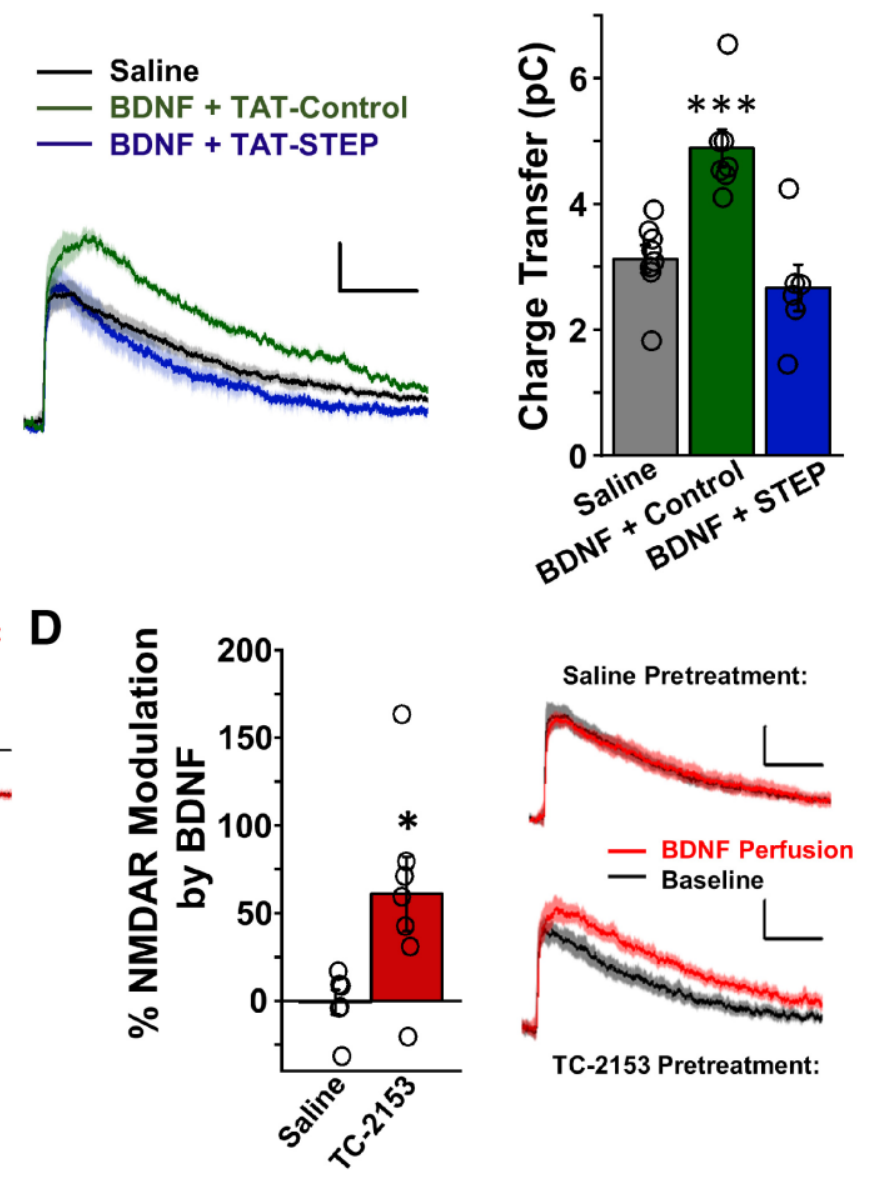

$\mathbf{E}$
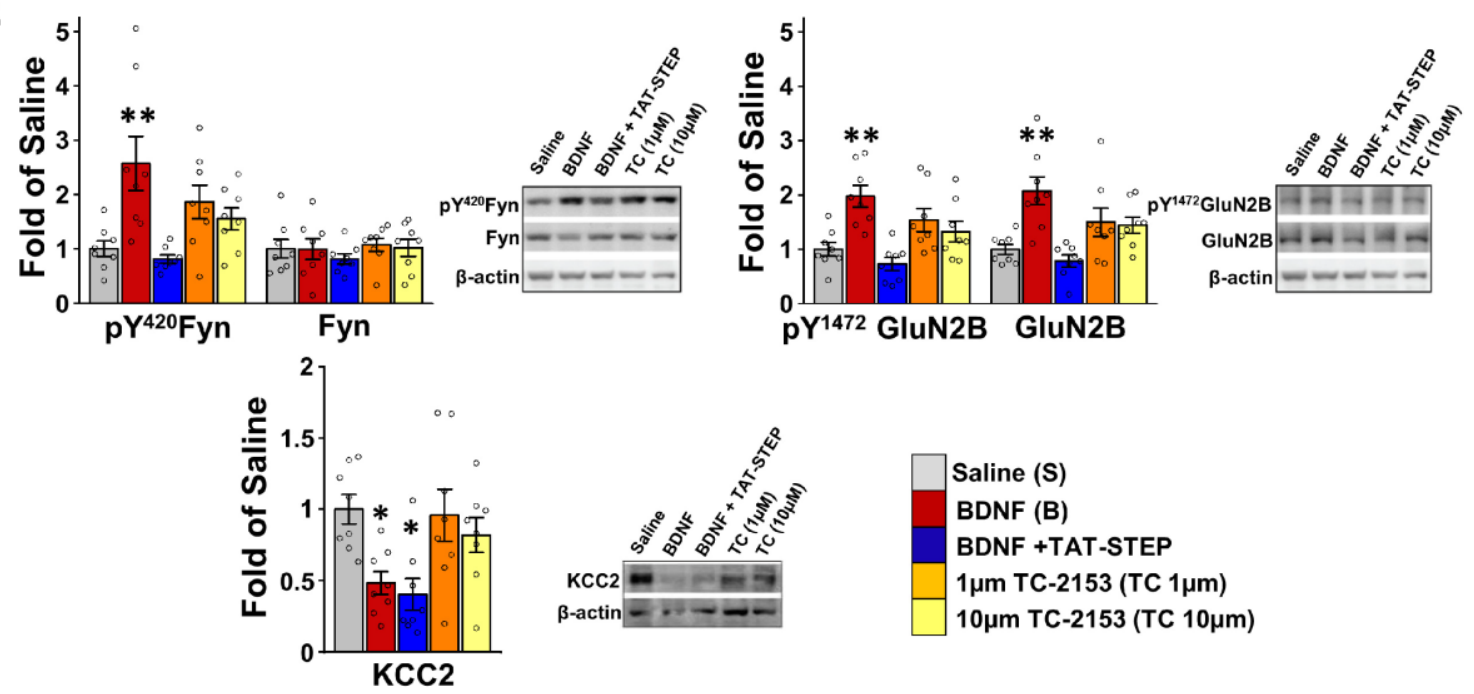

Chapter 2 Figure 2. STEP61 is necessary and sufficient to prime phosphorylation and potentiation of synaptic

GluN2B-NMDARs by BDNF. A) Illustration of the ex vivo BDNF model of pathological pain. Sections of lumbar spinal cord (window over rat's back illustrates the lumbar section used) from naïve rats are incubated in oxygenated saline with $50 \mathrm{ng} / \mathrm{ml}$ recombinant BDNF for $70 \mathrm{~min}$, before electrophysiology on individual lamina I neurons (B-D) 
or biochemistry on superficial dorsal horn tissue (E). B) Average NMDAR mEPSC traces (left) and associated charge transfer values (right) from lamina I neurons of naïve rat spinal slices pretreated with control saline (black, $n$ = 8), BDNF and a TAT-control peptide (green, $200 \mathrm{nM}, \mathrm{n}=7$ ), or BDNF and a membrane permeable active STEP peptide (TAT-STEP; blue, $200 \mathrm{nM}, \mathrm{n}=6$ ). Scale bars $=100 \mathrm{~ms}$ (x-axes); $5 \mathrm{pA}$ (y-axes). C) Treatment of naïve slices with $1 \mu \mathrm{M}$ TC-2153 for $70 \mathrm{~min}(\mathrm{red}, \mathrm{n}=7$ ) had no significant effect on NMDAR mEPSCs compared to saline-treated slices (grey, $\mathrm{n}=6)$. D) Administration of exogenous BDNF (100 ng/ml) during patch-clamp recording caused an increase in NMDAR mEPSC traces in TC-2153 pretreated slices (bottom right, $\mathrm{n}=7$ ), but not in saline pretreated slices (top right, $\mathrm{n}=6$ ). Plot on left calculated from NMDAR mEPSC charge transfer after BDNF normalized to baseline charge transfer, for each pretreatment condition. E) Plots and representative western blots from synaptosome fractions of superficial dorsal horn incubated in saline (grey), $50 \mathrm{ng} / \mathrm{ml} \mathrm{BDNF}$ (red), $50 \mathrm{ng} / \mathrm{ml}$ BDNF and $200 \mathrm{nM}$ TAT-STEP (blue), $1 \mu \mathrm{M}$ TC-2153 (orange), or $10 \mu \mathrm{M}$ TC-2153 (yellow) for 70 min (n = 8 animals/group). For quantification, all targets were normalized to beta actin as a loading control. $* \mathrm{P}<0.05, * * \mathrm{P}<$ $0.01,{ }^{* * *} \mathrm{P}<0.001$ compared to saline, exact $\mathrm{P}$-values for each comparison can be found in Supplementary Table 3.

We have shown that the protein tyrosine phosphatase $\mathrm{STEP}_{61}$ promotes dephosphorylation and inactivation of Fyn (at $\mathrm{pY}^{420}$ ) and that BDNF signalling leads to the degradation of $\mathrm{STEP}_{61}$ at brain synapses (Xu et al., 2015; Saavedra et al., 2016). Interestingly, we found here that active (dephosphorylated at Ser ${ }^{221}$ ) STEP61 was significantly decreased in superficial dorsal horn synaptosomes in the in vivo CFA and ex vivo BDNF models of pathological pain processing $(n=4, P=0.036$, Fig. $1 C ; n=8, P=0.013$; Fig. 1D). To test whether $\mathrm{STEP}_{61}$ downregulation is required for the potentiation of NMDAR responses at lamina I synapses, we co-treated spinal sections of naïve rats with both recombinant BDNF and a fusion peptide that contained a membrane-permeable TAT domain followed by the active STEP peptide (TAT-STEP) (Paul et al., 2007; Xu et al., 2009). Co-treatment of TAT-STEP (200 nM) with BDNF $(n=6)$ prevented the potentiation of NMDAR mEPSCs observed in neurons treated with 
BDNF and a TAT-fusion control peptide $(200 \mathrm{nM}, \mathrm{n}=7, \mathrm{P}=6.3 \times 10-4$; Fig. 2B). The NMDAR charge transfer for neurons treated with BDNF and TAT-STEP was not significantly different from that of neurons in untreated spinal sections $(\mathrm{n}=8, \mathrm{P}=0.28$; Fig. 2B), demonstrating that restoring STEP activity completely abolishes the BDNF-mediated potentiation of NMDAR responses at lamina I synapses. We next used the small organic STEP inhibitor, TC-2153 (Xu et al., 2014), to test whether blocking STEP activity itself is sufficient to induce potentiation of synaptic NMDAR responses. Pretreating spinal sections from naïve rats with TC-2153 $(1 \mu \mathrm{M}, \mathrm{n}=9)$ did not significantly alter NMDAR mEPSCs compared to control saline-treated slices $(n=6, P=0.33$; Fig. 2 C). However, following slice pretreatment with TC2153, administration of BDNF during recording significantly increased synaptic NMDAR responses ( $\mathrm{n}=7, \mathrm{P}=0.028$; Fig. 2D). Perfusion of BDNF had no effect on NMDAR mEPSCs in saline pretreated slices $(n=6, P=0.97)$. Similar to previous experiments using blockers and activators of KCC2-dependent inhibition (Hildebrand et al., 2016), our results suggest that loss of STEP $_{61}$ is necessary and sufficient to prime subsequent potentiation of synaptic NMDARs by BDNF.

Because disinhibition can trigger $\mathrm{STEP}_{61}$ downregulation and $\mathrm{STEP}_{61}$ regulates Fyn and GluN2B signalling in dorsal horn neurons (Li et al., 2015; Xu et al., 2015a; Azkona et al., 2016), we asked whether loss of $\mathrm{STEP}_{61}$ directly couples $\mathrm{KCC} 2$-dependent disinhibition to the potentiation of GluN2B NMDARs by Fyn in the ex vivo BDNF model of pathological pain. To investigate this, we explored the effects of $\mathrm{STEP}_{61}$ antagonists and agonists on superficial dorsal horn synaptosomes from saline- versus BDNF-treated rodent spinal cords. Co-treatment of active TAT-STEP with BDNF prevented the increases in $\mathrm{p}^{\mathrm{Y} 420} \mathrm{Fyn}\left(\mathrm{P}=9.9 \times 10^{-4}\right)$, total GluN2B $(\mathrm{P}=$ 
$\left.2.1 \times 10^{-4}\right)$, and $\mathrm{pY} 1472 \mathrm{GluN} 2 \mathrm{~B}\left(\mathrm{P}=1.1 \times 10^{-4}\right)$ that were induced by pretreating naïve spinal slices with BDNF alone $(\mathrm{n}=8$; Fig. 2E). In contrast, co-treatment of TAT-STEP with BDNF did not significantly affect the BDNF-mediated downregulation of KCC2 at superficial dorsal horn synaptosomes ( $\mathrm{n}=8 ; \mathrm{P}=0.99$; Fig. 2E). Unlike that observed in cortical neurons (Xu et al., 2014), pretreating spinal sections with the STEP antagonist, TC-2153 (1 $\mu \mathrm{M}$ or $10 \mu \mathrm{M})$, did not significantly increase $\mathrm{pY}^{420}$ Fyn $(\mathrm{P}=0.66$ for $10 \mu \mathrm{M})$, total GluN2B $(\mathrm{P}=0.46)$, or $\mathrm{pY} \mathrm{Y}^{1472}$ GluN2B $(\mathrm{P}=0.68)$ at superficial dorsal horn synaptosomes compared to saline-treated spinal sections $\left(\mathrm{n}=8\right.$; Fig. 2E). The finding that blocking $\mathrm{STEP}_{61}$ phosphatase activity does not directly induce phosphorylation and potentiation of GluN2B NMDARs by Fyn aligns with our previous observation of minimal basal Src family kinase activity in adult lamina I neurons (Hildebrand et al., 2016). We propose that BDNF is required for a subsequent activation of Fyn kinase. These biochemical experiments (Fig. 2E) suggest that the loss of $\mathrm{STEP}_{61}$ links upstream KCC2 downregulation to downstream Fyn-dependent phosphorylation and trafficking of GluN2B NMDARs to superficial dorsal horn synapses during pathological BDNF-dependent pain processing.

To test our working model further, we investigated whether disinhibition is required for BDNF-mediated STEP $61 /$ Fyn/GluN2B signalling. We used the carbonic acid anhydrase inhibitor, acetazolamide, which blocks disinhibition produced by KCC2-dependent chloride dysregulation without directly altering GABA or glycine receptor-mediated inhibition (Ferrini and De Koninck, 2013; Lee and Prescott, 2015). Co-treatment of spinal sections from naïve rats with acetazolamide $(10 \mu \mathrm{M})$ and BDNF abolished the BDNF-mediated increases in $\mathrm{pY}^{420} \mathrm{Fyn}(\mathrm{P}=$ 0.0014), total GluN2B $(\mathrm{P}=0.0071)$, and $\mathrm{pY}^{1472} \mathrm{GluN} 2 \mathrm{~B}(\mathrm{P}=8.1 \times 10-4)$ at superficial dorsal 
horn synaptosomes, while treatment with acetazolamide alone had no significant effects on these targets compared to saline-treated slices ( $\mathrm{n}=8$ per group; Fig. 3A and Supplementary Table 3 ). The effects of BDNF-mediated disinhibition on synaptic NMDARs were restricted to GluN2B, as neither total GluN2A nor tyrosine phosphorylated $\left(\mathrm{pY}^{1325}\right)$ GluN2A were altered in any of the treatment groups (Fig. 3A). Importantly, co-treatment of acetazolamide with BDNF prevented the decrease in total $\mathrm{STEP}_{61}$ at superficial dorsal horn synaptosomes compared to sections treated with BDNF alone ( $\mathrm{n}=8, \mathrm{P}=0.0047$; Fig. 3A). Thus, blocking KCC2-dependent disinhibition prevents the downregulation of $\mathrm{STEP}_{61}$ and subsequent increase in potentiated GluN2B NMDARs by Fyn at superficial dorsal horn synaptosomes in the ex vivo BDNF model of pathological pain processing. 
A
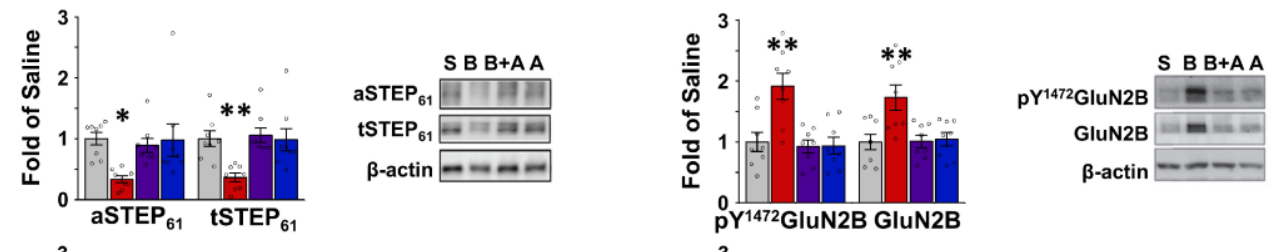

BDNF (B)

$B D N F+A C T Z(B+A)$
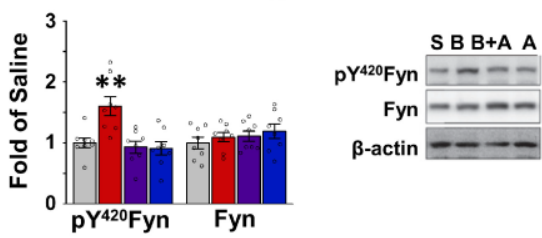

Fyn - - -

$\beta$-actin
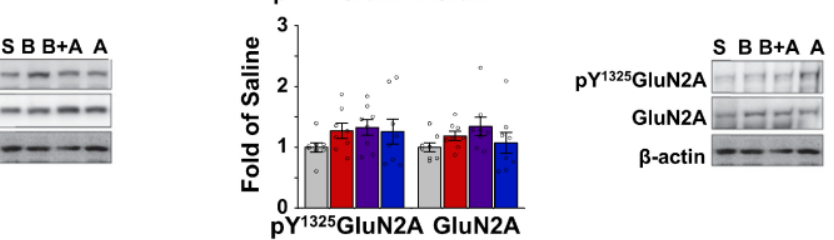

B
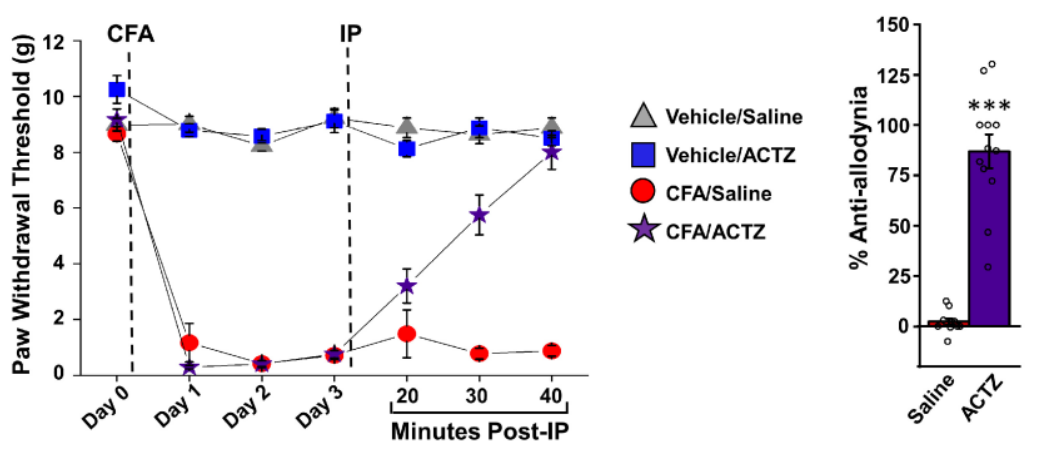

Chapter 2 Figure 3. Blocking KCC2-dependent disinhibition attenuates STEP 61 downregulation by BDNF at superficial dorsal horn synapses and reverses CFA-mediated tactile allodynia. A) Plots and representative western blots from synaptosome fractions of superficial dorsal horn treated with (from left to right) either saline (grey), BDNF (red), BDNF and acetazolamide (ACTZ) to block KCC2-dependent disinhibition (Lee and Prescott, 2015) (purple), or acetazolamide alone (blue) for $70 \mathrm{~min}(\mathrm{n}=8$ animals/group. For quantification, all targets were normalized to beta actin as a loading control. B) Left: Ipsilateral paw withdrawal threshold, in grams, of rats before (Day 0 ) and then 24, 48, and 72 (Day 3 ) h following a hindpaw injection of either vehicle or CFA. After the Day 3 behaviour testing, either $300 \mathrm{mg} / \mathrm{kg}$ acetazolamide or control saline were injected intraperitoneally with behaviour testing 20,30, and 40 min post-intraperitoneal injection [ $\mathrm{n}=8$ for vehicle/saline (grey) and vehicle/acetazolamide (blue), $\mathrm{n}=11$ for CFA/saline (red), and $\mathrm{n}=12$ for CFA/acetazolamide (purple)]. Right: Per cent anti-allodynia (reversal of the CFA-dependent decrease in paw withdrawal threshold) 40 min post-intraperitoneal injection of either saline or acetazolamide in CFA-injected rats. ${ }^{*} \mathrm{P}<0.05,{ }^{*} \mathrm{P}<0.01,{ }^{* * *} \mathrm{P}<0.001$ compared to saline; exact P-values can be found in Supplementary Table 3. 
We reasoned that if disinhibition is required for a conserved feed-forward pathological pathway, then acetazolamide should also reverse behavioural hypersensitivity induced by persistent inflammation. In CFA-injected rats, administration of acetazolamide significantly reversed the decrease in ipsilateral paw withdrawal threshold $\left(n=9, P=4.3 \times 10^{-7}\right)$, while no reversal effect was observed in saline-treated CFA rats $(n=9, P=0.22$; Fig. 3B). From the totality of our past (Xu et al., 2015a; Azkona et al., 2016; Hildebrand et al., 2016; Saavedra et al., 2016) and present evidence, we conclude that loss of $\mathrm{STEP}_{61}$ links BDNF/KCC2-dependent disinhibition to the potentiation of GluN2B NMDARs at lamina I synapses in a pathological spinal mechanism that is conserved between chronic inflammatory and neuropathic pain states.

With the exception of a study revealing increased glial activation in the spinal cord of a patient with neuropathic pain (Del Valle et al., 2009), very little is known regarding mechanisms of central sensitization in the human spinal cord. To compound this problem, typical human postmortem studies rely on delayed tissue collection from autopsy specimens, with associated molecular and cellular degradation. Here we collected lumbar spinal cord tissue from adult male neurological determination of death organ donors within 1 to $3 \mathrm{~h}(114 \pm 25 \mathrm{~min}, \mathrm{n}=14)$ after aortic cross-clamping, thereby maximizing tissue viability (Fig. 4A). Tissue from donors that had spinal cord damage or medical conditions that could impact spinal pain processing or that were taking chronic pain medications were excluded from the study. 
A

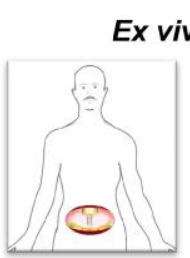

B

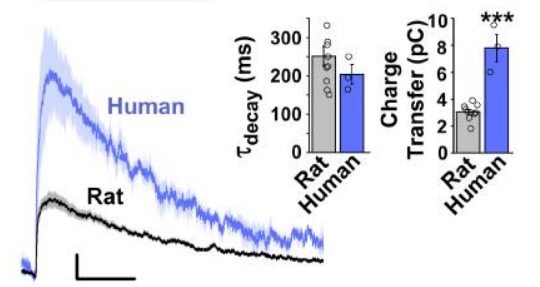

C

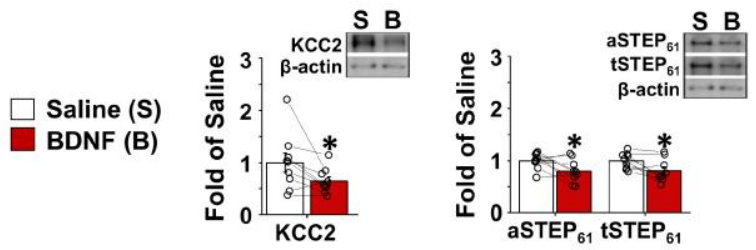

S B

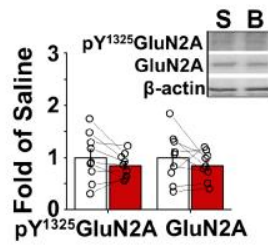

D
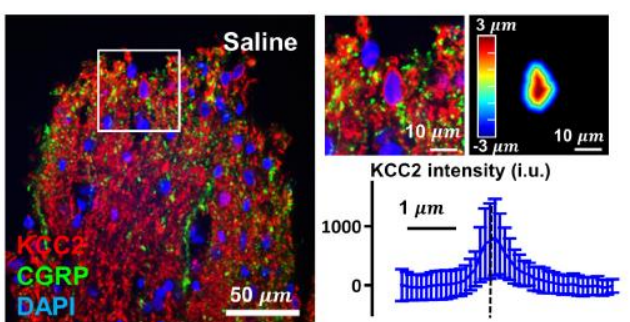

E
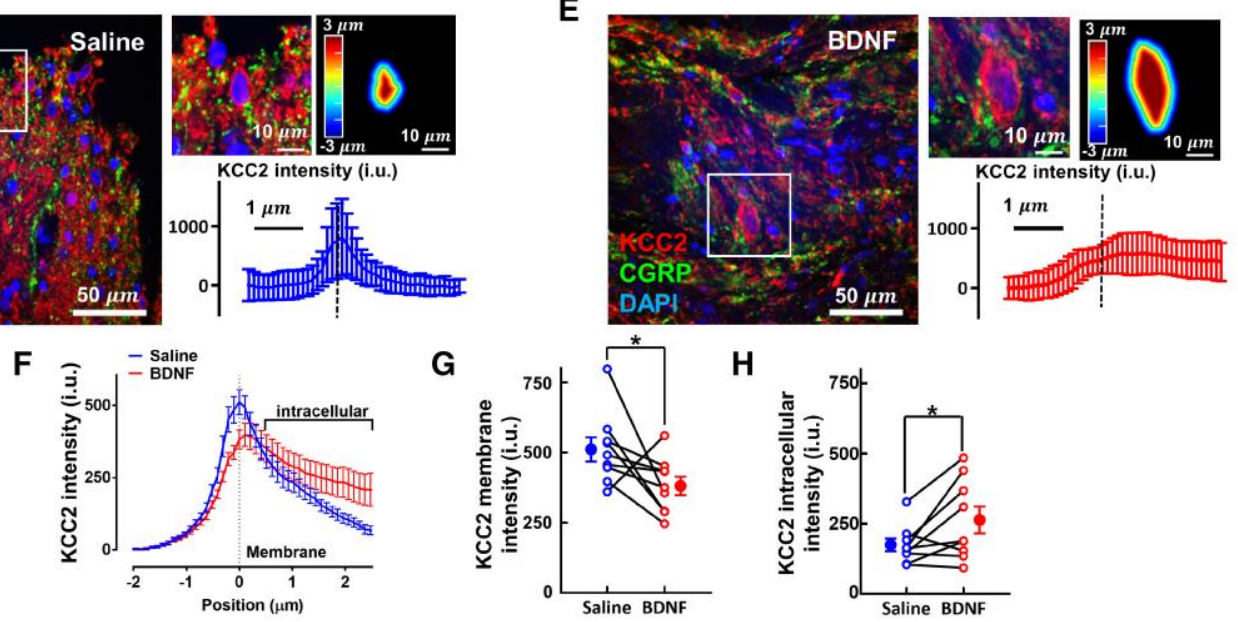

G

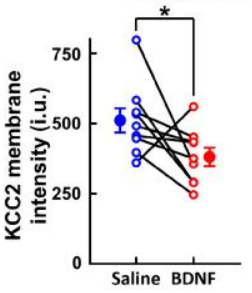

H

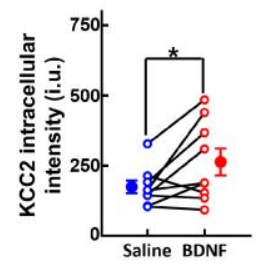

Chapter 2 Figure 4. The ex vivo BDNF treatment model of pathological pain processing drives a downregulation of KCC2 and STEP $_{61}$ and an increase in pFyn and pGluN2B NMDARs at superficial dorsal horn synapses of viable adult human spinal cord. A) Illustration of the human ex vivo BDNF model, highlighting the lumbar section (window). B) Average NMDAR mEPSCs at $+60 \mathrm{mV}$ (left) from lamina I neurons of human (18 and 52 years old) lumbar or thoracic spinal cord ( $\mathrm{n}=3$ neurons) or naïve adult rat lumbar spinal cord $(\mathrm{n}=8$ neurons), with associated decay constant (middle) and charge transfer (right) values. Scale bars $=100 \mathrm{~ms}$ ( $\mathrm{x}$-axes); 5 pA (y-axes). C) Plots and representative western blots from synaptosome fractions of human (30 to 70 years old) superficial dorsal horn treated with either control saline (white, $\mathrm{n}=9$ ) or $100 \mathrm{ng} / \mathrm{ml}$ recombinant BDNF for $70 \mathrm{~min}$ (red, $\mathrm{n}=9$ ). For quantification, all targets were normalized to beta actin as a loading control. (D and $\mathbf{E})$ Left: Representative confocal image of the human superficial dorsal horn incubated in saline (D) or BDNF (E). KCC2 (red), CGRP (green) and DAPI (blue). A zoomed region (top middle) shows a neuron expressing KCC2 together with the delineation of the membrane and the distance to the membrane of each pixel analyzed in a colour-coded distance map (top right) and $\mathrm{KCC} 2$ intensity versus distance to the membrane profile (bottom). (F) Average KCC2 
intensity profiles from superficial dorsal horn neurons incubated in saline (blue) versus BDNF-treated (red) spinal segments of nine male human donors (18-64 years old). (G and H) Averaged membrane KCC2 intensity values (G) and averaged intracellular KCC2 intensity values $(\mathrm{H})$ of superficial dorsal horn spinal cord neurons incubated in saline versus BDNF. ${ }^{*} \mathrm{P}<0.05$; exact $\mathrm{P}$-values can be found in Supplementary Table 3.

We recorded excitatory synaptic responses from lamina I neurons within transverse sections of human (18- and 52-year-old males; see Supplementary Table 1) spinal cord. NMDAR mEPSCs were significantly $(\mathrm{P}=7.0 \times 10-6)$ larger at adult human lamina I synapses $(\mathrm{n}=3)$ compared to adult rodent synapses $(\mathrm{n}=10)$, with amplitudes and total charge that were more than double that of rodents (Fig. 4B). The decay constant of $204 \pm 25 \mathrm{~ms}(\mathrm{n}=3)$ for NMDAR responses at human lamina I synapses was not significantly different from rodent lamina I synapses $(252 \pm 26, \mathrm{n}=10, \mathrm{P}=0.36)$, and is consistent with GluN2B-mediated NMDAR responses (Hildebrand et al., 2014) (Fig. 4B). In one recording, we were able to sequentially apply antagonists of GluN2A (10 $\mu \mathrm{M}$ TCN-201; (Hansen et al., 2012)) and then GluN2B (1 $\mu \mathrm{M}$ Ro25-6981; (Fischer et al., 1997)), which revealed a selective sensitivity of human NMDAR mEPSCs to Ro25-6981 but not TCN-201, as well as a GluN2B-like Ro25-6981-sensitive current (decay rate $=203 \mathrm{~ms}$; Supplementary Fig. 3). Thus, lamina I neurons in the collected human spinal tissue are viable and contain robust synaptic NMDAR responses that are partially mediated by GluN2B-containing receptors.

To test whether the disinhibition-STEP ${ }_{61}$ downregulation-NMDAR potentiation feedforward pathway occurs at human lamina I synapses, we developed an ex vivo BDNF treatment model of human pathological pain processing. As carried out for rodents (Fig. 2A; (Coull et al., 
2005; Hildebrand et al., 2016), we pretreated adjacent regions of male human (18 to 69 years old) lumbar spinal tissue with saline versus recombinant BDNF (100 ng/ml) for $70 \mathrm{~min}$ (Fig. 4A and Supplementary Table 1). Following this, we flash-froze the treated spinal segments and tested for BDNF-mediated changes in expression and phosphorylation of synaptosomal proteins in the superficial dorsal horn versus the remainder of the human spinal cord. Consistent with the rodent ex vivo BDNF model (Fig. 1D), we found that BDNF treatment resulted in a significant decrease in $\operatorname{KCC} 2(\mathrm{P}=0.044)$, total $\operatorname{STEP}_{61}(\mathrm{P}=0.019)$ and active $\mathrm{STEP}_{61}(\mathrm{P}=0.048)$ and a significant increase in active Fyn $(\mathrm{P}=0.040)$ and $\mathrm{pY}^{1472} \mathrm{GluN2B}(\mathrm{P}=0.031)$ at human superficial dorsal horn synaptosomes ( $\mathrm{n}=9$; Fig. 4C, Supplementary Table 3 and Supplementary Fig. 4). This BDNF-mediated pathway was restricted to superficial dorsal horn synaptosomes, as it was not observed in the homogenate fraction of superficial dorsal horn tissue nor at synaptosomes from the remainder of the spinal cord (Supplementary Figs 5 and 6, respectively). Further, we investigated the effect of BDNF on neuronal $\mathrm{KCC} 2$ in human ex vivo spinal cords of nine male subjects aged from 18 to 64 years old (Fig. 4D-H, Supplementary Fig. 7 and Supplementary Table 1). Superficial dorsal horn neurons were specifically examined based on CGRP immunostaining (Fig. 4D and E). Treatment of human spinal sections with BDNF induced a significant decrease in $\mathrm{KCC} 2$ immunostaining at neuronal membranes compared to saline-treated controls (Fig. 4D-G). The BDNF-mediated decrease in membrane KCC2 was paired with a concomitant increase in intracellular $\mathrm{KCC} 2$ immunostaining (Fig. 4H). Finally, BDNF treatment increased the proportion of superficial dorsal horn neurons that had KCC2 primarily localized to the intracellular compartment compared to saline-treated tissue (36/108 versus $8 / 116$ respectively, $\chi 2$ test $=61.71$; Supplementary Fig. 7 ). 


\subsection{Discussion}

We have discovered that $\mathrm{STEP}_{61}$ is the molecular brake that is lost following BDNFmediated disinhibition at lamina I synapses and subsequently drives the increase in excitatory GluN2B NMDAR responses by BDNF in both rodent and human spinal cords. The coupling between KCC2-dependent disinhibition and NMDAR potentiation is conserved between nerve injury (Hildebrand et al., 2016) and inflammatory models of chronic pain. We therefore propose that this spinal pathway represents a core pathophysiological mechanism that may drive pain hypersensitivity across divergent pain syndromes. Indeed, pharmacological, genetic and biochemical studies have demonstrated that key elements of the pathway-BDNF, KCC2dependent disinhibition, and GluN2B NMDARs — are essential mediators of pain hypersensitivity in other models of chronic pain, including spinal cord injury (Boulenguez et al., 2010; Hama and Sagen, 2012; Kim et al., 2012) and cancer-induced bone pain (Wang et al., 2012; Bao et al., 2014; Hou et al., 2018). However, as a putative molecular hub for spinal pain pathology, heterogeneous signalling factors and mechanisms may differentially interact with the pathway's components. These divergence points mean that no individual molecular player or target is likely to represent a therapeutic solution for all chronic pain syndromes, which may include underlying neuropathic and/or inflammatory mechanisms (Ratté and Prescott, 2016). For example, BDNF is proposed to be primarily released from primary nociceptive afferents following persistent inflammation (Mannion et al., 1999; Zhao et al., 2006; Sikandar et al., 2018) and from activated microglia following nerve injury (Coull et al., 2005; Ulmann et al., 2008), and microglia themselves only mediate lamina I hyperexcitability in the male spinal cord while other immune cells are proposed to drive hyperexcitability in the female spinal cord (Sorge et al., 
2015). Discriminating between divergent and convergent pain signalling elements will be needed to develop generalized versus syndrome-specific therapeutic strategies.

Given the plethora of molecular targets that continue to emerge as spinal mechanisms of pain are investigated, an essential missing step in the clinical development of novel therapeutics is to validate which candidates contribute to human spinal pain pathology. To address this, we have developed an ex vivo human BDNF treatment model of pathological pain processing that is based on an established approach for rodent spinal cord. In the rodent model, ex vivo BDNF treatment induces the same changes in protein composition and excitatory responses at lamina I synapses as that observed in the in vivo rodent models of chronic inflammatory and neuropathic pain (Coull et al., 2005; Hildebrand et al., 2016). Similar to this ex vivo rodent model, we are able to collect human spinal tissue that is neuro-protected right up until the point of collection. Using sectioned human dorsal horn tissue, we have characterized functional excitatory synaptic responses from individual lamina I neurons and have shown that phosphorylation-dependent intracellular signalling pathways can be activated by BDNF ex vivo, supporting the conclusion that the dorsal horn nociceptive network remains highly viable. Indeed, by combining the human ex vivo $\mathrm{BDNF}$ model with rodent ex vivo and in vivo pain models, we have shown that $\mathrm{STEP}_{61}$ is the molecular determinant that is lost to drive coupling between BDNF-mediated disinhibition and facilitation of GluN2B NMDARs at both rodent and human lamina I synapses. Future experiments will test what molecular players link $\mathrm{KCC} 2$-dependent disinhibition to decreased STEP $_{61}$ activity, and whether the role of candidates such as PKA (Xu et al., 2015b; Poddar et al., 2016) are conserved in pathological coupling mechanisms between rodent and human pain models. Using this approach, we can validate the role of identified molecular candidates in 
human spinal tissue and then test how targeting these players in vivo impacts pain hypersensitivity in rodent models of specific pain syndromes.

\subsection{Acknowledgements}

We thank Drs. Tuan Trang and Hongyu Sun for providing critical feedback on the manuscript. We thank the families that consented to donation of their loved one's spinal tissue for research; this gift is greatly appreciated. We also thank Dr. Suzan Chen and Lei Zhou, our clinical research coordinators, for their help organizing the human tissue collection, and Dr. Diana Ghinda and the operating room staff at The Ottawa Hospital for aiding with human tissue collection. 


\subsection{Supplementary Figures and Tables}
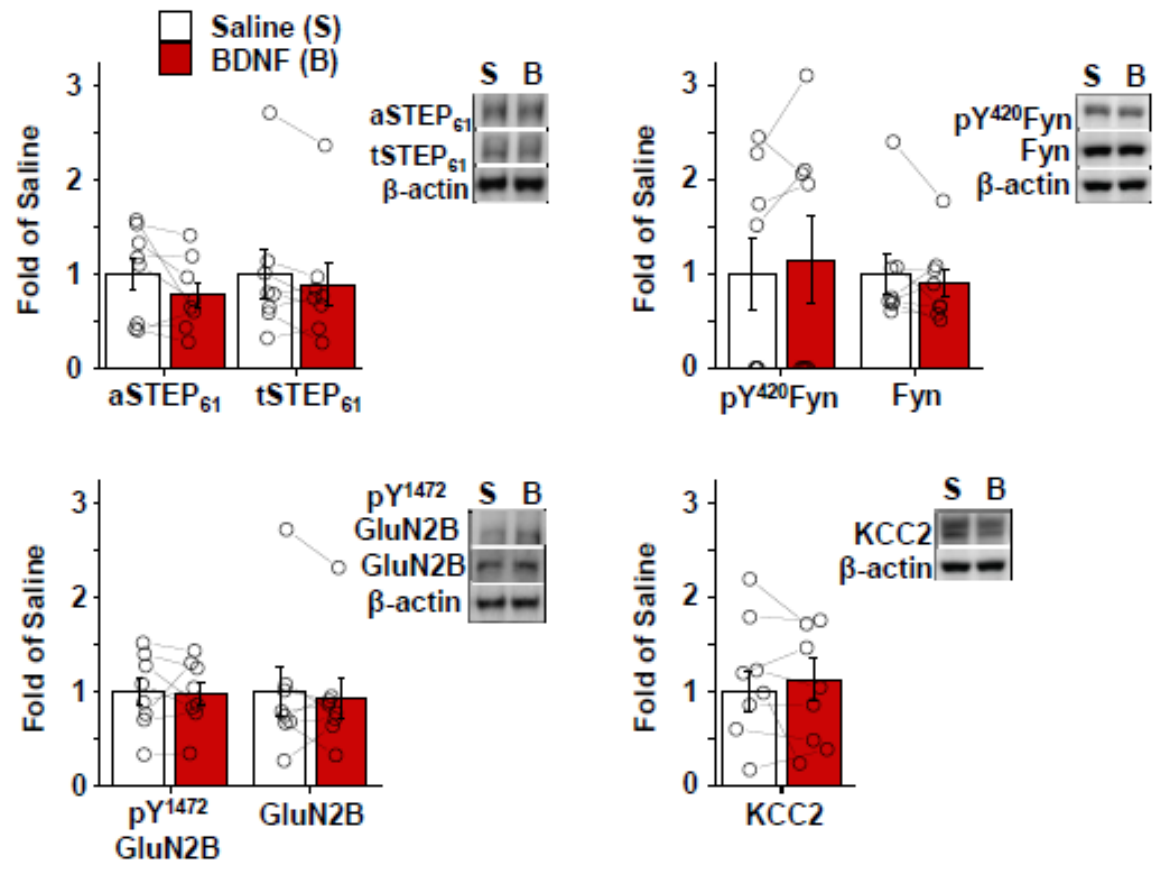

Chapter 2 Supplementary Figure 1. ex vivo BDNF treatment model elicits no effect in SDH homogenate.

Plots (left) and representative western blots (right) from rat SDH homogenate treated with either control saline (white, $\mathrm{n}=8$ ) or $50 \mathrm{ng} / \mathrm{mL}$ recombinant $\mathrm{BDNF}$ for 70 minutes (red, $\mathrm{n}=8$ ). 

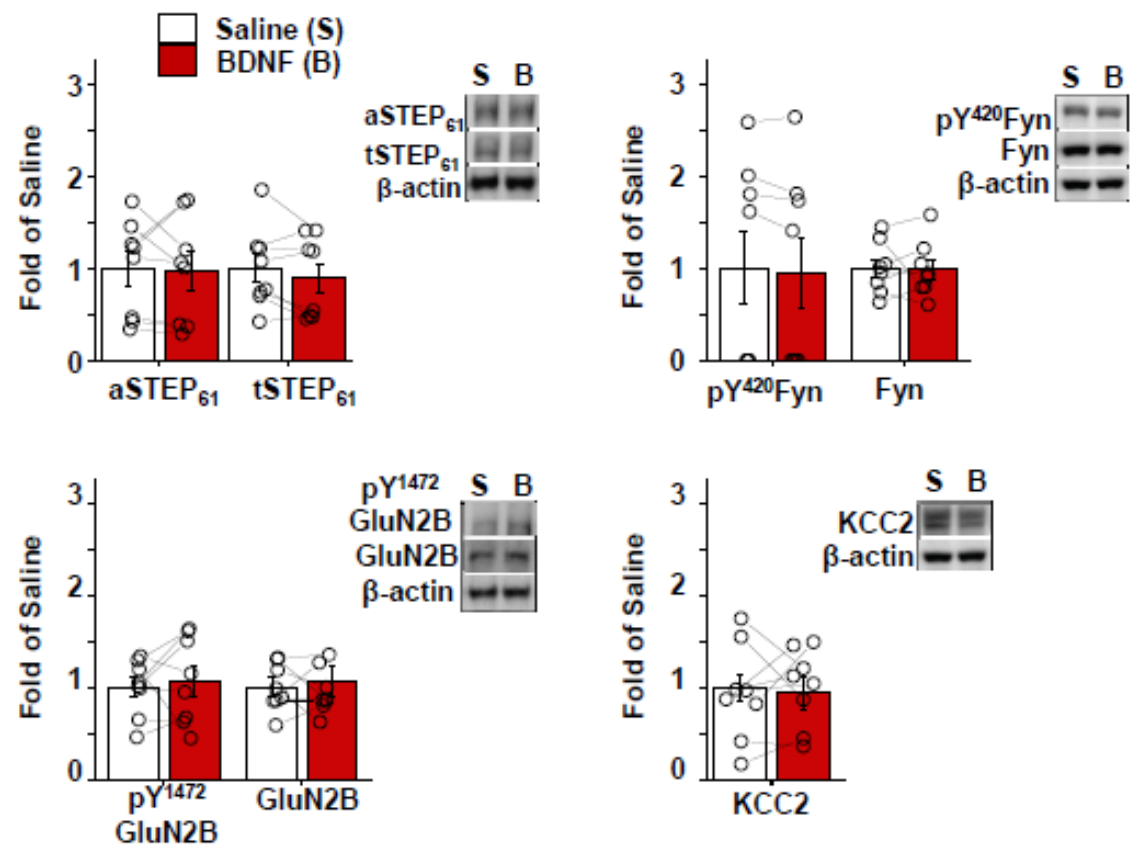

Chapter 2 Supplementary Figure 2. Ex vivo BDNF treatment model elicits no effect in non-SDH synaptosome fractions. Plots (left) and representative western blots (right) from rat non-SDH synaptosome fractions treated with either control saline (white, $\mathrm{n}=8$ ) or $50 \mathrm{ng} / \mathrm{mL}$ recombinant BDNF for 70 minutes (red, $\mathrm{n}=8$ ). Tissue was collected from the remainder of the spinal cord, after the SDH was removed.

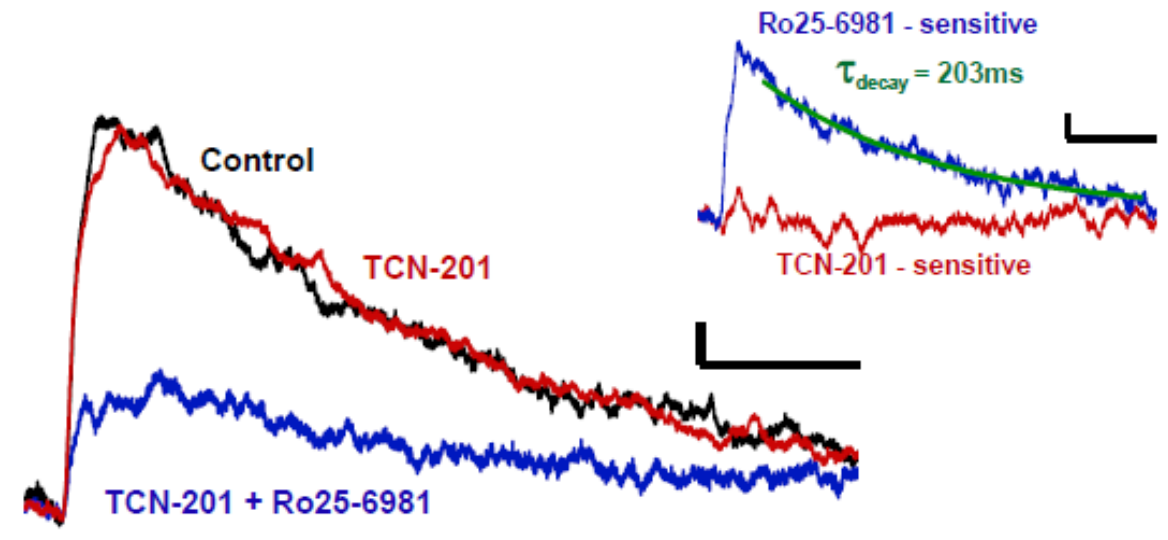

Chapter 2 Supplementary Figure 3. GluN2B dominates human lamina I mEPSCs. Left, NMDAR mEPSCs from a human lamina I neuron treated with a GluN2A antagonist (10 $\mu \mathrm{M}$ TCN-201; red trace) for 12 minutes followed by co-administration of a GluN2B antagonist (1 $\mu \mathrm{M}$ Ro25-6981; blue trace) for 22 minutes. Right, 
difference currents for the component of NMDAR mEPSCs altered by TCN-201 (red) and Ro25-6981 (blue). n=1.

\begin{tabular}{|c|c|c|c|c|c|c|c|c|c|c|}
\hline & & & & & & N2A & ICC2 & STEP & tSTEP & actin \\
\hline & B & & V B & V B & V B & V B & V B & V B & V B & V B \\
\hline P1 & - & & -1 & $=$ & & - & & n m & $=m$ & - \\
\hline P2 & & - & -1 & -1 & & -- & a ra & 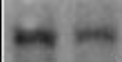 & - & - \\
\hline P3 & 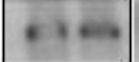 & 68 & +4 & $=0$ & 1 & 64 & B日e & ㅂ. & $\theta \rightarrow$ & \\
\hline P4 & 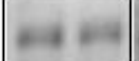 & $m$ & |ter & & & & 64 & HA & $B A$ & $\approx-$ \\
\hline P5 & -- & - & $7=$ & & . & & & - & & 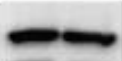 \\
\hline P6 & $-\infty$ & - & witis & & & $x_{1}$ & 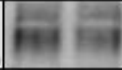 & -1. & & $-\infty$ \\
\hline P7 & $=$ & $=$ & $-1-1$ & $=$ & 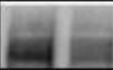 & 배무 & 目成 & -- & & $-\infty$ \\
\hline P9 & $=-$ & $=$ & 19 & $-1-$ & & & EE & $-m$ & -- & -0 \\
\hline P10 & $=-1$ & -- & & -- & & -1 & & -- & -- & -- \\
\hline
\end{tabular}

Chapter 2 Supplementary Figure 4. Western blots used for quantification in Figure 4C. Each row contains all samples quantified for each patient. Vehicle is abbreviated as ' $\mathrm{V}$,' on the left, while BDNF as 'B' on the right. 

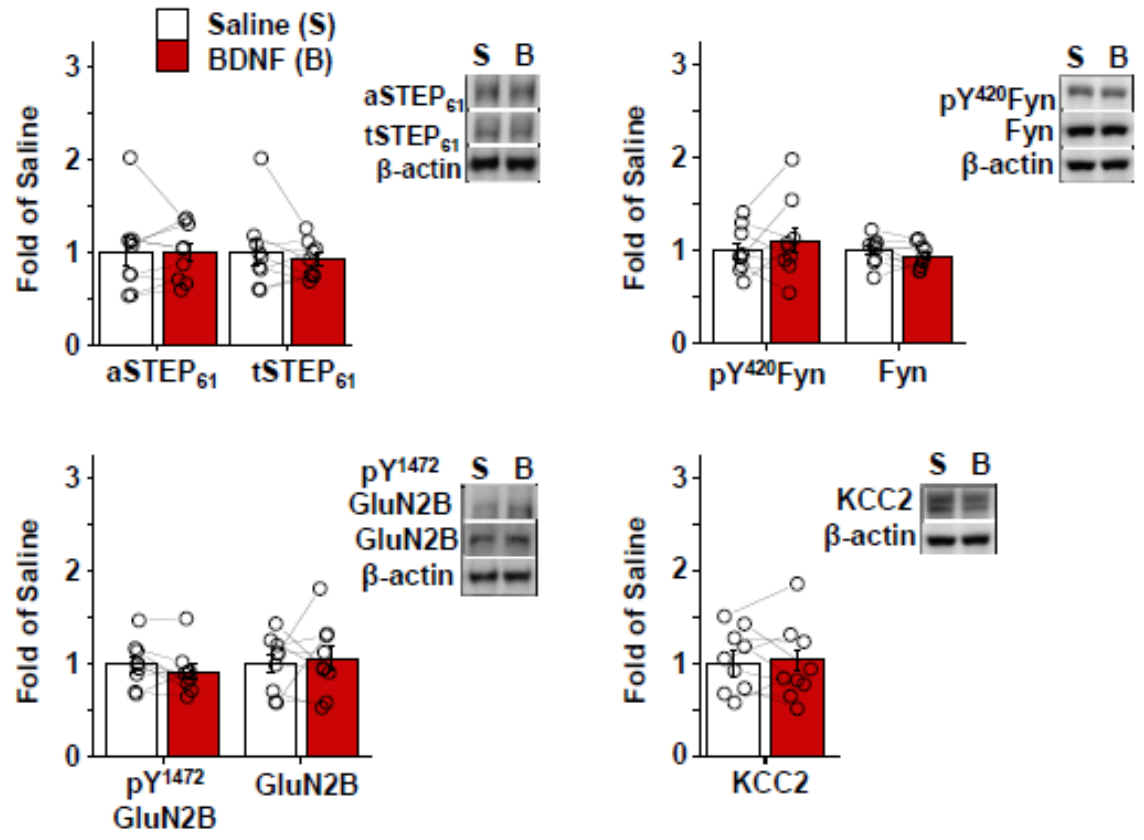

Chapter 2 Supplementary Figure 5. Ex vivo BDNF treatment model elicits no effect in human SDH homogenate. Plots (left) and representative western blots (right) from human SDH homogenates treated with either control saline (white, $\mathrm{n}=9$ ) or $50 \mathrm{ng} / \mathrm{mL}$ recombinant BDNF for 70 minutes (red, $\mathrm{n}=9$ ).
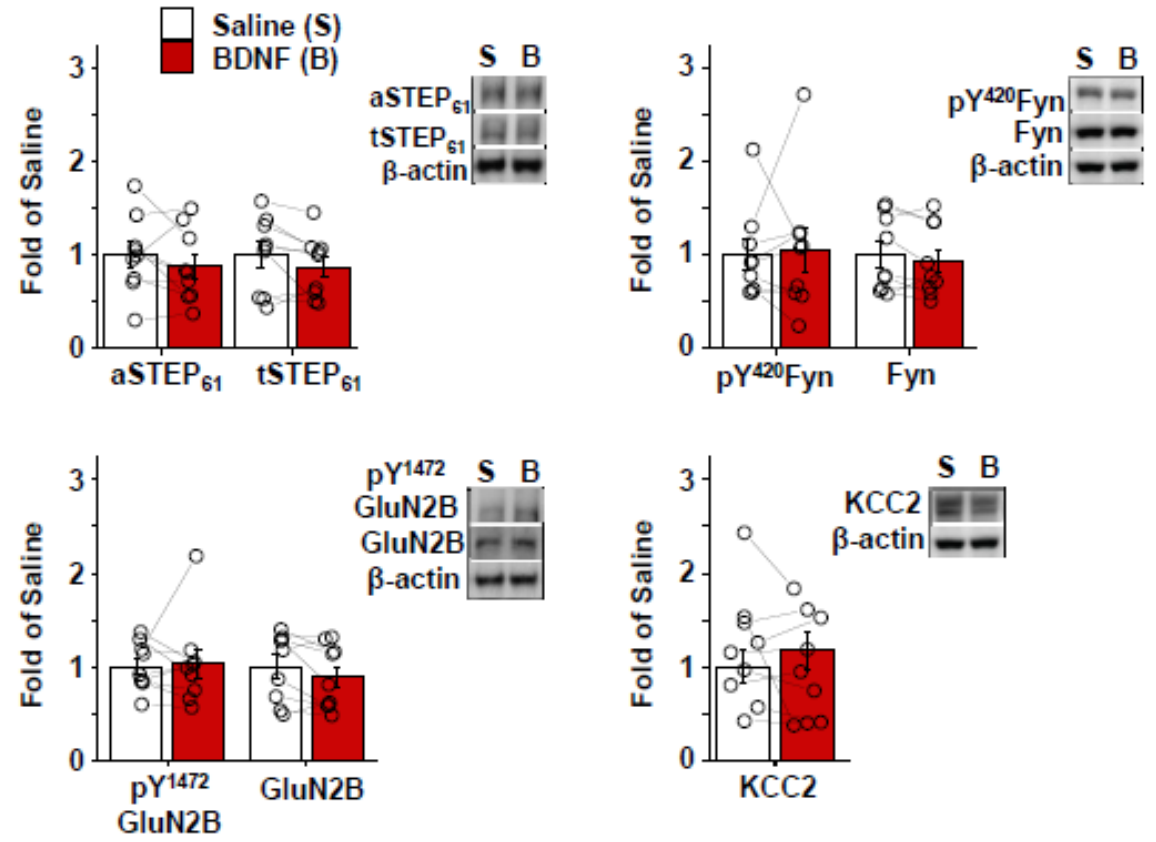

Chapter 2 Supplementary Figure 6. Ex vivo BDNF treatment model elicits no effect in human non-SDH synaptosome fractions. Plots (left) and representative western blots (right) from human non-SDH synaptosome 
fractions treated with either control saline (white, $\mathrm{n}=9$ ) or $50 \mathrm{ng} / \mathrm{mL}$ recombinant BDNF for 70 minutes (red, $\mathrm{n}=9$ ). Tissue was collected from the remainder of the spinal cord, after the SDH was removed.
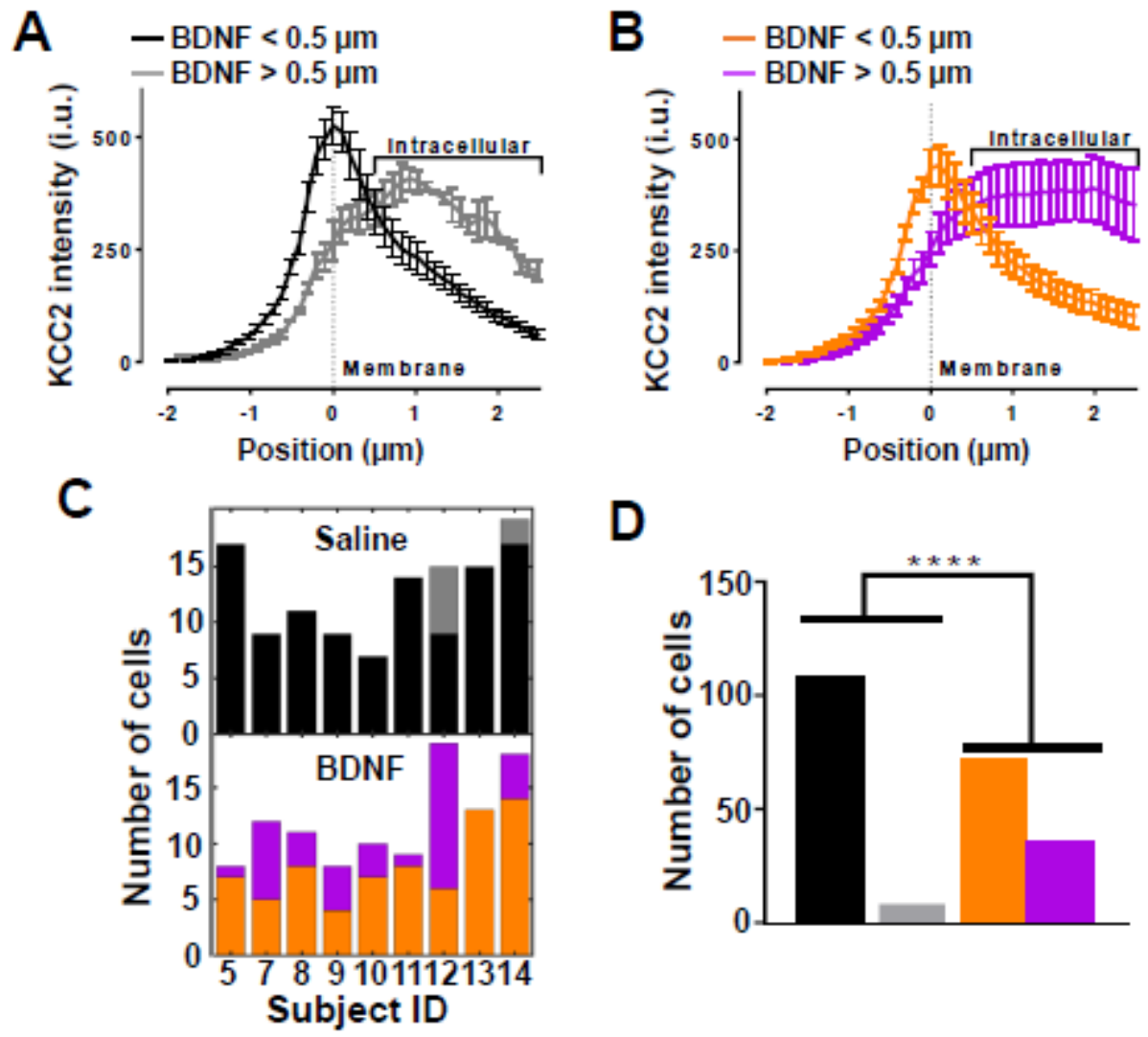

Chapter 2 Supplementary Figure 7. (A,B) SDH neurons were also separated into two groups: the neurons in which KCC2 expression peaked close to the membrane delineation (from 0 to $0.5 \mu \mathrm{m}$ ) or after $(>0.5 \mu \mathrm{m})$ in saline or BDNF conditions. (C) Distributions of neurons analyzed for each human subject in this study. 108 (black) of 116 neurons incubated in saline, compared to 72 neurons (orange) out of 108 neurons incubated in BDNF, were part of the $<0.5 \mu \mathrm{m}$ group. For saline, 8/116 neurons (grey) displayed a peak after $0.5 \mu \mathrm{m}$ and for BDNF 36/108 neurons (purple) displayed a peak after $0.5 \mu \mathrm{m}-($ Chi-2 test=61.71). D) KCC2 intensity values present at the neuron membrane $(0 \mu \mathrm{m})$ and in the intracellular compartment $(0.5 \mu \mathrm{m}<$ position $<2.5 \mu \mathrm{m})$ for the 4 groups presented in $(\mathrm{A}-\mathrm{C}) * * * * \mathrm{p}<0.0001$ 
Chapter 2 Supplementary Table 1. Use of tissue samples collected from human organ donors

\begin{tabular}{|l|l|l|}
\hline Donor & Age & Experiments Performed \\
\hline P1 & 56 & Western Blotting \\
\hline P2 & 42 & Western Blotting \\
\hline P3 & 53 & Western Blotting \\
\hline P4 & 69 & Western Blotting \\
\hline P5 & 33 & $\begin{array}{l}\text { Western Blotting, KCC2 } \\
\text { imaging }\end{array}$ \\
\hline P6 & 60 & Western Blotting \\
\hline P7 & 63 & $\begin{array}{l}\text { Western Blotting, KCC2 } \\
\text { imaging }\end{array}$ \\
\hline P8 & 22 & KCC2 imaging \\
\hline P9 & 52 & $\begin{array}{l}\text { Western Blotting, } \\
\text { Electrophysiology, KCC2 } \\
\text { imaging }\end{array}$ \\
\hline P10 & 64 & $\begin{array}{l}\text { Western Blotting, KCC2 } \\
\text { imaging }\end{array}$ \\
\hline P11 & $\begin{array}{l}\text { Electrophysiology, KCC2 } \\
\text { imaging }\end{array}$ \\
\hline P12 & 18 & KCC2 imaging \\
\hline P13 & 58 & KCC2 imaging \\
\hline P14 & 53 & KCC2 imaging \\
\hline Average Age: & 55 & \\
\hline & 50 & \\
\hline
\end{tabular}

Chapter 2 Supplementary Table 2. Antibodies used for western blots

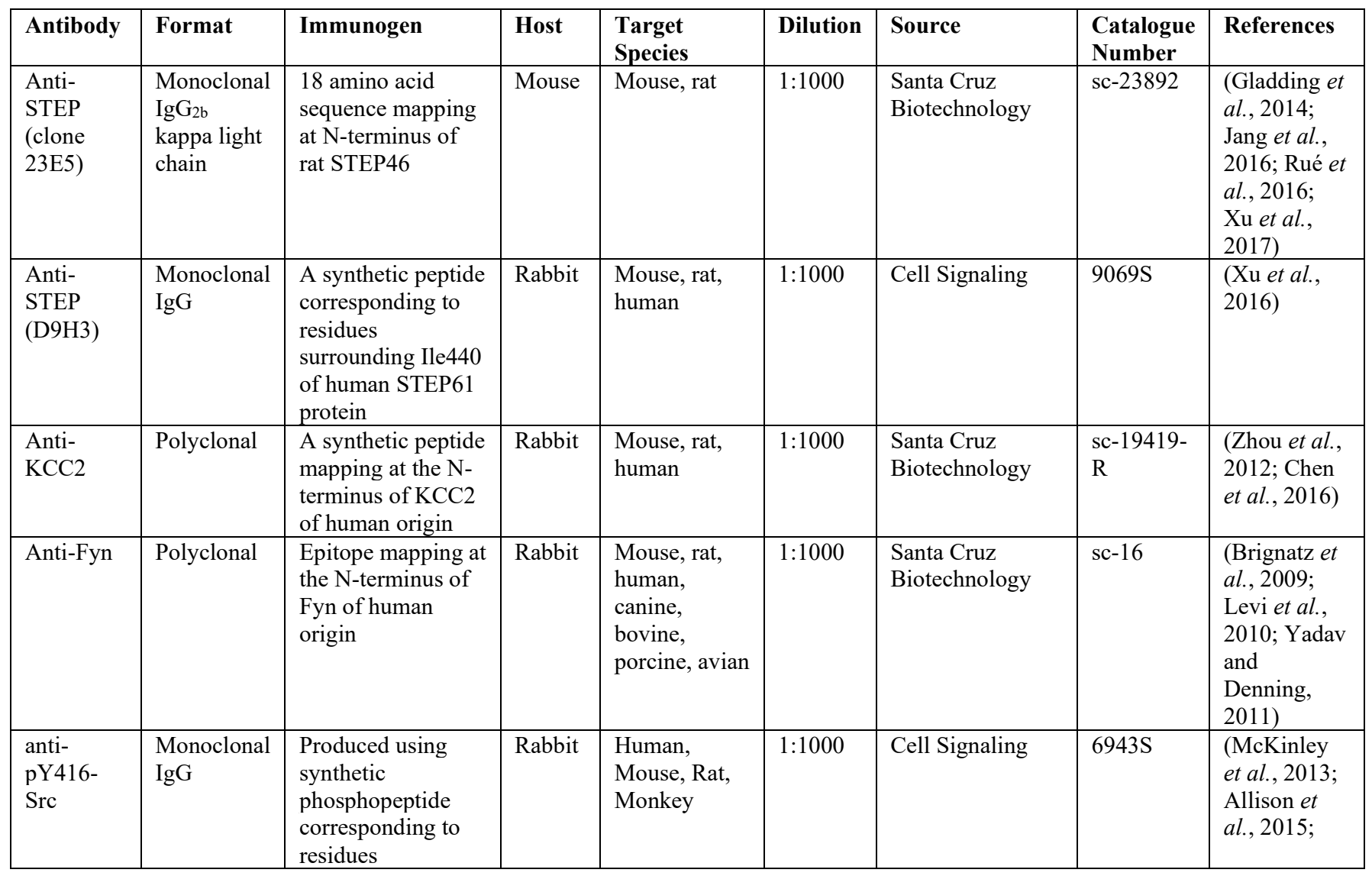




\begin{tabular}{|c|c|c|c|c|c|c|c|c|}
\hline & & $\begin{array}{l}\text { surrounding } \\
\text { Tyr419 of human } \\
\text { Src protein. } \\
\text { Detects } \\
\text { endogenous levels } \\
\text { of Src only when } \\
\text { phosphorylated at } \\
\text { Tyr416. May } \\
\text { cross-react with } \\
\text { other Src family } \\
\text { members (Lyn, } \\
\text { Fyn, Lck, Yes and } \\
\text { Hck) when } \\
\text { phosphorylated at } \\
\text { equivalent sites. } \\
\text { May cross react } \\
\text { with overexpressed } \\
\text { phosphorylated } \\
\text { RTKs. }\end{array}$ & & & & & & $\begin{array}{l}\text { Bieerkehazhi } \\
\text { et al., 2017) }\end{array}$ \\
\hline $\begin{array}{l}\text { anti-non- } \\
\text { phospho- } \\
\text { STEP }\end{array}$ & $\begin{array}{l}\text { Monoclonal } \\
\text { IgG }\end{array}$ & $\begin{array}{l}\text { Produced using } \\
\text { synthetic } \\
\text { nonphosphopeptide } \\
\text { corresponding to } \\
\text { residues } \\
\text { surrounding } \\
\text { Ser221 of human } \\
\text { STEP61 protein. } \\
\text { Detects STEP61 } \\
\text { protein only when } \\
\text { dephosphorylated } \\
\text { at Ser221 and of } \\
\text { STEP46 protein } \\
\text { when } \\
\text { dephosphorylated } \\
\text { at Ser49 }\end{array}$ & Rabbit & $\begin{array}{l}\text { Human, } \\
\text { Mouse, Rat }\end{array}$ & $1: 1000$ & $\begin{array}{l}\text { Cell Signaling } \\
\text { Technology }\end{array}$ & $5659 \mathrm{~S}$ & $\begin{array}{l}\text { (Castonguay } \\
\text { et al., 2018) }\end{array}$ \\
\hline $\begin{array}{l}\text { anti- } \\
\text { pY } \\
\text { GluN2B }\end{array}$ & Polyclonal & $\begin{array}{l}\text { Affinity Purified } \\
\text { from Pooled } \\
\text { Serum. } \\
\text { Phosphopeptide } \\
\text { corresponding to } \\
\text { amino acid } \\
\text { residues } \\
\text { surrounding the } \\
\text { phospho-Tyr1472 } \\
\text { of NMDA NR2B. }\end{array}$ & Rabbit & $\begin{array}{l}\text { Rat, Mouse, } \\
\text { Human, } \\
\text { Bovine, } \\
\text { Chicken, } \\
\text { Non-human } \\
\text { primate, } \\
\text { Zebra fish, } \\
\text { Canine }\end{array}$ & $1: 1000$ & PhosphoSolutions & $\begin{array}{l}\text { p1516- } \\
1472\end{array}$ & $\begin{array}{l}\text { (Castillo et } \\
\text { al., 2011; } \\
\text { Jang et al., } \\
2016)\end{array}$ \\
\hline $\begin{array}{l}\text { anti- } \\
\text { pY' } \\
\text { GluN2A }\end{array}$ & Polyclonal & $\begin{array}{l}\text { Affinity Purified } \\
\text { from Pooled } \\
\text { Serum. } \\
\text { Phosphopeptide } \\
\text { corresponding to } \\
\text { amino acid } \\
\text { residues }\end{array}$ & Rabbit & $\begin{array}{l}\text { Rat, Mouse, } \\
\text { Bovine, } \\
\text { Canine, } \\
\text { Hamster, } \\
\text { Sheep }\end{array}$ & $1: 1000$ & PhosphoSolutions & $\begin{array}{l}\text { p1514- } \\
1325\end{array}$ & $\begin{array}{l}\text { (Staples et } \\
\text { al., 2015; } \\
\text { Takashima } \\
\text { et al., 2018) }\end{array}$ \\
\hline
\end{tabular}




\begin{tabular}{|c|c|c|c|c|c|c|c|c|}
\hline & & $\begin{array}{l}\text { surrounding the } \\
\text { phospho-Tyr1325 } \\
\text { of rat NMDA } \\
\text { NR2A }\end{array}$ & & & & & & \\
\hline $\begin{array}{l}\text { anti- } \\
\text { GluN2B }\end{array}$ & $\begin{array}{l}\text { Monoclonal } \\
\text { IgG }\end{array}$ & $\begin{array}{l}6 \text { His-tagged fusion } \\
\text { protein } \\
\text { corresponding to } \\
\text { amino acids } 1265- \\
1464 \text { of mouse } \\
\text { NMDA receptor } \\
\text { 2A (NR2A) }\end{array}$ & Rabbit & Mouse, Rat & $1: 2000$ & Millipore & $06-600$ & $\begin{array}{l}\text { (Fenster } \text { et } \\
\text { al., 2012; } \\
\text { Wei et al., } \\
\text { 2014) }\end{array}$ \\
\hline $\begin{array}{l}\text { anti- } \\
\text { GluN2A }\end{array}$ & Monoclonal & $\begin{array}{l}\text { Immunogen from } \\
\text { amino acids } 1437- \\
1456 \text { of mature } \\
\text { mouse NR2B or } \\
1463-1482 \text { of } \\
\text { NR2B precursor. } \\
\text { This sequence is } \\
\text { identical to amino } \\
\text { acids } 1437-1456 \text { of } \\
\text { mature rat NR2B } \\
\text { and amino acids } \\
1465-1484 \text { of } \\
\text { human NR3, } \\
\text { containing an N- } \\
\text { terminal lysine. }\end{array}$ & Rabbit & Mouse, rat, & $1: 1000$ & Millipore & $04-901$ & $\begin{array}{l}\text { (Li et al., } \\
2015 ; \\
\text { Cabrera- } \\
\text { Pastor et al., } \\
\text { 2016; Xing } \\
\text { et al., 2018) }\end{array}$ \\
\hline
\end{tabular}

\section{References for Supplementary Table 2:}

Allison P, Espiritu D, Barnett J V., Camenisch TD. Type III TGF $\beta$ receptor and Src direct hyaluronan-mediated invasive cell motility. Cell. Signal. 2015; 27: 453-459.

Bieerkehazhi S, Chen Z, Zhao Y, Yu Y, Zhang H, Vasudevan SA, et al. Novel Src/Abl tyrosine kinase inhibitor bosutinib suppresses neuroblastoma growth via inhibiting Src/Abl signaling. Oncotarget 2017; 8: 1469-1480.

Brignatz C, Paronetto MP, Opi S, Cappellari M, Audebert S, Feuillet V, et al. Alternative splicing modulates autoinhibition and SH3 accessibility in the Src kinase Fyn. Mol. Cell. Biol. 2009; 29: 6438-48.

Cabrera-Pastor A, Hernandez-Rabaza V, Taoro-Gonzalez L, Balzano T, Llansola M, Felipo V. In vivo administration of extracellular cGMP normalizes TNF- $\alpha$ and membrane expression of AMPA receptors in hippocampus and spatial reference memory but not IL-1 $\beta$, NMDA receptors in membrane and working memory in hyperammonemic rats. Brain. Behav. Immun. 2016; 57: $360-370$.

Castillo C, Norcini M, Baquero-Buitrago J, Levacic D, Medina R, Montoya-Gacharna JV, et al. The N-methyl-D-aspartate-evoked cytoplasmic calcium increase in adult rat dorsal root ganglion neuronal somata was potentiated by substance $\mathrm{P}$ pretreatment in a protein kinase C-dependent manner. Neuroscience 2011; 177: 308-320. 
Castonguay D, Dufort-Gervais J, Ménard C, Chatterjee M, Quirion R, Bontempi B, et al. The Tyrosine Phosphatase STEP Is Involved in Age-Related Memory Decline. Curr. Biol. 2018; 28 : 1079-1089.e4.

Chen M, Wang J, Jiang J, Zheng X, Justice NJ, Wang K, et al. APP modulates KCC2 expression and function in hippocampal GABAergic inhibition [Internet]. 2016[cited 2019 Jan 30] Available from: https:/cdn.elifesciences.org/articles/20142/elife-20142-v2.pdf

Fenster C, Vullhorst D, Buonanno A. Acute neuregulin-1 signaling influences AMPA receptor mediated responses in cultured cerebellar granule neurons. Brain Res. Bull. 2012; 87: 21-29.

Gladding CM, Fan J, Zhang LYJ, Wang L, Xu J, Li EHY, et al. Alterations in STriatal-Enriched protein tyrosine Phosphatase expression, activation, and downstream signaling in early and late stages of the YAC128 Huntington's disease mouse model. J. Neurochem. 2014; 130: 145-159.

Jang S-S, Royston SE, Lee G, Wang S, Chung HJ. Seizure-Induced Regulations of Amyloid- $\beta$, STEP $_{61}$, and STEP ${ }_{61}$ Substrates Involved in Hippocampal Synaptic Plasticity. Neural Plast. 2016; 2016: 1-13.

Levi M, Maro B, Shalgi R. The involvement of Fyn kinase in resumption of the first meiotic division in mouse oocytes. Cell Cycle 2010; 9: 1577-1589.

Li M-L, Yang S-S, Xing B, Ferguson BR, Gulchina Y, Li Y-C, et al. LY395756, an mGluR2 agonist and mGluR3 antagonist, enhances NMDA receptor expression and function in the normal adult rat prefrontal cortex, but fails to improve working memory and reverse MK801induced working memory impairment. Exp. Neurol. 2015; 273: 190-201.

McKinley ET, Liu H, McDonald WH, Luo W, Zhao P, Coffey RJ, et al. Global Phosphotyrosine Proteomics Identifies PKC $\delta$ as a Marker of Responsiveness to Src Inhibition in Colorectal Cancer. PLoS One 2013; 8: e80207.

Rué L, Bañez-Coronel M, Creus-Muncunill J, Giralt A, Alcalá-Vida R, Mentxaka G, et al. Targeting CAG repeat RNAs reduces Huntington's disease phenotype independently of huntingtin levels. J. Clin. Invest. 2016; 126: 4319-4330.

Staples MC, Somkuwar SS, Mandyam CD. Developmental effects of wheel running on hippocampal glutamate receptor expression in young and mature adult rats. Neuroscience 2015; 305: 248-256.

Takashima Y, Fannon MJ, Galinato MH, Steiner NL, An M, Zemljic-Harpf AE, et al. Neuroadaptations in the dentate gyrus following contextual cued reinstatement of methamphetamine seeking. Brain Struct. Funct. 2018; 223: 2197-2211.

Ti S-C, Pollard TD. Purification of actin from fission yeast Schizosaccharomyces pombe and characterization of functional differences from muscle actin. J. Biol. Chem. 2011; 286: 5784-92.

Wei J, Yuen EY, Liu W, Li X, Zhong P, Karatsoreos IN, et al. Estrogen protects against the detrimental effects of repeated stress on glutamatergic transmission and cognition. Mol. Psychiatry 2014; 19: 588-598.

Wu Z, Zhu Y, Cao X, Sun S, Zhao B. Mitochondrial Toxic Effects of A $\beta$ Through Mitofusins in the Early Pathogenesis of Alzheimer's Disease. Mol. Neurobiol. 2014; 50: 986-996. 
Xing B, Han G, Wang M-J, Snyder MA, Gao W-J. Juvenile treatment with mGluR2/3 agonist prevents schizophrenia-like phenotypes in adult by acting through GSK3 $\beta$. Neuropharmacology 2018; 137: 359-371.

Xu J, Kurup P, Baguley TD, Foscue E, Ellman JA, Nairn AC, et al. Inhibition of the tyrosine phosphatase STEP61 restores BDNF expression and reverses motor and cognitive deficits in phencyclidine-treated mice. Cell. Mol. Life Sci. 2016; 73: 1503-14.

Xu J, Kurup P, Nairn AC, Lombroso PJ. Synaptic NMDA Receptor Activation Induces Ubiquitination and Degradation of STEP61 [Internet]. Mol. Neurobiol. 2017[cited 2018 Feb 28] Available from: http://www.ncbi.nlm.nih.gov/pubmed/28466270

Yadav V, Denning MF. Fyn is induced by Ras/PI3K/Akt signaling and is required for enhanced invasion/migration. Mol. Carcinog. 2011; 50: 346-352.

Zhou H-Y, Chen S-R, Byun H-S, Chen H, Li L, Han H-D, et al. N-methyl-D-aspartate receptorand calpain-mediated proteolytic cleavage of $\mathrm{K}+-\mathrm{Cl}$ - cotransporter-2 impairs spinal chloride homeostasis in neuropathic pain. J. Biol. Chem. 2012; 287: 33853-64.

Zuo H, Liu Z, Liu X, Yang J, Liu T, Wen S, et al. CD151 gene delivery after myocardial infarction promotes functional neovascularization and activates FAK signaling. Mol. Med. 2009; 15: 307-15.

Chapter 2 Supplementary Table 3. Statistical analyses performed in all Figures

\begin{tabular}{|c|c|c|c|}
\hline Figure and Section & Statistical test & Comparison & $\mathrm{p}$ value \\
\hline 1.A) & Student's unpaired $t$ test & $\begin{array}{l}\text { Vehicle/CFA paw } \\
\text { withdrawal threshold } 3 \\
\text { days post-injection }\end{array}$ & $4.97688 \mathrm{E}-09$ \\
\hline 1.B) & Student's unpaired $t$ test & $\begin{array}{l}\text { Naïve/CFA charge } \\
\text { transfer }\end{array}$ & $2.92086 \mathrm{E}-08$ \\
\hline 1.C) & Student's unpaired t test & Vehicle/CFA - pFyn & 0.032161 \\
\hline 1.C) & Student's unpaired $t$ test & Vehicle/CFA - Fyn & 0.418719 \\
\hline 1.C) & Student's unpaired t test & $\begin{array}{l}\text { Vehicle/CFA - } \\
\text { pGluN2B }\end{array}$ & 0.016426 \\
\hline 1.C) & Student's unpaired t test & $\begin{array}{l}\text { Vehicle/CFA - } \\
\text { GluN2B }\end{array}$ & 0.046825 \\
\hline 1.C) & Student's unpaired $t$ test & Vehicle/CFA - tSTEP & 0.503494 \\
\hline 1.C) & Student's unpaired $t$ test & Vehicle/CFA - aSTEP & 0.035955 \\
\hline 1.C) & Student's unpaired $t$ test & Vehicle/CFA - KCC2 & 0.023393 \\
\hline 1.D) & Student's paired t test & Saline/BDNF - pFyn & 0.001133 \\
\hline 1.D) & Student's paired t test & Saline/BDNF - Fyn & 0.543715 \\
\hline 1.D) & Student's paired $t$ test & $\begin{array}{l}\text { Saline/BDNF - } \\
\text { pGluN2B }\end{array}$ & 0.01621 \\
\hline 1.D) & Student's paired t test & $\begin{array}{l}\text { Saline/BDNF - } \\
\text { GluN2B }\end{array}$ & 0.026334 \\
\hline 1.D) & Student's paired $t$ test & Saline/BDNF - tSTEP & 0.012608 \\
\hline 1.D) & Student's paired $t$ test & Saline/BDNF - aSTEP & 0.013479 \\
\hline 1.D) & Student's paired $t$ test & Saline/BDNF - KCC2 & 0.006359 \\
\hline
\end{tabular}




\begin{tabular}{|c|c|c|c|}
\hline 2.B) & Student's unpaired $t$ test & $\begin{array}{l}\text { BDNF + TAT- } \\
\text { STEP/BDNF + Control } \\
\text { charge transfer }\end{array}$ & 0.000632 \\
\hline 2.B) & Student's unpaired $t$ test & $\begin{array}{l}\text { Saline/BDNF + TAT- } \\
\text { STEP charge transfer }\end{array}$ & 0.283378 \\
\hline 2.C) & Student's unpaired $t$ test & $\begin{array}{l}\text { Saline pretreatment/TC- } \\
2153 \text { pretreatment charge } \\
\text { transfer }\end{array}$ & 0.330171 \\
\hline 2.D) & Student's unpaired $t$ test & $\begin{array}{l}\text { Saline pretreatment, } \\
\text { Baseline/BDNF Perfusion }\end{array}$ & 0.974967 \\
\hline 2.D) & Student's unpaired $t$ test & $\begin{array}{l}\text { TC-2153 pretreatment, } \\
\text { Baseline/BDNF Perfusion }\end{array}$ & 0.027606 \\
\hline 2.E) & $\begin{array}{l}\text { One-way ANOVA, with } \\
\text { Tukey's }\end{array}$ & Saline/BDNF for $\mathrm{pFyn}$ & 0.0038 \\
\hline 2.E) & $\begin{array}{l}\text { One-way ANOVA, with } \\
\text { Tukey's }\end{array}$ & $\begin{array}{l}\text { Saline/BDNF + TAT- } \\
\text { STEP for pFyn }\end{array}$ & 0.9892 \\
\hline 2.E) & $\begin{array}{l}\text { One-way ANOVA, with } \\
\text { Tukey's }\end{array}$ & $\begin{array}{l}\text { Saline } / 1 \mu \mathrm{m} \text { TC-2153 for } \\
\text { pFyn }\end{array}$ & 0.2308 \\
\hline 2.E) & $\begin{array}{l}\text { One-way ANOVA, with } \\
\text { Tukey's }\end{array}$ & $\begin{array}{l}\text { Saline } / 10 \mu \mathrm{m} \text { TC-2153 for } \\
\text { pFyn }\end{array}$ & 0.6587 \\
\hline 2.E) & $\begin{array}{l}\text { One-way ANOVA, with } \\
\text { Tukey's }\end{array}$ & $\begin{array}{l}\text { BDNF/BDNF + TAT- } \\
\text { STEP for pFyn }\end{array}$ & 0.0009942 \\
\hline 2.E) & $\begin{array}{l}\text { One-way ANOVA, with } \\
\text { Tukey's }\end{array}$ & Saline/BDNF for Fyn & 1.0000 \\
\hline 2.E) & $\begin{array}{l}\text { One-way ANOVA, with } \\
\text { Tukey's }\end{array}$ & $\begin{array}{l}\text { Saline/BDNF + TAT- } \\
\text { STEP for Fyn }\end{array}$ & 0.9042 \\
\hline 2.E) & $\begin{array}{l}\text { One-way ANOVA, with } \\
\text { Tukey's }\end{array}$ & $\begin{array}{l}\text { BDNF/BDNF + TAT- } \\
\text { STEP for Fyn }\end{array}$ & 0.9195 \\
\hline 2.E) & $\begin{array}{l}\text { One-way ANOVA, with } \\
\text { Tukey's }\end{array}$ & $\begin{array}{l}\text { Saline/1 } \mu \mathrm{m} \text { TC-2153 for } \\
\text { Fyn }\end{array}$ & 0.9968 \\
\hline 2.E) & $\begin{array}{l}\text { One-way ANOVA, with } \\
\text { Tukey's }\end{array}$ & $\begin{array}{l}\text { Saline } / 10 \mu \mathrm{m} \text { TC-2153 for } \\
\text { Fyn }\end{array}$ & 1.0000 \\
\hline 2.E) & $\begin{array}{l}\text { One-way ANOVA, with } \\
\text { Tukey's }\end{array}$ & $\begin{array}{l}\text { Saline/BDNF for } \\
\text { pGluN2B }\end{array}$ & 0.0029 \\
\hline 2.E) & $\begin{array}{l}\text { One-way ANOVA, with } \\
\text { Tukey's }\end{array}$ & $\begin{array}{l}\text { Saline/BDNF + TAT- } \\
\text { STEP for pGluN2B }\end{array}$ & 0.7975 \\
\hline 2.E) & $\begin{array}{l}\text { One-way ANOVA, with } \\
\text { Tukey's }\end{array}$ & $\begin{array}{l}\text { Saline/1 } \mu \mathrm{m} \text { TC-2153 for } \\
\text { pGluN2B }\end{array}$ & 0.2073 \\
\hline 2.E) & $\begin{array}{l}\text { One-way ANOVA, with } \\
\text { Tukey's }\end{array}$ & $\begin{array}{l}\text { Saline/10 } \mu \mathrm{m} \text { TC-2153 for } \\
\text { pGluN2B }\end{array}$ & 0.6823 \\
\hline 2.E) & $\begin{array}{l}\text { One-way ANOVA, with } \\
\text { Tukey's }\end{array}$ & $\begin{array}{l}\text { BDNF/BDNF + TAT- } \\
\text { STEP for pGluN2B }\end{array}$ & 0.0001127 \\
\hline 2.E) & $\begin{array}{l}\text { One-way ANOVA, with } \\
\text { Tukey's }\end{array}$ & Saline/BDNF for GluN2B & 0.0023 \\
\hline 2.E) & $\begin{array}{l}\text { One-way ANOVA, with } \\
\text { Tukey's }\end{array}$ & $\begin{array}{l}\text { Saline/BDNF + TAT- } \\
\text { STEP for GluN2B }\end{array}$ & 0.9226 \\
\hline 2.E) & $\begin{array}{l}\text { One-way ANOVA, with } \\
\text { Tukey's }\end{array}$ & $\begin{array}{l}\text { BDNF/BDNF + TAT- } \\
\text { STEP for GluN2B }\end{array}$ & 0.0002147 \\
\hline 2.E) & $\begin{array}{l}\text { One-way ANOVA, with } \\
\text { Tukey's }\end{array}$ & $\begin{array}{l}\text { Saline/1 } \mu \mathrm{m} \text { TC-2153 for } \\
\text { GluN2B }\end{array}$ & 0.3479 \\
\hline 2.E) & $\begin{array}{l}\text { One-way ANOVA, with } \\
\text { Tukey's }\end{array}$ & $\begin{array}{l}\text { Saline/10 } \mu \mathrm{m} \text { TC-2153 for } \\
\text { GluN2B }\end{array}$ & 0.4636 \\
\hline 2.E) & $\begin{array}{l}\text { One-way ANOVA, with } \\
\text { Tukey's }\end{array}$ & Saline/BDNF for KCC2 & 0.4326 \\
\hline 2.E) & $\begin{array}{l}\text { One-way ANOVA, with } \\
\text { Tukey's }\end{array}$ & $\begin{array}{l}\text { Saline/BDNF + TAT- } \\
\text { STEP for KCC2 }\end{array}$ & 0.0140 \\
\hline 2.E) & $\begin{array}{l}\text { One-way ANOVA, with } \\
\text { Tukey's }\end{array}$ & $\begin{array}{l}\text { Saline/1 } \mu \mathrm{m} \text { TC-2153 for } \\
\mathrm{KCC} 2\end{array}$ & 0.9998 \\
\hline 2.E) & $\begin{array}{l}\text { One-way ANOVA, with } \\
\text { Tukey's }\end{array}$ & $\begin{array}{l}\text { Saline/10 } \mu \mathrm{m} \text { TC-2153 for } \\
\text { KCC2 }\end{array}$ & 0.8423 \\
\hline
\end{tabular}




\begin{tabular}{|c|c|c|c|}
\hline 2.E) & $\begin{array}{l}\text { One-way ANOVA, with } \\
\text { Tukey's }\end{array}$ & $\begin{array}{l}\text { BDNF/BDNF + TAT- } \\
\text { STEP for KCC2 }\end{array}$ & 0.991 \\
\hline 3.A) & $\begin{array}{l}\text { One-way ANOVA, with } \\
\text { Tukey’s }\end{array}$ & Saline/BDNF for aSTEP & 0.0252 \\
\hline 3.A) & $\begin{array}{l}\text { One-way ANOVA, with } \\
\text { Tukey's }\end{array}$ & $\begin{array}{l}\text { Saline/BDNF + ACTZ for } \\
\text { aSTEP }\end{array}$ & 0.9580 \\
\hline 3.A) & $\begin{array}{l}\text { One-way ANOVA, with } \\
\text { Tukey's }\end{array}$ & Saline/ACTZ for aSTEP & 0.9995 \\
\hline 3.A) & $\begin{array}{l}\text { One-way ANOVA, with } \\
\text { Tukey's }\end{array}$ & Saline/BDNF for tSTEP & 0.0100 \\
\hline 3.A) & $\begin{array}{l}\text { One-way ANOVA, with } \\
\text { Tukey's }\end{array}$ & $\begin{array}{l}\text { ACTZ+BDNF/BDNF for } \\
\text { tSTEP }\end{array}$ & 0.00471 \\
\hline 3.A) & $\begin{array}{l}\text { One-way ANOVA, with } \\
\text { Tukey's }\end{array}$ & $\begin{array}{l}\text { Saline/BDNF + ACTZ for } \\
\text { tSTEP }\end{array}$ & 0.9904 \\
\hline 3.A) & $\begin{array}{l}\text { One-way ANOVA, with } \\
\text { Tukey's }\end{array}$ & Saline/ACTZ for tSTEP & 0.9997 \\
\hline 3.A) & $\begin{array}{l}\text { One-way ANOVA, with } \\
\text { Tukey's }\end{array}$ & Saline/BDNF for $\mathrm{pFyn}$ & 0.0044 \\
\hline 3.A) & $\begin{array}{l}\text { One-way ANOVA, with } \\
\text { Tukey's }\end{array}$ & $\begin{array}{l}\text { Saline/BDNF + ACTZ for } \\
\text { pFyn }\end{array}$ & 0.9732 \\
\hline 3.A) & $\begin{array}{l}\text { One-way ANOVA, with } \\
\text { Tukey's }\end{array}$ & $\begin{array}{l}\text { BDNF/BDNF + ACTZ for } \\
\text { pFyn }\end{array}$ & 0.00144 \\
\hline 3.A) & $\begin{array}{l}\text { One-way ANOVA, with } \\
\text { Tukey's }\end{array}$ & Saline/ACTZ for $\mathrm{pFyn}$ & 0.9426 \\
\hline 3.A) & $\begin{array}{l}\text { One-way ANOVA, with } \\
\text { Tukey's }\end{array}$ & Saline/BDNF for Fyn & 0.8976 \\
\hline 3.A) & $\begin{array}{l}\text { One-way ANOVA, with } \\
\text { Tukey's }\end{array}$ & $\begin{array}{l}\text { Saline/BDNF + ACTZ for } \\
\text { Fyn }\end{array}$ & 0.8397 \\
\hline 3.A) & $\begin{array}{l}\text { One-way ANOVA, with } \\
\text { Tukey's }\end{array}$ & Saline/ACTZ for Fyn & 0.4917 \\
\hline 3.A) & $\begin{array}{l}\text { One-way ANOVA, with } \\
\text { Tukey's }\end{array}$ & $\begin{array}{l}\text { Saline/BDNF for } \\
\text { pGluN2B }\end{array}$ & 0.0020 \\
\hline 3.A) & $\begin{array}{l}\text { One-way ANOVA, with } \\
\text { Tukey's }\end{array}$ & $\begin{array}{l}\text { Saline/BDNF + ACTZ for } \\
\text { pGluN2B }\end{array}$ & 0.9863 \\
\hline 3.A) & $\begin{array}{l}\text { One-way ANOVA, with } \\
\text { Tukey's }\end{array}$ & $\begin{array}{l}\text { Saline/ACTZ for } \\
\text { pGluN2B }\end{array}$ & 0.9918 \\
\hline 3.A) & $\begin{array}{l}\text { One-way ANOVA, with } \\
\text { Tukey's }\end{array}$ & $\begin{array}{l}\text { BDNF/BDNF + ACTZ for } \\
\text { pGluN2B }\end{array}$ & 0.000809 \\
\hline 3.A) & $\begin{array}{l}\text { One-way ANOVA, with } \\
\text { Tukey's }\end{array}$ & Saline/BDNF for GluN2B & 0.0063 \\
\hline 3.A) & $\begin{array}{l}\text { One-way ANOVA, with } \\
\text { Tukey's }\end{array}$ & $\begin{array}{l}\text { Saline/BDNF + ACTZ for } \\
\text { GluN2B }\end{array}$ & 1.0000 \\
\hline 3.A) & $\begin{array}{l}\text { One-way ANOVA, with } \\
\text { Tukey's }\end{array}$ & $\begin{array}{l}\text { BDNF/BDNF + ACTZ for } \\
\text { GluN2B }\end{array}$ & 0.00710 \\
\hline 3.A) & $\begin{array}{l}\text { One-way ANOVA, with } \\
\text { Tukey's }\end{array}$ & Saline/ACTZ for GluN2B & 0.9962 \\
\hline 3.A) & $\begin{array}{l}\text { One-way ANOVA, with } \\
\text { Tukey's }\end{array}$ & $\begin{array}{l}\text { Saline/BDNF for } \\
\text { pGluN2A }\end{array}$ & 0.5357 \\
\hline 3.A) & $\begin{array}{l}\text { One-way ANOVA, with } \\
\text { Tukey's }\end{array}$ & $\begin{array}{l}\text { Saline/BDNF + ACTZ for } \\
\text { pGluN2A }\end{array}$ & 0.3823 \\
\hline 3.A) & $\begin{array}{l}\text { One-way ANOVA, with } \\
\text { Tukey's }\end{array}$ & $\begin{array}{l}\text { Saline/ACTZ for } \\
\text { pGluN2A }\end{array}$ & 0.5768 \\
\hline 3.A) & $\begin{array}{l}\text { One-way ANOVA, with } \\
\text { Tukey's }\end{array}$ & Saline/BDNF for GluN2A & 0.7271 \\
\hline 3.A) & $\begin{array}{l}\text { One-way ANOVA, with } \\
\text { Tukey's }\end{array}$ & $\begin{array}{l}\text { Saline/BDNF + ACTZ for } \\
\text { GluN2A }\end{array}$ & 0.2481 \\
\hline 3.A) & $\begin{array}{l}\text { One-way ANOVA, with } \\
\text { Tukey's }\end{array}$ & Saline/ACTZ for GluN2A & 0.9768 \\
\hline 3.B) & Student's unpaired t test & $\begin{array}{l}\text { CFA-Saline/CFA- } \\
\text { Acetazolamide } 40 \text { minutes } \\
\text { post-IP }\end{array}$ & 7.97002E-10 \\
\hline
\end{tabular}




\begin{tabular}{|c|c|c|c|}
\hline 3.B) & Student's paired t test & $\begin{array}{l}\text { CFA-Saline: Pre-IP vs } 40 \\
\text { minutes post-IP }\end{array}$ & 0.215839 \\
\hline 3.B) & Student's paired t test & $\begin{array}{l}\text { CFA-Acetazolamide: Pre- } \\
\text { IP vs } 40 \text { minutes post-IP }\end{array}$ & $4.2612 \mathrm{E}-07$ \\
\hline 4.B) & Student's unpaired t test & $\begin{array}{l}\text { Human/Rat charge } \\
\text { transfer }\end{array}$ & $6.97953 \mathrm{E}-06$ \\
\hline 4.B) & Student's unpaired t test & Human/Rat decay constant & 0.358285 \\
\hline 4.C) & Student's paired t test & Saline/BDNF - aSTEP & 0.048406 \\
\hline 4.C) & Student's paired t test & Saline/BDNF - tSTEP & 0.019056 \\
\hline 4.C) & Student's paired t test & Saline/BDNF - pGluN2B & 0.030548 \\
\hline 4.C) & Student's paired t test & Saline/BDNF - GluN2B & 0.490335 \\
\hline 4.C) & Student's paired t test & Saline/BDNF - pGluN2A & 0.166487 \\
\hline 4.C) & Student's paired t test & Saline/BDNF - GluN2A & 0.266342 \\
\hline 4.C) & Student's paired t test & Saline/BDNF - pFyn & 0.03987 \\
\hline 4.C) & Student's paired t test & Saline/BDNF - Fyn & 0.094406 \\
\hline 4.C) & Student's paired t test & Saline/BDNF - KCC2 & 0.043605 \\
\hline 4.G) & Student's paired t test & $\begin{array}{l}\text { Membrane: Saline/BDNF } \\
>0.5 \mu \mathrm{m}\end{array}$ & 0.05 \\
\hline 4.H) & Student's paired t test & $\begin{array}{l}\text { Intracellular: } \\
\text { Saline/BDNF }\end{array}$ & 0.0432 \\
\hline SF1. & Student's paired t test & Saline/BDNF - aSTEP & 0.111864 \\
\hline SF1. & Student's paired t test & Saline/BDNF - tSTEP & 0.112782 \\
\hline SF1. & Student's paired t test & Saline/BDNF - pGluN2B & 0.895413 \\
\hline SF1. & Student's paired t test & Saline/BDNF - GluN2B & 0.570437 \\
\hline SF1. & Student's paired t test & Saline/BDNF - pFyn & 0.293997 \\
\hline SF1. & Student's paired t test & Saline/BDNF - Fyn & 0.385260 \\
\hline SF1. & Student's paired t test & Saline/BDNF - KCC2 & 0.303066 \\
\hline SF2. & Student's paired t test & Saline/BDNF - aSTEP & 0.825054 \\
\hline SF2. & Student's paired t test & Saline/BDNF - tSTEP & 0.210702 \\
\hline SF2. & Student's paired t test & Saline/BDNF - pGluN2B & 0.637850 \\
\hline SF2. & Student's paired t test & Saline/BDNF - GluN2B & 0.662928 \\
\hline SF2. & Student's paired t test & Saline/BDNF - pFyn & 0.176693 \\
\hline SF2. & Student's paired t test & Saline/BDNF - Fyn & 0.876844 \\
\hline SF2. & Student's paired t test & Saline/BDNF - KCC2 & 0.733139 \\
\hline SF5. & Student's paired t test & Saline/BDNF - aSTEP & 0.24845 \\
\hline SF5. & Student's paired t test & Saline/BDNF - tSTEP & 0.181536 \\
\hline SF5. & Student's paired t test & Saline/BDNF - pGluN2B & 0.109915 \\
\hline SF5. & Student's paired t test & Saline/BDNF - GluN2B & 0.884676 \\
\hline SF5. & Student's paired t test & Saline/BDNF - pFyn & 0.936631 \\
\hline SF5. & Student's paired t test & Saline/BDNF - Fyn & 0.055142 \\
\hline SF5. & Student's paired t test & Saline/BDNF - KCC2 & 0.202092 \\
\hline SF6. & Student's paired t test & Saline/BDNF - aSTEP & 0.27162 \\
\hline SF6. & Student's paired t test & Saline/BDNF - tSTEP & 0.112001 \\
\hline SF6. & Student's paired t test & Saline/BDNF - pGluN2B & 0.823088 \\
\hline SF6. & Student's paired t test & Saline/BDNF - GluN2B & 0.114532 \\
\hline SF6. & Student's paired t test & Saline/BDNF - pFyn & 0.836541 \\
\hline SF6. & Student's paired t test & Saline/BDNF - Fyn & 0.207316 \\
\hline SF6. & Student's paired t test & Saline/BDNF - KCC2 & 0.275293 \\
\hline SF7 & Chi-square test & Saline/BDNF & $<0.0001$ \\
\hline
\end{tabular}




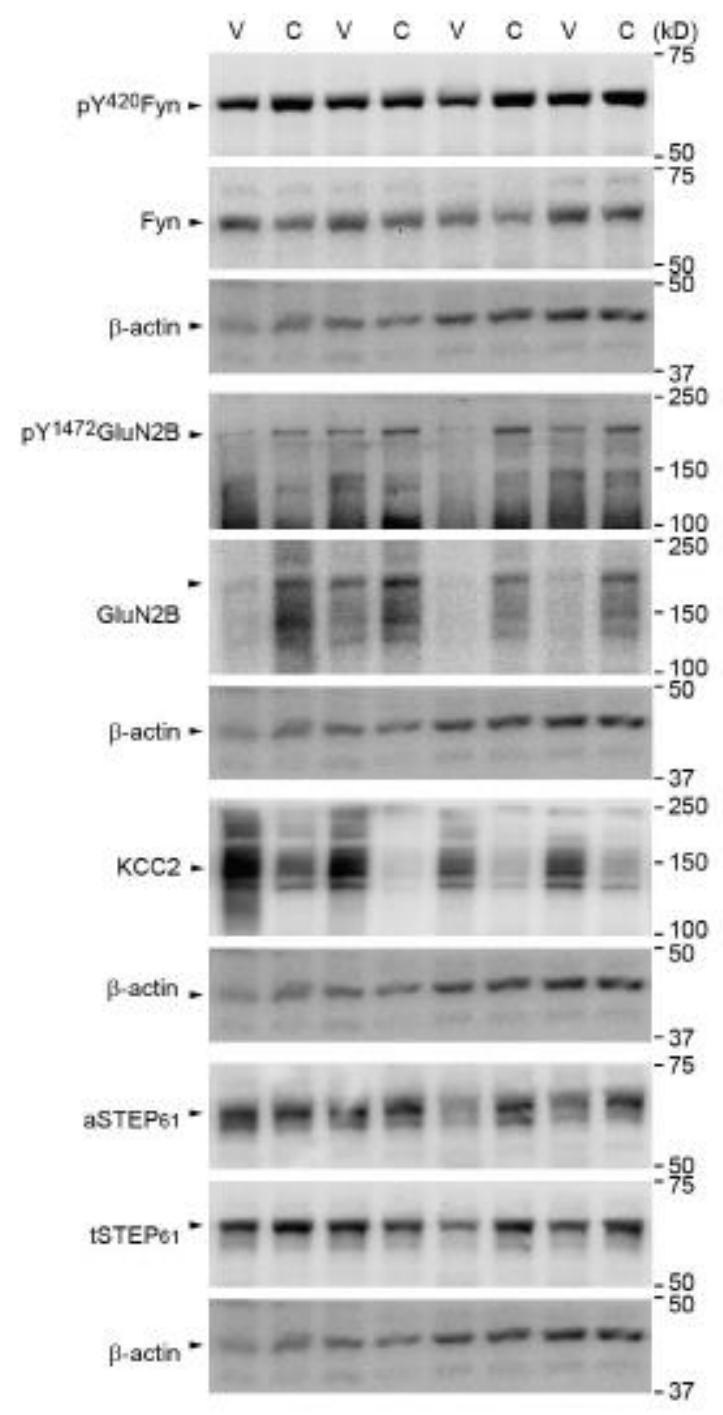

Chapter 2 Supplementary Figure 8. Western blots from 1C. Gels from animals treated with either vehicle (V) or CFA (C). Individual gels were cut into sections to allow for probing several targets concurrently $\beta$ actin, the loading control, can be seen under each set of targets (labelled on the left while weight of the target, in $\mathrm{kD}$ is on the right), $\mathrm{n}=4$. 


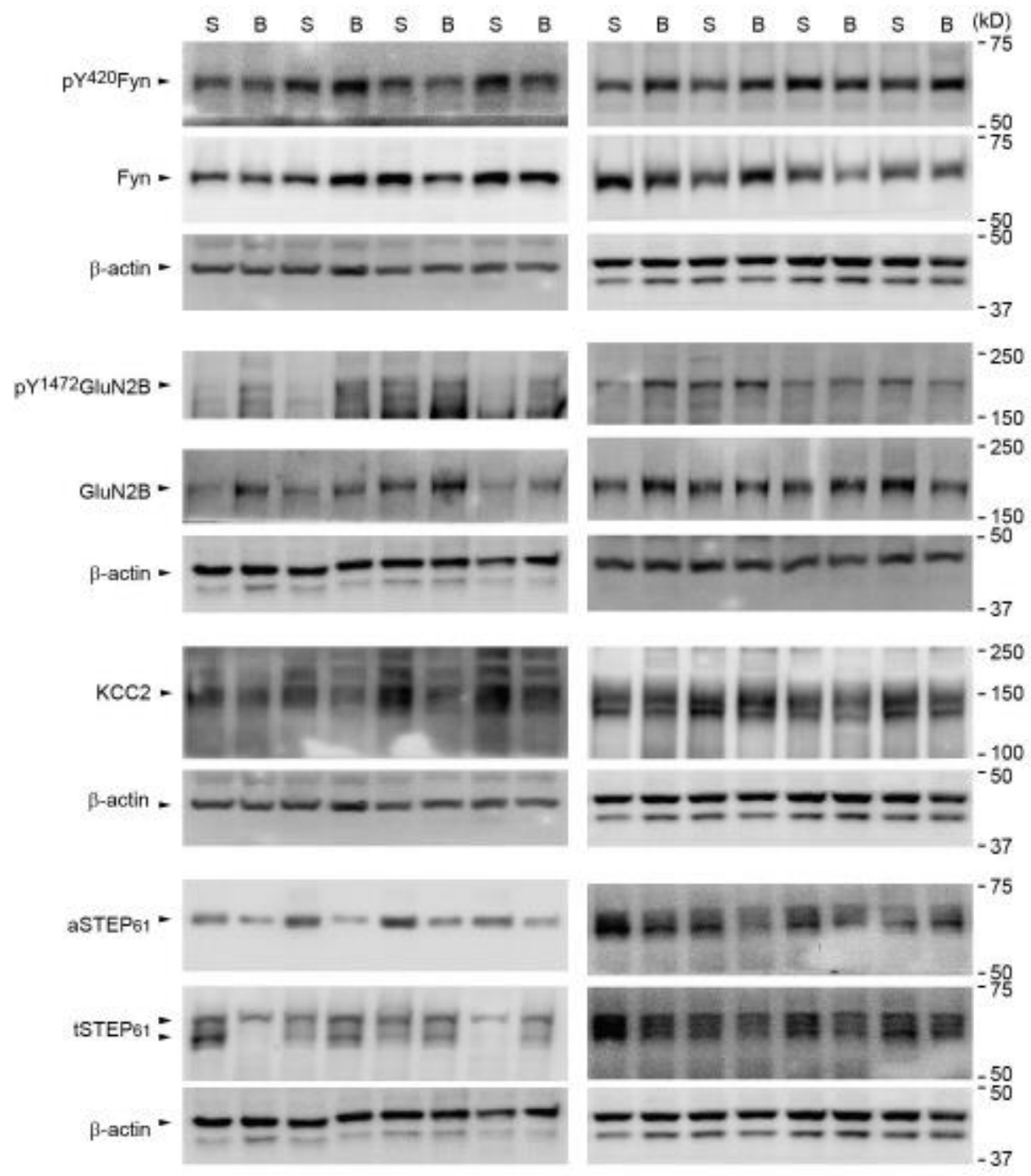

Chapter 2 Supplementary Figure 9. Western Blots from Figure 1D. Gels from animals treated with either saline (S) or BDNF (B). Individual gels were cut into sections to allow for probing several targets concurrently $\beta$ actin, the loading control, can be seen under each set of targets (labelled on the left while weight of the target, in $\mathrm{kD}$ is on the right), $\mathrm{n}=8$ gels were run in groups of 4 animals. 


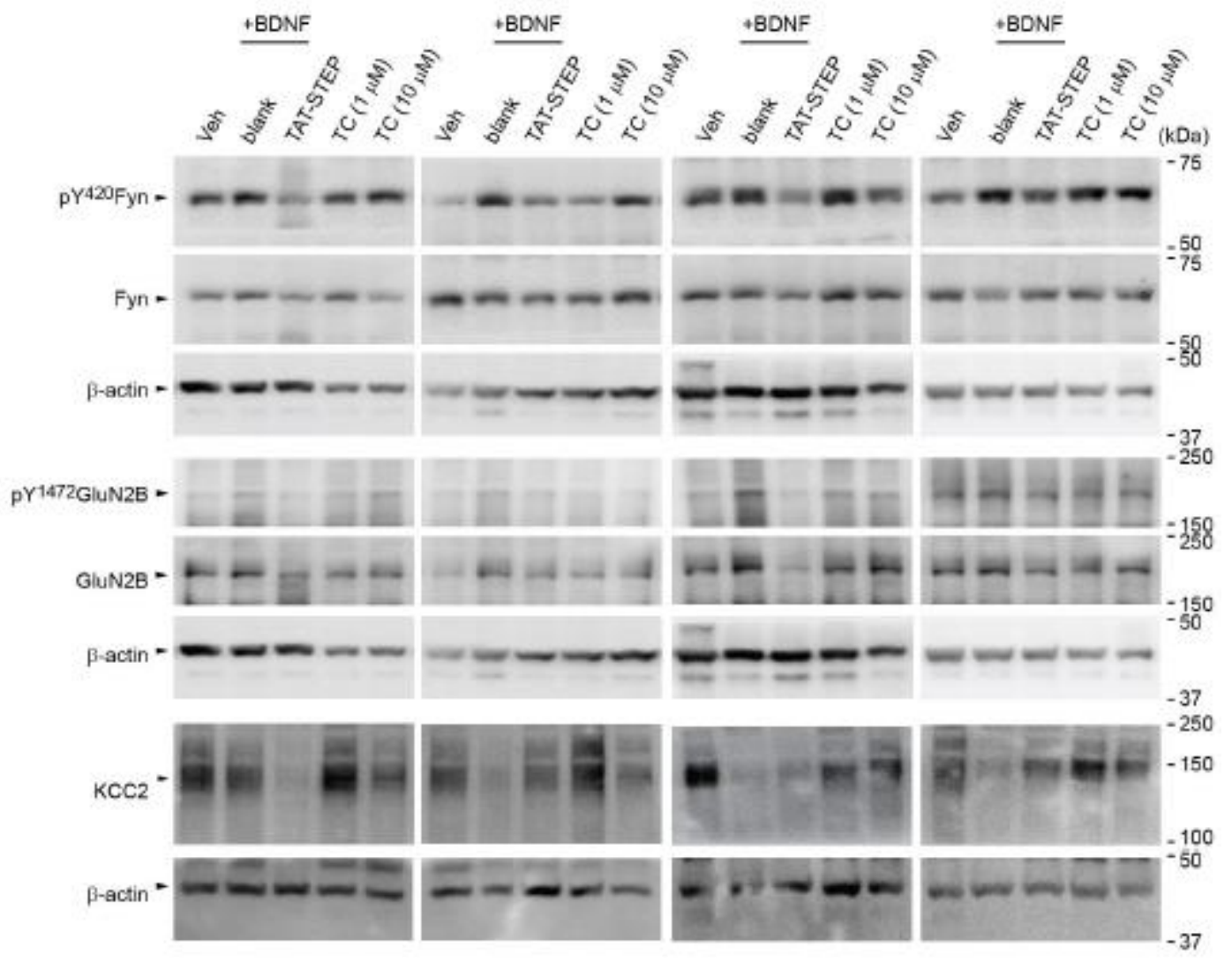

Chapter 2 Supplementary Figure 10. Western Blots from Figure 2E. Gels from animals 1-4 (out of 8 animals used in this figure) treated with either vehicle (Veh) BDNF (blank + BDNF), TAT-STEP + BDNF, $1 \mu \mathrm{M}$ TC-2153 or 10 $\mu \mathrm{M}$ TC-2153. Individual gels were cut into sections to allow for probing several targets concurrently. B-actin, the loading control, can be seen under each set of targets (labelled on the left while weight of the target, in $\mathrm{kD}$ is on the right), $\mathrm{n}=4$. 


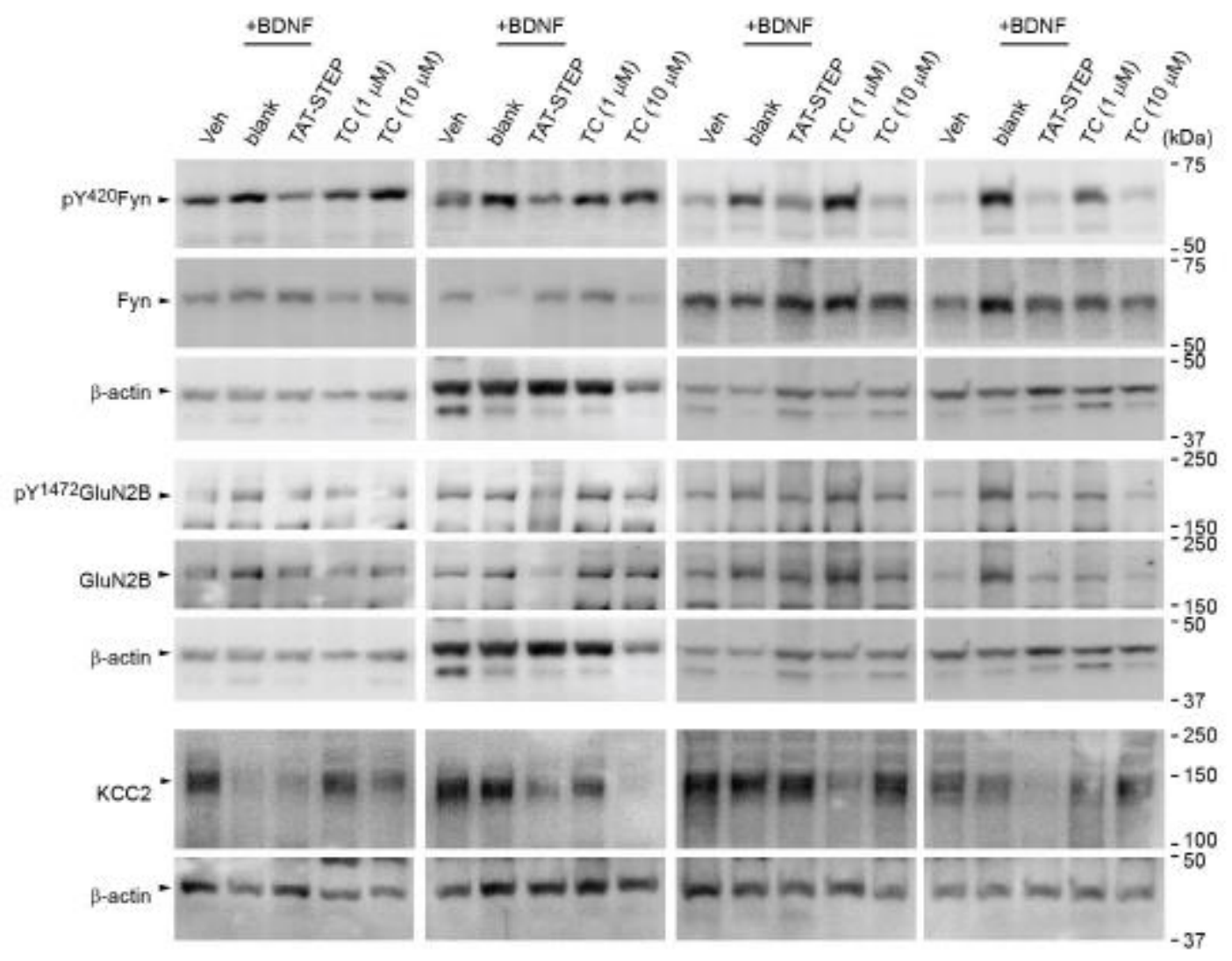

Chapter 2 Supplementary Figure 11. Western Blots from Figure 2E. Gels from animals 5-8 (out of 8 animals used in this figure) treated with either vehicle (Veh) BDNF (blank + BDNF), TAT-STEP + BDNF, $1 \mu \mathrm{M}$ TC-2153 or $10 \mu \mathrm{M}$ TC-2153. Individual gels were cut into sections to allow for probing several targets concurrently. B-actin, the loading control, can be seen under each set of targets (labelled on the left while weight of the target, in $\mathrm{kD}$ is on the right), $\mathrm{n}=4$. 


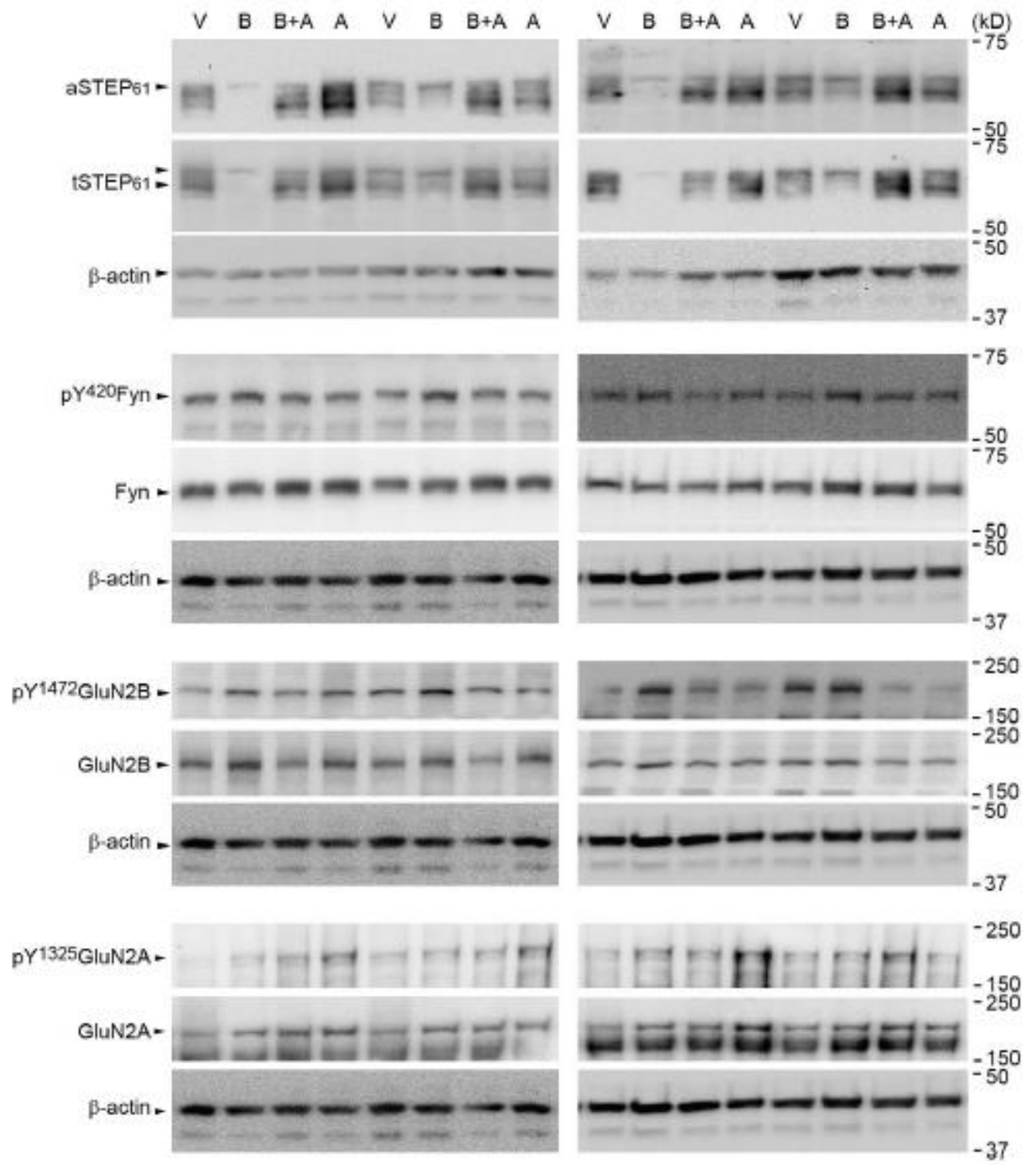

Chapter 2 Supplementary Figure 12. Western Blots from Figure 3A. Gels from animals 1-4 (out of 8 animals used in this figure) treated with either vehicle (V), BDNF (B), BDNF + acetazolamide (B+A), or acetazolamide (A). Individual gels were cut into sections to allow for probing several targets concurrently. B-actin, the loading control, can be seen under each set of targets (labelled on the left while weight of the target, in $\mathrm{kD}$ is on the right). Gels were run in groups of $2 \mathrm{n}=4$. 

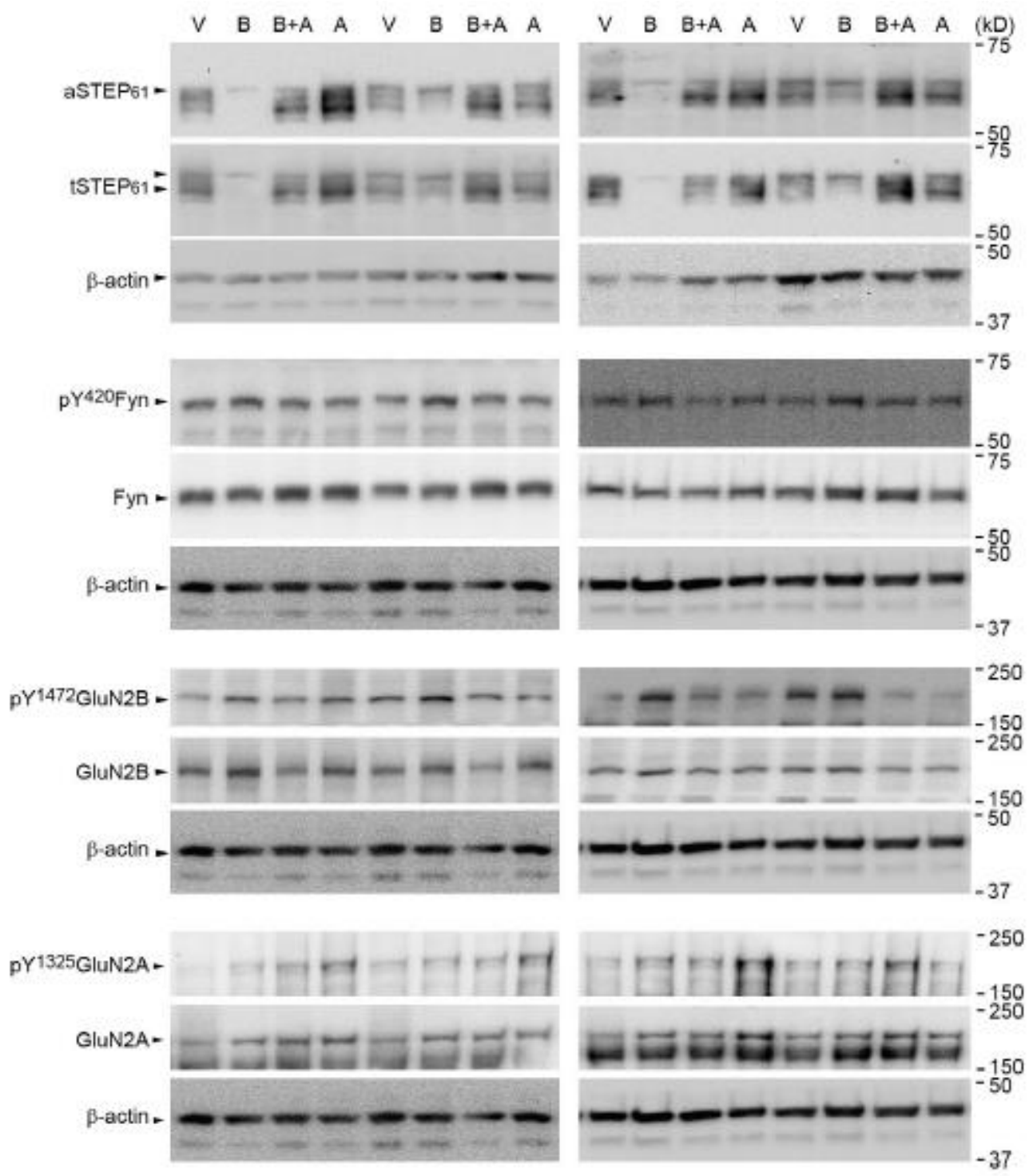

Chapter 2 Supplementary Figure 13. Western Blots from Figure 3A. Gels from animals 5-8 (out of 8 animals used in this figure) treated with either vehicle (V), BDNF (B), BDNF + acetazolamide (B+A), or acetazolamide (A). Individual gels were cut into sections to allow for probing several targets concurrently. B-actin, the loading control, can be seen under each set of targets (labelled on the left while weight of the target, in $\mathrm{kD}$ is on the right). Gels were run in groups of $2 \mathrm{n}=4$. 


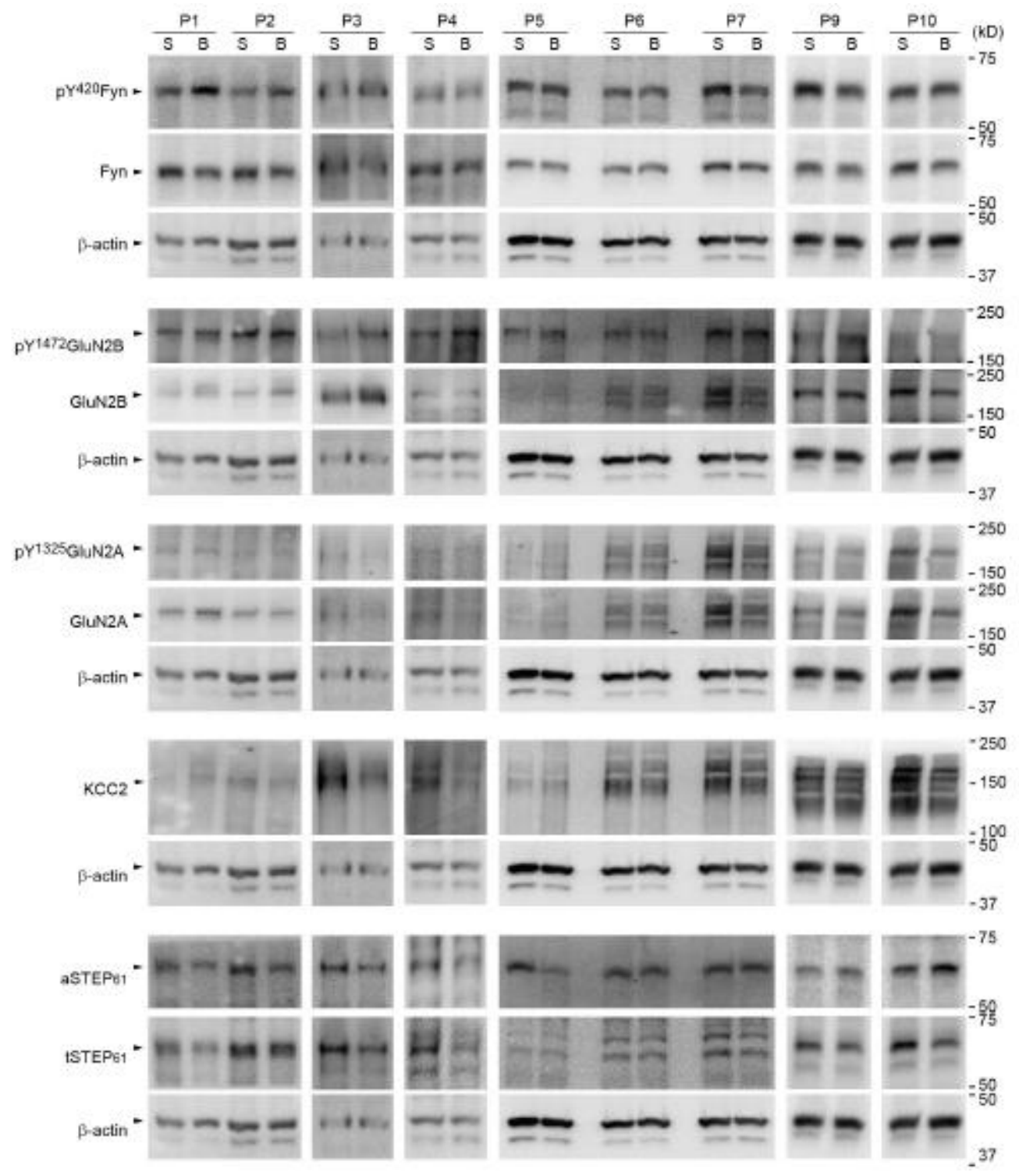

Chapter 2 Supplementary Figure 14. Western Blots from Figure 4C. Gels from human patients treated with either saline (S) or BDNF (B). Individual gels were cut into sections to allow for probing several targets concurrently. $\beta$-actin, the loading control, can be seen under each set of targets (labelled on the left while weight of the target, in $\mathrm{kD}$ is on the right). Gels were run in groups of $1-3 ; \mathrm{n}=9$. 


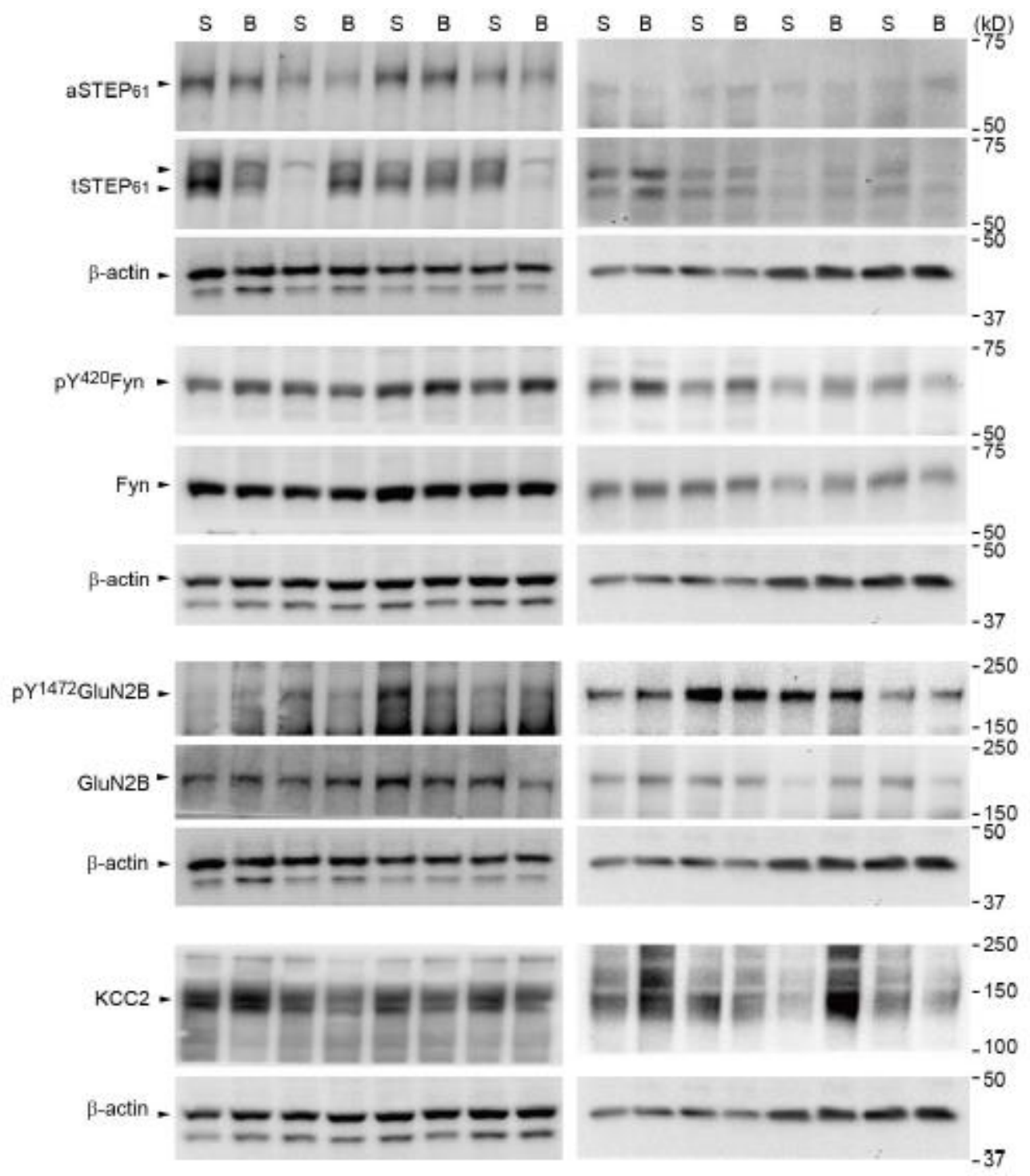

Chapter 2 Supplementary Figure 15. Western Blots from Supplementary Figure 1. Gels from animals treated with either saline (S) or BDNF (B). Individual gels were cut into sections to allow for probing several targets concurrently. $\beta$-actin, the loading control, can be seen under each set of targets (labelled on the left while weight of the target, in $\mathrm{kD}$ is on the right). $\mathrm{n}=8$ gels were run in groups of 4 animals. 


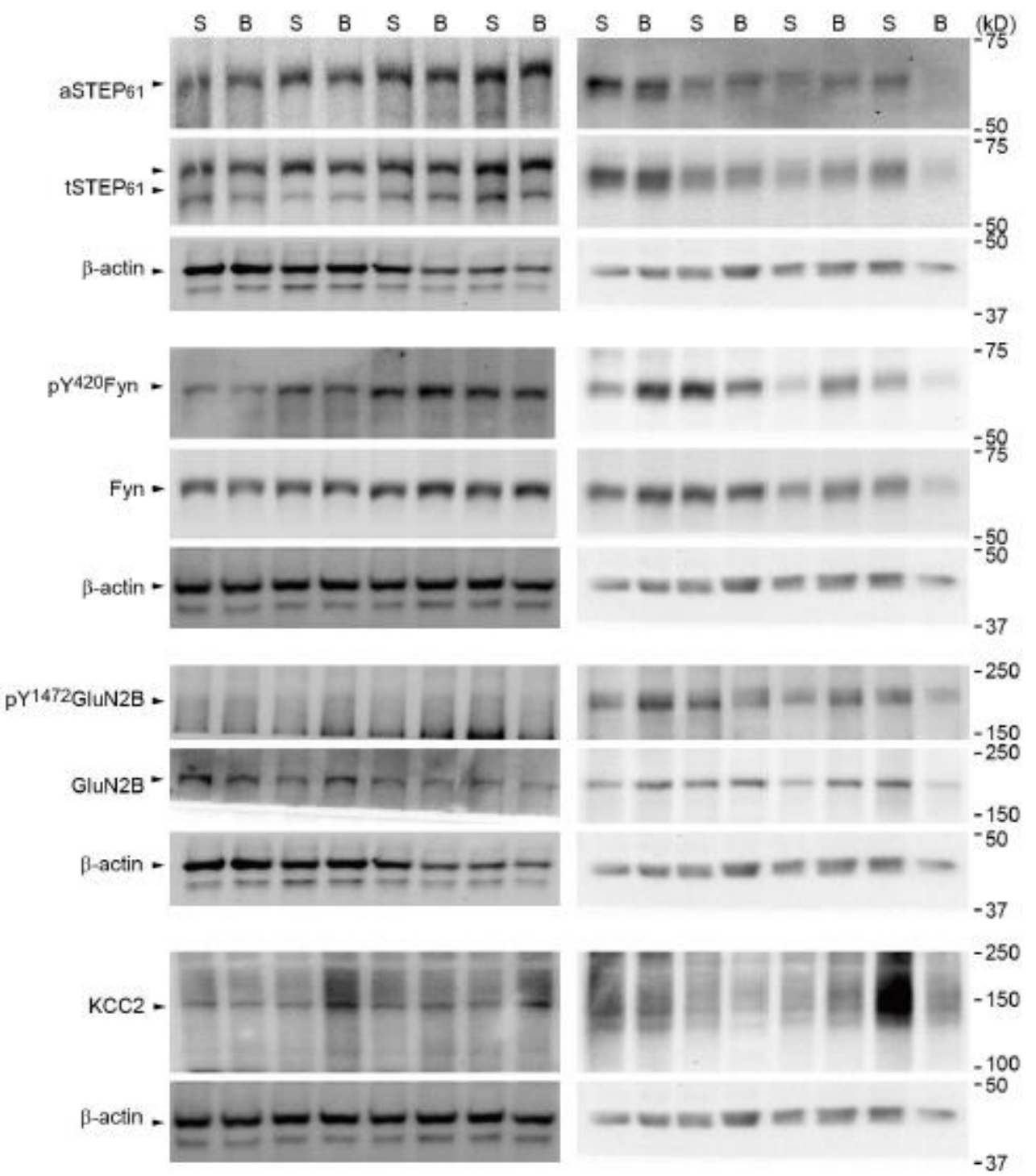

Chapter 2 Supplementary Figure 16. Western Blots from Supplementary Figure 2. Gels from animals treated with either saline (S) or BDNF (B). Individual gels were cut into sections to allow for probing several targets concurrently. $\beta$-actin, the loading control, can be seen under each set of targets (labelled on the left while weight of the target, in $\mathrm{kD}$ is on the right). $\mathrm{n}=8$ gels were run in groups of 4 animals. 


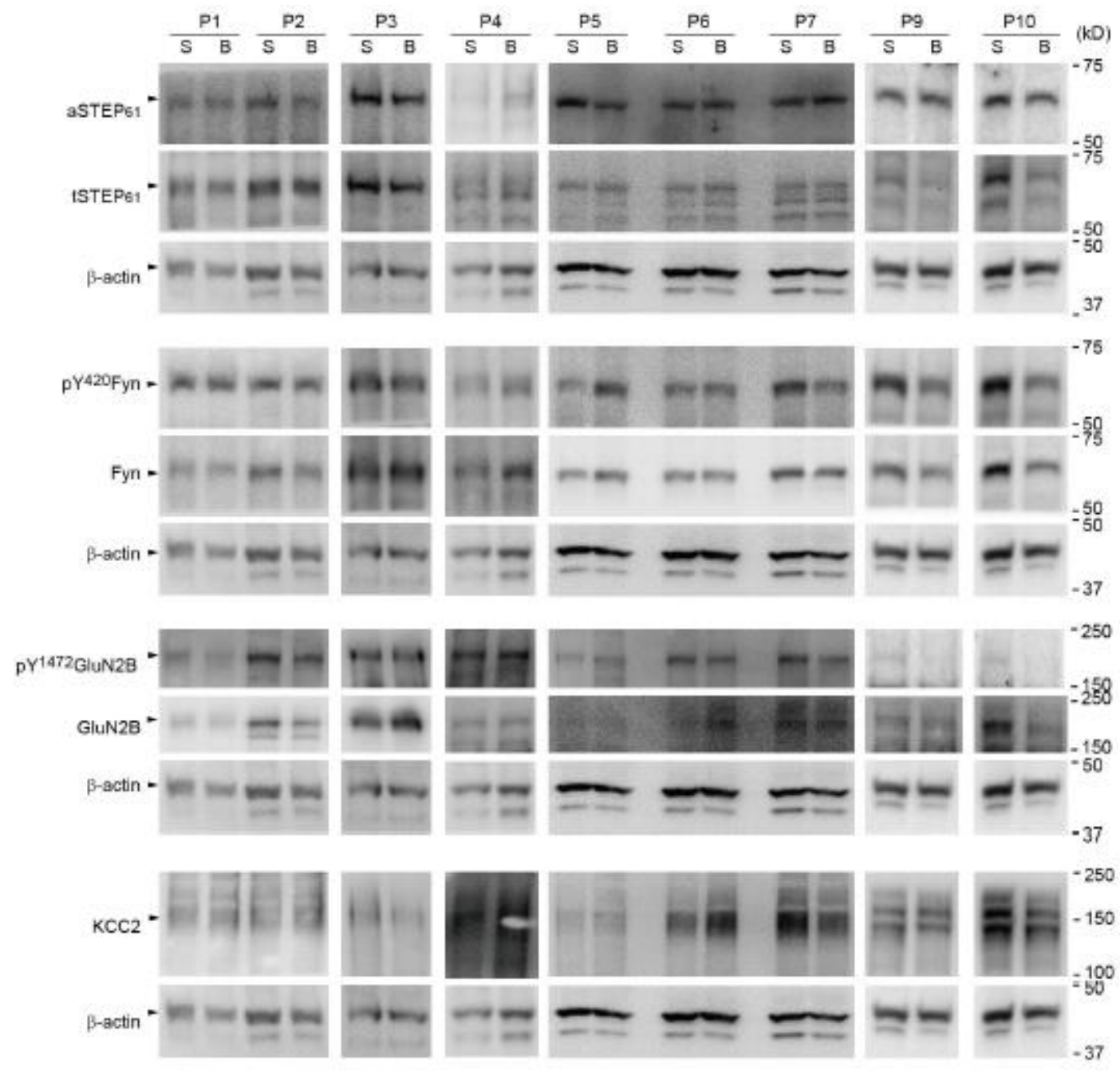

Chapter 2 Supplementary Figure 17. Western Blots from Supplementary Figure 5. Gels from human patients treated with either saline (S) or BDNF (B). Individual gels were cut into sections to allow for probing several targets concurrently. $\beta$-actin, the loading control, can be seen under each set of targets (labelled on the left while weight of the target, in $\mathrm{kD}$ is on the right). Gels were run in groups of 1-3 $\mathrm{n}=9$. 


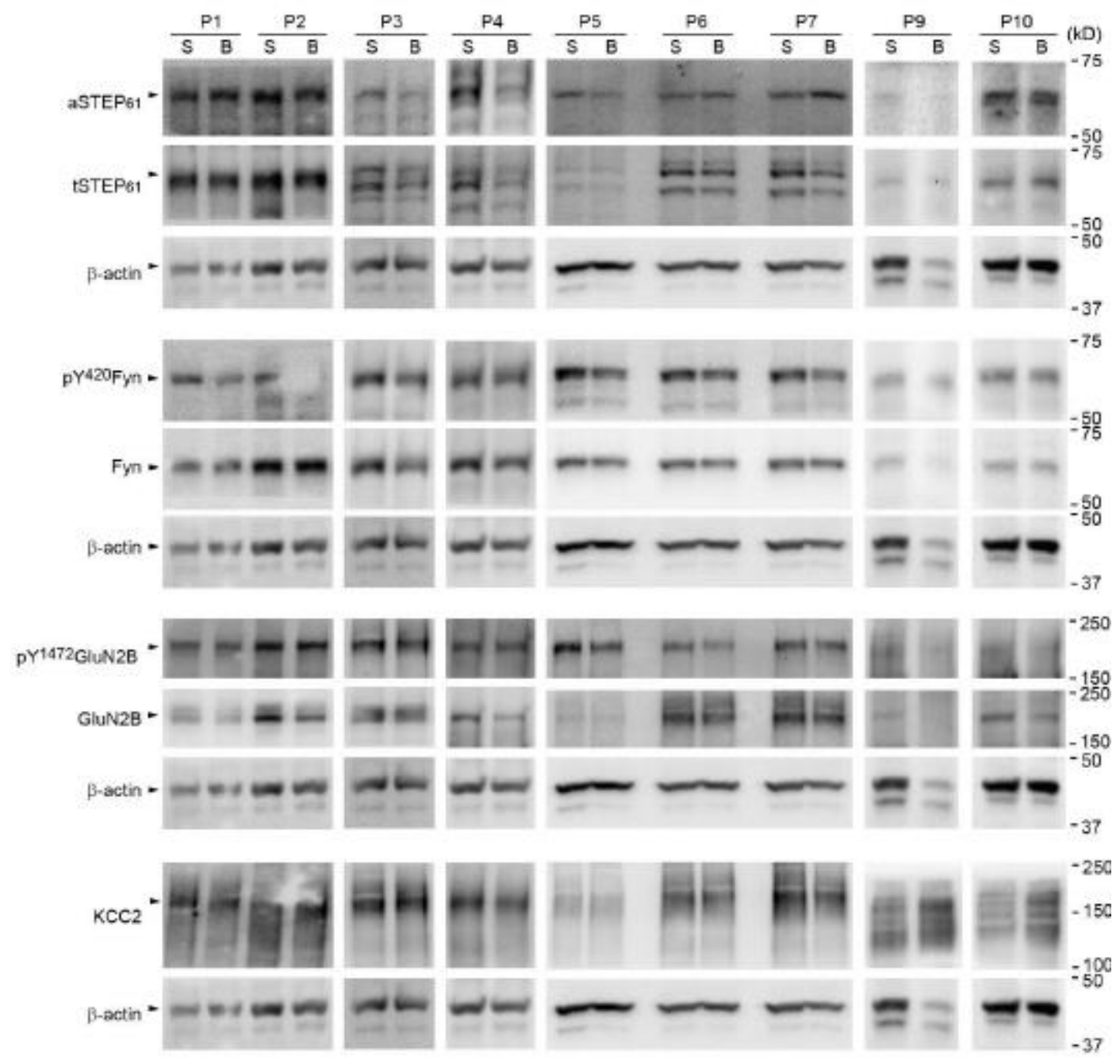

Chapter 2 Supplementary Figure 18. Western Blots from Supplementary Figure 6. Gels from human patients treated with either saline (S) or BDNF (B). Individual gels were cut into sections to allow for probing several targets concurrently. $\beta$-actin, the loading control, can be seen under each set of targets (labelled on the left while weight of the target, in $\mathrm{kD}$ is on the right). Gels were run in groups of 1-3 $\mathrm{n}=9$. 


\section{Chapter 3: A neuronal mechanism of spinal hyperexcitability is sexually dimorphic in rodent and human models of pathological pain.}

\subsection{Abstract:}

Clinical and epidemiological evidence suggests a sex difference in the mechanisms underlying chronic pain, with female chronic pain patients outnumbering their male counterparts by $2: 1$. Despite this, the neurobiological underpinnings of sexually dimorphic pain signalling remain unclear in rodents and virtually unexplored in human preclinical models. Within the nociceptive system, the superficial dorsal horn $\mathrm{SDH}$ ) is a critical site of pain modulation. We have recently characterized a pathological pain pathway in male rat and human SDH neurons where brain-derived neurotrophic factor (BDNF) mediates potentiation of excitatory GluN2B synaptic responses that depend on downregulation of inhibitory signalling factors (KCC2 and STEP $_{61}$ ) and upregulation of excitatory factors (Fyn) (Dedek et al., 2019). Here, we investigate whether molecular mechanisms of spinal hyperexcitability are conserved between sexes in both rodents and humans. In the Freund's Adjuvant in vivo model of inflammatory pain, both male and female rats display tactile allodynia, however, the NMDAR potentiation pathway is only activated in males. Further, ex vivo BDNF treatment results in synaptic NMDAR potentiation and changes in biochemical markers of the KCC2-STEP 61 -Fyn mechanism of NMDAR potentiation in males, but not in females. We find conserved results between species, with immunohistochemical evidence of disinhibition, marked by internalization of KCC2, as well as biochemical markers or facilitated excitation and disinhibition in the human male, but not female $\mathrm{SDH}$. Electrophysiological and biochemical investigations in ovariectomized rats suggest hormonal mediation of this sex difference. We therefore conclude that neuronal mechanisms of 
SDH hyperexcitability are sexually dimorphic in rats and humans. This sex difference in underpinning neurobiological mechanisms of chronic pain has profound implications for the development of novel pain therapeutics.

\section{Introduction:}

Chronic pain is a highly prevalent but difficult to treat disease. The development of safe and more effective treatment strategies is needed. Novel therapeutic development relies on fundamental research on nociception. For decades it was assumed that at a physiological level, nociceptive pathways are conserved between males and females, but epidemiological evidence suggests otherwise. Women are more likely to report low back pain, neck pain, orofacial pain, and neuropathic pain, and twice as many women report common migraines or headaches (Bouhassira et al., 2008; Pleis et al., 2010; Chen et al., 2017). In addition, studies of experimentally induced pain in humans indicate that across several modalities, including mechanical, electrical, thermal, and chemically-induced pain, women show more pain sensitivity than men (Bartley and Fillingim, 2013). This epidemiological difference is particularly problematic because to date, the foundational research in pain physiology has been conducted in male rodents only. To develop effective therapeutics for treating chronic pain, it is critical to understand the neurobiological underpinnings of chronic pain in females.

Although studies of female pain physiology are needed, there are barriers to understanding chronic pain in females. Our understanding of pain physiology is derived from studies of basic synaptic physiology and excitability in the brain. It was assumed that basic mechanisms of synaptic transmission and excitability are conserved between sexes, and thus these foundational neurophysiological studies were conducted in male or unsexed animals (Crair 
and Malenka, 1995; Bardoni et al., 1998; Hsia et al., 1998; Engelman et al., 1999; Hartmann et al., 2004; Tong and MacDermott, 2014). A second major barrier is a lack of understanding of sex differences across species. Without direct examination, it remains unknown if an identified sex difference is conserved across multiple species, especially humans. The combination of a lack of studies on basic synaptic physiology and excitability in females paired with uncertainty on how identified sex differences relate across species results in a large translational gap that requires preclinical study of human tissue from both sexes.

Recently, the push for female-inclusive biomedical research has highlighted differences in mechanisms of pain physiology and pathology between males and females. In males, microglial activation results in secretion of BDNF, which triggers a cascade that results in neuronal hyperexcitability (Coull et al., 2005). In contrast, hyperexcitability in females appears to be caused by T-lymphocyte activation and yet-to-be-identified downstream mechanisms (Sorge et al., 2015; Chen et al., 2017; Mapplebeck et al., 2017, 2018). In addition, immune-cell involvement differs between inflammatory and neuropathic pain models, with astroglial involvement in both sexes for models of neuropathic pain, but not for inflammatory pain models (Chen et al., 2017). Although sex differences in immune modulation of pain have been identified, the prevailing hypothesis is that these differences converge onto conserved neuronal determinants in the spinal cord (Mapplebeck et al., 2019). However, these mechanisms have not yet been probed in inflammatory pain, and it remains to be explicitly tested if molecular mechanisms of spinal hyperexcitability are conserved between sexes in rodents and humans.

Investigations in males have found that disrupting the balance of excitation and inhibition within the circuitry of the superficial spinal dorsal horn (SDH) leads to plastic changes that increase nociceptive outputs and result in pathological pain (Woolf and Salter, 2000; 
Latremoliere and Woolf, 2009; Todd, 2010). The $\mathrm{K}^{+}-\mathrm{Cl}^{-}$co-transporter $\mathrm{KCC} 2$ plays a fundamental role in mediating inhibition in the SDH by maintaining $\mathrm{Cl}^{-}$gradients that allow $\gamma$ aminobutyric acid $\mathrm{A}\left(\mathrm{GABA}_{\mathrm{A}}\right)$ and glycine receptors (GlyRs) to maintain inhibition (Kaila et al., 2014). In both inflammatory (Zhang et al., 2008; Hildebrand et al., 2016) and neuropathic(Coull et al., 2003, 2005; Zhang et al., 2008; Mapplebeck et al., 2019; Lorenzo et al., 2020) pain models, $\mathrm{KCC} 2$ downregulation, triggered by the release of brain-derived neurotrophic factor (BDNF) results in disinhibition of SDH neurons, leading to increased neuronal excitability. BDNF also drives facilitated excitation through the potentiation of GluN2B-containing NMDARs (Abe et al., 2005; Hildebrand et al., 2016; Dedek et al., 2019). We have recently identified that downregulation of a molecular brake, striatal-enriched protein tyrosine phosphatase-61 ( $\left.\mathrm{STEP}_{61}\right)$, serves as a feed-forward link between disinhibition and NMDAR potentiation in both male rodents and humans (Dedek et al., 2019). Although great headway has been made to understand the neuronal mechanisms of disinhibition and facilitated excitation in chronic pain using these preclinical male models, a burning question for potential translation is whether these mechanisms are conserved in females.

Although few investigations into female neuronal mechanisms of pathological pain exist, there is evidence of divergence in baseline neurophysiological features that could point towards sex differences in pathological pain states. Temi and colleagues show that NMDAR subunit distribution - basic molecular determinants of synaptic transmission, are not conserved between sexes, highlighting the urgent need to investigate neuronal mechanisms of pain processing in females (Temi et al., 2021). It remains unexplored if there are sex differences in human pain processing as well. Here, we pair ex vivo BDNF and in vivo inflammatory rodent models of pain with translational, ex vivo human tissue approaches. To probe the mechanisms of spinal 
hyperexcitability in the rat and human superficial dorsal horn, we measured behavioural pain responses, changes in synaptic proteins linked to excitability, functional synaptic NMDAR responses, and protein distribution across SDH neurons in both sexes. Finally, we explore whether identified sex differences are hormonally mediated using ovariectomized female rats.

\subsection{Methods:}

\subsubsection{Animals}

Rodent experiments were carried out on male or female adult (3-4-month-old) Sprague Dawley rats ordered from Charles River Laboratories. Animals were housed in same-sex pairs, on a 12-hour day/night cycle and had access to food and water ad libitum. Animals were randomly assigned to their respective treatment groups and were cared for in accordance with guidelines from the Canadian Council for Animal Care, Carleton University, and the University of Ottawa Heart Institute. Ovariectomized animals were ordered from Charles River Laboratories; surgery was performed at Charles River Laboratories on postnatal day 21. After animals recovered from surgery, they were shipped to the University of Ottawa Heart Institute and left to mature to age-match the intact animals.

\subsubsection{Freund's adjuvant model of inflammatory pain and von Frey behaviour testing}

To model persistent inflammatory pain, complete Freund's adjuvant (CFA, Sigma) was injected into the intraplantar surface of the rats' hindpaw. Rats were anesthetized using isoflurane gas, and the $0.4 \mathrm{~mL}$ injection of either phosphate-buffered saline vehicle (PBS) or 50\% $\mathrm{v} / \mathrm{v}$ PBS+CFA was injected once the rat was deeply anesthetized. 
Von Frey filaments were used to measure mechanical paw withdrawal threshold (PWT). PWT was measured at approximately the same time each day for all animals throughout the study. Rats were placed in testing chambers and left to acclimate for 45 minutes. PWT was measured by the simplified up-down method (SUDO) (Bonin et al., 2014) to ensure that the number of stimulus applications were standardized across subjects. Baseline testing was performed directly before vehicle/CFA injection, and was followed by testing every 24 hours until the end of the study (up to five days post-injection). Animals used for biochemical experiments were sacrificed five days post-injection, while animals used for electrophysiological recording were sacrificed three-five days post-injection. Only the ipsilateral side of the lumbar spinal cord from CFAinjected rats was used for all experiments.

\subsubsection{Rat spinal cord isolation and preparation}

Intraperitoneal injection of $3 \mathrm{~g} / \mathrm{kg}$ urethane (Sigma) was used to deeply anesthetize rats. Spinal cords were then quickly dissected by ventral laminectomy and placed in ice-cold oxygenated protective sucrose solution (referred to as 'saline': $50 \mathrm{mM}$ sucrose, $92 \mathrm{mM} \mathrm{NaCl}, 15$ $\mathrm{mM}$ D-glucose, $26 \mathrm{mM} \mathrm{NaHCO}_{3}, 5 \mathrm{mM} \mathrm{KCl}, 1.25 \mathrm{mM} \mathrm{NaH}_{2} \mathrm{PO}_{4}, 0.5 \mathrm{mM} \mathrm{CaCl}_{2}, 7 \mathrm{mM}$ $\mathrm{MgSO}_{4}, 1 \mathrm{mM}$ kynurenic acid, bubbled with $5 \% \mathrm{CO}_{2} / 95 \% \mathrm{O}_{2}$ ). Dorsal and ventral roots were removed and the lumbar region (L3-L6) was isolated under a dissection microscope. Spinal cords were sliced to $300 \mu \mathrm{m}$ thickness in the parasagittal plane for electrophysiological recording using a Leica VT1200S vibratome at an amplitude of $2.75 \mathrm{~mm}$ and a speed of $0.1-0.2$ $\mathrm{mm} / \mathrm{s}$ through the dorsal horn. Slices were incubated in $34^{\circ} \mathrm{C}$ kynurenic acid-free saline for 40 min to wash out kynurenic acid. Previous control experiments have shown no difference in NMDAR synaptic responses in lamina I neurons from slices that were sectioned in saline with or 
without kynurenic acid, provided that slices had recovered in kynurenic acid-free saline (data not shown). Following kynurenic acid washout, the incubation chamber was removed from the heated water bath and allowed to come to room temperature.

For biochemical analysis, an approximately $400 \mu \mathrm{m}$ horizontal section containing the dorsal horn was removed using a Leica VT1200S vibratome. The next $1200 \mu \mathrm{m}$ of spinal tissue containing the deep dorsal horn and ventral horn was used for control comparisons. Following slicing, tissue for biochemical analysis was either immediately flash frozen (CFA-treated tissue) using histo-freeze (Fisher Super Friendly Freeze'It) or was treated with ex vivo BDNF or saline (see below) and was subsequently flash frozen.

\subsubsection{Human spinal cord isolation and preparation}

We collected tissue from male and female adult (20-75-year-old (rounded to the nearest five for privacy)) neurological determination of death (NDD) organ donors identified by the Trillium Gift of Life Network. Candidates for donation are screened for communicable diseases (HIV/AIDS, syphilis) and conditions that could negatively affect the health of organs, such as morbid obesity. Causes of death resulted from a disruption of blood flow to/in the brain (hemorrhage or ischemia). In Canada, female deceased organ donors make up only $40 \%$ of all deceased donors (Anon, n.d.). Further, only 13 of the 40 total samples we have collected to date have been from female donors, resulting in a smaller sample size of female donors shown here. This difficulty in obtaining female human spinal cord tissue was one of the driving factors in why our previous study characterizing spinal determinants of pain hypersensitivity in rodents and humans (Dedek et al., 2019) included males only. Approval was obtained from the Ottawa Health Science Network Research Ethics Board for the collection of and experimentation on 
human spinal cord tissue. To prepare the patient for donation, hypothermia was induced using a cooling bed and the patient was perfused with a high magnesium protective solution (Celsior or Belzer UW). Following removal of organs for transplant, the spinal cord was extracted by ventral laminectomy, within 3 hours of aortic cross-clamp. Thoracic and lumbar regions were isolated and placed in ice-cold, oxygenated saline. After treatment with either BDNF or saline, tissue for western blot analysis was flash-frozen in liquid nitrogen and the dorsal horn was separated using a scalpel. Donors that had chronic pain, were taking prescription-only analgesics, or who had damaged or malformed spinal cords were excluded from western blot analysis. For immunohistochemical analysis, tissue was fixed in freshly made 4\% paraformaldehyde. Donors who had damaged or malformed spinal cords were excluded from immunohistochemical analysis.

\subsubsection{Ex vivo BDNF treatment}

After tissue preparation, rat or human spinal tissue was placed in oxygenated, room temperature saline containing 50-100 ng/mL recombinant BDNF (Alomone Labs) or control saline for 70 minutes. A subset of female rat experiments was carried out using an incubation time of 2-4.5 hours. Treatment of spinal tissue with BDNF and PP2 (Calbiochem) was carried out using the same approach.

\subsubsection{Lamina I electrophysiological recordings}

Spinal cord slices were viewed under brightfield optics. Lamina I neurons were identified as being situated dorsal to the substantia gelatinosa and within $50 \mu \mathrm{m}$ ventral to superficial white matter tracts. As previously described (Hildebrand et al., 2016; Dedek et al., 2019), the 
extracellular recording solution, an artificial CSF solution, contained (in mM): $125 \mathrm{NaCl}, 20 \mathrm{D}$ glucose, $26 \mathrm{NaHCO}_{3}, 3 \mathrm{KCl}, 1.25 \mathrm{NaH}_{2} \mathrm{PO}_{4}, 2 \mathrm{CaCl}_{2}$, and $1 \mathrm{MgCl}_{2}$ in addition to $500 \mathrm{nM} \mathrm{TTX}$, $10 \mu \mathrm{M} \mathrm{Cd}^{2+}, 10 \mu \mathrm{M}$ strychnine and $10 \mu \mathrm{M}$ bicuculline to block voltage-gated $\mathrm{Na}^{+}$channel, voltage-gated $\mathrm{Ca}^{2+}$ channel, glycinergic and GABAergic currents, respectively. We used borosilicate glass patch-clamp pipettes with resistances of 6-12 M $\Omega$. The internal patch pipette solution contained (in mM): 105 Cs-gluconate, $17.5 \mathrm{CsCl}$, 10 BAPTA or 10 EGTA, 10 HEPES, $2 \mathrm{MgATP}, 0.5 \mathrm{Na}_{2} \mathrm{GTP}$ and had a $\mathrm{pH}$ of 7.25 and an osmolarity of $295 \mathrm{mOsm}$.

Neurons within lamina I were selected by size, favouring large neurons that had an increased probability of being projection neurons (Ikeda et al., 2003a). Whole-cell patch was established at $-60 \mathrm{mV}$. Neurons were required to have an access resistance below $30 \mathrm{M} \Omega$, and leakage currents less than $-80 \mathrm{pA}$ at a holding potential $\left(\mathrm{V}_{\mathrm{h}}\right)$ of $-60 \mathrm{mV}$. Holding potential was slowly increased to $+60 \mathrm{mV}$ to record miniature excitatory postsynaptic currents (mEPSCs) that contained a dominant NMDAR-mediated component (Hildebrand et al., 2014). mEPSC traces were detected and averaged together for each treatment in Clampfit 10.7 (Molecular Devices). Selection criteria for events required that events did not completely decay within $100 \mathrm{~ms}$, had an amplitude $<100$ pA, and decayed to at least $50 \%$ of their overall amplitude by $500 \mathrm{~ms}$. For analysis, charge transfer was measured as the area under the curve from $40-500 \mathrm{~ms}$ after the start of the event. Peak amplitude of NMDAR mEPSCs was measured as the average amplitude from 18-24 ms after event onset (near the NMDAR peak, but where the AMPAR component is negligible (Hildebrand et al., 2014). The decay constant, $\tau$, was derived using exponential one-term standard fitting from just after the peak of the NMDAR component to $500 \mathrm{~ms}$, or where the decaying current reached steady-state if earlier. For all measurements of mEPSCs, measurements 
were taken for each cell and reported as an average of cells within a given treatment. After analysis, traces were transferred to Origin Pro (Northampton) for graphing.

\subsubsection{Western blot analysis on synaptosome fractions of rat and human spinal tissue}

Synaptosomal fractions were isolated as previously described (Xu et al., 2009). Briefly, tissue was homogenized using Wheaton dounce tissue grinders in $300 \mu \mathrm{L}$ of ice-cold TEVP-320 mM sucrose buffer containing (in mM): 320 sucrose, 10 Tris-HCl (pH 7.4), 1 EDTA, 1 EGTA, 5 $\mathrm{NaF}$, and $1 \mathrm{Na}_{3} \mathrm{VO}_{4}$ with complete protease inhibitor and phosphatase inhibitor cocktails (Roche) to obtain total homogenates. The remaining total homogenate lysates were centrifuged at $4{ }^{\circ} \mathrm{C}$ for $10 \mathrm{~min}$ at $1000 \mathrm{~g}$, followed by $15 \mathrm{~min}$ at $12 \mathrm{000 \textrm {g }}$ to obtain the crude synaptosome pellet. The pellet was resuspended in TEVP $320 \mathrm{mM}$ sucrose buffer by brief sonication.

The Pierce BCA protein assay kit (Thermo Scientific) was used to determine the protein content of the synaptosomal fractions. Thirty micrograms of total protein from each sample were loaded on $8 \%$ SDS-PAGE and transferred to PVDF membranes (Bio-Rad).

Membranes were blocked in 5\% bovine serum albumin (BSA) in Tris-buffered saline (TBS) $+0.1 \%$ TWEEN-20 (TBS-T) and incubated overnight in 5\% BSA + TBS-T plus primary antibodies [anti-STEP (1:1000), anti-KCC2 (1:1000), anti-Fyn (1:1000) and anti- $\beta$-actin (1:10 000) from Santa Cruz; anti-non-phospho-STEP (1:1000) and anti-pY416-Src (or pY420-Fyn) (1:1000) from Cell Signaling; and anti-pY1472GluN2B (1:1000) from PhosphoSolutions; antiGluN2B (1:2000) and anti-GluN2A (1:1000) from Millipore; for further details on antibodies used in western blots, see Supplementary Table 1. Membranes were washed three times with TBS-T and incubated in horseradish peroxidase (HRP)-conjugated secondary antibodies (antimouse and anti-rabbit (1:5000) from Pierce for 2 hours at room temperature. Membranes were 
developed using Chemiluminescent Substrate kit (Pierce) and visualized using ChemiDoc XRS+ system (Bio-Rad, Hercules, CA, USA). All densitometric bands were quantified using ImageJ (NIH).

\subsubsection{Immunohistochemistry}

A sledge freezing Vibratome Leica VT1200S (Leica Microsystems) was used to cut 25 $\mu \mathrm{m}$ transverse sections of paraformaldehyde-fixed human spinal tissue. Sections were permeabilized in PBS ( $\mathrm{pH} 7.4$ ) with $0.2 \%$ Triton (PBS+T) for $10 \mathrm{~min}$, washed twice in PBS, then incubated for $12 \mathrm{~h}$ at $4^{\circ} \mathrm{C}$ in primary anti-KCC2 antibody, anti-CGRP antibody and antiNeuN antibody (see below) diluted in PBS+T containing 10\% normal goat serum. Tissue was washed in PBS, and subsequently incubated for $2 \mathrm{~h}$ at room temperature in goat-Cy3 anti-rabbit purified secondary antibody (1:500, Jackson ImmunoResearch Laboratories, Cat. \#111-165144) goat anti-chicken Alexa Fluor ${ }^{\circledR} 647$ secondary antibody (1:500, Invitrogen Cat. \#AB2535866) diluted in PBS+T (pH 7.4) containing 10\% normal goat serum. Spinal sections

were mounted on SuperFrost ${ }^{\mathrm{TM}}$ gelatin-subbed slides (Fisherbrand) and were cover-slipped using fluorescence mounting medium (Dako, Cat. \#S3023).

Nociceptive peptidergic afferent terminals, which are not present in any other types of axons in the dorsal horn (Rosenfeld et al., 1983; Gibson et al., 1984; Hunt and Rossi, 1985; Ju et al., 1987), were labelled by calcitonin gene-related peptide (CGRP) immunoreactivity using a monoclonal anti-CGRP antibody (1:5000; Sigma \#C7113) raised in mouse. This antiserum detects human $\alpha$-CGRP and $\beta$-CGRP but does not cross-react with any other peptide (data supplied by Sigma). 
We used a rabbit polyclonal antibody (1:1000, Millipore/Upstate, Cat. \#07-432) to label KCC2. This antibody was raised against a His-tag fusion protein corresponding to residues 9321043 of the rat KCC2 intracellular C-terminal (Williams et al., 1999; Mercado et al., 2006). This antibody is highly specific for rat $\mathrm{KCC} 2$ ( $\mathrm{KCC} 2 \mathrm{a}$ and $\mathrm{KCC} 2 \mathrm{~b}$ isoforms) and does not share any homologous sequences with other KCCs or co-transporters.

We used a chicken polyclonal anti-NeuN antibody (1:1000, MilliporeSigma Cat. \#6B9155 to reveal neuron cell bodies and thus to better distinguish $\mathrm{KCC} 2$ positive neuronal membrane.

A Zeiss LSM 880 Confocal Laser Scanning Microscope was used to acquire all confocal images. Acquisitions were 12-bit images, $2048 \times 2048$ pixels with a pixel dwell time of $10 \mu \mathrm{s}$. An oil-immersion $\times 63$ plan-apochromatic objective was used for high magnification confocal laser scanning microscopy images, which were processed for quantification. Laser power was adequately chosen to avoid saturation and limit photobleaching. All acquisitions were performed using the same laser settings (laser, power, photomultiplier tube (PMT) settings, image size, pixel size and scanning time). The experimenter was blind to the slice treatment (saline versus BDNF treatment) during acquisition.

We have previously developed MATLAB code to quantify and monitor the changes in the KCC2 intensity distributions in subcellular compartments (Dedek et al., 2019). A user must delineate the membrane of neuronal cell bodies present in the acquired confocal image. CGRP served as our marker of the SDH, and only neurons present in the regions of the SDH expressing CGRP were considered. For both imaging and analysis, the experimenter was blinded to the experimental conditions. For each pixel in the region of interest, the distance to the closest membrane segment was calculated. Using this distance map, the mean pixel intensity and 
standard deviation of $\mathrm{KCC} 2$ fluorescence signal were quantified as a function of the distance to the neuron membrane. A negative position value represents the region outside of the labelled neuron. In male humans, 143 saline-treated neurons and 205 BDNF-treated neurons from 12 adult donors were analyzed. In female humans, 221 saline-treated neurons and 297 BDNFtreated neurons from 10 adult donors were analyzed. Averaged profiles were obtained for each subject and condition (saline and BDNF) and from those averages, the global $\mathrm{KCC} 2$ intensity profiles were obtained for each condition in each sex. The KCC2 membrane intensity $(-0.5 \mu \mathrm{m}<$ position $<0.5 \mu \mathrm{m})$ and the $\mathrm{KCC} 2$ intracellular intensity $(0.6 \mu \mathrm{m}<$ position $<2.5 \mu \mathrm{m})$ were extracted from each subject's averaged $\mathrm{KCC} 2$ intensity profiles.

\subsubsection{Statistical Analysis}

For all statistical analyses, $\mathrm{p}<0.05$ was used as the threshold for statistical significance, and in all experiments, the declared group size is the number of independent values, and statistical analysis was performed upon these values. Comparison of means for Figures 1, 2, 3E, 4, and all supplementary data was carried out using IBM SPSS Statistics 27. Parametric tests (t tests and ANOVAs) were used when assumptions of normality (tested by Shapiro-Wilk) and homogeneity of variance (tested by Levene's test) were met. One-way repeated measures ANOVAs were performed to test for differences in PWT. Before running each ANOVA, we examined Mauchly's test of sphericity. When the assumption of sphericity was violated, the Greenhouse-Geisser epsilon adjustment of degrees of freedom was used to determine the pvalue. Pairwise comparisons with Bonferroni adjustment followed repeated measures ANOVAs when ANOVA achieved statistical significance $(\mathrm{p}<0.05)$ and showed no significant variance in homogeneity. Paired t-tests were used when comparing samples from the same rat or human (e.g. 
- saline versus BDNF). Prior to running paired t-tests, the normality of the data was tested (Shapiro-Wilk test), and if the data failed this test of normality $(\mathrm{p}<0.05)$, a Wilcoxon signedrank test was performed instead. T-tests comparing the means from different rats were performed as independent samples t-tests. If the data failed a test of normality (Shapiro-Wilk test), then a Mann-Whitney rank-sum test was performed instead. One-way ANOVAs were used when data passed assumptions of normality and homogeneity of variance and were followed by Tukey's HSD when ANOVA achieved statistical significance $(\mathrm{p}<0.05)$. If the ANOVA assumption of normality was violated, the nonparametric Kruskal Wallis test was performed instead. If the assumption of equal variances was violated, Welch's test was performed instead, followed by Games-Howell post hoc test is Welch's achieved statistical significance.

Comparison of means for Figure 3A-D was carried out using GraphPad Prism 9. To compare KCC2 membrane values, a region ranging from $-0.5 \mu \mathrm{m}$ to $+0.5 \mu \mathrm{m}$ from the $\mathrm{KCC} 2$ peak intensity was taken. Gaussian curves were determined as the best equations to characterize this region. Amplitudes, means and standard deviations were compared using the extra sum-ofsquares F-test method. For males KCC2 membrane intensities, two different Gaussian curves were required to fit the values $(\mathrm{P}=0.0002)$. For female KCC2 membrane intensities, two Gaussian curves were not required to fit the values $(\mathrm{P}=0.2551)$.

KCC2 Membrane fits: Gaussian distribution

$$
Y=\text { Amplitude } * e^{-0.5 *\left(\frac{X-M e a n}{S D}\right)^{2}}
$$

with $\mathrm{Y}=\mathrm{I}_{\mathrm{KCC} 2} ; \mathrm{KCC} 2$ intensity; and $\mathrm{X}$ is the intensity of $\mathrm{KCC} 2$ for each distance from the membrane. Mean = Mean KCC2 intensity (peak of the Gaussian) 
To compare $\mathrm{KCC} 2$ intracellular values, a region ranging from $0.6 \mu \mathrm{m}$ to $2.5 \mu \mathrm{m}$ from the KCC2 peak intensity was taken. One phase exponential decays were determined as the best equations to characterize this region. Amplitude, means and standard deviation were compared using the extra sum-of-squares F-test method. For males $\mathrm{KCC} 2$ intracellular intensities, two exponential decay curves were required to fit the values $(\mathrm{P}<0.0001)$. For female $\mathrm{KCC} 2$ membrane intensities, two exponential decay curves were not required to fit the values $(\mathrm{P}=0.7235)$.

KCC2 intracellular fit: exponential decay

$$
Y(X)=Y_{0} e^{-\lambda X}+C
$$

with $\mathrm{Y}=\mathrm{IKCC2}_{\mathrm{K}}(\mathrm{KCC} 2$ intensity), $\mathrm{X}=$ distance form the membrane, $\mathrm{C}=$ the baseline KCC2 intensity

For a complete list of statistical tests, comparisons made, and exact $\mathrm{p}$ values, see Supplementary Table 2).

\subsection{Results:}

\subsubsection{The CFA inflammatory pain model induces tactile allodynia in male and female adult rats, but only triggers the STEP61/pFyn/pGluN2B spinal hyperexcitability pathway in males}

To investigate spinal mechanisms underlying pathological pain in males versus female rats, we tested behavioural pain responses in the CFA inflammatory pain model, which uses an intraplantar injection of CFA to establish sustained inflammatory pain (Ren et al., 1992). Both male and female rats that received an intraplantar injection of CFA displayed significant 
reduction in PWT one through five days following CFA injection, while vehicle-injected rats showed no significant change in PWT (Figure 1A and C, Supplementary Table 2). We found that PWT in CFA-injected males and females remained decreased one-five days post-CFA injection (Supplementary Figure 1), demonstrating persistent mechanical allodynia throughout this time range. Examining ipsilateral SDH spinal cord tissue from male CFA- versus saline-injected animals revealed a trend toward a decrease in $\operatorname{KCC} 2(p=0.053)$, a marker of disinhibition, and a decrease in active $\operatorname{STEP}_{61}(p=0.0085)$, which links disinhibition to facilitated excitation. We also saw an increase in active Fyn ( $p=0.013)$, the GluN2B-phosphorylating kinase, and an increase in overall and phosphorylated GluN2B $(p=0.023 ; p=5.61 \mathrm{E}-4)$, the dominant NMDAR GluN2 subunit at adult lamina I excitatory synapses (Hildebrand et al., 2014), in SDH crude synaptosome fractions ( $\mathrm{n}=8$ for all targets, Figure 1B, Supplementary Table 2$)$. These results show that a disinhibition-STEP 61 downregulation-NMDAR potentiation feed-forward pathway occurs in male CFA-treated animals, as we have previously reported (Dedek et al., 2019). In sharp contrast to males, when examining ipsilateral SDH tissue from female rats, no changes were seen in the above markers of disinhibition $\left(\mathrm{KCC}_{2}: \mathrm{p}=0.78 ; \operatorname{aSTEP}_{61}: \mathrm{p}=0.96 ; \mathrm{tSTEP}_{61}: \mathrm{p}\right.$ $=0.29$ ) and facilitated excitation ( $\mathrm{pY}^{420} \mathrm{Fyn}: \mathrm{p}=0.13$; Fyn: $\mathrm{p}=0.85 ; \mathrm{pY}^{1472}$ GluN2B: $\mathrm{p}=0.60$; GluN2B: $\mathrm{p}=0.43)$ between vehicle- and CFA-treated rats $(\mathrm{n}=8$ for all targets, Figure 1D, Supplementary Table 2). In addition, no significant CFA-mediated changes in any of the above targets were found in the crude synaptosome fractions of the remainder of the spinal cord for either sex (Supplementary Figures 2,5, Supplementary Table 2). The lack of changes in markers of this spinal mechanism of hyperexcitability in female CFA rats suggests that neuronal mechanisms of pathological pain may diverge between male and female rats. 


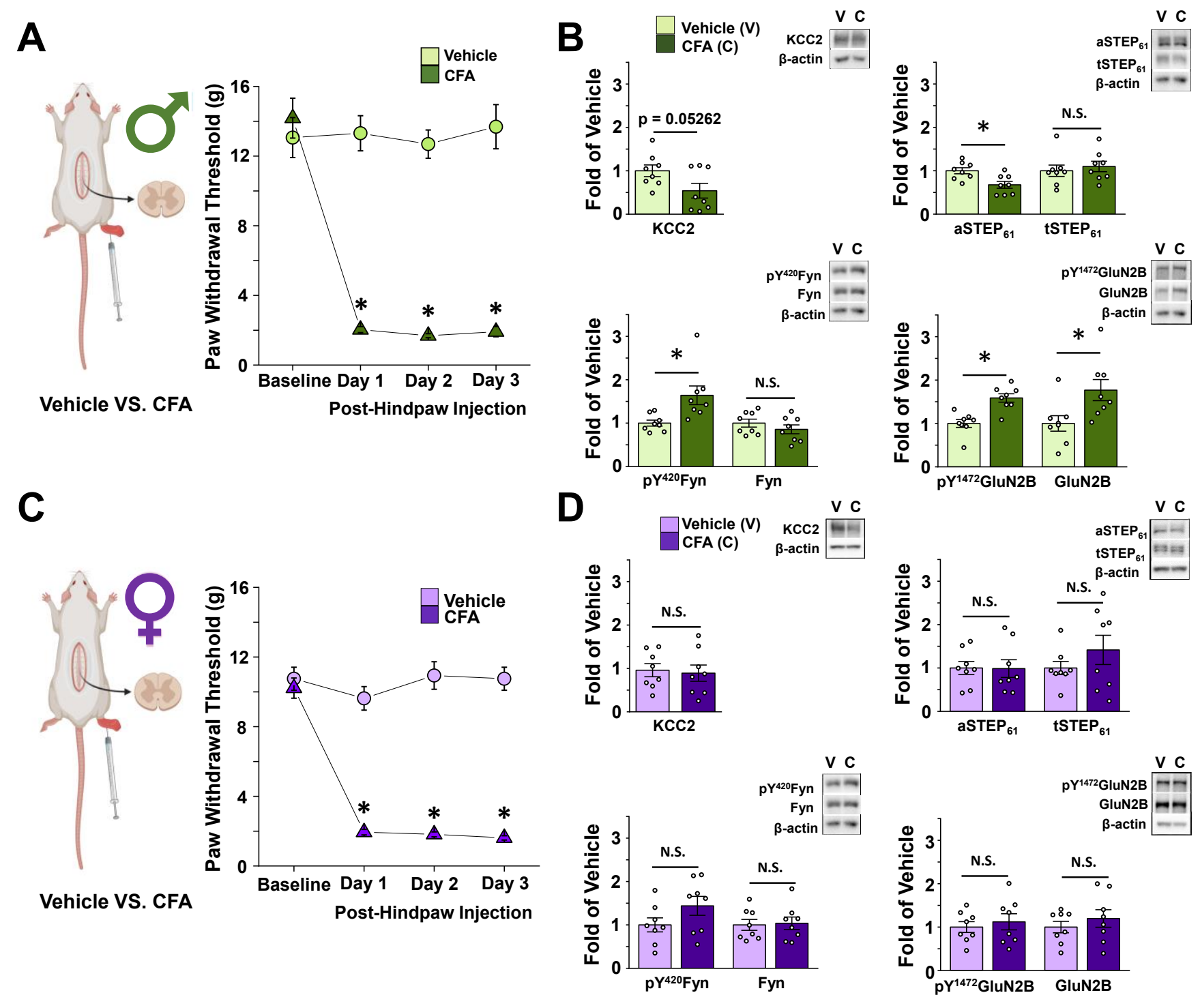

Chapter 3 Figure 1. CFA elicits tactile allodynia in male and female adult rats, but only activates the

STEP61/pFyn/pGluN2B spinal hyperexcitability pathway in males. A) Left: In vivo CFA inflammatory pain model. Right: CFA elicits tactile allodynia in adult male rats. Von Frey behaviour testing shows that rats given CFA $(\mathrm{n}=14)$ to their right hindpaw have decreased paw withdrawal threshold compared to vehicle-injected animals $(\mathrm{n}=8)$. Animals' tissue was collected for use in either western blot analysis or electrophysiological recordings. B) CFA inflammatory pain model drives upregulation of pFyn, pGluN2B, GluN2B, and downregulation of aSTEP ${ }_{61}$ in male rat SDH synaptosomes. Plots (left) and representative western blots (right) from male rat SDH synaptosomes treated 
with either vehicle (light green, $\mathrm{n}=8$ for all targets) or CFA (dark green, $\mathrm{n}=8$ for all targets) for 5 days. C) Left: In vivo CFA inflammatory pain model. Right: Von Frey behaviour testing shows that rats given CFA $(\mathrm{n}=14)$ to their right hindpaw have decreased paw withdrawal threshold compared to vehicle-injected animals $(\mathrm{n}=8)$. Animals' tissue was collected for use in either western blot analysis or electrophysiological recordings. D) CFA inflammatory pain model elicits no effect in the STEP61/pFyn/pGluN2B spinal hyperexcitability pathway of female rat SDH synaptosomes. Plots (left) and representative western blots (right) from female rat SDH synaptosomes treated with either vehicle (lilac, $\mathrm{n}=8$ ) or CFA (purple, $\mathrm{n}=8$ ) for 5 days. $* \mathrm{P}<0.05$

\subsubsection{NMDARs in the female SDH are not potentiated or upregulated by ex vivo BDNF treatment or in the in vivo CFA model of pathological pain}

Given observed differences in spinal markers of hyperexcitability between sexes, we next investigated excitatory synaptic NMDAR responses in male and female rats. To investigate baseline properties of excitatory NMDAR responses at lamina I synapses in male versus female rats, we measured NMDAR mEPSCs to compare spontaneous synaptic activity in lamina I, the outermost layer of the SDH. We compared mEPSCs at a holding potential of $+60 \mathrm{mV}$ and found that mEPSCs from untreated lamina I neurons do not differ between sex in peak amplitude (male $\mathrm{I}_{\text {peak }}=14.66 \pm 1.54 \mathrm{pA}$, female $\mathrm{I}_{\text {peak }}=17.03 \pm 1.02 \mathrm{pA}$ ), as measured from the average amplitude between 18-22 ms after the start of the mEPSC $(p=0.22, n=10$ cells from eight animals for males, $\mathrm{n}=10$ cells from six animals for females, Figure 2A). Furthermore, we observed no difference in decay constant (male $\tau$-decay $=251.64 \pm 25.61 \mathrm{~ms}$, female $\tau$-decay $=219.99 \pm$ 19.32 ms), or NMDAR charge transfer, measured as AUC from 40-500 ms after the start of the mEPSC, after AMPARs have decayed (Hildebrand et al., 2016) (male $=3.06 \pm 0.18 \mathrm{pC}$, female $=3.73 \pm 0.29 \mathrm{pC}$ ), indicating that overall biophysical properties of slow-decaying NMDAR responses at lamina I synapses are conserved $(p=0.063 ; p=0.32, n=10$ cells from eight 
animals for males, $n=10$ cells from six animals for females, Figure 2A) between male and female rats. These results suggest that baseline synaptic NMDAR physiology is conserved between sexes in rats.

We next compared NMDAR mEPSCs in both the ex vivo BDNF pathological pain model and in the CFA inflammatory pain model in both sexes. In male rats, NMDAR charge transfer from lamina I neurons of CFA animals with validated pain hypersensitivity (PWT measured in Figure 1A) is robustly potentiated in comparison to NMDAR mEPSCs from untreated animals ( $p$ $=0.032, \mathrm{n}=10$ cells from eight animals for control, 8 cells from six animals for CFA, Figure 2B, Supplementary Table 2). Furthermore, using our previously validated rodent ex vivo BDNF model, where slices from naïve rats are incubated in $50 \mathrm{ng} / \mathrm{mL}$ recombinant BDNF for a minimum of 70 minutes (Dedek et al., 2019), we found robust, significant potentiation of NMDAR responses at lamina I synapses $(p=2.90 \mathrm{E}-6, \mathrm{n}=10$ control cells from eight animals, $\mathrm{n}$ $=6$ BDNF cells from five animals, Figure 2B, Supplementary Table 2, control and CFA data originally published by Dedek et al (Dedek et al., 2019)).

The NMDAR potentiation seen in male lamina I neurons differs greatly from the findings in females. Under the same conditions as males, charge transfer through NMDAR mEPSCs from spinal cord slices of CFA-treated females was not different from control NMDAR mEPSCs $(\mathrm{p}=$ $0.25, \mathrm{n}=10$ control cells from six animals, $\mathrm{n}=8$ CFA cells from five animals, Figure $2 \mathrm{C}$, Supplementary Table 2). As was the case in males (Figure 1A), female CFA-treated animals showed significantly decreased PWT (Figure 1C), however, unlike males, females did not have potentiated NMDAR synaptic responses. Furthermore, in female rats, synaptic NMDAR responses from spinal cord slices treated with BDNF ex vivo were not significantly different from control-treated slices $(\mathrm{p}=0.25, \mathrm{n}=10$ control cells from six animals, $\mathrm{n}=8$ BDNF cells 
from five animals, Figure 2C, Supplementary Table 2), demonstrating a lack of potentiation of synaptic NMDARs by ex vivo BDNF treatment in female rats. A previous study has found a slower onset of BDNF-mediated hypersensitivity in female mice compared to males following in vivo BDNF administration (Mapplebeck et al., 2019). To investigate whether more prolonged exposure to BDNF would induce NMDAR potentiation at female lamina I synapses, we performed a subset of experiments using slices incubated in $50 \mathrm{ng} / \mathrm{mL}$ BDNF for 2-4.5 hours. As was the case in slices incubated for a minimum of 70 minutes, NMDAR charge transfer from this experiment did not differ from control mEPSCs, and thus were grouped into the overall BDNFtreated female cells $(\mathrm{p}=0.89, \mathrm{n}=10$ control cells from six animals, $\mathrm{n}=6$ long-BDNF cells from 3 animals, Supplementary Figure 8, Supplementary Table 2). Furthermore, in another subset of neurons, slices were incubated in $100 \mathrm{ng} / \mathrm{mL}$ BDNF for 2+ hours and showed no difference in NMDAR charge transfer compared to control-treated recordings (data not shown). Taken together, our findings indicate that although male and female baseline NMDAR responses do not differ, NMDAR potentiation at lamina I synapses is observed in males but not females in both the BDNF ex vivo model and the in vivo CFA inflammatory pain model.

As was seen in the CFA inflammatory pain model, the BDNF ex vivo pain model did not elicit significant changes in a marker of disinhibition, $\mathrm{KCC} 2(\mathrm{p}=0.88)$, in active or total $\mathrm{STEP}_{61}$ $(\mathrm{p}=0.74, \mathrm{p}=0.47$, respectively) or markers of increased excitability (pFyn $\mathrm{p}=0.64$, Fyn $\mathrm{p}=$ 0.60 , pGluN2B $\mathrm{p}=0.70$, GluN2B $\mathrm{p}=0.62$ ) in female rat SDH crude synaptosomes (Figure 2D, Supplementary Table 2, $\mathrm{n}=8$ for all targets). This differs from previous findings in male rats, where we found that the BDNF ex vivo pathological pain model resulted in a significant downregulation of $\mathrm{KCC} 2$, and active and total $\mathrm{STEP}_{61}$, and an upregulation of pFyn. pGluN2B and GluN2B (Dedek et al., 2019). As we have seen in both CFA-treated male tissue (Figure 1B) 
and previously in the male BDNF ex vivo pathological pain model (Dedek et al., 2019), we saw no BDNF-mediated changes in the synaptosome fractions of the remainder of the spinal cord (Supplementary Figure 9, Supplementary Table 2) of female rats. From these data, we gather that, unlike in males, ex vivo BDNF treatment does not induce the pathological KCC2-STEP $61^{-}$ Fyn-GluN2B signalling pathway at SDH synapses of female rats. 
A

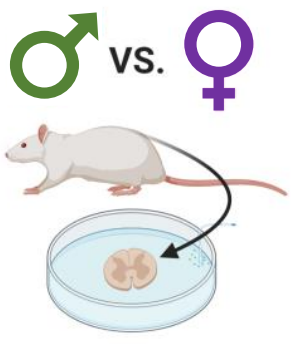

Naïve Control

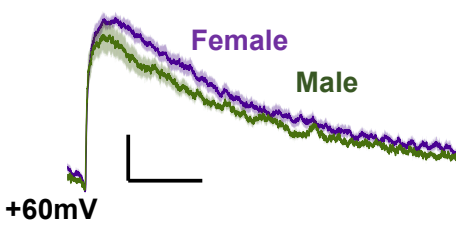

B

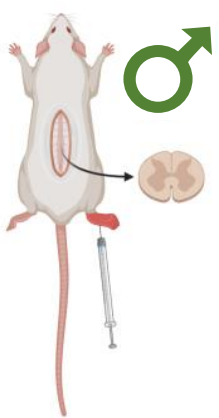

CFA vS. BDNF vS. Naïve Control

C

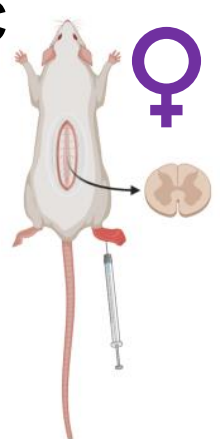

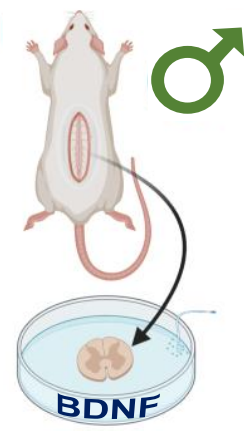

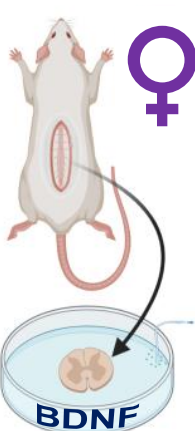

CFA VS. BDNF VS. Naïve Control

D

Saline (S)
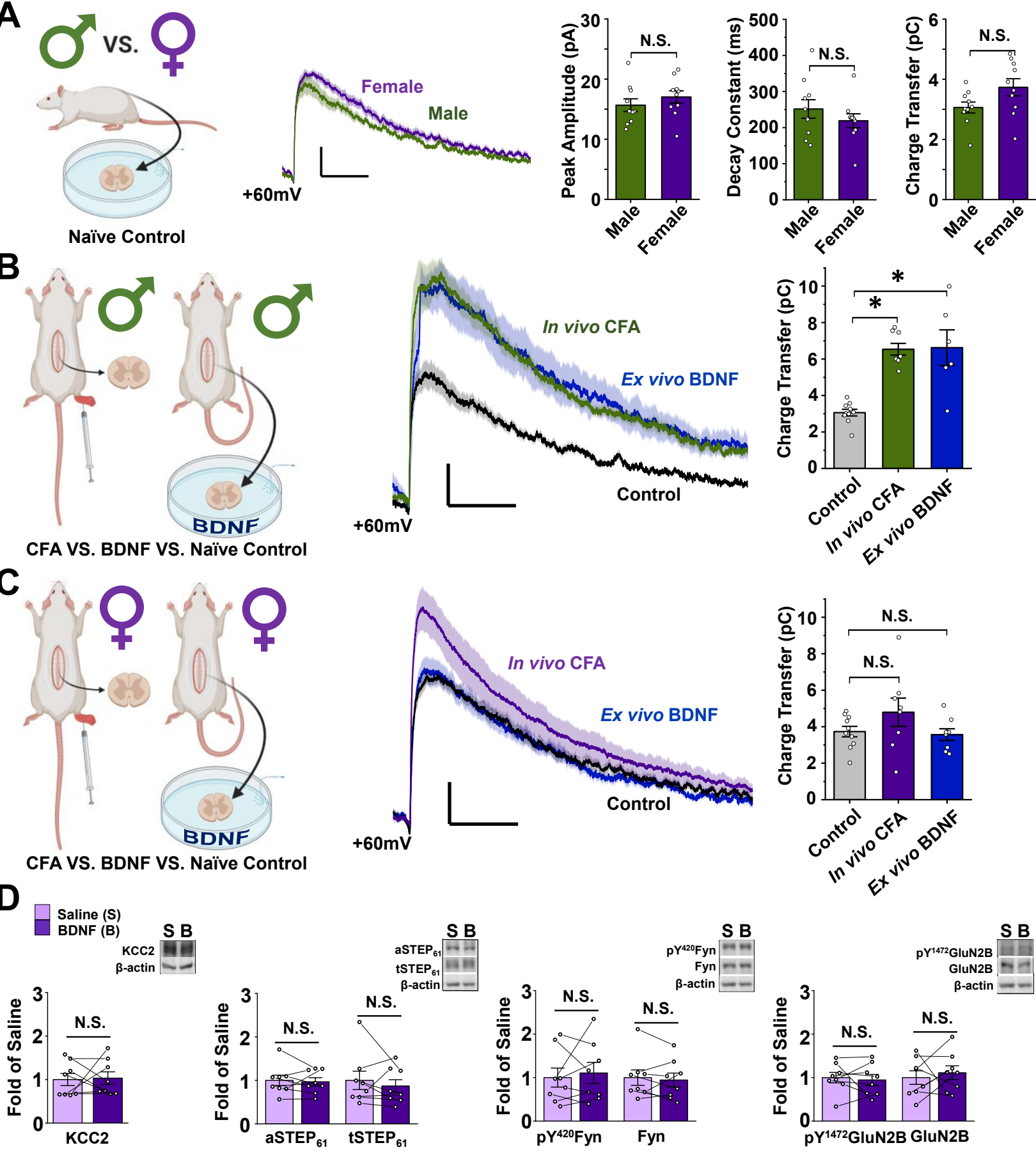

Chapter 3 Figure 2. Female SDH NMDARs are not potentiated or upregulated by the ex vivo BDNF or in vivo CFA models of pathological pain. A) Baseline lamina I mEPSCs do not differ between male and female adult SD 
rats. Left: saline-treated male vs. female lamina I neurons. Middle: NMDAR lamina I mEPSCs comparing male (green) and female (purple) synaptic responses. Right: charge transfer, decay constant and peak amplitude of NMDAR mEPSCs do not differ between male and female SD rats. $n=10$ for males and 9 for females. B) Male lamina I NMDARs are potentiated following CFA hindpaw injection and ex vivo BDNF treatment. Left: Experimental paradigm showing male in vivo CFA vs. ex vivo BDNF models. Middle: NMDAR mEPSCs from male rat lamina I neurons; control in black, CFA in green, BDNF in blue. Right, charge transfer of NMDAR mEPSCs is shown on left. $\mathrm{n}=10$ for control, 8 for CFA, and 6 for BDNF. C) Female lamina I NMDARs are not potentiated following CFA hindpaw injection or ex vivo BDNF treatment. Left, Experimental paradigm showing male in vivo CFA vs. ex vivo BDNF models. Middle: NMDAR mEPSCs from female rat lamina I neurons; control in black, CFA in purple, BDNF in blue. Right, charge transfer of NMDAR mEPSCs is shown on left. $\mathrm{n}=10$ for control, 8 for CFA, and 8 for BDNF. D) Ex vivo BDNF treatment model elicits no effect in female rat SDH synaptosomes. Plots (left) and representative western blots (right) from female rat SDH synaptosomes treated with either control saline (lilac, $\mathrm{n}=8$ ) or $50 \mathrm{ng} / \mathrm{mL}$ recombinant $\mathrm{BDNF}$ for 70 minutes (purple, $\mathrm{n}=8$ ). $* \mathrm{P}<0.05$

\subsubsection{Ex vivo BDNF treatment results in sexually dimorphic effects on markers of disinhibition and facilitated excitation at synapses in the human SDH}

A major barrier to understanding human spinal physiology is a lack of human preclinical pain models (Gereau et al., 2014). To address this, we have previously developed a human ex vivo BDNF model of pathological pain using tissue from human organ donors (Dedek et al., 2019). In this model, tissue was collected from adult neurological determination of death organ donors. Tissue was incubated in oxygenated solution containing either $100 \mathrm{ng} / \mathrm{mL}$ recombinant BDNF or a saline control for 70 minutes (Dedek et al., 2019). Previously, using male tissue, we have found that our human ex vivo BDNF model recapitulates mechanisms seen in rodents (Dedek et al., 2019). Here, we first probed for a marker of BDNF-mediated disinhibition in human SDH neurons using KCC2 immunostaining (Dedek et al., 2019; Ferrini et al., 2020). 
CGRP immunostaining was used to identify the SDH (Shiers et al., 2021). Within the CGRPpositive $\mathrm{SDH}$, we measured $\mathrm{KCC} 2$ immunostaining at neuronal membranes and within neurons and compared between BDNF- versus saline-treated spinal tissue from each organ donor. In a previous study using male tissue, we have shown that BDNF treatment results in a significant reduction in $\mathrm{KCC} 2$ at the neuronal membrane, as well as an increase in intracellular $\mathrm{KCC} 2$ in SDH neurons (Dedek et al., 2019). Here, with an expanded male tissue sample size, we found that BDNF treatment resulted in a significant decrease in membrane $\mathrm{KCC} 2$ intensity $(\mathrm{p}=2.09 \mathrm{E}$ $4, \mathrm{n}=12$, Figure $3 \mathrm{~A}$, Supplementary Table 2) and a significant increase of intracellular KCC2 intensity $(\mathrm{p}=1.75 \mathrm{E}-4, \mathrm{n}=12$, Figure $3 \mathrm{~A})$ in human SDH neurons. In female humans, however, we saw no change in $\mathrm{KCC} 2$ intensity at neuronal membranes or intracellularly following BDNF treatment $(p=0.26, p=0.72, n=10$, Figure 3B, Supplementary Table 2), demonstrating that $e x$ vivo BDNF treatment does not result in internalization of $\mathrm{KCC} 2$ from neuronal membranes. This suggests that, unlike in males, the ex vivo treatment with $\mathrm{BDNF}$ does not result in $\mathrm{KCC} 2-$ dependent disinhibition in SDH neurons of human females.

Examination of human spinal cord tissue by western blot shows further differences between responses in human males and females to ex vivo BDNF treatment. In SDH synaptosomes of human males, we have previously shown that BDNF treatment triggers downregulation of $\mathrm{KCC} 2$ and total and active $\mathrm{STEP}_{61}$, indicating disinhibition, and upregulation of phosphorylated Fyn and phosphorylated GluN2B, markers of facilitated excitation (Dedek et al., 2019). In contrast, female human tissue subjected to BDNF treatment displayed no changes in markers of disinhibition $\left(\mathrm{KCC} 2 \mathrm{p}=0.47, \mathrm{aSTEP}_{61} \mathrm{p}=0.60, \mathrm{tSTEP}_{61} \mathrm{p}=0.77\right)$ or facilitated excitation ( $p$ Fyn $p=0.49$, Fyn $p=0.63$, pGluN2B $p=0.60$, GluN2B $p=0.82$ ) when compared to saline-treated controls in either the SDH crude synaptosome fraction $(n=6$, Figure $3 \mathrm{C}$, 
Supplementary Table 2). Similar to male and female rats, no changes in these targets were seen in the crude synaptosome fraction from the remainder of the spinal cord as a result of BDNF treatment, except for a change in aSTEP 61 ( $p=0.025, \mathrm{n}=6$, Supplementary Figure 12, Supplementary Table 2). We thus demonstrate our findings of sexual divergence in neuronal mechanisms underlying pathological pain are conserved between rats and humans. 


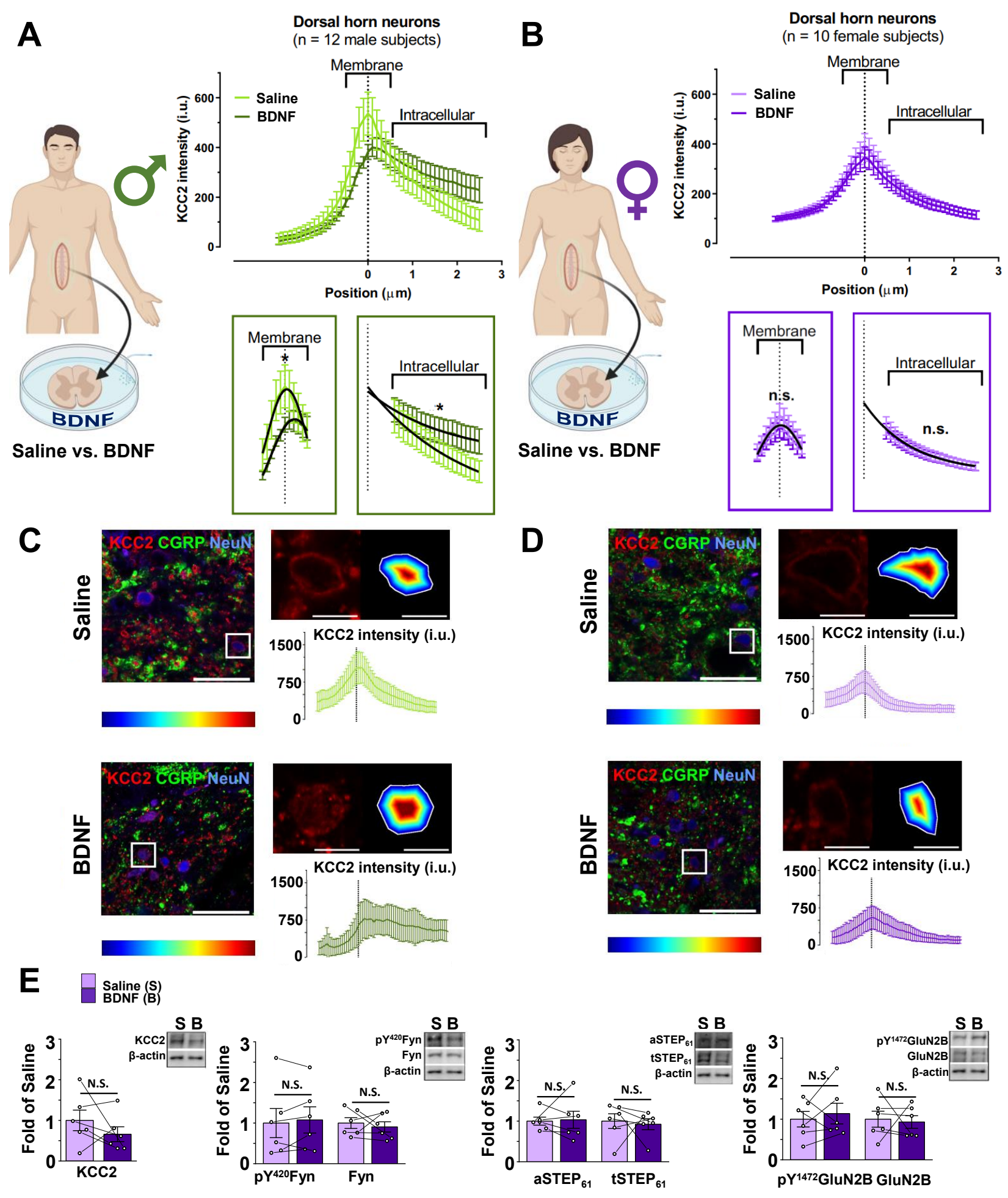

Chapter 3 Figure 3. Unlike in males, human female lamina I neurons do not show evidence of disinhibition or facilitated excitation following ex vivo BDNF treatment. A) The ex vivo BDNF model elicits KCC2 internalization in adult male human SDH neurons. Left: experimental paradigm showing treatment of human SDH 
tissue in either saline or BDNF. Right: Average KCC2 intensity values from SDH neurons incubated in saline (light green) versus BDNF-treated (dark green) spinal segments of 12 male human donors, with comparisons of membrane and intracellular regions below. B) The ex vivo BDNF model has no effect on $\mathrm{KCC} 2$ internalization in adult female human SDH neurons. Left: experimental paradigm showing treatment of human SDH tissue in either saline or BDNF. Right: Average KCC2 intensity values from SDH neurons incubated in saline (light green) versus BDNFtreated (dark green) spinal segments of 12 male human donors, with comparisons of membrane and intracellular regions below. C and D) Representative confocal images of male (C) and female (D) human superficial dorsal horn incubated in saline or BDNF. KCC2 (red), CGRP (green) and DAPI (blue). A zoomed region (top right) shows a neuron expressing $\mathrm{KCC} 2$ together with the delineation of the membrane and the distance to the membrane of each pixel analyzed in a colour-coded distance map. KCC2 intensity (i.u.) versus distance to the membrane profile (bottom). E) Ex vivo BDNF treatment model elicits no effect in human female SDH synaptosomes. Plots (left) and representative western blots (right) from human female SDH synaptosomes treated with either control saline (lilac, $\mathrm{n}=6$ ) or $100 \mathrm{ng} / \mathrm{mL}$ recombinant BDNF for 70 minutes (purple, $\mathrm{n}=6$ ). ${ }^{*} \mathrm{p}<0.05$

\subsubsection{Ovariectomy initiates BDNF-sensitivity in female SDH neurons}

After establishing a sex difference in pathological spinal pain processing that is conserved from rodents to humans, we aimed to understand the underpinnings of this sex difference. Since sexually dimorphic NMDAR signalling has previously been found to be controlled by sex hormones in a model of swim stress-induced analgesia (Mogil et al., 1993), we next explored whether removal of the effects of sex hormones during development would alter sensitivity to BDNF at adult female SDH synapses. We used animals that were ovariectomized on postnatal day 21 , before reaching sexual maturity, that were then left to age-match the adult animals used throughout the study. We first compared the effects of ovariectomy on baseline spontaneous lamina I synaptic NMDAR activity from adult rats. As was seen in when comparing baseline male and female lamina I NMDAR mEPSCs at $+60 \mathrm{mV}$ (Figure 2A), we found no 
difference in NMDAR mEPSC peak amplitude between age-matched naïve $\left(\mathrm{I}_{\text {peak }}=17.03 \pm 1.02\right.$ $\mathrm{pA})$ and ovariectomized $\left(\mathrm{I}_{\mathrm{peak}}=18.44 \pm 2.07 \mathrm{pA}\right)$ females $(\mathrm{p}=0.97, \mathrm{n}=10$ naïve control cells from six animals, $n=8$ ovariectomized control cells from five animals, Figure 4A). This indicates that the overall amplitude of synaptic NMDAR responses is not affected by the absence of sex hormones during development. Charge transfer (naïve $=3.73 \pm 0.29 \mathrm{pC}$, ovariectomized $=$ $3.55 \pm 0.27 \mathrm{pC}$ ), and decay constant (naïve $\tau$-decay $=219.99 \pm 19.32 \mathrm{~ms}$, ovariectomized $\tau$ decay $=212.37 \pm 30.56 \mathrm{~ms}$ ), were also not different between naïve and age-matched ovariectomized females, $(\mathrm{p}=0.66 ; \mathrm{p}=0.85, \mathrm{n}=10$ naïve control cells from six animals, $\mathrm{n}=8$ ovariectomized control cells from five animals, Figure 4A, Supplementary Table 2), suggesting that female sex hormones during early development do not significantly change the overall NMDAR subunit-driven (Hildebrand et al., 2014) biophysical properties of NMDAR responses at adult lamina I synapses at baseline.

Next, we tested the effects of $e x$ vivo BDNF treatment on adult spinal tissue from ovariectomized rats. Surprisingly, we found that charge transfer from lamina I NMDAR mEPSCs recorded at a holding potential of $+60 \mathrm{mV}$ from slices incubated in BDNF was significantly increased in comparison to ovariectomized control NMDAR charge transfer $(p=$ 5.01E-4, $\mathrm{n}=8$ control cells from five ovariectomized animals, $\mathrm{n}=9$ ovariectomized BDNF cells from seven animals, Figure 4B, Supplementary Table 2). Previously, we have shown that PP2, a Src family kinase inhibitor (Hanke et al., 1996), blocks BDNF-mediated potentiation of GluN2B-containing NMDARs at male rodent lamina I synapses (Hildebrand et al., 2016). Here, we tested whether PP2 could block BDNF-mediated NMDAR potentiation in tissue from ovariectomized animals. NMDAR charge transfer from BDNF $+1 \mu \mathrm{M}$ PP2-treated lamina I neurons did not differ from ovariectomized controls $(p=0.65, n=8$ ovariectomized control cells 
from five animals, $\mathrm{n}=9 \mathrm{BDNF}+\mathrm{PP} 2$ cells from five animals, Figure 4B, Supplementary Table 2), but did differ significantly with BDNF-treatment $(\mathrm{p}=0.0034, \mathrm{n}=9$ for both groups, Figure 4B, Supplementary Table 2), indicating that PP2 treatment blocked BDNF-mediated potentiation of NMDAR responses at lamina I synapses of ovariectomized rats.

When using western blot to compare levels of SDH synaptic proteins linked to the disinhibition/facilitated excitation pathway from ovariectomized rat tissue with and without $e x$ vivo BDNF treatment, we saw a marked difference compared to the results in naïve females reported in Figure 2D. BDNF treatment in ovariectomized rats resulted in downregulation of $\mathrm{KCC} 2(\mathrm{p}=0.040)$ and total $\mathrm{STEP}_{61}(\mathrm{p}=6.16 \mathrm{E}-4)$, and upregulation of $\mathrm{pY}^{420} \mathrm{Fyn}(\mathrm{p}=0.046)$ in the crude synaptosome fraction (no changes in: $\operatorname{aSTEP}_{61}: \mathrm{p}=0.41$, Fyn: $\mathrm{p}=0.87$, $p Y^{1472}$ GluN2B: $p=0.24$, and GluN2B: $p=0.33 ; n=8$ for all targets, Figure $4 C$, Supplementary Table 2). In the crude synaptosome fractions from the remainder of the spinal cord, we observed significant changes in Fyn $(p=0.011)$ and total $\operatorname{STEP}_{61}(p=0.0014$; Supplementary Figures 15 , Supplementary Table 2). In previous studies, results in males have only shown significant differences in the SDH crude synaptosome fractions (Hildebrand et al., 2016; Dedek et al., 2019). Ovariectomy changes the response to BDNF not only at SDH synapses, but throughout synaptosome fractions in the entire spinal cord. Together, these findings suggest that female rats ovariectomized before reaching sexual maturity respond in a male-like manner to BDNF treatment, switching from the non-BDNF-sensitive naïve female phenotype to the male disinhibition-linked-to-NMDAR-potentiation phenotype in SDH neurons. 
A

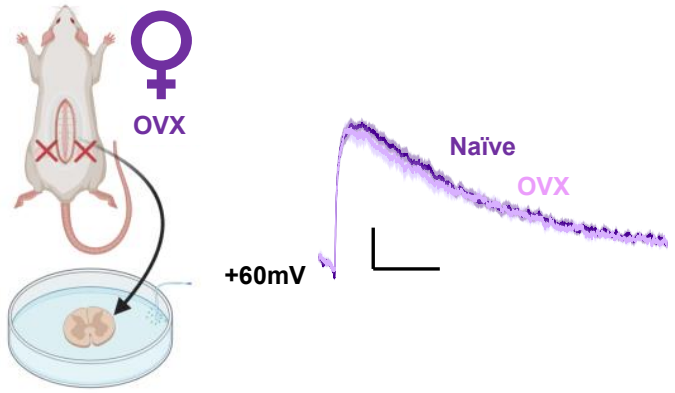

Naïve vs. OVX

B

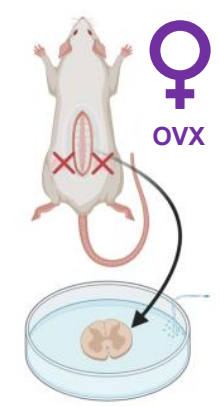

OVX Saline vs. BDNF
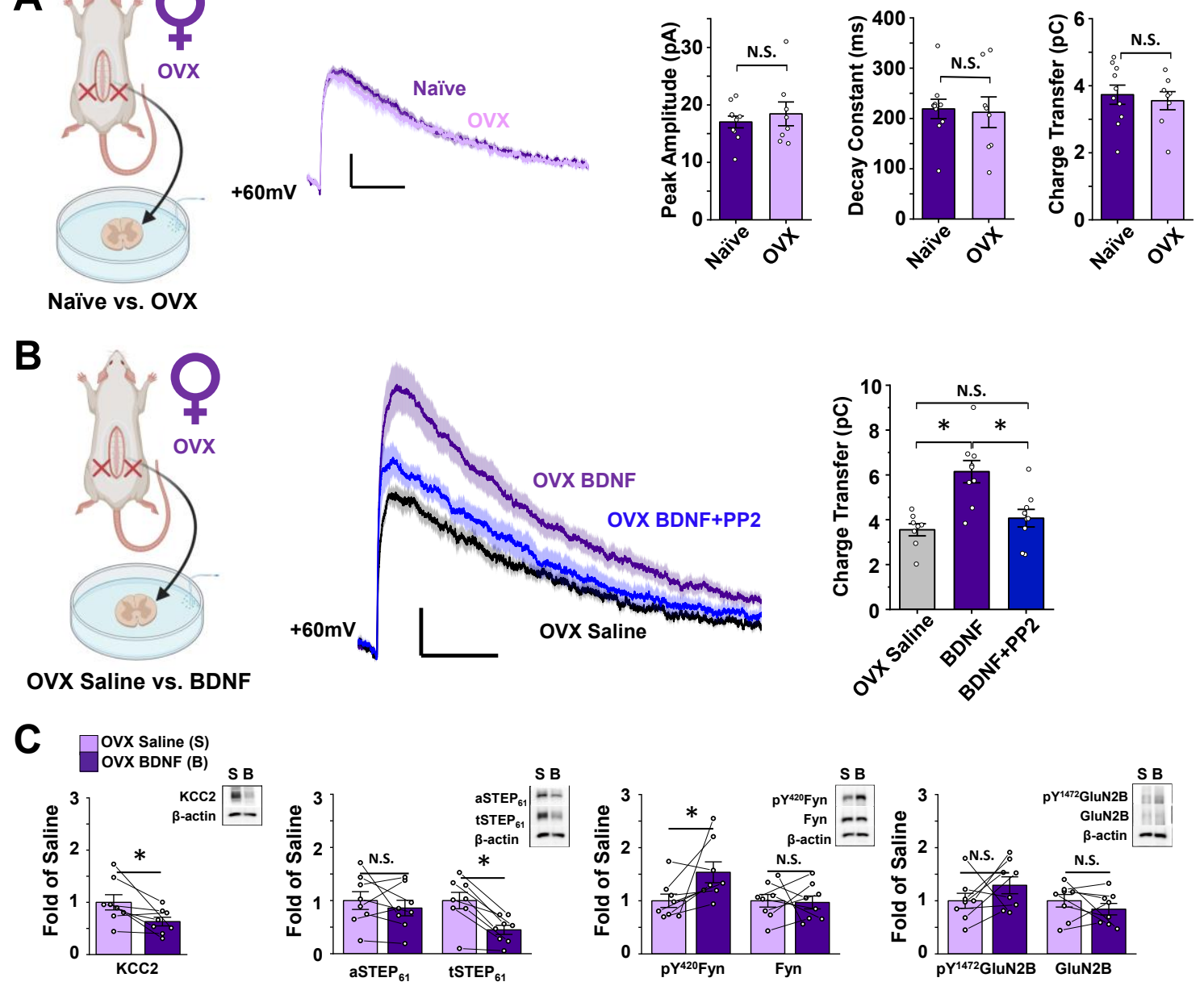

Chapter 3 Figure 4. Ovariectomy triggers BDNF-sensitivity in SDH neurons. A) Ovariectomy has no effect on baseline lamina I mEPSCs of female SD rats. Left: saline-treated naïve female vs. ovariectomized female lamina I neurons. Middle: NMDAR lamina I mEPSCs comparing naïve (purple) and ovariectomized (lilac) synaptic responses. Right: charge transfer, decay constant and peak amplitude of NMDAR mEPSCs do not differ between naïve and ovariectomized female SD rats. $\mathrm{n}=10$ for naïve and $\mathrm{n}=8$ for ovariectomized females. B) Ovariectomized female lamina I NMDARs are potentiated following ex vivo BDNF treatment. This potentiation is blocked using the SFK inhibitor PP2. Left: saline-treated naïve female vs. ovariectomized female lamina I neurons. Middle: NMDAR mEPSCs from ovariectomized female rat lamina I neurons; control in black, BDNF in purple, BDNF+PP2 in blue. Right: charge transfer of NMDAR mEPSCs shown on left. $\mathrm{n}=8$ for control, 9 for BDNF and BDNF + PP2. C) Ex vivo BDNF treatment model in ovariectomized rat $\mathrm{SDH}$ synaptosomes results in upregulation of $\mathrm{pY}^{420} \mathrm{Fyn}$ and downregulation of $\mathrm{KCC} 2$ and $\mathrm{STEP}_{61}$. Plots (left) and representative western blots (right) from ovariectomized 
female rat SDH synaptosomes treated with either control saline (lilac, $\mathrm{n}=8$ ) or $50 \mathrm{ng} / \mathrm{mL}$ recombinant BDNF for 70 minutes (purple, $\mathrm{n}=8$ ). $* \mathrm{P}<0.05$

\subsection{Discussion:}

We investigated mechanisms of spinal hyperexcitability in the rat and human superficial dorsal horn. Both male and female rats subjected to the in vivo CFA inflammatory pain model displayed tactile allodynia, and yet, we found that the STEP 61 -pFyn-pGluN2B spinal hyperexcitability pathway was only activated in males, and not in females. Unlike males, CFAinjected female rats did not show potentiated lamina I synaptic NMDAR responses. In addition, BDNF-treated rat slices showed potentiated synaptic lamina I NMDAR responses in male, but not female rats. Building on our previous work establishing the ex vivo BDNF model of pathological pain in male humans (Dedek et al., 2019), we tested the effects of ex vivo BDNF treatment on female human SDH tissue. We found that, like female rats, female human SDH tissue showed no changes in synaptic protein expression linked to hyperexcitability. Furthermore, we showed that, unlike in males, ex vivo BDNF treatment does not result in internalization of $\mathrm{KCC} 2$ from neuronal membranes, indicating that BDNF treatment does not result in disinhibition in human SDH neurons. Taken together, our female rat and human experiments show that $\mathrm{BDNF}$ does not drive a pathological $\mathrm{KCC}_{2} / \mathrm{STEP}_{61} /$ Fyn-mediated potentiation of synaptic NMDARs within the female SDH, as it does in males. We conclude that this sex difference in response to BDNF is hormonally mediated, with rats ovariectomized before reaching sexual maturity displaying a male-like response to BDNF treatment characterized by SFK-dependent NMDAR potentiation and synaptic markers of disinhibition and facilitated excitation. 
Although differences in immune mediation of pain have been discovered, the current hypothesis is that neuronal mechanisms are conserved, with sexually dimorphic immune mediators driving the divergence in pain phenotypes. In males, microglia-neuron interactions are required for mechanical pain hypersensitivity, while females instead rely on T-lymphocytes (Sorge et al., 2015; Mapplebeck et al., 2018). A neuronal mechanism long known to promote spinal hyperexcitability is the potentiation of NMDARs (Costigan and Woolf, 2000; Petrenko et al., 2003). We find that although baseline synaptic NMDAR responses are conserved between sex, we see a divergence in synaptic NMDAR responses to BDNF treatment and CFA-mediated

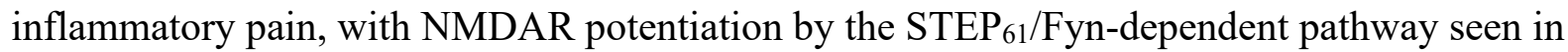
males, but not females. This contrasts with decades of research using male or unsexed animals that implicate NMDAR-mediated spinal hyperexcitability in inflammatory pain models (Chapman et al., 1995; Zhao et al., 2006; Liu et al., 2008; Dedek et al., 2019). A series of experiments from Sorge, Mapplebeck and colleagues showed that the development of SNIinduced mechanical allodynia was impaired in BDNF-null males, but not females, suggesting that in female mice BDNF is not required for the development of tactile allodynia (Sorge et al., 2015). It remains unknown if NMDARs are potentiated in the SDH in female neuropathic pain models, and future studies are needed to address this question.

Whilst we find no synaptic NMDAR potentiation at female lamina I synapses following either BDNF treatment or in the CFA inflammatory pain models, evidence supports that spinal hyperexcitability occurs in females. In female SNI rats (Luo et al., 1994) and human fibromyalgia patients (Tanwar et al., 2019), there is a decrease in nociceptive flexion reflex threshold, which has been used as a measure of central sensitization (Skljarevski and Ramadan, 2002). Windup, measured by electromyography (Redondo-Castro et al., 2013) and in vivo 
electrophysiology (Zhang et al., 2005), has also been observed in female animals with spinal cord injury. Additionally, LTP can be established in chronic opioid-treated rats (Haugan et al., 2008). Despite this evidence, the molecular mechanisms underlying SDH hyperexcitability in females remain unclear. One possible candidate is CGRP, which has been shown to alter excitatory postsynaptic responses within higher-order pain processing circuits of the amygdala (Han et al., 2010) as well as in SDH neurons (Bird et al., 2006). The roles of CGRP in mediating pain pathology may also be sexually dimorphic. In low doses, dural CGRP induces pain hypersensitivity only in females in rodent preclinical models of migraine (Avona et al., 2019). Recent developments show that this female-specific effect of CGRP may be dependant on prolactin (Avona et al., 2021). Further studies are needed to understand the role of CGRP in male and female pain signalling in the SDH.

Despite the differences in immune function, it has been difficult to uncover sexually dimorphic neuronal nociceptive mechanisms. This is because pharmacologically blocking neuronal targets in both sexes has been found to alleviate behavioural hypersensitivity, and thus, it has been assumed that different immune mechanisms converge at the neuronal level. For example, the NMDAR antagonist APV blocks SNI-induced allodynia in both sexes (Sorge et al., 2015), however, this indicates only that NMDAR inhibition is sufficient to cause anti-allodynia, not that NMDARs themselves underlie allodynia in females. This highlights the importance of performing mechanistic studies in both sexes (Seydel, 2021; Shansky and Murphy, 2021).

A small number of studies have previously performed in vivo intrathecal administration of BDNF to female rodents and found BDNF administration resulted in behavioural hypersensitivity in mice and rats (Lee-Kubli and Calcutt, 2013; Mapplebeck et al., 2019) and an associated downregulation of KCC2 (Lee-Kubli and Calcutt, 2013). In particular, Mapplebeck et 
al. show that intrathecal BDNF administration results in tactile allodynia in both sexes, but with a longer time required for the sensitizing effect in females (Mapplebeck et al., 2019). For this reason, we treated a subset of female rat spinal slices with BDNF for 2-4.5 hours (Supplementary Figure 1) and found no difference in NMDAR mEPSC charge transfer between the long-BDNF incubation or control-treated mEPSCs. Given that intrathecal BDNF administration has been found to cause tactile allodynia (Lee-Kubli and Calcutt, 2013; Mapplebeck et al., 2019), but we see no effect of BDNF on our targets in ex vivo spinal cord slices, we propose that BDNF induces other signalling pathways through trophic mechanisms (Lu et al., 2012; Boakye et al., 2019) in vivo that rely on factors outside our mechanism under study in the SDH. An important finding in previous studies is that intrathecal BDNF injection (Lee-Kubli and Calcutt, 2013), as well as neuropathic pain (Mapplebeck et al., 2019), can trigger disinhibition in both sexes. In an SNI neuropathic pain model, $\mathrm{KCC} 2$ was found to be downregulated in male and female mice, and enhancing chloride extrusion using CLP-290 in SNI mice partially reversed SNI-induced allodynia (Mapplebeck et al., 2019). Given that some neurochemical factors mediating allodynia differ between neuropathic and inflammatory pain (Honore et al., 2000; Zhao et al., 2006; Xu and Yaksh, 2011) it is unclear if the KCC2-STEP 61 -pFyn-pGluN2B spinal hyperexcitability pathway is activated in neuropathic pain states in female rats.

Foundational basic science studies are the basis for novel drug development. To bridge the gap between these basic science studies in rodent models and clinical drug trials in humans, we examined the effects of ex vivo BDNF treatment on the male and female SDH. Like in female rodents and unlike male rats and humans, ex vivo BDNF treatment did not activate the KCC2-

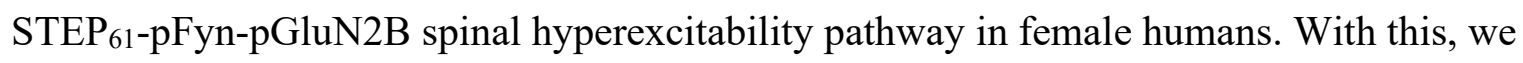
conclude that sexually dimorphic pain signalling is conserved across species. Further studies that 
capture the overall nociceptive output from the human SDH pain processing network, such as voltage-sensitive dye imaging or multielectrode array recordings, are also needed to capture the dynamic and degenerate signalling pathways involved in human pain signalling (Gereau et al., 2014; Ratté and Prescott, 2016). These approaches will enable investigation into how changes in individual targets impact the output of spinal nociceptive circuits (Greenspon et al., 2019). An understanding of how differential regulation of excitability across subpopulations of SDH neurons shapes the output of the spinal nociceptive network, in combination with emerging single-cell sequencing efforts (Sathyamurthy et al., 2018), will give insight into differences in neuronal subpopulation processing of pain across sex.

Finally, in this study, we explored the mechanisms that underlie the observed sex difference in response to BDNF in the SDH. Sex differences can result from either genetic, epigenetic or hormonally mediated processes. Because NMDAR-dependant sex differences have previously been identified to be hormonally mediated (Mogil et al., 1993), we investigated the effects of ex vivo BDNF treatment on ovariectomized rats. We found that sex hormones do not affect baseline NMDAR synaptic properties. However, unlike naïve females, ovariectomized females' SDH showed increased markers of facilitated excitation and disinhibition at SDH synapses, as well as robust NMDAR potentiation in response to BDNF treatment. With this, we conclude that ovariectomy results in a switch from the female BDNF-insensitive phenotype of SDH neurons, to the $\mathrm{KCC}_{2}-\mathrm{STEP}_{61}$-pFyn-pGluN2B spinal hyperexcitability pathway seen in male rats and humans (Dedek et al., 2019). These findings suggest the female sex hormones have an organizational effect on spinal mechanisms of female pain signalling, with persistent effects lasting into adulthood. Further study is needed to identify which female sex hormones regulate this dimorphism by isolating the effects that estrogen (Craft, 2007; Tang et al., 2008; 
Amandusson and Blomqvist, 2013) and progesterone (Ren et al., 2000) have on the development of the nociceptive pathway. Our results highlight the critical importance of foundational studies using both sexes, as well as the importance of using human preclinical models to improve translation between basic science and clinical medicine in the field of pain research and beyond.

\subsection{Acknowledgements:}

We thank the organ and tissue donors and their families for their generous, selfless gift. We thank the Trillium Gift of Life Network, the surgical staff at The Ottawa Hospital Civic Campus, and Dr. Suzan Chen, Lei Zhou, Santina Temi, Dr. Christopher Rudyk, Ahmad Galuta, Christopher Dedek, and Dr. Sara Ameri for their help with human spinal cord collection. 


\subsection{Supplementary Figures and Tables}
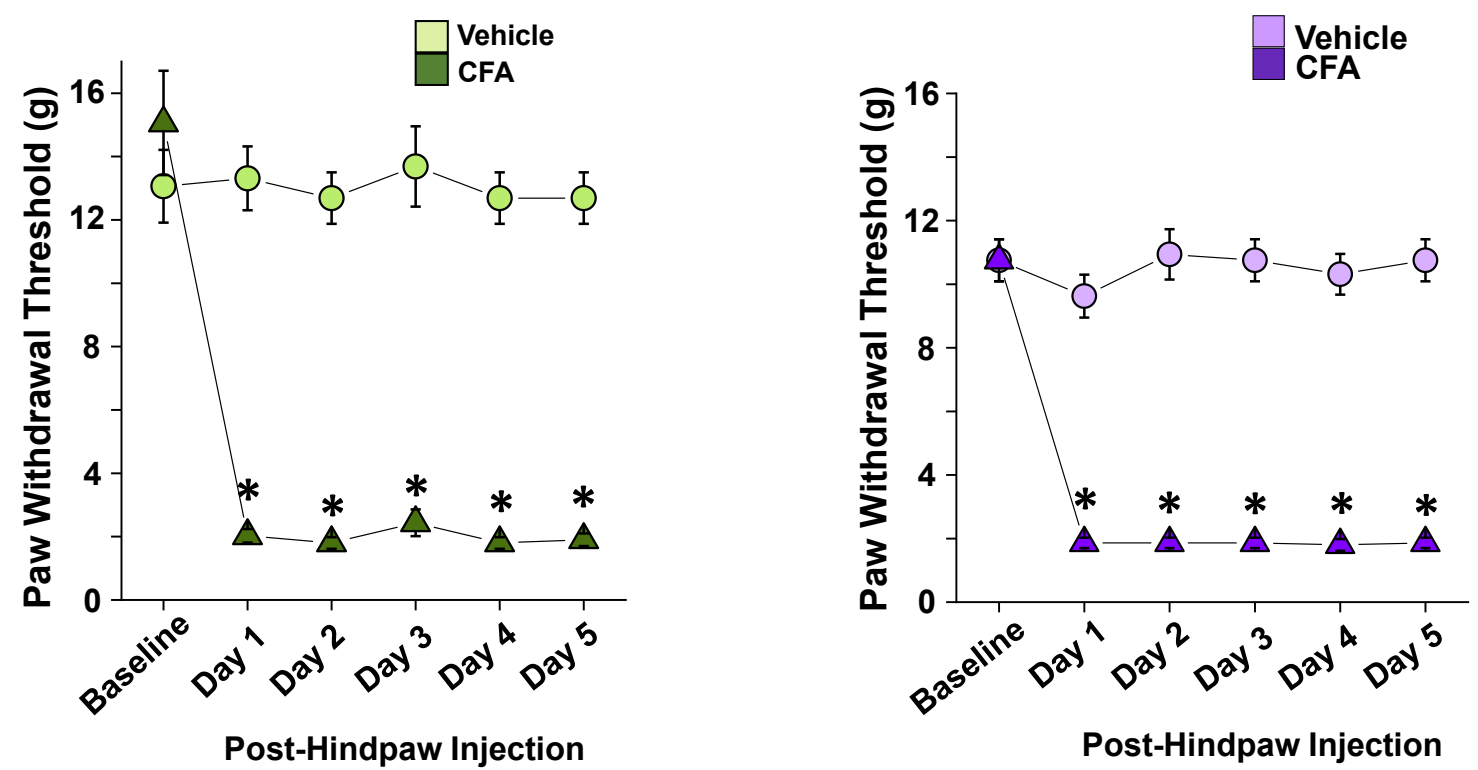

Chapter 3 Supplementary Figure 1. Paw withdrawal threshold is decreased in male and female rats receiving

CFA. Male rats, left, and female rats, right, that receive CFA injection display significantly decreased paw

withdrawal threshold $1-5$ days post-injection. Tissue from animals in this figure was used in Figures 1B and 1D. N $=8$ animals per sex per group $* \mathrm{p}<0.05$. 

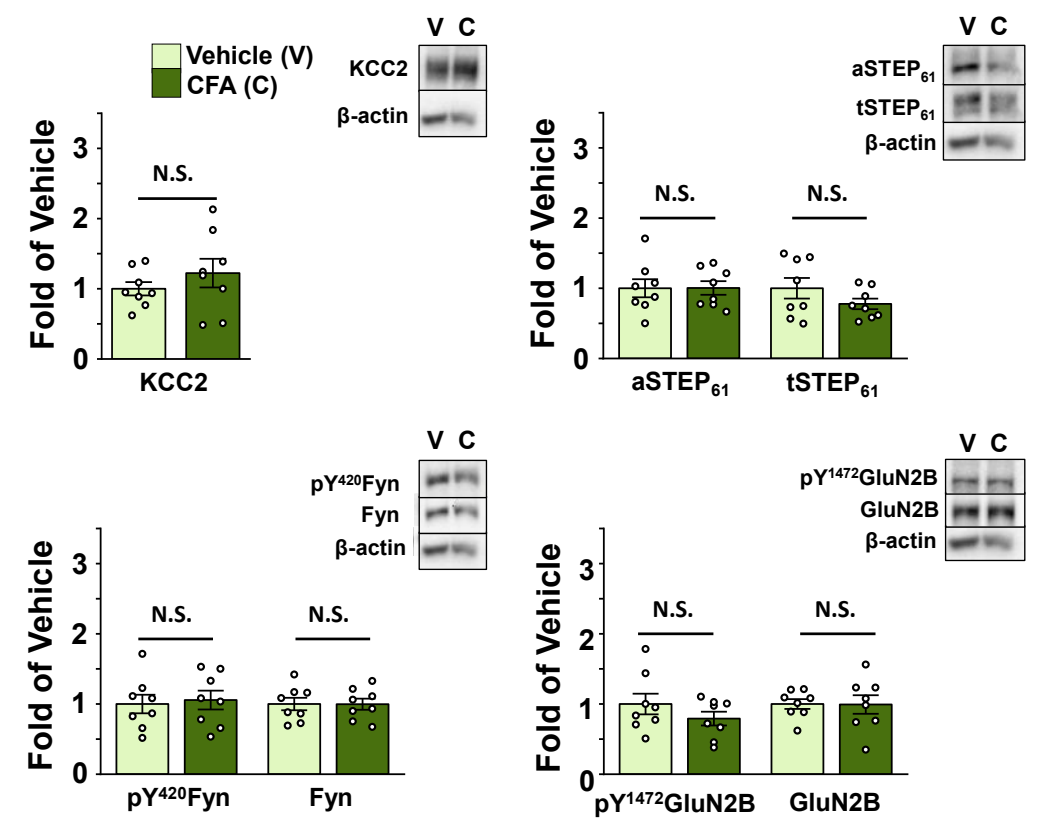

Chapter 3 Supplementary Figure 2. The CFA model of inflammatory pain elicits no change in our targets in crude synaptosome fractions of the portion of the spinal cord just ventral of the SDH in male rats. Vehicle in light green, CFA in dark green; $\mathrm{n}=8$ animals per group. 


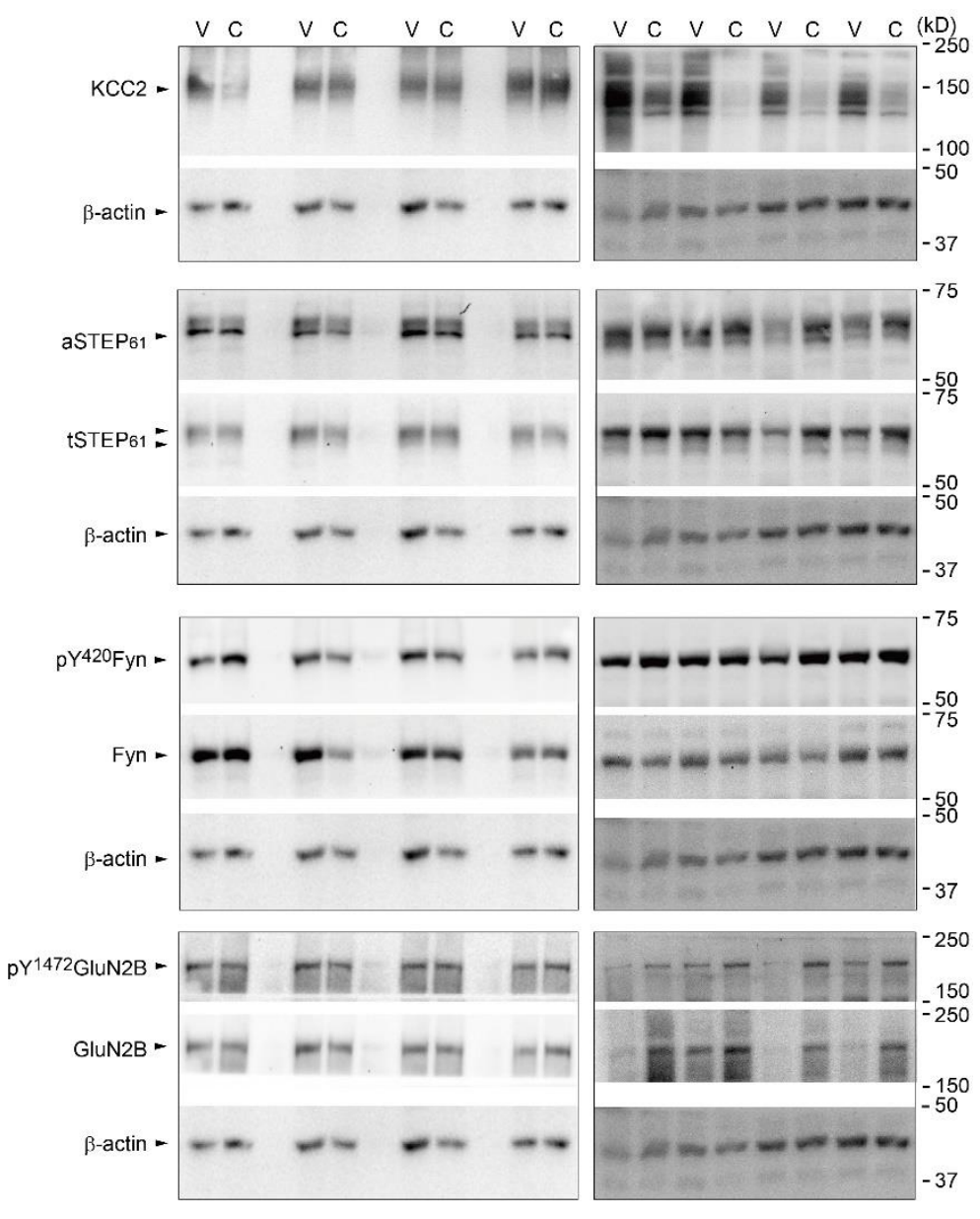

Chapter 3 Supplementary Figure 3. Male CFA SDH synaptosome gels from animals treated with either vehicle (V) or CFA (C). Individual gels were cut into sections to allow for probing several targets concurrently $\beta$ actin, the loading control, can be seen under each set of targets (labelled on the left while the weight of the target, in $\mathrm{kD}$ is on the right. $\mathrm{n}=8$ 


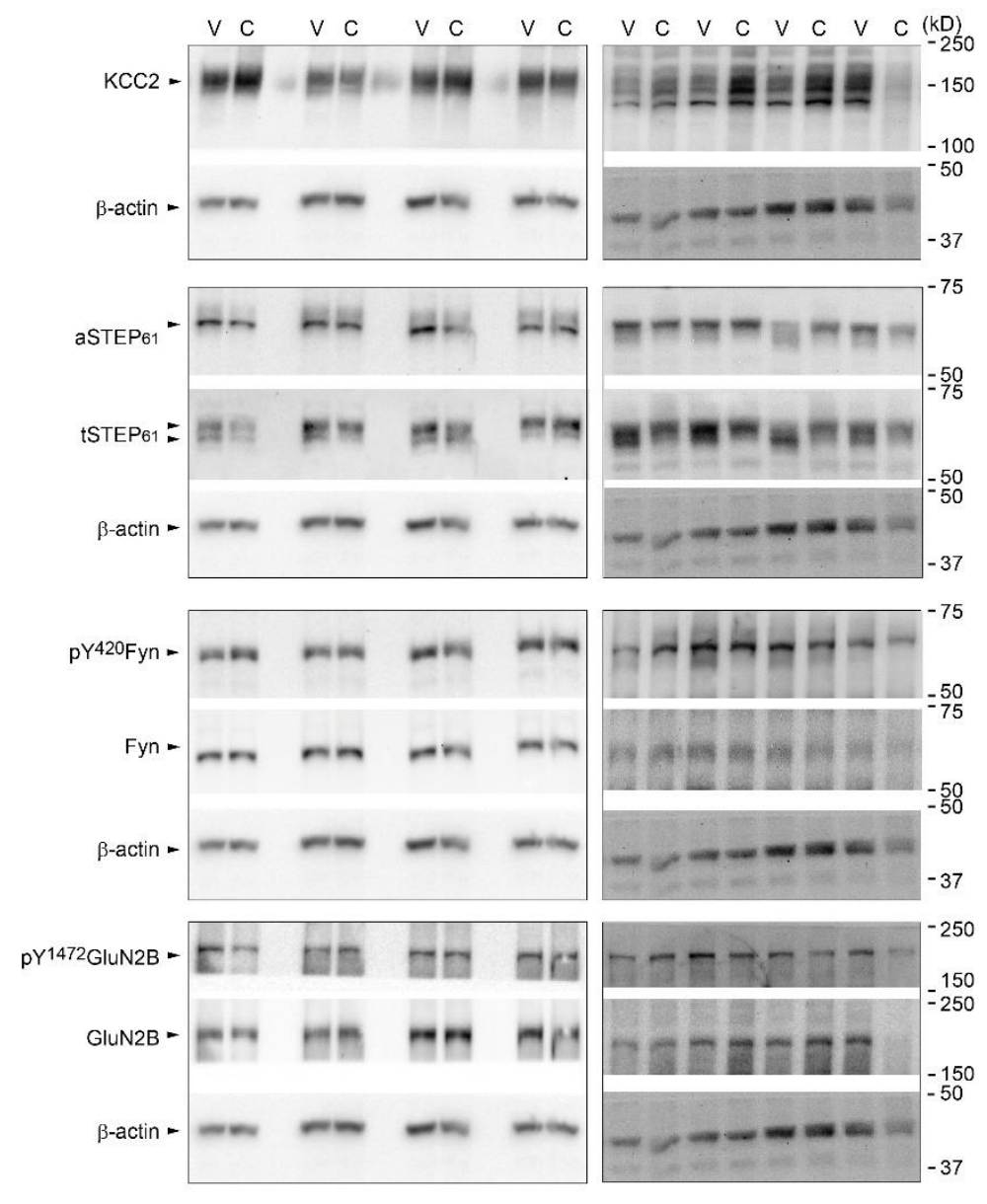

Chapter 3 Supplementary Figure 4. Male CFA VH synaptosome gels from animals treated with either vehicle

(V) or CFA (C). Individual gels were cut into sections to allow for probing several targets concurrently $\beta$-actin, the loading control, can be seen under each set of targets (labelled on the left while the weight of the target, in $\mathrm{kD}$ is on the right. $\mathrm{n}=8$ 

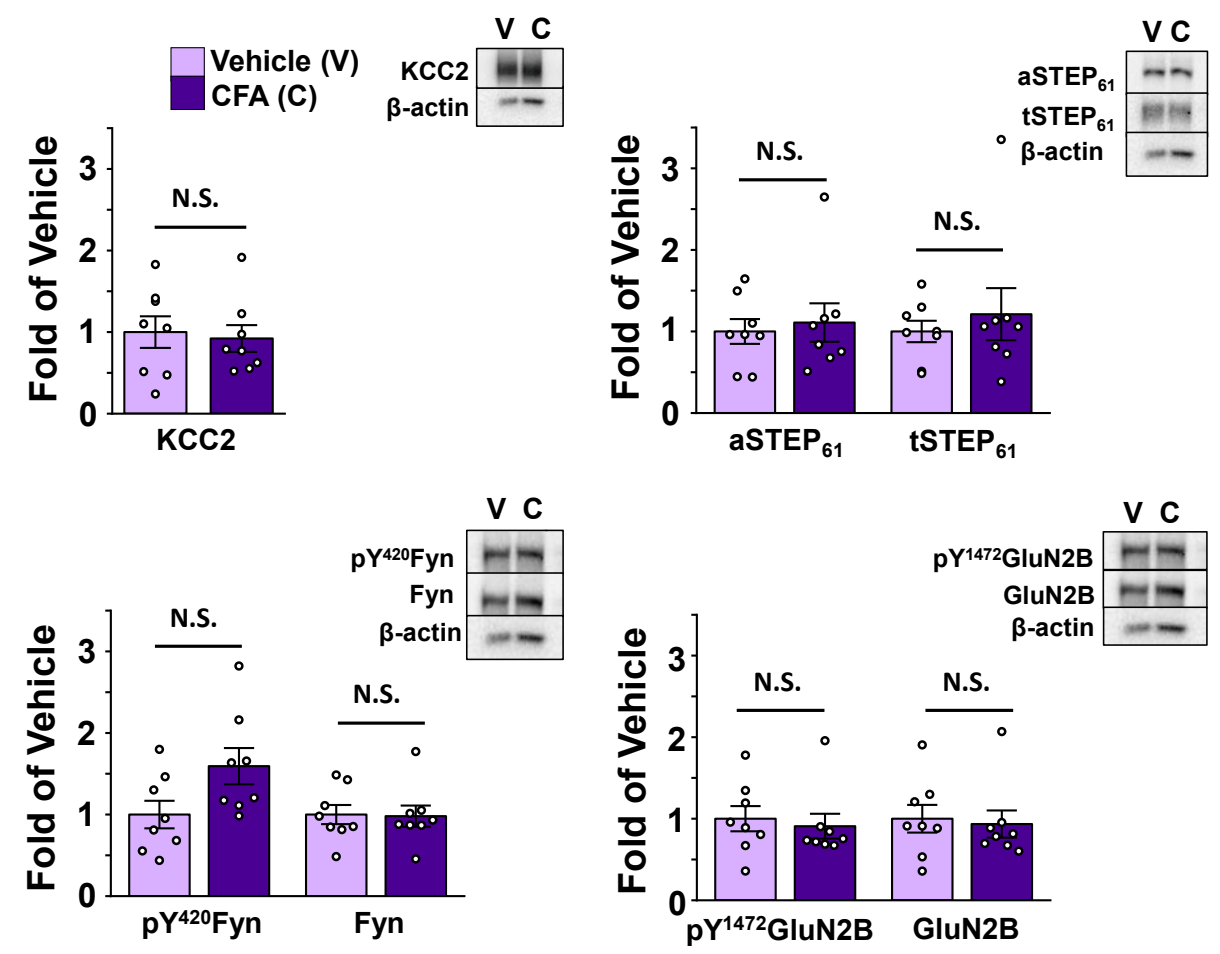

Chapter 3 Supplementary Figure 5. The CFA model of inflammatory pain elicits no change in our targets in crude synaptosome fractions of the portion of the spinal cord just ventral of the SDH in female rats. Vehicle in lilac, CFA in dark purple; $\mathrm{n}=8$ animals per group. 


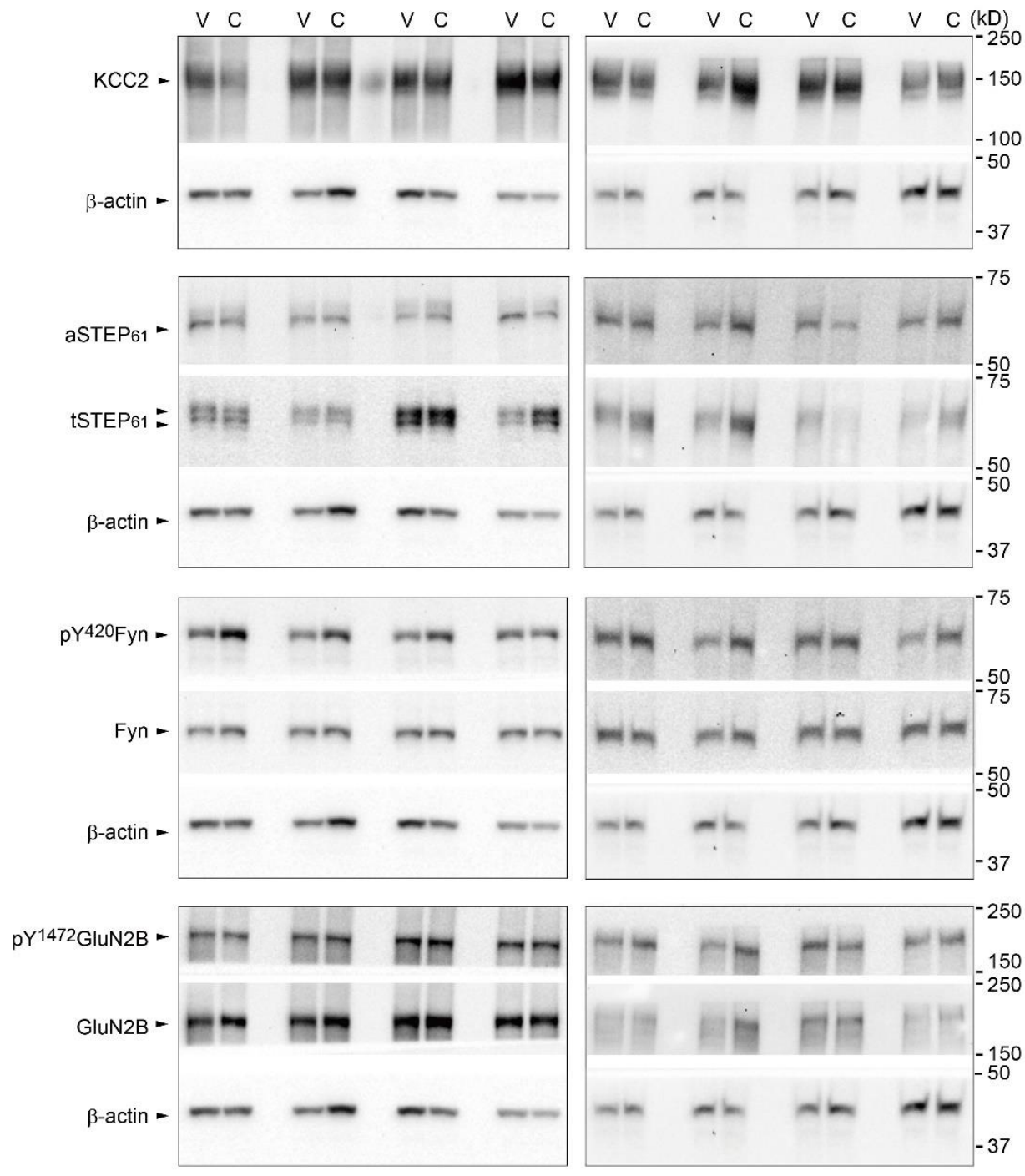

\section{Chapter 3 Supplementary Figure 6. Female CFA SDH synaptosome gels from animals treated with either}

vehicle (V) or CFA (C). Individual gels were cut into sections to allow for probing several targets concurrently $\beta$ actin, the loading control, can be seen under each set of targets (labelled on the left while the weight of the target, in $\mathrm{kD}$ is on the right. $\mathrm{n}=8$ 


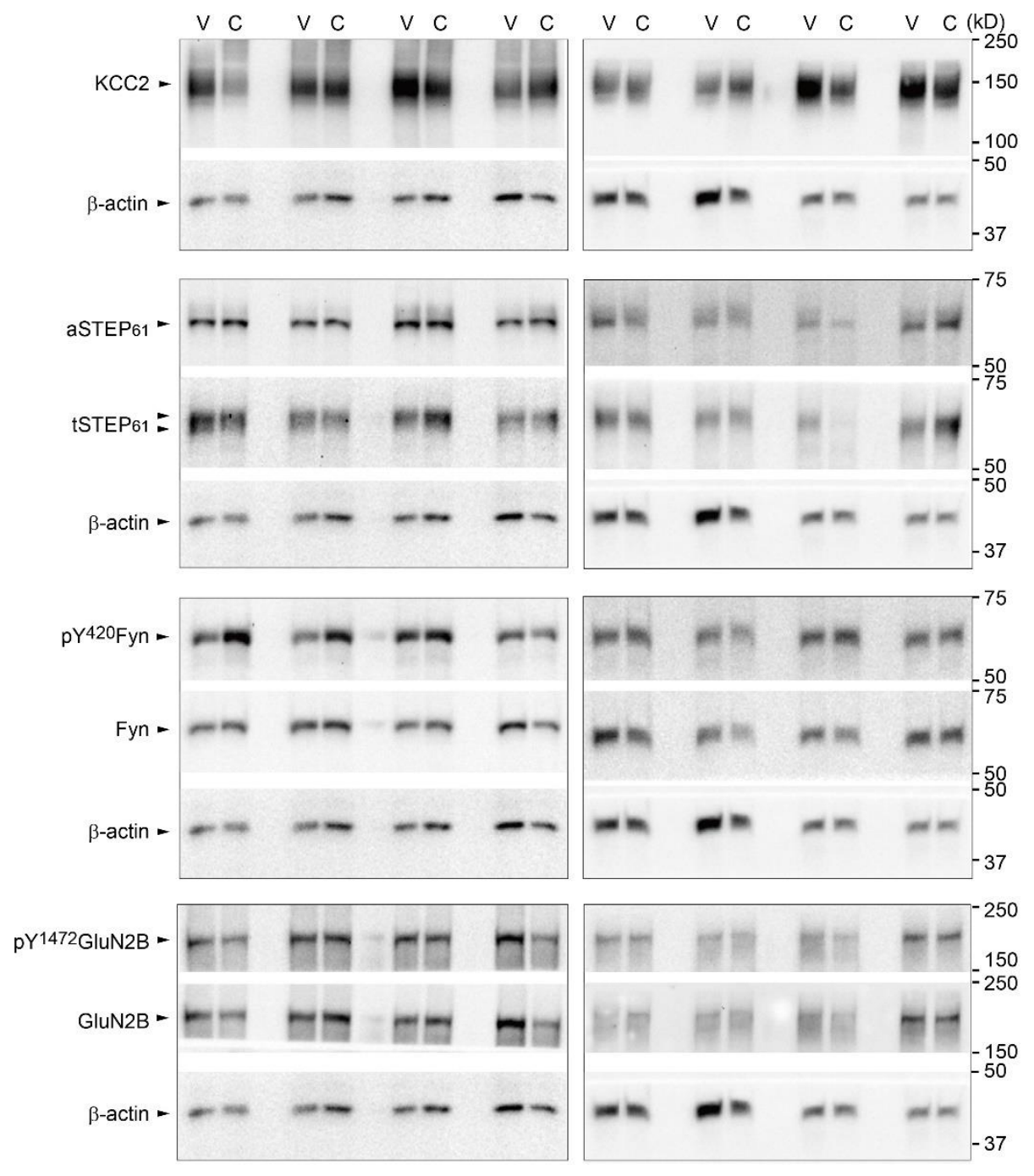

\section{Chapter 3 Supplementary Figure 7. Female CFA VH synaptosome gels from animals treated with either}

vehicle (V) or CFA (C). Individual gels were cut into sections to allow for probing several targets concurrently $\beta$ actin, the loading control, can be seen under each set of targets (labelled on the left while the weight of the target, in $\mathrm{kD}$ is on the right. $\mathrm{n}=8$ 

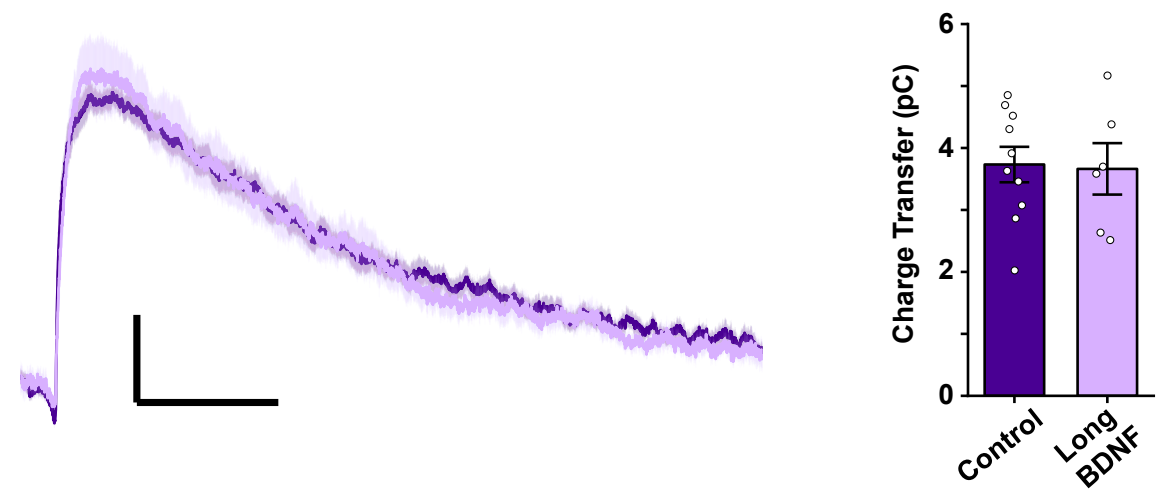

Chapter 3 Supplementary Figure 8. BDNF incubation for 2-4 hours does not result in potentiation of female rat lamina I mEPSCs. Lamina I mEPSCs from female rat tissue incubated for 2-4 hours in 50ng/mL BDNF (lilac) show no change in charge transfer when compared to control-treated slices (dark purple). $\mathrm{N}=10$ cells from 5 animals for control, 6 cells from 3 animals for long-BDNF.

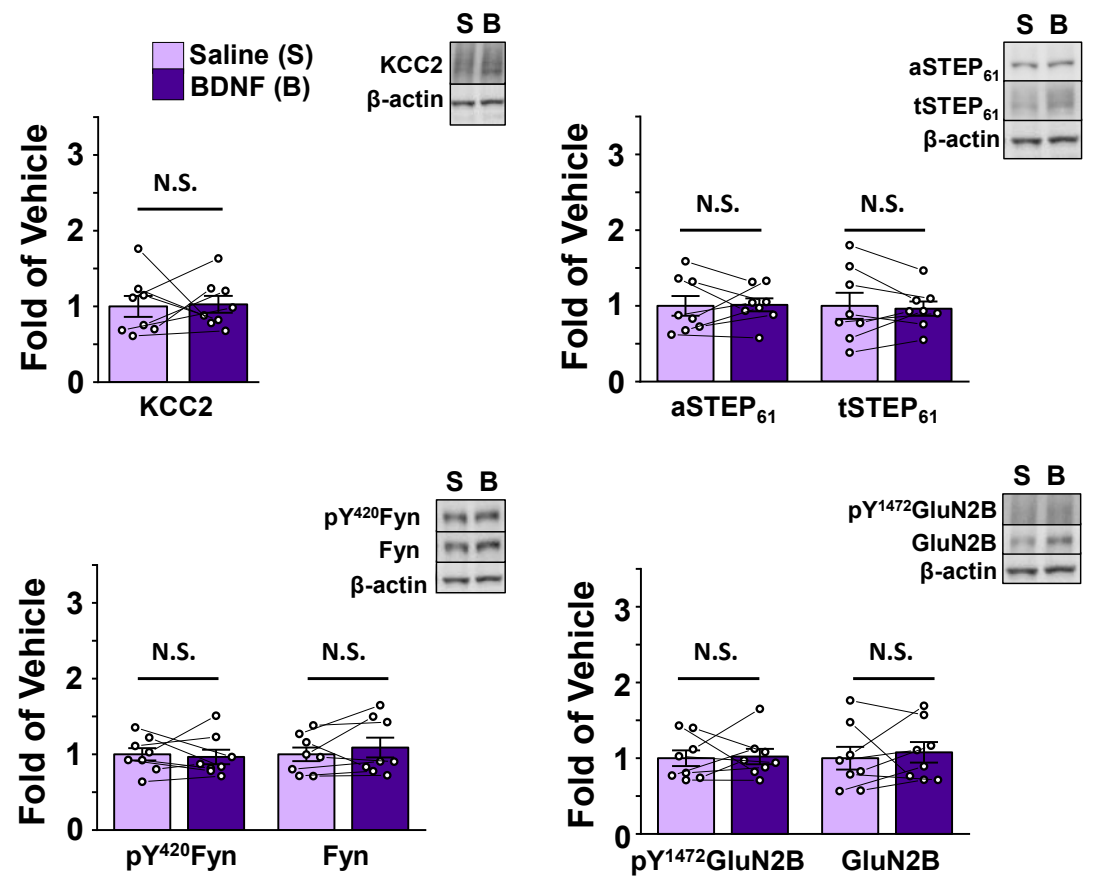

Chapter 3 Supplementary Figure 9. Incubating female rat tissue in $50 \mathrm{ng} / \mathrm{mL}$ BDNF elicits no change in our targets in crude synaptosome fractions of the portion of the spinal cord just ventral of the SDH. Saline in lilac, BDNF in dark purple; $\mathrm{n}=8$ animals per group. 

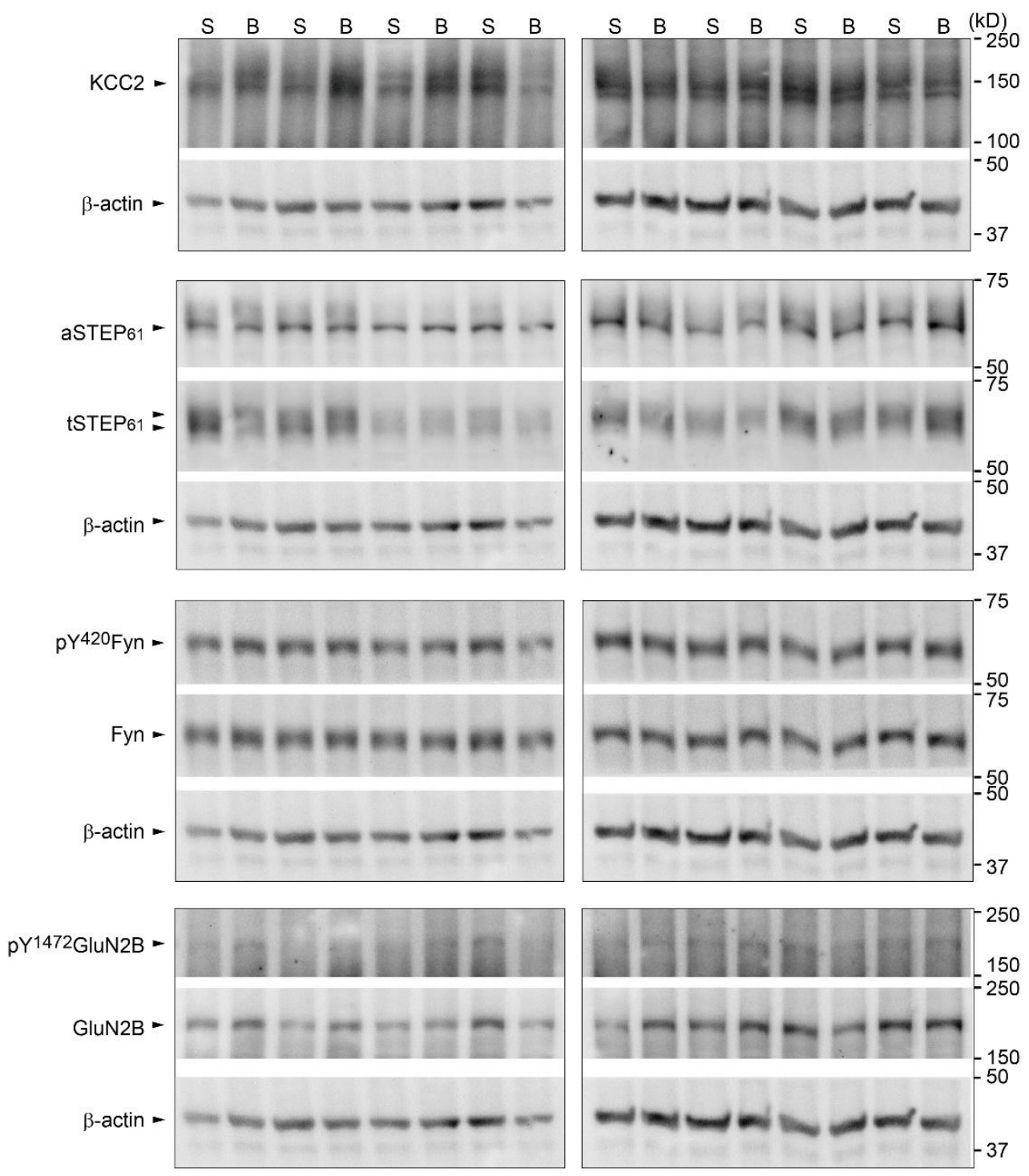

Chapter 3 Supplementary Figure 10. Female BDNF SDH synaptosome gels from animals treated with either saline (S) or BDNF (B). Individual gels were cut into sections to allow for probing several targets concurrently $\beta$ actin, the loading control, can be seen under each set of targets (labelled on the left while the weight of the target, in $\mathrm{kD}$ is on the right. $\mathrm{n}=8$ 


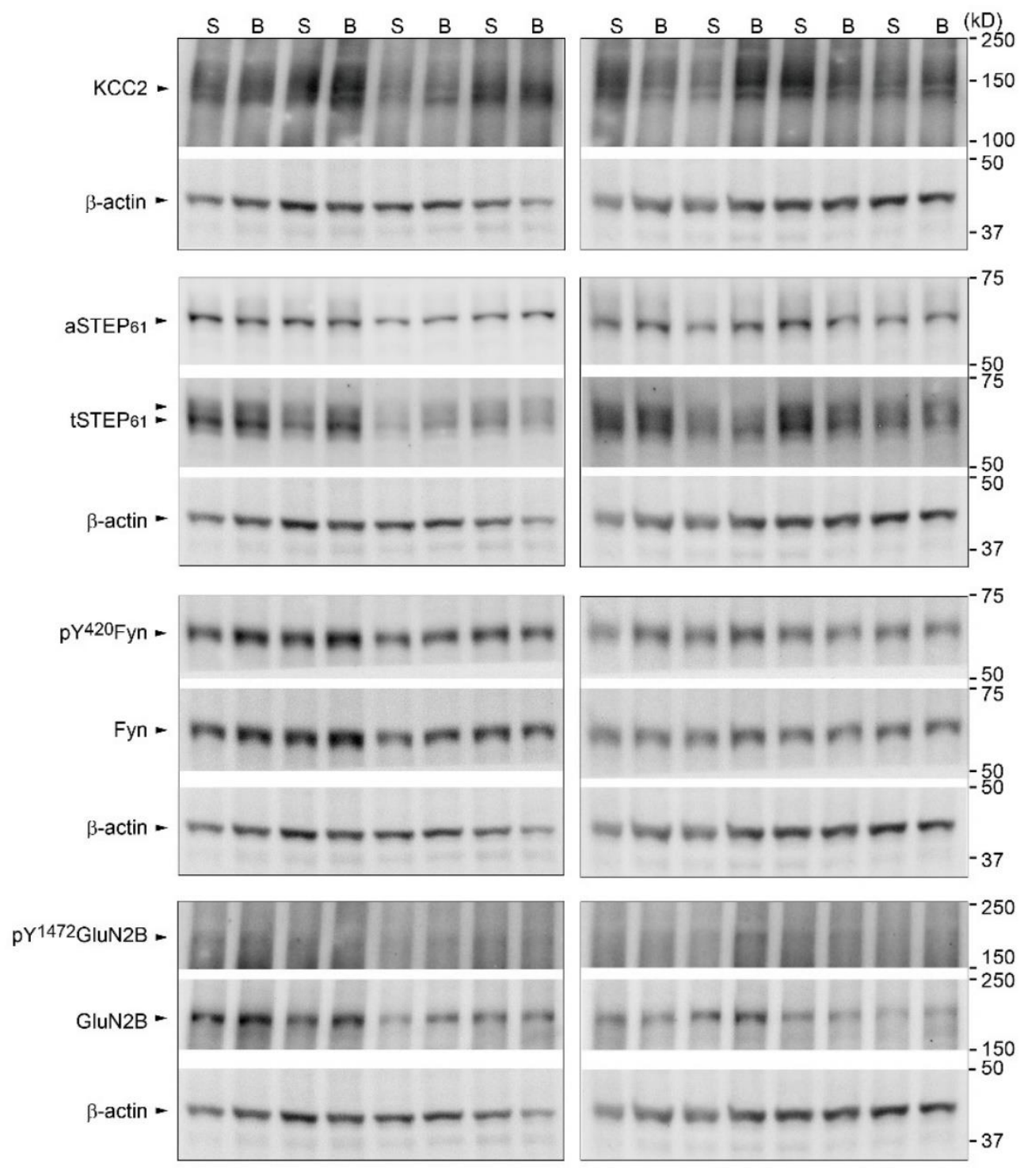

Chapter 3 Supplementary Figure 11. Female BDNF VH synaptosome gels from animals treated with either

saline (S) or BDNF (B). Individual gels were cut into sections to allow for probing several targets concurrently $\beta$ actin, the loading control, can be seen under each set of targets (labelled on the left while the weight of the target, in $\mathrm{kD}$ is on the right. $\mathrm{n}=8$ 

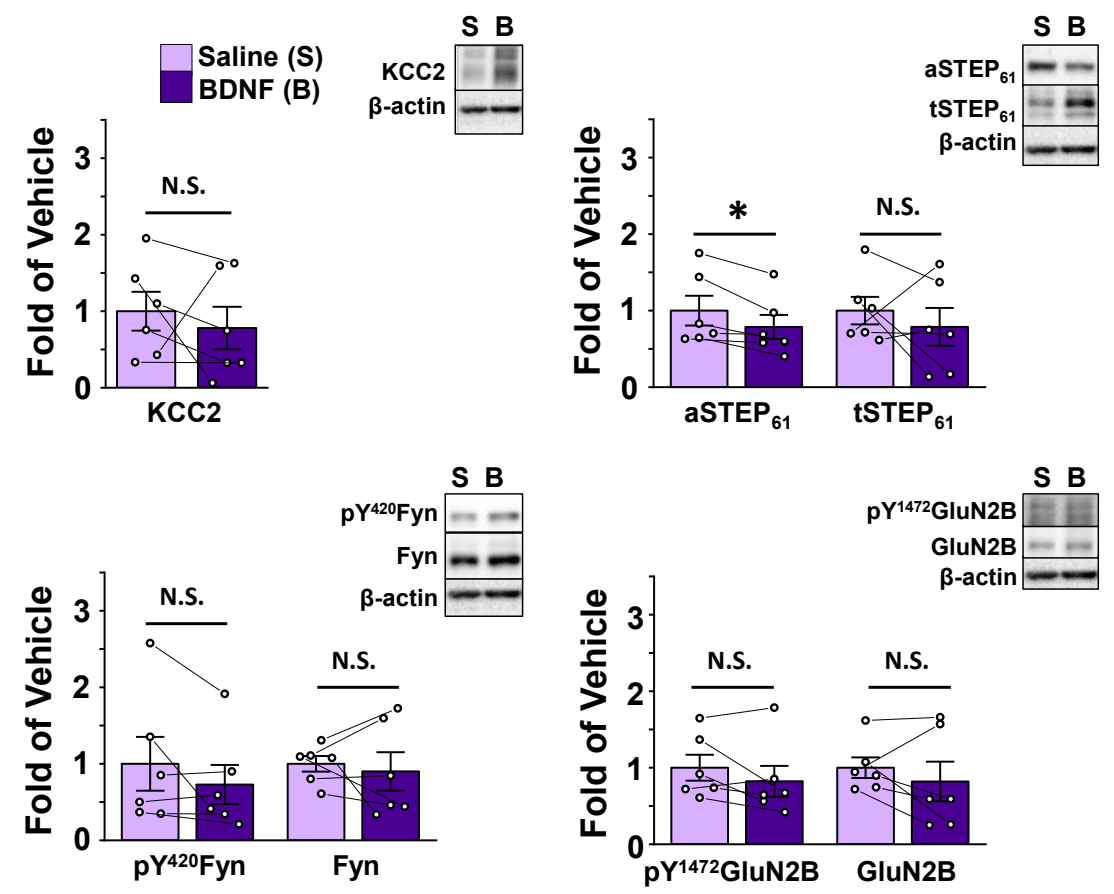

Chapter 3 Supplementary Figure 12. Incubating female human tissue in $100 \mathrm{ng} / \mathrm{mL}$ BDNF elicits no change in KCC2, tSTEP, pFyn, Fyn, pGluN2B, or GluN2B in crude synaptosome fractions of the portion of the spinal cord just ventral to the SDH. aSTEP is significantly decreased. Saline in lilac, BDNF in dark purple; $\mathrm{n}=$ 6. ${ }^{*} \mathrm{p}<0.05$ 


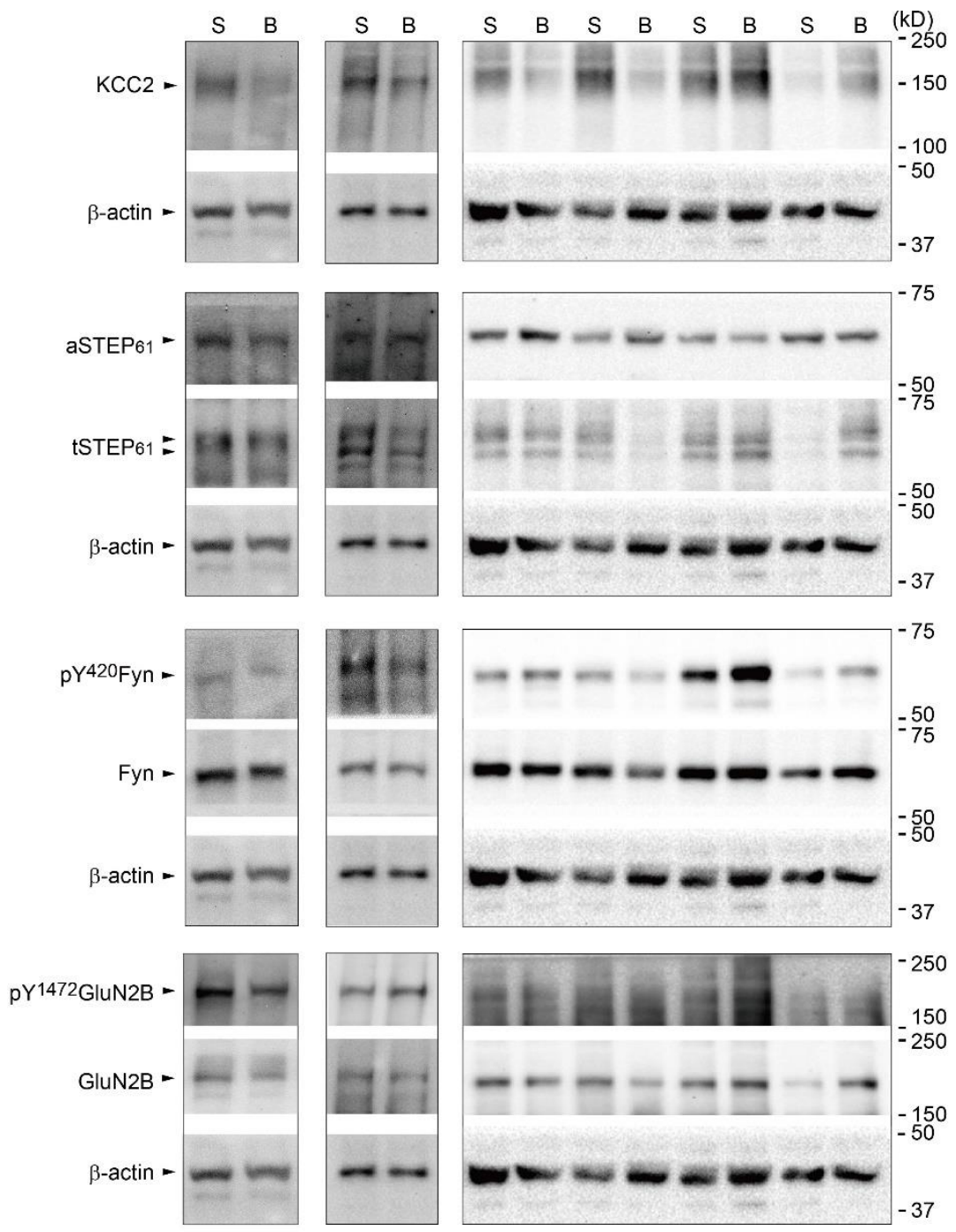

Chapter 3 Supplementary Figure 13. Female BDNF human SDH synaptosome gels from human spinal samples treated with either saline (S) or BDNF (B). Individual gels were cut into sections to allow for probing several targets concurrently $\beta$-actin, the loading control, can be seen under each set of targets (labelled on the left while the weight of the target, in $\mathrm{kD}$ is on the right. $\mathrm{n}=6$ 


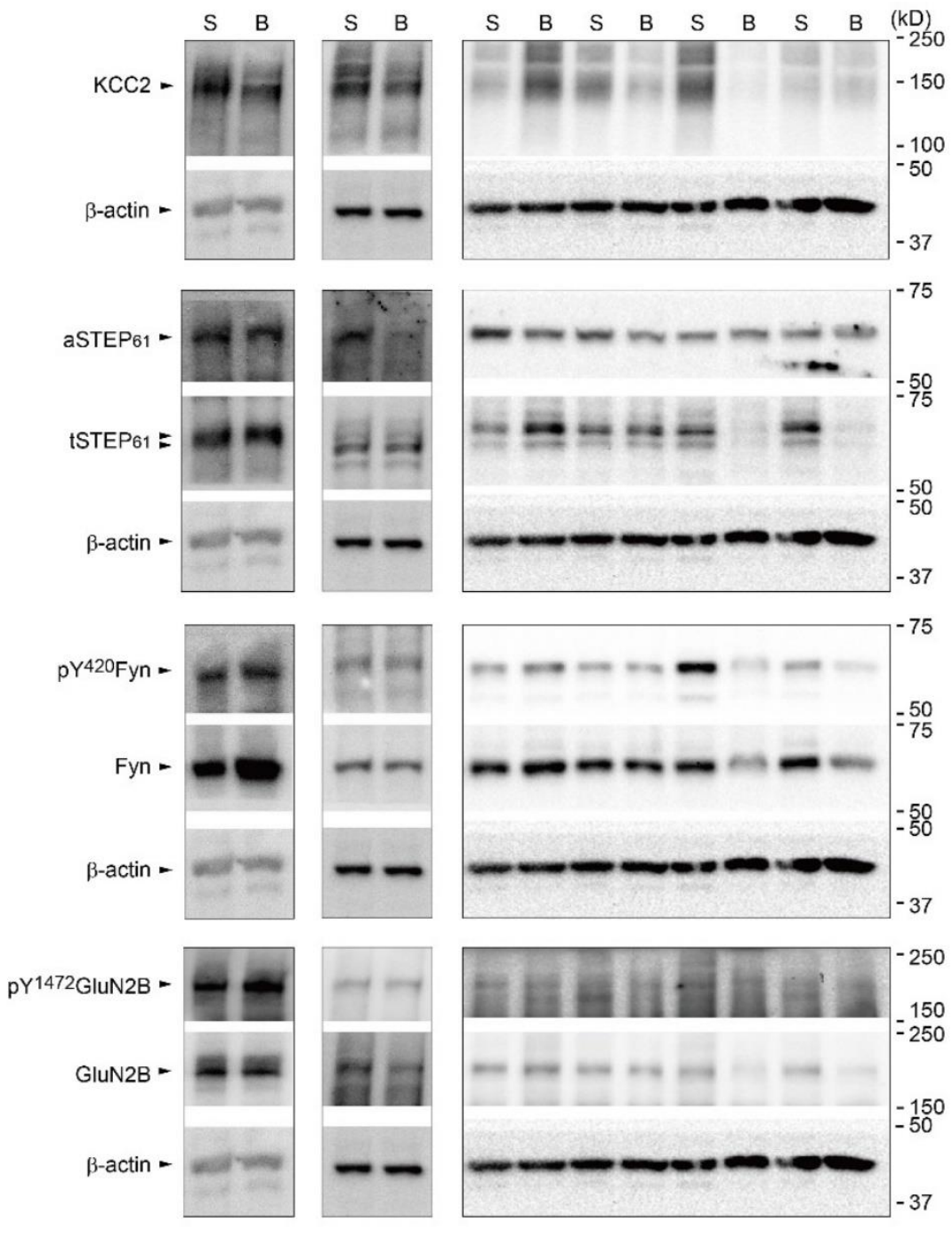

Chapter 3 Supplementary Figure 14. Female BDNF human VH synaptosome gels from human spinal samples treated with either saline (S) or BDNF (B). Individual gels were cut into sections to allow for probing several targets concurrently $\beta$-actin, the loading control, can be seen under each set of targets (labelled on the left while the weight of the target, in $\mathrm{kD}$ is on the right. $\mathrm{n}=6$ 

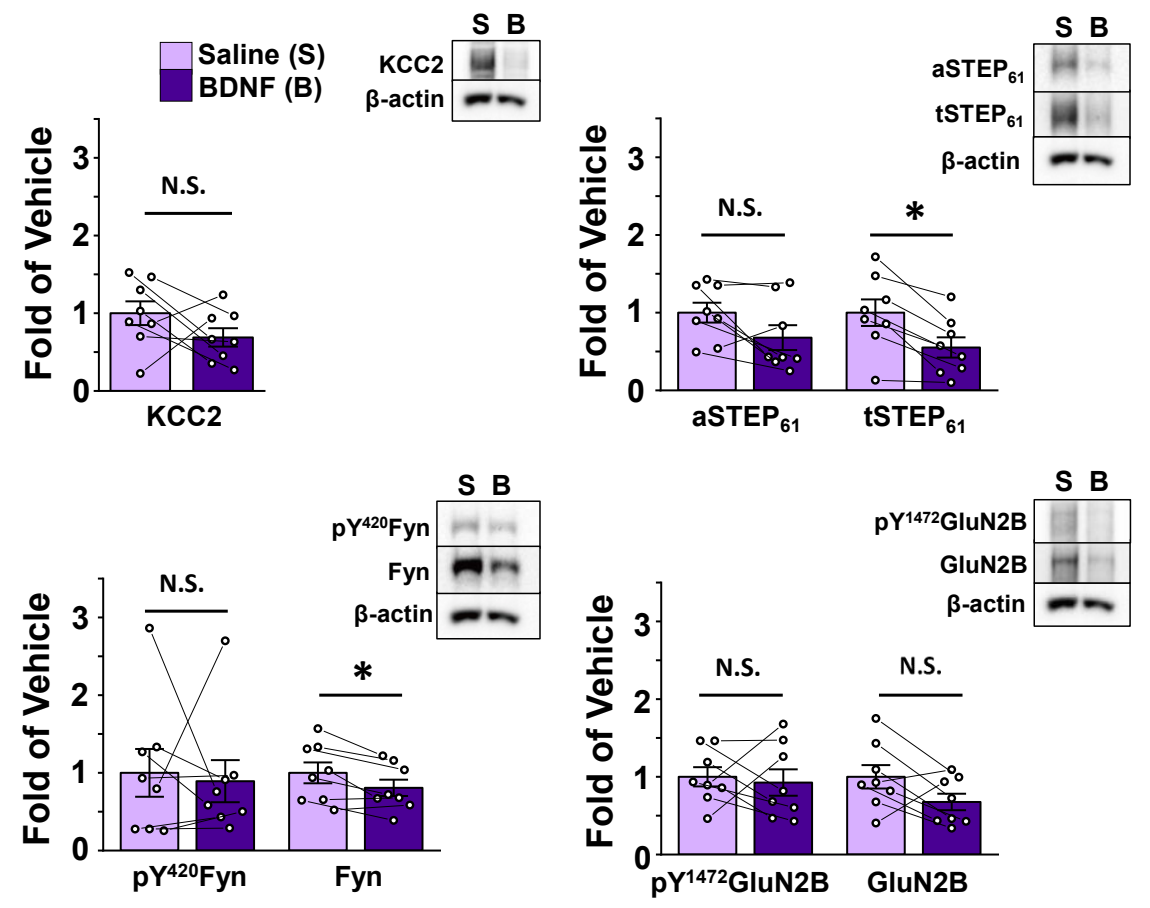

Chapter 3 Supplementary Figure 15. Incubating female OVX rat tissue in $50 \mathrm{ng} / \mathrm{mL}$ BDNF elicits decreases in tSTEP and Fyn, and no change in KCC2, aSTEP, pFyn, pGluN2B, and GluN2B in crude synaptosome fractions of the portion of the spinal cord just ventral of the SDH. Saline in lilac, BDNF in dark purple; $\mathrm{n}=8$ animals per group. 

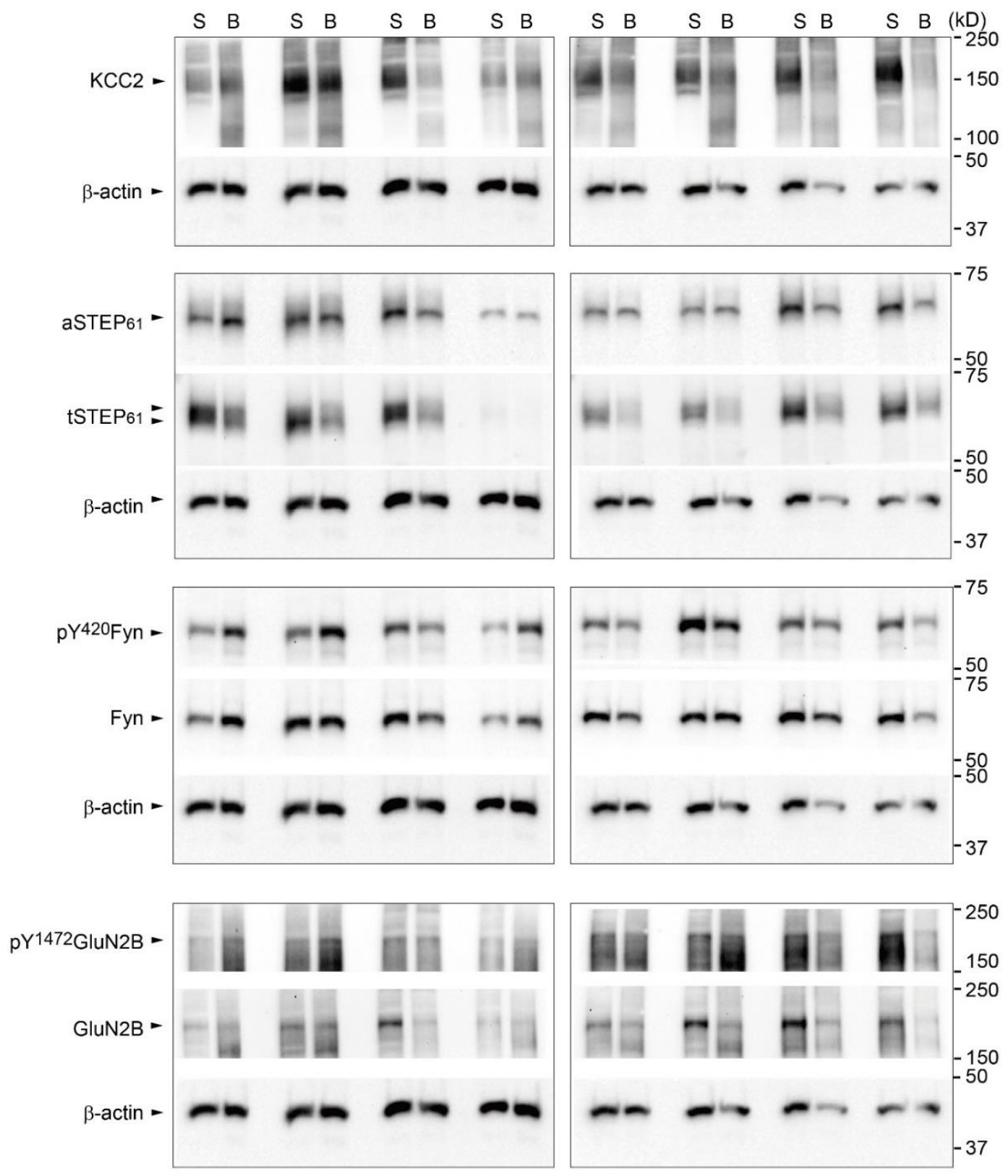

Chapter 3 Supplementary Figure 16. Female OVX rat SDH synaptosome gels from animals treated with either saline (S) or BDNF (B). Individual gels were cut into sections to allow for probing several targets concurrently $\beta$-actin, the loading control, can be seen under each set of targets (labelled on the left while the weight of the target, in $\mathrm{kD}$ is on the right. $\mathrm{n}=8$ 


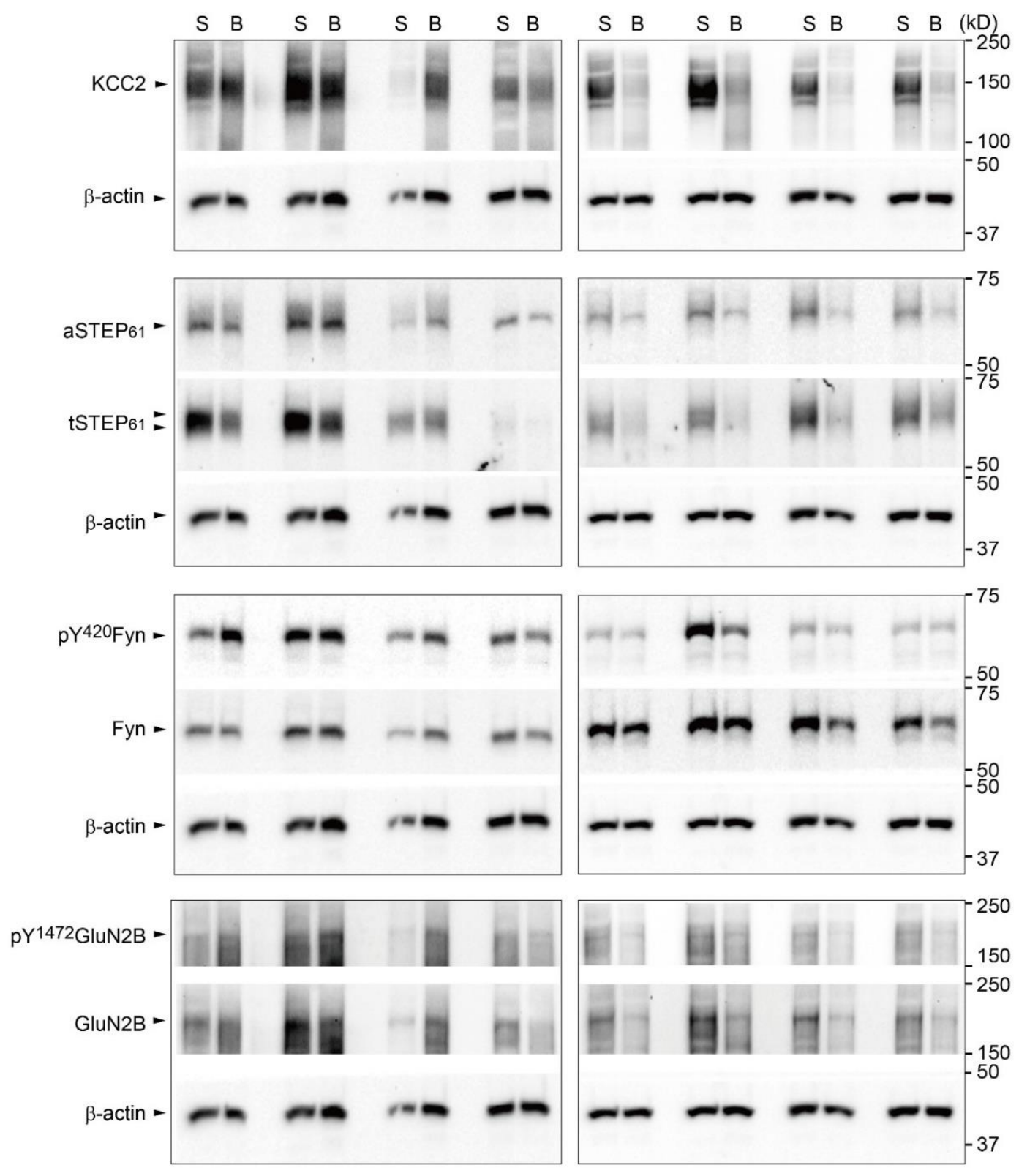

Chapter 3 Supplementary Figure 17. Female OVX rat VH synaptosome gels from animals treated with either saline (S) or BDNF (B). Individual gels were cut into sections to allow for probing several targets concurrently $\beta$ actin, the loading control, can be seen under each set of targets (labelled on the left while the weight of the target, in $\mathrm{kD}$ is on the right. $\mathrm{n}=8$ 
Chapter 3 Supplementary Table 1. Antibodies used for Western Blots.

\begin{tabular}{|c|c|c|c|c|c|c|c|c|}
\hline Antibody & Format & Immunogen & Host & $\begin{array}{l}\text { Target } \\
\text { Species }\end{array}$ & Dilution & Source & Cat \# & References \\
\hline $\begin{array}{l}\text { Anti- } \\
\text { STEP } \\
\text { (clone } \\
\text { 23E5) }\end{array}$ & $\begin{array}{l}\text { Monoclonal } \\
\text { IgG }_{2 b} \text { kappa light } \\
\text { chain }\end{array}$ & $\begin{array}{l}18 \text { amino acid } \\
\text { sequence mapping at } \\
\text { N-terminus of rat } \\
\text { STEP46 }\end{array}$ & Mouse & $\begin{array}{l}\text { Mouse, } \\
\text { rat }\end{array}$ & $1: 1000$ & $\begin{array}{l}\text { Santa Cruz } \\
\text { Biotechnology }\end{array}$ & $\begin{array}{l}\text { sc- } \\
23892\end{array}$ & $\begin{array}{l}\text { (Gladding et } \\
\text { al., 2014; } \\
\text { Jang et al., } \\
\text { 2016; Rué et } \\
\text { al., 2016; Xu } \\
\text { et al., 2017) }\end{array}$ \\
\hline $\begin{array}{l}\text { Anti- } \\
\text { STEP } \\
\text { (D9H3) }\end{array}$ & Monoclonal IgG & $\begin{array}{l}\text { A synthetic peptide } \\
\text { corresponding to } \\
\text { residues surrounding } \\
\text { Ile } 440 \text { of human } \\
\text { STEP } 61 \text { protein }\end{array}$ & Rabbit & $\begin{array}{l}\text { Mouse, } \\
\text { rat, } \\
\text { human }\end{array}$ & $1: 1000$ & Cell Signaling & $9069 \mathrm{~S}$ & $\begin{array}{l}\text { (Xu et al., } \\
2016)\end{array}$ \\
\hline $\begin{array}{l}\text { Anti- } \\
\text { KCC2 }\end{array}$ & Polyclonal & $\begin{array}{l}\text { A synthetic peptide } \\
\text { mapping at the } \mathrm{N}- \\
\text { terminus of } \mathrm{KCC} 2 \\
\text { of human origin }\end{array}$ & Rabbit & $\begin{array}{l}\text { Mouse, } \\
\text { rat, } \\
\text { human }\end{array}$ & $1: 1000$ & $\begin{array}{l}\text { Santa Cruz } \\
\text { Biotechnology }\end{array}$ & $\begin{array}{l}\text { Sc- } \\
19419- \\
\text { R }\end{array}$ & $\begin{array}{l}\text { (Zhou et al., } \\
\text { 2012; Chen et } \\
\text { al., 2016) }\end{array}$ \\
\hline Anti-Fyn & Polyclonal & $\begin{array}{l}\text { Epitope mapping at } \\
\text { the N-terminus of } \\
\text { Fyn of human origin }\end{array}$ & Rabbit & $\begin{array}{l}\text { Mouse, } \\
\text { rat, } \\
\text { human, } \\
\text { canine, } \\
\text { bovine, } \\
\text { porcine, } \\
\text { avian }\end{array}$ & $1: 1000$ & $\begin{array}{l}\text { Santa Cruz } \\
\text { Biotechnology }\end{array}$ & sc-16 & $\begin{array}{l}\text { (Brignatz et } \\
\text { al., 2009; } \\
\text { Levi et al., } \\
\text { 2010; Yadav } \\
\text { and Denning, } \\
\text { 2011) }\end{array}$ \\
\hline $\begin{array}{l}\text { anti- } \\
\text { pY416- } \\
\text { Src }\end{array}$ & Monoclonal IgG & $\begin{array}{l}\text { Produced using } \\
\text { synthetic } \\
\text { phosphopeptide } \\
\text { corresponding to } \\
\text { residues surrounding } \\
\text { Tyr419 of human } \\
\text { Src protein. Detects } \\
\text { endogenous levels } \\
\text { of Src only when } \\
\text { phosphorylated at } \\
\text { Tyr416. May cross- } \\
\text { react with other Src } \\
\text { family members } \\
\text { (Lyn, Fyn, Lck, Yes } \\
\text { and Hck) when } \\
\text { phosphorylated at } \\
\text { equivalent sites. } \\
\text { May cross-react } \\
\text { with overexpressed } \\
\text { phosphorylated } \\
\text { RTKs. }\end{array}$ & Rabbit & $\begin{array}{l}\text { Human, } \\
\text { Mouse, } \\
\text { Rat, } \\
\text { Monkey }\end{array}$ & $1: 1000$ & Cell Signaling & $6943 \mathrm{~S}$ & $\begin{array}{l}\text { (McKinley et } \\
\text { al., 2013; } \\
\text { Allison et al., } \\
\text { 2015; } \\
\text { Bieerkehazhi } \\
\text { et al., 2017) }\end{array}$ \\
\hline $\begin{array}{l}\text { Anti- } \beta- \\
\text { actin }\end{array}$ & $\begin{array}{l}\text { Monoclonal } \mathrm{IgG}_{1} \\
\text { kappa light chain }\end{array}$ & $\begin{array}{l}\text { Chicken gizzard } \\
\text { actin }\end{array}$ & Mouse & $\begin{array}{l}\text { mouse, } \\
\text { rat, } \\
\text { human, } \\
\text { avian, } \\
\text { bovine, } \\
\text { canine, } \\
\text { porcine, } \\
\text { rabbit, } \\
\text { Dictyoste } \\
\text { lium } \\
\text { discoideu } \\
\text { m, } \\
\text { Physarum } \\
\text { polyceph } \\
\text { alum }\end{array}$ & $1: 10000$ & $\begin{array}{l}\text { Santa Cruz } \\
\text { Biotechnology }\end{array}$ & $\begin{array}{l}\text { sc- } \\
47778\end{array}$ & $\begin{array}{l}\text { (Zuo et al., } \\
\text { 2009; Ti and } \\
\text { Pollard, } \\
\text { 2011; Wu et } \\
\text { al., 2014) }\end{array}$ \\
\hline $\begin{array}{l}\text { anti-non- } \\
\text { phospho- } \\
\text { STEP }\end{array}$ & Monoclonal IgG & $\begin{array}{l}\text { Produced using } \\
\text { synthetic } \\
\text { nonphosphopeptide } \\
\text { corresponding to } \\
\text { residues surrounding } \\
\text { Ser221 of human } \\
\text { STEP61 protein. }\end{array}$ & Rabbit & $\begin{array}{l}\text { Human, } \\
\text { Mouse, } \\
\text { Rat }\end{array}$ & $1: 1000$ & $\begin{array}{l}\text { Cell Signaling } \\
\text { Technology }\end{array}$ & $5659 \mathrm{~S}$ & $\begin{array}{l}\text { (Castonguay } \\
\text { et al., 2018) }\end{array}$ \\
\hline
\end{tabular}




\begin{tabular}{|c|c|c|c|c|c|c|c|c|}
\hline & & $\begin{array}{l}\text { Detects STEP61 } \\
\text { protein only when } \\
\text { dephosphorylated at } \\
\text { Ser221 and of } \\
\text { STEP46 protein } \\
\text { when } \\
\text { dephosphorylated at } \\
\text { Ser49 }\end{array}$ & & & & & & \\
\hline $\begin{array}{l}\text { anti- } \\
\text { pY }{ }^{1472} \\
\text { GluN2B }\end{array}$ & Polyclonal & $\begin{array}{l}\text { Affinity Purified } \\
\text { from Pooled Serum. } \\
\text { Phosphopeptide } \\
\text { corresponding to } \\
\text { amino acid residues } \\
\text { surrounding the } \\
\text { phospho-Tyr1472 of } \\
\text { NMDA NR2B. }\end{array}$ & Rabbit & $\begin{array}{l}\text { Rat, } \\
\text { Mouse, } \\
\text { Human, } \\
\text { Bovine, } \\
\text { Chicken, } \\
\text { Non- } \\
\text { human } \\
\text { primate, } \\
\text { Zebrafish } \\
\text { Canine }\end{array}$ & $1: 1000$ & PhosphoSolutions & $\begin{array}{l}\text { p1516- } \\
1472\end{array}$ & $\begin{array}{l}\text { (Castillo et } \\
\text { al., 2011; } \\
\text { Jang et al., } \\
2016 \text { ) }\end{array}$ \\
\hline $\begin{array}{l}\text { anti- } \\
\text { GluN2B }\end{array}$ & Monoclonal IgG & $\begin{array}{l}6 \text { His-tagged fusion } \\
\text { protein } \\
\text { corresponding to } \\
\text { amino acids } 1265- \\
1464 \text { of mouse } \\
\text { NMDA receptor } 2 A \\
\text { (NR2A) }\end{array}$ & Rabbit & $\begin{array}{l}\text { Mouse, } \\
\text { Rat }\end{array}$ & $1: 2000$ & Millipore & $06-600$ & $\begin{array}{l}\text { (Fenster et } \\
\text { al., 2012; } \\
\text { Wei et al., } \\
\text { 2014) }\end{array}$ \\
\hline
\end{tabular}

\section{References for Supplementary Table 2:}

Allison P, Espiritu D, Barnett J V., Camenisch TD. Type III TGF $\beta$ receptor and Src direct hyaluronan-mediated invasive cell motility. Cell. Signal. 2015; 27: 453-459.

Bieerkehazhi S, Chen Z, Zhao Y, Yu Y, Zhang H, Vasudevan SA, et al. Novel Src/Abl tyrosine kinase inhibitor bosutinib suppresses neuroblastoma growth via inhibiting Src/Abl signalling. Oncotarget 2017; 8: 1469-1480.

Brignatz C, Paronetto MP, Opi S, Cappellari M, Audebert S, Feuillet V, et al. Alternative splicing modulates autoinhibition and SH3 accessibility in the Src kinase Fyn. Mol. Cell. Biol. 2009; 29: 6438-48.

Castillo C, Norcini M, Baquero-Buitrago J, Levacic D, Medina R, Montoya-Gacharna JV, et al. The N-methyl-Daspartate-evoked cytoplasmic calcium increase in adult rat dorsal root ganglion neuronal somata was potentiated by substance P pretreatment in a protein kinase C-dependent manner. Neuroscience 2011; 177: 308-320.

Castonguay D, Dufort-Gervais J, Ménard C, Chatterjee M, Quirion R, Bontempi B, et al. The Tyrosine Phosphatase STEP Is Involved in Age-Related Memory Decline. Curr. Biol. 2018; 28: 1079-1089.e4.

Chen M, Wang J, Jiang J, Zheng X, Justice NJ, Wang K, et al. APP modulates KCC2 expression and function in hippocampal GABAergic inhibition [Internet]. 2016[cited 2019 Jan 30] Available from:

https://cdn.elifesciences.org/articles/20142/elife-20142-v2.pdf 
Fenster C, Vullhorst D, Buonanno A. Acute neuregulin-1 signaling influences AMPA receptor mediated responses in cultured cerebellar granule neurons. Brain Res. Bull. 2012; 87: 21-29.

Gladding CM, Fan J, Zhang LYJ, Wang L, Xu J, Li EHY, et al. Alterations in STriatal-Enriched protein tyrosine Phosphatase expression, activation, and downstream signaling in early and late stages of the YAC128 Huntington's disease mouse model. J. Neurochem. 2014; 130: 145-159.

Jang S-S, Royston SE, Lee G, Wang S, Chung HJ. Seizure-Induced Regulations of Amyloid- $\beta$, STEP 61 , and STEP ${ }_{61}$ Substrates Involved in Hippocampal Synaptic Plasticity. Neural Plast. 2016; 2016: 1-13.

Levi M, Maro B, Shalgi R. The involvement of Fyn kinase in resumption of the first meiotic division in mouse oocytes. Cell Cycle 2010; 9: 1577-1589.

McKinley ET, Liu H, McDonald WH, Luo W, Zhao P, Coffey RJ, et al. Global Phosphotyrosine Proteomics Identifies PKC $\delta$ as a Marker of Responsiveness to Src Inhibition in Colorectal Cancer. PLoS One 2013; 8: e80207.

Rué L, Bañez-Coronel M, Creus-Muncunill J, Giralt A, Alcalá-Vida R, Mentxaka G, et al. Targeting CAG repeat RNAs reduces Huntington's disease phenotype independently of huntingtin levels. J. Clin. Invest. 2016; 126: 43194330.

Ti S-C, Pollard TD. Purification of actin from fission yeast Schizosaccharomyces pombe and characterization of functional differences from muscle actin. J. Biol. Chem. 2011; 286: 5784-92.

Wei J, Yuen EY, Liu W, Li X, Zhong P, Karatsoreos IN, et al. Estrogen protects against the detrimental effects of repeated stress on glutamatergic transmission and cognition. Mol. Psychiatry 2014; 19: 588-598.

Wu Z, Zhu Y, Cao X, Sun S, Zhao B. Mitochondrial Toxic Effects of A $\beta$ Through Mitofusins in the Early Pathogenesis of Alzheimer's Disease. Mol. Neurobiol. 2014; 50: 986-996.

Xu J, Kurup P, Baguley TD, Foscue E, Ellman JA, Nairn AC, et al. Inhibition of the tyrosine phosphatase STEP61 restores BDNF expression and reverses motor and cognitive deficits in phencyclidine-treated mice. Cell. Mol. Life Sci. 2016; 73: 1503-14. 
Xu J, Kurup P, Nairn AC, Lombroso PJ. Synaptic NMDA Receptor Activation Induces Ubiquitination and Degradation of STEP61 [Internet]. Mol. Neurobiol. 2017[cited 2018 Feb 28] Available from:

http://www.ncbi.nlm.nih.gov/pubmed/28466270

Yadav V, Denning MF. Fyn is induced by Ras/PI3K/Akt signaling and is required for enhanced invasion/migration.

Mol. Carcinog. 2011; 50: 346-352.

Zhou H-Y, Chen S-R, Byun H-S, Chen H, Li L, Han H-D, et al. N-methyl-D-aspartate receptor- and calpainmediated proteolytic cleavage of $\mathrm{K}+-\mathrm{Cl}$ - cotransporter-2 impairs spinal chloride homeostasis in neuropathic pain. J.

Biol. Chem. 2012; 287: 33853-64.

Zuo H, Liu Z, Liu X, Yang J, Liu T, Wen S, et al. CD151 gene delivery after myocardial infarction promotes functional neovascularization and activates FAK signaling. Mol. Med. 2009; 15: 307-15.

Chapter 3 Supplementary Table 2. Statistics Summary Table

\begin{tabular}{|c|c|c|c|c|}
\hline Figure & Comparison & Test & P value & Significant? \\
\hline $1 \mathrm{~A}$ & PWT - Vehicle & $\begin{array}{l}\text { One-way repeated } \\
\text { measures ANOVA }\end{array}$ & $\begin{array}{c}\text { Sphericity established: } \\
0.8508\end{array}$ & No \\
\hline $1 \mathrm{~A}$ & PWT - CFA & $\begin{array}{l}\text { One-way repeated } \\
\text { measures ANOVA }\end{array}$ & $\begin{array}{c}\text { Sphericity violated, } \\
\text { Greenhouse-Geisser: } \\
\text { 3.17E-8 }\end{array}$ & Yes \\
\hline $1 \mathrm{~A}$ & $\begin{array}{c}\text { PWT - CFA: Baseline } \\
\text { vs. Day } 1\end{array}$ & $\begin{array}{l}\text { Pairwise Comp, } \\
\text { Bonferroni adj. }\end{array}$ & $1.4895 \mathrm{E}-6$ & Yes \\
\hline $1 \mathrm{~A}$ & $\begin{array}{c}\text { PWT - CFA: Baseline } \\
\text { vs. Day } 2\end{array}$ & $\begin{array}{l}\text { Pairwise Comp, } \\
\text { Bonferroni adj. }\end{array}$ & $5.1905 \mathrm{E}-7$ & Yes \\
\hline $1 \mathrm{~A}$ & $\begin{array}{c}\text { PWT - CFA: Baseline } \\
\text { vs. Day } 3\end{array}$ & $\begin{array}{l}\text { Pairwise Comp, } \\
\text { Bonferroni adj. }\end{array}$ & $3.5969 \mathrm{E}-7$ & Yes \\
\hline 1B & $\begin{array}{c}\text { WB: KCC2 Vehicle } \\
\text { vs. CFA }\end{array}$ & $\begin{array}{l}\text { Independent samples t } \\
\text { test }\end{array}$ & 0.05262 & No \\
\hline $1 \mathrm{~B}$ & $\begin{array}{c}\text { WB: aSTEP } 61 \text { Vehicle } \\
\text { vs. CFA }\end{array}$ & $\begin{array}{c}\text { Independent samples t } \\
\text { test }\end{array}$ & 0.008495 & Yes \\
\hline $1 \mathrm{~B}$ & $\begin{array}{c}\text { WB: tSTEP } 61 \text { Vehicle } \\
\text { vs. CFA }\end{array}$ & $\begin{array}{l}\text { Independent samples t } \\
\text { test }\end{array}$ & 0.5854 & No \\
\hline $1 \mathrm{~B}$ & $\begin{array}{c}\text { WB: } \mathrm{pY}^{420} \text { Fyn } \\
\text { Vehicle vs. CFA }\end{array}$ & $\begin{array}{c}\text { Independent samples } \mathrm{t} \\
\text { test }\end{array}$ & 0.01325 & Yes \\
\hline $1 \mathrm{~B}$ & $\begin{array}{l}\text { WB: Fyn Vehicle vs. } \\
\text { CFA }\end{array}$ & $\begin{array}{c}\text { Independent samples t } \\
\text { test }\end{array}$ & 0.314269 & No \\
\hline $1 \mathrm{~B}$ & $\begin{array}{l}\text { WB: } \mathrm{pY}^{1472} \text { GluN2B } \\
\text { Vehicle vs. CFA }\end{array}$ & $\begin{array}{c}\text { Independent samples t } \\
\text { test }\end{array}$ & $5.6060 \mathrm{E}-4$ & Yes \\
\hline $1 \mathrm{~B}$ & $\begin{array}{c}\text { WB: GluN2B Vehicle } \\
\text { vs. CFA }\end{array}$ & $\begin{array}{c}\text { Independent samples t } \\
\text { test }\end{array}$ & 0.02319 & Yes \\
\hline $1 \mathrm{C}$ & PWT - Vehicle & $\begin{array}{c}\text { One-way repeated } \\
\text { measures ANOVA }\end{array}$ & $\begin{array}{l}\text { Sphericity established: } \\
0.1328\end{array}$ & No \\
\hline $1 \mathrm{C}$ & PWT - CFA & $\begin{array}{l}\text { One-way repeated } \\
\text { measures ANOVA }\end{array}$ & $\begin{array}{c}\text { Sphericity violated, } \\
\text { Greenhouse-Geisser: } \\
6.2441 \mathrm{E}-11\end{array}$ & Yes \\
\hline
\end{tabular}




\begin{tabular}{|c|c|c|c|c|}
\hline $1 \mathrm{C}$ & $\begin{array}{c}\text { PWT - CFA: Baseline } \\
\text { vs. Day } 1\end{array}$ & $\begin{array}{l}\text { Pairwise Comp, } \\
\text { Bonferroni adj. }\end{array}$ & $6.1413 \mathrm{E}-8$ & Yes \\
\hline $1 \mathrm{C}$ & $\begin{array}{c}\text { PWT - CFA: Baseline } \\
\text { vs. Day } 2\end{array}$ & $\begin{array}{l}\text { Pairwise Comp, } \\
\text { Bonferroni adj. }\end{array}$ & $5.7102 \mathrm{E}-9$ & Yes \\
\hline $1 \mathrm{C}$ & $\begin{array}{c}\text { PWT - CFA: Baseline } \\
\text { vs. Day } 3 \\
\end{array}$ & $\begin{array}{l}\text { Pairwise Comp, } \\
\text { Bonferroni adj. }\end{array}$ & $1.2285 \mathrm{E}-8$ & Yes \\
\hline 1D & $\begin{array}{c}\text { WB: KCC2 Vehicle } \\
\text { vs. CFA }\end{array}$ & $\begin{array}{l}\text { Independent samples t } \\
\text { test }\end{array}$ & 0.7838 & No \\
\hline $1 \mathrm{D}$ & $\begin{array}{c}\text { WB: aSTEP } 61 \text { Vehicle } \\
\text { vs. CFA }\end{array}$ & $\begin{array}{c}\text { Independent samples t } \\
\text { test }\end{array}$ & 0.9580 & No \\
\hline $1 \mathrm{D}$ & $\begin{array}{c}\text { WB: } \text { tSTEP }_{61} \text { Vehicle } \\
\text { vs. CFA }\end{array}$ & $\begin{array}{c}\text { Independent samples } \mathrm{t} \\
\text { test, equal variances } \\
\text { not assumed }\end{array}$ & 0.2862 & No \\
\hline 1D & $\begin{array}{c}\text { WB: } \mathrm{pY}^{420} \mathrm{Fyn} \\
\text { Vehicle vs. CFA }\end{array}$ & $\begin{array}{c}\text { Independent samples t } \\
\text { test }\end{array}$ & 0.1279 & No \\
\hline $1 \mathrm{D}$ & $\begin{array}{c}\text { WB: Fyn Vehicle vs. } \\
\text { CFA }\end{array}$ & $\begin{array}{c}\text { Independent samples t } \\
\text { test }\end{array}$ & 0.8471 & No \\
\hline $1 \mathrm{D}$ & $\begin{array}{l}\text { WB: } \mathrm{pY}^{1472} \text { GluN2B } \\
\text { Vehicle vs. CFA }\end{array}$ & $\begin{array}{l}\text { Independent samples t } \\
\text { test }\end{array}$ & 0.5962 & No \\
\hline $1 \mathrm{D}$ & $\begin{array}{c}\text { WB: GluN2B Vehicle } \\
\text { vs. CFA }\end{array}$ & $\begin{array}{c}\text { Independent samples t } \\
\text { test }\end{array}$ & 0.4250 & No \\
\hline $2 \mathrm{~A}$ & $\begin{array}{l}\text { Male vs. Female } \\
\text { Charge Transfer }\end{array}$ & $\begin{array}{c}\text { Independent samples } \mathrm{t} \\
\text { test }\end{array}$ & 0.06258 & No \\
\hline $2 \mathrm{~A}$ & $\begin{array}{l}\text { Male vs. Female } \\
\text { Decay Constant }\end{array}$ & $\begin{array}{c}\text { Independent samples t } \\
\text { test }\end{array}$ & 0.3221 & No \\
\hline $2 \mathrm{~A}$ & $\begin{array}{c}\text { Male vs. Female Peak } \\
\text { Amplitude }\end{array}$ & $\begin{array}{c}\text { Independent samples t } \\
\text { test }\end{array}$ & 0.2183 & No \\
\hline $2 \mathrm{~B}$ & $\begin{array}{c}\text { Male Charge Transfer } \\
\text { Control vs. CFA vs. } \\
\text { BDNF }\end{array}$ & Welch's & $1.4040 \mathrm{E}-5$ & Yes \\
\hline $2 \mathrm{~B}$ & $\begin{array}{c}\text { Male Charge Transfer } \\
\text { Control vs. CFA }\end{array}$ & $\begin{array}{c}\text { Welch's, Games- } \\
\text { Howell }\end{array}$ & 0.03188 & Yes \\
\hline $2 \mathrm{~B}$ & $\begin{array}{l}\text { Male Charge Transfer } \\
\text { Control vs. BDNF }\end{array}$ & $\begin{array}{l}\text { Welch's, Games- } \\
\text { Howell }\end{array}$ & $2.9022 \mathrm{E}-6$ & Yes \\
\hline $2 \mathrm{C}$ & $\begin{array}{c}\text { Female Charge } \\
\text { Transfer Control vs. } \\
\text { CFA vs. BDNF }\end{array}$ & Kruskal Wallis & 0.2540 & No \\
\hline $2 \mathrm{D}$ & $\begin{array}{c}\text { WB: KCC2 Saline vs. } \\
\text { BDNF }\end{array}$ & Paired samples $t$ test & 0.8796 & No \\
\hline $2 \mathrm{D}$ & $\begin{array}{c}\text { WB: aSTEP }{ }_{61} \text { Saline } \\
\text { vs. BDNF }\end{array}$ & Paired samples $t$ test & 0.7451 & No \\
\hline $2 \mathrm{D}$ & $\begin{array}{c}\text { WB: } \text { tSTEP }_{61} \text { Saline } \\
\text { vs. BDNF }\end{array}$ & Paired samples $t$ test & 0.4751 & No \\
\hline $2 \mathrm{D}$ & $\begin{array}{c}\text { WB: } \mathrm{pY}^{420} \text { Fyn Saline } \\
\text { vs. BDNF }\end{array}$ & Paired samples $t$ test & 0.6427 & No \\
\hline $2 \mathrm{D}$ & $\begin{array}{l}\text { WB: Fyn Saline vs. } \\
\text { BDNF }\end{array}$ & Paired samples $t$ test & 0.6001 & No \\
\hline $2 \mathrm{D}$ & $\begin{array}{l}\text { WB: } \text { pY }^{1472} \text { GluN2B } \\
\text { Saline vs. BDNF }\end{array}$ & Paired samples $t$ test & 0.6979 & No \\
\hline $2 \mathrm{D}$ & $\begin{array}{l}\text { WB: GluN2B Saline } \\
\text { vs. BDNF }\end{array}$ & Paired samples $\mathrm{t}$ test & 0.6175 & No \\
\hline $3 \mathrm{~A}$ & $\begin{array}{c}\text { Male Membrane } \\
\text { KCC2 Average } \\
\text { Intensity Vehicle vs. } \\
\text { BDNF } \\
\end{array}$ & $\begin{array}{l}\text { Extra sum-of-squares } \\
\text { F-test method }\end{array}$ & $2.0919 \mathrm{E}-4$ & Yes \\
\hline $3 \mathrm{~A}$ & $\begin{array}{l}\text { Male Intracellular } \\
\text { KCC2 Average } \\
\text { Intensity Vehicle vs. } \\
\text { BDNF }\end{array}$ & $\begin{array}{c}\text { Extra sum-of-squares } \\
\text { F-test method }\end{array}$ & $1.7548 \mathrm{E}-6$ & Yes \\
\hline $3 B$ & $\begin{array}{l}\text { Female Membrane } \\
\text { KCC2 Average }\end{array}$ & $\begin{array}{c}\text { Extra sum-of-squares } \\
\text { F-test method }\end{array}$ & 0.2511 & No \\
\hline
\end{tabular}




\begin{tabular}{|c|c|c|c|c|}
\hline & $\begin{array}{c}\text { Intensity Vehicle vs. } \\
\text { BDNF }\end{array}$ & & & \\
\hline $3 \mathrm{~B}$ & $\begin{array}{c}\text { Female Intracellular } \\
\text { KCC2 Average } \\
\text { Intensity Vehicle vs. } \\
\text { BDNF } \\
\end{array}$ & $\begin{array}{c}\text { Extra sum-of-squares } \\
\text { F-test method }\end{array}$ & 0.7235 & No \\
\hline $3 \mathrm{E}$ & $\begin{array}{l}\text { Human WB: KCC2 } \\
\text { Saline vs. BDNF }\end{array}$ & Paired samples $t$ test & 0.4691 & No \\
\hline $3 \mathrm{E}$ & $\begin{array}{l}\text { Human WB: }_{\text {aSTEP }} 61 \\
\text { Saline vs. BDNF }\end{array}$ & $\begin{array}{c}\text { Wilcoxon signed-rank } \\
\text { test }\end{array}$ & 0.6002 & No \\
\hline $3 \mathrm{E}$ & $\begin{array}{l}\text { Human WB: } \text { tSTEP }_{61} \\
\text { Saline vs. BDNF }\end{array}$ & Paired samples t test & 0.7730 & No \\
\hline $3 \mathrm{E}$ & $\begin{array}{l}\text { Human WB: } \mathrm{pY}^{420} \mathrm{Fyn} \\
\text { Saline vs. BDNF }\end{array}$ & Paired samples $t$ test & 0.4945 & No \\
\hline $3 \mathrm{E}$ & $\begin{array}{l}\text { Human WB: Fyn } \\
\text { Saline vs. BDNF } \\
\end{array}$ & Paired samples $t$ test & 0.6278 & No \\
\hline $3 \mathrm{E}$ & $\begin{array}{c}\text { Human WB: } \\
\text { pY }{ }^{1472} \text { GluN2B Saline } \\
\text { vs. BDNF }\end{array}$ & Paired samples $t$ test & 0.6002 & No \\
\hline $3 \mathrm{E}$ & $\begin{array}{l}\text { Human WB: GluN2B } \\
\text { Saline vs. BDNF }\end{array}$ & Paired samples $t$ test & 0.8166 & No \\
\hline $4 \mathrm{~A}$ & $\begin{array}{c}\text { Female: Naive vs. } \\
\text { OVX Charge Transfer }\end{array}$ & $\begin{array}{c}\text { Independent samples t } \\
\text { test }\end{array}$ & 0.6602 & No \\
\hline $4 \mathrm{~A}$ & $\begin{array}{c}\text { Female: Naive vs. } \\
\text { OVX Decay Constant }\end{array}$ & $\begin{array}{c}\text { Independent samples t } \\
\text { test }\end{array}$ & 0.8513 & No \\
\hline $4 \mathrm{~A}$ & $\begin{array}{c}\text { Female: Naive vs. } \\
\text { OVX Peak Amplitude }\end{array}$ & Mann-Whitney test & 0.9654 & No \\
\hline 4B & $\begin{array}{c}\text { Female OVX Charge } \\
\text { Transfer ANOVA }\end{array}$ & One-way ANOVA & $3.5011 \mathrm{E}-4$ & Yes \\
\hline $4 \mathrm{~B}$ & $\begin{array}{c}\text { Female OVX Charge } \\
\text { Transfer Control vs. } \\
\text { BDNF }\end{array}$ & $\begin{array}{c}\text { One-way ANOVA, } \\
\text { Tukey HSD }\end{array}$ & $5.0100 \mathrm{E}-4$ & Yes \\
\hline $4 \mathrm{~B}$ & $\begin{array}{c}\text { Female OVX Charge } \\
\text { Transfer Control vs. } \\
\text { BDNF+PP2 }\end{array}$ & $\begin{array}{c}\text { One-way ANOVA, } \\
\text { Tukey HSD }\end{array}$ & .6517 & No \\
\hline $4 \mathrm{~B}$ & $\begin{array}{c}\text { Female OVX Charge } \\
\text { Transfer BDNF vs. } \\
\text { BDNF+PP2 }\end{array}$ & $\begin{array}{c}\text { One-way ANOVA, } \\
\text { Tukey HSD }\end{array}$ & 0.003389 & Yes \\
\hline $4 \mathrm{C}$ & $\begin{array}{c}\text { WB: KCC2 Saline vs. } \\
\text { BDNF }\end{array}$ & Paired samples $t$ test & 0.03953 & yes \\
\hline $4 \mathrm{C}$ & $\begin{array}{c}\text { WB: aSTEP } 61 \text { Saline } \\
\text { vs. BDNF }\end{array}$ & Paired samples $t$ test & 0.4111 & No \\
\hline $4 \mathrm{C}$ & $\begin{array}{c}\text { WB: } \text { tSTEP }_{61} \text { Saline } \\
\text { vs. BDNF }\end{array}$ & Paired samples $t$ test & $6.1642 \mathrm{E}-4$ & Yes \\
\hline $4 \mathrm{C}$ & $\begin{array}{c}\text { WB: } \mathrm{pY}^{420} \text { Fyn Saline } \\
\text { vs. BDNF }\end{array}$ & Paired samples $t$ test & 0.04553 & Yes \\
\hline $4 \mathrm{C}$ & $\begin{array}{c}\text { WB: Fyn Saline vs. } \\
\text { BDNF }\end{array}$ & Paired samples $t$ test & 0.8685 & No \\
\hline $4 \mathrm{C}$ & $\begin{array}{l}\text { WB: } \mathrm{pY}^{1472} \text { GluN2B } \\
\text { Saline vs. BDNF }\end{array}$ & Paired samples $t$ test & 0.2441 & No \\
\hline $4 \mathrm{C}$ & $\begin{array}{l}\text { WB: GluN2B Saline } \\
\text { vs. BDNF }\end{array}$ & Paired samples $t$ test & 0.3252 & No \\
\hline $\begin{array}{c}\text { Supplementary Figure } \\
1\end{array}$ & Male PWT - Vehicle & $\begin{array}{l}\text { One-way repeated } \\
\text { measures ANOVA }\end{array}$ & $\begin{array}{c}\text { Sphericity violated, } \\
\text { Greenhouse-Geisser: } \\
0.7816\end{array}$ & No \\
\hline $\begin{array}{c}\text { Supplementary Figure } \\
1\end{array}$ & Male PWT - CFA & $\begin{array}{l}\text { One-way repeated } \\
\text { measures ANOVA }\end{array}$ & $\begin{array}{c}\text { Sphericity violated, } \\
\text { Greenhouse-Geisser: } \\
5.5165 \mathrm{E}-5\end{array}$ & Yes \\
\hline $\begin{array}{c}\text { Supplementary Figure } \\
1\end{array}$ & $\begin{array}{l}\text { Male PWT - CFA: } \\
\text { Baseline vs. Day } 1\end{array}$ & $\begin{array}{l}\text { Pairwise Comp, } \\
\text { Bonferroni adj. }\end{array}$ & $2.3591 \mathrm{E}-3$ & Yes \\
\hline
\end{tabular}




\begin{tabular}{|c|c|c|c|c|}
\hline $\begin{array}{c}\text { Supplementary Figure } \\
1\end{array}$ & $\begin{array}{l}\text { Male PWT - CFA: } \\
\text { Baseline vs. Day } 2\end{array}$ & $\begin{array}{l}\text { Pairwise Comp, } \\
\text { Bonferroni adj. }\end{array}$ & $1.7286 \mathrm{E}-3$ & Yes \\
\hline $\begin{array}{c}\text { Supplementary Figure } \\
1\end{array}$ & $\begin{array}{l}\text { Male PWT - CFA: } \\
\text { Baseline vs. Day } 3\end{array}$ & $\begin{array}{l}\text { Pairwise Comp, } \\
\text { Bonferroni adj. }\end{array}$ & $1.7338 \mathrm{E}-3$ & Yes \\
\hline $\begin{array}{c}\text { Supplementary Figure } \\
1\end{array}$ & $\begin{array}{l}\text { Male PWT - CFA: } \\
\text { Baseline vs. Day } 4\end{array}$ & $\begin{array}{l}\text { Pairwise Comp, } \\
\text { Bonferroni adj. }\end{array}$ & $1.4770 \mathrm{E}-3$ & Yes \\
\hline $\begin{array}{c}\text { Supplementary Figure } \\
1\end{array}$ & $\begin{array}{l}\text { Male PWT - CFA: } \\
\text { Baseline vs. Day } 5\end{array}$ & $\begin{array}{l}\text { Pairwise Comp, } \\
\text { Bonferroni adj. }\end{array}$ & $1.2091 \mathrm{E}-3$ & Yes \\
\hline $\begin{array}{c}\text { Supplementary Figure } \\
1\end{array}$ & $\begin{array}{c}\text { Female PWT - } \\
\text { Vehicle } \\
\end{array}$ & $\begin{array}{l}\text { One-way repeated } \\
\text { measures ANOVA }\end{array}$ & $\begin{array}{c}\text { Sphericity established: } \\
0.3515 \\
\end{array}$ & No \\
\hline $\begin{array}{c}\text { Supplementary Figure } \\
1\end{array}$ & Female PWT - CFA & $\begin{array}{l}\text { One-way repeated } \\
\text { measures ANOVA }\end{array}$ & $\begin{array}{c}\text { Sphericity violated, } \\
\text { Greenhouse-Geisser: } \\
1.2645 \mathrm{E}-7\end{array}$ & Yes \\
\hline $\begin{array}{c}\text { Supplementary Figure } \\
1\end{array}$ & $\begin{array}{c}\text { Female PWT - CFA: } \\
\text { Baseline vs. Day } 1\end{array}$ & $\begin{array}{l}\text { Pairwise Comp, } \\
\text { Bonferroni adj. }\end{array}$ & $9.3423 \mathrm{E}-5$ & Yes \\
\hline $\begin{array}{c}\text { Supplementary Figure } \\
1\end{array}$ & $\begin{array}{c}\text { Female PWT - CFA: } \\
\text { Baseline vs. Day } 2 \\
\end{array}$ & $\begin{array}{l}\text { Pairwise Comp, } \\
\text { Bonferroni adj. }\end{array}$ & $2.8260 \mathrm{E}-5$ & Yes \\
\hline $\begin{array}{c}\text { Supplementary Figure } \\
1\end{array}$ & $\begin{array}{c}\text { Female PWT - CFA: } \\
\text { Baseline vs. Day } 3\end{array}$ & $\begin{array}{l}\text { Pairwise Comp, } \\
\text { Bonferroni adj. }\end{array}$ & $9.3423 \mathrm{E}-5$ & Yes \\
\hline $\begin{array}{c}\text { Supplementary Figure } \\
1 \\
\end{array}$ & $\begin{array}{c}\text { Female PWT - CFA: } \\
\text { Baseline vs. Day } 4\end{array}$ & $\begin{array}{l}\text { Pairwise Comp, } \\
\text { Bonferroni adj. }\end{array}$ & $1.1176 \mathrm{E}-4$ & Yes \\
\hline $\begin{array}{c}\text { Supplementary Figure } \\
1 \\
\end{array}$ & $\begin{array}{l}\text { Female PWT - CFA: } \\
\text { Baseline vs. Day } 5\end{array}$ & $\begin{array}{l}\text { Pairwise Comp, } \\
\text { Bonferroni adj. }\end{array}$ & $9.33423 \mathrm{E}-5$ & Yes \\
\hline $\begin{array}{c}\text { Supplementary Figure } \\
2\end{array}$ & $\begin{array}{c}\text { Male CFA VH: } \\
\text { aSTEP }_{61} \text { Vehicle vs. } \\
\text { CFA }\end{array}$ & $\begin{array}{c}\text { Independent samples t } \\
\text { test }\end{array}$ & 0.9804 & No \\
\hline $\begin{array}{c}\text { Supplementary Figure } \\
2\end{array}$ & $\begin{array}{c}\text { Male CFA VH: } \\
\text { tSTEP61 Vehicle vs. } \\
\text { CFA }\end{array}$ & Mann-Whitney test & 0.3823 & No \\
\hline $\begin{array}{c}\text { Supplementary Figure } \\
2\end{array}$ & $\begin{array}{c}\text { Male CFA VH: } \\
\text { pY }{ }^{420} \text { Fyn Vehicle vs. } \\
\text { CFA }\end{array}$ & $\begin{array}{c}\text { Independent samples t } \\
\text { test }\end{array}$ & 0.7686 & No \\
\hline $\begin{array}{l}\text { Supplementary Figure } \\
2 \\
\end{array}$ & $\begin{array}{c}\text { Male CFA VH: Fyn } \\
\text { Vehicle vs. CFA }\end{array}$ & $\begin{array}{c}\text { Independent samples t } \\
\text { test }\end{array}$ & 0.9794 & No \\
\hline $\begin{array}{c}\text { Supplementary Figure } \\
2\end{array}$ & $\begin{array}{c}\text { Male CFA VH: } \\
\text { pY }{ }^{1472} \text { GluN2B } \\
\text { Vehicle vs. CFA }\end{array}$ & $\begin{array}{c}\text { Independent samples t } \\
\text { test }\end{array}$ & 0.2592 & No \\
\hline $\begin{array}{c}\text { Supplementary Figure } \\
2\end{array}$ & $\begin{array}{c}\text { Male CFA VH: } \\
\text { GluN2B Vehicle vs. } \\
\text { CFA } \\
\end{array}$ & $\begin{array}{c}\text { Independent samples t } \\
\text { test }\end{array}$ & 0.9633 & No \\
\hline $\begin{array}{c}\text { Supplementary Figure } \\
5\end{array}$ & $\begin{array}{c}\text { Female CFA VH: } \\
\text { KCC2 Vehicle vs. } \\
\text { CFA } \\
\end{array}$ & $\begin{array}{c}\text { Independent samples t } \\
\text { test }\end{array}$ & 0.7619 & No \\
\hline $\begin{array}{c}\text { Supplementary Figure } \\
5\end{array}$ & $\begin{array}{c}\text { Female CFA VH: } \\
\text { aSTEP }_{61} \text { Vehicle vs. } \\
\text { CFA }\end{array}$ & Mann-Whitney test & 1.0000 & No \\
\hline $\begin{array}{c}\text { Supplementary Figure } \\
5\end{array}$ & $\begin{array}{c}\text { Female CFA VH: } \\
\text { tSTEP }_{61} \text { Vehicle vs. } \\
\text { CFA }\end{array}$ & Mann-Whitney test & 1.0000 & No \\
\hline $\begin{array}{l}\text { Supplementary Figure } \\
5\end{array}$ & $\begin{array}{c}\text { Female CFA VH: } \\
\text { pY }{ }^{420} \text { Fyn Vehicle vs. } \\
\text { CFA }\end{array}$ & $\begin{array}{l}\text { Independent samples t } \\
\text { test }\end{array}$ & 0.0519 & No \\
\hline $\begin{array}{l}\text { Supplementary Figure } \\
5 \\
\end{array}$ & $\begin{array}{c}\text { Female CFA VH: Fyn } \\
\text { Vehicle vs. CFA }\end{array}$ & $\begin{array}{c}\text { Independent samples t } \\
\text { test }\end{array}$ & 0.1921 & No \\
\hline $\begin{array}{c}\text { Supplementary Figure } \\
5\end{array}$ & $\begin{array}{c}\text { Female CFA VH: } \\
\text { pY }^{1472} \text { GluN2B } \\
\text { Vehicle vs. CFA }\end{array}$ & Mann-Whitney test & 0.5054 & No \\
\hline $\begin{array}{l}\text { Supplementary Figure } \\
5\end{array}$ & $\begin{array}{c}\text { Female CFA VH: } \\
\text { GluN2B Vehicle vs. } \\
\text { CFA }\end{array}$ & Mann-Whitney test & 0.5737 & No \\
\hline
\end{tabular}




\begin{tabular}{|c|c|c|c|c|}
\hline $\begin{array}{c}\text { Supplementary Figure } \\
8\end{array}$ & $\begin{array}{l}\text { Charge transfer: } \\
\text { Female Saline Vs. } \\
\text { Long BDNF }\end{array}$ & $\begin{array}{c}\text { Independent samples t } \\
\text { test }\end{array}$ & 0.8880 & No \\
\hline $\begin{array}{c}\text { Supplementary Figure } \\
9\end{array}$ & $\begin{array}{c}\text { Female VH: KCC2 } \\
\text { Saline vs. BDNF }\end{array}$ & Paired samples t test & 0.8904 & No \\
\hline $\begin{array}{c}\text { Supplementary Figure } \\
9 \\
\end{array}$ & $\begin{array}{c}\text { Female VH: aSTEP } 61 \\
\text { Saline vs. BDNF }\end{array}$ & Paired samples $t$ test & 0.9093 & No \\
\hline $\begin{array}{c}\text { Supplementary Figure } \\
9 \\
\end{array}$ & $\begin{array}{c}\text { Female VH: } \text { tSTEP }_{61} \\
\text { Saline vs. BDNF }\end{array}$ & Paired samples $t$ test & 0.7180 & No \\
\hline $\begin{array}{c}\text { Supplementary Figure } \\
9 \\
\end{array}$ & $\begin{array}{c}\text { Female VH: } \mathrm{pY}^{420} \mathrm{Fyn} \\
\text { Saline vs. BDNF }\end{array}$ & Paired samples t test & 0.7425 & No \\
\hline $\begin{array}{l}\text { Supplementary Figure } \\
9\end{array}$ & $\begin{array}{l}\text { Female VH: Fyn } \\
\text { Saline vs. BDNF }\end{array}$ & Paired samples $t$ test & 0.3572 & No \\
\hline $\begin{array}{c}\text { Supplementary Figure } \\
9\end{array}$ & $\begin{array}{c}\text { Female VH: } \\
\mathrm{pY}^{1472} \text { GluN2B Saline } \\
\text { vs. BDNF }\end{array}$ & Paired samples $t$ test & 0.8717 & No \\
\hline $\begin{array}{c}\text { Supplementary Figure } \\
9 \\
\end{array}$ & $\begin{array}{l}\text { Female VH: GluN2B } \\
\text { Saline vs. BDNF }\end{array}$ & Paired samples $t$ test & 0.6093 & No \\
\hline $\begin{array}{l}\text { Supplementary Figure } \\
12 \\
\end{array}$ & $\begin{array}{c}\text { Female VH: KCC2 } \\
\text { Saline vs. BDNF }\end{array}$ & Paired samples $t$ test & 0.5397 & No \\
\hline $\begin{array}{l}\text { Supplementary Figure } \\
12\end{array}$ & $\begin{array}{l}\text { Female VH: } \text { aSTEP }_{61} \\
\text { Saline vs. BDNF }\end{array}$ & Paired samples $t$ test & 0.02462 & Yes \\
\hline $\begin{array}{c}\text { Supplementary Figure } \\
12 \\
\end{array}$ & $\begin{array}{c}\text { Female VH: } \text { tSTEP }_{61} \\
\text { Saline vs. BDNF }\end{array}$ & Paired samples $t$ test & 0.4950 & No \\
\hline $\begin{array}{l}\text { Supplementary Figure } \\
12\end{array}$ & $\begin{array}{c}\text { Female VH: } \mathrm{pY}^{420} \mathrm{Fyn} \\
\text { Saline vs. BDNF }\end{array}$ & Paired samples t test & 0.1819 & No \\
\hline $\begin{array}{c}\text { Supplementary Figure } \\
12 \\
\end{array}$ & $\begin{array}{l}\text { Female VH: Fyn } \\
\text { Saline vs. BDNF } \\
\end{array}$ & Paired samples $t$ test & 0.6616 & No \\
\hline $\begin{array}{c}\text { Supplementary Figure } \\
12\end{array}$ & $\begin{array}{c}\text { Female VH: } \\
\mathrm{pY}^{1472} \text { GluN2B Saline } \\
\text { vs. BDNF }\end{array}$ & $\begin{array}{l}\text { Wilcoxon signed } \\
\text { ranks test }\end{array}$ & 0.2489 & No \\
\hline $\begin{array}{l}\text { Supplementary Figure } \\
12\end{array}$ & $\begin{array}{l}\text { Female VH: GluN2B } \\
\text { Saline vs. BDNF }\end{array}$ & Paired samples t test & 0.4107 & No \\
\hline $\begin{array}{c}\text { Supplementary Figure } \\
15\end{array}$ & $\begin{array}{c}\text { OVX Female VH: } \\
\text { KCC2 Saline vs. } \\
\text { BDNF }\end{array}$ & Paired samples $t$ test & 0.1779 & No \\
\hline $\begin{array}{l}\text { Supplementary Figure } \\
15\end{array}$ & $\begin{array}{c}\text { OVX Female VH: } \\
\text { aSTEP }_{61} \text { Saline vs. } \\
\text { BDNF } \\
\end{array}$ & $\begin{array}{l}\text { Wilcoxon signed } \\
\text { ranks test }\end{array}$ & 0.06870 & No \\
\hline $\begin{array}{l}\text { Supplementary Figure } \\
15\end{array}$ & $\begin{array}{c}\text { OVX Female VH: } \\
\text { tSTEP }_{61} \text { Saline vs. } \\
\text { BDNF }\end{array}$ & Paired samples t test & 0.001430 & Yes \\
\hline $\begin{array}{l}\text { Supplementary Figure } \\
15\end{array}$ & $\begin{array}{c}\text { OVX Female VH: } \\
\text { pY }{ }^{420} \text { Fyn Saline vs. } \\
\text { BDNF }\end{array}$ & $\begin{array}{l}\text { Wilcoxon signed } \\
\text { ranks test }\end{array}$ & 0.8886 & No \\
\hline $\begin{array}{l}\text { Supplementary Figure } \\
15\end{array}$ & $\begin{array}{l}\text { OVX Female VH: Fyn } \\
\text { Saline vs. BDNF }\end{array}$ & Paired samples $t$ test & 0.01102 & Yes \\
\hline $\begin{array}{l}\text { Supplementary Figure } \\
15\end{array}$ & $\begin{array}{c}\text { OVX Female VH: } \\
\text { pY }{ }^{1472} \text { GluN2B Saline } \\
\text { vs. BDNF }\end{array}$ & Paired samples t test & 0.7237 & No \\
\hline $\begin{array}{l}\text { Supplementary Figure } \\
15\end{array}$ & $\begin{array}{c}\text { OVX Female VH: } \\
\text { GluN2B Saline vs. } \\
\text { BDNF }\end{array}$ & Paired samples t test & 0.08205 & No \\
\hline
\end{tabular}




\section{Chapter 4: Integrated Discussion}

\subsection{Introduction:}

Our understanding of the mechanisms that underlie spinal hyperexcitability in pathological pain is incomplete: these mechanisms have been predominantly studied in male rodents. To expand our understanding of spinal hyperexcitability in both sexes and across species, we used in vivo rat models and ex vivo rat and human spinal cord models to investigate the molecular mechanisms underlying pathological changes in chronic pain.

We first investigated whether $\mathrm{STEP}_{61}$ is a molecular linker between disinhibition and potentiation of NMDARs in males. We found that STEP $_{61}$ downregulation is both necessary and sufficient to prime the potentiation of NMDARs by BDNF at SDH synapses in rodent ex vivo (spinal BDNF incubation) and in vivo (CFA-induced inflammation) chronic pain models. To bridge the gap between rodent models and clinical applications in humans, we next developed a spinal cord ex vivo human model of pathological pain using BDNF incubation. Using our human ex vivo BDNF pathological pain model, we found that the $\mathrm{STEP}_{61}$-linked pathway of disinhibition and NMDAR potentiation is conserved between rats and humans. Further, we performed the first-ever patch-clamp electrophysiology recordings of human lamina I neurons and found that, like rats (Hildebrand et al., 2014), human lamina I NMDAR mEPSCs are dominated by GluN2B-containing NMDARs.

Next, we turned our attention to mechanisms of spinal hyperexcitability in females. We found that, unlike males, NMDARs were not potentiated in either the in vivo CFA rodent inflammatory pain model or following ex vivo BDNF treatment in spinal cord tissue from female rats of human organ donors. Furthermore, we did not observe biochemical changes associated with disinhibition or facilitated excitation in in vivo CFA-treated rats or ex vivo BDNF treated 
female rats or humans. Collectively, these findings demonstrate that neuronal mechanisms underlying spinal hyperexcitability differ between sexes and that this sexual dimorphism is conserved between species. Finally, we demonstrated that female rats that had undergone ovariectomy before reaching sexual maturity displayed both NMDAR potentiation and biochemical changes in synaptic proteins indicative of disinhibition and facilitated excitation. Thus, we conclude that the sex difference in lamina I neuronal mechanisms of spinal hyperexcitability is hormonally mediated. In the sections that follow, we will further discuss these findings, put them in the broader context of neuroscience research, and will highlight opportunities to expand the research contributions discussed in this thesis.

\subsection{The role of $\mathrm{STEP}_{61}$ as a molecular linker of disinhibition and NMDAR potentiation:}

The first key finding of this thesis is the role of $\mathrm{STEP}_{61}$ as the molecular linker between disinhibition and facilitated excitation in male rats and humans. Our previous work (see Appendix A), described a mechanism where neither direct BDNF-TrkB signalling nor disinhibition alone were sufficient to potentiate lamina I NMDAR mEPSCs (Hildebrand et al., 2016). However, we found that disinhibition was required for lamina I NMDAR mEPSC potentiation by BDNF (Hildebrand et al., 2016). Findings from other groups demonstrate that NMDAR activity and an increase in intracellular calcium are required for the downregulation of KCC2 in the brain and spinal cord (Lee et al., 2011; Zhou et al., 2012; Chamma et al., 2013). Together, this leads to the proposition that there may be a molecular linker between NMDAR potentiation and disinhibition. Indeed, we investigated this hypothesis in Chapter 2 (Dedek et al., 2019) and find that STEP $_{61}$ downregulation is both necessary and sufficient to prime the potentiation of synaptic lamina I NMDAR mEPSCs by BDNF in rodent ex vivo and in vivo CFA- 
induced inflammatory pain models. In addition, we found that STEP $_{61}$ was downregulated by BDNF at human SDH synapses, indicating that this mechanism is conserved between species.

A missing piece of the $\mathrm{STEP}_{61}$ puzzle is an understanding of the role that $\mathrm{STEP}_{61}$ plays across various types of chronic pain. In Appendix A (Hildebrand et al., 2016), we used a PNI model of neuropathic pain as well as the ex vivo BDNF pathological pain model to demonstrate the conservation of disinhibition (BDNF signalling resulting in $\mathrm{KCC} 2$ downregulation) and facilitated excitation (BDNF acting through activated Fyn to result in NMDAR potentiation) across both pain models. In Chapter 2 (Dedek et al., 2019), we paired the ex vivo BDNF pathological pain model with in vivo CFA-induced inflammatory pain and found conserved roles for disinhibition and NMDAR potentiation in mediating spinal hyperexcitability. This led to the general conclusion that both disinhibition and NMDAR potentiation resulting from KCC2 downregulation play roles in mediating both neuropathic pain as well as inflammatory pain. Since BDNF is involved in both neuropathic (Coull et al., 2005; Trang et al., 2009; Chen et al., 2014b) and inflammatory pain (Mannion et al., 1999; Zhao et al., 2006), it is reasonable to hypothesize that the role of $\mathrm{STEP}_{61}$ is conserved across BDNF-dependant pain pathologies.

However, it remains untested whether $\mathrm{STEP}_{61}$ is the linker between disinhibition and NMDAR potentiation in neuropathic pain. A first step to definitively investigate the role of STEP $_{61}$ in neuropathic pain includes using western blot analysis to determine whether PNIinduced neuropathic pain drives down $\mathrm{STEP}_{61}$ at $\mathrm{SDH}$ synapses, as is the case in inflammatory pain (Azkona et al., 2016a; Dedek et al., 2019). Further, restoring STEP ${ }_{61}$ levels using cellmembrane permeable TAT-STEP 61 administered through intrathecal injection to PNI rats would determine whether restoring $\mathrm{STEP}_{61}$ in vivo can reverse tactile allodynia resulting from 
neuropathic pain. Finally, using tissue from animals that received intrathecal $\mathrm{STEP}_{61}$ to probe the effects of synaptic markers of disinhibition and facilitated excitation could elucidate the role of STEP $_{61}$ in neuropathic pain.

The discovery of the role of $\mathrm{STEP}_{61}$ in male rat and human models of pathological pain is an exciting development towards understanding the molecular forces driving chronic pain; however, systemically targeting $\mathrm{STEP}_{61}$ for pharmacological development in the clinic is a dubious prospect. Although we found BDNF's effect on $\mathrm{STEP}_{61}$ to be limited to the SDH, with no BDNF-mediated effects observed in spinal sections ventral to the SDH (Chapter 2 and 3: Dedek et al., 2019, Dedek et al., in preparation) research on $\mathrm{STEP}_{61}$ in the brain implicates STEP $_{61}$ signalling in other pathologies. For example, STEP $_{61}$ has been implicated in age-related memory decline (Castonguay et al., 2018), Alzheimer's disease (Xu et al., 2014; Lee et al., 2021), and schizophrenia (Xu et al., 2016). The crux is that high STEP 61 levels are associated with pathology in the brain, while low $\mathrm{STEP}_{61}$ levels are associated with pathological pain within the SDH. For that reason, globally manipulating $\mathrm{STEP}_{61}$ levels within the CNS may lead to undesired pathological effects.

An option to bypass the effects of systemic administration of a STEP 61 -targeting drug is intrathecal administration via an intrathecal pump system. This technique is currently used to administer ziconotide to treat severe chronic pain, however, it is associated with severe CNS side-effects (Schmidtko et al., 2010). This is because some drugs will diffuse out of the spinal CSF and to the brain. Additionally, this is a highly invasive treatment that is reserved for only a limited number of patients with debilitating pain (Schmidtko et al., 2010). Although it is a possible delivery mechanism for a $\mathrm{STEP}_{61}$-restoring drug, this strategy has practical limitations. 
One possibility to side-step the problems of global-CNS pharmacological targeting is to develop chemogenetic treatment to specifically target SDH neurons. Chemogenetic approaches allow for regulation of neuronal function in a specific neuronal population (Iyer et al., 2016) while relying on a less invasive strategy than optogenetics, which requires implantation of a light to the targeted area (Copits et al., 2016). Advances in chemogenetics have already allowed researchers to reduce microglial-induced neuroinflammation and allodynia in mouse models of neuropathic pain (Saika et al., 2020; Yi et al., 2020). Other studies have used chemogenetic approaches to reduce mechanical allodynia in early-stage osteoarthritis in rodents (Miller et al., 2017), as well as to silence sensory neurons to reverse thermal and mechanical hypersensitivity in a neuropathic pain model (Weir et al., 2017). Chemogenetic approaches could be used to selectively increase inhibition in SDH neurons to reduce spinal hyperexcitability. This would require the development of a mammalian ligand-gated chloride channel. Several groups have used a modified version of an invertebrate glutamate-gated chloride channel ( $\mathrm{GluCl})$; however, since this channel is of invertebrate origin, it would likely elicit an immune response in the subject/patient (Matthew Soleiman, 2017). A promoter for chemogenetic modulation could preferentially target excitatory subpopulations of SDH neurons to drive down excitability and output of the SDH nociceptive network. Using a chemogenetic approach to increase inhibitory tone within the SDH could, in the future, break the cycle of feed-forward hyperexcitability.

\subsection{Future directions for studying NMDAR function in spinal nociceptive networks:}

A limitation in the electrophysiological methods used in this thesis to study the molecular pathways that lead to SDH hyperexcitability is the use of only NMDAR mEPSCs to study 
NMDAR responses. Although we observed consistent potentiation of NMDAR mEPSCs in our pain models in males across the very heterogeneous lamina I neuronal population (Todd, 2010), questions remain about the involvement of extrasynaptic NMDARs, the contribution of presynaptic NMDARs to SDH hyperexcitability, and the effects of ex vivo BDNF and CFAinflammatory pain on afferent evoked NMDAR responses.

A starting point for examining the involvement of extrasynaptic NMDARs is to compare glutamate-evoked total NMDAR responses and synaptic NMDAR responses in lamina I neurons. Glutamate-evoked responses will include both synaptic and extrasynaptic NMDARs. Additionally, pre-treatment with MK-801 (a non-reversible use-dependant NMDAR antagonist) could be used to block synaptic NMDARs that are activated by spontaneous vesicle release primarily at synapses. MK-801 could then be washed out before recording, leaving extrasynaptic NMDARs largely unaffected (McQuate and Barria, 2020). With this experimental design, the amplitudes of evoked currents from extrasynaptic NDMARs can be compared between pain models to determine if there are differences in the contributions of extrasynaptic NMDARs between inflammatory and neuropathic pain models. In addition, NMDAR-subunit blockers can be used to identify the contributions of different GluN2-NMDAR subunit types to these extrasynaptic responses. These experiments would expand on our work examining synaptic NMDAR responses to include the contributions of extrasynaptic NMDARs.

To examine the effects of presynaptic NMDARs in the BDNF-NMDAR pathway, pairedpulse ratio can be used to examine neurons from BDNF or CFA-treated rats. Paired-pulse ratio examines the probability of neurotransmitter release from presynaptic vesicles using a ratio of the amplitudes of two evoked currents (Poncer and Malinow, 2001). A decrease in paired-pulse 
ratio indicates that there is an increase in the probability of vesicle release. There is already evidence that potentiation of presynaptic GluN2B-NMDARs at primary afferent terminals contributes to facilitated excitation in neuropathic pain (Yan et al., 2013; Chen et al., 2014b). Furthermore, like postsynaptic NMDARs described in Chapter 2 and Appendix A, the potentiation of presynaptic NMDARs depends on the activity of SFKs (Chen et al., 2010). The evidence from other groups on the contributions of presynaptic NMDARs in pain models necessitates further investigation into the mechanisms of their potentiation.

Finally, optogenetic approaches can be used to address the effects of nociceptive afferentevoked NMDAR responses on BDNF or CFA-mediated hyperexcitability. Because SDH neurons receive input from many types of afferents carrying distinct sensory information, it is important to understand the contributions of the different subtypes of afferents (Peirs and Seal, 2016). It is unclear whether afferent-evoked NMDAR responses differ from mEPSCs; some evidence in adult male rats suggests that lamina I mEPSCs and minimally-evoked unitary EPSCs show similar GluN2B-dominant contributions of GluN2-NMDARs (Hildebrand et al., 2014); however, another study that used juvenile rats of both sex found that C-fiber evoked NMDAR responses were primarily composed of GluN2A responses, with moderate contributions from GluN2BNMDARs (Tong and MacDermott, 2014). Using optogenetic approaches allows researchers to target specific types of primary afferents in an ex vivo transgenic mouse preparation. Examining NMDAR responses from optogenetically-evoked afferents can characterize the contributions of individual primary afferent populations on SDH hyperexcitability across models of pathological pain (Tashima et al., 2018; Warwick et al., 2020). 


\subsection{GluN3-NMDARs: a role in spinal hyperexcitability?}

Historically, it was thought that two main types of subunits can compose an NMDAR: two obligatory GluN1 subunits (which have eight different functional splice variants) and two GluN2 subunits (which have four isoforms) (Schüler et al., 2008; Yao et al., 2013). In recent decades, a third subtype was discovered: GluN3 (with two subtypes: GluN3A and GluN3B) (Dunah et al., 1999; Cavara and Hollmann, 2008; Grand et al., 2018). Because the contribution of GluN3-containing NMDARs to synaptic physiology has been poorly understood, the focus in this thesis, and largely within the field, has remained on GluN2-containing NMDARs. This is despite evidence that GluN3-NMDARs are expressed in both human (Aguet et al., 2019) and rodent (Chamessian et al., 2018; Russ et al., 2020) spinal cord.

GluN3-containing receptors' dominant-negative modulation of NMDAR signalling has been implicated in several neuropathologies. This includes Huntington's disease (Wesseling and Pérez-Otaño, 2015), excitotoxic cell death (as in the case of ischemic stroke) (Wang et al., 2013; Belov Kirdajova et al., 2020), and cocaine addiction (Huang et al., 2013; Yuan et al., 2013). Importantly, GluN3A knockout mice have shorter latency to thermal pain response, as well as increased duration of pain response in phase II of formalin-induced inflammatory pain (Mohamad et al., 2013). Further research is needed to elucidate the role played by GluN3containing NMDARs in pathological pain. Such future studies include the use of GluN3-specific antagonists such as EU1180-438 (Zhu et al., 2020), GluN3 enhancer CGP-78608 (Grand et al., 2018), and genetic knockdown models (Mohamad et al., 2013) in pain models. Because GluN3NMDARs have been implicated in several known NMDAR-related pathologies, they are 
expressed in both the human and rodent spinal cord, and because they have a regulatory role in neuroplasticity, it is essential that their role in spinal nociceptive physiology be further explored.

\subsection{Lack of NMDAR potentiation in female CFA and BDNF treatment:}

Arguably the most important finding in this thesis is the lack of NMDAR mEPSC potentiation and upregulation in female rat CFA inflammatory pain, and ex vivo BDNF treatment pain models in rats and humans. The next step is to determine how far-reaching this novel, nonNMDAR-dependant mechanism of spinal hyperexcitability is by using female rodent models of neuropathic pain. Because a previous study has found that microglial-BDNF-knockout female, but not male, mice develop tactile allodynia in response to SNI (Sorge et al., 2015), and microglia secrete BDNF in nerve injury models (Coull et al., 2005), we hypothesize that development of spinal hyperexcitability in females may be NMDAR-independent in neuropathic pain. We hypothesize that this difference is conserved between inflammatory and neuropathic pain states. This is because nociceptors are postulated to be the source of BDNF in inflammatory pain (Zhao et al., 2006), thus suggesting that the source of BDNF is irrelevant for triggering spinal hyperexcitability. In considering the response of female SDH neurons to BDNF, detailed experiments are required to understand BDNF's role in excitability in females. Chronic BDNF administration in cultured neurons from unsexed animals has been found to elicit distinct effects across different populations of SDH neurons (Lu et al., 2007; Alles et al., 2021), but it is unclear whether there are sex differences in this response. 
Further studies in females are required to understand the molecular cascade that results in spinal hyperexcitability in females. Experiments that can shed light on this topic include the use of single-cell RNA-seq on rodent spinal tissue to identify changes in the expression of specific proteins across diverse pain models. Because sex differences in immune factors mediating chronic pain have been at the forefront of spinal pain research, a starting point would be to look at proteins that interact with $\mathrm{T}$ cells, which have been linked to the development of allodynia in females (Mapplebeck et al., 2017; Laumet et al., 2019). Other potential targets include the neuropeptides CGRP, PACAP and NGF, which have all been linked to spinal hyperexcitability (Dickinson et al., 1999; Schou et al., 2017; Sun et al., 2020) and are discussed further in Section 4.7 below.

The discovery of sex differences in neuronal nociceptive signalling may come as a surprise, but current scientific standards of practice result in experimental designs that facilitate overlooking female-specific physiological mechanisms. Both the NIH and CIHR mandate that basic science studies include both sexes, but many researchers, under pressure to save time and resources, perform mechanistic investigations in male tissue and then test behavioural outcomes in both sexes (Seydel, 2021; Shansky and Murphy, 2021). This seemingly harmless shortcut lends itself to missing divergent neuronal mechanisms that can be reversed by a common pharmacological target. It is this very phenomenon that led to the assumption that NMDAR dysregulation is involved in pain in females: APV was found to reverse tactile allodynia in female mice, leading to the conclusion that NMDARs were involved in female neuropathic pain (Sorge et al., 2015). This, however, only demonstrates that blocking NMDARs is sufficient to reverse tactile allodynia in female PNI mice, not that NMDARs themselves are directly involved in the pathological molecular cascades that initiate the allodynia. This realization indicates that 
we need to fundamentally rethink how we perform basic research in both sexes (Seydel, 2021; Shansky and Murphy, 2021). Indeed, the fundamental building blocks of pain research are derived from male-only or unsexed animal studies on synaptic plasticity. Studies are showing at sexually divergent molecular mechanisms of synaptic plasticity (Dina et al., 2001; Joseph and Levine, 2003; Hucho et al., 2006; Jain et al., 2019), indicating we as pain researchers must start from square one to examine basic physiological pathways in females.

\subsection{First-ever patch-clamp electrophysiological recordings on human SDH tissue:}

In an effort to understand the functional contribution of NMDARs to synaptic responses in human lamina I neurons, we have performed the first-ever patch-clamp electrophysiological recordings on human SDH tissue. This important advance is the first functional evidence of NMDAR activity in the human SDH. We used a combination of fitting decay constant, $\tau$, to control-treated human lamina I synaptic NMDARs, as well as using pharmacological blockers TCN-201 (Hansen et al., 2012), a blocker of GluN2A, and Ro-6981 (Fischer et al., 1997), a blocker of GluN2B NMDARs. In this sample, we conclude that, like male rats, GluN2Bcontaining NMDARs dominate synaptic NMDAR responses in male human lamina I neurons (Dedek et al., 2019). This investigation is ongoing, with efforts to characterize these synaptic responses in a larger sample of human lamina I neurons.

A major difference between NMDAR mEPSCs in male rats and humans is the amplitude of NMDAR responses. We observed that the amplitude of human male NMDAR mEPSCs was almost double that of male rats (Dedek et al., 2019). This suggests that human lamina I neurons have a higher density of synaptic NMDARs than rats (Jonas et al., 1993; Prybylowski et al., 
2002; Patrizio and Specht, 2016). A secondary method that could be used to test the postsynaptic NMDAR density is using immune labelled NMDAR subunits paired with immunogold labelling (Nusser et al., 1995, 1997). When imaged using an electron microscope, these experiments would have sufficient resolution to examine the receptor density of NMDAR subunits at human lamina I synapses.

Of the three GluN2-containing NMDARs expressed in the spinal cord, we did not directly test the involvement of GluN2D, despite the finding in male rodents that GluN2B and GluN2D dominate synaptic responses in lamina I (Hildebrand et al., 2014). This is because our previous work (Hildebrand et al., 2016) and others' (Tong and MacDermott, 2014; Wu et al., 2014; Xie et al., 2016) implicate GluN2B-NMDARs in mediating SDH hyperexcitability, and thus we first aimed to tease apart the contributions of GluN2A and GluN2B-containing NMDARs. The analysis of decay constants of control-treated human lamina I mEPSCs was biased against GluN2D-NMDARs by rejecting individual events that did not decay to at least $50 \%$ of their maximum amplitude by $1000 \mathrm{~ms}$. This rejection criterium was based on earlier studies in rat models (Hildebrand et al., 2014), and was made in an effort to compare rat and human lamina I mEPSCs side-by-side. Our study concludes that contributions of GluN2BNMDARs make up a large portion of male human lamina I NMDAR responses, but further studies are required to understand the contributions of GluN2D NMDARs fully.

However, future work is needed to characterize human and rat lamina I responses in an unbiased manner. To address this issue, we are following up with an expanded analysis of patchclamp recordings of male and female rat and human synaptic mEPSCs. All NMDAR events, regardless of decay time, would need to be included. Events could then be individually fit to 
determine the decay constants, and the distributions of these decay constants could be used to interpret whether the NMDAR subunit composition is conserved between sex and species. Expanding on this idea, a subset of recordings in each species and sex could test the effects of sequential GluN2-subunit antagonists, including DQP-1105 (Acker et al., 2011) to test for the contribution of GluN2subunit-containing NMDARs.

Although we have been able to target synaptic responses from individual neurons, it remains unclear how these contribute to spinal-circuit level nociceptive processing. Examining spinal circuitry becomes a pressing issue when considering degeneracy of multiple separate pathways that result in spinal hyperexcitability; examining a single player in this degenerate circuit will repeatedly lead to the same outcome: spinal hyperexcitability (Ratté and Prescott, 2016).

New tools such as multielectrode arrays (MEAs) and voltage-sensitive dye imaging are valuable tools that can be used to examine the electrophysiological properties of spinal circuits. This technology allows high throughput analysis with a higher success rate than patch-clamp electrophysiology. In addition, MEAs and voltage-sensitive dye imaging provide more information about general excitability; this information is easier to extrapolate to a population of SDH neurons than patch-clamp recordings, where activity is recorded from a very small subset of neurons. This new technology has already been applied in several rodent spinal cord preparations. For example, MEAs have been used to characterize the spontaneous firing patterns of the mouse SDH (Lucas-Romero et al., 2018). Another study used in vivo rat MEA recordings paired with histology to map cervical spine interneuron populations in circuits regulating phrenic motor output (Streeter et al., 2017). Recently, in vivo MEA recordings were used to categorize 
the processing of both innocuous and noxious stimuli across dorsal horn laminae in rats

(Greenspon et al., 2019). Voltage-sensitive dye imaging can also be used in similar applications. For example, voltage-sensitive dye imaging was used to discover mediolateral asymmetry in synaptic connectivity in the rat SDH (Mizuno et al., 2019). To expand on the work done here, a logical next step is to use MEAs to examine the time-course of the onset of BDNF-mediated hyperexcitability. This type of analysis would also identify if regions of the SDH form regionspecific microcircuits of excitability in response to BDNF or other neuropeptides. Paired with antagonists of specific NMDAR subunits, MEAs and voltage-sensitive dye imaging can be used to identify how individual NMDAR subunits contribute to overall SDH excitability. In the future, MEA and voltage-sensitive dye imaging can be used in acute and organotypic slice preparations (which are described in Section 4.7 below) to better understand how differential regulation of excitability across subpopulations of dorsal horn neurons shapes the output of the spinal nociceptive network in the male and female human spinal cord.

\subsection{Ex vivo human spinal cord pathological pain models:}

In Chapter 2, we pioneered a novel human tissue model of chronic pain using ex vivo BDNF incubation. Using this model, we discovered that BDNF drives a loss of KCC2-dependent inhibition, which is followed by potentiation of GluN2B-containing NMDARs at human SDH synapses: a phenomenon that is conserved across male rats and humans (Dedek et al., 2019). Using a similar approach in spinal cord tissue from female organ donors, we find that ex vivo BDNF treatment does not constitute a pathological pain model in females (Chapter 3). The use of human ex vivo pain models is an exciting development that can establish clinical relevance for 
potential therapeutic targets; using BDNF to model pain is just the beginning of such developments.

Important next steps include the investigation of other neuropeptides' effects on spinal plasticity. Neuropeptides and growth factors such as calcitonin gene-related peptide (CGRP), nerve growth factor (NGF) and pituitary adenylate cyclase-activating peptide (PACAP) have been well documented to have pain-amplifying effects (Dickinson et al., 1999; Schou et al., 2017; Sun et al., 2020). However, the specific roles that these signalling molecules may play in altering SDH excitability remain poorly understood in rats and completely unexplored in humans. All three of these pain amplifying factors have been either directly or indirectly linked to mechanisms of central sensitization within the dorsal horn. The neuropeptide CGRP is expressed in A $\delta$ - and C-fiber nociceptive afferents (Ju et al., 1987; Hill and Elde, 1988) and levels of CGRP are associated with visceral, somatic, neuropathic and inflammatory pain conditions in the clinic (Schou et al., 2017). CGRP has been shown to alter excitatory postsynaptic responses within higher-order pain processing circuits of the amygdala (Han et al., 2010) as well as in SDH neurons (Bird et al., 2006), but the molecular determinants of this potentiation pathway have not been fully identified. The roles of CGRP in mediating pain pathology may also be sexually dimorphic, as low dose dural CGRP induces pain hypersensitivity only in females in both rat and mouse preclinical models of migraine (Avona et al., 2019). It is therefore critically important to investigate whether CGRP directly alters synaptic responses and excitability within subpopulations of SDH neurons in male and female rodents and humans. 
In contrast to CGRP, very little is known on how NGF may or may not alter SDH excitability. NGF is a critical modulator of the excitability of peripheral nociceptive neurons and has been implicated in peripheral sensitization and pathological pain processing (Barker et al., 2020). Given that intrathecal administration of NGF triggers BDNF release in the SDH (Lever et al., 2001), it is also possible that NGF release from nociceptive afferents either directly or indirectly alters excitability to mediate central sensitization. However, this hypothesis remains to be tested. Similarly, intrathecal injection of PACAP induces long-lasting nociceptive behaviours (Ohnou et al., 2016; Yokai et al., 2016), while PACAP antagonists inhibit noxious responses and SDH excitability (Dickinson et al., 1999). Given that PACAP immunoreactivity is concentrated in laminae I and II and not other spinal cord laminae (Dun et al., 1996), it will be important to investigate the molecular mechanisms by which PACAP preferentially alters the excitability of SDH neurons, which may include NMDAR potentiation (Liu and Madsen, 1997).

Using a similar approach to the BDNF ex vivo model, it is possible to adapt this spinal incubation model to test the effects of CGRP, NGF and PACAP on human spinal tissue. These investigations have the potential to lead to the development of new ex vivo human pathological pain models. In addition to the biochemical and immunohistochemical tools used in Chapters 2 and 3, future studies in ex vivo pain models can use organotypic slice culture to expand the possible manipulations and investigations possible from using acute slices alone. For example, organotypic slice culture would allow for increased time for patch-clamp experiments from a single organ donor, allowing for an increased number of treatments to be performed on the same donor tissue, thus serving as a within-subject control. Another use of organotypic slice culture is combining the technique with adeno-associated viral vectors and optogenetics (Elfarrash et al., 2019; Griffin et al., 2019). This would allow for the first-ever manipulations of human spinal 
nociceptive circuits and would allow for the elucidation of the factors that lead to spinal hyperexcitability. Organotypic slice cultures can be used for either patch-clamp investigations, as was done in Chapters 2 and 3, or can be used for MEA recording, as was described in Section 4.6. Importantly, the sample of organ donors includes both sexes, meaning that these targets can be examined in males and females.

\subsection{Hormones in spinal hyperexcitability:}

In Chapter 3 of this thesis, we explored the factors driving the observed sex difference in neuronal nociceptive signalling. By using SDH tissue from female rats that were ovariectomized before reaching sexual maturity, we discovered that the sex difference in response to BDNF is hormonally mediated. We found that ex vivo BDNF treatment in these ovariectomized animals resulted in the potentiation of synaptic mEPSC NMDAR responses. The next step in this research question is to determine which gonadal hormone is responsible for this switch to the male-like BDNF-NMDAR sensitive phenotype. This could be achieved by performing ovariectomy at P21, like was done in Chapter 3, but then supplementing either progesterone or estrogen and observing the effects of BDNF on NMDARs. If NMDARs are potentiated when a hormone is supplemented in ovariectomized animals, that will demonstrate that the supplemented hormone is responsible for mediating the sex difference. An interesting question that remains unexplored is whether ovariectomized females subjected to the CFA inflammatory pain model would also display potentiation of synaptic NMDAR mEPSC responses since naïve CFA-females did not.

Hormonally mediated effects can be broadly categorized as activational or organizational. Activational sex differences are transient effects that require the presence or absence of a 
hormone for the resulting physiological change or behaviour (Kawata, 1995). In contrast, organizational effects occur when hormonal signalling confers changes to structures or circuits that persist from that point throughout the subject's development (Kawata, 1995). In Chapter 3, we see that the effect of removing female sex hormones before reaching sexual maturity had a long-lasting effect into adulthood, in altering the neuronal response to BDNF treatment in adult rats. Further evidence of an organizational effect can be found in our human female data. The age range of female donors was from mid-twenty-years-old to early-seventy-years-old. Of our female donors, $25 \%$ were under the age of 51, the median age of onset of menopause (McKinlay et al., 1985). Despite the drop in circulating female sex hormones that we predict based on the age of the majority of female donors (Dalal and Agarwal, 2015), we did not observe a switch to male-like BDNF sensitivity in the SDH, as we saw with ovariectomized animals. Therefore, we propose that the organizational effect of female sex hormones is life-long.

Because we only examined females, it remains unclear how reaching sexual maturity affects male BDNF-inducible pathological changes. Juvenile prepubescent animals may not have the same response to BDNF, or other pain-mediating factors, as their adult counterparts. Indeed, we have found that BDNF treatment did not result in potentiation of synaptic NMDAR responses in male juvenile rats (unpublished observations). This gap in understanding affects not only our understanding of neurodevelopment and plasticity on a basic scientific level but also has implications for children living with chronic pain. Although chronic pain is often thought of as a disease targeting primarily adults, it has a substantial prevalence in children (Perquin et al., 2000; Huguet and Miró, 2008; King et al., 2011). Interestingly, one study found a sharp increase in reports of chronic pain in girls aged 12-14 years (Perquin et al., 2000), perhaps indicating pubertal effects on pain in the clinical population. Because of ethical implications and risks in 
including children in clinical trials, it is particularly important to use animal and human preclinical models to investigate how hormonal changes affect the development of spinal nociceptive circuitry. Indeed, of 61 new drug approvals by the FDA in 2018, only 4 were for the pediatric age group (Dinh et al., 2021). Thus, the effectiveness of pharmacological targets must be examined at the basic science level in juvenile models.

Thus far, we have discussed the topic of sex as a binary matter; however, sex exists on a spectrum (Ainsworth, 2015). Although we typically base genetic sex on the presence or absence of a Y chromosome, new research suggests even this basic view of sex is complex. For example, male microchimerisms have been discovered in the brains of adult female humans (Chan et al., 2012). Cells from male offspring are transferred to their mother during pregnancy, resulting in a persistent XY genetic profile of cells in some brain areas (Chan et al., 2012). The current steps taken to be female-inclusive in basic science research do not begin to consider the physiology of transgender and intersex people. It is likely that the sex differences described here exist on a spectrum, but this research question has yet to be probed in humans or rodent models. In rodents, it is possible to supplement gonadal hormones from the opposite sex in adult animals and examine their effects on nociception to model hormone therapy in transgender people. To further explore the complex and important research questions regarding sex differences, human tissuebased approaches offer a solution to tackle these questions in ways rodent-based research simply cannot address.

Much like the spectrum of sex, there is natural variation in circulating levels of sex hormones between individuals (Bartley et al., 2015). In women, naturally varying testosterone levels are associated with changes in sensitivity to experimentally induced pain, with women with higher testosterone displaying increased pain thresholds (Bartley et al., 2015). Another 
interesting hormonally-mediated finding is that of widely reported baseline difference in PWT in naïve rodents: females have lower PWT than males (Li et al., 2009; Sorge et al., 2015; Dedek et al., 2019; Mapplebeck et al., 2019). Interestingly, this difference in baseline PWT is abolished in estrogen receptor $\alpha$ or $\beta$ knockouts (Li et al., 2009). To add to the complexity of hormonal regulation of pain, the pro-or-anti-nociceptive effects of estrogen on pain sensitivity have been hotly debated (Mogil, 2012). Several studies, using a variety of techniques in both sexes, find estrogen to be pronociceptive (LaCroix-Fralish et al., 2005; Hucho et al., 2006; Li et al., 2009), while others find antinociceptive effects (Kuba and Quinones-Jenab, 2005; Mannino et al., 2007; Martin, 2009; Bieerkehazhi et al., 2017). Craft has postulated that the reasons for these differences are due to methodological differences between studies, widespread but variable levels of estrogen receptors across the CNS, and possible biphasic dose-response relationships (Craft, 2007). Of the gonadal hormones, the role of progesterone, especially during pregnancy, is most clear. Progesterone is antinociceptive, increasing pain thresholds to electric foot shock during pregnancy (Gintzler, 1980; Liu and Gintzler, 2000) and relieving mechanical allodynia in temporomandibular joint inflammation (Hornung et al., 2020). Notably, progesterone has been found to reduce inflammatory pain in females in an NMDAR-linked mechanism (Ren et al., 2000). Regardless of the specific effect of each gonadal hormone, the pain readout from an individual will be a summation of the transient and organizational effects of all hormones on nociceptive circuitry. Further research on the contribution of each gonadal hormone on nociception is needed to understand how these effects will vary between individuals. 


\subsection{Conclusions:}

In this collective work, we examined mechanisms of SDH hyperexcitability across sex and species. We find that neurons in the male rat and human SDH display a mechanism of spinal hyperexcitability where BDNF drives KCC2-dependent disinhibition, which is linked to NMDAR potentiation by downregulation of the phosphatase, STEP 61 . Surprisingly, we find that spinal hyperexcitability in females is not driven by this BDNF-driven mechanism in the SDH. We observed that the sex difference in neuronal mechanisms of spinal hyperexcitability is hormonally mediated. We also developed the first ex vivo human model of pathological pain. Using our ex vivo human model of pathological pain, we found that the sexually divergent neuronal mechanisms of SDH neuronal hyperexcitability are conserved across species. Further, we show preliminary evidence suggesting that overall kinetic properties, but not amplitude, of lamina I synaptic NMDAR responses are conserved between male rodents and humans.

This research has several exciting future directions. The first includes the development of novel ex vivo human pain models using additional neuropeptides. Further, MEA and voltagesensitive dye imaging techniques will bring our findings from a detailed single-cell approach to a holistic global picture of network mechanisms of spinal hyperexcitability. Such novel ex vivo human tissue approaches, in combination with MEA recordings or voltage-sensitive dye imaging, can serve as preclinical screening tools for the discovery of novel pain therapeutics.

\subsection{Disciplinary implications:}

This work generates important implications for the broader field of neuroscience research. It is critically important that basic science research be sex inclusive, including in basic mechanistic studies. Furthermore, basic scientists should be mindful that sex differences 
observed in genetically similar rodents may exist as a spectrum in the diverse human population. Our work also highlights the use of preclinical pain models using human tissue. The use of such preclinical models in basic science research can be used to bridge the gap between basic science rodent models and future treatment approaches for humans. 


\section{Appendices:}

\section{Appendix A - Potentiation of Synaptic GluN2B NMDAR Currents by Fyn Kinase Is Gated through BDNF-Mediated Disinhibition in Spinal Pain Processing}

\section{Summary}

In chronic pain states, the neurotrophin brain-derived neurotrophic factor (BDNF) transforms the output of lamina I spinal neurons by decreasing synaptic inhibition. Pain hypersensitivity also depends on N-methyl-D-aspartate receptors (NMDARs) and Src-family kinases, but the locus of NMDAR dysregulation remains unknown. Here, we show that NMDAR-mediated currents at lamina I synapses are potentiated in a peripheral nerve injury model of neuropathic pain. We find that BDNF mediates NMDAR potentiation through activation of TrkB and phosphorylation of the GluN2B subunit by the Src-family kinase Fyn. Surprisingly, we find that $\mathrm{Cl}^{-}$-dependent disinhibition is necessary and sufficient to prime potentiation of synaptic NMDARs by BDNF. Thus, we propose that spinal pain amplification is mediated by a feedforward mechanism whereby loss of inhibition gates the increase in synaptic excitation within individual lamina I neurons. Given that neither disinhibition alone nor BDNF-TrkB signalling is sufficient to potentiate NMDARs, we have discovered a form of molecular coincidence detection in lamina I neurons. 


\section{Pain Hypersensitivity}

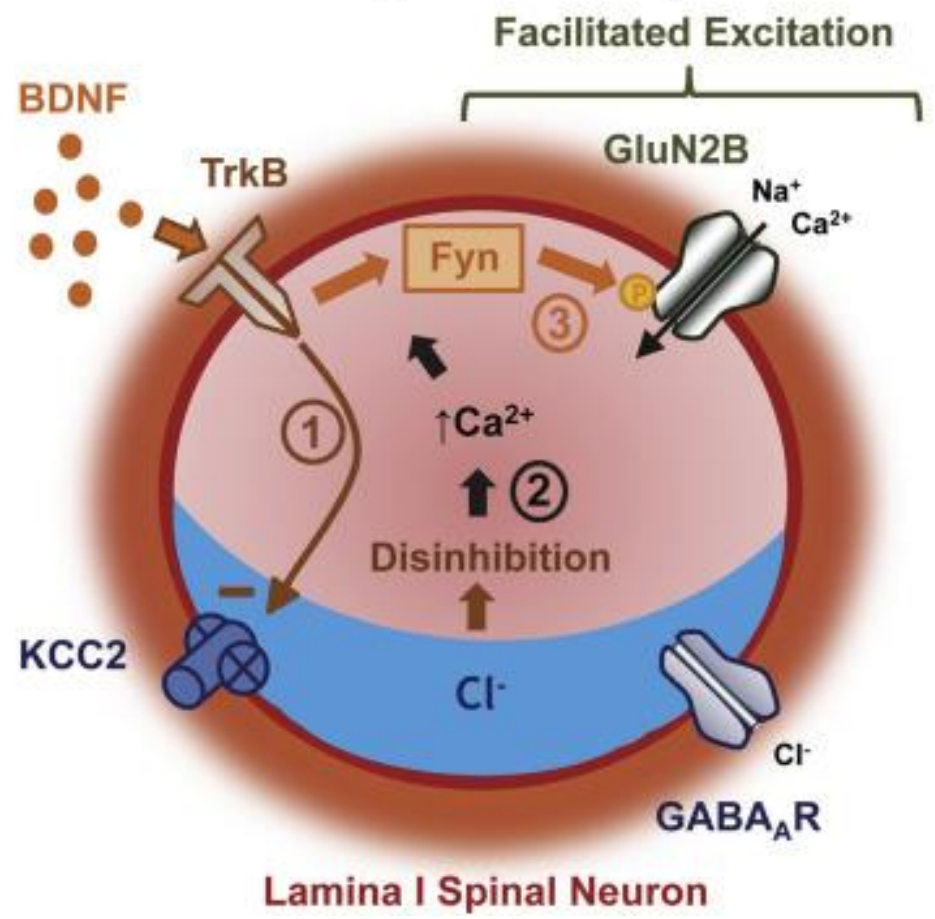

\section{Introduction}

Pain hypersensitivity depends on maladaptive changes in neuronal activity within the peripheral, spinal, and brain nociceptive networks. The spinal nociceptive network in the dorsal horn is an interconnected matrix of inputs from primary afferents as well as from inhibitory and excitatory local circuit neurons and descending efferent fibres. Neurons in dorsal horn lamina I are an essential component of the spinal nociceptive network and are critical for acute as well as chronic pain signalling (Braz et al., 2014; Prescott et al., 2014).

The output of lamina I neurons is controlled by a delicate balance between synaptic excitation and inhibition and shifting the balance toward excitation leads to chronic pain (Woolf and Salter, 2000). This balance may be disrupted by reducing synaptic inhibition (disinhibition). A growing body of evidence has demonstrated that brain-derived neurotrophic factor (BDNF) is a major driver of disinhibition at lamina I synapses (Beggs and Salter, 2013; Ferrini and De 
Koninck, 2013). In the context of peripheral nerve injury (PNI), BDNF is released from activated microglia and binds to TrkB receptors on lamina I neurons (Coull et al., 2005; Beggs et al., 2012). Activated TrkB receptors downregulate the potassium-chloride co-transporter, KCC2, resulting in increased intracellular $\mathrm{Cl}^{-}$, attenuated $\mathrm{GABAergic}$ inhibition, and increased action potential firing in lamina I neurons (Coull et al., 2003, 2005; Keller et al., 2007).

Pain-producing changes in the balance between excitation and inhibition may also be mediated by enhancing excitation within the spinal nociceptive network. Excitation is driven by glutamatergic synaptic transmission, and the N-methyl-D-aspartate receptor (NMDAR) class of glutamate receptors is prominently implicated in spinal mechanisms of chronic pain (Bourinet et al., 2014). Blocking NMDAR activity of receptors containing the GluN2B subunit using pharmacological or genetic approaches blocks pain hypersensitivity in animal models of chronic pain. Moreover, behavioural and biochemical studies show that enhancement of NMDAR function by Src family kinases is critical for pain hypersensitivity (Abe et al., 2005; Liu et al., 2008).

A major open question is where is the critical locus of change in glutamate receptor function within the highly interconnected matrix that is the spinal nociceptive network. Here, we tested the hypothesis that in pain hypersensitivity, there is enhanced glutamatergic synaptic transmission onto neurons in lamina I. In order to properly characterize the postsynaptic responses at lamina I glutamatergic synapses, it was necessary to avoid activating the nociceptive network, which includes prolonged polysynaptic excitatory responses that contaminate direct excitatory synaptic responses (Todd, 2010). Thus, we studied miniature excitatory postsynaptic currents (mEPSCs) in lamina I neurons, as these mEPSCs are direct synaptic responses (Hildebrand et al., 2014). We found that NMDAR-mediated mEPSCs were enhanced in a PNI 
model of chronic pain. Because the enhancement was mediated by BDNF-TrkB signalling, we tested whether there is functional crosstalk between potentiation of NMDAR-mediated excitation and loss of GABAergic inhibition. We found that BDNF-mediated disinhibition led to enhancement of GluN2B NMDAR responses via Fyn activation in naive animals and, conversely, blocking disinhibition reversed the enhancement after PNI. Thus, our findings show that enhanced excitation of lamina I neurons is gated by disinhibition.

\section{Experimental Procedures}

\section{Animals}

All experiments involving rats and their care were performed in accordance with the recommendations of the Canadian Council on Animal Care and were according to the animal care regulations and policies of the Hospital for Sick Children, Toronto. We performed all experiments on male adult (350-450 g) Sprague Dawley (SD) rats, except for the experiments in Figure 3A, which utilized juvenile (P20-P22) SD rats.

\section{Peripheral Nerve Injury and Behavioral Testing}

As previously described (Mosconi and Kruger, 1996; Pitcher et al., 1999), we used a PNI model of neuropathic pain to study pathological pain signalling. Under isoflurane anesthesia, we implanted a 2-mm long polyethylene cuff around the sciatic nerve of adult SD rats. Ten to 17 days after surgery, animals were tested for behavioural hypersensitivity compared to baseline by measuring mechanical paw withdrawal threshold with von Frey filaments. Animals used for spinal slice electrophysiology typically had a reduction in paw withdrawal threshold from $15 \mathrm{~g}$ to $4 \mathrm{~g}$, which was restricted to the ipsilateral paw.

\section{Spinal Cord Isolation}


We anesthetized male SD rats through intraperitoneal (i.p.) injection of $3 \mathrm{~g} / \mathrm{kg}$ urethane (Sigma). As previously described (Hildebrand et al., 2014), we rapidly dissected out the lumbar spinal cord and immediately placed the cord in an ice-cold, oxygenated protective modified artificial cerebrospinal fluid (pACSF) solution. Parasagittal spinal slices $(300 \mu \mathrm{m})$ were cut from an L3-L6 section of lumbar spinal cord.

\section{Electrophysiological Recordings on Lamina I Spinal Cord Neurons}

We visualized cells using infrared differential interference contrast (IR-DIC) optics and neurons from lamina I were selected based upon their location dorsal to the substantia gelatinosa layer, within $50 \mu \mathrm{m}$ of the white matter (Hildebrand et al., 2014). Although this selection criteria enriches for a population of mainly lamina I neurons, we cannot formally exclude the possibility that a smaller fraction of recorded neurons were in lamina II.

The extracellular recording solution consisted of an artificial cerebrospinal fluid (ACSF) solution containing (in mM): $125 \mathrm{NaCl}, 20 \mathrm{D}$-glucose, $26 \mathrm{NaHCO}_{3}, 3 \mathrm{KCl}, 1.25 \mathrm{NaH}_{2} \mathrm{PO}_{4}, 2$ $\mathrm{CaCl}_{2}$, and $1 \mathrm{MgCl}_{2}$ as well as $500 \mathrm{nM}$ TTX, $10 \mu \mathrm{M} \mathrm{Cd}^{2+}, 10 \mu \mathrm{M}$ strychnine and $10 \mu \mathrm{M}$ bicuculline to block voltage-gated $\mathrm{Na}^{+}$channel, voltage-gated $\mathrm{Ca}^{2+}$ channel, glycinergic and GABAergic currents, respectively. We pulled patch-clamp pipettes with resistances of 4-8 M $\Omega$. The internal patch pipette solution contained (in mM): 105 Cs-gluconate, $17.5 \mathrm{CsCl}, 10$ BAPTA, 10 HEPES, 5 QX-314, 2 MgATP, $0.5 \mathrm{Na}_{2} \mathrm{GTP}(\mathrm{pH}=7.25,295 \mathrm{mOsm})$, except in a subset of experiments where 10 BAPTA was replaced with 0.1 EGTA (Supplementary Figure 7).

Neurons typically had access resistances below $20 \mathrm{M} \Omega$ and leakage currents $>-100 \mathrm{pA}$ at a holding potential $\left(\mathrm{V}_{\mathrm{h}}\right)$ of $-70 \mathrm{mV}$. As previously described (Hildebrand et al., 2014), we generated averaged mEPSCs for time intervals ranging between 5 and 10 min, typically with 30 to 50 events per averaged trace. For recordings of NMDAR-mEPSCs at $+60 \mathrm{mV}$, we broke 
through to the whole-cell configuration at $-60 \mathrm{mV}$ and gradually adjusted the holding potential to $+60 \mathrm{mV}$. Thus, analysis of "initial" NMDAR-mEPSC currents was based on averages from 5-15 min after whole-cell configuration. We selected for mEPSCs containing an NMDAR component at $+60 \mathrm{mV}$ by excluding mEPSCs that exhibited complete decay within $100 \mathrm{~ms}$. For experiments with conditions that promoted a rise in intracellular $\mathrm{Ca}^{2+}$ (Supplementary Figures 6 and 7), recordings were done at a holding potential of $-40 \mathrm{mV}$, on neurons with an mEPSC frequency $>1 \mathrm{~Hz}$.

\section{Compounds and Perfusion}

For electrophysiology experiments, D-AP5, bicuculline methochloride, K252a, Ro256981, TCN-201. and VU 0240551 were from Tocris Bioscience. TTX was from Alomone Labs. Recombinant BDNF protein was from Abcam, while TrkB-Fc and TrkB-IgG were from R\&D Systems. Ionomycin, PP2 and SU6656 were from Calbiochem. The Src40-58 and Fyn39-57 inhibitor peptides as well as their TAT-linked versions (Liu et al., 2008; Yang et al., 2012) were synthesized by GenScript. Unless otherwise indicated, all other compounds were ordered from Sigma-Aldrich. We applied $50 \mathrm{ng} / \mathrm{mL}$ BDNF in slice pre-treatment experiments, where prolonged treatment would allow for permeation of BDNF throughout the slice. A higher concentration of BDNF (100 ng/mL) was used for acute perfusion to ensure that sufficient levels of BDNF reached the neuron under study during the shorter time course of the recordings.

\section{Isolation of Synaptic Fractions and Biochemical Analyses}

For biochemical studies, the lumbar spinal cord was sectioned horizontally with a vibratome into an approximately $300 \mu \mathrm{m}$ thick horizontal SDH section and a second section containing the remainder of the spinal cord. Isolation of synaptic fractions was performed as 
previously described (Xu et al., 2009). Briefly, tissues were lysed in Dounce tissue grinders (Wheaton) in $1 \mathrm{~mL}$ ice-cold TEVP buffer supplemented with complete protease inhibitor cocktail (Roche). Aliquots of lysates were saved for further analyses as total homogenates. The rest of lysates went through two spins $(10 \mathrm{~min}$ at $1,000 \times \mathrm{g}$ and $15 \mathrm{~min}$ at $12,000 \times g)$ to obtain synaptic fractions. Samples $(30 \mu \mathrm{g})$ were loaded on $8 \%$ SDS-PAGE and transferred to nitrocellulose membrane (Bio-Rad). Membranes were blocked in 5\% BSA in TBS $+1 \%$ Tween-20 (TBS-T) and incubated with primary antibodies overnight (anti-pY ${ }^{416}$ Src family $[1: 1,000]$ and anti-pY ${ }^{527}$ Src family $[1: 1,000]$ from Upstate; anti-Fyn [1:2,000], anti-Src [1:2,000], anti-KCC2 [1:2,000] and anti-actin [1:5,000] from Santa Cruz; anti-pY ${ }^{1472}$ GluN2B $[1: 1,000]$ and anti-pY ${ }^{1325}$ GluN2A [1:1,000] from PhosphoSolutions; anti-GluN2B [1:2,000] and anti-GluN2A [1:2,000] from Millipore; anti-fodrin [1:5,000] from Enzo Life Sciences) in 5\% BSA+TBS-T. Membranes were washed three times with TBS-T and then incubated with horseradish peroxidase (HRP)-coupled secondary antibodies (anti-mouse or anti-rabbit, Pierce, 1:5,000) for $2 \mathrm{hr}$ at room temperature. Membranes were developed using Chemiluminescent Substrate kit (Pierce) and visualized by a G:BOX with the GeneSnap software (Syngene). All densitometric bands were quantified using Image $(\mathrm{NIH})$.

\section{Data Analysis}

We used Microcal Origin 8.5 (Northampton) and Clampfit10 (Molecular Devices) for data analysis. All data are given as means \pm SEM. We performed statistical comparisons of data using both Student's paired and unpaired t tests as well as one-way ANOVAs followed by a Tukey's test for means comparisons. We considered $\mathrm{p}<0.05$ to be statistically significant. 


\section{Results}

\section{The NMDAR but Not the AMPAR Component of mEPSCs in Lamina I Neurons Is Increased following Peripheral Nerve Injury}

To investigate synaptic glutamatergic responses in lamina I neurons, we made whole-cell patchclamp recordings on visualized neurons in lamina I in acute spinal slices from adult rats. When we recorded at a holding potential of $-60 \mathrm{mV}$, we observed spontaneously occurring, rapidly rising, and decaying inward currents that were eliminated by the AMPAR antagonist CNQX, demonstrating that these were mEPSCs. Holding the membrane potential at $+60 \mathrm{mV}$ revealed a slowly decaying NMDAR component of the mEPSCs that was blocked by $100 \mu \mathrm{M}$ APV (Figure 1A). Thus, the currents we recorded here corresponded to bi-component, AMPAR/NMDAR mEPSCs described previously (Hildebrand et al., 2014). To clearly separate the NMDAR-mediated component from the AMPAR-mediated component during recordings at $+60 \mathrm{mV}$, we calculated the NMDAR component as that beginning $40 \mathrm{~ms}$ after the onset of the mEPSC and extending to $500 \mathrm{~ms}$ after mEPSC onset (Supplementary Table 1). AMPAR currents were studied at a holding potential of $-60 \mathrm{mV}$. 

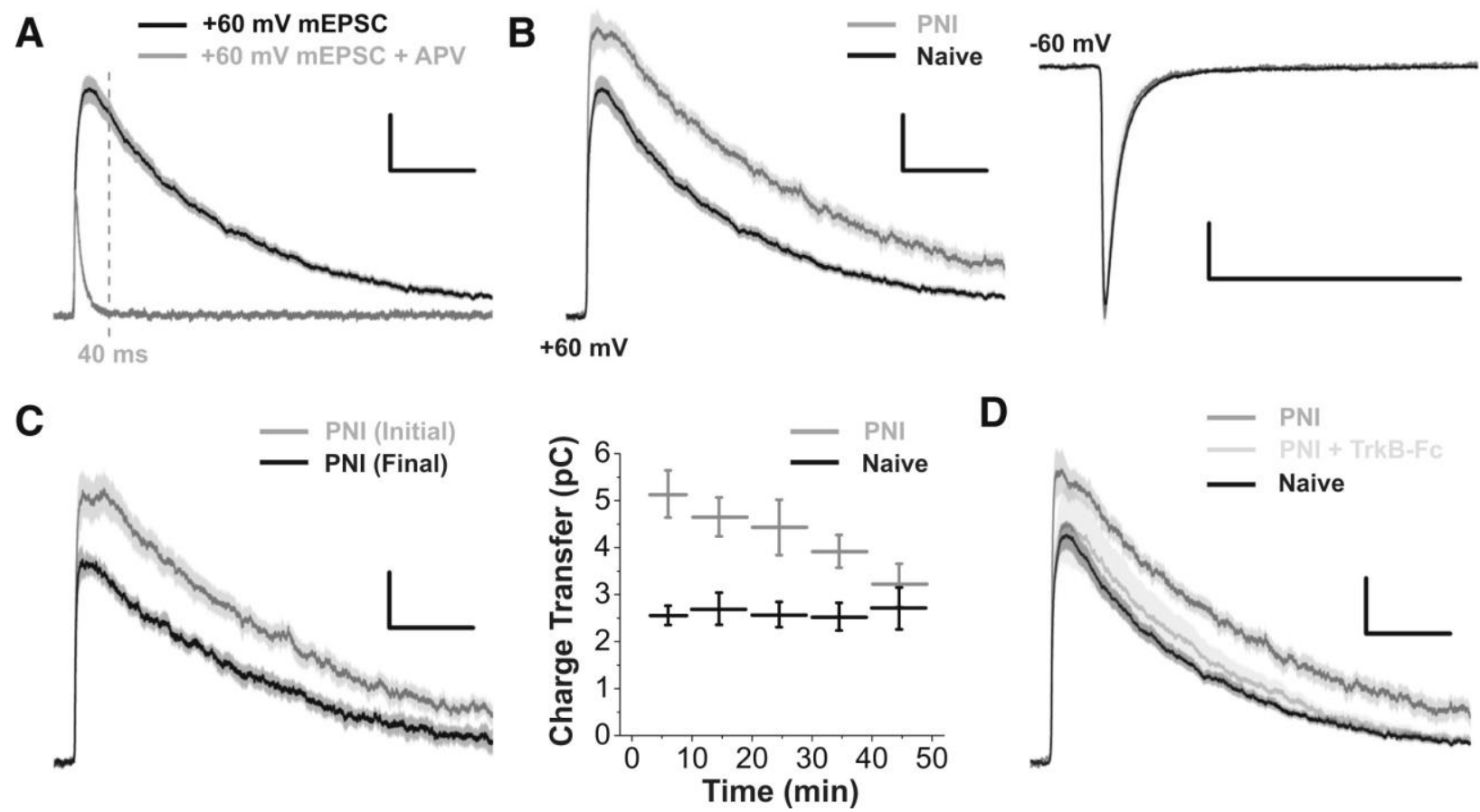

D

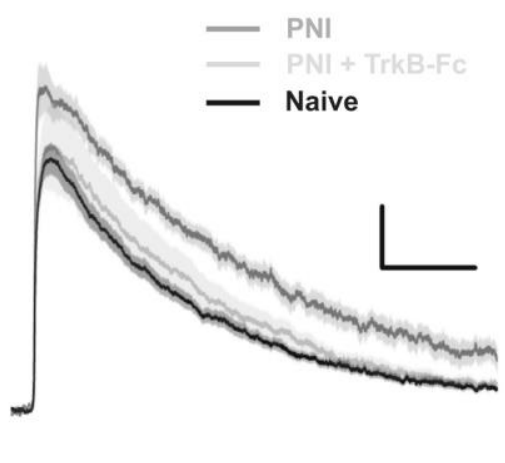

Appendix A Figure 1. NMDAR mEPSCs in Lamina I Neurons Are Reversibly Potentiated by BDNF in the

PNI Model of Neuropathic Pain. (A) Average outward mEPSC traces at $+60 \mathrm{mV}$ from lamina I neurons in spinal slices of naive rats in the absence (black, $\mathrm{n}=20$ ) and presence (gray, $\mathrm{n}=3$ ) of $100 \mu \mathrm{M}$ APV. (B) Left: average mEPSC traces at $+60 \mathrm{mV}$ in rats with PNI (gray, $\mathrm{n}=9$ ) compared to naive rats (black, $\mathrm{n}=20$ ). Right: average mEPSC traces at $-60 \mathrm{mV}$ in rats with PNI (gray, $\mathrm{n}=5$ ) compared to naive rats (black, $\mathrm{n}=13$ ). (C) Left: averaged mEPSC traces from rats with PNI during an initial recording period (gray) and during a final period (30-50 min) of recording (black, $\mathrm{n}=7$ ). Right: time course of NMDAR charge transfer (averaged every $10 \mathrm{~min}, \mathrm{n}=4$ each). (D) Average mEPSC traces from naive rats (black) and rats with PNI (gray) as well as from rats with PNI that had slices treated with $200 \mathrm{ng} / \mathrm{mL}$ TrkB-F $\mathrm{F}_{\mathrm{c}}$ before recording (light gray, $\mathrm{n}=9$ ). Current traces are represented as means $($ dark lines) \pm SEM (lighter bars) of mEPSCs from all averaged cells. Scale bars, $100 \mathrm{~ms}$ (x axes); 5 pA (y axes).

To determine whether these glutamatergic synaptic responses of lamina I neurons are affected during pathological pain processing, we studied mEPSCs in neurons from rats that had received a PNI (Mosconi and Kruger, 1996; Pitcher et al., 1999). PNI is widely used as a model of peripheral neuropathic pain and pain hypersensitivity is inferred by a lowering of the 
mechanical paw withdrawal threshold ipsilateral to the nerve injury. We included only animals in which the paw withdrawal threshold was significantly reduced compared to the same paw before PNI ( $\mathrm{n}=23$ animals). The recordings were made from slices taken from animals 10-17 days after PNI, when the mechanical hypersensitivity has reached maximum. We found that the frequency of mEPSCs was not affected by PNI (PNI: $4.5 \pm 1.8 \mathrm{~Hz}, \mathrm{n}=5$; naive $3.6 \pm 0.9, \mathrm{n}=13$, $\mathrm{p}=0.61)$. Moreover, we found that the AMPAR mEPSCs in lamina I neurons were not altered in neurons from animals with PNI (Figure 1B): peak $(-23.6 \pm 1.2 \mathrm{pA})$, rise time $(0.9 \pm 0.2 \mathrm{~ms})$, and decay time constant $(5.9 \pm 0.9 \mathrm{~ms})$ of the AMPAR mEPSCs after PNI ( $\mathrm{n}=5$ neurons) were not significantly different than those from naive rats $(-22.0 \pm 1.2 \mathrm{pA}, \mathrm{p}=0.45 ; 1.2 \pm 0.1 \mathrm{~ms}, \mathrm{p}=$ $0.27 ; 6.7 \pm 0.5 \mathrm{~ms}, \mathrm{p}=0.43$, respectively; $\mathrm{n}=13$ neurons). By contrast, we found that the NMDAR component of the mEPSCs was significantly increased after PNI (Figure 1B). We used charge transfer as a robust measure of the NMDAR component of the mEPSCs (Hildebrand et al., 2014) and observed that the charge transfer in neurons from rats with PNI $(5.27 \pm 0.35 \mathrm{pC}$, $\mathrm{n}=9$ neurons) was greater than that in naive rats $(3.20 \pm 0.22 \mathrm{pC}, \mathrm{n}=20$ neurons; $\mathrm{p}=0.000021)$ The NMDAR component of the mEPSCs in sham-operated rats $(2.75 \pm 0.52 \mathrm{pC}, \mathrm{n}=6)$ was not different from that in naive rats $(\mathrm{p}=0.37)$. From these findings, we conclude that the NMDAR component, but not the AMPAR component, of mEPSCs in lamina I neurons was significantly potentiated following PNI.

\section{BDNF-TrkB Signaling Mediates the Potentiation of NMDAR mEPSCs by PNI}

Strikingly, we observed a progressive decrease in the NMDAR component of mEPSCs during recordings of lamina I neurons from rats with PNI but not during recordings in neurons from naive rats (Figure 1C; Supplementary Table 2). By comparison, the AMPAR component of the mEPSCs was stable during recording from either PNI or naive rats (not illustrated). In 
neurons from rats with PNI, the NMDAR charge transfer decreased from an initial value of 5.17 $\pm 0.47 \mathrm{pC}$ to $3.08 \pm 0.37 \mathrm{pC}(\mathrm{n}=7$ neurons, $\mathrm{p}=0.000030)$ after $30 \mathrm{~min}$ of recording. The NMDAR charge transfer at the end of the recordings from these neurons was not different from the charge transfer in neurons from naive rats $(\mathrm{p}=0.47$; Figure 1C). These findings indicate that the potentiation of the NMDAR component of the mEPSCs in neurons from rats with PNI was gradually lost during the recordings and that the NMDAR mEPSCs returned to the basal level observed in neurons from naive animals.

The neurons were continuously superfused with extracellular recording solution during the recording period, and we questioned whether the decrease in NMDAR mEPSCs in neurons from rats with PNI was due to progressive loss of an endogenous, extracellular potentiating substance. Given the key role of BDNF in pain hypersensitivity (Beggs and Salter, 2013; Ferrini and De Koninck, 2013), we considered the possibility that BDNF might be such a potentiating substance. To test this possibility, we treated spinal cord slices from rats with PNI with a BDNFsequestering fusion protein, TrkB-Fc (Ninkina et al., 1997), beginning at least 10 min prior to recording and continuing throughout the recording period. In contrast to the recordings without TrkB-Fc treatment, we found that the NMDAR charge transfer was stable during recordings $(\mathrm{n}=$ $6, \mathrm{p}=0.30)$ in neurons from PNI rats treated with TrkB-Fc $(200 \mathrm{ng} / \mathrm{mL})$. Moreover, the initial NMDAR charge transfer in neurons from PNI rats treated with TrkB-Fc was significantly less than that in PNI neurons not treated with TrkB-Fc ( $n=9, p=0.014$, Figure 1D). In addition, the NMDAR charge transfer in PNI neurons treated with TrkB-Fc was not different from the final NMDAR charge transfer in neurons from PNI rats ( $\mathrm{n}=9$ and 7 neurons, respectively, $\mathrm{p}=0.62$ ) nor from that in neurons from naive rats $(n=20$ neurons, $p=0.62)$. Together, these findings indicate that prior to the beginning of the recordings in neurons from PNI animals, the pre- 
treatment with TrkB-Fc occluded the decline in NMDAR mEPSCs. The observation that the NMDAR charge transfer in neurons from PNI rats remained constant or increased during the first 10 min of recording suggests that the decline in NMDAR responses was not due to loss of an intracellular signal through dialysis of the patch pipette solution. Therefore, we conclude that the potentiation of NMDAR mEPSCs in lamina I neurons following PNI depends upon endogenously released BDNF.

To test whether BDNF is sufficient to cause potentiation of NMDAR mEPSCs, we pretreated spinal slices from naive animals with recombinant BDNF (50 ng/mL BDNF, $75 \pm 3 \mathrm{~min}$, $\mathrm{n}=14$ neurons). We found that the initial NMDAR charge transfer in BDNF-pre-treated slices $(5.03 \pm 0.37 \mathrm{pC} ; \mathrm{n}=14$ neurons) was significantly larger than that in neurons from untreated slices $(3.20 \pm 0.22 \mathrm{pC} ; \mathrm{n}=20$ neurons; $\mathrm{p}=0.000088$; Figures $2 \mathrm{~A})$. During these experiments, we stopped the BDNF treatment just prior to recording and observed a progressive decrease in the NMDAR mEPSCs (Figure 2B) comparable to the decline in the NMDAR component during recordings in neurons from PNI rats (cf. Figure 1C). To determine whether we could reverse the loss of NMDAR mEPSC charge transfer during recordings from PNI animals, we applied BDNF after the currents had declined (Figure 2C; Supplementary Table 3). NMDAR currents significantly increased by $55 \% \pm 14 \%(\mathrm{n}=5$ neurons, $\mathrm{p}=0.0086)$ following BDNF administration (100 $\mathrm{ng} / \mathrm{mL}, 7-20 \mathrm{~min}$ ), indicating that the decline in NMDAR mEPSCs was rescued by exogenous BDNF. The finding that NMDAR responses were potentiated by exogenous BDNF at this later time point further supports the conclusion that the initial decline in NMDARs was not due to whole-cell dialysis. Together, these findings indicate that BDNF is not only necessary for the potentiation following PNI but is also sufficient to induce a potentiation of synaptic NMDAR currents in lamina I neurons. 
A NAIVE

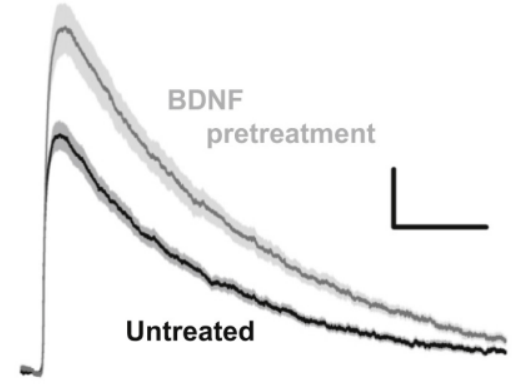

D

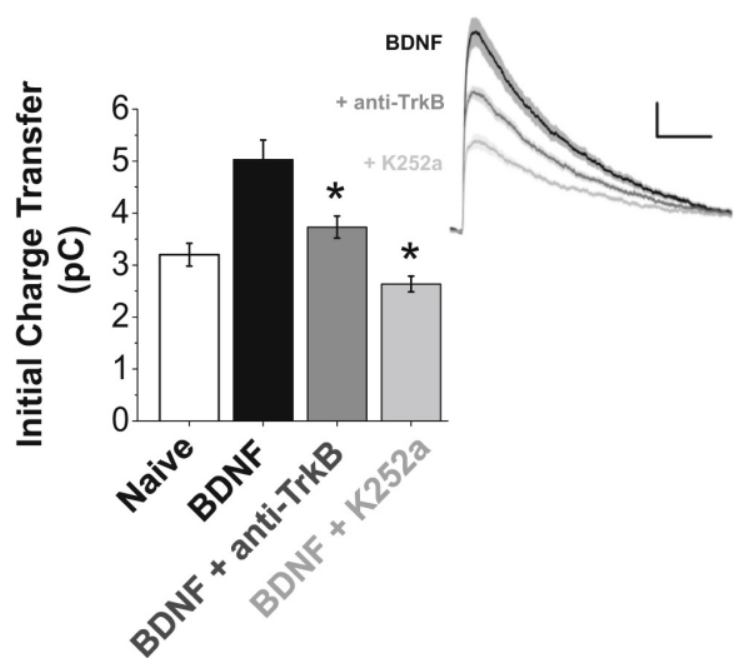

NAIVE

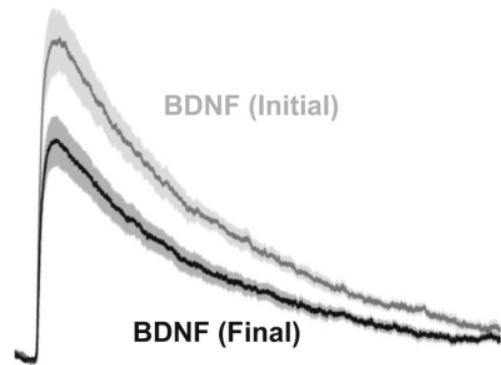

E
C PNI
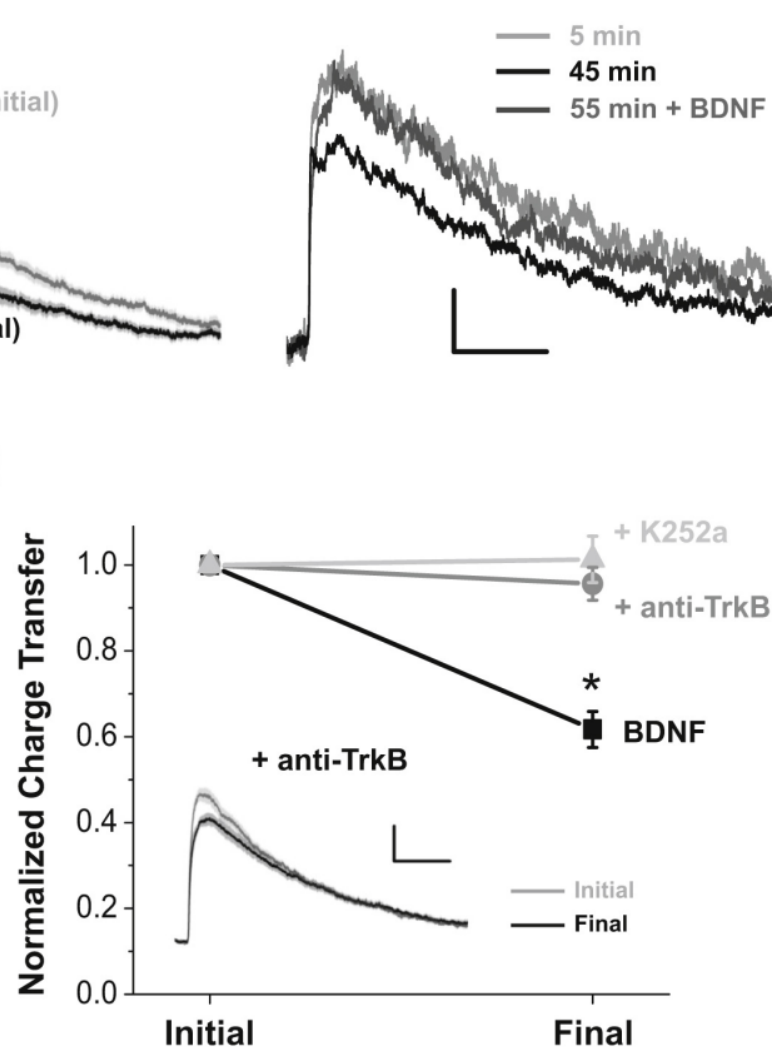

Appendix A Figure 2. BDNF Is Sufficient to Facilitate NMDAR mEPSCs. (A) Average initial mEPSC traces in slices of naive rats treated with (gray, $\mathrm{n}=14$ ) or without (black, $\mathrm{n}=20$ ) $50 \mathrm{ng} / \mathrm{mL}$ BDNF. (B) Average mEPSC traces from BDNF-treated slices during initial (gray) and final (black, 20 to 50 min) periods of recording $(\mathrm{n}=10)$. (C) mEPSC traces from a rat with PNI during an initial recording period (gray), a later recording period (black), and subsequent perfusion of $100 \mathrm{ng} / \mathrm{mL}$ recombinant BDNF protein for 5-15 min (dark gray). (D) Average initial mEPSC traces (top) and associated NMDAR charge transfer values (bottom) from spinal slices of naive rats pretreated with BDNF alone (black, $\mathrm{n}=14$ ), BDNF and $2 \mathrm{mg} / \mathrm{mL}$ anti-TrkB (gray, $\mathrm{n}=7$ ), or BDNF followed by intracellular $100 \mathrm{nM} \mathrm{K252a}$ during recording (light gray, $\mathrm{n}=8$ ). ${ }^{*} \mathrm{p}<0.05$ compared to BDNF pre-treatment alone. (E) Plot of final charge transfer values normalized to initial values. ${ }^{*} \mathrm{p}<0.05$ compared to initial charge transfer values for given treatment. Inset, average mEPSC traces at $+60 \mathrm{mV}$ during initial (gray) and final (black) recording periods for slices pre-treated with BDNF and anti-TrkB $(\mathrm{n}=5)$. Scale bars, $100 \mathrm{~ms}(\mathrm{x}$ axes); $5 \mathrm{pA}$ (y axes). All current traces and plots are represented as means \pm SEM. 
To determine whether the potentiation of NMDAR mEPSCs by BDNF is mediated through activation of its cognate receptor, TrkB, we utilized a function-blocking anti-TrkB antibody (anti-TrkB) (Coull et al., 2005; Prescott et al., 2014). In spinal slices from naive rats, co-treatment of anti-TrkB $(2 \mu \mathrm{g} / \mathrm{mL})$ with BDNF ( $\mathrm{n}=7$ neurons) prevented the potentiation of NMDAR mEPSCs (Figure 2D) and the subsequent decline in NMDAR mEPSCs (Figure 2E) seen in neurons pre-treated with BDNF but not anti-TrkB (cf. Figure 2A). To test whether TrkB was activated directly in the neuron in which NMDAR mEPSCs were potentiated, we intracellularly administered the TrkB inhibitor, K252a (Tapley et al., 1992), into the recorded neuron through the patch pipette. Because we administered K252a directly into the cell, we used near $\mathrm{IC}_{50}$ concentrations $(100 \mathrm{nM})$ to minimize inhibition of TrkB in neighbouring neurons. In neurons with intracellularly administered K252a ( $\mathrm{n}=8$ neurons), we observed neither the BDNF-induced increase in NMDAR charge transfer (Figure 2D) nor the subsequent decline in NMDAR mEPSCs during recordings (Figure 2E). Therefore, we conclude that the potentiation by BDNF of synaptic NMDAR responses in a given lamina I neuron is mediated by activating TrkB on that neuron. 

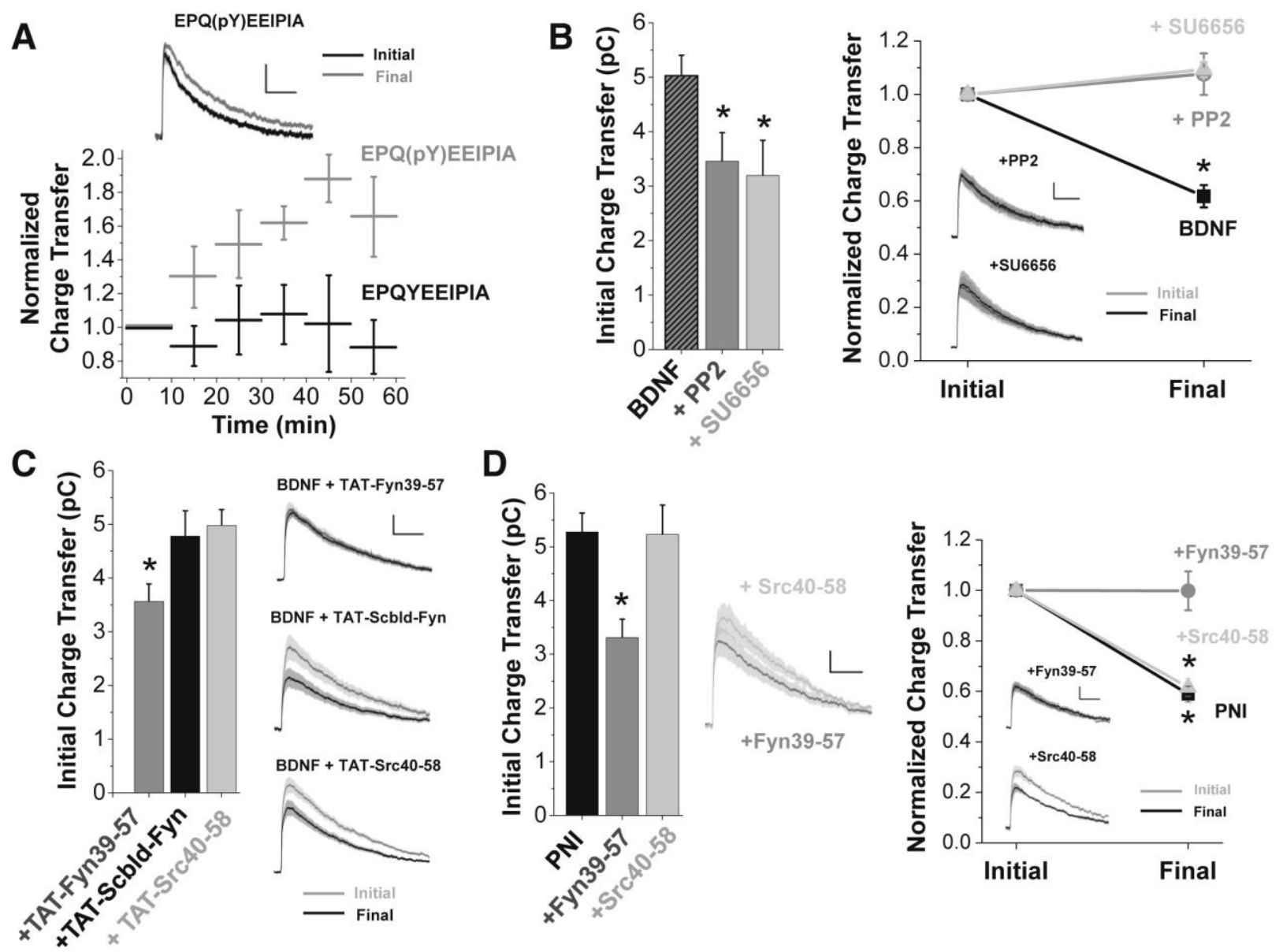

Appendix A Figure 3. The Potentiation of NMDAR mEPSCs by BDNF/ PNI Requires Fyn but Not Src. (A)

Time course of NMDAR charge transfer with either $1 \mathrm{mM}$ EPQ(pY)EEIPIA (gray, $\mathrm{n}=4$ ) or $1 \mathrm{mM}$ EPQYEEIPIA (black, $\mathrm{n}=3$ ) in the intracellular pipette solution. Inset, average mEPSC traces during initial (black) and final (gray, 40+ min) recording periods, with $1 \mathrm{mM} \mathrm{EPQ(pY)EEIPIA} \mathrm{in} \mathrm{the} \mathrm{intracellular} \mathrm{solution.} \mathrm{(B)} \mathrm{Left:} \mathrm{plot} \mathrm{of} \mathrm{initial}$ NMDAR charge transfer for slices pre-treated with BDNF (shaded, cf. Figure 3A), BDNF and $1 \mu \mathrm{M}$ PP2 (gray, $\mathrm{n}=$ 6), or BDNF and $10 \mu \mathrm{M}$ SU6656 (light gray, $\mathrm{n}=5$ ). ${ }^{*} \mathrm{p}<0.05$ compared to BDNF pre-treatment alone. Right: plot of normalized charge transfer values for the same pre-treatment conditions as left, with inset average mEPSC traces for pre-treatment with BDNF and PP2 (top) or BDNF and SU6656 (bottom). ${ }^{*} \mathrm{p}<0.05$ compared to initial charge transfer value for given treatment. (C) Left: plot of initial NMDAR charge transfer for slices pre-treated with BDNF and $10 \mu \mathrm{M}$ TAT-Fyn39-57 (gray, $\mathrm{n}=9$ ), BDNF and $10 \mu \mathrm{M}$ TAT-Scbld-Fyn (black, $\mathrm{n}=7$ ), or BDNF and $10 \mu \mathrm{M}$ TAT-Src40-58 (light gray, $\mathrm{n}=9$ ). ${ }^{*} \mathrm{p}<0.05$ compared to BDNF pre-treatment alone. Right: average mEPSC traces during initial (gray) and final (black) recording periods for the pre-treatment conditions to the left. (D) Left: plot of 
initial NMDAR charge transfer from PNI rats during control recordings (black, $\mathrm{n}=9$ ) or recordings with intracellular dialysis of Fyn39-57 (gray, $\mathrm{n}=6$ ) or Src40-58 (light gray, $\mathrm{n}=7$ ). Middle: average initial mEPSCs of PNI rats with intracellular Src40-58 (light gray) or intracellular Fyn39-57 (gray). Right: plot of normalized charge transfer values during initial and final recording periods for the same treatment conditions in PNI rats as left, with inset average mEPSC traces. Scale bars, $100 \mathrm{~ms}$ (x axes); 5 pA (y axes). All current traces and plots are represented as means \pm SEM.

\section{Potentiation of NMDAR mEPSCs by BDNF Requires Fyn Kinase Activity}

As upregulation of NMDARs by Src family protein tyrosine kinases (Wang and Salter, 1994) is implicated in models of pain hypersensitivity (Bourinet et al., 2014), we investigated whether Src family kinases (SFKs) participate in the BDNF-mediated potentiation of NMDAR mEPSCs in lamina I neurons. First, we found that the phosphopeptide SFK activator EPQ(pY)EEIPIA ( $1 \mathrm{mM}, \mathrm{n}=4$ neurons, $\mathrm{p}=0.0056)$, but not the inactive EPQYEEIPIA ( $1 \mathrm{mM}$, $\mathrm{n}=3$ neurons, $\mathrm{p}=0.47$ ), caused a progressive increase in NMDAR charge transfer when administered intracellularly through the patch pipette (Figure 3A). Thus, in lamina I neurons synaptic NMDAR currents can be upregulated by activating SFKs.

Next, we tested for SFK involvement in BDNF-mediated potentiation of NMDAR mEPSCs. We found that the BDNF-induced increase in NMDAR charge transfer was prevented (Figure 3B, left) by pre-administering the SFK inhibitor PP2 $(1 \mu \mathrm{M} ; \mathrm{n}=6$ neurons). Pre-treating with PP2 also precluded the in-recording decline in NMDAR charge transfer normally observed in neurons pre-treated with BDNF without PP2 (Figure 3B, right). Administering PP2 acutely to spinal slices from naive animals had no effect on NMDAR charge transfer $(n=4$ neurons, $p=$ 0.46; Supplementary Figure 1), implying that PP2 does not directly inhibit NMDARs. Also, the lack of effect of PP2 on NMDAR mEPSCs indicates that in lamina I neurons under basal 
conditions there is no ongoing upregulation of NMDAR mEPSCs by SFKs. The effects of PP2 on BDNF potentiation of NMDARs were mimicked by a structurally distinct SFK inhibitor, SU6656 (10 $\mu \mathrm{M} ; \mathrm{n}=5$ neurons; Figure 3B $)$. Thus, we conclude that SFK activity is necessary for the BDNF-mediated potentiation of NMDAR mEPSCs in lamina I neurons.

Because two individual SFK members, Src and Fyn, are implicated in chronic pain hypersensitivity (Abe et al., 2005; Liu et al., 2008), we tested whether Src or Fyn is required for the potentiation of NMDAR mEPSCs by BDNF. We utilized membrane-permeable TAT-fusion peptide inhibitors that differentially block the upregulation of NMDAR currents by Src kinase itself, TAT-Src40-58 (Liu et al., 2008), or by Fyn kinase, TAT-Fyn39-57 (Yang et al., 2012). We examined the effects of the TAT peptides $(10 \mu \mathrm{M})$ on the BDNF-induced potentiation in lamina I neurons from naive rats. Pre-treating with TAT-Fyn39-57 together with BDNF ( $\mathrm{n}=9$ neurons) prevented the potentiation of NMDAR mEPSCs (Figure 3C) and the subsequent decline in NMDAR mEPSCs (Figure 3C) observed in neurons pre-treated with BDNF alone. However, pretreatment with a TAT-fusion peptide in which the Fyn39-57 sequence was scrambled (TATScbld-Fyn, $\mathrm{n}=7$ neurons) did not prevent the BDNF potentiation: the NMDAR charge transfer in neurons treated with TAT-Scbld-Fyn was not different from that in neurons treated with BDNF alone and the characteristic in-recording decline in the NMDAR mEPSCs was observed (Figure 3C). In contrast to TAT-Fyn39-57, TAT-Src40-58 ( $\mathrm{n}=9$ neurons) did not prevent the BDNF-induced increase in NMDAR charge transfer nor the subsequent in-recording decline in the NMDAR mEPSCs (Figure 3C).

As these findings indicate that the potentiation of NMDAR mEPSCs by BDNF depends upon Fyn kinase but not Src kinase, we tested whether Fyn, but not Src, is required for the potentiation of NMDAR mEPSCs following PNI. In these experiments, we utilized membrane- 
impermeant versions of the peptides - Fyn39-57 and Src40-58 (30 $\mu \mathrm{g} / \mathrm{mL})$-which were administered directly into the neurons during recordings to allow us to determine whether Fyn, or Src, was required in the neuron under study. We found in recordings from PNI animals that the NMDAR charge transfer in neurons in which Fyn39-57 was administered intracellularly $(\mathrm{n}=$ 6 neurons) was significantly less than that in neurons without Fyn39-57 (Figure 3D, left). In addition, there was no in-recording decline in NMDAR mEPSCs in neurons recorded with Fyn39-57 (Figure 3D, right). Moreover, after PNI the NMDAR charge transfer in neurons recorded with Fyn39-57 was not different from that in neurons from naive rats not receiving Fyn39-57 (PNI + Fyn $3.31 \pm 0.34$ pC, $n=6$ neurons; naive $3.20 \pm 0.22$ pC, $n=20$ neurons, $\mathrm{p}=$ 0.81). The effects of Fyn39-57 on NMDAR charge transfer in neurons from PNI rats were not produced by intracellularly administering Src40-58 ( $\mathrm{n}=7$ neurons): NMDAR charge transfer and subsequent in-recording decline were not different from those in neurons without peptide administration (Figure 3D).

\section{BDNF Activates Fyn but Not Src to Drive the Phosphorylation and Potentiation of GluN2B NMDARs}

Because Fyn was required for the potentiation of synaptic NMDARs by either BDNF or nerve injury, we next tested whether activation of Fyn was sufficient to potentiate NMDARs at adult lamina I synapses. We found that activation of intracellular SFKs with EPQ(pY)EEIPIA significantly $(\mathrm{p}<0.05)$ increased NMDAR mEPSCs when Src was blocked with intracellular Src40-58 $(n=5)$ but not when Fyn was blocked with intracellular Fyn39-57 ( $n=6)($ Figure 4A). We previously found that SFKs are differentially coupled to specific NMDAR GluN2 subunits, with Src potentiating GluN2A-containing receptors and Fyn potentiating GluN2B-containing receptors (Yang et al., 2012). Here, we found that the component of NMDAR mEPSCs that was 
potentiated by BDNF had a decay time constant of $276 \mathrm{~ms}$, which is consistent with NMDARs containing GluN1 together with GluN2B (Figure 4B). We therefore tested whether GluN2A and/or GluN2B is required for the potentiation of NMDAR mEPSCs by BDNF. In slices treated with BDNF and a GluN2A selective antagonist, TCN-201 (3 $\mu \mathrm{M})$, the initial NMDAR charge transfer $(4.24 \pm 0.22 \mathrm{pC} ; \mathrm{n}=8)$ was significantly greater than slices treated with the GluN2A antagonist alone $(2.86 \pm 0.25 \mathrm{pC} ; \mathrm{n}=12 ; \mathrm{p}=0.0012)$, with the same decline in NMDAR mEPSCs observed for BDNF pre-treatment alone (Figure 4C). In contrast, treating slices with BDNF and a GluN2B selective antagonist, Ro25-6981 (1 $\mu \mathrm{M})$, prevented the potentiation and subsequent decline of NMDAR mEPSCs (Figure 4C): the initial NMDAR charge transfer was not significantly different for BDNF and Ro25-6981 (1.54 $\pm 0.22 \mathrm{pC} ; \mathrm{n}=6)$ compared to treatment with Ro25-6981 alone $(1.23 \pm 0.17 \mathrm{pC} ; \mathrm{n}=12 ; \mathrm{p}=0.30)$. 
A

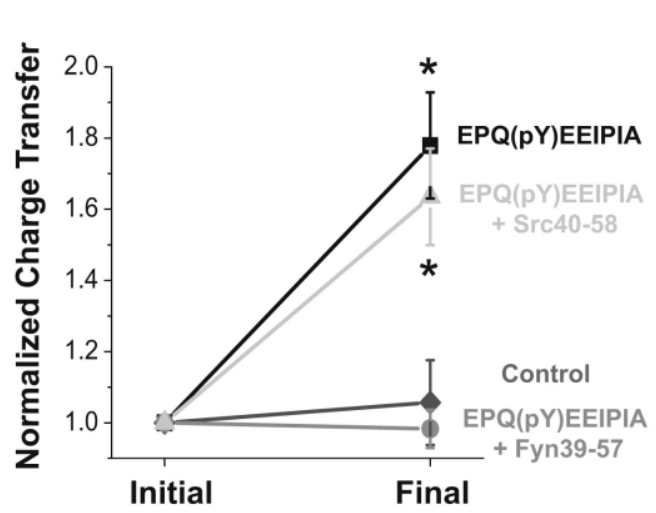

Control

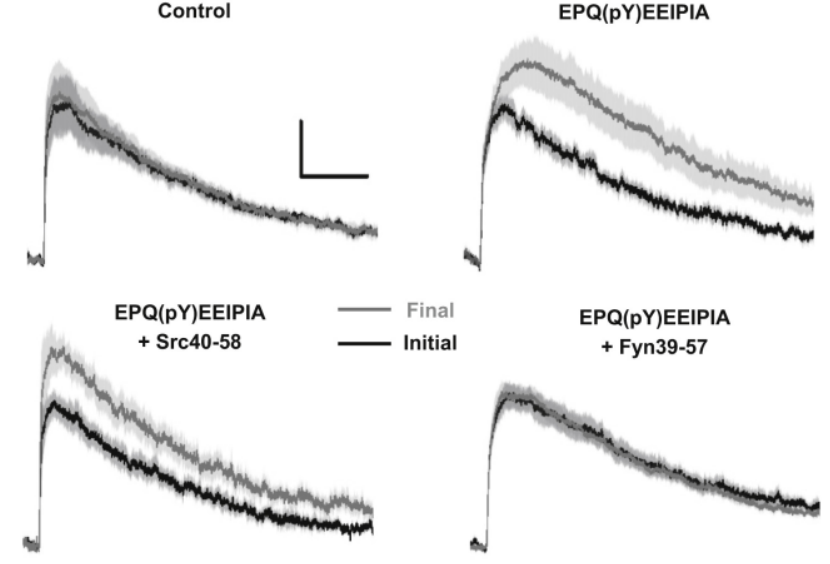

B

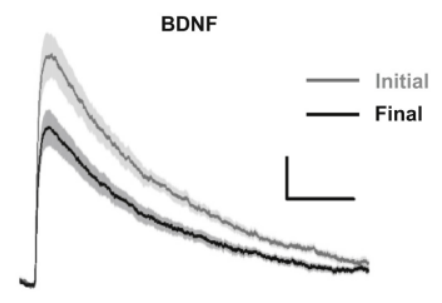

BDNF - Sensitive

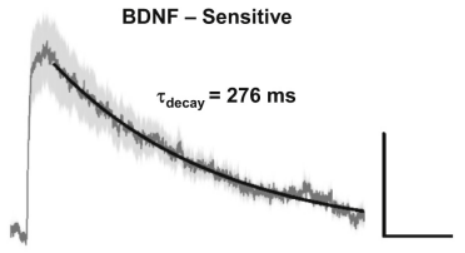

C
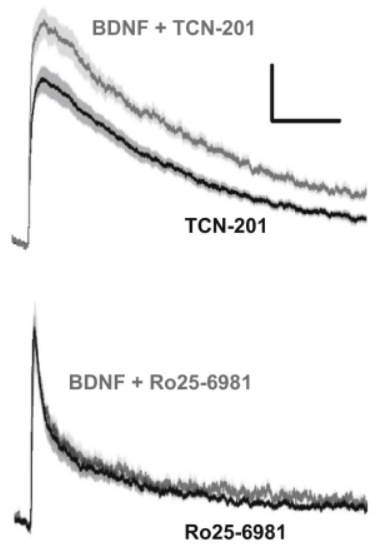

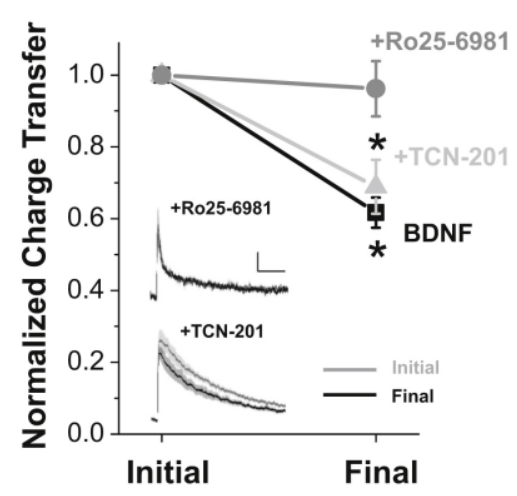

Appendix A Figure 4. Fyn but Not Src Directly Mediates the Potentiation of GluN2B-Containing NMDAR

Responses at Lamina I Synapses. (A) Plot of initial versus final NMDAR charge transfer values (left) and averaged traces (right) for recordings on naive lamina I neurons with control internal solution $(n=5)$,

EPQ(pY)EEIPIA $(n=6)$, EPQ(pY)EEIPIA + Src40-58 $(n=5)$, or EPQ(pY)EEIPIA + Fyn39-57 $(n=6)$ in the patch pipette. (B) Subtraction of final mEPSCs from initial mEPSCs in BDNF pre-treated slices yields a BDNF-sensitive mEPSC component $(n=10)$. (C) Left: average initial mEPSC traces from slices pre-treated with TCN-201 $(n=12)$ versus BDNF and TCN-201 ( $\mathrm{n}=8)$ (top) or Ro25-6981 ( $\mathrm{n}=12)$ versus BDNF and Ro25-6981 ( $\mathrm{n}=6)$ (bottom). Right: plot of normalized charge transfer values during initial and final recording periods for slices pre-treated with BDNF $(n=10)$, BDNF and Ro25-6981 ( $n=4)$, or BDNF and TCN-201 $(n=6)$, with inset average mEPSC traces. ${ }^{*} \mathrm{p}<0.05$ compared to initial charge transfer value for given treatment. Scale bars, $100 \mathrm{~ms}$ (x axes); 5 pA (y axes). All current traces and plots are represented as means \pm SEM. 
Next, we used biochemical approaches to investigate whether BDNF treatment altered Fyn-meditated phosphorylation and synaptic localization of NMDAR subunits in the superficial dorsal horn (SDH). We first immunoprecipitated Fyn or Src from the synaptosomal fraction of SDH tissue, followed by detection with phospho-SFK antibodies. Phosphorylation of two tyrosine residues regulate the activity of these kinases, with phosphorylation of $\mathrm{Tyr}^{20}$ activating Fyn (Tyr ${ }^{416}$ in Src) and phosphorylation of Tyr ${ }^{531}$ inhibiting Fyn (Tyr ${ }^{527}$ in Src) (Salter and Kalia, 2004; Ingley, 2008). Treatment of SDH tissue with BDNF led to a significant increase in Fyn phosphorylation at $\operatorname{Tyr}^{20}(p=0.012)$ and a concomitant decrease at $\operatorname{Tyr}^{531}(p=0.014)$, indicating increased activation of Fyn at SDH synapses ( $\mathrm{n}=6$ slices, Figure 5A). In contrast, we found no changes in $\operatorname{Src}$ phosphorylation at either $\operatorname{Tyr}^{116}(\mathrm{p}=0.58)$ or $\operatorname{Tyr}^{527}(\mathrm{p}=0.48)(\mathrm{n}=6$, Figure 5A) as well as no change in total Src or Fyn protein levels (Figure 5B, data not shown). 
A
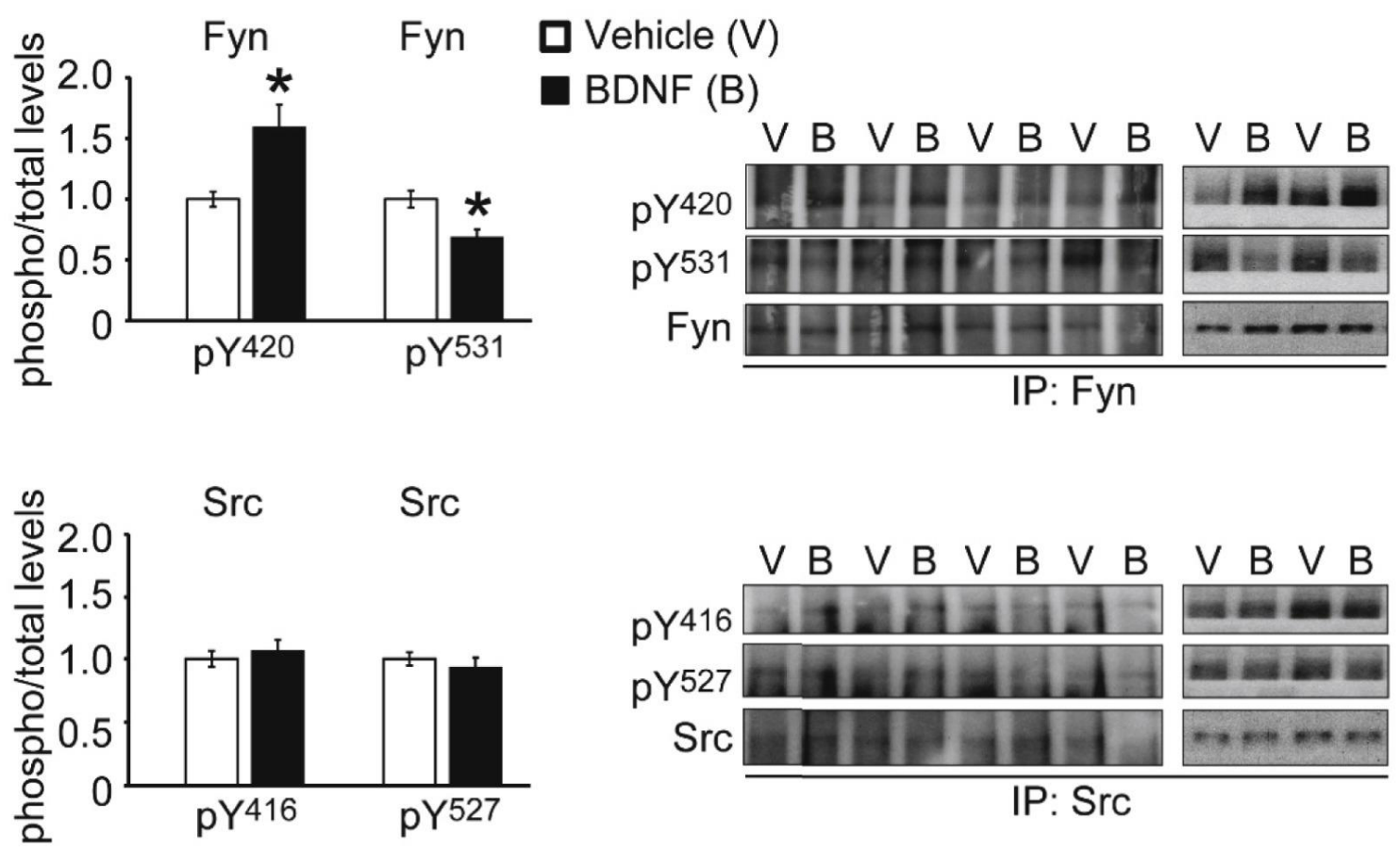

B
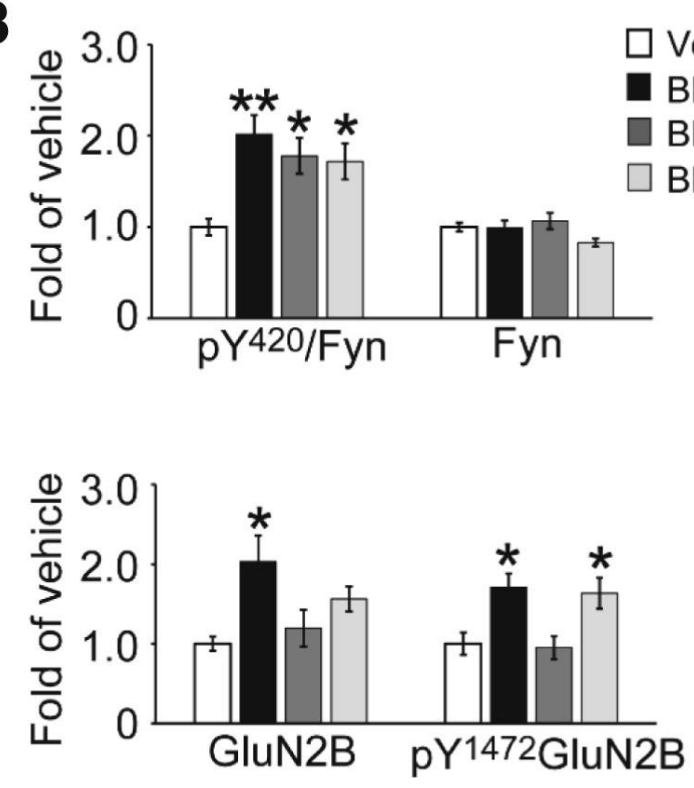

Vehicle (V)

BDNF (B)

BDNF+TAT-Fyn 39-57 (B+Fyn)

BDNF+TAT-Scbld Fyn (B+sc-Fyn)
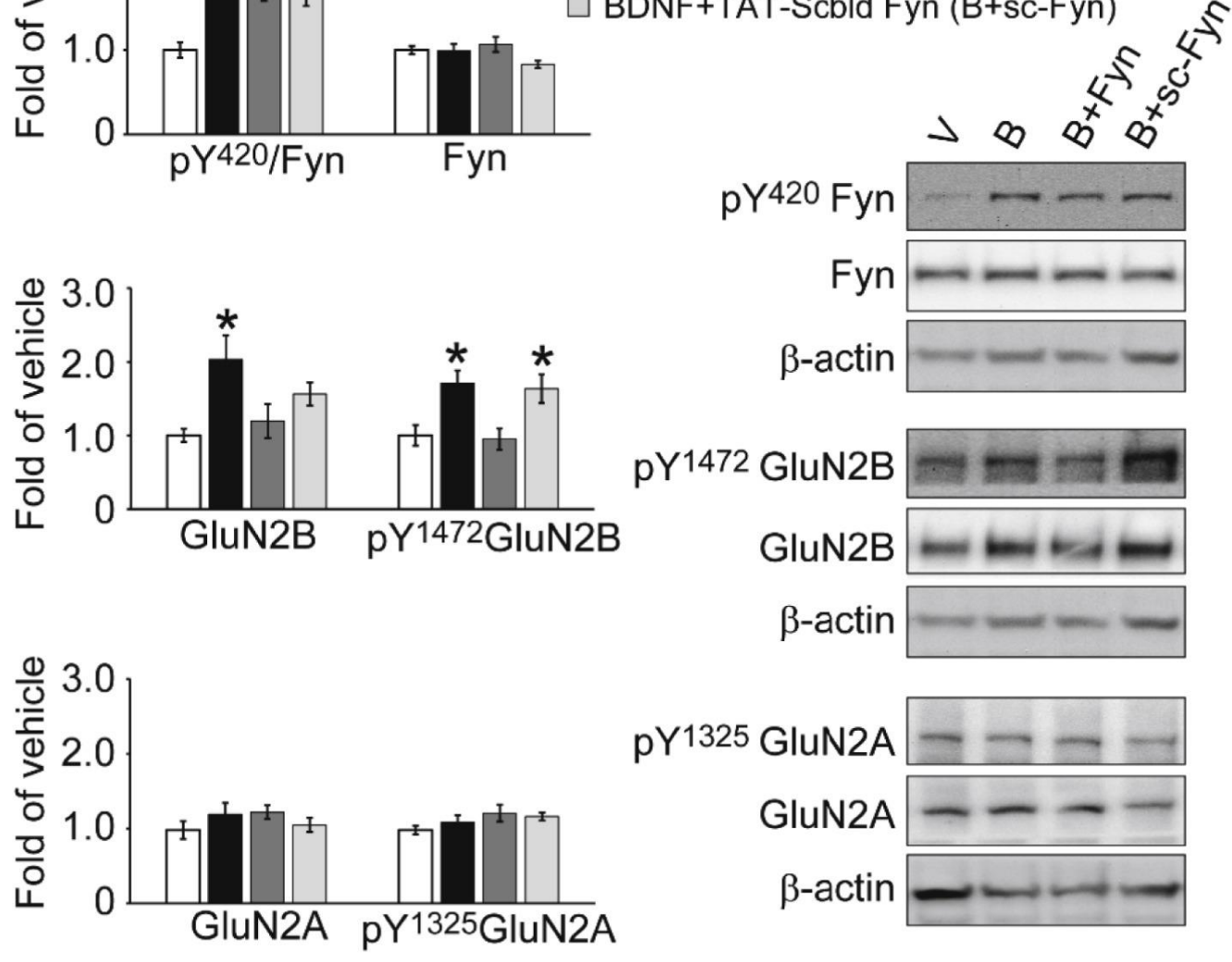

pY1325 GluN2A

GluN2A

$\beta$-actin 
Appendix A Figure 5. BDNF Activates Fyn but Not Src to Drive Trafficking and Phosphorylation of GluN2B NMDARs Selectively at Superficial Dorsal Horn Synapses. (A) Fyn or Src were immunoprecipitated from synaptic fractions of SDH. Western blots using phospho-specific antibodies (right), with phosphorylation levels normalized to total protein levels (left). ${ }^{*} \mathrm{p}<0.05, \mathrm{n}=6$. (B) Western blots from synaptic fractions of SDH treated with either saline, BDNF, BDNF and TAT-Fyn39-57, or BDNF and TAT-Scbld-Fyn for 70 min. Blots $(\mathrm{n}=8$, right) were probed with the antibodies indicated. Phospho-Fyn was normalized to Fyn and then to beta-actin as a loading control while all other targets were normalized to beta-actin directly (left). ${ }^{*} p<0.05$ using one-way ANOVA with Tukey's post hoc test. All plots are represented as means \pm SEM.

We also tested the phospho-SFK antibody without immunoprecipitation and found a significant increase in SFK phosphorylation at this Tyr residue $\left(\mathrm{Tyr}^{420} / \mathrm{Tyr}^{416}\right.$, Figure 5B, top; Supplementary Data 1). As phosphorylation of Src was not altered by BDNF (Figure 5A), we conclude that this likely reflects an increase in Fyn phosphorylation at $\mathrm{Tyr}^{420}$. Activated Fyn potentiates GluN2B-containing NMDARs by phosphorylating Tyr ${ }^{1472}$ on the GluN2B subunit (Salter and Kalia, 2004). Consistent with our findings of a Fyn-dependent potentiation of GluN2B-mediated synaptic responses, we found increased phosphorylation of GluN2B at $\mathrm{Tyr}^{1472}$ upon BDNF treatment $(\mathrm{p}=0.023)$ as well as increased localization of GluN2B protein in SDH synaptosomes $(p=0.012)(n=8$ slices, Figure $5 B$, middle). In contrast, we found that BDNF treatment did not alter the phosphorylation of GluN2A at an analogous residue $\left(\operatorname{Tyr}^{1325}\right)$ $(p=0.84)$ nor total GluN2A levels $(p=0.59)(n=8$ slices, Figure 5B, bottom $)$. Moreover, BDNF treatment had no effect on Fyn or GluN2B phosphorylation or expression in synaptosomes from deep dorsal horn and ventral horn tissue (Supplementary Figure 2A) nor in SDH total homogenates (Supplementary Figure 2B). 
To test whether Fyn kinase was required for the BDNF-mediated increase in GluN2B phosphorylation and synaptic localization, we treated SDH tissue with BDNF and TAT-Fyn3957. We found that co-administration of TAT-Fyn39-57 abolished the increase in phosphorylation of GluN2B at $\operatorname{Tyr}^{1472}(p=0.99)$ and the increase in total synaptosomal GluN2B $(p=0.93)$ observed for BDNF alone ( $\mathrm{n}=8$ slices, Figure 5B, middle). In slices treated with TAT-ScbldFyn and BDNF, we found an increase in phosphorylated GluN2B $(p=0.049)$ and total GluN2B $(p=0.28)$ compared to control-treated slices, albeit below the significance threshold for the latter ( $\mathrm{n}=8$ slices). Importantly, the increase in phosphorylation of Fyn at $\mathrm{Tyr}^{420}$ was preserved when slices were treated with BDNF and TAT-Fyn39-57 ( $n=8$ slices, $p=0.025$; Figure 5B, top), showing that TAT-Fyn39-57 blocks Fyn-GluN2B interactions rather than the regulation of Fyn activity itself. These findings together suggest that synaptic NMDAR currents in lamina I neurons are potentiated following PNI by BDNF-induced stimulation of TrkB, activation of Fyn kinase, and subsequent phosphorylation of GluN2B-containing NMDARs.

\section{BDNF-Mediated Disinhibition Is Required for the Potentiation of GluN2B NMDARs by} Fyn after PNI

BDNF is a well-known mediator of PNI-induced pain hypersensitivity via TrkB-mediated blockade of $\mathrm{KCC} 2$ and subsequent suppression of $\mathrm{GABA}_{\mathrm{A}}$ - and glycine-mediated inhibition of neurons in spinal lamina I (Beggs and Salter, 2013; Ferrini and De Koninck, 2013). Hence, we wondered whether this type of disinhibition may be required for the potentiation of NMDAR mEPSCs in lamina I neurons. As $\mathrm{GABA}_{\mathrm{A}}$ and glycine receptors are permeable to $\mathrm{HCO}_{3}{ }^{-}$, in addition to $\mathrm{Cl}^{-}$, disinhibition driven by blockade of $\mathrm{KCC} 2$ is dependent upon $\mathrm{HCO}_{3}{ }^{-}$efflux (Staley et al., 1995; Price et al., 2009) and can be reversed by lowering intracellular $\mathrm{HCO}_{3}{ }^{-}$via inhibiting carbonic anhydrase (Kaila et al., 2014). Therefore, we examined the potential 
involvement of disinhibition in the BDNF-mediated potentiation of NMDAR currents by using the membrane-permeant inhibitor of carbonic anhydrase, acetazolamide. Acetazolamide has been found to reverse depolarizing shifts in $\mathrm{GABA}_{\mathrm{A}}$-receptor currents in lamina I neurons (Ferrini and De Koninck, 2013).

Pre-treating slices from naive rats with acetazolamide $(10 \mu \mathrm{M})$ together with BDNF prevented the potentiation of NMDAR mEPSCs induced by BDNF pre-treatment alone (Figure 6A, left). Specifically, the NMDAR charge transfer in neurons pre-treated with BDNF and acetazolamide ( $3.24 \pm 0.34 \mathrm{pC}, \mathrm{n}=8$ neurons) was significantly less than that in neurons treated with BDNF alone $(p=0.0045)$ and was not significantly different from that in untreated neurons from naive rats $(3.20 \pm 0.22 \mathrm{pC}, \mathrm{n}=20$ neurons, $\mathrm{p}=0.92)$. Also, the in-recording decline of NMDAR mEPSCs, characteristic of neurons pre-treated with BDNF alone was not seen in neurons pre-treated with BDNF together with acetazolamide (Figure 6A, right). We found that pre-treating slices from naive rats with acetazolamide alone had no significant effect on NMDAR mEPSCs, suggesting that disinhibition does not regulate NMDAR currents under basal conditions (Figure 6B). However, in recordings from rats with PNI, treating slices with acetazolamide prior to recording significantly reduced the amplitude of the initial NMDAR mEPSCs to $3.94 \pm 0.46 \mathrm{pC}$ ( $\mathrm{n}=7$ neurons; $\mathrm{p}=0.035$ compared to PNI alone) - $\mathrm{a}$ level not different from that of the charge transfer in neurons from rats without PNI (Figure 6C, left, $p=$ 0.88). Acetazolamide pre-treatment also prevented the in-recording decline of NMDAR mEPSCs in rats with PNI (Figure 6C, right). It is conceivable that the effects of acetazolamide might have been due to blockade of BDNF activation of TrkB receptors. However, we found that acetazolamide $(10 \mu \mathrm{M})$ did not affect activation of TrkB by BDNF (Figure S3) and thus acetazolamide was not acting to prevent BDNF from activating TrkB. 
A

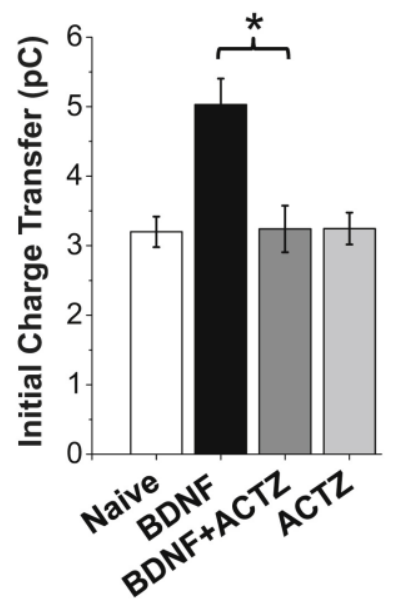

BDNF

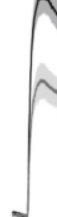

BDNF

$\int$

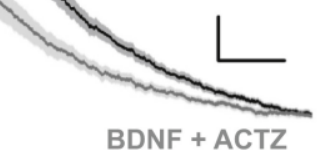

BDNF + ACTZ (Initial)

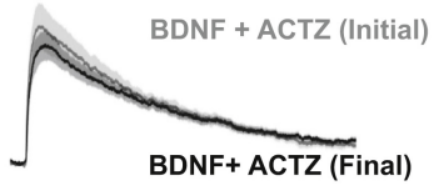

B

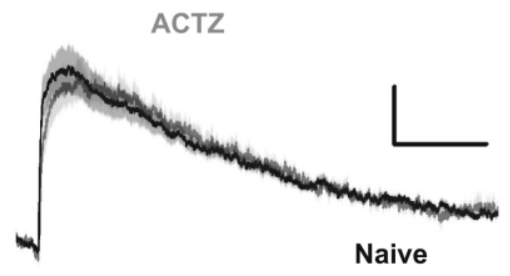

C

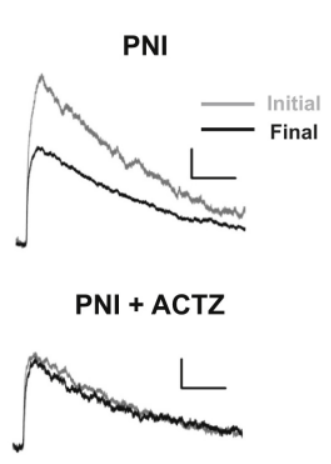

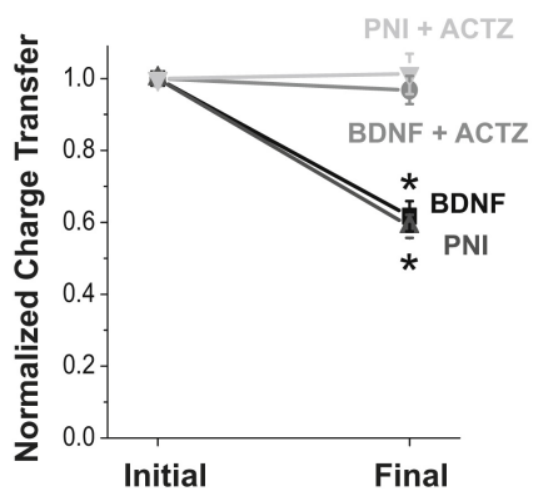

D

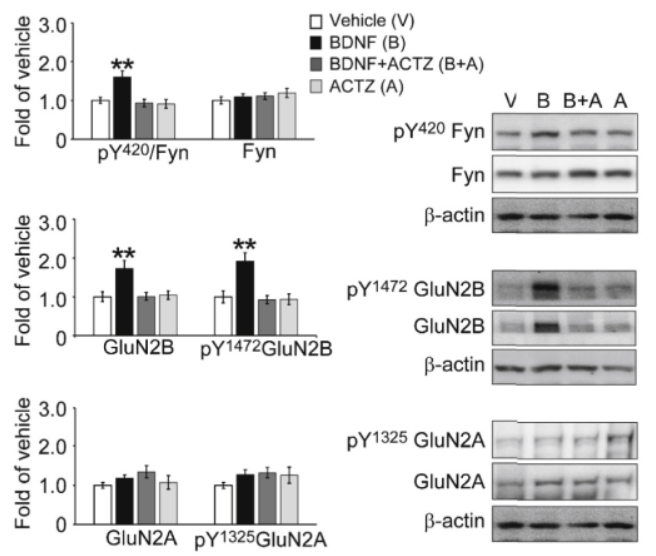

Appendix A Figure 6. The Potentiation of NMDAR mEPSCs by BDNF/PNI Requires Disinhibition. (A) Left: plot of initial NMDAR charge transfer for slices pretreated with saline (white), BDNF (black), BDNF and $10 \mu \mathrm{M}$ acetazolamide (gray), or acetazolamide alone (light gray). Right top: average initial mEPSCs from naive slices pretreated with BDNF (black) or with BDNF and $10 \mu \mathrm{M}$ acetazolamide (gray). Right bottom: average mEPSCs for slices pre-treated with BDNF and $10 \mu \mathrm{M}$ acetazolamide, during initial (gray) and final (black) recording periods. (B) Initial average mEPSCs for slices pre-treated with $10 \mu \mathrm{M}$ acetazolamide $(\mathrm{n}=7)$ compared to saline pre-treated slices $(\mathrm{n}=20)$. (C) Left: representative mEPSC traces during initial (gray) and final (black) recording periods from lamina I neurons of the same PNI rat whereby one slice was pre-treated with control solution (top) and a separate slice was pre-treated with $10 \mu \mathrm{M}$ acetazolamide for $72 \mathrm{~min}$ (bottom). Right: plot of normalized charge transfer values during initial and final recording periods. ${ }^{*} \mathrm{p}<0.05$ compared to initial charge transfer value for a given treatment. Scale bars, $100 \mathrm{~ms}$ (x axes); 5 pA (y axes). (D) Western blots from synaptic fraction of SDH treated with either saline, BDNF, BDNF and acetazolamide, or acetazolamide alone. Statistical significance $\left({ }^{*} \mathrm{p}<0.05, \mathrm{n}=8\right.$ for all 
treatments) was determined using one-way ANOVA with Tukey's post hoc test. All current traces and plots are represented as means $\pm \mathrm{SEM}$.

In contrast to SDH tissue treated with BDNF alone, treatment of slices with BDNF and acetazolamide prevented the BDNF-dependent increase in phosphorylation of Fyn at $\operatorname{Tyr}^{420}(\mathrm{p}=0.97)$ as well as the increase in phosphorylated GluN2B $\left(\mathrm{pY}^{1472}, \mathrm{p}=0.99\right)$ and total GluN2B $(\mathrm{p}=0.99)$ in SDH synaptosomes $(\mathrm{n}=8$ slices, Figure 6D; Data S1). Moreover, treatment of slices with acetazolamide alone had no effect on Fyn phosphorylation at $\operatorname{Tyr}^{420}(\mathrm{p}=$ 0.94), GluN2B phosphorylation at $\operatorname{Tyr}^{1472}(\mathrm{p}=0.99)$, or total synaptic GluN2B levels $(\mathrm{p}=0.99$, $\mathrm{n}=8$ slices, Figure 6D), suggesting there is no basal level of disinhibition within the naive SDH. Similarly, treatment with either acetazolamide or acetazolamide and BDNF had no effect on levels of $\mathrm{pY}^{420}$ Fyn, $\mathrm{pY} \mathrm{Y}^{1472}$ GluN2B, or GluN2B at deep dorsal horn and ventral horn synapses (Figure S4). Thus, we conclude that BDNF-induced disinhibition is necessary for the Fynmediated potentiation of GluN2B-containing NMDARs produced by exogenous BDNF or by PNI.

\section{KCC2 Downregulation Primes Lamina I Neurons for NMDAR Potentiation by BDNF}

To test whether the disinhibition mediated by suppressing KCC2 is sufficient to potentiate synaptic NMDAR responses, we used the pharmacological inhibitor of $\mathrm{KCC} 2$, VU0240551 (Delpire et al., 2009). We applied VU0240551 to spinal slices from naive rats at a concentration $(50 \mu \mathrm{M})$ known to increase intracellular $\left[\mathrm{Cl}^{-}\right]$and decrease GABA-mediated inhibition (Doyon et al., 2011). Pre-treating slices with VU0240551 ( $\mathrm{n}=12$ neurons) did not significantly alter the initial NMDAR mEPSCs: charge transfer $(3.63 \pm 0.31 \mathrm{pC}, \mathrm{p}=0.25)$ was 
not different from slices with no VU0240551 pre-treatment (Figure 7A, left). Also, VU0240551treated neurons did not display any time-dependent change in NMDAR charge transfer during recordings (Figure 7A, right).

A

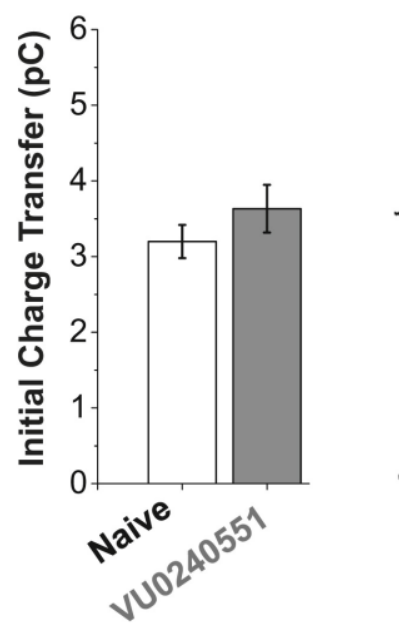

B

VU0240551 Pretreatment

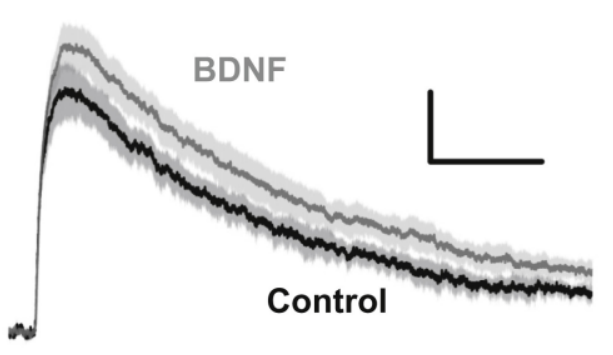

Naive

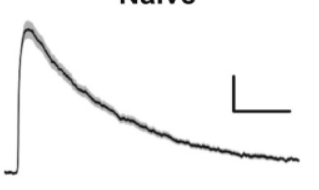

VU0240551
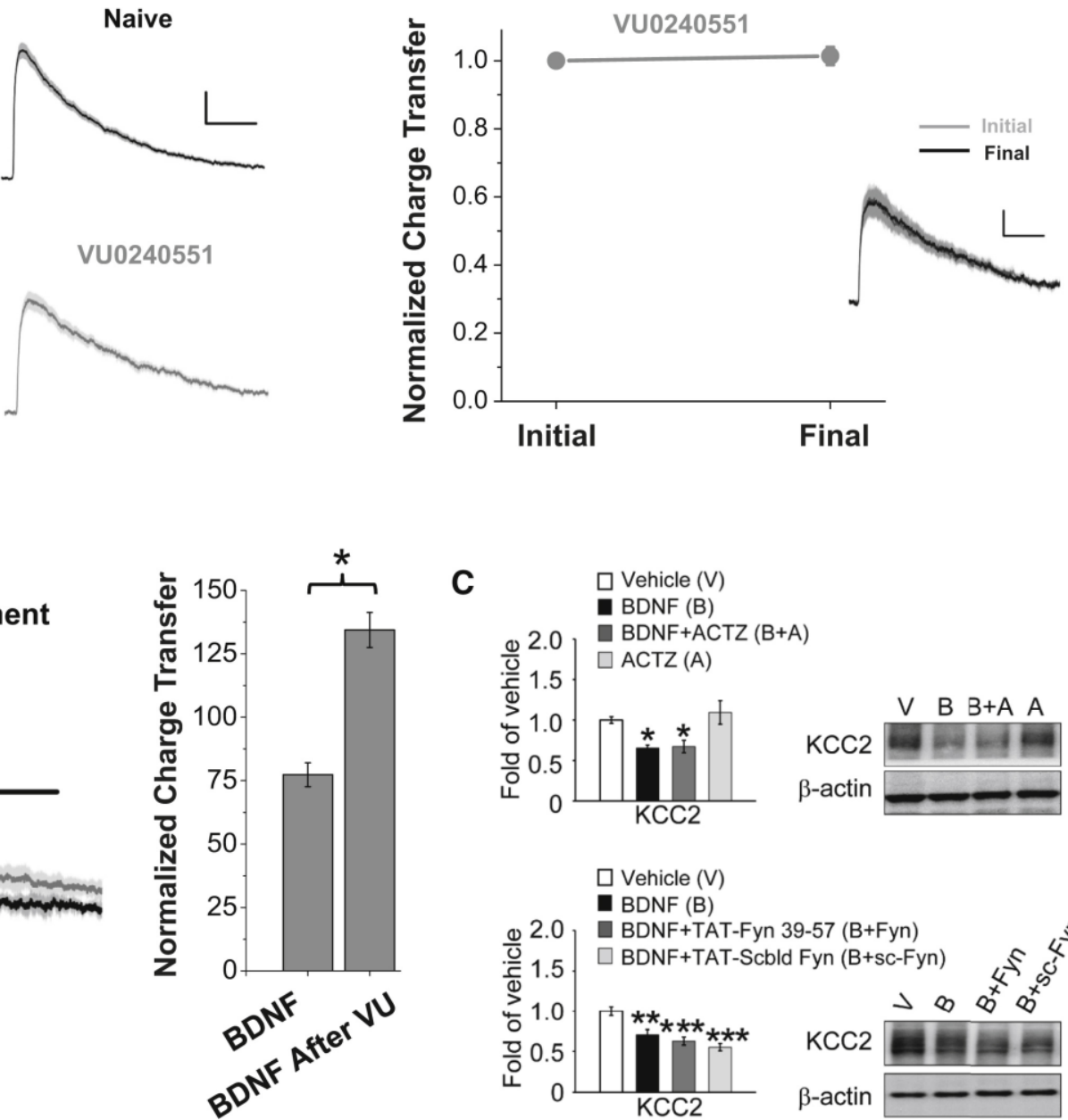
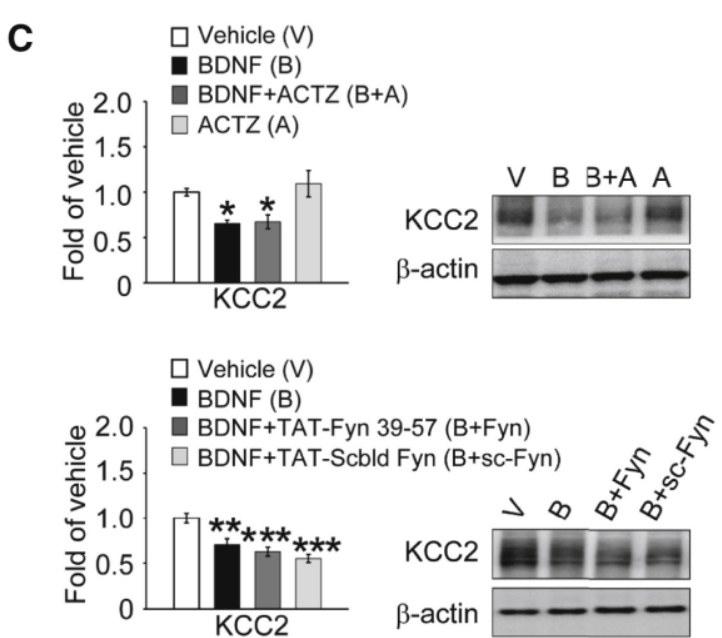

Appendix A Figure 7. Downregulation of KCC2 Activity Gates Potentiation of NMDAR mEPSCs by

Exogenous BDNF. (A) Average initial mEPSC traces (middle) and associated NMDAR charge transfer values (left) in control-treated slices (white) or slices pre-treated with $50 \mu \mathrm{M}$ VU0240551 for $80 \pm 3 \mathrm{~min}$ (gray, $\mathrm{n}=12$ ). Right: average mEPSC traces (inset) and normalized charge transfer values for slices pre-treated with VU0240551 during initial and final recording periods. (B) Left: average mEPSC traces from slices pre-treated VU0240551 for 60+ min $(\mathrm{n}=5)$. Following a control period of recording (black trace), $100 \mathrm{ng} / \mathrm{mL}$ BDNF was acutely perfused during recording for $10+\min$ (gray trace). Right: NMDAR charge transfer during acute administration of BDNF 
normalized to a control recording period for untreated (left bar) and VU0240551 pre-treated (right bar) slices. ${ }^{*} \mathrm{p}<$ 0.05. Scale bars, $100 \mathrm{~ms}$ (x axes); $5 \mathrm{pA}$ (y axes). (C) Synaptic fraction from SDH treated with the indicated agents for $70 \mathrm{~min}$ KCC2 protein levels were normalized to beta-actin as a loading control (left). Statistical significance $\left({ }^{*} \mathrm{p}<0.05, \mathrm{n}=8\right)$ was determined one-way ANOVA with Tukey's post hoc test. All current traces and plots are represented as means $\pm \mathrm{SEM}$.

As suppressing KCC2 function did not cause a potentiation of synaptic NMDAR responses per se, we asked whether this form of disinhibition may gate the potentiation of NMDAR mEPSCs by BDNF. In lamina I neurons from vehicle-treated slices of naive rats, bathapplying exogenous BDNF (100 ng/mL) after the start of whole-cell recording failed to produce potentiation of NMDAR mEPSCs and instead caused a small inhibition of synaptic NMDAR responses $(n=11$ neurons, Figure 7B). However, in recordings from neurons in spinal slices that had been pre-treated with VU0240551, bath-applying BDNF (100 ng/mL) during the recording significantly increased NMDAR charge transfer $(n=5$ neurons, $p=0.0072$, Figure 7B). In biochemical experiments, we found that BDNF pre-treatment reduced the level of KCC2 in the SDH ( $\mathrm{n}=8$ slices, $\mathrm{p}=0.042 / 0.0034$, Figure 7C, top/bottom; Data S1) but not in the deep dorsal horn and ventral horn (Supplementary Figure 4). Moreover, this reduction was not abolished by pre-treatment of BDNF with acetazolamide $(n=8$ slice, $p=0.047$, Figure $7 C$, top) nor TAT-Fyn39-57 ( $\mathrm{n}=8$ slices, $\mathrm{p}=0.00020$, Figure 7C, bottom). As acetazolamide and TATFyn39-57 did block the phosphorylation and potentiation of GluN2B NMDARs by BDNF, this finding further supports that KCC2 downregulation alone is not sufficient to induce potentiation of NMDARs. Thus, we conclude that not only does BDNF suppress KCC2-dependent inhibition 
but that the resultant disinhibition permits the action of this neurotrophin to potentiate synaptic GluN2B NMDARs via Fyn activation in lamina I neurons.

KCC2 level is regulated by several mechanisms following induction of neuronal activity (Lee et al., 2011; Chamma et al., 2013). To investigate whether calpain-mediated cleavage may be responsible for the loss of KCC2 upon BDNF treatment, we measured levels of fodrin-a well-characterized calpain substrate (Siman et al., 1984) — as a readout for calpain activity. We

found no changes in full-length $(250 \mathrm{kDa})$ or calpain-cleaved $(150 / 145 \mathrm{kDa})$ fragments of fodrin after BDNF treatment in SDH synaptosomes (Supplementary Figure 5A) or SDH homogenates (Supplementary Figure 5B), suggesting calpain was not activated by BDNF under these conditions.

\section{Raising Intracellular Ca2+ Permits BDNF-Induced Potentiation of NMDAR mEPSCs}

We next investigated how disinhibition may gate subsequent potentiation of synaptic NMDARs by BDNF. The acetazolamide experiments suggest that a rise in intracellular $\mathrm{Cl}^{-}$does not permit the potentiation of synaptic NMDARs by the TrkB/Fyn pathway. As disinhibition also induces an increase in intracellular $\left[\mathrm{Ca}^{2+}\right]\left(\left[\mathrm{Ca}^{2+}\right]_{\mathrm{i}}\right)$ in spinal neurons (Darbon et al., 2002), it is possible that a rise in $\left[\mathrm{Ca}^{2+}\right]_{\text {i }}$ gates the effect of BDNF on NMDARs at lamina I synapses. Hence, we studied NMDAR mEPSCs in lamina I neurons of naive rats under conditions that permit, or prevent, an increase in $\left[\mathrm{Ca}^{2+}\right]_{\mathrm{i}}$. To facilitate increasing $\left[\mathrm{Ca}^{2+}\right]_{\mathrm{i}}$ we used an extracellular recording solution lacking $\mathrm{Cd}^{2+}$ and $\mathrm{Mg}^{2+}$ to permit and enhance calcium influx through voltagegated calcium channels and NMDARs, respectively. Moreover, we reduced intracellular $\mathrm{Ca}^{2+}$ buffering by using $100 \mu \mathrm{M}$ EGTA in the intracellular pipette solution. Under these recording conditions, NMDAR mEPSCs at $-40 \mathrm{mV}$ remained constant during $20 \mathrm{~min}$ of wholecell recording: the charge transfer of inward NMDAR mEPSCs was not significantly different 
between the 10-15 min recording period $(\mathrm{Q}=-0.45 \pm 0.11, \mathrm{n}=6)$ and the $15-20$ min recording period $(\mathrm{Q}=-0.44 \pm 0.11 \mathrm{pC}, \mathrm{n}=6, \mathrm{p}=0.75$; Supplementary Figure 6). However, we found under these recording conditions that administrating exogenous BDNF induced an increase in NMDAR mEPSCs (Supplementary Figure 7): average NMDAR charge transfer significantly increased from $-0.45 \pm 0.11 \mathrm{pC}$ during baseline recording to $-0.83 \pm 0.16 \mathrm{pC}$ during administration of BDNF ( $n=6$ neurons, $p=0.0021$, Supplementary Figure 7B, left). This BDNF-induced increase in NMDAR charge transfer was not observed in neurons where we greatly increased intracellular $\mathrm{Ca}^{2+}$ buffering by replacing EGTA $(100 \mu \mathrm{M})$ with the more rapid buffer BAPTA at the higher concentration of $10 \mathrm{mM}$ (Supplementary Figure 7B, right; baseline $\mathrm{Q}=-0.45 \pm 0.10 \mathrm{pC}, \mathrm{BDNF} \mathrm{Q}=-0.51 \pm 0.10 \mathrm{pC}, \mathrm{n}=5$ ). Thus, an increase in $\left[\mathrm{Ca}^{2+}\right]_{\mathrm{i}}$ within the lamina I neuron recorded is permissive for the subsequent potentiation of synaptic NMDARs by BDNF.

\section{Discussion}

Given the importance of lamina I neurons to the integration and output of spinal nociceptive signals (Braz et al., 2014; Prescott et al., 2014), we investigated whether glutamatergic responses at lamina I synapses are altered in a model of neuropathic pain. We found that unitary synaptic NMDAR currents in lamina I spinal neurons are potentiated following traumatic peripheral nerve injury. The AMPAR component of the mEPSCs, by contrast, was unchanged by nerve injury indicating that the quantal release of glutamate onto lamina I neurons is not altered by PNI, consistent with previous reports (Chen et al., 2014a; Li et al., 2016). Rather, our findings indicate that the increase in the NMDAR component of the mEPSCs in the nerve-injured state is mediated post-synaptically by enhancement of NMDARs in the lamina I neurons themselves. The most parsimonious signalling cascade suggested by our results is that activation of TrkB by 
its cognate ligand BDNF leads to activation of Fyn kinase and potentiation of GluN2Bcontaining synaptic NMDARs. Unexpectedly, the potentiation of NMDARs by the BDNF-TrkBFyn signalling pathway requires prior and sustained KCC2-dependent disinhibition, as we found that blocking disinhibition with acetazolamide abolished the enhancement of lamina I NMDARs by exogenously administered BDNF or nerve injury. Thus, taking our evidence together suggests that within individual lamina I neurons, BDNF-mediated disinhibition leads to a post-synaptic rise in $\left[\mathrm{Ca}^{2+}\right]_{i}$ that gates potentiation of synaptic GluN2B NMDARs, by the TrkB-Fyn signalling pathway, in a feed-forward cycle that is activated by nerve injury.

Our findings indicate that ongoing release of BDNF and continuous activation of TrkB-Fyn signalling is required to maintain the basal potentiation of NMDAR responses at lamina I synapses. Specifically, we found that NMDAR mEPSCs in lamina I neurons from animals with PNI were robustly potentiated even several hours after sectioning — potentiation that was reversed by acute blockade of TrkB, Fyn, or disinhibition. From these findings, we conclude that following nerve injury, a BDNF-mediated signalling cascade that includes spinal disinhibition and activation of TrkB and Fyn within individual lamina I neurons is being continuously activated and is required for basal NMDAR potentiation. Thus, the potentiation of NMDARs at lamina I synapses is not an event induced by PNI and maintained independently, like activityinduced long-term potentiation. A form of long-term potentiation has been shown at lamina I synapses following peripheral inflammation (Ikeda et al., 2006), and it is possible that the basal potentiation of synaptic NMDARs observed here can lead to a facilitation of synaptic AMPAR responses following a train of afferent firing. However, unlike canonical long-term potentiation, the basal potentiation of synaptic NMDARs in lamina I neurons is maintained by ongoing signalling. This conclusion is consistent with the behavioural sensitization in PNI models that is 
reversed by interfering with specific elements of the signalling cascade. For example, PNIinduced behavioural sensitization is reversed by BDNF-TrkB blockers (Coull et al., 2005; Wang et al., 2009), NMDAR antagonists (Bourinet et al., 2014), SFK inhibitors (Peng et al., 2010), and blockers of KCC2-dependent spinal disinhibition (Asiedu et al., 2010; Gagnon et al., 2013; Li et al., 2016).

Potentiation of NMDAR by SFKs is known to depend upon tyrosine residues on the GluN2 subunits (Salter and Kalia, 2004) with different SFKs preferentially potentiating NMDARs comprised of different GluN2 subunits (Salter et al., 2009.). The GluN2 subunit dependency has been worked out most extensively with NMDAR responses in hippocampal CA1 neurons. In CA1 neurons, Fyn selectively phosphorylates and enhances the activity of GluN2B-containing NMDARs, while Src phosphorylates and enhances GluN2A-containing receptors (Yang et al., 2012). In spinal lamina I neurons, NMDAR mEPSCs are dominated by GluN2B subunits with little contribution from GluN2A-containing receptors (Hildebrand et al., 2014). This GluN2B dominance is consistent with our findings showing that the potentiation of NMDAR mEPSCs after PNI is dependent on Fyn, but not Src activity. Indeed, we show that Fyn directly potentiates GluN2B NMDAR responses at lamina I synapses and that BDNF mediates an increase in the activated/phosphorylated forms of Fyn and GluN2B proteins selectively at SDH synapses. Thus, we conclude that GluN2B-containing NMDARs in lamina I neurons are selectively enhanced by Fyn kinase, activated downstream of TrkB. Conversely, although phosphorylation of NMDARs by Src has been implicated in pain hypersensitivity (Liu et al., 2008), GluN2A-containing NMDARs contribute little to NMDAR mEPSCs in lamina I neurons (Hildebrand et al., 2014). We observe here that the Src inhibitor peptide had no effect on the potentiation of NMDAR mEPSCs after PNI and that BDNF did not alter GluN2A expression or phosphorylation at SDH 
synapses. We therefore conclude that the regulation of NMDARs by Src that is linked to pathological pain occurs at a locus in the pain transmission pathway other than in lamina I spinal neurons. Potential sites where Src-dependent potentiation of NMDARs may mediate pain hypersensitivity include presynaptic afferents (Chen et al., 2014b) and lamina II neurons (Wang et al., 2016).

The reversal of PNI-induced potentiation of NMDAR mEPSCs by acetazolamide implies that spinal disinhibition is required for the potentiation of NMDAR responses at lamina I synapses. One possible mechanism is that the increase in $\mathrm{Cl}$ - that is produced by decreased $\mathrm{KCC} 2$ activity in individual lamina I neurons (Coull et al., 2003) gates the potentiation of NMDARs by Fyn. However, acetazolamide should not alter intracellular $\mathrm{Cl}^{-}$concentration, as it blocks intracellular $\mathrm{HCO}_{3}{ }^{-}$production to reverse disinhibition (Staley et al., 1995). We also found that treating naive slices with the KCC2 antagonist, VU0240551, had no effect on NMDAR mEPSCs in lamina I neurons. Thus, an increase in intracellular $\mathrm{Cl}^{-}$is not sufficient for the potentiation of NMDARs at lamina I synapses. One potential player in this process is the protein tyrosine phosphatase STEP $_{61}$. STEP $_{61}$ dephosphorylates and inactivates Fyn, and not Src, and dephosphorylates GluN2B, leading to internalization of GluN1/GluN2B receptor complexes (Xu et al., 2015a; Azkona et al., 2016a). Disinhibition may thereby drive a downregulation of $\mathrm{STEP}_{61}$ to gate NMDAR potentiation by BDNF. As neither the disinhibition pathway alone nor the BDNF-TrkB signalling pathway alone is sufficient to cause potentiation of synaptic NMDAR currents, these processes appear to require coincident activation to cause potentiation of NMDARs. Together, the data indicate that we have discovered a form of molecular coincidence detection in lamina I neurons that requires ongoing inputs—BDNF-TrkB signalling and spinal disinhibition-to mediate the potentiation of synaptic excitation. 
The relationship between disinhibition and NMDAR potentiation is a feed-forward mechanism for accelerating enhancement of overall excitability of lamina I neurons. The relationship may also be reciprocal. It has recently been shown that NMDAR activity and an increase in $\left[\mathrm{Ca}^{2+}\right]_{\mathrm{i}}$ are required for the downregulation of $\mathrm{KCC} 2$ in the brain and spinal cord (Lee et al., 2011; Zhou et al., 2012; Chamma et al., 2013), implying a vicious cycle within lamina I neurons whereby loss of inhibition gates an increase in excitation and vice versa. The potential therapeutic implications of such a cycle might be that blocking disinhibition may secondarily relieve the permissive effect that is needed for NMDAR potentiation, while blocking NMDAR activity may relieve the drive to downregulate $\mathrm{KCC} 2$. In support, blockade of the individual molecular players in this pathway has been shown to reverse nerve-injury induced hypersensitivity in vivo, including BDNF (Coull et al., 2005), KCC2 downregulation (Li et al., 2016), disinhibition (Coull et al., 2003; Lee and Prescott, 2015), Fyn activation (Abe et al., 2005; Katsura et al., 2006; Liu et al., 2014), interactions between Fyn and pY1472-GluN2B (Matsumura et al., 2010), and GluN2B function (Kim et al., 2012). Thus, our results provide a biologically plausible model of molecular coupling within lamina I neurons that may explain how multiple signalling pathways might each be necessary to sustain nerve-injury induced hypersensitivity.

We speculate that our findings have implications beyond understanding nerve-injury induced hypersensitivity, as BDNF has been implicated in other types of pain hypersensitivity. In inflammatory hypersensitivity, BDNF appears to be released from nociceptive primary afferents, and knocking down nociceptor BDNF selectively attenuates behavioural hypersensitivity induced by peripheral inflammation (Mannion et al., 1999; Zhao et al., 2006) and burst stimulation of afferents drives BDNF release (Lever et al., 2001). In morphine-induced 
hyperalgesia, BDNF released from activated spinal microglia mediates a disinhibition in lamina I neurons that drives sensitization (Ferrini and De Koninck, 2013). Thus, our present findings raise the possibility that BDNF-mediated disinhibition may drive NMDAR potentiation in a number of hypersensitivity states in the spinal cord. Finally, both BDNF-evoked disinhibition and potentiation of synaptic NMDARs can occur at synapses throughout the brain, and coupling between these pathways could underlie neuronal hyperexcitability in disorders ranging from addiction to traumatic brain injury to epilepsy (Kalia et al., 2008; Ferrini and De Koninck, 2013; Vargas-Perez et al., 2014; Semaan et al., 2015). We therefore propose that our model of coincident detection between facilitation of NMDAR-mediated excitation and loss of KCC2dependent inhibition may be broadly relevant to synaptic signalling throughout the CNS. 


\section{Supplementary Figures}

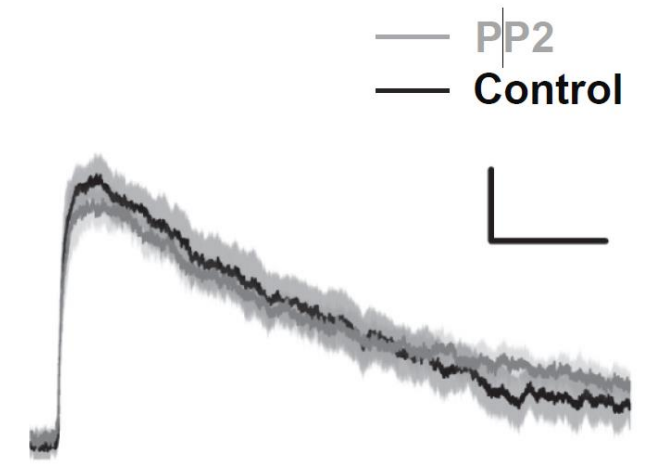

Appendix A Supplementary Figure 1. Application of PP2 does not affect NMDAR mEPSCs. Averaged mEPSCs at $+60 \mathrm{mV}$ in lamina I neurons from untreated slices of naive rats before (black) and during (grey) perfusion of $1 \mathrm{OM}$ PP2 $($ Control $\mathrm{Q}=3.85+/-0.41 ; 1 \mathrm{uM} \mathrm{PP} 2 \mathrm{Q}=3.73+/-0.28, \mathrm{n}=4)$. Scale bar y axis $=5 \mathrm{pA}, \mathrm{X}$ axis $=100 \mathrm{~ms}$. 
A

Synapses from deep dorsal horn and ventral horn
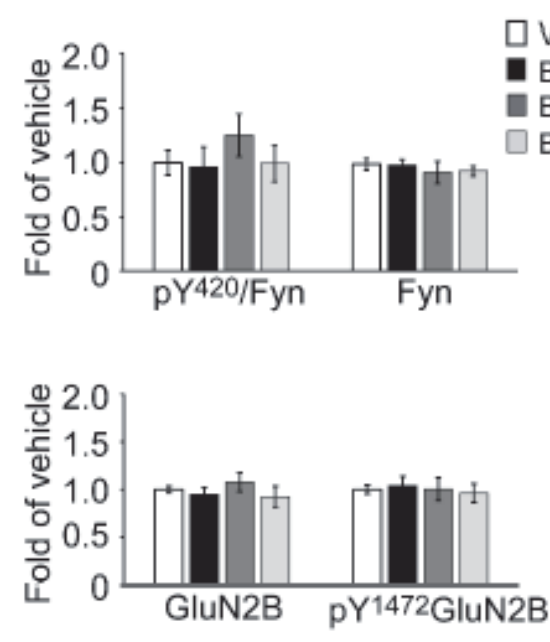

$\square$ Vehicle (V)

BDNF (B)

- BDNF+TAT-Fyn 39-57 (B+Fyn)

$\square$ BDNF+TAT-Scbld Fyn (B+sC-Fyn)
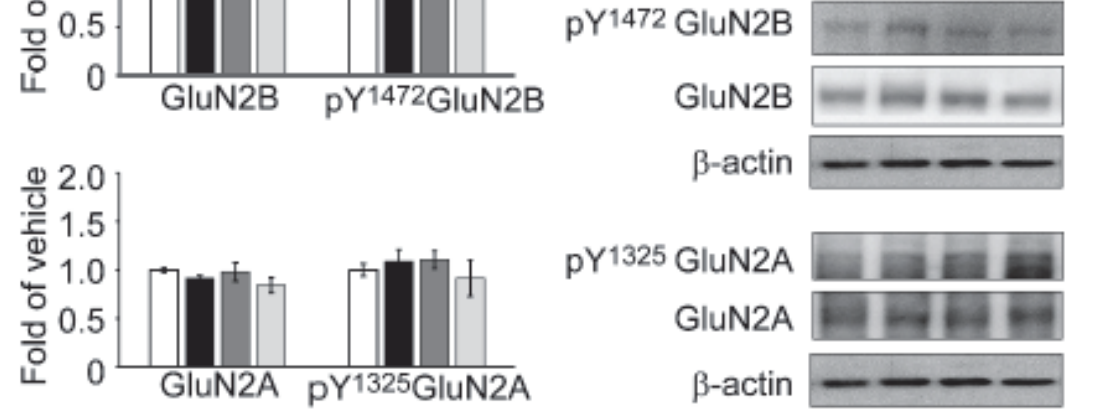

B

\section{Superficial dorsal horn - cell homogenates}

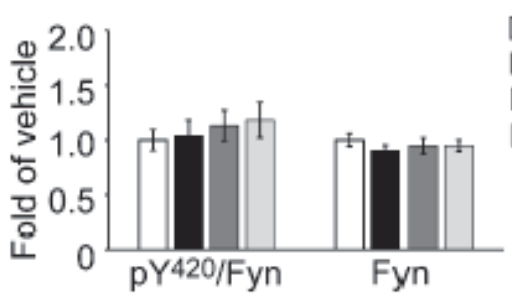

$\square$ Vehicle ( $V$ ) - BDNF (B)

BDNF+TAT-Fyn 39-57 (B+Fyn)

$\square$ BDNF+TAT-Scbld Fyn (B+sC-Fyn)

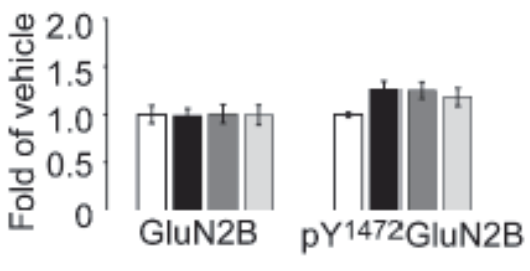

pY1472 GluN2B

GluN2B

$\beta$-actin
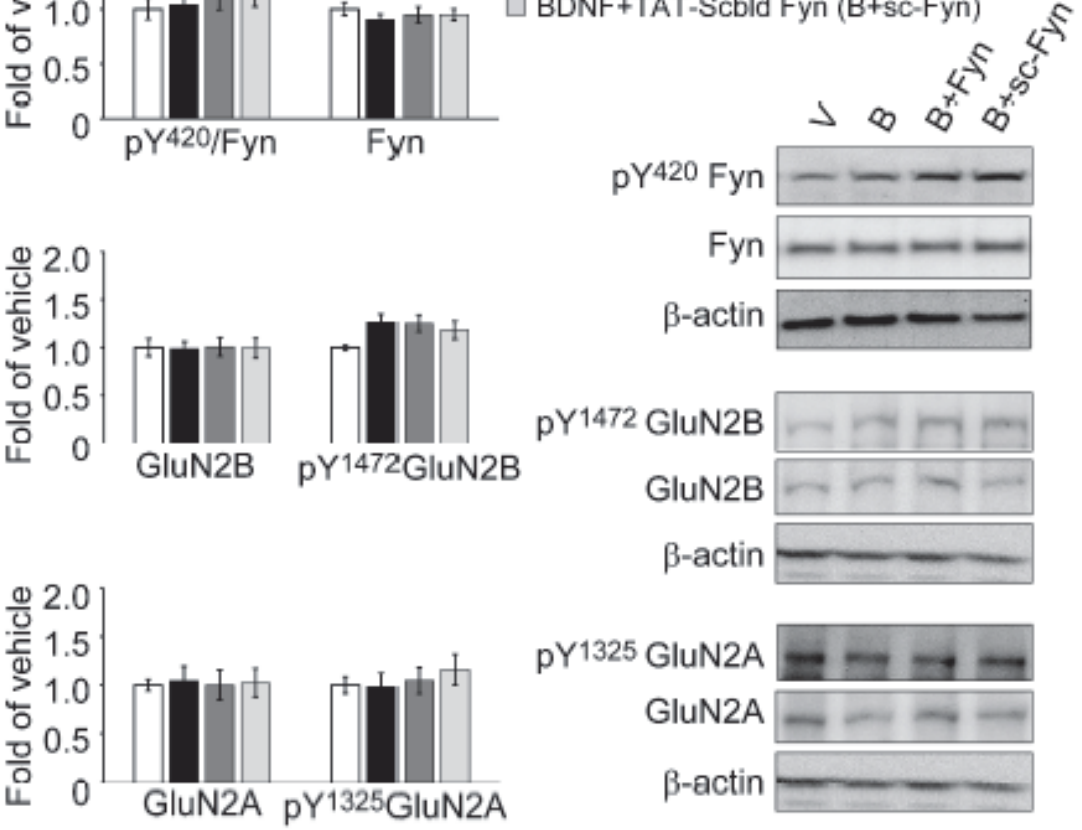

Appendix A Supplementary Figure 2. BDNF does not alter phosphorylation of Fyn and GluN2B NMDARs in deep dorsal horn and ventral horn synaptosomes or total homogenates of superficial dorsal horn. Synaptic 
fractions of deep dorsal horn and ventral horn (A) and total homogenates from superficial dorsal horn (B) were analyzed on Western blotting. Blots were probed with phospho-specific antibodies and total antibodies, respectively. Representative blots from eight independent slices are shown ( $\mathrm{n}=8$ for bar graphs).
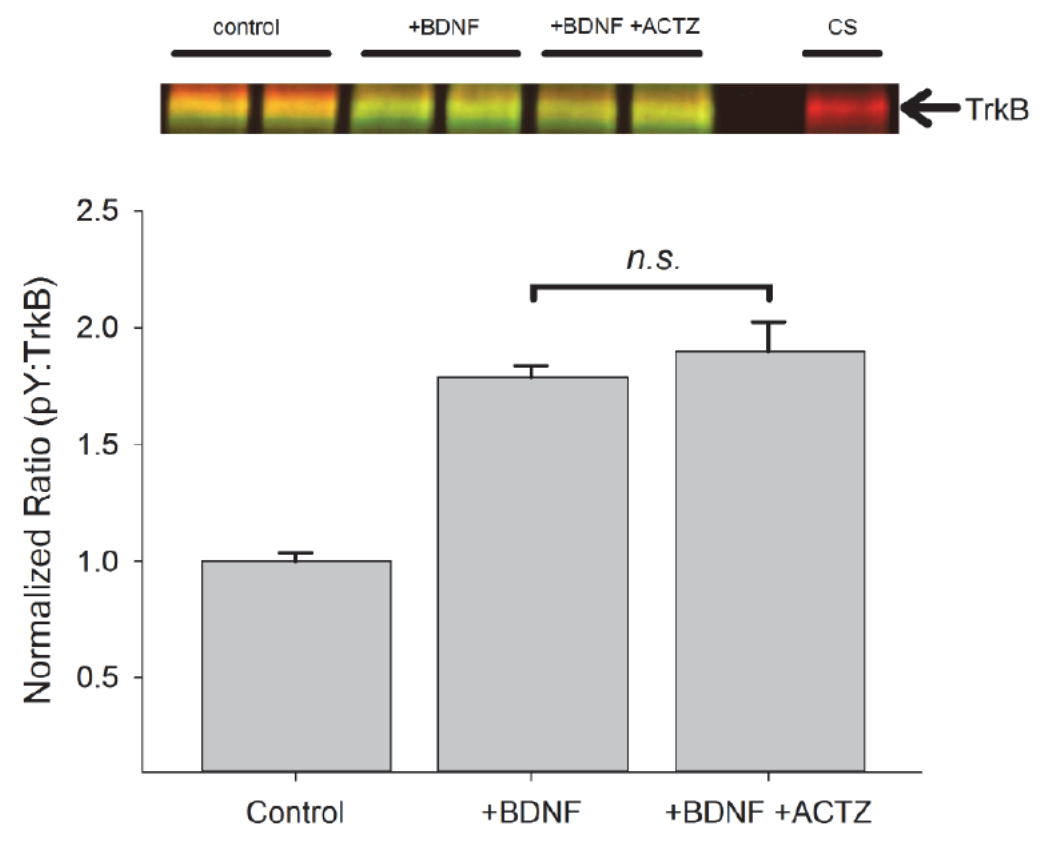

Appendix A Supplementary Figure 3. Tyrosine phosphorylation of TrkB by BDNF is not affected by the addition of $\boldsymbol{\mu M}$ acetazolamide (ACTZ). Top, protein lysates from cultured rat cortical neurons (6-7 DIV) treated with either BDNF or BDNF+ACTZ were co-immunoblotted with anti-phosphotyrosine (green) and anti-TrkB (red). Superimposed signals appear as yellow. Representative TrkB bands $(\sim 130 \mathrm{kDa})$ from parallel treatment groups from a single blot are shown, along with a phosphorylation control lane labelled 'CS' from adult rat cortical synaptosome lysate treated with calf intestinal phosphatase. Bottom, histogram of TrkB phosphorylation levels normalized to control levels from the same blots. Five to six independent culture dishes were used for each treatment group. $\mathrm{p}<0.001$ when comparing each treatment group versus control using one-way ANOVA (Holm-Sidak method). 

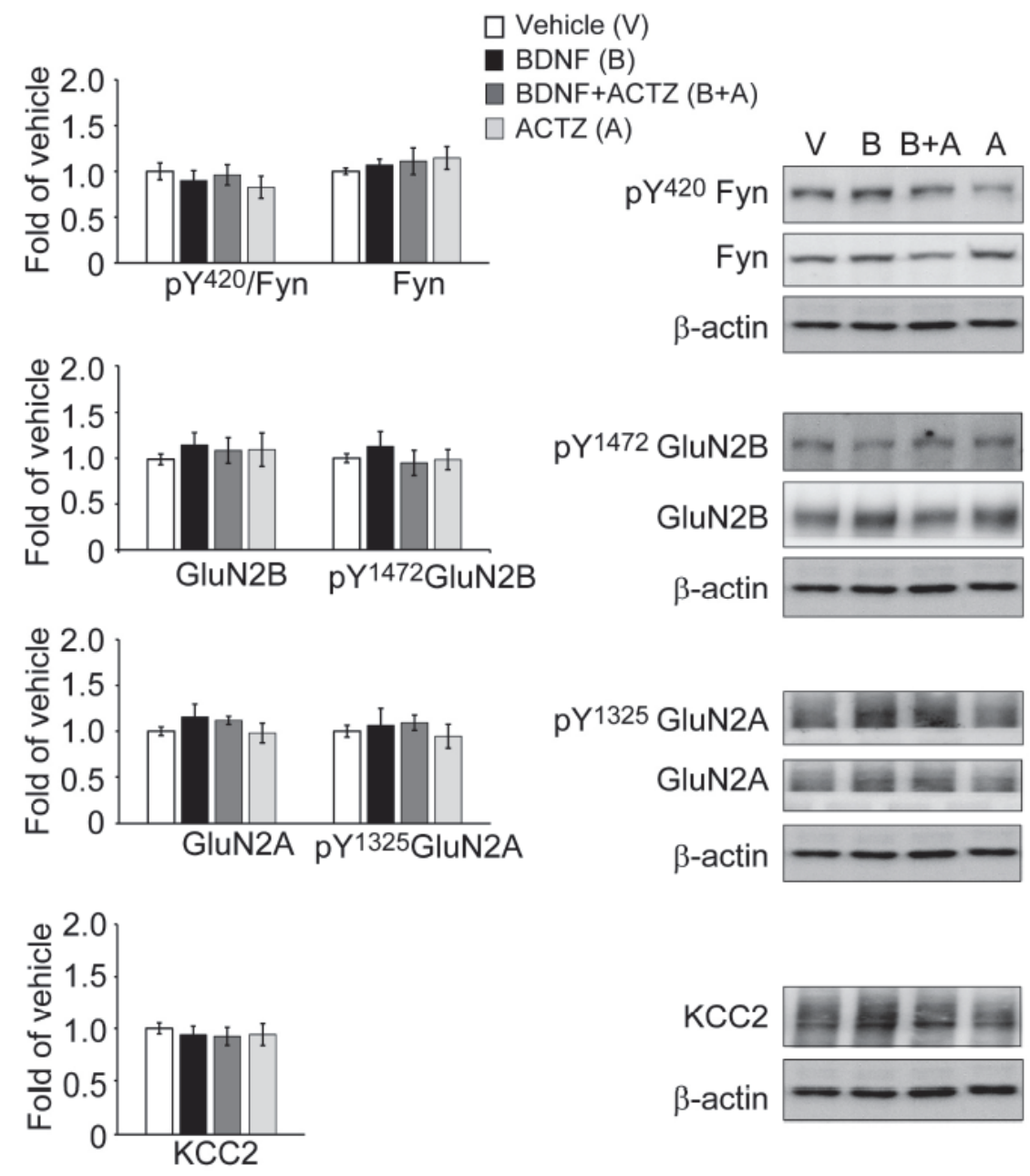

Appendix A Supplementary Figure 4. BDNF, BDNF + acetazolamide, nor acetazolamide alone affects Fyn activation and GluN2B phosphorylation in the synaptic fraction from deep dorsal horn and ventral horn spinal tissue. Synaptic fraction from tissue was used to examine phosphorylation and total protein levels of targets as indicated in the bar graphs and representative blots $(n=8)$. 
A

Superficial dorsal horn synaptosomes

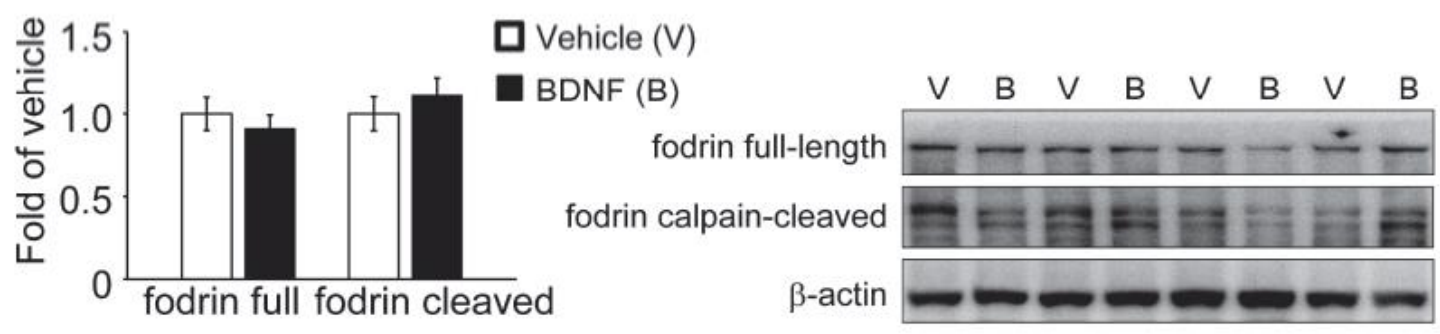

B

Superficial dorsal horn - cell homogenates

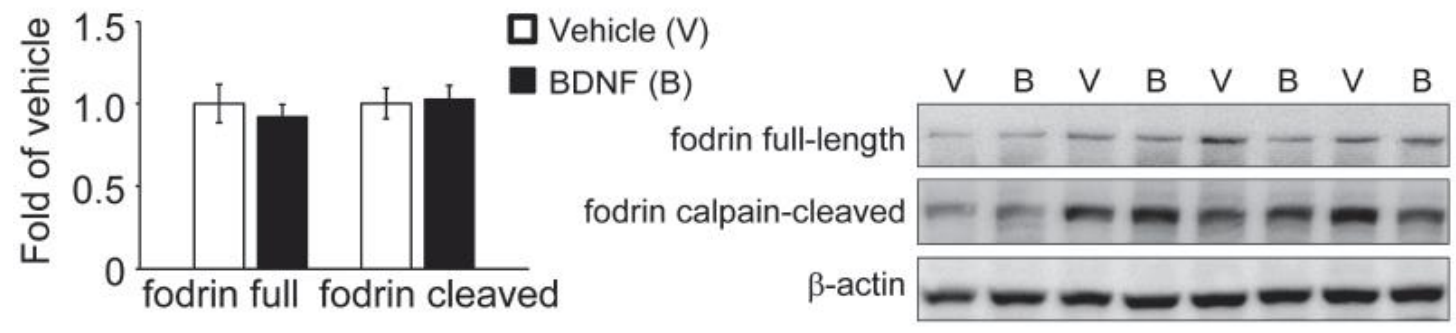

Appendix A Supplementary Figure 5. BDNF pretreatment did not increase calpain activity. Synaptic fraction (A) and total homogenates (B) were isolated from superficial dorsal horn and processed on Western blotting. Blots were probed for fodrin cleavage, a well-established calpain substrate, as a readout for calpain activity. Eight slices were used for each treatment group. Protein levels were normalized to $\square$-actin as a loading control. $\mathrm{N}=8$, but only 4 representative blots are shown for each treatment. 
$100 \mu$ M EGTA, Baseline

-10 to 15 min Control

- 15 to 20 min Control

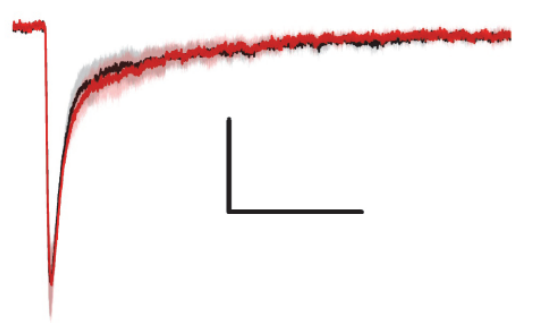

Appendix A Supplementary Figure 6. Inward NMDAR mEPSCs remain constant during baseline records. Averaged mEPSC traces from lamina I neurons of naïve rats during baseline recordings at $-40 \mathrm{mV}$, with $10 \mu \mathrm{M}$ EGTA in the intracellular pipette solution and no added extracellular $\mathrm{Mg} 2+$ and $\mathrm{Cd} 2+$ in the extracellular solution. NMDAR mEPSC charge transfer was not significantly different between 10 to $15 \mathrm{~min}$ and 15 to 20 min recording periods. 10 to $15 \min \mathrm{Q}=-0.45+/-0.11 \mathrm{pC} ; 15$ to $20 \min \mathrm{Q}=-0.44+/-0.11 \mathrm{pC}, \mathrm{n}=6, \mathrm{p}=0.75$. Scale bar $\mathrm{y}$ axis $=$ $5 \mathrm{pA}, \mathrm{X}$ axis $=100 \mathrm{~ms}$ 

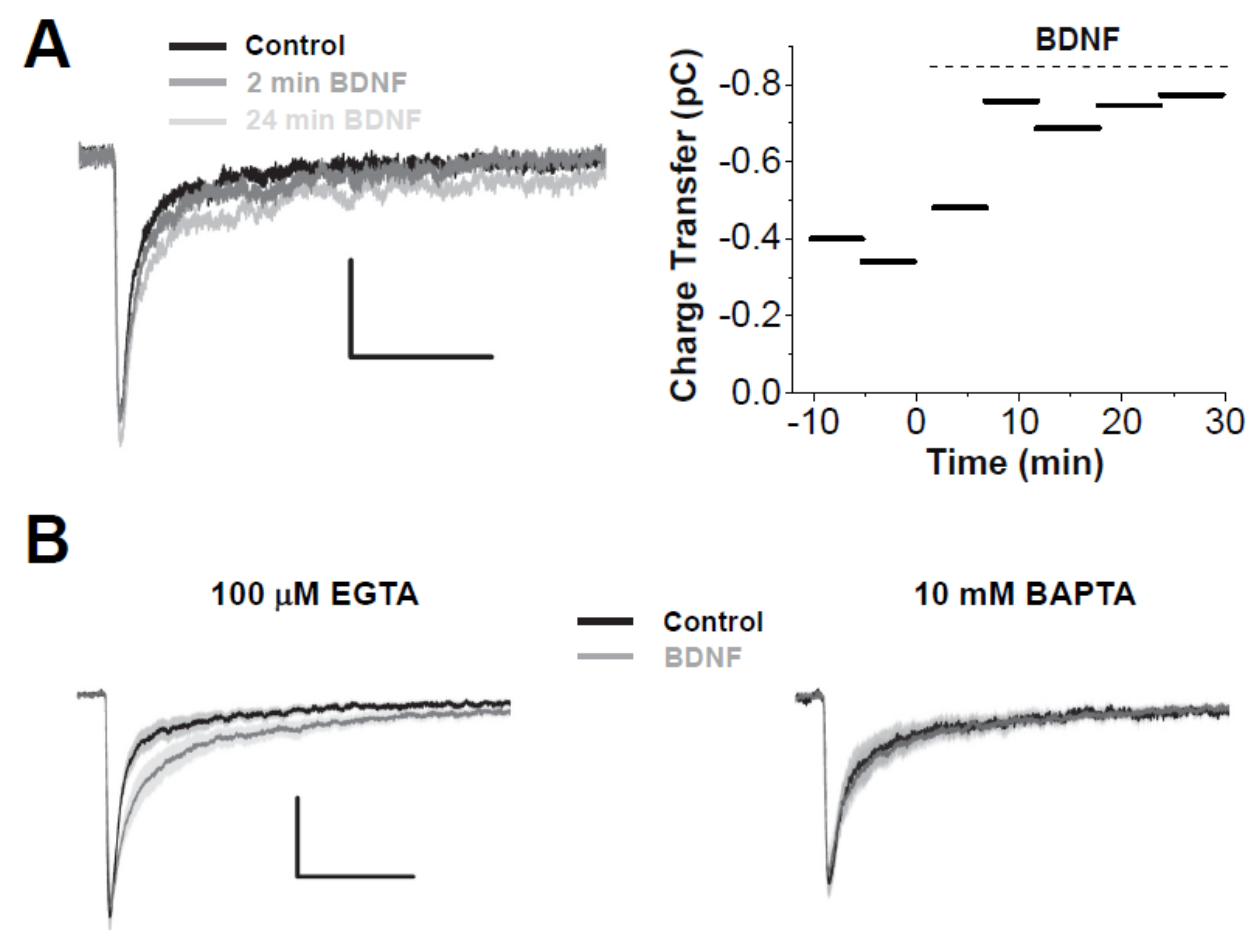

Appendix A Supplementary Figure 7. An increase in intracellular calcium is sufficient to gate potentiation of NMDAR mEPSCs by BDNF. A) Representative mEPSC traces (left) and charge transfer time course (right) demonstrating that under recording conditions that mimic the hyperexcitability produced by disinhibition (baseline mEPSC recording at $-40 \mathrm{mV}$ for 20 minutes with no added extracellular $\mathrm{Mg} 2+$ or $\mathrm{Cd} 2+$ ) and that do not tightly buffer intracellular calcium (100 uM intracellular EGTA instead of $10 \mathrm{mM}$ BAPTA) acute perfusion of $100 \mathrm{ng} / \mathrm{mL}$ exogenous BDNF causes a potentiation of inward NMDAR mEPSCs.

B) Averaged - $40 \mathrm{mV}$ mEPSC traces (no added extracellular $\mathrm{Mg} 2+$ or $\mathrm{Cd} 2+$ ) demonstrating that acute perfusion of $100 \mathrm{ng} / \mathrm{mL}$ BDNF induces a significant $(\mathrm{p}<0.05)$ potentiation of inward NMDAR mEPSCs when intracellular calcium is lightly buffered (100 uM EGTA, left) but not when it is tightly buffered (10 mM BAPTA, right). $100 \mathrm{uM}$ EGTA, $+92+/-12 \%$ BDNF modulation, $\mathrm{n}=6 ; 10$ mM BAPTA, +13 +/- $9 \%$ BDNF modulation, $\mathrm{n}=6 . \mathrm{Scale}$ bar $\mathrm{x}$ axes $=100 \mathrm{~ms}, \mathrm{y}$ axes $=5 \mathrm{pA}$. 


\section{Appendix A Supplementary Table 1. Initial NMDAR charge transfer values for various treatment}

conditions. Initial NMDAR charge transfer values calculated between $40 \mathrm{~ms}$ to $500 \mathrm{~ms}$ from the onset of averaged mEPSCs at $+60 \mathrm{mV}$. Initial NMDAR mEPSC events were averaged between 2 and 10 minutes from the start of recording at $+60 \mathrm{mV}$. Animal types and treatment conditions are indicated in the left column of the table.

\begin{tabular}{|r|c|c|c|}
\hline Treatment Type & Charge Transfer $(\mathbf{p F})$ & SE & $\mathbf{n}$ \\
\hline Naïve & 3.20 & 0.22 & 20 \\
\hline 100 uM APV & 0.03 & 0.02 & 5 \\
\hline Slice Pre-treatment: & & & \\
\hline BDNF +2 ug/mL anti-TrkB & 5.03 & 0.37 & 14 \\
\hline BDNF + 100 nM K252a & 3.73 & 0.21 & 7 \\
\hline BDNF + 1 uM PP2 & 2.64 & 0.15 & 8 \\
\hline BDNF + 10 uM SU6656 & 3.46 & 0.52 & 6 \\
\hline BDNF + 10 uM TAT-Fyn39-57 & 3.19 & 0.65 & 5 \\
\hline BDNF + 10 uM TAT-Scbld-Fyn & 3.56 & 0.32 & 9 \\
\hline BDNF +10 uM Acetazolamide & 4.78 & 0.48 & 7 \\
\hline 10 uM Acetazolamide & 4.98 & 0.29 & 9 \\
\hline 50 uM VU0240551 & 3.24 & 0.34 & 8 \\
\hline PNI Animals: & 3.25 & 0.23 & 7 \\
\hline PNI & 3.63 & 0.31 & 12 \\
\hline Sham & 5.27 & & \\
\hline PNI + 200 ng/mL TrkB-Fc & 2.75 & 0.35 & 9 \\
\hline PNI + 30 ug/mL Fyn39-57 & 3.45 & 0.52 & 6 \\
\hline PNI + 30 ug/mL Src40-58 & 3.31 & 0.56 & 9 \\
\hline PNI + 10 uM Acetazolamide & 3.23 & 0.34 & 6 \\
\hline
\end{tabular}




\section{Appendix A Supplementary Table 2. NMDAR charge transfer at two distinct points in patch-clamp}

recordings at $+60 \mathrm{mV}$. NMDAR charge transfer values calculated during the "initial" period, 2 and 10 minutes from the start of recording at $+60 \mathrm{mV}$, versus a later "final" period of recording taken from $30+$ minutes for PNI animal experiments and $20+$ minutes for pre-treatment experiments.

\begin{tabular}{|r|c|c|c|c|c|}
\hline Treatment Type & Initial Charge Transfer (pF) & SE & Final Charge Transfer (pF) & SE & $\mathbf{n}$ \\
\hline Naïve & 2.63 & $0 . .26$ & 2.62 & 0.36 & 4 \\
\hline Slice Pre-treatment: & & & & & \\
\hline $50 \mathrm{ng} / \mathrm{mL}$ BDNF & 4.54 & 0.33 & 2.83 & 0.30 & 10 \\
\hline BDNF + 2 ug/mL anti-TrkB & 3.76 & 0.15 & 3.59 & 0.21 & 5 \\
\hline BDNF + 100 nM K252a & 2.61 & 0.18 & 2.65 & 0.25 & 5 \\
\hline BDNF + 1 uM PP2 & 3.44 & 0.82 & 3.55 & 0.66 & 4 \\
\hline BDNF + 10 uM SU6656 & 3.51 & 0.73 & 3.81 & 0.74 & 4 \\
\hline BDNF + 10 uM TAT-Fyn39-57 & 3.72 & 0.37 & 3.63 & 0.40 & 7 \\
\hline BDNF + 10 uM TAT-Scbld-Fyn & 4.81 & 0.68 & 2.81 & 0.53 & 5 \\
\hline BDNF + 10 uM TAT-Src40-58 & 5.08 & 0.31 & 3.46 & 0.32 & 8 \\
\hline BDNF +10 uM Acetazolamide & 3.37 & 0.37 & 3.22 & 0.30 & 6 \\
\hline 50 uM VU0240551 & 4.28 & 0.62 & 4.33 & 0.63 & 4 \\
\hline PNI Animals: & & & & & \\
\hline PNI & 5.17 & 0.47 & 3.08 & 0.37 & 7 \\
\hline Sham & 2.39 & 0.45 & 2.71 & 0.50 & 5 \\
\hline PNI + 200 ng/mL TrkB-Fc & 4.23 & 0.57 & 4.52 & 0.56 & 6 \\
\hline PNI + 30 ug/mL Src40-58 & 3.41 & 0.40 & 3.52 & 0.46 & 5 \\
\hline PNI + 10 uM Acetazolamide & 5.27 & 0.10 & 3.24 & 0.16 & 4 \\
\hline
\end{tabular}

Appendix A Supplementary Table 3. NMDAR charge transfer values before and after intracellular or extracellular pharmacological treatment during recording at $+60 \mathrm{mV}$.

\begin{tabular}{|c|c|c|c|c|c|}
\hline Treatment Type & Initial Charge Transfer (pF) & SE & Final Charge Transfer (pF) & SE & $\mathbf{n}$ \\
\hline \multicolumn{6}{|l|}{ Intracellular treatments: } \\
\hline Control Internal Solution & 2.95 & 0.31 & 3.05 & 0.40 & 5 \\
\hline $1 \mathrm{mMEPQ}(\mathrm{pY}) \mathrm{EEIPIA}$ & 2.85 & 0.30 & 5.11 & 0.82 & 6 \\
\hline $1 \mathrm{mMEPQ}(\mathrm{pY})$ EEIPIA + $30 \mathrm{ug} / \mathrm{mL}$ Src40-58 & 2.24 & 0.36 & 3.59 & 0.48 & 5 \\
\hline $1 \mathrm{mM}$ EPQ(pY)EEIPIA + 30 ug/mL Fyn39-57 & 3.49 & 0.37 & 3.36 & 0.23 & 6 \\
\hline $\begin{array}{ll}\text { Treatment Type } \\
\end{array}$ & Control - Charge Transfer (pF) & SE & BDNF - Charge Transfer (pF) & SE & $\mathbf{n}$ \\
\hline \multicolumn{6}{|c|}{ PNI animals } \\
\hline After initial rundown: & 2.29 & 0.37 & 3.47 & 0.48 & 5 \\
\hline Treatment Type & Control - Charge Transfer (pF) & SE & BDNF - Charge Transfer (pF) & SE & $\mathbf{n}$ \\
\hline \multicolumn{6}{|c|}{ Pre-treatments: } \\
\hline Saline & 3.16 & 0.37 & 2.45 & 0.29 & 11 \\
\hline 50 uM VU0240551 & 3.23 & 0.43 & 4.30 & 0.57 & 5 \\
\hline
\end{tabular}




\section{Acknowledgments}

We dedicate this paper to our dear friend Dr. John F. MacDonald, who was taken from us much too soon. This study was supported by a grant from CIHR to M.W.S. ( MT-12682 ) and grants from NSERC (Discovery Grant), IASP (Early Career Research Grant), the Canadian Pain Society, and Pfizer Canada (Early Career Investigator Pain Research Grant) to M.E.H. M.W.S. hold the Northbridge Chair in Paediatric Research. M.E.H. was initially supported by a postdoctoral fellowship from CIHR and a Pain Scientist Award from the University of Toronto Centre for the Study of Pain. 


\title{
Appendix B - The T-type calcium channel antagonist, Z944, reduces spinal excitability and
} pain hypersensitivity

\begin{abstract}
Background and Purpose: T-type voltage-gated calcium channels are an emerging potential therapeutic target for neurological disorders including epilepsy and pain. Inhibition of T-type channels reduces the excitability of peripheral nociceptive sensory neurons and reverses pain hypersensitivity in male rodent pain models. However, administration of peripherally restricted T-type antagonists has failed to show efficacy in multiple clinical and preclinical pain trials, suggesting that inhibition of peripheral T-type channels alone may be insufficient for pain relief. Experimental Approach: We utilized the selective and CNS-penetrant T-type channel antagonist, Z944, in electrophysiological, calcium imaging, and behavioural paradigms to determine its effect on lamina I neuron excitability and inflammatory pain behaviours.
\end{abstract}

Key Results: Voltage-clamp recordings from lamina I spinal neurons of adult rats revealed that approximately $80 \%$ of neurons possess a low threshold T-type current, which was blocked by Z944. Due to this highly prevalent T-type current, Z944 potently blocked action-potential evoked somatic and dendritic calcium transients in lamina I neurons. Moreover, application of Z944 to spinal cord slices attenuated action potential firing rates in over half of laminae I/II neurons. Finally, we found that intraperitoneal injection of Z944 (1-10 mg/kg) dose-dependently reversed mechanical allodynia in the complete Freund's adjuvant model of persistent inflammatory pain, with a similar magnitude and time course of analgesic effects between male and female rats.

Conclusion and Implications: Our findings demonstrate that T-type calcium channels critically shape the excitability of lamina I pain processing neurons, and inhibition of these channels by the clinical-stage antagonist Z944 potently reverses pain hypersensitivity across sexes. 


\section{Introduction}

T-type voltage-gated calcium channels are an important emerging molecular target for the treatment of pain (Bourinet et al., 2014; Todorovic \& Jevtovic-Todorovic, 2011; Weiss \& Zamponi, 2019). Given their unique gating kinetics and low voltage activation near resting membrane potentials, T-type channels regulate subthreshold excitability as well as action potential firing patterns in both physiological and pathological states (Cheong and Shin, 2013).

For example, T-type currents promote low threshold spikes and rebound burst firing in subsets of thalamic neurons, which are implicated in both slow-wave sleep oscillations as well as in absence seizures (Cain and Snutch, 2013; Cheong and Shin, 2013; Cain et al., 2018). Several antiepileptic drugs including ethosuximide are thought to act in part through the inhibition of Ttype channels (Weiss and Zamponi, 2019) and, interestingly, these same T-type targeting clinical compounds have analgesic effects in rodents (Dogrul et al., 2003; Flatters and Bennett, 2004). More broadly, selective knockdown or block of T-type channels through genetic and pharmacological approaches reverses pain hypersensitivity in rodent models of pain including bladder pain (Tsubota et al., 2018), post-surgical pain (Joksimovic et al., 2019), inflammatory pain (Watanabe et al., 2015), nerve injury-induced pain (Bourinet et al., 2005; Feng et al., 2019), and chemotherapy-induced and diabetic peripheral neuropathies (Jacus et al., 2012; Li et al., 2017b).

The involvement of T-type channels in mediating pain processing has been extensively studied in the periphery. Of the three T-type voltage-gated calcium channel subtypes - $\mathrm{Ca}_{\mathrm{v}} 3.1$, $\mathrm{Ca}_{\mathrm{v}} 3.2$, and $\mathrm{Ca}_{\mathrm{v}} 3.3$ - the $\mathrm{Ca}_{\mathrm{v}} 3.2$ isoform has been shown to be selectively upregulated within dorsal root ganglion (DRG) peripheral sensory neurons in rodent models of chronic pain (GarcíaCaballero et al., 2014; Watanabe et al., 2015; Fukami et al., 2017; Li et al., 2017b; Gomez et al., 
2020). Interfering with the various pathological mechanisms that upregulate $\mathrm{Ca}_{\mathrm{v}} 3.2$ in $\mathrm{DRG}$ neurons reverses pain hypersensitivity within these rodent models. In terms of subcellular localization, $\mathrm{Ca}_{\mathrm{v}} 3.2$ channels are now known to be functionally expressed throughout DRG neurons, from nerve endings in skin hair follicles to the presynaptic terminals of primary afferents (Jacus et al., 2012; François et al., 2015). However, peripherally-restricted T-type channel antagonists such as ABT-639 have failed to show efficacy in multiple clinical pain trials (Serra et al., 2015; Wallace et al., 2016) as well as in some rodent studies (Picard et al., 2019). These findings raise the possibility that T-type channels within the spinal cord could be an important target for the treatment of pain.

The superficial dorsal horn (SDH) of the spinal cord consists of lamina I and lamina II, which together receive the bulk of nociceptive information from the periphery and are highly involved in the processing and relay of nociceptive information to the brain (Bourinet et al., 2014; Todd, 2010). Although intrathecal spinal injections of T-type antagonists reverse pain hypersensitivity in rodent pain models (Wen et al., 2010; Feng et al., 2019; Picard et al., 2019), the specific contributions of T-type channels to pain processing in the SDH are poorly understood. T-type mediated calcium currents have recently been found to be expressed in a subset (approximately 45 to 60\%) of lamina II neurons of both mice and rats (Wu et al., 2018; Candelas et al., 2019). However, the potential contributions of T-type channels in regulating excitability within lamina I neuron subpopulations remain unexplored. This represents a significant knowledge gap given that lamina I contains the output projection neurons for the SDH nociceptive network (Todd, 2010), and hyperexcitability of lamina I neurons has been implicated in the development and maintenance of pathological pain (Ikeda et al., 2003b; Keller et al., 2007; Liu et al., 2008). 
The small organic compound, Z944, is a CNS-penetrant, high-affinity T-type calcium channel antagonist originally under clinical development for the treatment of absence seizures (Tringham et al., 2012). Z944 selectively blocks T-type channels at nanomolar concentrations, with minimal effects on other voltage-gated calcium channels, cardiovascular-related hERG channels, and Nav1.5 channels up to the low micromolar concentrations produced by systemic administration (Tringham et al., 2012). Cryo-electron microscopy has demonstrated a direct physical interaction between Z944 and the central cavity of the T-type channel pore domain (Zhao et al., 2019). Recent evidence has suggested that Z944 has analgesic potential in both rodents and humans. In male rodents, systemic injection of Z944 alleviated behavioural measures of chronic neuropathic pain (Leblanc et al., 2016), while in human phase Ia and Ib clinical studies, both oral and systemic injection of Z944 were found to be well-tolerated and reduced pain sensitization as well as Visual Analog Scale pain ratings in an experimental pain model (Lee, 2014). To explore both the mechanism of action and the efficacy of Z944 as a potential novel pain therapeutic, we tested the effects of Z944 on putative T-type currents, activityinduced calcium transients, and overall membrane excitability in individual lamina I neurons, and assessed the analgesic effects of Z944 across doses in a CFA model of inflammatory pain in both male and female adult rats.

\section{Methods}

\section{Data Availability:}

The data that support the findings of this study are available from the corresponding author upon reasonable request.

\section{Study Approval:}


These experiments were approved by the institutional Animal Care Committees where experiments were performed (Hospital for Sick Children, University of Toronto, University of British Columbia, Carleton University, and the University of Ottawa Heart Institute; Animal Use Protocol\# 111497), and performed in accordance with animal care regulation and policies of the Canadian Council on Animal Care. All animals were housed and cared for in accordance with the recommendations of the Canadian Council for Animal Care.

\section{Animals:}

All experiments were performed on male or female rats supplied by Charles River Laboratories. Sprague Dawley and Wistar rats were selected for this study, as rat spinal tissue provides increased area in comparison to mice, and Sprague Dawley rats have a calm demeanour, making them well-suited for behavioural experiments. Animals for electrophysiological and calcium imaging experiments were completed using male juvenile Wistar rats (P11 to P21, Figure 3 only) or adult (P60-90) Sprague Dawley (SD) rats (325-400g).

Sprague Dawley rats for behavioural experiments were delivered at 3 months of age to account for the weight differences between male and female animals and were housed at the testing facility for a minimum of 5 days before beginning the study.

\section{Spinal cord isolation:}

Spinal cords were isolated from juvenile male Wistar rats or adult male SD rats as previously published (Hildebrand et al, 2011, Pain; Hildebrand et al, 2014). Briefly, animals were anesthetized with intraperitoneal (IP) injection of $20 \%(\mathrm{w} / \mathrm{v})$ urethane $(3 \mathrm{~g} / \mathrm{kg})$ and euthanized by severing of the cervical spinal cord and vertebrae. The lumbar region of the spinal cord was dissected from the rat and placed in a protective sucrose dissection solution. Dissection solution contained (mM): 50 sucrose, $92 \mathrm{NaCl}, 15$ D-Glucose, $26 \mathrm{NaHCO}, 2.5 \mathrm{KCl}, 1.25$ 
$\mathrm{NaH} 2 \mathrm{PO} 4,0.5 \mathrm{CaCl} 2,7 \mathrm{MgSO} 4,1$ kynurenic acid, bubbled with 5\% CO2/95\% O2 (pH 7.3, 310 mOsm). Dorsal roots and dura were removed from the lumbar cord, and L4-L6 were removed from the rest of the cord. The L4-L6 piece was then glued against an agar block (4\% agarose in distilled water) and placed in a Leica VT 1000s or 1200s vibratome (Leica, Germany) containing ice-cold sucrose dissection solution. Parasagittal slices (300 $\mu \mathrm{m}$ thick) were obtained from the vibratome. Slices were then incubated in dissection solution lacking kynurenic acid at $34^{\circ} \mathrm{C}$ for $40 \mathrm{~min}$ and then cooled passively to room temperature ( 21 to $22{ }^{\circ} \mathrm{C}$ for $\geq 30 \mathrm{~min}$ ) before electrophysiological recording and calcium imaging.

\section{Electrophysiology of spinal cord lamina I neurons.}

For data in Figure 1, slices were placed under an upright Olympus BX51WI microscope (Olympus Corporation, USA) with a 40x water immersion objective. Lamina I neurons were identified based on location relative to myelin tracts and substantia gelatinosa, and patched into with recording pipettes of 6-8 M $\Omega$, pulled by a Sutter P97 puller (Sutter Instruments, USA). External recording solution was as above. The internal voltage-clamp patch pipette solution consisted of (mM): 105 D-gluconic acid, $105 \mathrm{CsOH}, 17.5 \mathrm{CsCl}$, 10 EGTA, 10 HEPES, $2 \mathrm{Mg}$ ATP, $0.5 \mathrm{Na}_{2}$-ATP ( $\mathrm{pH} 7.3,290 \mathrm{mOsm}$ ). The external recording solution (ACSF) consisted of (mM): $125 \mathrm{NaCl}, 20$ D-Glucose, $26 \mathrm{NaHCO}_{3}, 3 \mathrm{KCl}, 1.25 \mathrm{NaH}_{2} \mathrm{PO}_{4}, 2 \mathrm{CaCl}_{2}, 1 \mathrm{MgCl}_{2}$, and 0.04 Alexa Fluor-488 or -594 (pH 7.3, 310 mOsm). Perfusion speed was $1 \mathrm{~mL} / \mathrm{min}$, and the solution was continuously bubbled with $5 \% \mathrm{CO}_{2} / 95 \% \mathrm{O}_{2}$. The recording solution also contained $0.5 \mu \mathrm{M}$ TTX and $5 \mathrm{mM}$ TEA to block voltage-gated sodium channels and voltage-gated potassium channels, respectively. All recordings were performed at room temperature. Morphology of lamina I neurons was determined after recording by using the fine focus to move through the z- 
plane and identify the number of primary dendrites. Neurons with two primary dendrites were classified as fusiform, and similarly, neurons with three or four primary dendrites were classified as pyramidal or multipolar, respectively (Supplemental Figure 1) (Lima and Coimbra, 1986).

Patch-clamp recordings were made using a Multiclamp 700B amplifier (Molecular Devices, CA, USA) and a Digidata 1550B Digitizer (Molecular Devices, CA, USA) connected to a desktop computer with pClamp 10.7 software. Voltage-clamp recordings were digitized at 10 $\mathrm{kHz}$ and low-pass filtered at $2 \mathrm{kHz}$. Recordings were included for analysis only if they maintained a leak current of less than $-50 \mathrm{pA}$ at $-60 \mathrm{mV}$, and if access resistance began below 25 $\mathrm{M} \Omega$ and did not change by more than $30 \%$ during the recording period. The IV-curve protocol started at $-60 \mathrm{mV}$, with a $500 \mathrm{~ms}$ step hyperpolarization to $-100 \mathrm{mV}$, followed immediately by incremental steps from $-100 \mathrm{mV}$, increasing by $10 \mathrm{mV}$ (up to $-40 \mathrm{mV}$ ). For time-course experiments, steps began from $-70 \mathrm{mV}$, and were repeated continuously at a rate of once per min. For these experiments, drug wash-in experiments were randomized such that Z944 and DMSO vehicle trials were completed in alternating order where possible. Blinded analysis was not possible given that only the experimenter had the expertise required for this specific analysis. Statistical analysis in Figures 1A and 1E was performed on groups of unequal size, due to differing population sizes from an unbiased sampling of all neurons in lamina I, and due to exclusion of two neurons from analysis in the DMSO vehicle group due to significant changes in input resistance, respectively. Statistical analysis was not performed on the data in Figure 1C due to group sizes of $\mathrm{n}<5$, but was included as evidence that all morphological classes can either possess or lack a T-type component. 
For data within Figure 3, slices were placed under an Axioskop 2 FS plus microscope under IR-DIC optics (Zeiss, Germany). All other parameters were the same as Figure 1, except the internal current-clamp patch pipette solution consisted of (mM): $140 \mathrm{~K}$-gluconate, $4 \mathrm{NaCl}$, $0.5 \mathrm{MgCl}_{2}, 10$ HEPES, 4 Mg-ATP, 0.5 Na2-ATP, 1 EGTA (pH 7.3, $290 \mathrm{mOsm}$ ). The junction potential for current-clamp was calculated as $14.6 \mathrm{mV}$ (i.e. $-50 \mathrm{mV}=-64.6 \mathrm{mV}$ ) (Junction potential calculator, Clampex), and has been corrected in all experimental data. Recordings were included for analysis only if they maintained a resting membrane potential below $-50 \mathrm{mV}$, and if first action potentials reached a minimum of $+5 \mathrm{mV}$. Bridge balance and pipette capacitance compensation were performed in all neurons, and corrected prior to each recording. In all experiments, neurons were held between -60 and $-70 \mathrm{mV}$ (typically 0 to $-20 \mathrm{pA}$ holding current injection). VI-curves were run from a holding potential of -60 to $-70 \mathrm{mV}$, and incremental step current injections were performed from $-40 \mathrm{pA}$, with $10 \mathrm{pA}$ steps (1200 ms duration). Drug wash-in experiments were randomized such that Z944 and DMSO vehicle trials were completed in alternating order where possible. Statistical analysis in Figure 3E was performed on groups of unequal size, due to variability in the number of neurons that met the criteria for recordings and analysis. Statistical analysis was not performed on Figure 3B due to group sizes of $n<5$, but was included as a representative expansion of data presented in 3C.

For pharmacology experiments, Z944 was first dissolved in DMSO, and then added to the external recording solution, such that the final DMSO concentration was always below $0.1 \%$. Washout experiments are problematic in spinal slice recording assays, with the potential for hydrophobic compounds to stick to myelin and cell membranes as well as to change excitability measures over the longer time durations required for washout. Moreover, we have previously found that the Z944-mediated inhibition of T-type currents in nRT neurons of thalamic slices 
was largely non-reversible after 30 minutes of washout (Tringham et al., 2012). Here, we therefore tested for net effects of Z944 in SDH neuron recordings by comparing to cells that were treated with vehicle for the same duration and under the same experimental conditions. For vehicle experiments, DMSO without Z944 was added. For all electrophysiology experiments, perfusion of Z944 or vehicle was 10-15 minutes.

\section{Simultaneous electrophysiology and two-photon calcium imaging of lamina I neurons:}

Spinal cord lamina I neurons were recorded from in current-clamp configuration, with simultaneous two-photon calcium imaging as previously described (Harding et al, 2020). Briefly, slices were placed under a Zeiss 710 NLO system equipped with an AxioExaminar Z1 (Zeiss, Germany), and neurons were visualized under IR-DIC optics. Patch-clamp recordings were made with recording pipettes of 7-10 M $\Omega$, pulled by a Sutter P97 puller (Sutter Instruments, Navato, CA, USA). The external recording solution (ACSF) and perfusion speed were as above. The internal current-clamp patch pipette solution consisted of (mM): $112 \mathrm{~K}$-gluconate, $8 \mathrm{KCl}, 10$ HEPES, 4 Mg-ATP, 0.3 Na2-ATP, 10 Phosphocreatine, 0.3 EGTA, 0.04 Alexa Fluor-594, 0.11 Oregon Green Bapta-1 (pH 7.3, 290 mOsm). Neurons were allowed to dialyze for 20-30 min before imaging to allow the fluorescent dye concentration to equilibrate. All recordings were performed between $24-28^{\circ} \mathrm{C}$. Current-clamp recordings were performed as above. Bridge balance and pipette capacitance compensation were performed in all neurons. In all experiments, neurons were held between -70 and $-80 \mathrm{mV}$ (typically 0 to $-20 \mathrm{pA}$ holding current injection). Single action potentials were evoked with a 5 ms current injection of between 150-400 pA. Each recording was 15 seconds in duration, with the experimental current injection at $2 \mathrm{sec}$ into 
recording. A minimum of $15 \mathrm{sec}$ was given between the end of one recording and the beginning of the next to allow the neuron to return to baseline.

Two-photon dual excitation of OGB-1 (110 $\mu \mathrm{M}$ in recording pipette) and AF-594 (40 $\mu \mathrm{M}$ in recording pipette) was achieved using a Coherent Chameleon Ultra Ti:Sapphire laser tuned to $800 \mathrm{~nm}$ (Coherent, USA). OGB-1 and AF-594 fluorescence were split based on emission spectra using NDD filter cubes (500-550 nm, 565-610 nm; Carl Zeiss Microscopy, Germany), and sent into NDD detectors (Carl Zeiss Microscopy, Germany). Two-photon images were obtained using a 20x water-immersion objective lens (Carl Zeiss Microscopy, Germany) and the Zen 2009 acquisition program (Carl Zeiss Microscopy, Germany). Laser power was kept between 0.3$0.7 \%$, and gain was restrained to $650-850$ for all calcium imaging experiments. Fluorescence data were acquired using line scan acquisition (1024x1, 2x averaging) at a rate of $133 \mathrm{~Hz}$, and saved as LSM files from Zen 2009 (Carl Zeiss Microscopy, Germany). Calcium imaging data were analyzed as previously described, utilizing a custom-made, semi-automated MATLAB toolbox (Mathworks, USA) for analysis, entitled CIAT (Calcium Imaging Analysis Toolbox) (Harding et al, 2020). Use of the semi-automated analysis toolbox, CIAT, which automatically calculates peak response from averaged calcium imaging trials, was designed to remove experimental bias. For data in Figure 2, drug wash-in experiments were randomized such that Z944 and DMSO vehicle trials were completed in alternating order where possible. Statistical analysis within this figure was performed on groups with an $n>5$, of unequal size due to one neuron being removed for photobleaching. 


\section{Freund's adjuvant model of inflammatory pain and behaviour testing:}

Animals were housed in pairs, had free access to food and water and were randomly assigned to their respective experimental groups. Group sizes for behavioural experiments (Figure 4) were set to 10 animals per group based on previous study design (Dedek et al., 2019) and a priori sample size calculation for repeated measures ANOVA for within-group differences using $\mathrm{G}^{*}$ Power 3.1.9.7 (Heinrich-Heine-Universität Düsseldorf) based on the following parameters: a moderate effect size of $0.20, \alpha=0.05$, power $=0.99$, number of groups $=4$ (Figure $4 \mathrm{~A}$ and $\mathrm{C}$ ) and 5 (Figure 4E), 13 measurements, correlation among repeated measures $=0.5$, and nonsphericity correction $\varepsilon=1$. These parameters yielded a total sample size of 36 for Figure $4 \mathrm{~A}$ and $\mathrm{C}$, and 35 for Figure 4E. Total sample size was rounded up to 10 animals per group to ensure equal group samples and to ensure adequate power if an animal needed to be removed from the study. Two animals were withdrawn from the study and sacrificed early because they injured each other in a fight. Animal husbandry was performed exclusively by the tester for the entire duration of the animals' stay at the housing facility. Animals were housed in individually HEPAfiltered cages. Cohorts of male and female animals were run separately, however, in some instances male and female animals were housed in the same room concurrently. In cases when the housing room had both male and female rats, husbandry for female animals was performed before any male cages were opened. Cohorts were run in groups of 8-10 animals. Lab coats and gloves were changed between cohorts. The housing room was used exclusively for this study and was open to only the experimenter (female) and animal care staff (who did not open cages or handle the animals). 
Complete Freund's adjuvant (CFA, Sigma) was used to model inflammatory pain. Rats were given a $0.3 \mathrm{~mL}$ plantar injection of either phosphate-buffered saline (PBS: saline) or CFA (a $50 \%$ by volume mixture of CFA and PBS) under isoflurane anesthesia. Blinding was not performed for CFA injections, as the tester also performed injections and all animal husbandry, and the effects of CFA injection are obvious while handling the animals and performing testing. Animals were left to acclimate to their behaviour testing chambers for $45 \mathrm{~min}$ before testing each

morning. Tests were performed at approximately the same time each day, across cohorts, for the duration of the study. Behaviour testing for pain hypersensitivity was performed using von Frey filaments to measure mechanical paw withdrawal threshold (PWT). Withdrawal threshold was measured in the injected paw using the simplified up-down method (SUDO), as described by Bonin et al. (2014). Measurements were taken at baseline (pre-injection), and then at 24, 48, and $72 \mathrm{hr}$ post-CFA or PBS injection. We report PWT in grams, as labelled on each von Frey filament. As per the SUDO method, an adjustment factor is used to determine the PWT for each trial. We used an adjustment factor of $+/-0.5$ (difference, in grams, between filaments).

\section{Intraperitoneal injection:}

Seventy-two hours following sub-plantar injection of CFA and following Day 3 behaviour testing (see above), male and female adult Sprague-Dawley rats were given an IP injection of 1,3 , or $10 \mathrm{mg} / \mathrm{kg} \mathrm{Z944}$ or vehicle dissolved in $0.5 \%$ (weight/volume) of carboxymethylcellulose (Sigma Aldrich, USA). Z944 is highly bioavailable and CNS-penetrant, with pharmacokinetic studies demonstrating that oral and systemic administration produces low micromolar concentrations of Z944 in rodent plasma (unpublished observations and (CasillasEspinosa et al., 2019)). Moreover, IP injection of $10 \mathrm{mg} / \mathrm{kg}$ Z944 reduced brain epileptiform 
activity in adult rats (Tringham et al., 2012), demonstrating CNS penetrance and actions on central excitability at the dose and route of administration of Z944 used here. Z944 was dissolved in DMSO to create a stock solution $(100 \mathrm{mg} / \mathrm{mL}$ for animals receiving $10 \mathrm{mg} / \mathrm{kg}, 30$ $\mathrm{mg} / \mathrm{mL}$ for animals receiving $3 \mathrm{mg} / \mathrm{kg}$, and $10 \mathrm{mg} / \mathrm{mL}$ for animals receiving $1 \mathrm{mg} / \mathrm{kg}$ ) that was then suspended in the carboxymethylcellulose solution (1-part Z944 stock: 9-parts carboxymethylcellulose solution). Vehicle-treated animals received DMSO without Z944 in $0.5 \%$ (weight/volume) of carboxymethylcellulose solution. All solutions were prepared the day of injection, and the experimenter was blinded to the treatment by a lab-mate. All animals were allowed 20 min in their home cage between Day 3 testing and the administration of the IP injection. This allowed animals to eat or drink before being placed back in testing chambers for the time course. Animals received injections 1-1.5 min apart, with the exact time noted by the experimenter to ensure accurate readings during the time course. IP injection was performed by the experimenter alone using a surgical drape to restrain the animal. After IP injection, animals were placed in the behaviour testing chambers and allowed to acclimate for $20 \mathrm{~min}$. During the time course, measurements of PWT were taken every $15 \mathrm{~min}$, starting $20 \mathrm{~min}$ after IP injection. The time of the test was measured from the third stimulus administration (five stimuli were presented in each trial, and the third, the middle stimulus, occurred at the given time interval). We found that IP injection of Z944 (1 to $10 \mathrm{mg} / \mathrm{kg}$ ) did not induce any behavioural signs of sedation or motor deficits. Moreover, a previous in-depth analysis of potential non-specific in vivo effects of Z944 demonstrated that IP injection of Z944 at a dose above that used in the present study $(30 \mathrm{mg} / \mathrm{kg})$ did not induce any significant sedation or motor abnormalities (Tringham et al., 2012). 
The technique for measuring PWT differed during the time course. SUDO normally involves two trials of 5 stimuli that are then averaged to give PWT. For the time course, only one trial was performed every 15 min to ensure that the animals were not over-stimulated. A pilot study was used to determine an effective interval for the time course. One unsuccessful pilot was run using a testing interval of $10 \mathrm{~min}$. This pilot was deemed unsuccessful because animals were displaying freezing behaviour: not reflexively withdrawing their paw at weights normally far above the expected withdrawal threshold. We concluded that allowing animals more time to recover between trails was necessary, and thus increased the testing interval by $50 \%$. This decision, in turn, allowed more accurate timing of testing at each animal's given testing time. Animals were sacrificed immediately after the conclusion of the time course.

\section{Materials.}

Unless otherwise indicated, all compounds were obtained from Sigma Aldrich (USA). Z944 was synthesized as previously described (27).

\section{Data and Analysis.}

This manuscript complies with BJP's recommendations and requirements on experimental design and analysis (Curtis et al., 2018). Data in some figures of this manuscript have been normalized to reduce variability. In Figure 1E, data are plotted as \% peak current reduction within each neuron (post / pre) to allow direct comparison of Z944 effects as compared to a DMSO control. In Figure $1 \mathrm{G}$, data are plotted as normalized \% current, representing the percent change in average current for each neuron within the first three minutes of recording (post / pre). In Figure 2D and E, data are plotted as \% peak calcium transient remaining and \% 
AUC remaining (post / pre) to account for rundown effects in the DMSO control, allowing for better comparison of the true effect of Z944. For all experiments in all figures, no analysis was performed to determine the presence of outliers, and therefore all data points were included for analysis.

For all statistical analyses, $\mathrm{p}<0.05$ was used as the threshold for statistical significance, and in all experiments, the declared group size is the number of independent values, and statistical analysis was performed upon these values. Statistical analysis was only performed when group size was at least $\mathrm{n}=5$, which reflects the number of independent, non-technical replicate values. Sample sizes for experiments in Figures 1-3 were determined based on previous studies (Hildebrand et al., 2014; Harding et al., 2020), and data in these figures were analyzed with Sigmaplot 12.0 (Systat Software, USA). Prior to running paired t-tests, the normality of the data was tested (Shapiro-Wilk test), and if the data failed this test of normality $(p<0.05)$, a Wilcoxon signed-rank test was performed in lieu of a paired t-test. T-tests comparing the means from different neurons or animals were performed as unpaired t-tests. If the data failed a test of normality (Shapiro-Wilk test), then a Mann-Whitney rank-sum test was performed instead. Data in Figure 3B were analyzed by two-way repeated measure ANOVAs, with drug application and current injection as factors. These were performed within Sigmaplot, and both two-way ANOVAs passed the Shapiro-Wilk test for normality of data. All two-way ANOVA post-hoc comparisons were performed using the Holm-Sidak method when ANOVA achieved statistical significance $(\mathrm{p}<0.05)$ and showed no significant variance in homogeneity.

Within Figure 4, comparisons of means were performed using unpaired Mann-Whitney ttests, due to failure of the Shapiro-Wilk normality test (Sigmaplot 12.0, Systat Software, USA). 
One-way repeated-measures ANOVAs were performed using SPSS (IBM SPSS Statistics 25.0, see supplemental tables 1 and 3). Before running each ANOVA, we examined Mauchly's test of sphericity, which indicated that the assumption of sphericity was violated in each case (see supplemental tables 1 and 3). In each case, the Greenhouse-Geisser epsilon adjustment of degrees of freedom was deemed the most appropriate adjustment, since in each case it was below 0.75 (supplemental table 1 and 3 ) and was thus used to determine the p-value. Bonferroni's significant difference test followed ANOVAs when ANOVA achieved statistical significance (p $<0.05$ ) and showed no significant variance in homogeneity (Table 2 and 4). ${ }^{*}<0.05$ for all figures.

\section{Results}

\section{Z944 reduces inward current through low voltage-activated calcium channels in a subset of} lamina I neurons

To determine if T-type channels are present in lamina I spinal dorsal horn neurons, we first recorded calcium channel currents from visually identified lamina I neurons within parasagittal spinal cord slices of adult male rats. The voltage threshold for activation of calcium currents was identified for each recorded neuron by analyzing IV-curves. Of 34 recorded neurons, 27 exhibited a rapidly inactivating inward current beginning at $-60 \mathrm{mV}$, consistent with the presence of low voltage-activated (LVA) T-type calcium channels (Figure 1A-B) (Weiss and Zamponi, 2019). In 7 of 34 neurons, only a slower inactivating inward current was detected at depolarizations beginning at $-40 \mathrm{mV}$, consistent with the biophysical properties of high voltageactivated (HVA) calcium channels. As such, we defined this neuronal subpopulation as one with no measurable LVA T-type component. 
Distinct subpopulations of lamina I neurons can be delineated by morphology (Lima and Coimbra, 1986). We therefore classified the morphology of Alexa Fluor-filled neurons after recording (Supplementary Figure 1) and compared the distribution of morphologies for lamina I neurons that did and did not contain the LVA T-type component. Of the neurons with an LVA component where morphology could be definitively determined, 9/17 were fusiform, 6/17 were multipolar, and 2/17 were pyramidal (Figure 1C). Of those without an LVA component, 2/6 were fusiform, 1/6 was multipolar, and 3/6 were pyramidal. Although there was a bias towards fusiform neurons containing an LVA component, and pyramidal neurons not containing an LVA component, group sizes were too small to perform statistical analysis (Figure 1C). However, from these exploratory results, we can conclude that LVA currents are found in a large subset of lamina I neurons and are not restricted to specific morphological subtypes.

We next sought to determine whether Z944 application affects the LVA component of calcium currents present in $\sim 80 \%$ of lamina I neurons. While perfusion of ACSF with DMSO vehicle did not affect LVA currents at -60 and $-50 \mathrm{mV}$, administering $2 \mu \mathrm{M}$ Z944 reduced these LVA currents at both potentials (Figure 1D, E). In a subset of neurons, we analyzed the time course of LVA current block by Z944 using repeated test pulses to $-50 \mathrm{mV}$ (Figure $1 \mathrm{~F}, \mathrm{G})$. We found that within the first minute of Z944 administration, peak current dropped by $20 \%$, and reached a plateau inhibition of $40-50 \%$ within 5 minutes. Overall, Z944 treatment resulted in a significant reduction in calcium currents elicited at $-50 \mathrm{mV}$ as compared to DMSO controltreated neurons ( $\mathrm{n}=10$ neurons for both conditions). No significant effect of Z944 compared to control treatment was observed for HVA calcium currents elicited at $0 \mathrm{mV}(\mathrm{n}=10$ neurons for vehicle, 8 neurons for Z944) (Figure 1E), indicating specificity to T-type channels. Altogether, the presence of rapidly inactivating LVA calcium currents, and their selective inhibition by Z944 
strongly supports the conclusion that T-type currents are present in $\sim 80 \%$ of lamina I neurons of adult rats, encompassing all three main morphologically-defined neuron subpopulations.
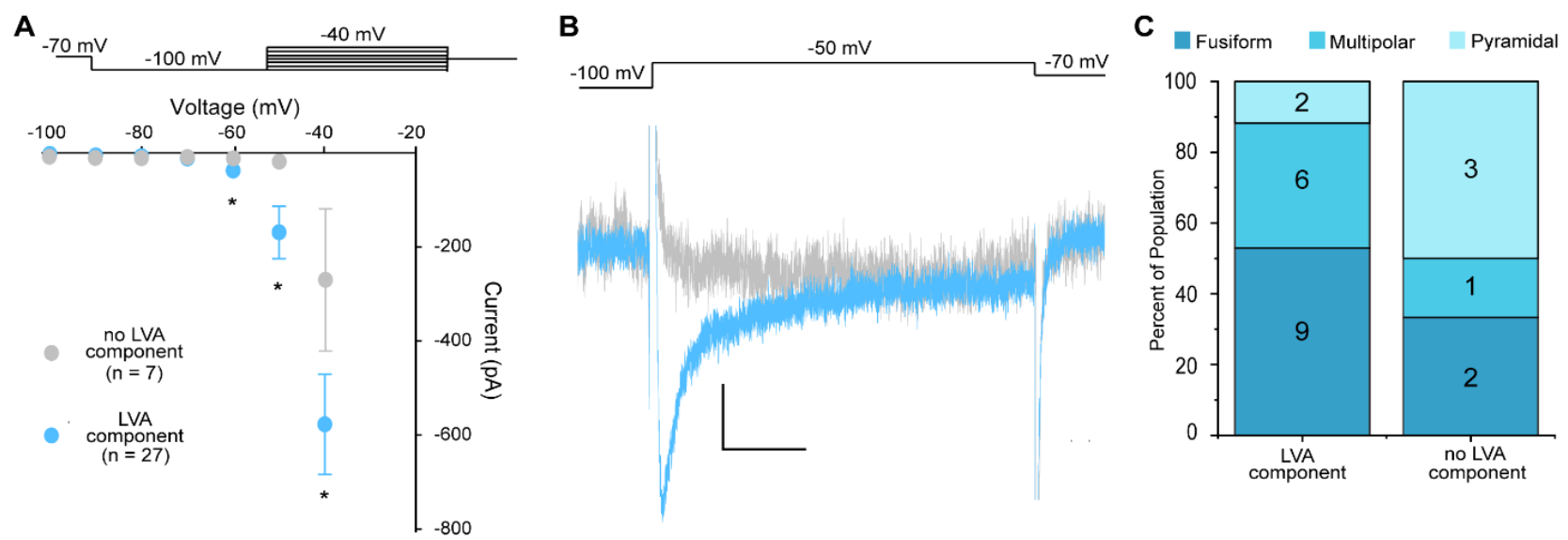

D

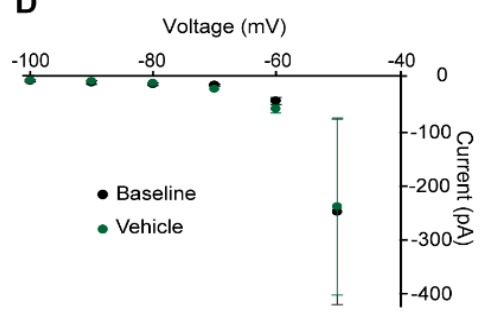

F

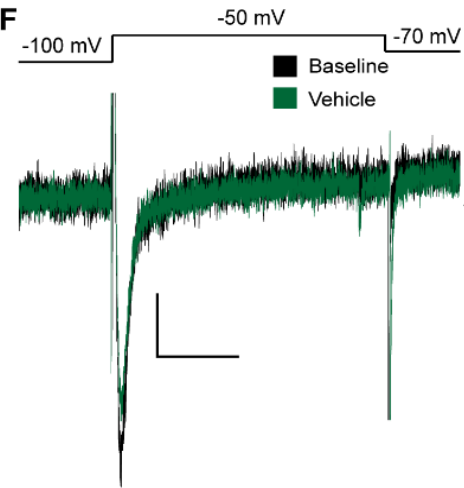

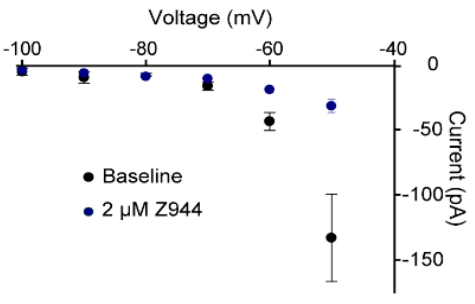

E
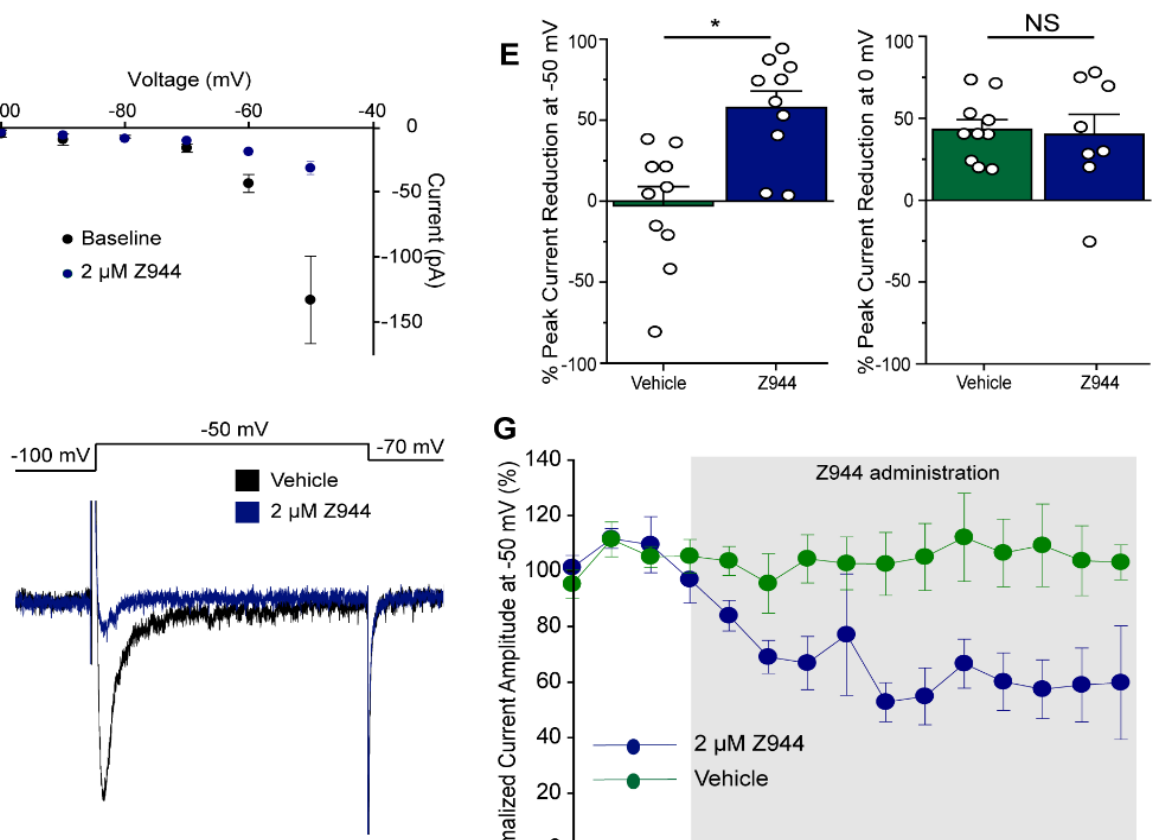

G

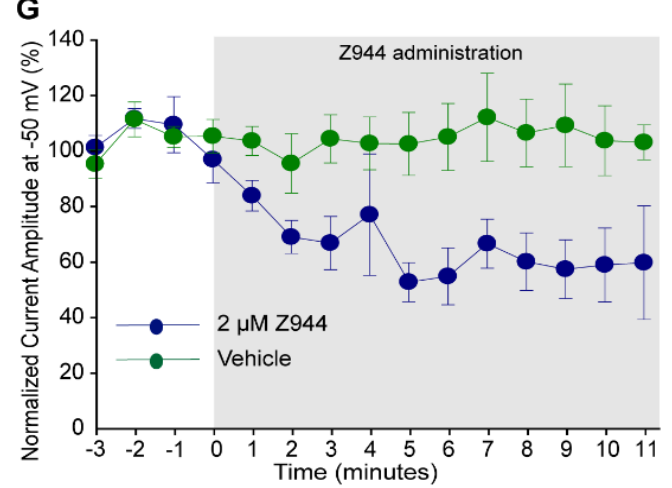


Appendix B Figure 1. Z944 selectively reduces inward current through low voltage-activated calcium channels in spinal cord lamina I neurons. A) Top: Voltage-clamp protocol used to measure calcium currents in lamina I neurons. Neurons were hyperpolarized to $-100 \mathrm{mV}$ for $500 \mathrm{~ms}$, before a $500 \mathrm{~ms}$ step current injection beginning at $-100 \mathrm{mV}$, and increasing by $10 \mathrm{pA}$ each sweep. Bottom: Analysis of calcium currents revealed two populations of lamina I neurons; those with a low voltage-activated (LVA) component (27/34, light blue), and those lacking an LVA component (7/34, light grey circles). Peak current with steps to $-60 \mathrm{mV},-50 \mathrm{mV}$, and $-40 \mathrm{mV}$ were compared between neurons with and without an LVA component using unpaired Mann-Whitney t-tests. (-60 mV: $\mathrm{p}=4.0 \times 10-4,-50 \mathrm{mV}: \mathrm{p}=9.0 \times 10-4,-40 \mathrm{mV}: \mathrm{p}=0.014)$. B) Example of current traces in an example neuron with an LVA component (light blue), and without an LVA component (light grey) for a voltage step from -100 mV to -50 $\mathrm{mV}$. Scale bar $\mathrm{x}$-axis=20 pA, y-axis=100 ms. C) Distribution of morphologies in a subset of recorded neurons. Where possible, neurons were classified into three main morphologies. D) In a subset of neurons with an LVA component, IV-curves were plotted for baseline recordings, and after vehicle ( $\mathrm{n}=8$ neurons, left, green) or Z944 ( $\mathrm{n}=10$ neurons, $2 \mu \mathrm{M}$, right, blue) administration. E) Left: Quantification of percent peak current reduction at -50

$\mathrm{mV}$ for vehicle (green) and Z944 (blue) administration (unpaired t-test, $\mathrm{p}<0.001, \mathrm{n}=10$ neurons for both conditions). Right: Quantification of percent peak current reduction at $0 \mathrm{mV}$ for vehicle (green) and Z944 (blue) administration (unpaired t-test, $\mathrm{p}=0.82, \mathrm{n}=10$ neurons for vehicle, 8 neurons for Z944). F) Sample current traces induced by depolarization from $-100 \mathrm{mV}$ to $-50 \mathrm{mV}$. Left: before (black) and after vehicle (green) administration. Right: before (black) and after Z944 administration. Scale bar x-axis=20 pA, y-axis=150 ms. G) Time course of peak current at $50 \mathrm{mV}$, normalized to first $3 \mathrm{~min}$ before administration of either vehicle (green, $\mathrm{n}=6$ neurons), or Z944 (blue, n=6 neurons). All error bars represent \pm SEM. ${ }^{*} \mathrm{p}<0.05$.

\section{Activity-induced calcium transients in spinal cord lamina I neurons are reduced by Z944}

Activity-dependent intracellular calcium signalling in lamina I neurons has been demonstrated to lead to hyperexcitability and pathological pain (Ikeda et al., 2003b; Wei et al., 2006). We have recently shown that single action potentials drive increases in intracellular calcium concentration at both the soma and dendrites of lamina I neurons, through a mechanism 
dependent upon voltage-gated calcium channels (Harding et al., 2020). Given our identification here of T-type currents in the majority of lamina I neurons, we next used a combination of current-clamp recordings and simultaneous two-photon calcium imaging to test whether Z944 can attenuate activity-induced calcium responses in these critical nociceptive spinal neurons.

Lamina I neurons were filled with the calcium-sensitive fluorophore Oregon Green Bapta-1 and the structural fluorophore Alexa Fluor-594 via the patch pipette and single action potential-induced calcium responses were measured in the soma and primary dendrites, as previously described (Figure 2A) (Harding et al., 2020). We found that, on average, perfusion of $2 \mu \mathrm{M}$ Z944 significantly decreased action potential-induced calcium responses in both the soma and dendrites, as compared to the DMSO vehicle control ( $\mathrm{n}=8$ neurons for vehicle, 7 neurons for Z944; Figure 2B-D). Interestingly, we also found that perfusion of Z944 significantly decreased the area under the curve for action potential waveforms, as compared to the DMSO vehicle control ( $\mathrm{n}=7$ neurons for Z944 and vehicle conditions; Figure 2D). This decrease in area under the curve corresponded to a visible reduction in the action potential afterdepolarization following Z944 treatment (Figure 2C). Our findings that Z944 significantly reduced action potential-evoked calcium responses by over $50 \%$ in both the soma and dendrites of lamina I neurons indicates that T-type calcium channels are the predominant mediators of this action potential-evoked calcium signal. 

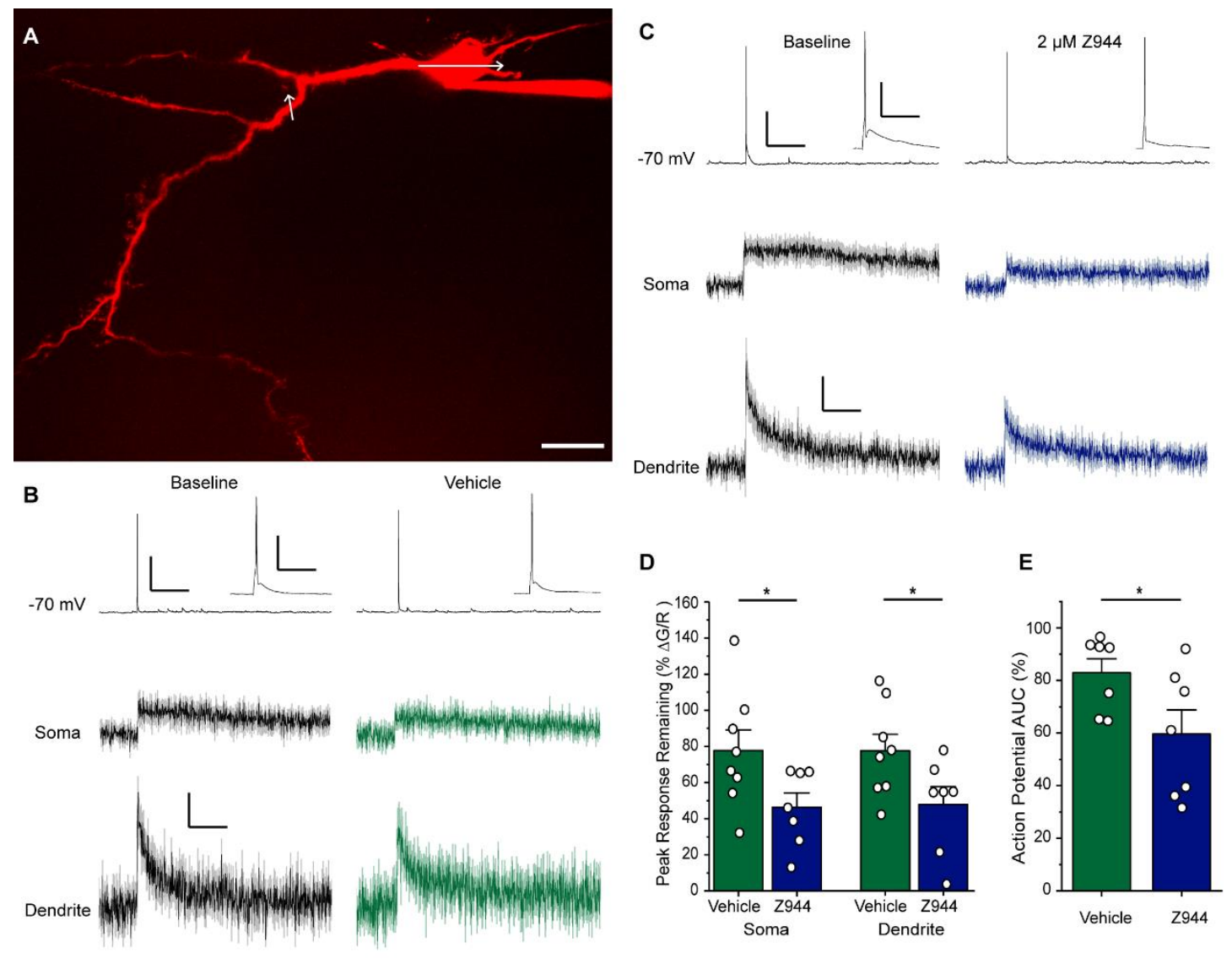

\section{Appendix B Figure 2. Activity-induced calcium transients in lamina I neurons are reduced by Z944. A)}

Flattened projection of a two-photon z-stack of a lamina I neuron filled with Alexa Fluor-594 via a patch pipette. White arrows represent line scan trajectory during calcium imaging. Scale bar represents $20 \mu \mathrm{m}$. B) Left: Single action potentials induced by current injection $(5 \mathrm{~ms}, 200 \mathrm{pA})$ from a membrane potential of $-70 \mathrm{mV}$ induce calcium transients in the somata and dendrites of lamina I neurons (black). Right: Recordings remain stable after vehicle administration (green, right). Traces are an average of 8 neurons for both somatic and dendritic compartments. C) Wash-in of Z944 ( $2 \mu \mathrm{M})$ greatly reduces calcium transients in both the somata and dendrites (dark blue), and decreases action potential afterdepolarization (insets). Traces are an average of 7 neurons for both somatic and dendritic compartments). For B and C, action potential traces are representative examples from a single neuron, calcium transients are average responses, \pm SEM. For $\mathrm{B}$ and $\mathrm{C}$, electrophysiology scale bar $\mathrm{x}$-axis $=30 \mathrm{mV}, \mathrm{y}$-axis $=2$ s. Inset scale bar $x$-axis $=30 \mathrm{mV}, 100 \mathrm{~ms}$. Calcium imaging scale bar $\mathrm{x}$-axis $=\Delta \mathrm{G} / \mathrm{R}$ 0.04, y-axis=2 s. D) Quantification of the percent remaining peak calcium transient, as displayed in B and C after the administration of Z944 or vehicle. Comparisons performed with unpaired $t$-tests ( $\mathrm{p}=0.045$ for soma, $\mathrm{p}=0.047$ for dendrite, $\mathrm{n}=8$ 
neurons for vehicle, 7 neurons for Z944 for both compartments). E) Quantification of the percent remaining action potential area under the curve after administration of $Z 944$ or vehicle ( $\mathrm{p}=0.048$, unpaired $\mathrm{t}$-test, $\mathrm{n}=7$ neurons for Z944 and vehicle conditions). ${ }^{*} \mathrm{p}<0.05$ for all t-tests. All error bars represent SEM.

\section{Z944 reduces excitability in a subset of SDH spinal cord neurons}

Given our findings that Z944 decreases activity-induced calcium responses and reduces action potential afterdepolarizations, we next sought to determine whether administration of Z944 affects the overall excitability of SDH nociceptive neurons. We therefore tested the effects of Z944 on trains of action potentials in a combined population of laminae I and II SDH neurons, with stepwise test current injections increasing by $+10 \mathrm{pA}$ increments and the number of evoked action potentials calculated for each depolarizing current step (Figure 3A, B). For each neuron, we identified a depolarizing test current injection step $(+10$ to $+50 \mathrm{pA})$ that evoked action potential firing in the $10-20 \mathrm{~Hz}$ frequency range, which corresponds to physiological firing rates induced by nociceptive input (Keller et al., 2007) (Figure 3C). Administration of vehicle DMSO control for 10 to 15 min did not significantly alter the frequency of action potential firing at these test current injection steps (Baseline 15.5 $\pm 1.6 \mathrm{~Hz}$, DMSO 17.0 $\pm 2.0 \mathrm{~Hz}, \mathrm{n}=5$ neurons, data not shown).

We next administered Z944 for 10 to $15 \mathrm{~min}$ at a concentration $(10 \mathrm{mM})$ that completely abolishes T-type currents in thalamic slices (Tringham et al., 2012), and measured the effects of Z944 on 10 to $20 \mathrm{~Hz}$ action potential firing (Tringham et al., 2012). We found that SDH neurons could be separated into those that responded to $10 \mu \mathrm{M}$ Z944 with a significant decrease in action potential firing frequency ( $48.7 \pm 10.6 \%$ reduction, $7 / 13$ neurons), and those that showed no response to this elevated concentration of Z944 (-5.1 $\pm 4.3 \%$ reduction, 6/13 neurons) (Figure 
3C, D). In a subset of recorded neurons, we investigated the full relationship between current injected and number of action potentials evoked and found that the Z944-mediated decrease in action potential number for Z944-sensitive neurons ( 3 of 6 neurons) was observed at all current injection amplitudes, but was most pronounced with larger current injections of +50 and $+60 \mathrm{pA}$ (Figure 3B). Importantly, the differential effects of Z944 between SDH neuron subpopulations were not due to differences in passive membrane properties, as both resting membrane potential and input resistance were not significantly different between Z944-sensitive and -insensitive neurons (Figure 3E). Our finding that administration of Z944 significantly reduces the firing frequency of over half of SDH neurons strongly suggests that postsynaptic T-type channels shape the excitability of these nociceptive spinal neurons and could therefore be an important target for reducing nociceptive input that ascends to the brain. 

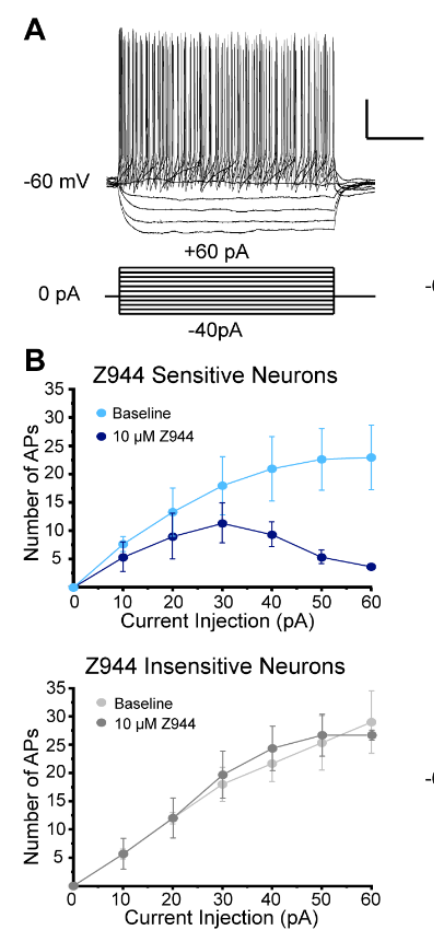

C

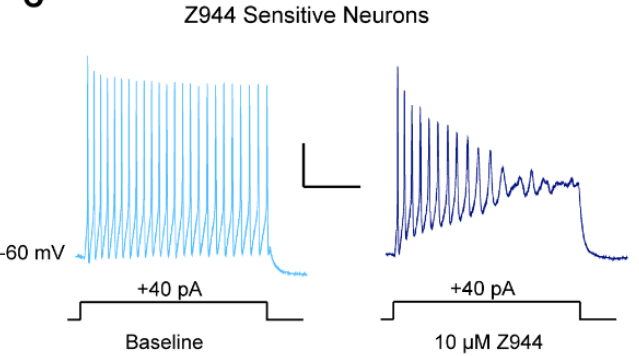

Baseline
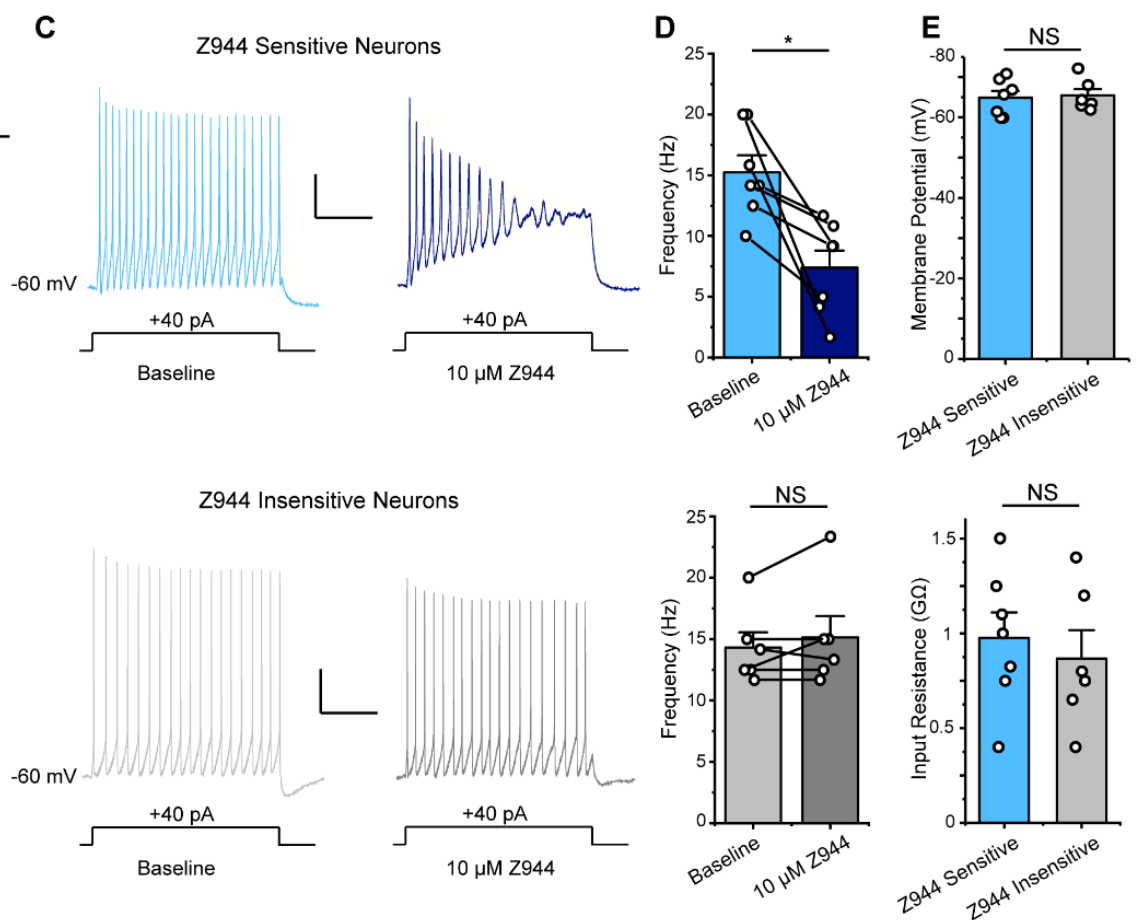

Appendix B Figure 3. Z944 reduces excitability in a subset of laminae I/II spinal cord neurons. A)

Representative membrane potential trace of a lamina I/II neuron in response to step current injection (1200 ms) beginning from $-40 \mathrm{pA}$, and increasing by $10 \mathrm{pA}$ each step. B) Top: Administration of Z944 (10 $\mu \mathrm{M})$ significantly inhibited action potential firing in a subset of laminae I/II neurons, while having no effect on action potential firing for the remaining laminae I/II neurons (bottom). Action potentials were counted during each step current injection in a subset of neurons, revealing a population of neurons with reduced firing after the administration of Z944 (baseline=light blue, Z944=dark blue; $\mathrm{n}=3$ neurons), and a population insensitive to $\mathrm{Z} 944$ administration (baseline=light grey, Z944=dark grey; n=3 neurons). Data in both graphs were analyzed by two-way repeatedmeasures ANOVA, with drug administration and the number of action potentials as factors. In the Z944-sensitive population, a significant interaction between the number of action potentials and drug administration was found $(\mathrm{p}=0.003)$. Post-hoc Holm-Sidak comparisons revealed significant differences between baseline and Z944 at 50 and $60 \mathrm{pA}$ current injection ( $\mathrm{p}=0.043$ and $\mathrm{p}=0.032$, respectively). No significant differences were found in the Z944insensitive neurons. C \& D) The effects of Z944 on excitability were evaluated for depolarizing current injection steps that elicited 10 to $20 \mathrm{~Hz}$ AP firing for each individual laminae I/II neuron (typically $\sim 40 \mathrm{pA}$ ). Administration of Z944 (10 $\mu \mathrm{M})$ significantly inhibited action potential firing in a subset of laminae I/II neurons (top, 7/13 neurons) ( $\mathrm{p}=0.01$, paired $\mathrm{t}$-test), while having no effect on action potential firing for the remaining laminae $\mathrm{I} / \mathrm{II}$ neurons 
(bottom, 6/13 neurons) ( $\mathrm{p}=0.28$, paired t-test). E) The input resistance and resting membrane potential of Z944sensitive laminae I/II neurons were not significantly different than Z944-insensitive neurons $(p=0.60$ and $p=0.81$ respectively, unpaired t-tests, $n=7$ neurons for Z944-sensitive group, 6 neurons for Z944-insensitive group. All error bars represent mean \pm SEM. ${ }^{*} p<0.05$.

\section{IP administration of Z944 reverses CFA-mediated tactile allodynia in a dose-dependent manner}

Having demonstrated that Z944 decreases SDH neuron excitability through inhibition of postsynaptic T-type channels, we next sought to determine whether Z944 can produce analgesia in a rodent complete Freund's adjuvant (CFA) model of inflammatory pain. The CFA model of inflammatory pain includes a spinal sensitization component that mediates prolonged pain hypersensitivity (Ren et al., 1992). Intraplantar injection of CFA induced a robust decrease in mechanical paw withdrawal threshold (PWT) compared to baseline measures (Day 0), corresponding to tactile allodynia, which was not observed in saline control-injected rats (Figure 4A). Three days after CFA injection, Z944 or a DMSO vehicle control were IP-injected and PWT was measured every $15 \mathrm{~min}$ for $140 \mathrm{~min}$ post-injection. The initial tested dose of Z944 (10 $\mathrm{mg} / \mathrm{kg}$ ) has previously been shown to cross the blood brain barrier and attenuate absence seizure activity in the brain (Tringham et al., 2012). We found that IP injection of Z944 significantly reversed the CFA-mediated decrease in PWT compared to vehicle injections from 50 to 125 min post-injection (Figure 4A). In males, IP injection of Z944 $(10 \mathrm{mg} / \mathrm{kg})$ resulted in $86 \pm 12 \%$ antiallodynia, while injection of a DMSO vehicle produced $3 \pm 1 \%$ anti-allodynia $(n=10$ animals for Z944, 9 animals for DMSO, Figure 4B), as measured at the peak effect ( 80 min post IP injection). 
A major barrier to the translation of potential pain therapeutics for use in humans is that the majority of preclinical investigations have typically been performed exclusively in male rodents (Mogil, 2012). As spinal mechanisms of chronic pain differ between males and females (Sorge et al., 2015) and the effects of T-type antagonists on pain sensitivity have not been compared between sexes, we next tested if inhibition of T-type channels with Z944 would reverse mechanical allodynia in CFA-injected female rats. In females, IP injection of $10 \mathrm{mg} / \mathrm{kg}$ Z944 resulted in $105 \pm 15 \%$ anti-allodynia, while injection of a DMSO vehicle produced $6 \pm 3 \%$ anti-allodynia ( $\mathrm{n}=10$ animals, Figure 4C-D). Both the time course and magnitude of antiallodynia produced by Z944 were similar between male and female CFA-injected rats (Figure 4A-D). Importantly, IP injection of Z944 had no effect on PWT in control male ( $\mathrm{n}=10$ animals, Figure 4A) and female ( $\mathrm{n}=10$ animals, Figure 4C) rats injected with intraplantar saline. This demonstrates that Z944 did not alter baseline mechanical sensitivity and thus, did not alter withdrawal responses through non-specific locomotor or sedative effects, but rather selectively reversed CFA-mediated mechanical allodynia in both sexes.

Finally, we sought to determine whether lower doses of IP-injected Z944 (1 mg/kg, 3 $\mathrm{mg} / \mathrm{kg}$ ) would reverse mechanical allodynia and whether Z944 exhibits a sigmoidal dosedependent analgesic relationship typical of many clinically available analgesics (Kuo et al., 2015). Overall, we found that all doses ( $1 \mathrm{mg} / \mathrm{kg}, 3 \mathrm{mg} / \mathrm{kg}, 10 \mathrm{mg} / \mathrm{kg})$ of Z944 produced antiallodynia in male rats $(38.6 \pm 3 \%$ for $1 \mathrm{mg} / \mathrm{kg}, 59 \pm 7 \%$ for $3 \mathrm{mg} / \mathrm{kg}, \mathrm{n}=10$ animals for both groups), but anti-allodynia was greatest for $10 \mathrm{mg} / \mathrm{kg} \mathrm{Z944,} \mathrm{indicating} \mathrm{that} \mathrm{this} \mathrm{was} \mathrm{the} \mathrm{most}$ efficacious dose examined (Figure 4E). We found that anti-allodynia could be fit to a sigmoidal curve, and obtained an $\mathrm{IC}_{50}$ of $1.76 \pm 0.14 \mathrm{mg} / \mathrm{kg}$ for the anti-allodynic effects of Z944 (Figure 
4F). Taken together, our results suggest that Z944 potently reverses tactile allodynia produced by persistent inflammation in a dose-dependent manner, across both sexes.

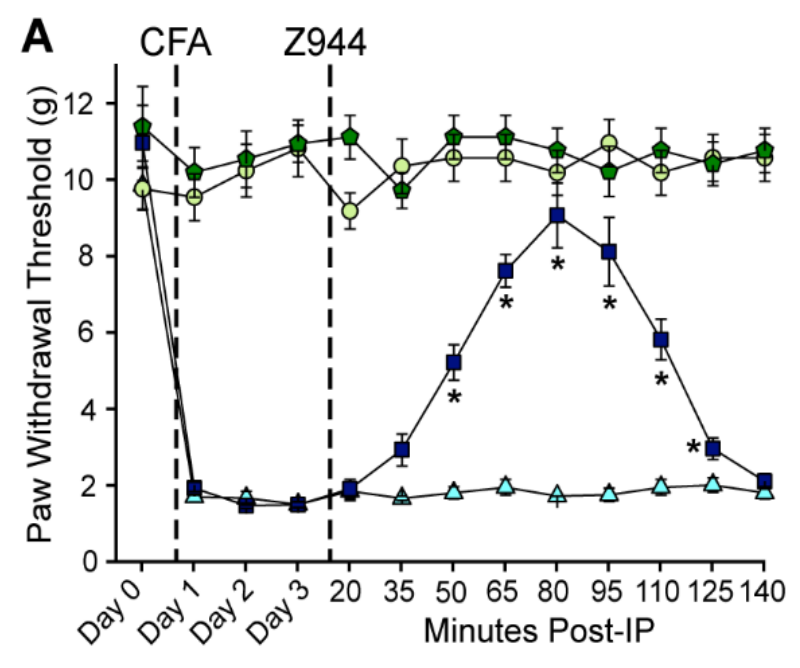

o Saline/Vehicle

- Saline/10 mg/kg Z944

$\triangle$ CFA/Vehicle

- CFA/10 mg/kg Z944

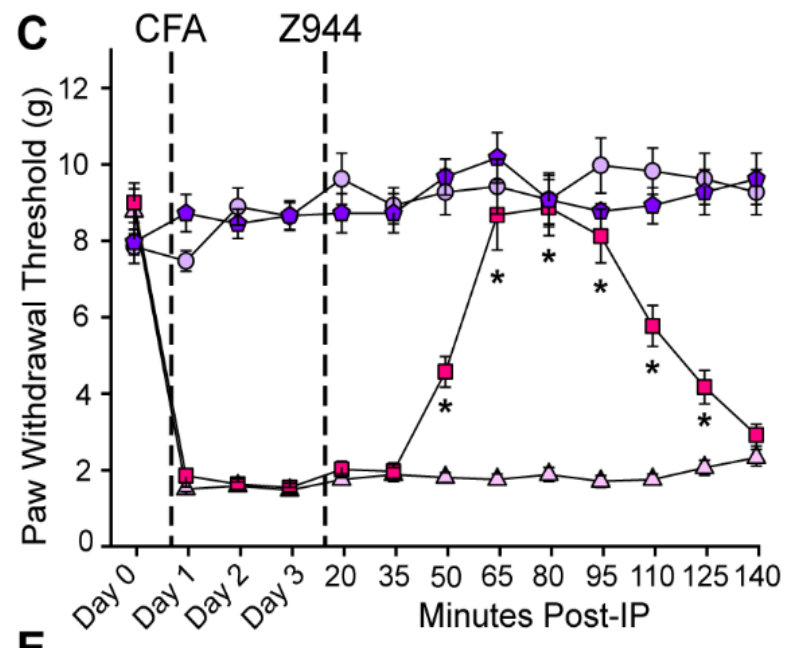

o Saline/Vehicle

- Saline/10 mg/kg Z944

$\triangle$ CFA/Vehicle

口 CFA/10 mg/kg Z944
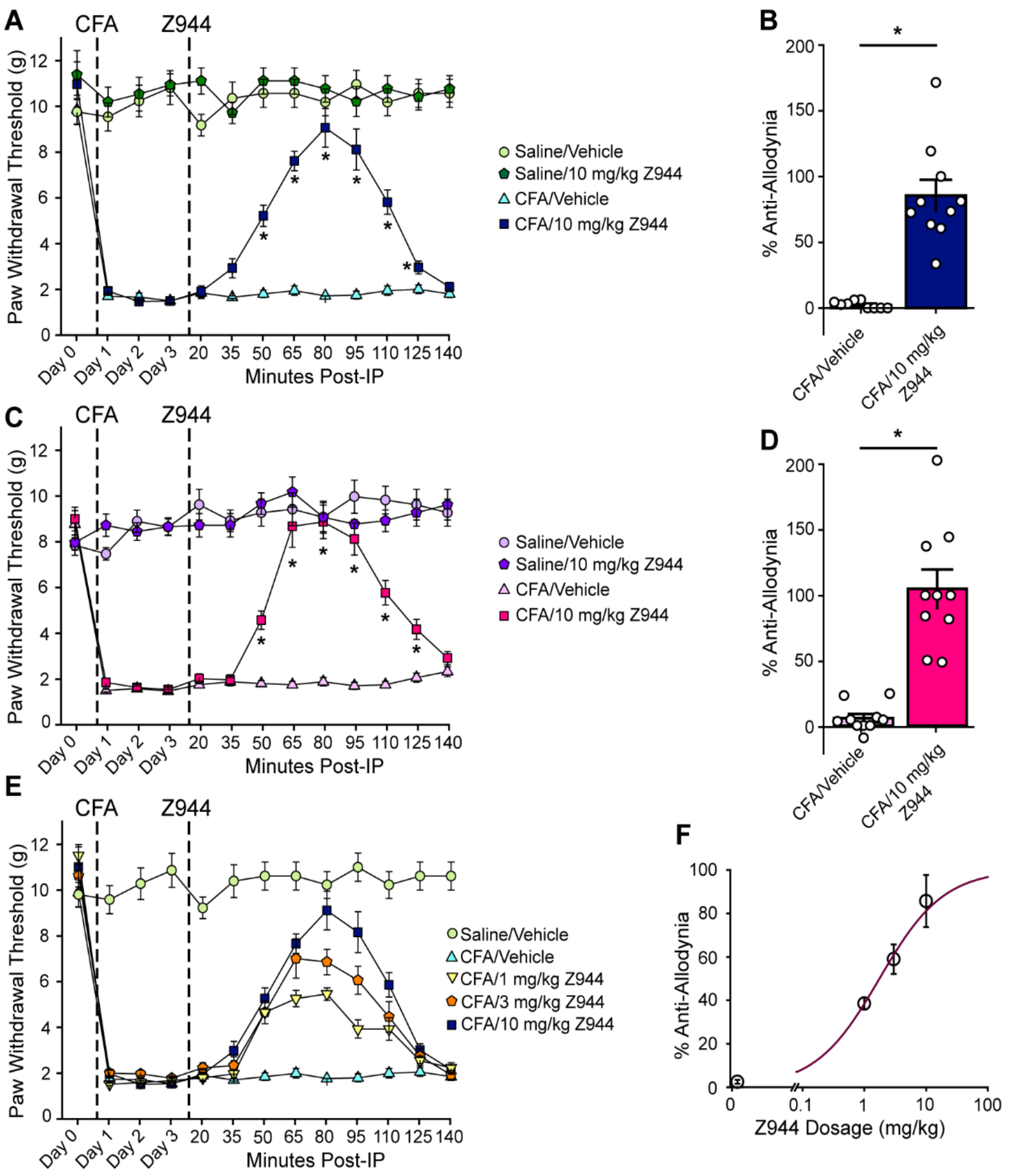


\section{Appendix B Figure 4. IP administration of Z944 reverses CFA-mediated tactile allodynia in both sexes, in a}

dose-dependent manner. A) Paw withdrawal threshold, in grams, of adult male SD rats before (Day 0$)$ and 1,2 , and 3 days following a hindpaw injection of either saline or CFA. Timepoints 20-140 min are following an IP injection of either $10 \mathrm{mg} / \mathrm{kg}$ Z944 or vehicle 3 days post-CFA injection. ( $\mathrm{n}=9$ animals for saline/vehicle and CFA/vehicle; $\mathrm{n}=10$ for saline/10 mg/kg Z944 and CFA/10 mg/kg Z944). The effect of Z944 on CFA-induced tactile allodynia (dark blue) was determined with a one-way repeated measures ANOVA $\left(\mathrm{p}=1.3 \times 10^{-11}\right)$, with Bonferroni post-hoc comparisons to the Day 3 post-CFA injection baseline paw withdrawal threshold. B) Percent anti-allodynia resulting from injection of either vehicle or $10 \mathrm{mg} / \mathrm{kg}$ Z944 in CFA injected male SD rats $\left(\mathrm{p}=4.9 \times 10^{-6}, \mathrm{Mann}-\right.$ Whitney unpaired t-test, $\mathrm{n}=9$ animals for vehicle, 10 animals for Z944). Measures taken 80 min post-IP injection. C) Paw withdrawal threshold, in grams, of adult female SD rats before (Day 0) and 1, 2, and 3 days following hindpaw injection of either saline or CFA. Timepoints 20-140 min are following IP injection of either $10 \mathrm{mg} / \mathrm{kg}$ Z944 or vehicle 3 days post-CFA injection. ( $\mathrm{n}=10$ animals for all groups). The effect of Z944 on CFA-induced tactile allodynia (dark pink) was determined with a one-way repeated measures ANOVA $\left(\mathrm{p}=3.2 \times 10^{-12}\right)$, with Bonferroni post-hoc comparisons to the Day 3 post-CFA injection paw withdrawal threshold. D) Percent anti-allodynia resulting from injection of either vehicle or $10 \mathrm{mg} / \mathrm{kg}$ Z944 in CFA injected female SD rats ( $\mathrm{p}=3.6 \times 10^{-6}, \mathrm{Mann}-$ Whitney unpaired t-test, $\mathrm{n}=10$ animals for vehicle, 10 animals for Z944). Measures taken 80 min post-IP injection.

E) Paw withdrawal threshold, in grams, of adult male SD rats before (Day 0) and 1, 2, and 3 days following a hindpaw injection of either saline or CFA. Timepoints 20-140 min are following IP injection of either 1,3 , or 10 $\mathrm{mg} / \mathrm{kg}$ Z944 or vehicle 3 days post-CFA injection. (n=10 animals for all groups). F) Percent anti-allodynia resulting from injection of either vehicle (0 mg/kg Z944) or 1, 3, or $10 \mathrm{mg} / \mathrm{kg}$ Z944 in CFA injected male SD rats. Measures taken 80 min post-IP injection. All error bars represent mean $\pm \mathrm{SEM}$. ${ }^{*} \mathrm{p}<0.05$ for all post-hoc comparisons.

\section{Discussion}

T-type calcium channels play a critical role in regulating neuronal excitability, but their role in lamina I pain processing neurons remains unclear. Furthermore, the role of T-type channels in mediating inflammatory pain across sexes has yet to be explored. Here we used a 
combination of electrophysiology, calcium imaging, and an in vivo CFA-induced inflammatory pain model in male and female rats to investigate the contributions of T-type calcium channels to pain signalling within lamina I neurons, including assessment of the effects of a selective T-type antagonist under clinical development, Z944, on lamina I neuronal excitability and CFAmediated pain hypersensitivity.

\section{T-type channels are present in a large majority of lamina I neurons}

We first sought to define the presence of T-type channels in lamina I neurons of adult rats. Voltage-clamp recordings of visually-identified lamina I neurons demonstrated a fast inactivating inward current beginning at $-60 \mathrm{mV}$ in over $80 \%$ of neurons, indicating the presence of LVA currents most likely mediated by some combination of the three isoforms of T-type channels (Cav3.1, 3.2, 3.3) (Weiss and Zamponi, 2019). Although we saw a bias towards neurons with T-type channels being of the fusiform morphology, our preliminary results could not determine if there is a significant morphological difference in neurons with and without Ttype channels, which could be used to infer population-type differences. Future studies could investigate this further by looking at the differential expression and function of T-type channel isoforms in genetically defined populations of lamina I neurons (Häring et al., 2018; Sathyamurthy et al., 2018).

In pharmacological experiments, we found that Z944 administration blocked over $50 \%$ of inward current at -60 and $-50 \mathrm{mV}$ within 5 minutes of perfusion, with no effect on inward current at $0 \mathrm{mV}$, demonstrating specificity to LVA T-type currents. These experiments provide compelling evidence for functional T-type channels in the majority of lamina I neurons. With a greater percentage of lamina I neurons (80\%) containing T-type currents compared to lamina II 
(45 to 60\%) (Wu et al., 2018; Candelas et al., 2019), these calcium channel variants may have a more prominent role in regulating the excitability of this critical subpopulation of nociceptive SDH neurons. Given that the $\mathrm{Ca}_{\mathrm{v}} 3.2$ isoform of T-type channel regulates peripheral pain processing (Bourinet et al., 2014; Todorovic \& Jevtovic-Todorovic, 2011; Weiss \& Zamponi, 2019), is preferentially expressed in the SDH of the spinal cord (Li et al., 2017b), and has recently been shown to control the excitability of lamina II spinal neurons (Candelas et al., 2019), it will also be important to investigate whether specific inhibition of postsynaptic $\mathrm{Ca}_{\mathrm{v}} 3.2$ channels in the spinal cord mediates robust analgesia.

\section{Inhibition of T-type channels in lamina I neurons reduces action potential-induced calcium transients}

Having demonstrated the presence of T-type channels in lamina I neurons, we next investigated how these channels contribute to neuronal excitability. Performing simultaneous two-photon calcium imaging while driving action potential firing in lamina I neurons showed that T-type channels contribute to over $50 \%$ of the action potential-evoked calcium response in both the soma and dendrites. In these experiments, since we were in current-clamp configuration we could not determine which neurons had an LVA component and therefore included all neurons for analysis, regardless of effect size. However, there was a high degree of variability in the percent peak remaining after the addition of Z944, ranging from $4 \%$ to $78 \%$ within the soma, and $13 \%$ to $67 \%$ within the dendrites. This heterogeneity in Z944 effect size raises the possibility that one or more neurons within this population may not have a substantial T-type component, after accounting for rundown present within the DMSO control. It is therefore likely that the true 
T-type contribution to action potential-evoked calcium responses is even larger in lamina I neurons containing T-type channels.

Our results indicate that T-type calcium channels are the predominant mediators of action potential-evoked calcium signals which, as we have previously demonstrated, represent actively backpropagating action potentials (Harding et al., 2020). Actively backpropagating action potentials shape the excitability of a neuron and have effects on both short-term and long-term plasticity. Dendritic T-type channels can drive LTP and LTD at specific hippocampal synapses (Udakis et al., 2020), and have also been shown to regulate dendritic excitability in cerebellar Purkinje neurons (Hildebrand et al., 2009) as well as in thalamocortical and thalamic reticular neurons (Connelly, W.M., Crunelli, V., and Errington, 2015). Here, we have presented the first evidence that T-type channels contribute to dendritic calcium transients in spinal lamina I neurons, suggesting that the use of Z944 could reduce the excitability of lamina I neurons, and decrease the likelihood of these neurons to undergo activity-dependent synaptic plasticity (Frick et al., 2004; Rosenkranz et al., 2009; Short et al., 2017). Our results therefore have wide implications for the understanding of spinal mechanisms of pain processing and amplification. Further studies using pharmacological inhibitors of the other VGCC classes are needed to systematically investigate the relative contribution of T-type versus HVA calcium channel classes in mediating these action potential-induced calcium responses in lamina I dendrites, as well as a potential role of dendritic T-type channels in governing lamina I neuron activitydependent plasticity.

\section{Z944 reduces superficial dorsal horn neuron excitability}

To directly examine the effect of Z944 on superficial dorsal horn neuron membrane excitability, we measured changes in rheobase in recordings from a mixed population of lamina I 
and II neurons. In other neuron types, T-type channels contribute to membrane excitability, and can promote burst firing through depolarizing the resting membrane potential, thus decreasing the current injection required to reach and sustain action potential threshold (Cain and Snutch, 2010). Here, we found that over half of superficial dorsal horn neurons responded to Z944 with a significant reduction in action potential firing in response to depolarization, suggesting that $\mathrm{T}$ type channels also contribute to membrane excitability within the superficial dorsal horn, including both lamina I and II neurons.

Together, the above experiments indicate that administration of Z944 decreases the excitability of superficial dorsal horn neurons, providing a novel, central mechanism through which T-type channel blockers may also exert their analgesic effects. These findings are of particular significance in light of recent findings demonstrating that the peripherally-restricted Ttype antagonist ABT-639 fails to show efficacy in clinical trials for pain (Serra et al., 2015; Wallace et al., 2016). This suggests that inhibition of T-type channels by Z944 in the SDH may hold future promise for effective clinical application.

\section{Z944 as a potential treatment for inflammatory pain}

Although previous studies have found that T-type antagonists can produce analgesia, these studies have relied on T-type channel blockers with significantly less selectivity, such as ethosuximide, mibefradil, and $\mathrm{NiCl}_{2}$, raising the possibility of action through inhibition of other ion channels such as HVA calcium channels and voltage-gated sodium channels (Dogrul et al., 2003; Flatters and Bennett, 2004; Feng et al., 2019). In contrast, Z944 is a high affinity T-type channel antagonist, with no significant block of HVA calcium channels or sodium channels at concentrations sufficient to block T-type channels (Tringham et al., 2012), and has been shown 
to be well tolerated in human clinical trials (Lee, 2014). We found that IP injection of Z944 produced a profound reversal of CFA-induced tactile allodynia in both male and female rats, in a clear dose-dependent manner without affecting baseline mechanical sensitivity. Z944 was well tolerated over a large therapeutic window with an $\mathrm{IC}_{50}$ of $1.76 \pm 0.14 \mathrm{mg} / \mathrm{kg}$. We are the first to demonstrate that Z944 reverses mechanical allodynia in both sexes, which is an important step in preclinical investigation.

T-type calcium channels are also expressed in peripheral DRG sensory neurons, presynaptic terminals of nociceptive primary afferents, and higher-order brain structures involved in nociceptive processing (Jacus et al., 2012; Bourinet et al., 2014; Leblanc et al., 2016). As IP injection of Z944 induces systemic exposure of Z944 to both the peripheral and central nervous systems, the analgesic efficacy of Z944 in reversing CFA-induced pain hypersensitivity could also be due to inhibition of T-type channels at these additional nociceptive loci. To directly confirm that Z944 exerts analgesic effects through acting on spinal cord T-type channels, pain thresholds and behaviours could be assessed after intrathecal injection of Z944 in rodent models of chronic pain. In combination with the above-suggested knockdown of T-type isoforms in dorsal horn neuron subpopulations, this future work would shed further light on spinal cord T-type channels as a specific target for the development of novel pain therapeutics.

In summary, we have identified low threshold calcium currents as well as somatic and dendritic calcium transients that are mediated by T-type channels in lamina I neurons of the spinal dorsal horn, which are effectively inhibited by the administration of the highly selective Ttype channel blocker Z944. Moreover, we have demonstrated that Z944 potently reverses tactile allodynia produced by persistent inflammation in a dose-dependent manner, across both sexes. Together, our findings indicate that the anti-allodynic effect of T-type channel block may be 
through inhibition of SDH neuron excitability, in addition to the previously defined peripheral mechanisms, implying a central mechanism for Z944's analgesic actions.

\section{Acknowledgements}

We thank Dr. Chris Rudyk, Rikesh Raichura, and Chris Dedek for preparing injections and blinding for IP injections. 


\section{Supplemental Figures and Tables}
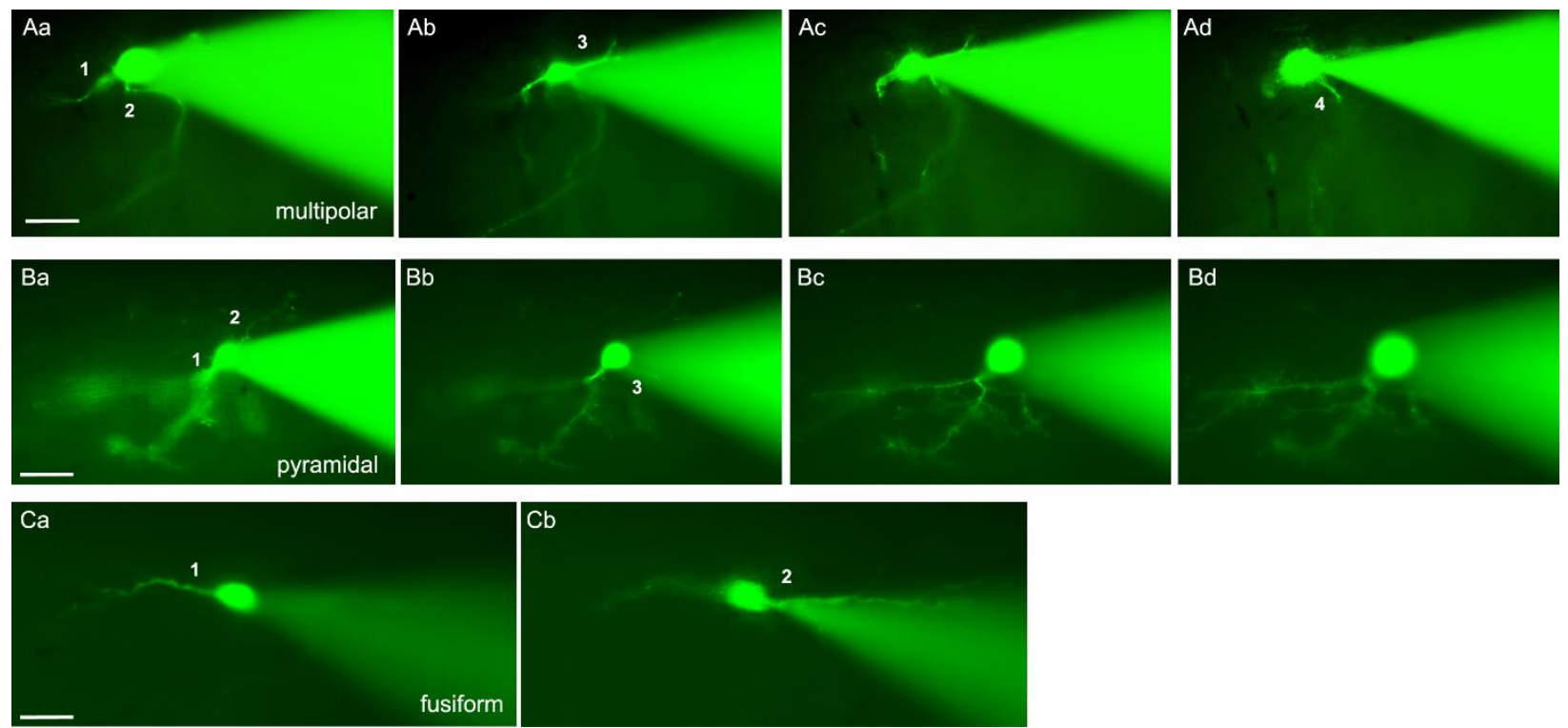

Appendix B Supplementary Figure1. Representative images of lamina I neuron morphology. Lamina I neuron morphology was defined after recordings by moving the fine focus of an epifluorescent microscope through the z-plane of AF-488-filled neurons. Samples of each morphology can be seen in A (multipolar), B (pyramidal), and C (fusiform), corresponding to four, three, and two primary dendrites, respectively. Scale bars represent $20 \mu \mathrm{m}$ (A and B), or $30 \mu \mathrm{m}(\mathrm{C})$.

Appendix B Supplementary Table 1. Repeated measures one-way ANOVAs comparing effect of treatment over time in males.

\begin{tabular}{|l|l|l|l|}
\hline & Mauchly's test of sphericity & Greenhouse-Geisser epsilon & $\begin{array}{l}\text { epsilon adjustment for test of } \\
\text { within-subjects effects }\end{array}$ \\
\hline Saline + Vehicle & Violated, $p<0.000 \mathrm{E}-36$ & 0.299 & $\mathrm{p}=0.243$ \\
\hline Saline $+10 \mathrm{mg} / \mathrm{kg}$ Z944 & Violated, $\mathrm{p}<0.000 \mathrm{E}-36$ & 0.382 & $\mathrm{p}=0.625$ \\
\hline $\mathrm{CFA}+$ Vehicle & Violated, $\mathrm{p}<0.000 \mathrm{E}-36$ & 0.187 & $\mathrm{p}=3.500 \mathrm{E}-10$ \\
\hline $\mathrm{CFA}+1 \mathrm{mg} / \mathrm{kg}$ Z944 & Violated, $\mathrm{p}<0.000 \mathrm{E}-36$ & 0.350 & $\mathrm{p}=1.329 \mathrm{E}-19$ \\
\hline $\mathrm{CFA}+3 \mathrm{mg} / \mathrm{kg}$ Z944 & Violated, $\mathrm{p}<0.000 \mathrm{E}-36$ & 0.265 & $\mathrm{p}=7.733 \mathrm{E}-12$ \\
\hline $\mathrm{CFA}+10 \mathrm{mg} / \mathrm{kg}$ Z944 & Violated, $\mathrm{p}<0.000 \mathrm{E}-36$ & 0.269 & $\mathrm{p}=1.255 \mathrm{E}-11$ \\
\hline
\end{tabular}


Appendix B Supplementary Table 2: Post-hoc Bonferroni-corrected tests for all significant one-way repeated measure ANOVAs in males, comparing day 3 (post-CFA injection) to each other time point.

\begin{tabular}{|c|c|c|c|c|}
\hline Timepoint & $\mathrm{CFA}+\mathrm{Veh}$ & $\mathrm{CFA}+1 \mathrm{mg} / \mathrm{kg}$ & $\mathrm{CFA}+3 \mathrm{mg} / \mathrm{kg}$ & $\mathrm{CFA}+10 \mathrm{mg} / \mathrm{kg}$ \\
\hline Baseline & $1.122 \mathrm{E}-4$ & $6.312 \mathrm{E}-8$ & $1.965 \mathrm{E}-6$ & $5.513 \mathrm{E}-4$ \\
\hline Day 1 & 1.000 & 1.000 & 1.000 & 1.000 \\
\hline Day 2 & 1.000 & 1.000 & 1.000 & 1.000 \\
\hline $20 \mathrm{~min}$ & 1.000 & 1.000 & 1.000 & 1.000 \\
\hline $35 \mathrm{~min}$ & 1.000 & 1.000 & 1.000 & 0.5241 \\
\hline $50 \mathrm{~min}$ & 1.000 & $1.254 \mathrm{E}-2$ & $9.896 \mathrm{E}-3$ & $1.955 \mathrm{E}-3$ \\
\hline $65 \mathrm{~min}$ & 1.000 & $1.590 \mathrm{E}-4$ & $1.248 \mathrm{E}-2$ & $1.562 \mathrm{E}-5$ \\
\hline $80 \mathrm{~min}$ & 1.000 & $4.983 \mathrm{E}-5$ & $2.964 \mathrm{E}-4$ & $5.719 \mathrm{E}-4$ \\
\hline $95 \mathrm{~min}$ & 1.000 & $5.632 \mathrm{E}-2$ & $3.035 \mathrm{E}-3$ & $3.244 \mathrm{E}-3$ \\
\hline $110 \mathrm{~min}$ & 1.000 & 0.1851 & 0.1365 & $2.041 \mathrm{E}-3$ \\
\hline $125 \mathrm{~min}$ & 1.000 & 0.5332 & 0.7234 & 4.297E-2 \\
\hline $140 \mathrm{~min}$ & 1.000 & 1.000 & 1.000 & 0.5757 \\
\hline
\end{tabular}

Appendix B Supplementary Table 3. Repeated measures one-way ANOVAs comparing effect of treatment over time in females.

\begin{tabular}{|l|l|l|l|}
\hline & Mauchly's test of sphericity & Greenhouse-Geisser epsilon & $\begin{array}{l}\text { epsilon adjustment for test of } \\
\text { within-subjects effects }\end{array}$ \\
\hline Saline + Vehicle & Violated, $\mathrm{p}<0.000 \mathrm{E}-36$ & 0.357 & $\mathrm{p}=0.057$ \\
\hline Saline $+10 \mathrm{mg} / \mathrm{kg} \mathrm{Z944}$ & Violated, $\mathrm{p}<0.000 \mathrm{E}-36$ & 0.384 & $\mathrm{p}=0.206$ \\
\hline $\mathrm{CFA}+$ Vehicle & Violated, $\mathrm{p}<0.000 \mathrm{E}-36$ & 0.232 & $\mathrm{p}=1.1508 \mathrm{E}-12$ \\
\hline $\mathrm{CFA}+10 \mathrm{mg} / \mathrm{kg} \mathrm{Z944}$ & Violated, $\mathrm{p}<0.000 \mathrm{E}-36$ & 0.310 & $\mathrm{p}=3.170 \mathrm{E}-12$ \\
\hline
\end{tabular}

Appendix B Supplementary Table 4. Post-hoc Bonferroni-corrected tests for all significant one-way repeated measure ANOVAs in females, comparing day 3 (post-CFA injection) to each other time point. 


\begin{tabular}{|l|l|l|}
\hline Timepoint & CFA + Veh & CFA $+10 \mathrm{mg} / \mathrm{kg}$ \\
\hline Baseline & $7.644 \mathrm{E}-5$ & $2.670 \mathrm{E}-5$ \\
\hline Day 1 & 1.000 & 1.000 \\
\hline Day 2 & 1.000 & 1.000 \\
\hline $20 \mathrm{~min}$ & 1.000 & 1.000 \\
\hline $35 \mathrm{~min}$ & 1.000 & 1.000 \\
\hline $50 \mathrm{~min}$ & 1.000 & $4.1648 \mathrm{E}-3$ \\
\hline $65 \mathrm{~min}$ & 1.000 & $1.2825 \mathrm{E}-3$ \\
\hline $80 \mathrm{~min}$ & 1.000 & $2.7584 \mathrm{E}-4$ \\
\hline $95 \mathrm{~min}$ & 1.000 & $6.5222 \mathrm{E}-4$ \\
\hline $110 \mathrm{~min}$ & 1.000 & $2.8250 \mathrm{E}-3$ \\
\hline $125 \mathrm{~min}$ & 1.000 & $2.8733 \mathrm{E}-2$ \\
\hline $140 \mathrm{~min}$ & 0.3724 & 0.1262 \\
\hline
\end{tabular}




\section{References}

Abe T, Matsumura S, Katano T, Mabuchi T, Takagi K, Xu L, Yamamoto A, Hattori K, Yagi T, Watanabe M, Nakazawa T, Yamamoto T, Mishina M, Nakai Y, Ito S (2005) Fyn kinasemediated phosphorylation of NMDA receptor NR2B subunit at Tyr1472 is essential for maintenance of neuropathic pain. Eur J Neurosci 22:1445-1454 Available at: http://www.ncbi.nlm.nih.gov/pubmed/16190898 [Accessed October 21, 2017].

Acker TM, Yuan H, Hansen KB, Vance KM, Ogden KK, Jensen HS, Burger PB, Mullasseril P, Snyder JP, Liotta DC, Traynelis SF (2011) Mechanism for noncompetitive inhibition by novel GluN2C/D N-methyl-D-aspartate receptor subunit-selective modulators. Mol Pharmacol 80:782-795 Available at: https://pubmed.ncbi.nlm.nih.gov/21807990/ [Accessed March 29, 2021].

Aguet F, Barbeira AN, Bonazzola R, Jo B, Kasela S, Liang Y, Parsana P, Aguet F, Battle A, Brown A, Castel SE, Engelhardt BE, Hormozdiari F, Im HK, Kim-Hellmuth S, Oliva M, Stranger BE, Wen X (2019) The GTEx Consortium atlas of genetic regulatory effects across human tissues The Genotype Tissue Expression Consortium. bioRxiv.

Ainsworth C (2015) Sex redefined. Nature 518:288-291 Available at:

http://www.nature.com/news/sex-redefined-1.16943 [Accessed April 6, 2021].

Aiyer R, Mehta N, Gungor S, Gulati A (2018) A Systematic Review of NMDA Receptor Antagonists for Treatment of Neuropathic Pain in Clinical Practice. Clin J Pain 34:450-467 Available at: https://pubmed.ncbi.nlm.nih.gov/28877137/ [Accessed April 4, 2021].

Al-Khater KM, Todd AJ (2009) Collateral projections of neurons in laminae I, III, and IV of rat spinal cord to thalamus, periaqueductal gray matter, and lateral parabrachial area. J Comp Neurol. 
Alles SRA, Odem MA, Lu VB, Cassidy RM, Smith PA (2021) Chronic BDNF simultaneously inhibits and unmasks superficial dorsal horn neuronal activity. Sci Rep 11:2249 Available at: https://doi.org/10.1038/s41598-021-81269-6 [Accessed March 9, 2021].

Alles SRA, Smith PA (2018) Etiology and Pharmacology of Neuropathic Pain Isom LL, ed. Pharmacol Rev 70:315-347.

Almeida TF, Roizenblatt S, Tufik S (2004) Afferent pain pathways: a neuroanatomical review. Brain Res 1000:40-56 Available at: https://www.sciencedirect.com/science/article/pii/S0006899304001052 [Accessed May 12, 2019].

Amandusson Å, Blomqvist A (2013) Estrogenic influences in pain processing. Front Neuroendocrinol 34:329-349.

Anon (n.d.) Organ replacement in Canada: CORR annual statistics, 2020 | CIHI. Available at: https://www.cihi.ca/en/organ-replacement-in-canada-corr-annual-statistics-2020 [Accessed March 10, 2021].

Asiedu M, Ossipov MH, Kaila K, Price TJ (2010) Acetazolamide and midazolam act synergistically to inhibit neuropathic pain. Pain 148:302-308 Available at: http://www.ncbi.nlm.nih.gov/pubmed/20007010 [Accessed October 23, 2017].

Asiedu MN, Mejia GL, Hübner CA, Kaila K, Price TJ (2014) Inhibition of carbonic anhydrase augments GABAA receptor-mediated analgesia via a spinal mechanism of action. J Pain 15:395-406 Available at: /pmc/articles/PMC3972347/ [Accessed April 2, 2021].

Avona A, Burgos-Ega C, Burton MD, Akopian AN, Price TJ, Dussor G (2019) Dural calcitonin gene-related peptide produces female-specific responses in rodent migraine models. J Neurosci 39:4323-4331 Available at: https://doi.org/10.1523/JNEUROSCI.0364-19.2019 
[Accessed March 9, 2021].

Avona A, Mason BN, Burgos-Vega C, Hovhannisyan AH, Belugin SN, Mecklenburg J, Goffin V, Wajahat N, Price TJ, Akopian AN, Dussor G (2021) Meningeal < scp >CGRPProlactin $</$ scp $>$ interaction evokes female-specific migraine behavior. Ann Neurol:ana.26070 Available at: https://onlinelibrary.wiley.com/doi/10.1002/ana.26070 [Accessed April 20, 2021].

Azkona G, Saavedra A, Aira Z, Aluja D, Xifró X, Baguley T, Alberch J, Ellman JA, Lombroso PJ, Azkue JJ, Pérez-Navarro E (2016a) Striatal-enriched protein tyrosine phosphatase modulates nociception: evidence from genetic deletion and pharmacological inhibition. PAIN® 157:377-386 Available at: https://insights.ovid.com/crossref?an=00006396201602000-00014 [Accessed March 14, 2018].

Bao Y, Hou W, Liu R, Gao Y, Kong X, Yang L, Shi Z, Li W, Zheng H, Jiang S, Li C, Qin Y, Hua B (2014) PAR2-mediated upregulation of BDNF contributes to central sensitization in bone cancer pain. Mol Pain 10:1744-8069-10-28.

Bardoni R, Magherini PC, MacDermott AB (1998) NMDA EPSCs at glutamatergic synapses in the spinal cord dorsal horn of the postnatal rat. J Neurosci 18:6558-6567 Available at: https://pubmed.ncbi.nlm.nih.gov/9698343/ [Accessed February 8, 2021].

Bartley EJ, Fillingim RB (2013) Sex differences in pain: a brief review of clinical and experimental findings. Br J Anaesth 111:52-58 Available at: http://www.ncbi.nlm.nih.gov/pubmed/23794645 [Accessed October 23, 2017].

Bartley EJ, Palit S, Kuhn BL, Kerr KL, Terry EL, Delventura JL, Rhudy JL (2015) Natural Variation in Testosterone is Associated with Hypoalgesia in Healthy Women. Clin J Pain 31:730-739 Available at: https://pubmed.ncbi.nlm.nih.gov/25185874/ [Accessed March 30, 
2021].

Beggs S, Currie G, Salter MW, Fitzgerald M, Walker SM (2012) Priming of adult pain responses by neonatal pain experience: Maintenance by central neuroimmune activity. Brain 135:404417 Available at: https://pubmed.ncbi.nlm.nih.gov/22102650/ [Accessed April 9, 2021].

Beggs S, Salter MW (2013) The known knowns of microglia-neuronal signalling in neuropathic pain. Neurosci Lett 557:37-42 Available at: https://pubmed.ncbi.nlm.nih.gov/23994389/ [Accessed April 9, 2021].

Belov Kirdajova D, Kriska J, Tureckova J, Anderova M (2020) Ischemia-Triggered Glutamate Excitotoxicity From the Perspective of Glial Cells. Front Cell Neurosci 14:51 Available at: www.frontiersin.org [Accessed March 29, 2021].

Bennett GJ, Xie YK (1988) A peripheral mononeuropathy in rat that produces disorders of pain sensation like those seen in man. Pain 33:87-107 Available at: https://pubmed.ncbi.nlm.nih.gov/2837713/ [Accessed April 1, 2021].

Bieerkehazhi S, Chen Z, Zhao Y, Yu Y, Zhang H, Vasudevan SA, Woodfield SE, Tao L, Yi JS, Muscal JA, Pang JC, Guan S, Zhang H, Nuchtern JG, Li H, Li H, Yang J (2017) Novel Src/Abl tyrosine kinase inhibitor bosutinib suppresses neuroblastoma growth via inhibiting Src/Abl signaling. Oncotarget 8:1469-1480 Available at: http://www.ncbi.nlm.nih.gov/pubmed/27903968 [Accessed January 30, 2019].

Bird GC, Han JS, Fu Y, Adwanikar H, Willis WD, Neugebauer V (2006) Pain-related synaptic plasticity in spinal dorsal horn neurons: Role of CGRP. Mol Pain 2:31 Available at: /pmc/articles/PMC1592081/?report=abstract [Accessed September 17, 2020].

Bliss TVP, Lømo T (1973) Long-lasting potentiation of synaptic transmission in the dentate area of the anaesthetized rabbit following stimulation of the perforant path. J Physiol 232:331- 
356 Available at: http://www.ncbi.nlm.nih.gov/pubmed/4727084 [Accessed May 13, 2019].

Boakye PA, Rancic V, Whitlock KH, Simmons D, Longo FM, Ballanyi K, Smith PA (2019) Receptor dependence of BDNF actions in superficial dorsal horn: Relation to central sensitization and actions of macrophage colony stimulating factor 1. J Neurophysiol 121:2308-2322 Available at: www.jn.org [Accessed March 9, 2021].

Bonin RP, Bories C, De Koninck Y (2014) A simplified up-down method (SUDO) for measuring mechanical nociception in rodents using von Frey filaments. Mol Pain 10:26 Available at: http://www.ncbi.nlm.nih.gov/pubmed/24739328 [Accessed October 21, 2017].

Bouhassira D, Lantéri-Minet M, Attal N, Laurent B, Touboul C (2008) Prevalence of chronic pain with neuropathic characteristics in the general population. Pain 136:380-387.

Boulenguez P, Liabeuf S, Bos R, Bras H, Jean-Xavier C, Brocard C, Stil A, Darbon P, Cattaert D, Delpire E, Marsala M, Vinay L (2010) Down-regulation of the potassium-chloride cotransporter KCC2 contributes to spasticity after spinal cord injury. Nat Med 16:302-307.

Bourinet E, Alloui A, Monteil A, Barrère C, Couette B, Poirot O, Pages A, McRory J, Snutch TP, Eschalier A, Nargeot J (2005) Silencing of the Cav3.2 T-type calcium channel gene in sensory neurons demonstrates its major role in nociception. EMBO J 24:315-324.

Bourinet E, Altier C, Hildebrand ME, Trang T, Salter MW, Zamponi GW (2014) CalciumPermeable Ion Channels in Pain Signaling. Physiol Rev 94:81-140 Available at: http://www.ncbi.nlm.nih.gov/pubmed/24382884 [Accessed October 23, 2017].

Braz J, Solorzano C, Wang X, Basbaum AI (2014) Transmitting Pain and Itch Messages: A Contemporary View of the Spinal Cord Circuits that Generate Gate Control. Neuron $82: 522-536$.

Cain SM, Snutch TP (2010) Contributions of T-type calcium channel isoforms to neuronal firing. 
Channels.

Cain SM, Snutch TP (2013) T-type calcium channels in burst-firing, network synchrony, and epilepsy. Biochim Biophys Acta - Biomembr 1828:1572-1578.

Cain SM, Tyson JR, Choi H-B, Ko R, Lin PJC, LeDue JM, Powell KL, Bernier L-P, Rungta RL, Yang Y, Cullis PR, O'Brien TJ, MacVicar BA, Snutch TP (2018) Ca v 3.2 drives sustained burst-firing, which is critical for absence seizure propagation in reticular thalamic neurons. Epilepsia 59:778-791.

Candelas M, Reynders A, Arango-Lievano M, Neumayer C, Fruquière A, Demes E, Hamid J, Lemmers C, Bernat C, Monteil A, Compan V, Laffray S, Inquimbert P, Le Feuvre Y, Zamponi GW, Moqrich A, Bourinet E, Méry P-F (2019) Cav3.2 T-type calcium channels shape electrical firing in mouse Lamina II neurons. Sci Rep 9:3112.

Cao J, Yang X, Liu YN, Suo ZW, Shi L, Zheng CR, Yang H Bin, Li S, Hu XD (2011) GABAergic disinhibition induced pain hypersensitivity by upregulating NMDA receptor functions in spinal dorsal horn. Neuropharmacology 60:921-929 Available at: https://pubmed.ncbi.nlm.nih.gov/21277878/ [Accessed April 2, 2021].

Casillas-Espinosa PM, Shultz SR, Braine EL, Jones NC, Snutch TP, Powell KL, O’Brien TJ (2019) Disease-modifying effects of a novel T-type calcium channel antagonist, Z944, in a model of temporal lobe epilepsy. Prog Neurobiol 182:101677.

Castonguay D, Dufort-Gervais J, Ménard C, Chatterjee M, Quirion R, Bontempi B, Schneider JS, Arnsten AFT, Nairn AC, Norris CM, Ferland G, Bézard E, Gaudreau P, Lombroso PJ, Brouillette J (2018) The Tyrosine Phosphatase STEP Is Involved in Age-Related Memory Decline. Curr Biol 28:1079-1089.e4 Available at: https://linkinghub.elsevier.com/retrieve/pii/S096098221830232X [Accessed January 30, 
2019].

Cavara NA, Hollmann M (2008) Shuffling the deck anew: how NR3 tweaks NMDA receptor function. Mol Neurobiol 38:16-26 Available at:

http://www.ncbi.nlm.nih.gov/pubmed/18654865 [Accessed April 14, 2016].

Chamessian A, Young M, Qadri Y, Berta T, Ji RR, Van De Ven T (2018) Transcriptional Profiling of Somatostatin Interneurons in the Spinal Dorsal Horn. Sci Rep 8.

Chamma I, Heubl M, Chevy Q, Renner M, Moutkine I, Eugène E, Poncer JC, Lévi S (2013) Activity-dependent regulation of the $\mathrm{K} / \mathrm{Cl}$ transporter $\mathrm{KCC} 2$ membrane diffusion, clustering, and function in hippocampal neurons. J Neurosci 33:15488-15503 Available at: https://www.jneurosci.org/content/33/39/15488 [Accessed March 28, 2021].

Chan WFN, Gurnot C, Montine TJ, Sonnen JA, Guthrie KA, Nelson JL (2012) Male Microchimerism in the Human Female Brain. PLoS One 7 Available at: https://pubmed.ncbi.nlm.nih.gov/23049819/ [Accessed April 7, 2021].

Chapman V, Honoré P, Buritova J, Besson J-M (1995) The contribution of NMDA receptor activation to spinal c-Fos expression in a model of inflammatory pain. Br J Pharmacol 116:1628-1634 Available at: http://doi.wiley.com/10.1111/j.1476-5381.1995.tb16383.x [Accessed March 2, 2021].

Chen G, Luo X, Qadri MY, Berta T, Ji R-R (2017) Sex-Dependent Glial Signaling in Pathological Pain: Distinct Roles of Spinal Microglia and Astrocytes. Neurosci Bull Available at: http://www.ncbi.nlm.nih.gov/pubmed/28585113 [Accessed October 22, 2017].

Chen S-R, Hu Y-M, Chen H, Pan H-L, Physiol J (2014a) Calcineurin inhibitor induces pain hypersensitivity by potentiating pre-and postsynaptic NMDA receptor activity in spinal cords Chen and others. J Physiol Neurosci C 592:215-227 Available at: 
https://physoc.onlinelibrary.wiley.com/doi/pdf/10.1113/jphysiol.2013.263814 [Accessed May 10, 2019].

Chen W, Walwyn W, Ennes HS, Kim H, McRoberts JA, Marvizón JCG (2014b) BDNF released during neuropathic pain potentiates NMDA receptors in primary afferent terminals. Eur J Neurosci 39:1439-1454 Available at: http://www.ncbi.nlm.nih.gov/pubmed/24611998 [Accessed March 18, 2019].

Chen W, Zhang G, Marvizón JCG (2010) NMDA receptors in primary afferents require phosphorylation by Src family kinases to induce substance P release in the rat spinal cord. Neuroscience 166:924-934 Available at: http://www.ncbi.nlm.nih.gov/pubmed/20074620 [Accessed May 15, 2019].

Cheong E, Shin H-S (2013) T-TYPE Ca 2 CHANNELS IN NORMAL AND ABNORMAL BRAIN FUNCTIONS. Physiol Rev 93:961-992.

Choi J Il, Svensson CI, Koehrn FJ, Bhuskute A, Sorkin LS (2010) Peripheral inflammation induces tumor necrosis factor dependent AMPA receptor trafficking and Akt phosphorylation in spinal cord in addition to pain behavior. Pain 149:243-253 Available at: https://www.sciencedirect.com/science/article/pii/S0304395910000837?casa_token=bKrCX RSjbesAAAAA:Htlfa_8o0YhCOIDVXpU5Z1TNw5vulRaFSnHgileFeV0O4sjt4TBxq2DZ1 KjvUZ3uIELwCE73cA [Accessed April 3, 2021].

Choi S, Hachisuka J, Brett MA, Magee AR, Omori Y, Iqbal N ul A, Zhang D, DeLisle MM, Wolfson RL, Bai L, Santiago C, Gong S, Goulding M, Heintz N, Koerber HR, Ross SE, Ginty DD (2020) Parallel ascending spinal pathways for affective touch and pain. Nature 587:258-263 Available at: https://www.nature.com/articles/s41586-020-2860-1 [Accessed April 4, 2021]. 
Chowdhury D, Marco S, Brooks IM, Zandueta A, Rao Y, Haucke V, Wesseling JF, Tavalin SJ, Perez-Otano I (2013) Tyrosine Phosphorylation Regulates the Endocytosis and Surface Expression of GluN3A-Containing NMDA Receptors. J Neurosci.

Collins S, Sigtermans MJ, Dahan A, Zuurmond WWA, Perez RSGM (2010) NMDA Receptor Antagonists for the Treatment of Neuropathic Pain. Pain Med 11:1726-1742 Available at: https://pubmed.ncbi.nlm.nih.gov/21044263/ [Accessed April 4, 2021].

Connelly, W.M., Crunelli, V., and Errington AC (2015) The Global Spike: Conserved Dendritic Properties Enable Unique Ca2 Spike Generation in Low-Threshold Spiking Neurons. J Neurosci 35:15505-15522.

Copits BA, Pullen MY, Gereau RW (2016) Spotlight on pain: Optogenetic approaches for interrogating somatosensory circuits. Pain 157:2424-2433 Available at: /pmc/articles/PMC5069102/ [Accessed March 28, 2021].

Costigan M, Woolf CJ (2000) Pain: Molecular mechanisms. In: Journal of Pain, pp 35-44. Churchill Livingstone Inc.

Coull JAM, Boudreau D, Bachand K, Prescott SA, Nault F, Sík A, De Koninck P, De Koninck Y (2003) Trans-synaptic shift in anion gradient in spinal lamina I neurons as a mechanism of neuropathic pain. Nature 424:938-942 Available at: www.nature.com/nature. [Accessed January 20, 2021].

Coull JAMM, Beggs S, Boudreau D, Boivin D, Tsuda M, Inoue K, Gravel C, Salter MW, De Koninck Y (2005) BDNF from microglia causes the shift in neuronal anion gradient underlying neuropathic pain. Nature 438:1017-1021 Available at: http://www.nature.com/articles/nature04223 [Accessed October 21, 2017]. Craft RM (2007) Modulation of pain by estrogens. Pain 132:S3. 
Crair MC, Malenka RC (1995) A critical period for long-term potentiation at thalamocortical synapses. Nature 375:325-328 Available at: https://pubmed.ncbi.nlm.nih.gov/7753197/ [Accessed February 8, 2021].

Curtis MJ, Alexander S, Cirino G, Docherty JR, George CH, Giembycz MA, Hoyer D, Insel PA, Izzo AA, Ji Y, MacEwan DJ, Sobey CG, Stanford SC, Teixeira MM, Wonnacott S, Ahluwalia A (2018) Experimental design and analysis and their reporting II: updated and simplified guidance for authors and peer reviewers. Br J Pharmacol 175:987-993 Available at: http://doi.wiley.com/10.1111/bph.14153 [Accessed February 11, 2021].

Dalal PK, Agarwal M (2015) Postmenopausal syndrome. Indian J Psychiatry 57:222-232 Available at: /pmc/articles/PMC4539866/ [Accessed March 30, 2021].

Darbon P, Pignier C, Niggli E, Streit J (2002) Involvement of calcium in rhythmic activity induced by disinhibition in cultured spinal cord networks. J Neurophysiol 88:1461-1468 Available at: https://pubmed.ncbi.nlm.nih.gov/12205166/ [Accessed April 9, 2021].

Davidson S, Copits BA, Zhang J, Page G, Ghetti A, Gereau RW (2014) Human sensory neurons: Membrane properties and sensitization by inflammatory mediators. Pain 155:1861-1870.

Decosterd I, Woolf CJ (2000) Spared nerve injury: An animal model of persistent peripheral neuropathic pain. Pain 87:149-158 Available at: https://pubmed.ncbi.nlm.nih.gov/10924808/ [Accessed April 1, 2021].

Dedek A, Xu J, Kandegedara CMCM, Lorenzo L-É, Godin AG, De Koninck Y, Lombroso PJ, Tsai EC, Hildebrand ME (2019) Loss of STEP61 couples disinhibition to N-methyl-Daspartate receptor potentiation in rodent and human spinal pain processing. Brain 142:15351546 Available at: https://academic.oup.com/brain/article/142/6/1535/5498995 [Accessed September 21, 2020]. 
Del Valle L, Schwartzman RJ, Alexander G (2009) Spinal cord histopathological alterations in a patient with longstanding complex regional pain syndrome. Brain Behav Immun 23:85-91 Available at: http://linkinghub.elsevier.com/retrieve/pii/S0889159108003413 [Accessed February 28, 2018].

Delpire E, Days E, Lewis LM, Mi D, Kim K, Lindsley CW, Weaver CD (2009) Small-molecule screen identifies inhibitors of the neuronal K-Cl cotransporter KCC2. Proc Natl Acad Sci 106:5383-5388 Available at: http://www.ncbi.nlm.nih.gov/pubmed/19279215 [Accessed November 17, 2017].

Dickie AC, Bell AM, Iwagaki N, Polgár E, Gutierrez-Mecinas M, Kelly R, Lyon H, Turnbull K, West SJ, Etlin A, Braz J, Watanabe M, Bennett DLH, Basbaum AI, Riddell JS, Todd AJ (2019) Morphological and functional properties distinguish the substance P and gastrinreleasing peptide subsets of excitatory interneuron in the spinal cord dorsal horn. Pain 160:442-462 Available at: /pmc/articles/PMC6330098/ [Accessed April 4, 2021].

Dickie AC, McCormick B, Lukito V, Wilson KL, Torsney C (2017) Inflammatory Pain Reduces C Fiber Activity-Dependent Slowing in a Sex-Dependent Manner, Amplifying Nociceptive Input to the Spinal Cord. J Neurosci 37:6488-6502 Available at: http://www.ncbi.nlm.nih.gov/pubmed/28576935 [Accessed October 21, 2017].

Dickinson T, Mitchell R, Robberecht P, Fleetwood-Walker SM (1999) The role of VIP/PACAP receptor subtypes in spinal somatosensory processing in rats with an experimental peripheral mononeuropathy. Neuropharmacology 38:167-180.

Diehl B, Hoheisel U, Mense S (1988) Histological and neurophysiological changes induced by carrageenan in skeletal muscle of cat and rat. Agents Actions 25:210-213 Available at: https://link.springer.com/article/10.1007/BF01965013 [Accessed April 1, 2021]. 
Dina OA, Aley KO, Isenberg W, Messing RO, Levine JD (2001) Sex hormones regulate the contribution of PKC $\varepsilon$ and PKA signalling in inflammatory pain in the rat. Eur J Neurosci 13:2227-2233 Available at: http://doi.wiley.com/10.1046/j.0953-816x.2001.01614.x [Accessed March 23, 2021].

Dina OA, Gear RW, Messing RO, Levine JD (2007) Severity of alcohol-induced painful peripheral neuropathy in female rats: Role of estrogen and protein kinase (A and $\mathrm{C} \varepsilon$ ). Neuroscience 145:350-356 Available at: /pmc/articles/PMC1817724/ [Accessed March 23, 2021].

Dinh A, Berger A, Krane E (2021) Analgesic Drug Development for Children: A History of Shortcomings ... Until Now. J Pain Res Volume 14:867-870 Available at: https://www.dovepress.com/analgesic-drug-development-for-children-a-history-ofshortcomings-hell-peer-reviewed-article-JPR [Accessed March 30, 2021].

Dogrul A, Gardell LR, Ossipov MH, Tulunay FC, Lai J, Porreca F (2003) Reversal of experimental neuropathic pain by T-type calcium channel blockers. Pain.

Doyon N, Prescott SA, Castonguay A, Godin AG, Kröger H, de Koninck Y (2011) Efficacy of synaptic inhibition depends on multiple, dynamically interacting mechanisms implicated in chloride homeostasis. PLoS Comput Biol 7 Available at: https://pubmed.ncbi.nlm.nih.gov/21931544/ [Accessed April 9, 2021].

Du H, Shi J, Wang M, An S, Guo X, Wang Z (2018) Analyses of gene expression profiles in the rat dorsal horn of the spinal cord using RNA sequencing in chronic constriction injury rats. J Neuroinflammation 15:280 Available at: https://jneuroinflammation.biomedcentral.com/articles/10.1186/s12974-018-1316-0 [Accessed April 3, 2021]. 
Dubuisson D, Dennis SG (1977) The formalin test: A quantitative study of the analgesic effects of morphine, meperidine, and brain stem stimulation in rats and cats. Pain 4:161-174.

Dunah AW, Yasuda RP, Luo J, Wang Y, Prybylowski KL, Wolfe BB (1999) Biochemical studies of the structure and function of the N-methyl-D-aspartate subtype of glutamate receptors. Mol Neurobiol 19:151-179 Available at: http://www.ncbi.nlm.nih.gov/pubmed/10371467 [Accessed April 14, 2016].

Dunah AW, Yasuda RP, Wang Y, Luo J, Dávila-García MI, Gbadegesin M, Vicini S, Wolfe BB (2002) Regional and Ontogenic Expression of the NMDA Receptor Subunit NR2D Protein in Rat Brain Using a Subunit-Specific Antibody. J Neurochem.

Dunah AW, Yasuda RP, Wolfe BB (1998) Developmental regulation of tyrosine phosphorylation of the NR2D NMDA glutamate receptor subunit in rat central nervous system. J Neurochem 71:1926-1934 Available at: http://www.ncbi.nlm.nih.gov/pubmed/9798917 [Accessed July 13, 2019].

Edwards RH (2007) The Neurotransmitter Cycle and Quantal Size. Neuron 55:835-858 Available at: http://www.cell.com/article/S089662730700668X/fulltext [Accessed April 3, 2021].

Elfarrash S, Jensen NM, Ferreira N, Betzer C, Thevathasan JV, Diekmann R, Adel M, Omar NM, Boraie MZ, Gad S, Ries J, Kirik D, Nabavi S, Jensen PH (2019) Organotypic slice culture model demonstrates inter-neuronal spreading of alpha-synuclein aggregates. Acta Neuropathol Commun 7:213 Available at: https://actaneurocomms.biomedcentral.com/articles/10.1186/s40478-019-0865-5 [Accessed March 29, 2021].

Engelman HS, Allen TB, MacDermott AB (1999) The distribution of neurons expressing 
calcium-permeable AMPA receptors in the superficial laminae of the spinal cord dorsal horn. J Neurosci 19:2081-2089 Available at: https://pubmed.ncbi.nlm.nih.gov/10066261/ [Accessed February 8, 2021].

Enright HA, Felix SH, Fischer NO, Mukerjee E V., Soscia D, McNerney M, Kulp K, Zhang J, Page G, Miller P, Ghetti A, Wheeler EK, Pannu S (2016) Long-term non-invasive interrogation of human dorsal root ganglion neuronal cultures on an integrated microfluidic multielectrode array platform. Analyst 141:5346-5357.

Erreger K, Dravid SM, Banke TG, Wyllie DJA, Traynelis SF (2005) Subunit-specific gating controls rat NR1/NR2A and NR1/NR2B NMDA channel kinetics and synaptic signalling profiles. J Physiol.

Ewald RC, Cline HT (2009) NMDA Receptors and Brain Development. Available at: http://www.ncbi.nlm.nih.gov/pubmed/21204419 [Accessed July 16, 2019].

Fauchon C, Meunier D, Rogachov A, Hemington KS, Cheng JC, Bosma RL, Osborne NR, Kim JA, Hung PS-P, Inman RD, Davis KD (2021) Sex differences in brain modular organization in chronic pain. Pain 162:1188-1200 Available at: https://journals.lww.com/10.1097/j.pain.0000000000002104 [Accessed March 23, 2021].

Feng XJ, Ma LX, Jiao C, Kuang HX, Zeng F, Zhou XY, Cheng XE, Zhu MY, Zhang DY, Jiang CY, Liu T (2019) Nerve injury elevates functional Cav3.2 channels in superficial spinal dorsal horn. Mol Pain 15:1744806919836569.

Ferrini F, De Koninck Y (2013) Microglia control neuronal network excitability via BDNF signalling. Neural Plast 2013.

Ferrini F, Lorenzo LE, Godin AG, Quang M Le, De Koninck Y (2017) Enhancing KCC2 function counteracts morphine-induced hyperalgesia. Sci Rep 7 Available at: 
https://pubmed.ncbi.nlm.nih.gov/28634406/ [Accessed March 24, 2021].

Ferrini F, Perez-Sanchez J, Ferland S, Lorenzo LE, Godin AG, Plasencia-Fernandez I, Cottet M, Castonguay A, Wang F, Salio C, Doyon N, Merighi A, De Koninck Y (2020) Differential chloride homeostasis in the spinal dorsal horn locally shapes synaptic metaplasticity and modality-specific sensitization. Nat Commun 11:1-18 Available at: https://doi.org/10.1038/s41467-020-17824-y [Accessed February 21, 2021].

Finnerup NB, Kuner R, Jensen TS (2021) Neuropathic pain: Frommechanisms to treatment. Physiol Rev 101:259-301 Available at: https://doi.org/10.1152/physrev.00045.2019 [Accessed April 3, 2021].

Fischer G, Mutel V, Trube G, Malherbe P, Kew JN, Mohacsi E, Heitz MP, Kemp JA (1997) Ro 25-6981, a highly potent and selective blocker of N-methyl-D-aspartate receptors containing the NR2B subunit. Characterization in vitro. J Pharmacol Exp Ther 283:12851292 Available at: http://www.ncbi.nlm.nih.gov/pubmed/9400004 [Accessed November 17, 2017].

Flatters SJL, Bennett GJ (2004) Ethosuximide reverses paclitaxel- and vincristine-induced painful peripheral neuropathy. Pain.

Fox CJ, Russell KI, Wang YT, Christie BR (2006) Contribution of NR2A and NR2B NMDA subunits to bidirectional synaptic plasticity in the hippocampus in vivo. Hippocampus 16:907-915 Available at: https://pubmed.ncbi.nlm.nih.gov/17024679/ [Accessed April 3, 2021].

François A, Schüetter N, Laffray S, Sanguesa J, Pizzoccaro A, Dubel S, Mantilleri A, Nargeot J, Noël J, Wood JN, Moqrich A, Pongs O, Bourinet E (2015) The Low-Threshold Calcium Channel Cav3.2 Determines Low-Threshold Mechanoreceptor Function. Cell Rep 10:370- 
382.

Frick A, Magee J, Johnston D (2004) LTP is accompanied by an enhanced local excitability of pyramidal neuron dendrites. Nat Neurosci 7:126-135 Available at:

https://pubmed.ncbi.nlm.nih.gov/14730307/ [Accessed October 28, 2020].

Fukami K, Sekiguchi F, Kawabata A (2017) Hydrogen Sulfide and T-Type Ca2+ Channels in Pain Processing, Neuronal Differentiation and Neuroendocrine Secretion. Pharmacology 99:196-203.

Gagnon M, Bergeron MJ, Lavertu G, Castonguay A, Tripathy S, Bonin RP, Perez-Sanchez J, Boudreau D, Wang B, Dumas L, Valade I, Bachand K, Jacob-Wagner M, Tardif C, Kianicka I, Isenring P, Attardo G, Coull JAM, De Koninck Y (2013) Chloride extrusion enhancers as novel therapeutics for neurological diseases. Nat Med 19:1524-1528

Available at: http://www.ncbi.nlm.nih.gov/pubmed/24097188 [Accessed October 23, 2017].

García-Caballero A, Gadotti VM, Stemkowski P, Weiss N, Souza IA, Hodgkinson V, Bladen C, Chen L, Hamid J, Pizzoccaro A, Deage M, François A, Bourinet E, Zamponi GW (2014) The deubiquitinating enzyme USP5 modulates neuropathic and inflammatory pain by enhancing Cav3.2 channel activity. Neuron 83:1144-1158.

Garraway SM, Xu Q, Inturrisi CE (2007) Design and Evaluation of Small Interfering RNAs That Target Expression of the N-Methyl-D-aspartate Receptor NR1 Subunit Gene in the Spinal Cord Dorsal Horn. J Pharmacol Exp Ther 322:982-988 Available at: http://www.ncbi.nlm.nih.gov/pubmed/17551091 [Accessed May 14, 2019].

Gatto G, Smith KM, Ross SE, Goulding M (2019) Neuronal diversity in the somatosensory system: bridging the gap between cell type and function. Curr Opin Neurobiol 56:167-174 Available at: /pmc/articles/PMC6583900/ [Accessed April 4, 2021]. 
Gereau RW, Sluka KA, Maixner W, Savage SR, Price TJ, Murinson BB, Sullivan MD, Fillingim RB (2014) A pain research agenda for the 21st century. J Pain 15:1203-1214.

Geus TJ, Patijn J, Joosten EAJ (2020) Qualitative review on N-methyl-D-aspartate receptor expression in rat spinal cord during the postnatal development: Implications for central sensitization and pain. Dev Neurobiol 80:443-455 Available at: https://onlinelibrary.wiley.com/doi/10.1002/dneu.22789 [Accessed April 6, 2021].

Gewandter JS et al. (2020) Improving Study Conduct and Data Quality in Clinical Trials of Chronic Pain Treatments: IMMPACT Recommendations. J Pain 21:931-942.

Gibson SJ, Polak JM, Bloom SR, Sabate IM, Mulderry PM, Ghatei MA, McGregor GP, Morrison JF, Kelly JS, Evans RM (1984) Calcitonin gene-related peptide immunoreactivity in the spinal cord of man and of eight other species. J Neurosci 4:3101-3111 Available at: http://www.ncbi.nlm.nih.gov/pubmed/6209366 [Accessed July 6, 2018].

Gintzler AR (1980) Endorphin-mediated increases in pain threshold during pregnancy. Science (80- ) 210:193-195 Available at: https://pubmed.ncbi.nlm.nih.gov/7414330/ [Accessed March 30, 2021].

Gladding CM, Fan J, Zhang LYJ, Wang L, Xu J, Li EHY, Lombroso PJ, Raymond LA (2014) Alterations in STriatal-Enriched protein tyrosine Phosphatase expression, activation, and downstream signaling in early and late stages of the YAC128 Huntington's disease mouse model. J Neurochem 130:145-159 Available at: http://www.ncbi.nlm.nih.gov/pubmed/24588402 [Accessed January 30, 2019]. Goebel-Goody SM, Baum M, Paspalas CD, Fernandez SM, Carty NC, Kurup P, Lombroso PJ (2012) Therapeutic implications for striatal-enriched protein tyrosine phosphatase (STEP) in neuropsychiatric disorders. Pharmacol Rev 64:65-87 Available at: 
https://pubmed.ncbi.nlm.nih.gov/22090472/ [Accessed April 3, 2021].

Goebel-Goody SM, Davies KD, Alvestad Linger RM, Freund RK, Browning MD (2009)

Phospho-regulation of synaptic and extrasynaptic N-methyl-d-aspartate receptors in adult hippocampal slices. Neuroscience 158:1446-1459 Available at:

http://www.ncbi.nlm.nih.gov/pubmed/19041929 [Accessed July 13, 2019].

Gold MS, Gebhart GF (2010) Nociceptor sensitization in pain pathogenesis. Available at: http://www.nature.com/articles/nm.2235 [Accessed March 18, 2019].

Gomez K, Calderón-Rivera A, Sandoval A, González-Ramírez R, Vargas-Parada A, OjedaAlonso J, Granados-Soto V, Delgado-Lezama R, Felix R (2020) Cdk5-Dependent Phosphorylation of CaV3.2 T-Type Channels: Possible Role in Nerve Ligation-Induced Neuropathic Allodynia and the Compound Action Potential in Primary Afferent C Fibers. J Neurosci 40:283-296.

Gong N, Hagopian G, Holmes TC, Luo ZD, Xu X (2019) Functional reorganization of local circuit connectivity in superficial spinal dorsal horn with neuropathic pain states. eNeuro 6 Available at: https://pubmed.ncbi.nlm.nih.gov/31533959/ [Accessed April 3, 2021].

Goswami SC, Thierry-Mieg D, Thierry-Mieg J, Mishra S, Hoon MA, Mannes AJ, Iadarola MJ (2014) Itch-Associated Peptides: RNA-Seq and Bioinformatic Analysis of Natriuretic Precursor Peptide B and Gastrin Releasing Peptide in Dorsal Root and Trigeminal Ganglia, and the Spinal Cord. Mol Pain 10:1744-8069-10-44 Available at: http://journals.sagepub.com/doi/10.1186/1744-8069-10-44 [Accessed October 23, 2017]. Gracely RH, Lynch SA, Bennett GJ (1992) Painful neuropathy: altered central processing maintained dynamically by peripheral input. Pain 51:175-194 Available at: https://www.sciencedirect.com/science/article/pii/030439599290259E [Accessed May 12, 
2019].

Grand T, Abi Gerges S, David M, Diana MA, Paoletti P (2018) Unmasking GluN1/GluN3A excitatory glycine NMDA receptors. Nat Commun 9:1-12 Available at: www.nature.com/naturecommunications [Accessed March 29, 2021].

Gray JA, Shi Y, Usui H, During MJ, Sakimura K, Nicoll RA (2011) Distinct Modes of AMPA Receptor Suppression at Developing Synapses by GluN2A and GluN2B: Single-Cell NMDA Receptor Subunit Deletion In Vivo. Neuron 71:1085-1101 Available at: http://www.cell.com/article/S089662731100688X/fulltext [Accessed April 6, 2021].

Greenspon CM, Battell EE, Devonshire IM, Donaldson LF, Chapman V, Hathway GJ (2019) Lamina-specific population encoding of cutaneous signals in the spinal dorsal horn using multi-electrode arrays. J Physiol 597:377-397 Available at: https://onlinelibrary.wiley.com/doi/abs/10.1113/JP277036 [Accessed March 12, 2021]. Grichnik KP, Ferrante FM (1991) The difference between acute and chronic pain. Mt Sinai J Med 58:217-220 Available at: http://www.ncbi.nlm.nih.gov/pubmed/1875958 [Accessed May 12, 2019].

Griffin JM, Fackelmeier B, Fong DM, Mouravlev A, Young D, O’Carroll SJ (2019) Astrocyteselective AAV gene therapy through the endogenous GFAP promoter results in robust transduction in the rat spinal cord following injury. Gene Ther 26:198-210 Available at: https://doi.org/10.1038/s41434-019-0075-6 [Accessed March 29, 2021].

Groveman BR, Feng S, Fang X-Q, Pflueger M, Lin S-X, Bienkiewicz EA, Yu X (2012) The regulation of N-methyl-d-aspartate receptors by Src kinase. FEBS J 279:20-28 Available at: http://www.ncbi.nlm.nih.gov/pubmed/22060915 [Accessed October 21, 2017].

Gutierrez-Mecinas M, Furuta T, Watanabe M, Todd AJ (2016) A quantitative study of 
neurochemically defined excitatory interneuron populations in laminae I-III of the mouse spinal cord. Mol Pain 12 Available at: /pmc/articles/PMC4946630/ [Accessed April 4, 2021].

Hachisuka J, Koerber HR, Ross SE (2020) Selective-cold output through a distinct subset of lamina I spinoparabrachial neurons. Pain 161:185-194 Available at: https://journals.lww.com/10.1097/j.pain.0000000000001710 [Accessed April 4, 2021].

Hama A, Sagen J (2012) Combinations of intrathecal gamma-amino-butyrate receptor agonists and N-methyl-d-aspartate receptor antagonists in rats with neuropathic spinal cord injury pain. Eur J Pharmacol 683:101-108.

Hama AT, Toide K, Takamatsu H (2013) Beyond rodent models of pain: non-human primate models for evaluating novel analgesic therapeutics and elaborating pain mechanisms. CNS Neurol Disord Drug Targets 12:1257-1270 Available at: http://www.ncbi.nlm.nih.gov/pubmed/24111837 [Accessed October 23, 2017].

Han JS, Adwanikar H, Li Z, Ji G, Neugebauer V (2010) Facilitation of synaptic transmission and pain responses by CGRP in the amygdala of normal rats. Mol Pain 6:10 Available at: /pmc/articles/PMC2829526/?report=abstract [Accessed September 22, 2020].

Hanke JH, Gardner JP, Dow RL, Changelian PS, Brissette WH, Weringer EJ, Pollok BA, Connelly PA (1996) Discovery of a novel, potent, and Src family-selective tyrosine kinase inhibitor: Study of Lck- and FynT-dependent T cell activation. J Biol Chem 271:695-701 Available at: http://www.jbc.org/article/S002192581895489X/fulltext [Accessed February 21, 2021].

Hansen KB, Ogden KK, Traynelis SF (2012) Subunit-selective allosteric inhibition of glycine binding to NMDA receptors. J Neurosci 32:6197-6208 Available at: 
http://www.ncbi.nlm.nih.gov/pubmed/22553026 [Accessed November 17, 2017].

Hansen KB, Yi F, Perszyk RE, Furukawa H, Wollmuth LP, Gibb AJ, Traynelis SF (2018)

Structure, function, and allosteric modulation of NMDA receptors. J Gen Physiol

150:1081-1105 Available at: http://www.ncbi.nlm.nih.gov/pubmed/30037851 [Accessed

May 13, 2019].

Harding EK, Boivin B, Salter MW (2020) Intracellular calcium responses encode action potential firing in spinal cord lamina I neurons. J Neurosci 40:4439-4456 Available at: https://doi.org/10.1523/JNEUROSCI.0206-20.2020 [Accessed February 23, 2021].

Häring M, Zeisel A, Hochgerner H, Rinwa P, Jakobsson JETT, Lönnerberg P, La Manno G, Sharma N, Borgius L, Kiehn O, Lagerström MC, Linnarsson S, Ernfors P (2018) Neuronal atlas of the dorsal horn defines its architecture and links sensory input to transcriptional cell types. Nat Neurosci 21:869-880 Available at: https://doi.org/10.1038/s41593-018-0141-1 [Accessed April 4, 2021].

Hartmann B, Ahmadi S, Heppenstall PA, Lewin GR, Schott C, Borchardt T, Seeburg PH, Zeilhofer HU, Sprengel R, Kuner R (2004) The AMPA receptor subunits GluR-A and GluR-B reciprocally modulate spinal synaptic plasticity and inflammatory pain. Neuron 44:637-650 Available at: https://pubmed.ncbi.nlm.nih.gov/15541312/ [Accessed February $8,2021]$.

Haugan F, Rygh LJ, Tjølsen A (2008) Ketamine blocks enhancement of spinal long-term potentiation in chronic opioid treated rats. Acta Anaesthesiol Scand 52:681-687.

Henson MA, Roberts AC, Pérez-Otaño I, Philpot BD (2010) Influence of the NR3A subunit on NMDA receptor functions. Prog Neurobiol 91:23-37 Available at:

http://www.pubmedcentral.nih.gov/articlerender.fcgi?artid=2883719\&tool=pmcentrez\&ren 
dertype $=$ abstract $[$ Accessed April 14, 2016].

Hildebrand ME, Isope P, Miyazaki T, Nakaya T, Garcia E, Feltz A, Schneider T, Hescheler J, Kano M, Sakimura K, Watanabe M, Dieudonné S, Snutch TP (2009) Functional coupling between mGluR1 and Cav3.1 T-type calcium channels contributes to parallel fiber-induced fast calcium signaling within Purkinje cell dendritic spines. J Neurosci 29:9668-9682.

Hildebrand ME, Pitcher GM, Harding EK, Li H, Beggs S, Salter MW (2014) GluN2B and GluN2D NMDARs dominate synaptic responses in the adult spinal cord. Sci Rep 4:4094 Available at: http://www.ncbi.nlm.nih.gov/pubmed/24522697 [Accessed October 21, 2017].

Hildebrand MEME, Xu J, Dedek A, Li YY, Sengar ASASAS, Beggs S, Lombroso PJPJ, Salter MWMWMW (2016) Potentiation of Synaptic GluN2B NMDAR Currents by Fyn Kinase Is Gated through BDNF-Mediated Disinhibition in Spinal Pain Processing. Cell Rep 17:27532765 Available at: http://www.ncbi.nlm.nih.gov/pubmed/27926876 [Accessed October 21, 2017].

Ho Kim S, Mo Chung J (1992) An experimental model for peripheral neuropathy produced by segmental spinal nerve ligation in the rat. Pain 50:355-363 Available at: https://pubmed.ncbi.nlm.nih.gov/1333581/ [Accessed April 1, 2021].

Honore P, Rogers SD, Schwei MJ, Salak-Johnson JL, Luger NM, Sabino MC, Clohisy DR, Mantyh PW (2000) Murine models of inflammatory, neuropathic and cancer pain each generates a unique set of neurochemical changes in the spinal cord and sensory neurons. Neuroscience 98:585-598.

Hornung RS, Benton WL, Tongkhuya S, Uphouse L, Kramer PR, Averitt DL (2020) Progesterone and Allopregnanolone Rapidly Attenuate Estrogen-Associated Mechanical Allodynia in Rats with Persistent Temporomandibular Joint Inflammation. Front Integr 
Neurosci 14:26 Available at: www.frontiersin.org [Accessed March 30, 2021].

Hou X, Weng Y, Wang T, Ouyang B, Li Y, Song Z, Pan Y, Zhang Z, Zou W, Huang C, Guo Q (2018) Suppression of HDAC2 in Spinal Cord Alleviates Mechanical Hyperalgesia and Restores KCC2 Expression in a Rat Model of Bone Cancer Pain. Neuroscience 377:138149.

Hsia AY, Malenka RC, Nicoll RA (1998) Development of Excitatory Circuitry in the Hippocampus. J Neurophysiol 79:2013-2024 Available at: https://www.physiology.org/doi/10.1152/jn.1998.79.4.2013 [Accessed February 8, 2021].

Hu HJ, Gereau IV RW (2003) ERK integrates PKA and PKC signaling in superficial dorsal horn neurons. II. Modulation of neuronal excitability. J Neurophysiol 90:1680-1688 Available at: www.jn.org [Accessed April 3, 2021].

Huang YH, Schlüter OM, Dong Y (2013) An Unusual Suspect in Cocaine Addiction. Neuron 80:835-836 Available at: /pmc/articles/PMC3883311/ [Accessed March 29, 2021].

Hucho TB, Dina OA, Kuhn J, Levine JD (2006) Estrogen controls PKCe-dependent mechanical hyperalgesia through direct action on nociceptive neurons. Eur J Neurosci 24:527-534 Available at: http://doi.wiley.com/10.1111/j.1460-9568.2006.04913.x [Accessed March 23, 2021].

Huguet A, Miró J (2008) The Severity of Chronic Pediatric Pain: An Epidemiological Study. J Pain 9:226-236.

Hunt SP, Rossi J (1985) Peptide- and non-peptide-containing unmyelinated primary afferents: the parallel processing of nociceptive information. Philos Trans R Soc Lond B Biol Sci 308:283-289 Available at: http://www.ncbi.nlm.nih.gov/pubmed/2580323 [Accessed July $6,2018]$. 
Iadarola MJ, Brady LS, Draisci G, Dubner R (1988) Enhancement of dynorphin gene expression in spinal cord following experimental inflammation: stimulus specificity, behavioral parameters and opioid receptor binding. Pain 35:313-326.

Ikeda H, Heinke B, Ruscheweyh R, Sandkühler J (2003a) No Title. Science (80- ) 299:12371240.

Ikeda H, Heinke B, Ruscheweyh R, Sandkühler J (2003b) Synaptic plasticity in spinal lamina I projection neurons that mediate hyperalgesia. Science (80- ) 299:1237-1240.

Ikeda H, Stark J, Fischer H, Wagner M, Drdla R, Jäger T, Sandkühler J (2006) Synaptic amplifier of inflammatory pain in the spinal dorsal horn. Science (80- ) 312:1659-1662 Available at: https://pubmed.ncbi.nlm.nih.gov/16778058/ [Accessed April 9, 2021].

Ilkjaer S, Petersen KL, Brennum J, Wernberg M, Dahl JB (1996) Effect of systemic N-methylD-aspartate receptor antagonist (ketamine) on primary and secondary hyperalgesia in humans. Br J Anaesth 76:829-834 Available at: https://linkinghub.elsevier.com/retrieve/pii/S000709121743443X [Accessed May 14, 2019]. Ingley E (2008) Src family kinases: Regulation of their activities, levels and identification of new pathways. Biochim Biophys Acta - Proteins Proteomics 1784:56-65 Available at: https://pubmed.ncbi.nlm.nih.gov/17905674/ [Accessed April 9, 2021].

Inoue H, Asaka T, Nagata N, Koshihara Y (1997) Mechanism of mustard oil-induced skin inflammation in mice. Eur J Pharmacol 333:231-240.

Ishikawa K, Ott K, Porter RW, Stuart D (1966) Low frequency depression of the H wave in normal and spinal man. Exp Neurol 15:140-156 Available at: https://pubmed.ncbi.nlm.nih.gov/5934660/ [Accessed April 8, 2021].

Iwagaki N, Garzillo F, Polgár E, Riddell JS, Todd AJ (2013) Neurochemical characterisation of 
lamina II inhibitory interneurons that express GFP in the PrP-GFP mouse. Mol Pain 9:56 Available at: /pmc/articles/PMC4228398/ [Accessed April 4, 2021].

Iyer SM, Vesuna S, Ramakrishnan C, Huynh K, Young S, Berndt A, Lee SY, Gorini CJ, Deisseroth K, Delp SL (2016) Optogenetic and chemogenetic strategies for sustained inhibition of pain. Sci Rep 6 Available at: /pmc/articles/PMC4971509/ [Accessed March $28,2021]$.

Jacus MO, Uebele VN, Renger JJ, Todorovic SM (2012) Presynaptic CaV3.2 channels regulate excitatory neurotransmission in nociceptive dorsal horn neurons. J Neurosci 32:9374-9382.

Jain A, Huang GZ, Woolley CS (2019) Latent sex differences in molecular signaling that underlies excitatory synaptic potentiation in the hippocampus. J Neurosci 39:1552-1565 Available at: /pmc/articles/PMC6391563/ [Accessed March 12, 2021].

Ji R-R, Kohno T, Moore KA, Woolf CJ (2003) Central sensitization and LTP: do pain and memory share similar mechanisms? Trends Neurosci 26:696-705 Available at: http://www.ncbi.nlm.nih.gov/pubmed/14624855 [Accessed October 21, 2017].

Ji RR, Nackley A, Huh Y, Terrando N, Maixner W (2018) Neuroinflammation and central sensitization in chronic and widespread pain. Anesthesiology 129:343-366 Available at: http://pubs.asahq.org/anesthesiology/article-pdf/129/2/343/382145/20180800_0-00029.pdf [Accessed April 3, 2021].

Joksimovic SL, Joksimovic SM, Manzella FM, Asnake B, Orestes P, Raol YH, Krishnan K, Covey DF, Jevtovic-Todorovic V, Todorovic SM (2019) Novel neuroactive steroid with hypnotic and T-type calcium channel blocking properties exerts effective analgesia in a rodent model of post-surgical pain. Br J Pharmacol.

Jonas P, Major G, Sakmann B (1993) Quantal components of unitary EPSCs at the mossy fibre 
synapse on CA3 pyramidal cells of rat hippocampus. J Physiol 472:615-663 Available at: http://doi.wiley.com/10.1113/jphysiol.1993.sp019965 [Accessed March 31, 2021].

Jones ML, Liao GY, Malecki R, Li M, Salazar NM, Leonard JP (2012) PI 3-kinase and PKCל mediate insulin-induced potentiation of NMDA receptor currents in Xenopus oocytes. Brain Res.

Joseph EK, Levine JD (2003) Sexual dimorphism in the contribution of protein kinase C isoforms to nociception in the streptozotocin diabetic rat. Neuroscience 120:907-913 Available at: https://pubmed.ncbi.nlm.nih.gov/12927197/ [Accessed March 23, 2021].

Ju G, Hökfelt T, Brodin E, Fahrenkrug J, Fischer JA, Frey P, Elde RP, Brown JC (1987) Primary sensory neurons of the rat showing calcitonin gene-related peptide immunoreactivity and their relation to substance P-, somatostatin-, galanin-, vasoactive intestinal polypeptide- and cholecystokinin-immunoreactive ganglion cells. Cell Tissue Res 247:417-431 Available at: http://www.ncbi.nlm.nih.gov/pubmed/2434236 [Accessed July 6, 2018].

Kaila K, Price TJ, Payne JA, Puskarjov M, Voipio J (2014) Cation-chloride cotransporters in neuronal development, plasticity and disease. Nat Rev Neurosci 15:637-654 Available at: www.nature.com/reviews/neuro [Accessed January 20, 2021].

Kalia L V., Kalia SK, Salter MW (2008) NMDA receptors in clinical neurology: excitatory times ahead. Lancet Neurol 7:742-755 Available at: https://pubmed.ncbi.nlm.nih.gov/18635022/ [Accessed April 9, 2021].

Kann O, Kovács R, Njunting M, Behrens CJ, Otáhal J, Lehmann T-N, Gabriel S, Heinemann U (2005) Metabolic dysfunction during neuronal activation in the ex vivo hippocampus from chronic epileptic rats and humans. Brain 128:2396-2407 Available at: http://academic.oup.com/brain/article/128/10/2396/274628/Metabolic-dysfunction-during- 
neuronal-activation [Accessed October 23, 2017].

Kato G, Yasaka T, Katafuchi T, Furue H, Mizuno M, Iwamoto Y, Yoshimura M (2006) Behavioral/Systems/Cognitive Direct GABAergic and Glycinergic Inhibition of the Substantia Gelatinosa from the Rostral Ventromedial Medulla Revealed by In Vivo PatchClamp Analysis in Rats.

Katsura H, Obata K, Mizushima T, Sakurai J, Kobayashi K, Yamanaka H, Dai Y, Fukuoka T, Sakagami M, Noguchi K (2006) Activation of Src-family kinases in spinal microglia contributes to mechanical hypersensitivity after nerve injury. J Neurosci 26:8680-8690 Available at: https://pubmed.ncbi.nlm.nih.gov/16928856/ [Accessed April 9, 2021].

Kawasaki Y, Kohno T, Zhuang ZY, Brenner GJ, Wang H, Van Der Meer C V., Befort K, Woolf CJ, Ji RR (2004) Ionotropic and metabotropic receptors, protein kinase A, protein kinase C, and Src contribute to C-fiber-induced ERK activation and cAMP response element-binding protein phosphorylation in dorsal horn neurons, leading to central sensitization. J Neurosci 24:8310-8321 Available at: https://www.jneurosci.org/content/24/38/8310 [Accessed April 3, 2021].

Kawata M (1995) Roles of steroid hormones and their receptors in structural organization in the nervous system. Neurosci Res 24:1-46.

Keller AF, Beggs S, Salter MW, De Koninck Y (2007) Transformation of the Output of Spinal Lamina I Neurons After Nerve Injury and Microglia Stimulation Underlying Neuropathic Pain. Mol Pain 3:1744-8069-3-27.

Kim JA, Bosma RL, Hemington KS, Rogachov A, Osborne NR, Cheng JC, Dunkley BT, Davis KD (2021) Sex-differences in network level brain dynamics associated with pain sensitivity and pain interference. Hum Brain Mapp 42:598-614 Available at: 
https://onlinelibrary.wiley.com/doi/10.1002/hbm.25245 [Accessed March 23, 2021].

Kim Y, Cho H, Ahn YJ, Kim J, Yoon YW (2012) Effect of NMDA NR2B antagonist on neuropathic pain in two spinal cord injury models. Pain 153:1022-1029.

King S, Chambers CT, Huguet A, MacNevin RC, McGrath PJ, Parker L, MacDonald AJ (2011) The epidemiology of chronic pain in children and adolescents revisited: A systematic review. Pain 152:2729-2738.

Kuba T, Quinones-Jenab V (2005) The role of female gonadal hormones in behavioral sex differences in persistent and chronic pain: Clinical versus preclinical studies. Brain Res Bull 66:179-188 Available at: https://pubmed.ncbi.nlm.nih.gov/16023915/ [Accessed March 30, 2021].

Kuner R (2010) Central mechanisms of pathological pain. Nat Med 16:1258-1266 Available at: https://www.nature.com/articles/nm.2231 [Accessed April 3, 2021].

Kuner R (2015) Spinal excitatory mechanisms of pathological pain. Pain 156:S11-S17.

Kuo A, Wyse BD, Meutermans W, Smith MT (2015) In vivo profiling of seven common opioids for antinociception, constipation and respiratory depression: No two opioids have the same profile. Br J Pharmacol 172:532-548.

LaCroix-Fralish ML, Tawfik VL, DeLeo JA (2005) The organizational and activational effects of sex hormones on tactile and thermal hypersensitivity following lumbar nerve root injury in male and female rats. Pain 114:71-80.

LaMotte RH, Shain CN, Simone DA, Tsai EFP (1991) Neurogenic hyperalgesia: Psychophysical studies of underlying mechanisms. J Neurophysiol 66:190-211 Available at: https://journals.physiology.org/doi/abs/10.1152/jn.1991.66.1.190 [Accessed April 1, 2021].

Larsson M, Broman J (2008) Translocation of GluR1-containing AMPA receptors to a spinal 
nociceptive synapse during acute noxious stimulation. J Neurosci 28:7084-7090 Available at: www.jneurosci.org [Accessed April 3, 2021].

Latremoliere A, Woolf CJ (2009) Central Sensitization: A Generator of Pain Hypersensitivity by Central Neural Plasticity. NIH Public Access. Available at: http://www.ncbi.nlm.nih.gov/pubmed/19712899 [Accessed October 23, 2017].

Lau CG, Zukin RS (2007) NMDA receptor trafficking in synaptic plasticity and neuropsychiatric disorders. Nat Rev Neurosci 8:413-426 Available at: www.nature.com/reviews/neuro [Accessed April 3, 2021].

Laumet G, Ma J, Robison AJ, Kumari S, Heijnen CJ, Kavelaars A (2019) T Cells as an Emerging Target for Chronic Pain Therapy. Front Mol Neurosci 12:216.

Leblanc BW, Lii TR, Huang JJ, Chao YC, Bowary PM, Cross BS, Lee MS, Vera-Portocarrero LP, Saab CY (2016) T-type calcium channel blocker Z944 restores cortical synchrony and thalamocortical connectivity in a rat model of neuropathic pain. Pain 157:255-263.

Lee-Kubli CAG, Calcutt NA (2013) ALTERED RATE-DEPENDENT DEPRESSION OF THE SPINAL H-REFLEX AS AN INDICATOR OF SPINAL DISINHIBITION IN MODELS OF NEUROPATHIC PAIN.

Lee HHC, Deeb TZ, Walker JA, Davies PA, Moss SJ (2011) NMDA receptor activity downregulates $\mathrm{KCC} 2$ resulting in depolarizing GABAA receptor-mediated currents. Nat Neurosci 14:736-743 Available at: https://www.nature.com/articles/nn.2806 [Accessed March 28, 2021].

Lee KY, Prescott SA (2015) Chloride dysregulation and inhibitory receptor blockade yield equivalent disinhibition of spinal neurons yet are differentially reversed by carbonic anhydrase blockade. Pain 156:2431-2437 Available at: 
http://content.wkhealth.com/linkback/openurl?sid=WKPTLP:landingpage\&an=00006396201512000-00008 [Accessed March 14, 2018].

Lee M (2014) Z944: A first in class T-type calcium channel modulator for the treatment of pain. In: Journal of the Peripheral Nervous System, pp S11-S12. Blackwell Publishing Inc.

Lee Z-F, Huang T-H, Chen S-P, Cheng IH (2021) Altered nociception in Alzheimer's disease is associated with STEP signaling. PAIN $®$ In Press Available at: https://journals.lww.com/pain/Abstract/9000/Altered_nociception_in_Alzheimer_s_disease _is.98164.aspx.

Lever IJ, Bradbury EJ, Cunningham JR, Adelson DW, Jones MG, McMahon SB, Marvizón JCG, Malcangio M (2001) Brain-derived neurotrophic factor is released in the dorsal horn by distinctive patterns of afferent fiber stimulation. J Neurosci 21:4469-4477 Available at: /pmc/articles/PMC6762751/?report=abstract [Accessed September 22, 2020].

Levitan IB (1994) MODULATION OF ION CHANNELS BY PROTEIN PHOSPHORYLATION AND DEPHOSPHORYLATION. Available at: www.annualreviews.org [Accessed April 3, 2021].

Li BS, Sun MK, Zhang L, Takahashi S, Ma W, Vinade L, Kulkarni AB, Brady RO, Pant HC (2001) Regulation of NMDA receptors by cyclin-dependent kinase-5. Proc Natl Acad Sci U S A 98:12742-12747 Available at: http://www.ncbi.nlm.nih.gov/pubmed/11675505 [Accessed July 13, 2019].

Li L, Chen S-R, Chen H, Wen L, Hittelman WN, Xie J-D, Pan H-L (2016) Chloride Homeostasis Critically Regulates Synaptic NMDA Receptor Activity in Neuropathic Pain. Cell Rep 15:1376-1383 Available at: https://www.sciencedirect.com/science/article/pii/S2211124716304661 [Accessed March 
$18,2019]$.

Li L, Fan X, Warner M, Xu XJ, Gustafsson JÅ, Wiesenfeld-Hallin Z (2009) Ablation of estrogen receptor $\alpha$ or $\beta$ eliminates sex differences in mechanical pain threshold in normal and inflamed mice. Pain 143:37-40.

Li L, Shi L, Xu Y-M, Yang X, Suo Z-W, Hu X-D (2015) GABAergic inhibition regulated pain sensitization through STEP61 signaling in spinal dorsal horn of mice. Anesthesiology 122:686-697.

Li S, Cai J, Feng ZB, Jin ZR, Liu BH, Zhao HY, Jing HB, Wei TJ, Yang GN, Liu LY, Cui YJ, Xing GG (2017a) BDNF Contributes to Spinal Long-Term Potentiation and Mechanical Hypersensitivity Via Fyn-Mediated Phosphorylation of NMDA Receptor GluN2B Subunit at Tyrosine 1472 in Rats Following Spinal Nerve Ligation. Neurochem Res 42:2712-2729 Available at: https://pubmed.ncbi.nlm.nih.gov/28497343/ [Accessed April 2, 2021].

Li Y, Tatsui CE, Rhines LD, North RY, Harrison DS, Cassidy RM, Johansson CA, Kosturakis AK, Edwards DD, Zhang H, Dougherty PM (2017b) Dorsal root ganglion neurons become hyperexcitable and increase expression of voltage-gated T-type calcium channels (Cav3.2) in paclitaxel-induced peripheral neuropathy. Pain 158:417-429.

Lima D, Coimbra A (1986) A Golgi study of the neuronal population of the marginal zone (lamina I) of the rat spinal cord. J Comp Neurol 244:53-71.

Lin CR, Chuang YC, Cheng JT, Wang CJ, Yang LC (2002) Intrathecal clonidine decreases spinal nitric oxide release in a rat model of Complete Freund's Adjuvant induced inflammatory pain. Inflammation 26:161-166 Available at: https://link.springer.com/article/10.1023/A:1016563628274 [Accessed April 2, 2021]. Ling X et al. (2021) Regulation of NMDA receptor trafficking and gating by activity-dependent 
CaMKII $\alpha$ phosphorylation of the GluN2A subunit. bioRxiv:2021.01.11.425709 Available at: https://doi.org/10.1101/2021.01.11.425709 [Accessed April 3, 2021].

Liu NJ, Gintzler AR (2000) Prolonged ovarian sex steroid treatment of male rats produces antinociception: Identification of sex-based divergent analgesic mechanisms. Pain 85:273281 Available at: https://pubmed.ncbi.nlm.nih.gov/10692628/ [Accessed March 30, 2021].

Liu XJ, Gingrich JR, Vargas-Caballero M, Dong YN, Sengar A, Beggs S, Wang S-H, Ding HK, Frankland PW, Salter MW (2008) Treatment of inflammatory and neuropathic pain by uncoupling Src from the NMDA receptor complex. Nat Med 14:1325-1332 Available at: http://www.ncbi.nlm.nih.gov/pubmed/19011637 [Accessed October 21, 2017].

Liu XJ, Salter MW (2010) Glutamate receptor phosphorylation and trafficking in pain plasticity in spinal cord dorsal horn. Eur J Neurosci 32:278-289 Available at: /pmc/articles/PMC3589563/ [Accessed April 3, 2021].

Liu YN, Yang X, Suo ZW, Xu YM, Hu XD (2014) Fyn kinase-regulated NMDA receptor- and AMPA receptor-dependent pain sensitization in spinal dorsal horn of mice. Eur J Pain (United Kingdom) 18:1120-1128.

Lorenzo LE, Godin AG, Ferrini F, Bachand K, Plasencia-Fernandez I, Labrecque S, Girard AA, Boudreau D, Kianicka I, Gagnon M, Doyon N, Ribeiro-da-Silva A, De Koninck Y (2020) Enhancing neuronal chloride extrusion rescues $\alpha 2 / \alpha 3$ GABAA-mediated analgesia in neuropathic pain. Nat Commun 11.

Low C-M, Wee KS-L (2010) New insights into the not-so-new NR3 subunits of N-methyl-Daspartate receptor: localization, structure, and function. Mol Pharmacol 78:1-11 Available at: http://molpharm.aspetjournals.org/content/78/1/1.long [Accessed March 11, 2016].

Lu VB, Ballanyi K, Colmers WF, Smith PA (2007) Neuron type-specific effects of brain-derived 
neurotrophic factor in rat superficial dorsal horn and their relevance to 'central sensitization.' J Physiol 584:543-563 Available at: http://doi.wiley.com/10.1113/jphysiol.2007.141267 [Accessed March 30, 2021].

Lu VB, Colmers WF, Smith PA (2012) Long-term actions of BDNF on inhibitory synaptic transmission in identified neurons of the rat substantia gelatinosa. J Neurophysiol 108:441452 Available at: https://www.physiology.org/doi/10.1152/jn.00457.2011 [Accessed March 9, 2021].

Lucas-Romero J, Rivera-Arconada I, Roza C, Lopez-Garcia JA (2018) Origin and classification of spontaneous discharges in mouse superficial dorsal horn neurons. In: Scientific Reports, pp 1-11. Nature Publishing Group. Available at: www.nature.com/scientificreports [Accessed March 29, 2021].

Luo C, Kuner T, Kuner R (2014) Synaptic plasticity in pathological pain. Trends Neurosci 37:343-355 Available at: http://dx.doi.org/10.1016/j.tins.2014.04.002 [Accessed March 11, 2019].

Luo L, Puke MJC, Wiesenfeld-Hallin Z (1994) The effects of intrathecal morphine and clonidine on the prevention and reversal of spinal cord hyperexcitability following sciatic nerve section in the rat. Pain 58:245-252.

Lüscher C, Malenka RC (2012) NMDA receptor-dependent long-term potentiation and longterm depression (LTP/LTD). Cold Spring Harb Perspect Biol 4:1-15 Available at: /pmc/articles/PMC3367554/ [Accessed April 3, 2021].

Mahmoud H, Martin N, Hildebrand ME (2020) Conserved contributions of NMDA receptor subtypes to synaptic responses in lamina II spinal neurons across early postnatal development. Mol Brain 13:31 Available at: 
https://molecularbrain.biomedcentral.com/articles/10.1186/s13041-020-00566-9 [Accessed April 6, 2021].

Maki BA, Cole R, Popescu GK (2013) Two serine residues on GluN2A C-terminal tails control NMDA receptor current decay times. Channels.

Mannino CA, South SM, Quinones-Jenab V, Inturrisi CE (2007) Estradiol Replacement in Ovariectomized Rats Is Antihyperalgesic in the Formalin Test. J Pain 8:334-342.

Mannion RJ, Costigan M, Decosterd I, Amaya F, Ma QP, Holstege JC, Ji RR, Acheson A, Lindsay RM, Wilkinson GA, Woolf CJ (1999) Neurotrophins: Peripherally and centrally acting modulators of tactile stimulus-induced inflammatory pain hypersensitivity. Proc Natl Acad Sci 96:9385-9390.

Mapplebeck JCS, Beggs S, Salter MW (2017) Molecules in pain and sex: a developing story. Mol Brain 10:9 Available at: http://www.ncbi.nlm.nih.gov/pubmed/28270169 [Accessed October 23, 2017].

Mapplebeck JCS, Dalgarno R, Tu Y, Moriarty O, Beggs S, Kwok CHT, Halievski K, Assi S, Mogil JS, Trang T, Salter MW (2018) Microglial P2X4R-evoked pain hypersensitivity is sexually dimorphic in rats. Pain:1 Available at: http://insights.ovid.com/crossref?an=00006396-900000000-98919 [Accessed November 28, 2018].

Mapplebeck JCS, Lorenzo LE, Lee KY, Gauthier C, Muley MM, De Koninck Y, Prescott SA, Salter MW (2019) Chloride Dysregulation through Downregulation of KCC2 Mediates Neuropathic Pain in Both Sexes. Cell Rep 28:590-596.e4.

Martin VT (2009) Ovarian hormones and pain response: A review of clinical and basic science studies. Gend Med 6:168-192 Available at: https://pubmed.ncbi.nlm.nih.gov/19406368/ 
[Accessed March 30, 2021].

Martins MA, De Castro Bastos L, Tonussi CR (2006) Formalin injection into knee joints of rats: Pharmacologic characterization of a deep somatic nociceptive model. J Pain 7:100-107 Available at: https://pubmed.ncbi.nlm.nih.gov/16459275/ [Accessed April 1, 2021].

Matsumura S, Kunori S, Mabuchi T, Katano T, Nakazawa T, Abe T, Watanabe M, Yamamoto T, Okuda-Ashitaka E, Ito S (2010) Impairment of CaMKII activation and attenuation of neuropathic pain in mice lacking NR2B phosphorylated at Tyr1472. Eur J Neurosci 32:798810 Available at: https://pubmed.ncbi.nlm.nih.gov/20722721/ [Accessed April 9, 2021].

Matthew Soleiman (2017) A Chemogenetic Approach to Alleviating Neuropathic Pain | Pain Research Forum. Available at: https://www.painresearchforum.org/news/87944chemogenetic-approach-alleviating-neuropathic-pain [Accessed March 28, 2021].

McKinlay SM, Bifano NL, McKinlay JB (1985) Smoking and age at menopause in women. Ann Intern Med 103:350-356.

McQuate A, Barria A (2020) Rapid exchange of synaptic and extrasynaptic NMDA receptors in hippocampal CA1 neurons. J Neurophysiol 123:1004-1014 Available at: https://journals.physiology.org/doi/10.1152/jn.00458.2019 [Accessed March 31, 2021].

Mecklenburg J, Zou Y, Wangzhou A, Garcia D, Lai Z, Tumanov A V., Dussor G, Price TJ, Akopian AN (2020) Transcriptomic sex differences in sensory neuronal populations of mice. Sci Rep 10.

Meinck HM (1976) Occurrence of the H reflex and the F wave in the rat. Electroencephalogr Clin Neurophysiol 41:530-533 Available at: https://pubmed.ncbi.nlm.nih.gov/61856/ [Accessed April 8, 2021].

Meloto CB, Benavides R, Lichtenwalter RN, Wen X, Tugarinov N, Zorina-Lichtenwalter K, 
Chabot-Doré AJ, Piltonen MH, Cattaneo S, Verma V, Klares R, Khoury S, Parisien M, Diatchenko L (2018) Human pain genetics database: A resource dedicated to human pain genetics research. Pain 159:749-763 Available at:

https://pubmed.ncbi.nlm.nih.gov/29300278/ [Accessed April 1, 2021].

Melzack R, Wall PD (1965) Number 3699 I. Available at:

http://pdfs.semanticscholar.org/38d2/be60471398c102c148b998b093a779773e3a.pdf [Accessed May 13, 2019].

Mercado A, Broumand V, Zandi-Nejad K, Enck AH, Mount DB (2006) A C-terminal domain in KCC2 confers constitutive K+-Cl- cotransport. J Biol Chem 281:1016-1026 Available at: http://www.ncbi.nlm.nih.gov/pubmed/16291749 [Accessed July 6, 2018].

Merskey H, Bogduk N (2002) Classification of chronic pain. Available at: http://www.iasppain.org/files/Content/ContentFolders/Publications2/FreeBooks/Classification-of-ChronicPain.pdf.

Miller RE, Ishihara S, Bhattacharyya B, Delaney A, Menichella DM, Miller RJ, Malfait AM (2017) Chemogenetic Inhibition of Pain Neurons in a Mouse Model of Osteoarthritis. Arthritis Rheumatol 69:1429-1439 Available at: http://doi.wiley.com/10.1002/art.40118 [Accessed March 28, 2021].

Mills EP, Di Pietro F, Alshelh Z, Peck CC, Murray GM, Vickers ER, Henderson LA (2018) Brainstem pain-control circuitry connectivity in chronic neuropathic pain. J Neurosci 38:465-473 Available at: https://www.jneurosci.org/content/38/2/465 [Accessed April 3, 2021].

Mizuno M, Kato G, Strassman AM (2019) Spatial organization of activity evoked by focal stimulation within the rat spinal dorsal horn as visualized by voltage-sensitive dye imaging 
in the slice. J Neurophysiol 122:1697-1707 Available at:

https://www.physiology.org/doi/10.1152/jn.00697.2018 [Accessed March 29, 2021].

Moechars D, Weston MC, Leo S, Callaerts-Vegh Z, Goris I, Daneels G, Buist A, Cik M, Van

Der Spek P, Kass S, Meert T, D'Hooge R, Rosenmund C, Hampson RM (2006) Vesicular glutamate transporter VGLUT2 expression levels control quantal size and neuropathic pain. J Neurosci 26:12055-12066 Available at: www.jneurosci.org [Accessed April 3, 2021].

Mogil JS (2012) Sex differences in pain and pain inhibition: multiple explanations of a controversial phenomenon. Nat Rev Neurosci 13:859-866 Available at: http://www.nature.com/articles/nrn3360 [Accessed April 28, 2019].

Mogil JS, Sternberg WF, Kest B, Marek P, Liebeskind JC (1993) Sex differences in the antagonism of swim stress-induced analgesia: effects of gonadectomy and estrogen replacement. Pain 53:17-25 Available at: https://www.sciencedirect.com/science/article/pii/030439599390050Y [Accessed April 28, 2019].

Mohamad O, Song M, Wei L, Yu SP (2013) Regulatory roles of the NMDA receptor GluN3A subunit in locomotion, Pain perception and cognitive functions in adult mice. J Physiol 591:149-168 Available at:/pmc/articles/PMC3630778/ [Accessed March 29, 2021].

Momiyama A (2000) Distinct synaptic and extrasynaptic NMDA receptors identified in dorsal horn neurones of the adult rat spinal cord. J Physiol 523:621-628 Available at: https://onlinelibrary.wiley.com/doi/10.1111/j.1469-7793.2000.t01-1-00621.x [Accessed April 8, 2021].

Monyer H, Sprengel R, Schoepfer R, Herb A, Higuchi M, Lomeli H, Burnashev N, Sakmann B, Seeburg PH (1992) Heteromeric NMDA receptors: Molecular and functional distinction of 
subtypes. Science (80- ) 256:1217-1221 Available at:

https://pubmed.ncbi.nlm.nih.gov/1350383/ [Accessed April 5, 2021].

Morgan P, Van Der Graaf PH, Arrowsmith J, Feltner DE, Drummond KS, Wegner CD, Street SDA (2012) Can the flow of medicines be improved? Fundamental pharmacokinetic and pharmacological principles toward improving Phase II survival. Drug Discov Today $17: 419-424$.

Mosconi T, Kruger L (1996) Fixed-diameter polyethylene cuffs applied to the rat sciatic nerve induce a painful neuropathy: Ultrastructural morphometric analysis of axonal alterations. Pain 64:37-57 Available at: https://pubmed.ncbi.nlm.nih.gov/8867246/ [Accessed April 9, 2021].

Nakazawa T, Komai S, Tezuka T, Hisatsune C, Umemori H, Semba K, Mishina M, Manabe T, Yamamoto T (2001) Characterization of Fyn-mediated Tyrosine Phosphorylation Sites on GluRe2 (NR2B) Subunit of the $N$-Methyl-d-aspartate Receptor. J Biol Chem 276:693-699 Available at: http://www.ncbi.nlm.nih.gov/pubmed/11024032 [Accessed March 18, 2019].

Nelson KA, Park KM, Robinovitz E, Tsigos C, Max MB (1997) High-dose oral dextromethorphan versus placebo in painful diabetic neuropathy and postherpetic neuralgia. Neurology 48:1212-1218 Available at: https://pubmed.ncbi.nlm.nih.gov/9153445/ [Accessed April 4, 2021].

Niesters M, Martini C, Dahan A (2014) Ketamine for chronic pain: Risks and benefits. Br J Clin Pharmacol 77:357-367 Available at: https://pubmed.ncbi.nlm.nih.gov/23432384/ [Accessed April 4, 2021].

Ninkina N, Grashchuck M, Buchman VL, Davies AM (1997) TrkB variants with deletions in the leucine-rich motifs of the extracellular domain. J Biol Chem 272:13019-13025 Available 
at: https://pubmed.ncbi.nlm.nih.gov/9148911/ [Accessed April 9, 2021].

Nusser Z, Cull-Candy S, Farrant M (1997) Differences in Synaptic GABAA Receptor Number Underlie Variation in GABA Mini Amplitude. Neuron 19:697-709 Available at: https://linkinghub.elsevier.com/retrieve/pii/S0896627300803827 [Accessed March 31, 2021].

Nusser Z, David J, Roberts,' B, Baude A, Grayson Richards J, Somogyil P (1995) Relative Densities of Synaptic and Extrasynaptic GAB!, Receptors on Cerebellar Granule Cells As Determined by a Quantitative lmmunogold Method. Available at: https://www.jneurosci.org/content/15/4/2948.short [Accessed March 31, 2021].

Osborne NR, Cheng JC, Rogachov A, Kim JA, Hemington KS, Bosma RL, Inman RD, Davis $\mathrm{KD}$ (2021) Abnormal subgenual anterior cingulate circuitry is unique to women but not men with chronic pain. Pain 162:97-108 Available at: https://journals.lww.com/10.1097/j.pain.0000000000002016 [Accessed March 23, 2021].

Pachernegg S, Strutz-Seebohm N, Hollmann M (2012) GluN3 subunit-containing NMDA receptors: not just one-trick ponies. Trends Neurosci 35:240-249 Available at: http://www.ncbi.nlm.nih.gov/pubmed/22240240 [Accessed April 14, 2016].

Palat G, Chary S (2018) Practical guide for using methadone in pain and palliative care practice. Indian J Palliat Care 24:S21-S29 Available at:/pmc/articles/PMC5806302/ [Accessed April 4, 2021].

Paoletti P, Bellone C, Zhou Q (2013) NMDA receptor subunit diversity: impact on receptor properties, synaptic plasticity and disease. Nat Rev Neurosci 14:383-400 Available at: http://www.nature.com/articles/nrn3504 [Accessed May 13, 2019].

Parisien M, Khoury S, Chabot-Doré AJ, Sotocinal SG, Slade GD, Smith SB, Fillingim RB, 
Ohrbach R, Greenspan JD, Maixner W, Mogil JS, Belfer I, Diatchenko L (2017) Effect of Human Genetic Variability on Gene Expression in Dorsal Root Ganglia and Association with Pain Phenotypes. Cell Rep 19:1940-1952 Available at:/pmc/articles/PMC5524461/ [Accessed April 1, 2021].

Patrizio A, Specht CG (2016) Counting numbers of synaptic proteins: absolute quantification and single molecule imaging techniques. Neurophotonics 3:041805 Available at: /pmc/articles/PMC4891561/ [Accessed March 31, 2021].

Paul S, Nairn AC, Wang P, Lombroso PJ (2003) NMDA-mediated activation of the tyrosine phosphatase STEP regulates the duration of ERK signaling. Nat Neurosci 6:34-42 Available at: https://pubmed.ncbi.nlm.nih.gov/12483215/ [Accessed April 3, 2021].

Paul S, Olausson P, Venkitaramani D V., Ruchkina I, Moran TD, Tronson N, Mills E, Hakim S, Salter MW, Taylor JR, Lombroso PJ (2007) The Striatal-Enriched Protein Tyrosine Phosphatase Gates Long-Term Potentiation and Fear Memory in the Lateral Amygdala. Biol Psychiatry 61:1049-1061 Available at: http://www.ncbi.nlm.nih.gov/pubmed/17081505 [Accessed June 3, 2018].

Peirs C, Seal RP (2016) Neural circuits for pain: Recent advances and current views. Science (80- ) 354:578-584 Available at: https://pubmed.ncbi.nlm.nih.gov/27811268/ [Accessed March 31, 2021].

Pelkey KA, Rand A, Surojit P, Kalia L V., Tri-Hung N, Pitcher GM, Salter MW, Lombroso PJ (2002) Tyrosine Phosphatase STEP Is a Tonic Brake on Induction of Long-Term Potentiation. Neuron 34:127-138 Available at: http://www.sciencedirect.com.proxy.library.carleton.ca/science/article/pii/S0896627302006 335?via\%3Dihub [Accessed October 22, 2017]. 
Peng HY, Chen G Den, Lai CH, Tung KC, Chang JL, Lin T Bin (2010) Endogenous ephrinB2 mediates colon-urethra cross-organ sensitization via Src kinase-dependent tyrosine phosphorylation of NR2B. Am J Physiol - Ren Physiol 298 Available at: https://pubmed.ncbi.nlm.nih.gov/19864302/ [Accessed April 9, 2021].

Pérez-Otaño I, Luján R, Tavalin SJ, Plomann M, Modregger J, Liu X-B, Jones EG, Heinemann SF, Lo DC, Ehlers MD (2006) Endocytosis and synaptic removal of NR3A-containing NMDA receptors by PACSIN1/syndapin1. Nat Neurosci 9:611-621 Available at: http://dx.doi.org/10.1038/nn1680 [Accessed April 15, 2016].

Perquin CW, Hazebroek-Kampschreur AAJM, Hunfeld JAM, Bohnen AM, Van Suijlekom-Smit LWA, Passchier J, Van Der Wouden JC (2000) Pain in children and adolescents: A common experience. Pain 87:51-58.

Petitjean H, Pawlowski SA, Fraine SL, Sharif B, Hamad D, Fatima T, Berg J, Brown CM, Jan LY, Ribeiro-da-Silva A, Braz JM, Basbaum AI, Sharif-Naeini R (2015) Dorsal Horn Parvalbumin Neurons Are Gate-Keepers of Touch-Evoked Pain after Nerve Injury. Cell Rep 13:1246-1257 Available at: https://pubmed.ncbi.nlm.nih.gov/26527000/ [Accessed April 3, 2021].

Petrenko AB, Yamakura T, Baba H, Shimoji K (2003) The Role of N-Methyl-d-Aspartate (NMDA) Receptors in Pain: A Review. Anesth Analg 97:1108-1116 Available at: http://journals.lww.com/00000539-200310000-00035 [Accessed March 8, 2021].

Picard E, Carvalho FA, Agosti F, Bourinet E, Ardid D, Eschalier A, Daulhac L, Mallet C (2019) Inhibition of $\mathrm{Ca} v 3.2$ calcium channels: A new target for colonic hypersensitivity associated with low-grade inflammation. Br J Pharmacol 176:950-963.

Pitcher GM, Ritchie J, Henry JL (1999) Paw withdrawal threshold in the von Frey hair test is 
influenced by the surface on which the rat stands. J Neurosci Methods 87:185-193

Available at: https://pubmed.ncbi.nlm.nih.gov/11230815/ [Accessed April 9, 2021].

Pleis JR, Ward BW, Lucas JW (2010) Summary health statistics for U.S. adults: National Health Interview Survey, 2009. Vital Health Stat 10:1-207 Available at: http://www.ncbi.nlm.nih.gov/pubmed/21905346 [Accessed October 23, 2017].

Poddar R, Rajagopal S, Shuttleworth CW, Paul S (2016) Zn2+-dependent activation of the trk signaling pathway induces phosphorylation of the brain-enriched tyrosine phosphatase STEP: Molecular basis for ZN2+-induced ERK MAPK activation. J Biol Chem 291:813825.

Poncer JC, Malinow R (2001) Postsynaptic conversion of silent synapses during LTP affects synaptic gain and transmission dynamics. Nat Neurosci 4:989-996.

Prescott SA, Ma Q, De Koninck Y (2014) Normal and abnormal coding of somatosensory stimuli causing pain. Nat Neurosci 17:183-191 Available at: http://www.ncbi.nlm.nih.gov/pubmed/24473266 [Accessed March 18, 2019].

Price TJ, Cervero F, Gold MS, Hammond DL, Prescott SA (2009) Chloride regulation in the pain pathway. Brain Res Rev 60:149-170.

Prybylowski K, Fu Z, Losi G, Hawkins LM, Luo JH, Chang K, Wenthold RJ, Vicini S (2002) Relationship between availability of NMDA receptor subunits and their expression at the synapse. J Neurosci 22:8902-8910.

Punnakkal P, Schoultz C, Haenraets K, Wildner H, Zeilhofer HU (2014) Morphological, biophysical and synaptic properties of glutamatergic neurons of the mouse spinal dorsal horn. J Physiol 592:759-776 Available at:

https://onlinelibrary.wiley.com/doi/10.1113/jphysiol.2013.264937 [Accessed April 4, 
2021].

Qu XX, Cai J, Li MJ, Chi YN, Liao FF, Liu FY, Wan Y, Han JS, Xing GG (2009) Role of the spinal cord NR2B-containing NMDA receptors in the development of neuropathic pain. Exp Neurol 215:298-307.

Radhakrishnan R, Moore SA, Sluka KA (2003) Unilateral carrageenan injection into muscle or joint induces chronic bilateral hyperalgesia in rats. Pain 104:567-577 Available at: https://pubmed.ncbi.nlm.nih.gov/12927629/ [Accessed April 1, 2021].

Raja SN, Carr DB, Cohen M, Finnerup NB, Flor H, Gibson S, Keefe FJ, Mogil JS, Ringkamp M, Sluka KA, Song X-J, Stevens B, Sullivan MD, Tutelman PR, Ushida T, Vader K (2020) The revised International Association for the Study of Pain definition of pain: concepts, challenges, and compromises. Pain 161:1976-1982 Available at: https://journals.lww.com/10.1097/j.pain.0000000000001939 [Accessed April 1, 2021].

Ran ZS, Zhang ET, Craig AD (1998) Nociceptive and thermoreceptive lamina I neurons are anatomically distinct. Nat Neurosci 1:218-225 Available at: https://pubmed.ncbi.nlm.nih.gov/10195146/ [Accessed April 8, 2021].

Ratté S, Prescott SA (2016) Afferent hyperexcitability in neuropathic pain and the inconvenient truth about its degeneracy. Curr Opin Neurobiol 36:31-37 Available at: https://pubmed.ncbi.nlm.nih.gov/26363576/ [Accessed March 9, 2021].

Redondo-Castro E, García-Alías G, Navarro X (2013) Plastic changes in lumbar segments after thoracic spinal cord injuries in adult rats: An integrative view of spinal nociceptive dysfunctions. Restor Neurol Neurosci 31:411-430.

Ren K, Hylden JLK, Williams GM, Ruda MA, Dubner R (1992) The effects of a noncompetitive NMDA receptor antagonist, MK-801, on behavioral hyperalgesia and dorsal 
horn neuronal activity in rats with unilateral inflammation. Pain 50:331-344.

Ren K, Wei F, Dubner R, Murphy A, Hoffman GE (2000) Progesterone attenuates persistent inflammatory hyperalgesia in female rats: Involvement of spinal NMDA receptor mechanisms. Brain Res 865:272-277.

Retchless BS, Gao W, Johnson JW (2012) A single GluN2 subunit residue controls NMDA receptor channel properties via intersubunit interaction. Nat Neurosci 15:406-413 Available at: https://www.nature.com/articles/nn.3025 [Accessed April 5, 2021].

Ro JY, Capra N, Masri R (2003) Development of a behavioral assessment of craniofacial muscle pain in lightly anesthetized rats. Pain 104:179-185 Available at: https://pubmed.ncbi.nlm.nih.gov/12855327/ [Accessed April 1, 2021].

Roberts AC, Díez-García J, Rodriguiz RM, López IP, Luján R, Martínez-Turrillas R, Picó E, Henson MA, Bernardo DR, Jarrett TM, Clendeninn DJ, López-Mascaraque L, Feng G, Lo DC, Wesseling JF, Wetsel WC, Philpot BD, Pérez-Otaño I (2009) Downregulation of NR3A-Containing NMDARs Is Required for Synapse Maturation and Memory Consolidation. Neuron 63:342-356.

Rose RD, Koerber HR, Sedivec MJ, Mendell LM (1986) Somal action potential duration differs in identified primary afferents. Neurosci Lett 63:259-264 Available at: https://pubmed.ncbi.nlm.nih.gov/3951752/ [Accessed April 8, 2021].

Rosenfeld MG, Mermod J-J, Amara SG, Swanson LW, Sawchenko PE, Rivier J, Vale WW, Evans RM (1983) Production of a novel neuropeptide encoded by the calcitonin gene via tissue-specific RNA processing. Nature 304:129-135 Available at: http://www.nature.com/doifinder/10.1038/304129a0 [Accessed July 6, 2018].

Rosenkranz JA, Frick A, Johnston D (2009) Kinase-dependent modification of dendritic 
excitability after long-term potentiation. J Physiol 587:115-125 Available at:

https://pubmed.ncbi.nlm.nih.gov/19001050/ [Accessed October 28, 2020].

Ross SE (2011) Pain and itch: Insights into the neural circuits of aversive somatosensation in health and disease. Curr Opin Neurobiol 21:880-887.

Rudjito R, Agalave NM, Farinotti AB, Lundbäck P, Szabo-Pardi TA, Price TJ, Harris HE, Burton MD, Svensson CI (2021) Sex- and cell-dependent contribution of peripheral high mobility group box 1 and TLR4 in arthritis-induced pain. Pain 162:459-470 Available at: /pmc/articles/PMC7808351/ [Accessed April 1, 2021].

Russ E, Patterson Cross RB, Li L, Koch SC, Matson KJ (2020) A Harmonized Atlas of Spinal Cord Cell Types and Their Computational Classification 12 Daniel. bioRxiv:2020.09.03.241760 Available at: https://doi.org/10.1101/2020.09.03.241760 [Accessed April 4, 2021].

Saavedra A, Puigdellívol M, Tyebji S, Kurup P, Xu J, Ginés S, Alberch J, Lombroso PJ, PérezNavarro E (2016) BDNF Induces Striatal-Enriched Protein Tyrosine Phosphatase 61 Degradation Through the Proteasome. Mol Neurobiol 53:4261-4273.

Saika F, Matsuzaki S, Kobayashi D, Ideguchi Y, Nakamura TY, Kishioka S, Kiguchi N (2020) Chemogenetic Regulation of CX3CR1-Expressing Microglia Using Gi-DREADD Exerts Sex-Dependent Anti-Allodynic Effects in Mouse Models of Neuropathic Pain. Front Pharmacol 11:925 Available at: https://www.frontiersin.org/article/10.3389/fphar.2020.00925/full [Accessed March 28, 2021].

Salter M, Dong Y, Kalia L, ... XL-B of the N, 2009 undefined (n.d.) Regulation of NMDA receptors by kinases and phosphatases. books.google.com Available at: 
https://books.google.com/books?hl=en\&lr=\&id=MWA5ur_jswC\&oi=fnd\&pg=PA123\&dq $=$ Salter + M.W. + Dong + Y. + Kalia + L.V. + Liu + X.J. + Pitcher + G. + Regulation + of + NMDA + recep tors+by+kinases+and+phosphatases.in:+Van+Dongen+A.M. + Biology + of + the + NMDA + Re ceptor.+CRC+Press/Taylor+\%26+Francis, $\% 0 \mathrm{~A}+2009 \&$ ots=tZklYYkLyL\&sig=q8bhm3LL mTXSc0ZjQ71Xed51CIM [Accessed April 9, 2021].

Salter MW, Kalia L V. (2004) Src kinases: a hub for NMDA receptor regulation. Nat Rev Neurosci 5:317-328 Available at: http://www.nature.com/doifinder/10.1038/nrn1368 [Accessed October 22, 2017].

Sang CN, Booher S, Gilron I, Parada S, Max MB (2002) Dextromethorphan and memantine in painful diabetic neuropathy and postherpetic neuralgia: Efficacy and dose-response trials. Anesthesiology 96:1053-1061 Available at: https://pubmed.ncbi.nlm.nih.gov/11981142/ [Accessed April 4, 2021].

Sanz-Clemente A, Gray JA, Ogilvie KA, Nicoll RA, Roche KW (2013a) Activated CaMKII couples GluN2B and casein kinase 2 to control synaptic NMDA receptors. Cell Rep 3:607614 Available at: http://www.ncbi.nlm.nih.gov/pubmed/23478024 [Accessed July 13, 2019].

Sanz-Clemente A, Nicoll RA, Roche KW (2013b) Diversity in NMDA receptor composition: many regulators, many consequences. Neuroscientist 19:62-75 Available at: http://www.pubmedcentral.nih.gov/articlerender.fcgi?artid=3567917\&tool=pmcentrez\&ren dertype $=$ abstract $[$ Accessed April 14, 2016].

Sathyamurthy A, Johnson KR, Matson KJE, Dobrott CI, Li L, Ryba AR, Bergman TB, Kelly MC, Kelley MW, Levine AJ (2018) Massively Parallel Single Nucleus Transcriptional Profiling Defines Spinal Cord Neurons and Their Activity during Behavior. Cell Rep 
$22: 2216-2225$.

Schmidtko A, Lötsch J, Freynhagen R, Geisslinger G (2010) Ziconotide for treatment of severe chronic pain. Lancet 375:1569-1577.

Schou WS, Ashina S, Amin FM, Goadsby PJ, Ashina M (2017) Calcitonin gene-related peptide and pain: a systematic review. J Headache Pain 18 Available at: /pmc/articles/PMC5355411/?report=abstract [Accessed September 22, 2020].

Schüler T, Mesic I, Madry C, Bartholomäus I, Laube B (2008) Formation of NR1/NR2 and NR1/NR3 heterodimers constitutes the initial step in N-methyl-D-aspartate receptor assembly. J Biol Chem 283:37-46 Available at: http://www.ncbi.nlm.nih.gov/pubmed/17959602 [Accessed April 14, 2016].

Scott DB, Blanpied TA, Swanson GT, Zhang C, Ehlers MD (2001) An NMDA receptor ER retention signal regulated by phosphorylation and alternative splicing. J Neurosci.

Seltzer Z, Dubner R, Shir Y (1990) A novel behavioral model of neuropathic pain disorders produced in rats by partial sciatic nerve injury. Pain 43:205-218 Available at: https://pubmed.ncbi.nlm.nih.gov/1982347/ [Accessed April 1, 2021].

Semaan S, Wu J, Gan Y, Jin Y, Li GH, Kerrigan JF, Chang YC, Huang Y (2015) Hyperactivation of BDNF-TrkB signaling cascades in human hypothalamic hamartoma (HH): A potential mechanism contributing to epileptogenesis. CNS Neurosci Ther 21:164172 Available at: https://pubmed.ncbi.nlm.nih.gov/25307426/ [Accessed April 9, 2021].

Serra J, Duan WR, Locke C, Solà R, Liu W, Nothaft W (2015) Effects of a T-type calcium channel blocker, ABT-639, on spontaneous activity in C-nociceptors in patients with painful diabetic neuropathy. Pain 156:2175-2183.

Seydel C (2021) The missing sex. Nat Biotechnol Available at: 
http://www.nature.com/articles/s41587-021-00844-4 [Accessed March 9, 2021].

Shansky RM, Murphy AZ (2021) Considering sex as a biological variable will require a global shift in science culture. Nat Neurosci:1-8 Available at:

http://www.ncbi.nlm.nih.gov/pubmed/33649507 [Accessed March 9, 2021].

Shibata T, Watanabe M, Ichikawa R, Inoue Y, Koyanagi T (1999) Different expressions of $\alpha$ amino-3-hydroxy-5-methyl-4-isoxazole propionic acid and N-methyl-D-aspartate receptor subunit mRNAS between visceromotor and somatomotor neurons of the rat lumbosacral spinal cord. J Comp Neurol 404:172-182 Available at:

https://pubmed.ncbi.nlm.nih.gov/9934992/ [Accessed April 6, 2021].

Shiers S, Sankaranarayanan I, Jeevakumar V, Cervantes A, Reese JC, Price TJ (2021)

Convergence of peptidergic and non-peptidergic protein markers in the human dorsal root ganglion and spinal dorsal horn. J Comp Neurol Available at: https://pubmed.ncbi.nlm.nih.gov/33550628/ [Accessed March 5, 2021].

Short SM, Oikonomou KD, Zhou W-L, Acker CD, Popovic MA, Zecevic D, Antic SD (2017) The stochastic nature of action potential backpropagation in apical tuft dendrites. $\mathrm{J}$ Neurophysiol 118:1394-1414 Available at: www.jn.org [Accessed October 28, 2020].

Siaw K, Wee L, Chee F, Tan K, Cheong Y-P, Khanna • Sanjay, Low C-M (2016) Ontogenic Profile and Synaptic Distribution of GluN3 Proteins in the Rat Brain and Hippocampal Neurons. Neurochem Res 41.

Sikandar S, Minett MS, Millet Q, Santana-Varela S, Lau J, Wood JN, Zhao J (2018) Brainderived neurotrophic factor derived from sensory neurons plays a critical role in chronic pain. 141:1028-1039 Available at: http://www.ncbi.nlm.nih.gov/pubmed/29394316 [Accessed September 18, 2018]. 
Siman R, Baudry M, Lynch G (1984) Brain fodrin: Substrate for calpain i, an endogenous calcium-activated protease. Proc Natl Acad Sci U S A 81:3572-3576 Available at: https://pubmed.ncbi.nlm.nih.gov/6328521/ [Accessed April 9, 2021].

Skljarevski V, Ramadan NM (2002) The nociceptive flexion reflex in humans - Review article. Pain 96:3-8 Available at: https://pubmed.ncbi.nlm.nih.gov/11932055/ [Accessed March 7, 2021].

Sluka KA, Kalra A, Moore SA (2001) Unilateral intramuscular injections of acidic saline produce a bilateral, long-lasting hyperalgesia. Muscle Nerve 24:37-46 Available at: https://onlinelibrary.wiley.com/doi/10.1002/1097-4598(200101)24:1\%3C37::AIDMUS4\%3E3.0.CO;2-8 [Accessed April 1, 2021].

Sorge RE et al. (2015) Different immune cells mediate mechanical pain hypersensitivity in male and female mice. Nat Neurosci 18:1081-1083 Available at: http://www.ncbi.nlm.nih.gov/pubmed/26120961 [Accessed October 23, 2017].

South SM, Kohno T, Kaspar BK, Hegarty D, Vissel B, Drake CT, Ohata M, Jenab S, Sailer AW, Malkmus S, Masuyama T, Horner P, Bogulavsky J, Gage FH, Yaksh TL, Woolf CJ, Heinemann SF, Inturrisi CE (2003) A conditional deletion of the NR1 subunit of the NMDA receptor in adult spinal cord dorsal horn reduces NMDA currents and injuryinduced pain. J Neurosci 23:5031-5040 Available at: http://www.ncbi.nlm.nih.gov/pubmed/12832526 [Accessed May 14, 2019].

Spike RC, Puskár Z, Andrew D, Todd AJ (2003) A quantitative and morphological study of projection neurons in lamina I of the rat lumbar spinal cord. Eur J Neurosci 18:2433-2448 Available at: http://www.ncbi.nlm.nih.gov/pubmed/14622144 [Accessed July 11, 2019]. Staahl C, Drewes AM (2004) Experimental Human Pain Models: A Review of Standardised 
Methods for Preclinical Testing of Analgesics. Pharmacol Toxicol 95:97-111 Available at: http://doi.wiley.com/10.1111/j.1742-7843.2004.950301.x [Accessed October 23, 2017].

Staley KJ, Soldo BL, Proctor WR (1995) Ionic mechanisms of neuronal excitation by inhibitory GABAA receptors. Science (80- ) 269:977-981 Available at: https://pubmed.ncbi.nlm.nih.gov/7638623/ [Accessed April 9, 2021].

Stratton HJ, Khanna R (2020) Sculpting dendritic spines during initiation and maintenance of neuropathic pain. J Neurosci 40:7578-7589 Available at: https://doi.org/10.1523/JNEUROSCI.1664-20.2020 [Accessed April 3, 2021].

Streeter KA, Sunshine MD, Patel SR, Liddell SS, Denholtz LE, Reier PJ, Fuller DD, Baekey DM (2017) Coupling multielectrode array recordings with silver labeling of recording sites to study cervical spinal network connectivity. J Neurophysiol 117:1014-1029 Available at: /pmc/articles/PMC5338613/ [Accessed March 29, 2021].

Sun S, Diggins NH, Gunderson ZJ, Fehrenbacher JC, White FA, Kacena MA (2020) No pain, no gain? The effects of pain-promoting neuropeptides and neurotrophins on fracture healing. Bone 131 Available at: https://pubmed.ncbi.nlm.nih.gov/31715336/ [Accessed March 31, 2021].

Sun S, Xu Q, Guo C, Guan Y, Liu Q, Dong X (2017) Leaky Gate Model: Intensity-Dependent Coding of Pain and Itch in the Spinal Cord. Neuron 93:840-853.e5 Available at: /pmc/articles/PMC5324710/ [Accessed April 3, 2021].

Tang B, Ji Y, Traub RJ (2008) Estrogen alters spinal NMDA receptor activity via a PKA signaling pathway in a visceral pain model in the rat. Pain 137:540-549.

Taniguchi S et al. (2009) Involvement of NMDAR2A tyrosine phosphorylation in depressionrelated behaviour. EMBO J 28:3717-3729 Available at: 
http://www.ncbi.nlm.nih.gov/pubmed/19834457 [Accessed July 13, 2019].

Tanwar S, Mattoo B, Kumar U, Bhatia R (2019) Can aberrant spinal nociception be a marker of chronicity of pain in fibromyalgia syndrome? J Clin Neurosci 65:17-22.

Tao YX (2012) AMPA receptor trafficking in inflammation-induced dorsal horn central sensitization. Neurosci Bull 28:111-120 Available at:/pmc/articles/mid/NIHMS355007/ [Accessed April 3, 2021].

Tapley P, Lamballe F, Barbacid M (1992) K252a is a selective inhibitor of the tyrosine protein kinase activity of the trk family of oncogenes and neurotrophin receptors. Oncogene 7:371381 Available at: https://europepmc.org/article/med/1312698 [Accessed April 9, 2021].

Tashima R, Koga K, Sekine M, Kanehisa K, Kohro Y, Tominaga K, Matsushita K, TozakiSaitoh H, Fukazawa Y, Inoue K, Yawo H, Furue H, Tsuda M (2018) Optogenetic activation of non-nociceptive $a \beta$ fibers induces neuropathic pain-like sensory and emotional behaviors after nerve injury in rats. eNeuro 5 Available at: /pmc/articles/PMC5819669/ [Accessed March 31, 2021].

Tavares-Ferreira D, Ray PR, Sankaranarayanan I, Mejia GL, Wangzhou A, Shiers S, Uttarkar R, Megat S, Barragan-Iglesias P, Dussor G, Akopian AN, Price TJ (2020) Sex Differences in Nociceptor Translatomes Contribute to Divergent Prostaglandin Signaling in Male and Female Mice. Biol Psychiatry.

Temi S, Rudyk C, Armstrong J, Landrigan JA, Dedek C, Salmaso N, Hildebrand ME (2021) Differential expression of GluN2 NMDA receptor subunits in the dorsal horn of male and female rats. Channels (Austin) 15:179-192 Available at: http://www.ncbi.nlm.nih.gov/pubmed/33509021 [Accessed February 19, 2021]. Tingley WG, Ehlers MD, Kameyama K, Doherty C, Ptak JB, Riley CT, Huganir RL (1997) 
Characterization of Protein Kinase A and Protein Kinase C Phosphorylation of the. Mol Biol.

Todd AJ (2010) Neuronal circuitry for pain processing in the dorsal horn. Nat Rev Neurosci 11:823-836 Available at: http://www.ncbi.nlm.nih.gov/pubmed/21068766 [Accessed October 23, 2017].

Todd AJ (2015) Plasticity of inhibition in the spinal cord. Handb Exp Pharmacol 227:171-190 Available at: https://link-springercom.proxy.library.carleton.ca/content/pdf/10.1007\%2F978-3-662-46450-2_9.pdf [Accessed March 11, 2019].

Todd AJ (2017) Identifying functional populations among the interneurons in laminae I-III of the spinal dorsal horn. Mol Pain 13 Available at:/pmc/articles/PMC5315367/ [Accessed April 4, 2021].

Todorovic SM, Jevtovic-Todorovic V (2011) T-type voltage-gated calcium channels as targets for the development of novel pain therapies. Br J Pharmacol 163:484-495.

Tong C-K, MacDermott AB (2014) Synaptic GluN2A and GluN2B containing NMDA receptors within the superficial dorsal horn activated following primary afferent stimulation. $\mathrm{J}$ Neurosci 34:10808-10820 Available at: http://www.ncbi.nlm.nih.gov/pubmed/25122884 [Accessed October 22, 2017].

Torsney C (2011) Inflammatory pain unmasks heterosynaptic facilitation in lamina I neurokinin 1 receptor-expressing neurons in rat spinal cord. J Neurosci 31:5158-5168 Available at: https://www.jneurosci.org/content/31/13/5158 [Accessed April 2, 2021].

Toyoda H, Zhao MG, Zhuo M (2009) Enhanced quantal release of excitatory transmitter in anterior cingulate cortex of adult mice with chronic pain. Mol Pain 5. 
Trang T, Beggs S, Salter MW (2012) Brain-derived neurotrophic factor from microglia: A molecular substrate for neuropathic pain. Neuron Glia Biol 7:99-108 Available at: /pmc/articles/PMC3748035/ [Accessed April 8, 2021].

Trang T, Beggs S, Wan X, Salter MW (2009) P2X4-receptor-mediated synthesis and release of brain-derived neurotrophic factor in microglia is dependent on calcium and p38-mitogenactivated protein kinase activation. J Neurosci 29:3518-3528 Available at: https://www.jneurosci.org/content/29/11/3518 [Accessed March 28, 2021].

Trepanier CH, Jackson MF, MacDonald JF (2012) Regulation of NMDA receptors by the tyrosine kinase Fyn. FEBS J 279:12-19 Available at: http://www.ncbi.nlm.nih.gov/pubmed/21985328 [Accessed October 21, 2017].

Tringham E, Powell KL, Cain SM, Kuplast K, Mezeyova J, Weerapura M, Eduljee C, Jiang X, Smith P, Morrison J-L, Jones NC, Braine E, Rind G, Fee-Maki M, Parker D, Pajouhesh H, Parmar M, O'Brien TJ, Snutch TP (2012) T-type calcium channel blockers that attenuate thalamic burst firing and suppress absence seizures. Sci Transl Med 4:121ra19.

Tsubota M, Okawa Y, Irie Y, Maeda M, Ozaki T, Sekiguchi F, Ishikura H, Kawabata A (2018) Involvement of the cystathionine- $\gamma$-lyase/Cav3.2 pathway in substance P-induced bladder pain in the mouse, a model for nonulcerative bladder pain syndrome. Neuropharmacology 133:254-263.

Udakis M, Pedrosa V, Chamberlain SEL, Clopath C, Mellor JR (2020) Interneuron-specific plasticity at parvalbumin and somatostatin inhibitory synapses onto CA1 pyramidal neurons shapes hippocampal output. Nat Commun.

Ulmann L, Hatcher JP, Hughes JP, Chaumont S, Green PJ, Conquet F, Buell GN, Reeve AJ, Chessell IP, Rassendren F (2008) Up-Regulation of P2X4 Receptors in Spinal Microglia 
after Peripheral Nerve Injury Mediates BDNF Release and Neuropathic Pain. J Neurosci 28:11263-11268 Available at: http://www.ncbi.nlm.nih.gov/pubmed/18971468 [Accessed October 21, 2017].

Valjent E, Pascoli V, Svenningsson P, Paul S, Enslen H, Corvol JC, Stipanovich A, Caboche J, Lombroso PJ, Nairn AC, Greengard P, Hervé D, Girault JA (2005) Regulation of a protein phosphatase cascade allows convergent dopamine and glutamate signals to activate ERK in the striatum. Proc Natl Acad Sci U S A 102:491-496 Available at: https://pubmed.ncbi.nlm.nih.gov/15608059/ [Accessed April 3, 2021].

Vargas-Perez H, Bahi A, Bufalino MR, Ting-A-Kee R, Maal-Bared G, Lam J, Fahmy A, Clarke L, Blanchard JK, Larsen BR, Steffensen S, Dreyer JL, van der Kooy D (2014) BDNF signaling in the VTA links the drug-dependent state to drug withdrawal aversions. J Neurosci 34:7899-7909 Available at: https://pubmed.ncbi.nlm.nih.gov/24899712/ [Accessed April 9, 2021].

Vicini S, Wang JF, Li JH, Zhu WJ, Wang YH, Luo JH, Wolfe BB, Grayson DR (1998) Functional and Pharmacological Differences Between Recombinant $N$-Methyl- d Aspartate Receptors. J Neurophysiol 79:555-566 Available at: http://www.physiology.org/doi/10.1152/jn.1998.79.2.555 [Accessed May 13, 2019]. Vilim FS (1999) Gene for Pain Modulatory Neuropeptide NPFF: Induction in Spinal Cord by Noxious Stimuli. Available at: http://www.molpharm.org [Accessed October 23, 2017]. Vinegar R, Schreiber W, Hugo R (1969) Biphasic development of carrageenan edema in rats. J Pharmacol Exp Ther 166.

Wallace M, Duan R, Liu W, Locke C, Nothaft W (2016) A Randomized, Double-Blind, PlaceboControlled, Crossover Study of the T-Type Calcium Channel Blocker ABT-639 in an 
Intradermal Capsaicin Experimental Pain Model in Healthy Adults. Pain Med 17:551-560.

Wang G, Erpelding N, Davis KD (2014a) Sex differences in connectivity of the subgenual anterior cingulate cortex. Pain 155:755-763.

Wang JQ, Guo ML, Jin DZ, Xue B, Fibuch EE, Mao LM (2014b) Roles of subunit phosphorylation in regulating glutamate receptor function. Eur J Pharmacol.

Wang L-N, Yang J-P, Ji F, Zhan Y, Jin X, Xu Q-N, Wang X-Y, Zuo J-L (2012) Brain-derived neurotrophic factor modulates N-methyl-D-aspartate receptor activation in a rat model of cancer-induced bone pain. J Neurosci Res 90:1249-1260.

Wang M, Yang Y, Wang C-J, Gamo NJJ, Jin LEE, Mazer JAA, Morrison JHH, Wang X-J, Arnsten AFTFT (2013) The GluN3A subunit exerts a neuroprotective effect in brain ischemia and the hypoxia process. Neuron 77:736-749 Available at: https://www.sciencedirect.com/science/article/pii/S089662731300038X [Accessed January 30, 2019].

Wang X, Ratnam J, Zou B, England PM, Basbaum AI (2009) TrkB signaling is required for both the induction and maintenance of tissue and nerve injury-induced persistent pain. J Neurosci 29:5508-5515 Available at: https://pubmed.ncbi.nlm.nih.gov/19403818/ [Accessed April 8, 2021].

Wang XT, Zheng R, Suo ZW, Liu YN, Zhang ZY, Ma ZA, Xue Y, Xue M, Yang X, Hu XD (2016) Activity-dependent dephosphorylation of paxillin contributed to nociceptive plasticity in spinal cord dorsal horn. Pain 157:652-665 Available at: https://pubmed.ncbi.nlm.nih.gov/26882347/ [Accessed April 9, 2021].

Wang YT, Salter MW (1994) Regulation of NMDA receptors by tyrosine kinases and phosphatases. Nature 369:233-235 Available at: http://www.nature.com/articles/369233a0 
[Accessed November 17, 2017].

Warwick CA, Cassidy C, Hachisuka J, Wright MC, Baumbauer KM, Adelman PC, Lee KH, Smith KM, Ross SE, Richard Koerber H (2020) Optogenetic evaluation of the ability of different cutaneous $\mathrm{C}$-fiber afferents to evoke aversive behaviors.

bioRxiv:2020.09.17.296384 Available at: https://doi.org/10.1101/2020.09.17.296384 [Accessed March 31, 2021].

Watanabe M, Ueda T, Shibata Y, Kumamoto N, Shimada S, Ugawa S (2015) Expression and regulation of Cav3.2 T-Type calcium channels during inflammatory hyperalgesia in mouse dorsal root ganglion neurons. PLoS One 10.

Waxman SG, Zamponi GW (2014) Regulating excitability of peripheral afferents: emerging ion channel targets. Nat Neurosci 17:153-163 Available at: http://www.nature.com/articles/nn.3602 [Accessed March 18, 2019].

Wee KSL, Zhang Y, Khanna S, Low CM (2008) Immunolocalization of NMDA receptor subunit NR3B in selected structures in the rat forebrain, cerebellum, and lumbar spinal cord. J Comp Neurol 509:118-135 Available at: https://pubmed.ncbi.nlm.nih.gov/18425811/ [Accessed March 29, 2021].

Wei F, Vadakkan KI, Toyoda H, Wu LJ, Zhao MG, Xu H, Shum FWF, Yong HJ, Zhuo M (2006) Calcium calmodulin-stimulated adenylyl cyclases contribute to activation of extracellular signal-regulated kinase in spinal dorsal horn neurons in adult rats and mice. $\mathrm{J}$ Neurosci 26:851-861.

Weir GA, Middleton SJ, Clark AJ, Daniel T, Khovanov N, McMahon SB, Bennett DL (2017) Using an engineered glutamate-gated chloride channel to silence sensory neurons and treat neuropathic pain at the source. Brain 140:2570-2585 Available at: 
https://academic.oup.com/brain/article/140/10/2570/4085760 [Accessed March 28, 2021].

Weiss N, Zamponi GW (2019) T-type calcium channels: From molecule to therapeutic opportunities. Int J Biochem Cell Biol 108:34-39.

Wen X-J, Xu S-Y, Chen Z-X, Yang C-X, Liang H, Li H (2010) The Roles of T-Type Calcium Channel in the Development of Neuropathic Pain following Chronic Compression of Rat Dorsal Root Ganglia. Pharmacology 85:295-300 Available at: https://www.karger.com/Article/FullText/276981 [Accessed May 10, 2020].

Wesseling JF, Pérez-Otaño I (2015) Modulation of GluN3A expression in Huntington disease: a new n-methyl-D-aspartate receptor-based therapeutic approach? JAMA Neurol 72:468-473 Available at: http://www.ncbi.nlm.nih.gov/pubmed/25686081 [Accessed April 15, 2016].

West SJ, Bannister K, Dickenson AH, Bennett DL (2015) Circuitry and plasticity of the dorsal horn - Toward a better understanding of neuropathic pain. Neuroscience 300:254-275 Available at: https://www.sciencedirect.com/science/article/pii/S0306452215004522?via\%3Dihub [Accessed February 27, 2019].

Williams JR, Sharp JW, Kumari VG, Wilson M, Payne JA (1999) The neuron-specific K-Cl cotransporter, KCC2. Antibody development and initial characterization of the protein. J Biol Chem 274:12656-12664 Available at: http://www.ncbi.nlm.nih.gov/pubmed/10212246 [Accessed July 6, 2018].

Won S, Roche KW (2021) Regulation of glutamate receptors by striatal-enriched tyrosine phosphatase 61 (STEP61). J Physiol 599:443-451 Available at: https://pubmed.ncbi.nlm.nih.gov/32170729/ [Accessed April 3, 2021].

Woolf CJ (1983) Evidence for a central component of post-injury pain hypersensitivity. Nature 
306:686-688 Available at: http://www.ncbi.nlm.nih.gov/pubmed/6656869 [Accessed May 13, 2019].

Woolf CJ (2011) Central sensitization: implications for the diagnosis and treatment of pain. Pain 152:S2-15 Available at: http://www.ncbi.nlm.nih.gov/pubmed/20961685 [Accessed May 13, 2019].

Woolf CJ, Mannion RJ (1999) Neuropathic pain: aetiology, symptoms, mechanisms, and management. Lancet 353:1959-1964 Available at: http://www.ncbi.nlm.nih.gov/pubmed/10371588 [Accessed October 21, 2017].

Woolf CJ, Salter MW (2000) Neuronal plasticity: increasing the gain in pain. Science 288:17651769 Available at: http://www.ncbi.nlm.nih.gov/pubmed/10846153 [Accessed March 18, 2019].

Wu F, Pan R, Chen J, Sugita M, Chen C, Tao Y, Yu W, Sun Y (2014) Lentivirus Mediated siRNA against GluN2B Subunit of NMDA Receptor Reduces Nociception in a Rat Model of Neuropathic Pain. Biomed Res Int 2014.

Wu J, Peng S, Xiao L, Cheng X, Kuang H, Zhu M, Zhang D, Jiang C, Liu T (2018) Cell-Type Specific Distribution of T-Type Calcium Currents in Lamina II Neurons of the Rat Spinal Cord. Front Cell Neurosci 12:370.

Wú Lin JQ, Lú Willis YW, Westlund K (1998) Changes in nitric oxide synthase isoforms in the spinal cord of rat following induction of chronic arthritis. Springer-Verlag.

Xie J-D, Chen S-R, Chen H, Zeng W-A, Pan H-L (2016) Presynaptic N-Methyl-d-aspartate (NMDA) Receptor Activity Is Increased Through Protein Kinase C in Paclitaxel-induced Neuropathic Pain. J Biol Chem 291:19364-19373 Available at: http://www.ncbi.nlm.nih.gov/pubmed/27458019 [Accessed March 18, 2019]. 
Xu J et al. (2014) Inhibitor of the Tyrosine Phosphatase STEP Reverses Cognitive Deficits in a Mouse Model of Alzheimer's Disease Khosla C, ed. PLoS Biol 12:e1001923 Available at: http://dx.plos.org/10.1371/journal.pbio.1001923 [Accessed November 17, 2017].

Xu J, Kurup P, Azkona G, Baguley TD, Saavedra A, Nairn AC, Ellman JA, Pérez-Navarro E, Lombroso PJ (2016) Down-regulation of BDNF in cell and animal models increases striatal-enriched protein tyrosine phosphatase 61 (STEP61 ) levels. J Neurochem 136:285294 Available at: http://www.ncbi.nlm.nih.gov/pubmed/26316048 [Accessed March 14, 2018].

Xu J, Kurup P, Baguley TD, Foscue E, Ellman JA, Nairn AC, Lombroso PJ (n.d.) Supplementary Material Inhibition of the tyrosine phosphatase STEP 61 restores BDNF expression and reverses motor and cognitive deficits in phencyclidine treated mice. Available at: https://static-content-springercom.proxy.library.carleton.ca/esm/art\%3A10.1007\%2Fs00018-015-20571/MediaObjects/18_2015_2057_MOESM1_ESM.pdf [Accessed January 30, 2019].

Xu J, Kurup P, Foscue E, Lombroso PJ (2015a) Striatal-enriched protein tyrosine phosphatase regulates the PTP $\alpha /$ Fyn signaling pathway. J Neurochem 134:629-641 Available at: http://doi.wiley.com/10.1111/jnc.13160 [Accessed October 23, 2017].

Xu J, Kurup P, Zhang Y, Goebel-Goody SM, Wu PH, Hawasli AH, Baum ML, Bibb JA, Lombroso PJ (2009) Extrasynaptic NMDA Receptors Couple Preferentially to Excitotoxicity via Calpain-Mediated Cleavage of STEP. J Neurosci 29:9330-9343 Available at: http://www.ncbi.nlm.nih.gov/pubmed/19625523 [Accessed October 21, 2017]. Xu Q, Yaksh TL (2011) A brief comparison of the pathophysiology of inflammatory versus neuropathic pain. Curr Opin Anaesthesiol 24:400-407 Available at: 
/pmc/articles/PMC3290396/ [Accessed March 3, 2021].

Xu YM, Wang XT, Zhang ZY, Suo ZW, Yang X, Hu XD (2015b) Noradrenergic ??2 receptor attenuated inflammatory pain through STEP61/ERK signalling. Eur J Pain (United Kingdom) 19:1298-1307.

Yamamoto T, Yaksh TL (1992a) Studies on the spinal interaction of morphine and the NMDA antagonist MK-801 on the hyperesthesia observed in a rat model of sciatic mononeuropathy. Neurosci Lett 135:67-70 Available at: http://www.ncbi.nlm.nih.gov/pubmed/1311824 [Accessed May 14, 2019].

Yamamoto T, Yaksh TL (1992b) Comparison of the antinociceptive effects of pre- and posttreatment with intrathecal morphine and MK801, an NMDA antagonist, on the formalin test in the rat. Anesthesiology 77:757-763 Available at: http://www.ncbi.nlm.nih.gov/pubmed/1416174 [Accessed May 14, 2019].

Yan X, Jiang E, Gao M, Weng H-R (2013) Endogenous activation of presynaptic NMDA receptors enhances glutamate release from the primary afferents in the spinal dorsal horn in a rat model of neuropathic pain. J Physiol 591:2001-2019 Available at: http://www.ncbi.nlm.nih.gov/pubmed/23359671 [Accessed March 18, 2019].

Yang H-B, Yang X, Cao J, Li S, Liu Y-N, Suo Z-W, Cui H-B, Guo Z, Hu X-D (2011) cAMPdependent protein kinase activated Fyn in spinal dorsal horn to regulate NMDA receptor function during inflammatory pain. J Neurochem 116:93-104 Available at: http://doi.wiley.com/10.1111/j.1471-4159.2010.07088.x [Accessed April 2, 2021].

Yang K, Trepanier C, Sidhu B, Xie Y-F, Li H, Lei G, Salter MW, Orser BA, Nakazawa T, Yamamoto T, Jackson MF, MacDonald JF (2012) Metaplasticity gated through differential regulation of GluN2A versus GluN2B receptors by Src family kinases. EMBO J 31:805- 
816 Available at: http://emboj.embopress.org/cgi/doi/10.1038/emboj.2011.453 [Accessed April 3, 2021].

Yang M, Leonard JP (2001) Identification of mouse NMDA receptor subunit NR2A C-terminal tyrosine sites phosphorylated by coexpression with v-Src. J Neurochem 77:580-588 Available at: http://www.ncbi.nlm.nih.gov/pubmed/11299320 [Accessed July 13, 2019].

Yao Y, Belcher J, Berger AJ, Mayer ML, Lau AY (2013) Conformational analysis of NMDA receptor GluN1, GluN2, and GluN3 ligand-binding domains reveals subtype-specific characteristics. Structure 21:1788-1799 Available at: http://www.pubmedcentral.nih.gov/articlerender.fcgi?artid=3814224\&tool=pmcentrez\&ren dertype $=$ abstract $[$ Accessed April 14, 2016].

Yasaka T, Tiong SYX, Hughes DI, Riddell JS, Todd AJ (2010) Populations of inhibitory and excitatory interneurons in lamina II of the adult rat spinal dorsal horn revealed by a combined electrophysiological and anatomical approach. Pain 151:475-488 Available at: https://pubmed.ncbi.nlm.nih.gov/20817353/ [Accessed April 4, 2021].

Yashiro K, Philpot BD (2008) Regulation of NMDA receptor subunit expression and its implications for LTD, LTP, and metaplasticity. Neuropharmacology 55:1081-1094 Available at: /pmc/articles/PMC2590778/ [Accessed April 3, 2021].

Yekkirala AS, Roberson DP, Bean BP, Woolf CJ (2017) Breaking barriers to novel analgesic drug development. Nat Rev Drug Discov 16:545-564.

Yi MH, Liu YU, Liu K, Chen T, Bosco DB, Zheng J, Xie M, Zhou L, Qu W, Wu LJ (2020) Chemogenetic manipulation of microglia inhibits neuroinflammation and neuropathic pain in mice. Brain Behav Immun 92:78-89.

Yuan T, Mameli M, O’Connor EC, O’ Connor EC, Dey PN, Verpelli C, Sala C, Perez-Otano I, 
Lüscher C, Bellone C (2013) Expression of cocaine-evoked synaptic plasticity by GluN3Acontaining NMDA receptors. Neuron 80:1025-1038 Available at:

http://www.ncbi.nlm.nih.gov/pubmed/24183704 [Accessed April 14, 2016].

Yung KKL (1998) Localization of glutamate receptors in dorsal horn of rat spinal cord.

Neuroreport 9:1639-1644 Available at: https://pubmed.ncbi.nlm.nih.gov/9631479/

[Accessed April 6, 2021].

Zhang H, Xie W, Xie Y (2005) Spinal cord injury triggers sensitization of wide dynamic range dorsal horn neurons in segments rostral to the injury. Brain Res 1055:103-110.

Zhang RX, Ren K (2011) Animal models of inflammatory pain. Neuromethods 49:23-40 Available at: https://link.springer.com/protocol/10.1007/978-1-60761-880-5_2 [Accessed April 1, 2021].

Zhang SB, Lin SY, Liu M, Liu CC, Ding HH, Sun Y, Ma C, Guo RX, Lv YY, Wu SL, Xu T, Xin WJ (2019a) CircAnks 1a in the spinal cord regulates hypersensitivity in a rodent model of neuropathic pain. Nat Commun 10:1-16 Available at: https://doi.org/10.1038/s41467019-12049-0 [Accessed April 3, 2021].

Zhang W, Liu LY, Xu TL (2008) Reduced potassium-chloride co-transporter expression in spinal cord dorsal horn neurons contributes to inflammatory pain hypersensitivity in rats. Neuroscience 152:502-510.

Zhang X, Hartung JE, Friedman RL, Koerber HR, Belfer I, Gold MS (2019b) Nicotine Evoked Currents in Human Primary Sensory Neurons. J Pain 20:810-818.

Zhang X, Priest BT, Belfer I, Gold MS (2017) Voltage-gated Na+ currents in human dorsal root ganglion neurons. Elife 6 .

Zhang XL, Lee KY, Priest BT, Belfer I, Gold MS (2015) Inflammatory mediator-induced 
modulation of GABAA currents in human sensory neurons. Neuroscience 310:401-409 Available at: https://pubmed.ncbi.nlm.nih.gov/26415765/ [Accessed March 20, 2021].

Zhang Y, Liu S, Zhang YQ, Goulding M, Wang YQ, Ma Q (2018) Timing Mechanisms Underlying Gate Control by Feedforward Inhibition. Neuron 99:941-955.e4.

Zhao J, Seereeram A, Nassar MA, Levato A, Pezet S, Hathaway G, Morenilla-Palao C, Stirling C, Fitzgerald M, McMahon SB, Rios M, Wood JN (2006) Nociceptor-derived brain-derived neurotrophic factor regulates acute and inflammatory but not neuropathic pain. Mol Cell Neurosci 31:539-548.

Zhao Y, Huang G, Wu Q, Wu K, Li R, Lei J, Pan X, Yan N (2019) Cryo-EM structures of apo and antagonist-bound human Cav3.1. Nature 576:492-497.

Zhong Y, Zhou L-J, Ren W-J, Xin W-J, Li Y-Y, Zhang T, Liu X-G (2010) The direction of synaptic plasticity mediated by C-fibers in spinal dorsal horn is decided by Src-family kinases in microglia: The role of tumor necrosis factor- $\alpha$. Brain Behav Immun 24:874-880 Available at: http://www.ncbi.nlm.nih.gov/pubmed/20116424 [Accessed March 18, 2019].

Zhou H-Y, Chen S-R, Byun H-S, Chen H, Li L, Han H-D, Lopez-Berestein G, Sood AK, Pan HL (2012) N-methyl-D-aspartate receptor- and calpain-mediated proteolytic cleavage of K+Cl- cotransporter-2 impairs spinal chloride homeostasis in neuropathic pain. J Biol Chem 287:33853-33864 Available at: http://www.ncbi.nlm.nih.gov/pubmed/22854961 [Accessed January 30, 2019].

Zhou HY, Chen SR, Pan HL (2011) Targeting N-methyl-D-aspartate receptors for treatment of neuropathic pain. Expert Rev Clin Pharmacol 4:379-388.

Zhou XL, Zhang CJ, Peng YN, Wang Y, Xu HJ, Liu CM (2019) ROR2 modulates neuropathic pain via phosphorylation of NMDA receptor subunit GluN2B in rats. Br J Anaesth 
123:e239-e248.

Zhu Z, Yi F, Epplin MP, Liu D, Summer SL, Mizu R, Shaulsky G, XiangWei W, Tang W, Burger PB, Menaldino DS, Myers SJ, Liotta DC, Hansen KB, Yuan H, Traynelis SF (2020) Negative allosteric modulation of GluN1/GluN3 NMDA receptors. Neuropharmacology 176:108117.

Zoli M, Jansson A, Syková E, Agnati LF, Fuxe K (1999) Volume transmission in the CNS and its relevance for neuropsychopharmacology. Trends Pharmacol Sci 20:142-150 Available at: http://www.ncbi.nlm.nih.gov/pubmed/10322499 [Accessed July 11, 2019]. 